\title{
Cellular Targets and Tumour Suppressive Mechanisms of Peloruside A
}

\author{
By \\ Ariane Chan
}

A thesis submitted to the Victoria University of Wellington in fulfilment of the requirements for the degree of Doctor of Philosophy

Victoria University of Wellington 2012 


\begin{abstract}
Peloruside A (PelA) is a novel secondary metabolite isolated from the New Zealand marine sponge Mycale hentscheli. It is a potent microtubule-stabilizer and binds to a distinct site on $\beta$-tubulin compared to the widely used anti-cancer drug paclitaxel. PelA has clear potential benefits over paclitaxel, including increased solubility, reduced sensitivity to multiple drug resistance in cancer cell lines, and improved tolerability and efficacy in non-small cell lung cancer xenografts in mice. Using several established cancer cell lines, we investigated potential tumour suppressive effects of PelA.
\end{abstract}

1A9 human ovarian carcinoma cells treated with PelA were screened using several protease inhibitors to determine whether these inhibitors could protect against the induction of apoptosis. The greatest protection was conferred by the pan-caspase inhibitor zVAD-fmk, and subsequent biochemical assays suggested that caspases-8, 9 and $-3 / 7$ were activated after prolonged treatment with PelA $(>24 \mathrm{~h})$. These results indicate a predominant role for caspases in PelA-induced cell death. Additionally, decreased protein expression levels of stathmin and c-Myc, two proteins that have previously been shown to influence sensitivity to microtubule-targeting agents (MTAs), were observed after treatment of HL-60 cells with PelA, suggesting that they may also mediate some of PelA's cytotoxic effects.

Flow cytometric analysis of 1A9 human ovarian carcinoma cells demonstrated that low concentrations of PelA could induce chromosome mis-segregation in up to $14 \%$ of cells. Cells treated with $40 \mathrm{nM}$ PelA displayed a delayed mitotic entry (by $\sim 1 \mathrm{~h}$ ), but an otherwise fairly normal cell cycle progression profile. Western blot analyses of spindle activation checkpoint proteins found no change in expression levels of MAD2, BUBR1 or p55CDC at this drug concentration. In response to $100 \mathrm{nM}$ PelA, cyclin B1 expression levels remained elevated, corresponding with the mitotic arrest that occurred at this concentration. The cell cycle kinetics of MAD2 and BUBR1 dissociation from p55CDC was investigated by co-immunoprecipitation. Despite a concentration-dependent increase in MAD2/p55CDC association by PelA, at $40 \mathrm{nM}$ PelA the timing of MAD2/p55CDC dissociation was similar to that of controls. This 
contrasted with sustained MAD2/p55CDC complexes at $100 \mathrm{nM}$ PelA. Changes in the levels of BUBR1 associated with p55CDC were observed in response to $40 \mathrm{nM}$ and $100 \mathrm{nM}$ PelA compared to controls. Dissociation of BUBR1/p55CDC complexes still occurred in the presence of $40 \mathrm{nM}$ PelA, which contrasted with the sustained presence of BUBR1/p55CDC complexes at $100 \mathrm{nM}$ PelA. These results suggested that at low concentrations of PelA, the spindle activation checkpoint is being silenced (or bypassed) despite the presence of erroneous microtubule-kinetochore attachments.

Studies using the MCF7 human breast cancer cell line indicated that in addition to apoptosis, PelA induced cells to adopt a morphological and biochemical phenotype that is indicative of premature senescence (a permanent cell cycle arrest). The induction of premature senescence involved activation of the $\mathrm{p} 53$ and $\mathrm{pRb}$ tumour suppressor pathways, and also correlated with reduced clonogenicity.

PelA was also screened for anti-angiogenic activity by assessing its ability to inhibit crucial endothelial cell functions. PelA potently inhibited human umbilical vein endothelial cell proliferation and migration, and also affected the number and quality of 3-dimensional capillary-like structures that could form on Matrigel ${ }^{\mathrm{TM}}$, a basement membrane matrix.

Despite the acquired resistance and undesirable side effects (e.g. allergic reactions, neutropenia and neuropathy) associated with the use of MTAs in the clinic, tubulin still represents one of the most successful drug targets for cancer; thus, there is an ongoing need for new MTAs with improved therapeutic profiles. The work that we have presented here highlights the fact that PelA can induce multiple cell fates, some of which are potentially tumour suppressive. Additionally, the findings in this study further support its development as a candidate anti-cancer chemotherapeutic. 


\section{Acknowledgements}

Firstly, I would like to thank my primary supervisor, Prof. John Miller, not only for his invaluable support and guidance throughout my $\mathrm{PhD}$, but also for his endless humour. I could not have asked for a better supervisor. I must also acknowledge my secondary supervisor, Assoc. Prof. Paul Teesdale-Spittle, for much appreciated advice and feedback. I am also very grateful for the financial support of the Genesis Oncology Trust who provided me with a postrgraduate scholarship during my $\mathrm{PhD}$, as well as funding to support conference travel.

This research would not have been possible without the infallible support, encouragement, and advice from many people, of whom there are far too many to all name individually. To the following people I offer my most heartfelt thanks:

My fellow JHM lab members (both past and present), in particular Arun Kanakkanthara, Viswanath Das, Jessica Field, Anja Wilmes, and Dora Leahy, who have intimately shared in the many challenges and successes over the past few years I am forever grateful.

My talented colleagues in SBS, for sharing their knowledge and offering much needed support, encouragement and discussions. A special thank you to Bridget Simonson, David O’Sullivan, Marie Kharkrang, Kirsty Danielson, Peter Bosch, Magalie Boucher, James Matthews, Ryan Steel and to the wider LAF, Chem Gen and Proteomics groups. I have cherished the many friendships that I have made, as long or transient as they may be.

The wonderful team of academics, technicians and support staff, in particular: Assoc. Prof. Peter Northcote and Jonathan Singh, for ample supplies of peloruside A; Sushila Pillai, Lee Botes (Olympus) and Ray Gilbert (Auckland University) for their assistance with confocal; and Craig Doney, Neville Higgison, and Cameron Jack for going out of their way to fix anything and everything that broke along the way.

My family and friends - Sally, Jyoti, Ruth, Kim S., Kim R., Laura, Lisa, and Maz - I am lucky to know such amazing people. Finally, I owe my deepest gratitude to Patrick Tuapola, for his loving support and incredible patience during this epic journey. 
For Claire Wilson and Yoshimi Hayashi

With all its sham, drudgery, and broken dreams,

it is still a beautiful world.

- Max Ehrmann 


\section{Table of Contents}

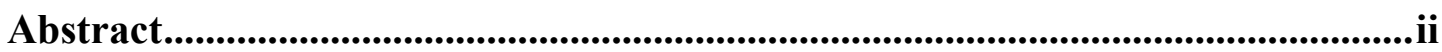

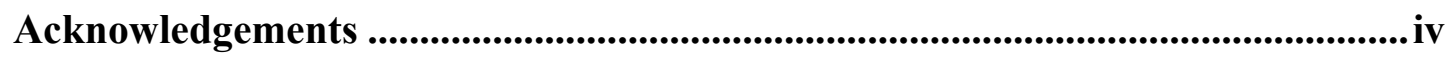

Table of Contents .................................................................................................. vi

List of Figures....................................................................................................................................xiii

List of Tables ..............................................................................................................

List of Equations ........................................................................................................

List of major abbreviations........................................................................................

Chapter 1. General Introduction .......................................................... 1

1.1 Anti-cancer drug development ................................................................... 1

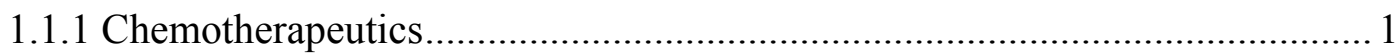

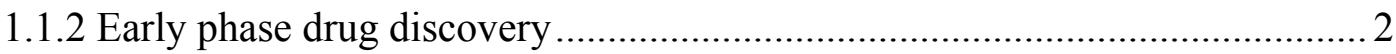

1.2 Structure and function of microtubules.........................................................6

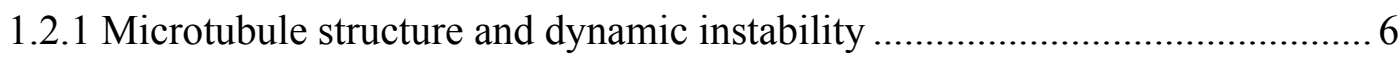

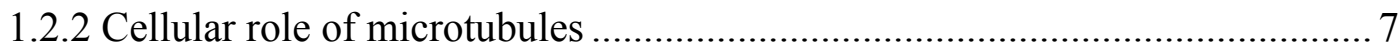

1.3 Regulation of microtubules and their dynamics ................................................8

1.3.1 Tubulin isotypes and post-translational modifications ................................ 8

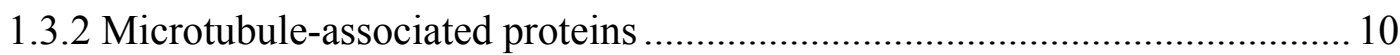

1.4 Targeting mitosis as a chemotherapeutic strategy.......................................11

1.4.1 Regulating cell cycle and mitotic progression ......................................... 11

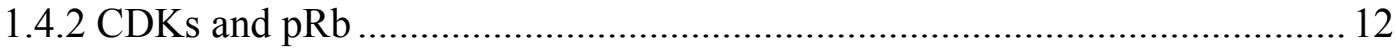

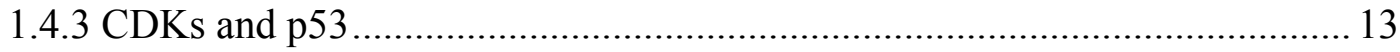

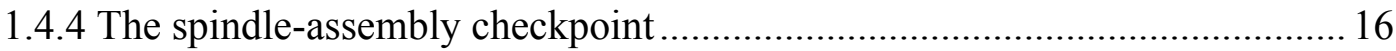

1.5 Microtubule-targeting agents .................................................................17

1.6 Tumour suppressive mechanisms of microtubule-targeting agents................23

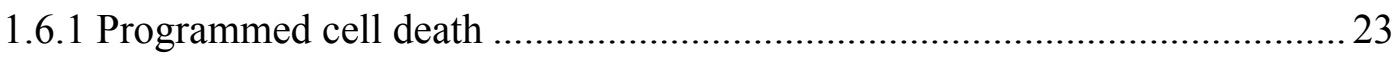

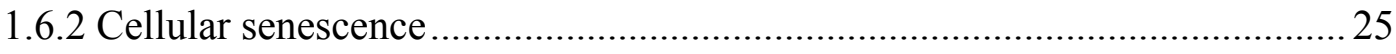

1.6.3 Tumour-induced angiogenesis and anti-angiogenic drugs ..........................2 28

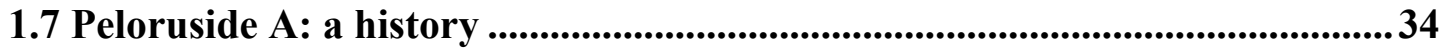

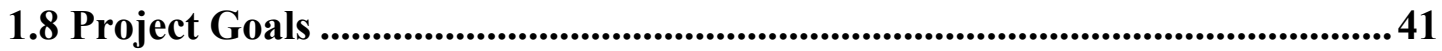

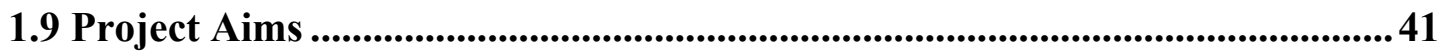


Chapter 2. General Methods

2.1 Chemical compounds.............................................................................................44

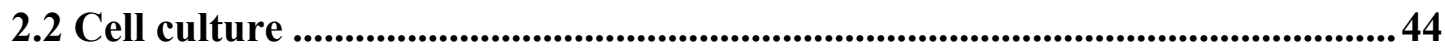

2.3 MTT cell proliferation assay ....................................................................45

2.4 Cell cycle analysis by flow cytometry .........................................................46

2.5 Western blotting...............................................................................................47

2.6 Staining protein gels with Coomassie blue .......................................................48

\section{Chapter 3. Mechanisms underlying the induction of apoptosis by}

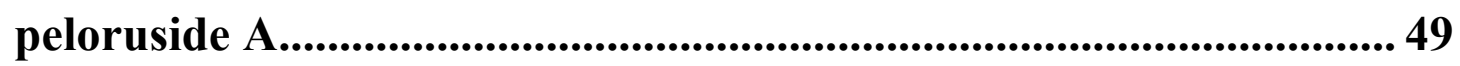

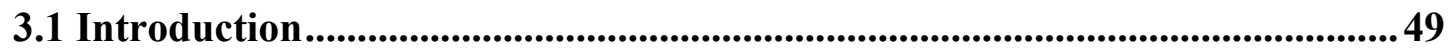

3.1.1 The extrinsic (death receptor) and intrinsic (mitochondrial) pathways ......... 49

3.1.2 The link between microtubules and apoptosis ............................................. 51

3.1.3 Apoptosis induced by secondary metabolites of Mycale hentscheli..............54

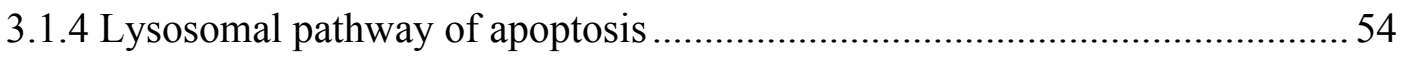

3.1.5 Caspase-independent cell death by MSAs ...................................................56

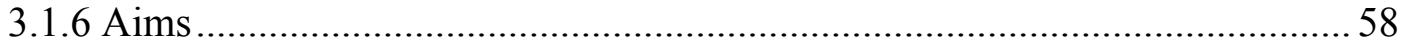

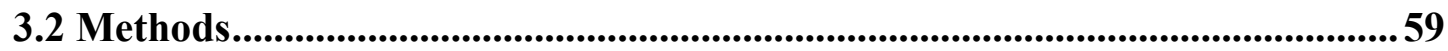

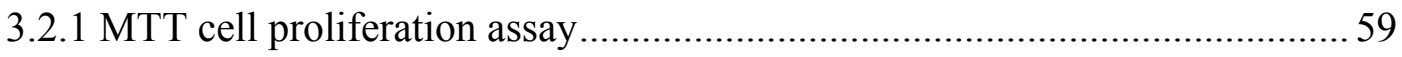

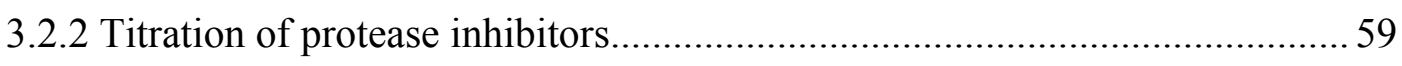

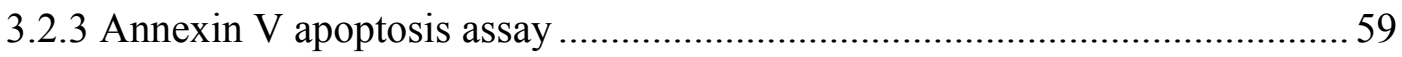

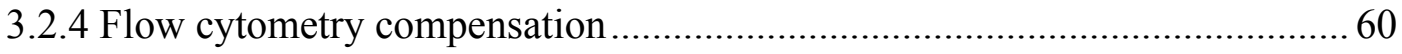

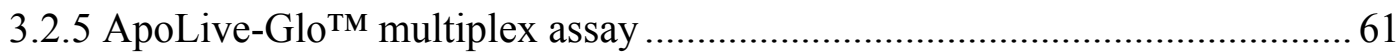

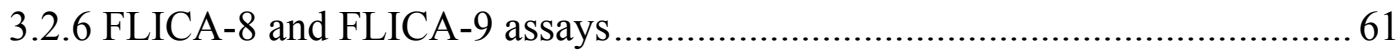

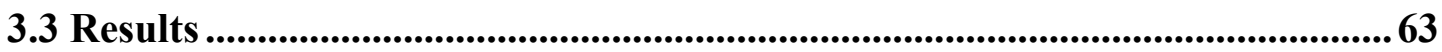

3.3.1 MTT assays to titrate the effects of different protease inhibitors .................63

3.3.2 Protective effects of different protease inhibitors on PelA-, MycA- or

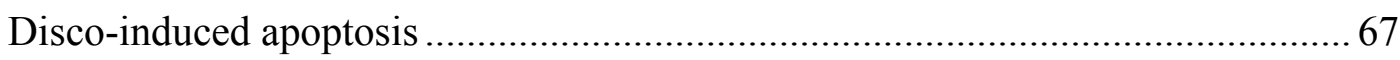

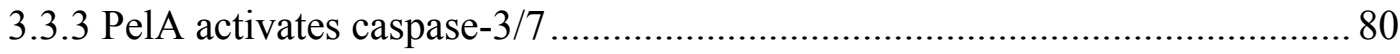

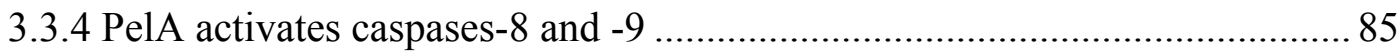

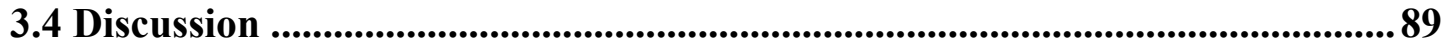

3.4.1 Protective effects of a panel of protease inhibitors against PelA, MycA and

Disco 
3.4.2 Activation of caspase-3/7 by PelA ............................................................ 92

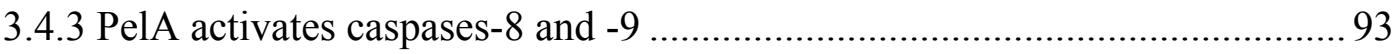

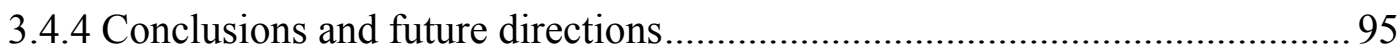

\section{Chapter 4. Chromosome mis-segregation induced by low}

concentrations of peloruside $A$................................................................99

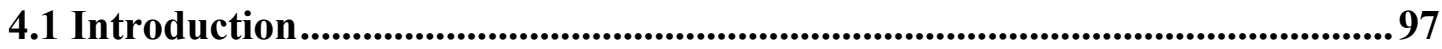

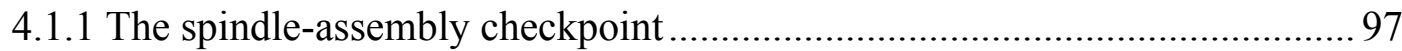

4.1.2 Function of MAD2 and BUBR1 in the MCC …...........................................98

4.1.3 Erroneous microtubule-kinetochore attachments that are not detected by the $\mathrm{SAC}$ 100

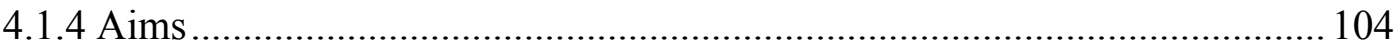

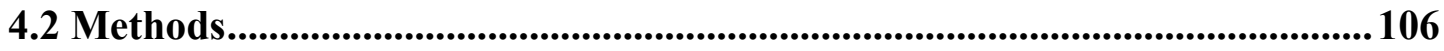

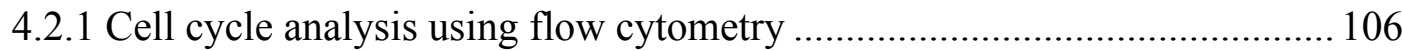

4.2.2 Western blot analysis of cell cycle proteins............................................. 107

4.2.3 Harvesting cells for co-immunoprecipitation ........................................... 107

4.2.4 Crosslinking of protein G Dynabeads ${ }^{\circledR}$ to anti-p55CDC antibody ............. 108

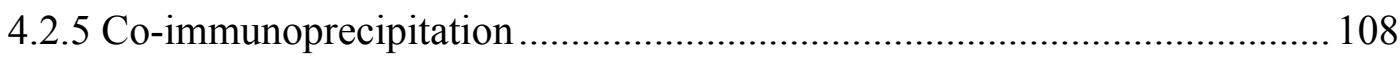

4.2.6 Immunostaining to detect possible merotelic attachments ........................ 109

4.2.7 Confocal microscopy and image acquisition ........................................... 110

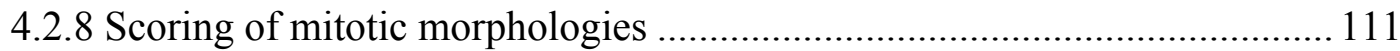

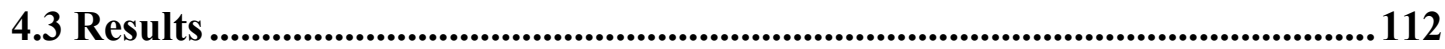

4.3.1 Induction of aneuploidy by PelA in unsynchronized 1A9 cells .................. 112

4.3.2 Induction of aneuploidy at low concentrations of PelA in synchronized

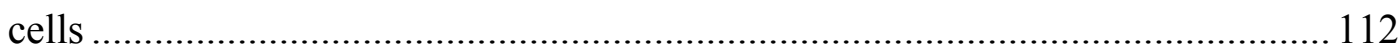

4.3.3 Differences in cell cycle protein expression in response to PelA ................ 116

4.3.4 Cell cycle kinetics of MAD2 and BUBR1 association with p55CDC........ 116

4.3.5 PelA treatment does not increase the frequency of lagging chromosomes but does impair chromosome alignment ............................................................... 123

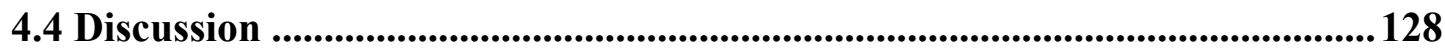

4.4.1 PelA induces aneuploidy at low concentrations in the absence of mitotic arrest. 
4.4.2 PelA does not affect the expression of proteins involved in the SAC, but high concentrations lead to prolonged, elevated cyclin B1 expression levels...... 128 4.4.3 MAD2 and BUBR1 dissociation from $\mathrm{p} 55 \mathrm{CDC}$ differs at low versus high concentrations of PelA

4.4.4 Increased frequency of pseudo-metaphase cells as a possible mechanism underlying the induction of aneuploidy at low PelA concentrations.

4.4.5 Conclusions and future directions

\section{Chapter 5. Potential anti-angiogenic activity of peloruside A.......... 135}

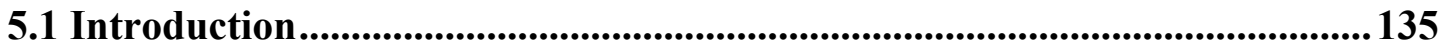

5.1.1 Cytoskeletal involvement in cell migration ............................................. 135

5.1.2 In vitro assays to screen for anti-angiogenic compounds .......................... 137

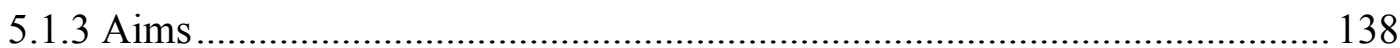

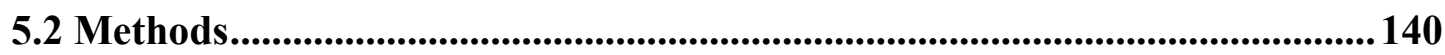

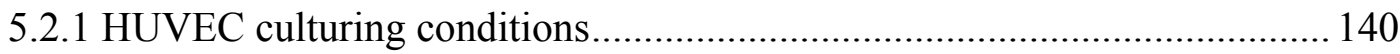

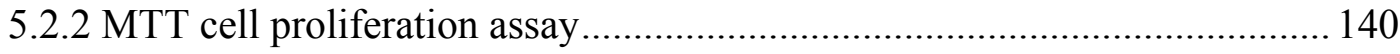

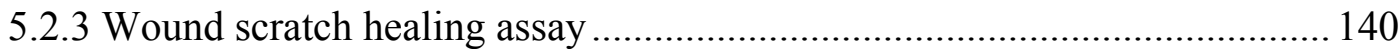

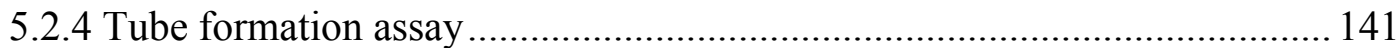

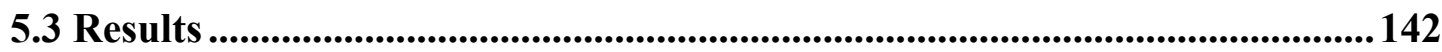

5.3.1 Inhibition of EC proliferation by the microtubule-stabilizing drugs PelA

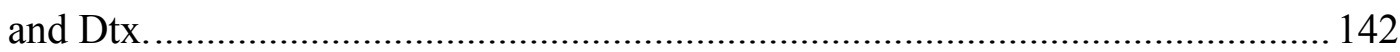

5.3.2 Inhibition of HUVEC migration by PelA and Dtx. ................................. 142

5.3.3 PelA and Dtx inhibit HUVEC capillary-like tube formation...................... 145

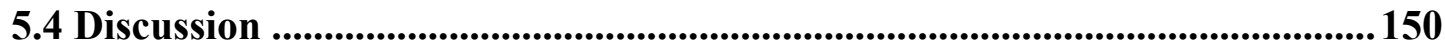

5.4.1 PelA inhibits HUVEC proliferation........................................................ 150

5.4.2 PelA inhibits EC migration....................................................................... 150

5.4.3 PelA inhibits capillary-like tube formation.............................................. 152

5.4.4 Possible mechanisms linking microtubule-binding to anti-angiogenic

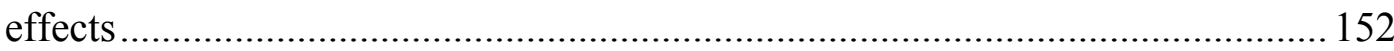

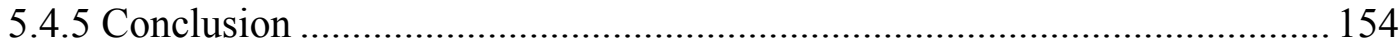

Chapter 6. Induction of premature senescence by peloruside A..... 156

6.1 Introduction.................................................................................................................156 


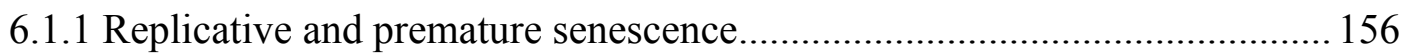

6.1.2 Characteristics of senescent cells............................................................ 156

6.1.3 Mechanisms underlying cellular senescence induction ............................ 159

6.1.4 Senescence as a tumour suppressive mechanism......................................... 161

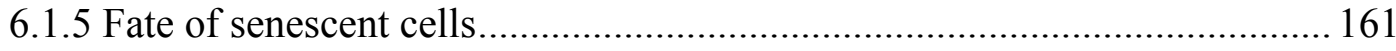

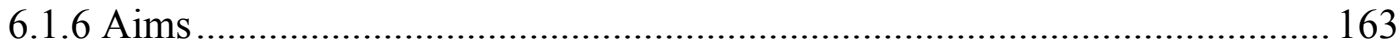

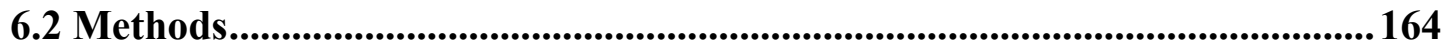

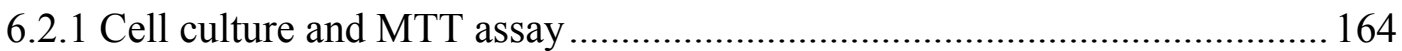

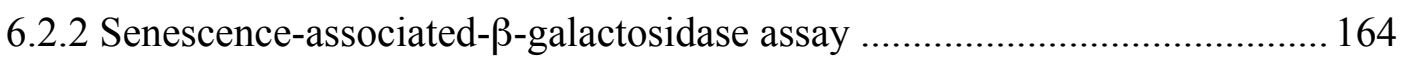

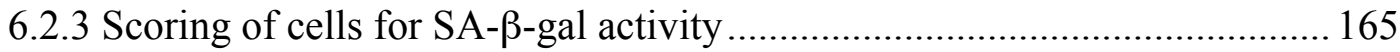

6.2.4 Click-iT EdU incorporation assay ......................................................... 165

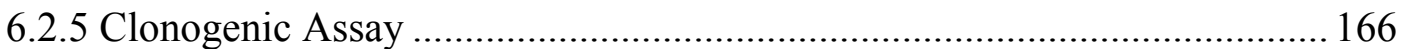

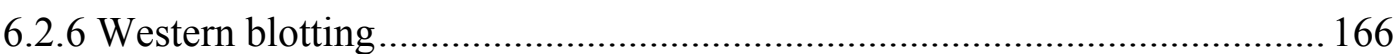

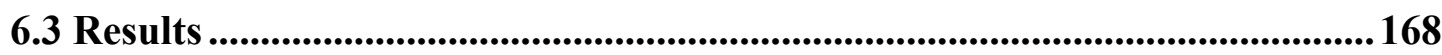

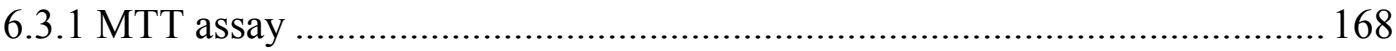

6.3.2 Senescence-associated $\beta$-galactosidase assay .......................................... 168

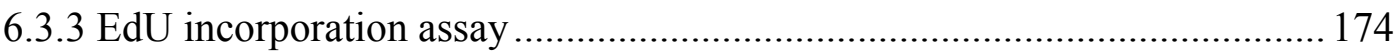

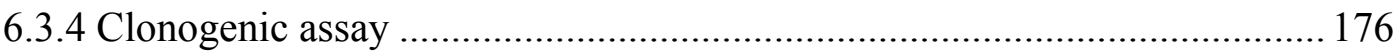

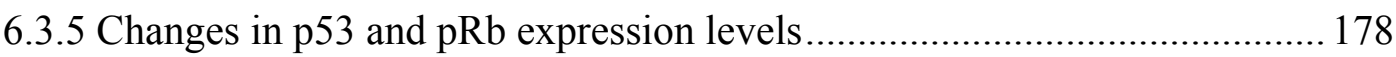

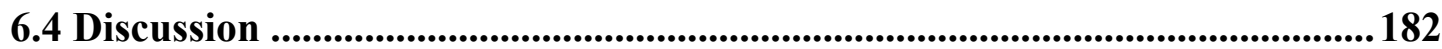

6.4.1 Increased SA- $\beta$-gal activity in response to MSAs .................................... 182

6.4.2 Cell cycle changes after prolonged exposure to chemotherapeutic agents.. 183

6.4.3 Activation of p53 and down-regulation of pRb ...................................... 184

6.4.4 Premature senescence versus other cell fates............................................. 186

6.4.5 Mechanisms underlying MSA-induced senescence.................................. 188

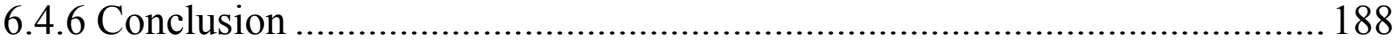

\section{Chapter 7. Stathmin, c-Myc and topoisomerase I role in the action of}

peloruside A.......................................................................................... 190

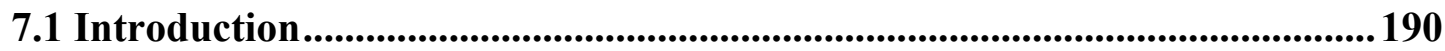

7.1.1 Identifying drug targets and elucidating mode of action ........................... 190

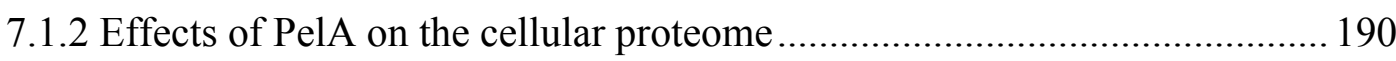

7.1.3 HIP and HOP microarray analyses of PelA ............................................... 193 
7.1.4 Aims

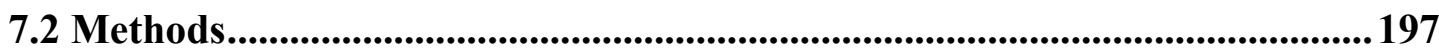

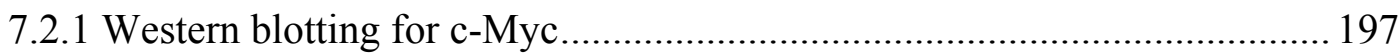

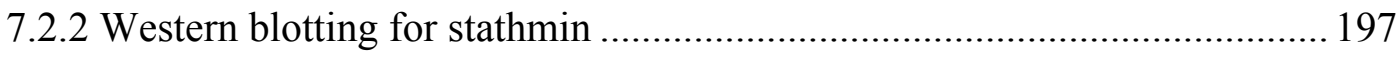

7.3 Results ............................................................................................................................... 198

7.3.1 c-Myc expression is decreased by both PelA and Ptx ................................ 198

7.3.2 PelA and Ptx reduce stathmin expression levels......................................... 198

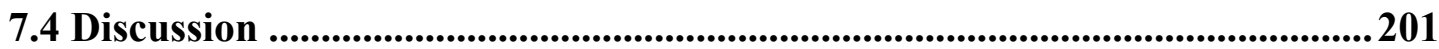

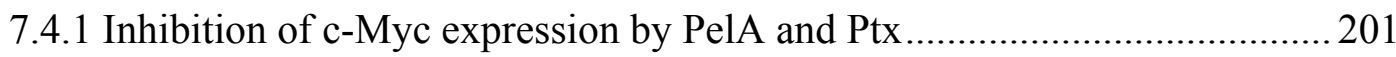

7.4.2 Inhibition of stathmin by PelA and Ptx................................................... 203

Chapter 8. Final Discussion ................................................................... 204

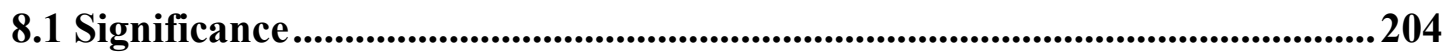

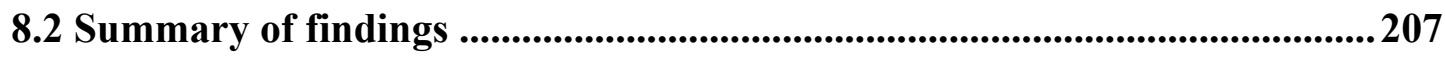

8.2.1 PelA induces apoptosis via a caspase-dependent mechanism in 1A9 cells . 207

8.2.2 Low concentrations of PelA induce aneuploidy and may involve incorrect microtubule-kinetochore attachments. 208

8.2.3 Potential tumour suppressive mechanism of PelA: Induction of premature senescence and inhibition of angiogenesis .......................................................20 209

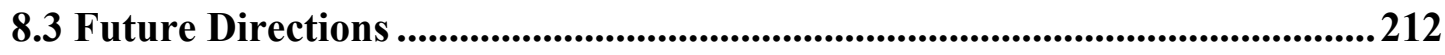

8.3.1 Confirmation of the primary apoptotic signalling pathway induced by PelA212

8.3.2 Quantification of the frequencies of erroneous microtubule-kinetochore attachments at low PelA concentrations

8.3.3 Confirmation of whether PelA decreases c-Myc expression in additional cancer cell lines and potential implications for angiogenesis

8.3.4 Confirming a lack of DNA replication in response to senescence-inducing conditions

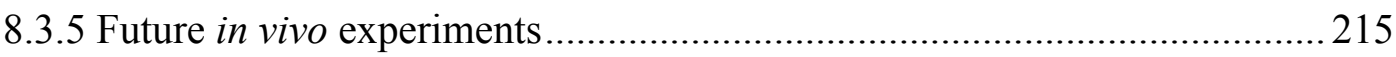

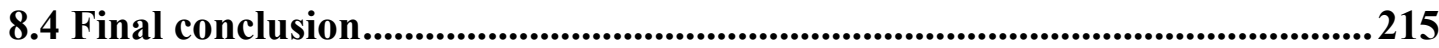

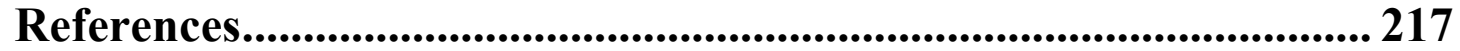


Appendices

Appendix A: General Recipes...................................................................................... I

Appendix B: Chapter 3..............................................................................................

Appendix C: Chapter 4 ................................................................................................XI

Appendix D: Chapter 5 .................................................................................................XII

Appendix E: Chapter 6.................................................................................... XV

Appendix F: Chapter 7...........................................................................................XVIII

Appendix G: Topoisomerase I methods, results and discussion .........................XIX 


\section{List of Figures}

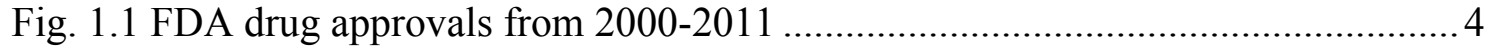

Fig. 1.2 Microtubule polymerization and depolymerisation......................................... 5

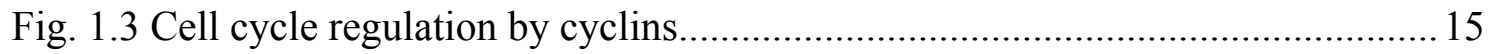

Fig. 1.4 Angiogenesis can lead to tumour growth and metastasis................................30

Fig. 1.5 Structure of the microtubule-stabilizing agent PelA …..................................... 36

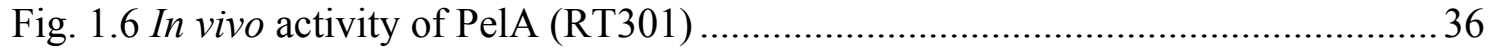

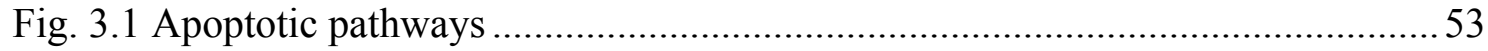

Fig. 3.2 Effects of individual protease inhibitors on cell proliferation ..........................65

Fig. 3.3 Effects of protease inhibitors on MTT assay responses to PelA, MycA, and

Disco in $1 \mathrm{~A} 9$ cells 66

Fig. 3.4 Protective effects of protease inhibitors on PelA-, MycA- and Disco-induced

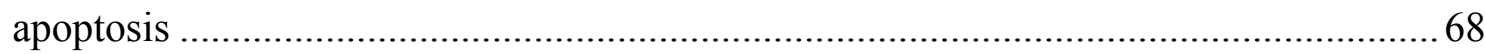

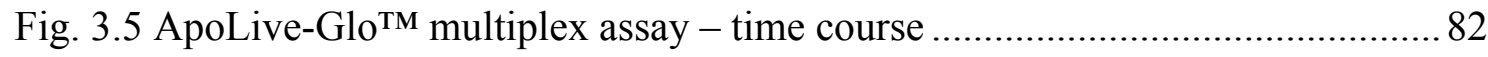

Fig. 3.6 ApoLive-Glo ${ }^{\mathrm{TM}}$ multiplex assay - concentration-response.............................. 83

Fig. 3.7 Protease inhibitor effects on caspase-3/7 activation ...................................... 84

Fig. 3.8 FLICA assay for detecting caspase- 8 and -9 activation by PelA ...................... 86

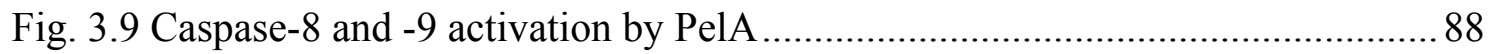

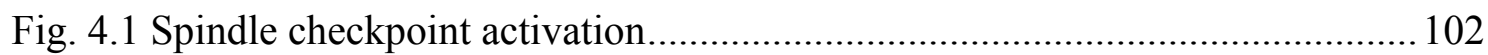

Fig. 4.2 Kinetochores undergo various changes in orientation during spindle assembly103

Fig. 4.3 Induction of aneuploidy by PelA in unsynchronized cells ............................. 113

Fig. 4.4 Aneuploidy induction in synchronized 1A9 cells over time ........................... 114

Fig. 4.5 Cell cycle progression at low and high concentrations of PelA ..................... 115

Fig. 4.6 Representative Western blots showing changes in total protein expression of cell cycle proteins during cell cycle progression

Fig. 4.7 Expression of the cell cycle proteins, p55CDC, BUBR1, MAD2, and cyclin

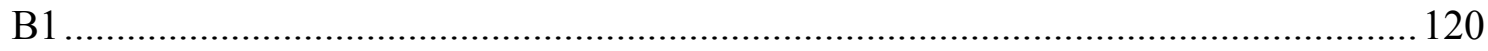

Fig. 4.8 Western blots following Co-IP of p55CDC, MAD2 and BUBR1 during cell cycle progression

Fig. 4.9 Changes in association of MAD2 and BUBR1 with p55CDC as determined by Co-IP and Western blotting

Fig. 4.10 Untreated cells undergo normal mitosis 124 
Fig. 4.11 Examples of pseudo-metaphase cells after treatment with low concentration PelA 125

Fig. 4.12 Abnormal mitosis at high concentrations of PelA....................................... 126

Fig. 5.1 Inhibition of HUVEC proliferation by PelA and Dtx................................... 143

Fig. 5.2 Inhibition of HUVEC migration by PelA and Dtx in a wound scratch assay . 144

Fig. 5.3 Inhibition of capillary tube formation by PelA and Dtx................................. 146

Fig. 5.4 Effects of PelA and Dtx on total tube length and total tube area .................... 148

Fig. 5.5 Inhibition of HUVEC proliferation over $16 \mathrm{~h}$.............................................. 149

Fig. 5.6 MSA effects on endothelial cell functions .................................................. 155

Fig. 6.1 Features of cellular senescence and typical signalling pathways ................... 158

Fig. 6.2 Photomicrographs of premature senescence induction ............................... 171

Fig. 6.3 Enlarged photomicrographs of senescent cells............................................. 172

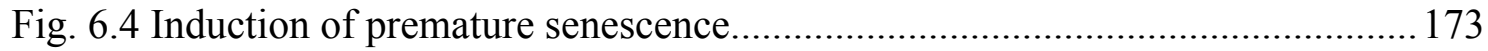

Fig. 6.5 Cell cycle histograms after 6 day incubations with MSAs or Doxo ............... 175

Fig. 6.6 Effects of chemotherapeutic drugs on clonogenic survival.......................... 177

Fig. 6.7 Relationship between induction of senescence and clonogenic survival ........ 177

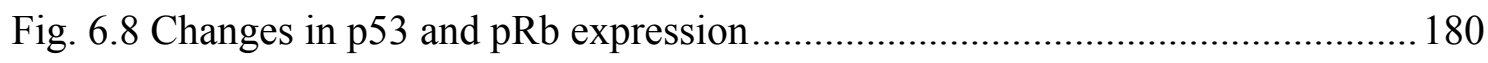

Fig. 6.9 Graphs showing relative changes in p53 and pRb expression....................... 181

Fig. 7.1 PelA and Ptx reduce c-Myc expression levels................................................ 199

Fig. 7.2 PelA and Ptx reduce stathmin expression levels .........................................200

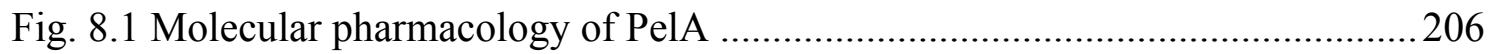

Appendix Fig. B.1 Protective effects of combinations of protease inhibitors against

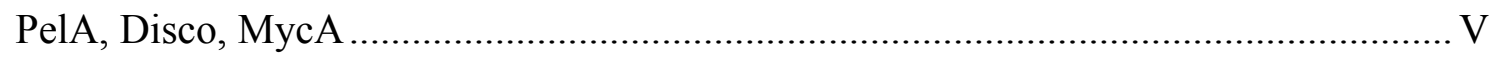

Appendix Figure B.2. Effects of protease inhibitors on MTT assay responses to PelA,

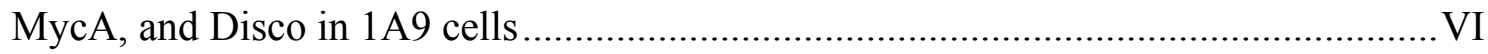

Appendix Fig. B.3 Protective effects of zVAD-fmk against PelA, MycA and Disco.. VII Appendix Fig. B.4 Positive shift in autofluorescence in response to PelA ................ VIII Appendix Fig. B.5 Positive control for caspase- 8 and -9 activation ............................. Appendix Fig. B.6 Caspase- 8 and -9 activation by PelA at later timepoints.................. X Appendix Fig. C.1 Co-immunoprecipitation (Co-IP) optimization ................................ Appendix Fig. D.1 Multiwell plate layout for wound scratch assay ........................... XII Appendix Fig. D.2 Plate insert for generating more consistent wound scratches ....... XIII Appendix Fig. D.3 Transwell migration assay results .............................................. XIV 
Appendix Fig. E.1 EdU incorporation assay XVI

Appendix Fig. E.2 Clonogenic assay plates XVII

Appendix Fig. F.1 Stathmin regulates mitotic entry XVIII

Appendix Fig. G.1 Topoisomerase I (TOPO I) relaxation assay XXII

\section{List of Tables}

Table 1.1 Summary of microtubule-targeting agents, their binding sites, and stage of development 19

Table 3.1. $\mathrm{IC}_{50}$ or $\mathrm{IC}_{80}$ values for $1 \mathrm{~A} 9$ cells treated with various three different drugs .63 Table 3.2. Summary of findings from the protease inhibitor screen and caspase activation assays for PelA, MycA and Disco. 90

Table 4.1. Frequency of cells exhibiting different mitotic morphologies in response to PelA

Table 6.1. Growth inhibitory concentration values for MCF7 cells treated with various drugs 168

\section{List of Equations}

Equation 2.1 Four parameter logistic curve equation 46 


\section{List of major abbreviations}

$\begin{array}{ll}\text { 2-DE } & \text { 2-dimensional gel electrophoresis } \\ \text { 2-ME } & \text { 2-methoxyestradiol/Panzem }{ }^{\circledR} \\ { }^{3} \text { H } & \text { tritiated } \\ \text { ADME } & \text { adsorption, distribution, metabolism, excretion } \\ \text { ADP } & \text { adenosine diphosphate } \\ \text { AIF } & \text { apoptosis-inducing factor } \\ \text { APC/C } & \text { anaphase promoting complex/cyclosome } \\ \text { APS } & \text { ammonium persulfate } \\ \text { ATP } & \text { adenosine-5'-triphosphate } \\ \text { BAG2 } & \text { BCL-2 associated anthanogene 2 } \\ \text { BCA kit } & \text { bicinchoninic acid kit } \\ \text { BCL-2 } & \text { B cell lymphoma 2 } \\ \text { bFGF } & \text { basic fibroblast growth factor } \\ \text { BH1-4 } & \text { BCL-2 homology domains 1-4 } \\ \text { BLA } & \text { biologic license applications } \\ \text { BSA } & \text { bovine serum albumin } \\ \text { BUB } & \text { budding uninhibited by benzimidizoles } \\ \text { BUBR1 } & \text { budding uninhibited by benzimidizoles related 1 } \\ \text { CA-1-P } & \text { combretastatin A-1 diphosphate } \\ \text { CA-4-P } & \text { combretastatin A-4 phosphate } \\ \text { CARD } & \text { caspase recruitment domain } \\ \text { CDC20 } & \text { cell division cycle 20 } \\ \text { CDK } & \text { cyclin dependent kinase } \\ \text { CENP } & \text { centromere-associated protein } \\ \text { CHAPS } & \text { 3-[(3-Cholamidopropyl) dimethylammonio]-1-propanesulfonate } \\ \text { CIN } & \text { chromosomal instability } \\ \text { CKI } & \text { cyclin dependent kinase inhibitor } \\ \text { Co-IP } & \text { co-immunoprecipitation } \\ \text { CPC } & \text { chromosome passenger complex } \\ \text { CPT } & \text { camptothecin } \\ \text { eIF4A } & \text { eukaryotic initiation factor 4A } \\ \text { ECM } & \text { extracellular matrix } \\ \text { DAPI } & \text { 4',6-diamidino-2-phenylindole } \\ \text { DDR } & \text { DNA-damage response } \\ \text { DIGE } & \text { differential in-gel electrophoresis } \\ \text { DISC } & \text { death inducing signalling complex } \\ \text { Disco } & \text { discodermolide } \\ \text { DJF } & \text { Dean-Jett-Fox } \\ \text { DMF } & \text { N, N-dimethylformamide } \\ \text { DMP } & \text { dimethyl pimelimidate } \\ \text { DMSO } & \text { dimethyl sulfoxide } \\ \text { Doxo } & \text { doxorubicin } \\ \text { DSB } & \text { double-strand break } \\ \text { Dtx } & \text { docetaxel/Taxotere } ~ \\ \text { EC } & \text { endothelial cell } \\ \text { ECM } & \text { extracellular matrix } \\ \text { EdU } & \text { 5-ethynyl-2'-deoxyuridine } \\ & \\ & \end{array}$




\begin{tabular}{|c|c|}
\hline EGF & epidermal growth factor \\
\hline EHS & Engelbreth-Holm-Swarm \\
\hline eIF4A & eukaryotic initiation factor $4 \mathrm{~A}$ \\
\hline $\mathrm{EpoA} / \mathrm{B} / \mathrm{C}$ & epothilone $\mathrm{A}, \mathrm{B}, \mathrm{C}$ \\
\hline E-site & exchangeable site \\
\hline $\mathrm{EtOH}$ & ethanol \\
\hline FADD & Fas associated death domain \\
\hline FAK & focal adhesion kinase \\
\hline FasL & Fas ligand \\
\hline FCS & fetal calf serum \\
\hline FDA & US Food and Drug Administration \\
\hline FLICA & fluorescent labelled inhibitor of caspases \\
\hline FSC & forward scatter \\
\hline GAPs & GDP-dissociation inhibitors \\
\hline GDP & guanosine 5'-diphosphate \\
\hline GEF & guanine nucleotide exchange factor \\
\hline GFP & green fluorescent protein \\
\hline GTP & guanosine-5'-triphosphate \\
\hline $\mathrm{HC}$ & antibody heavy chain \\
\hline $\mathrm{HCl}$ & hydrochloric acid \\
\hline HDAC6 & histone deacetylase- 6 \\
\hline HIF-1 & hypoxia inducible factor 1 \\
\hline HIP & haploid insufficiency profiling \\
\hline HOP & homozygous deletion profiling \\
\hline HPLC & high-performance liquid chromatography \\
\hline Hsp90 & heat shock protein 90 \\
\hline HUVEC & human umbilical vein endothelial cell \\
\hline IC & inhibitory concentration \\
\hline $\operatorname{IgG}$ & immunoglobulin \\
\hline IL & interleukin \\
\hline INCENP & inner-centromere protein \\
\hline INK4 & inhibitor of kinase 4 \\
\hline i.p. & intraperitoneal \\
\hline i.v. & intravenous \\
\hline IPA & ingenuity pathway analysis \\
\hline KIF5 & kinesin-1 \\
\hline kMTs & kinetochore microtubules \\
\hline $\mathrm{LC}$ & antibody light chain \\
\hline LMP & lysosomal membrane permeabilization \\
\hline Lum & luminescent \\
\hline MAD & mitotic arrest deficiency \\
\hline MALDI-TOF & matrix assisted laser desorption ionization-time of flight \\
\hline MAP & microtubule-associated protein \\
\hline MAPK & mitogen activated protein kinase \\
\hline $\mathrm{MCC}$ & mitotic checkpoint complex \\
\hline MDA & microtubule-destabilizing agent \\
\hline MDR & multiple drug resistance \\
\hline MEF & mouse embryonic fibroblast \\
\hline MINUS & microtubule nucleation suppressor \\
\hline MOMP & mitochondrial outer membrane permeabilization \\
\hline
\end{tabular}




\begin{tabular}{|c|c|}
\hline MPS1 & multipolar spindle-1 \\
\hline mRNA & messenger RNA \\
\hline MSA & microtubule-stabilizing agent \\
\hline MS & mass spectrometry \\
\hline $\mathrm{MS} / \mathrm{MS}$ & tandem mass spectrometry \\
\hline MTA & microtubule-targeting agent \\
\hline MTT & 2-(4,5-dimethyl-2thiazolyl)-3,5-diphenyl-2H-tetrazolium bromide \\
\hline MTOC & microtubule organizing centre \\
\hline MQ & milli-Q water \\
\hline MycA & mycalamide A \\
\hline NEB & nuclear envelope breakdown \\
\hline NIWA & National Institute of Water and Atmospheric Research \\
\hline NME & new molecular entities \\
\hline NSCLC & non-small cell lung carcinoma \\
\hline N-site & non-exchangeable site \\
\hline $\mathrm{NuMa}$ & nuclear mitotic apparatus protein \\
\hline (OC)DNA & open circular DNA \\
\hline OIS & oncogene-induced senescence \\
\hline Op 18 & oncoprotein 18 \\
\hline P1GF & placenta growth factor \\
\hline PARP & poly (ADP) ribose polymerase \\
\hline PBS & phosphate buffered saline \\
\hline PCD & programed cell death \\
\hline PCR & polymerase chain reaction \\
\hline PDL & poly-D-lysine \\
\hline PelA & peloruside A \\
\hline PCNA & proliferating cell nuclear antigen \\
\hline PDGF & platelet-derived growth factor \\
\hline PFA & paraformaldehyde \\
\hline Pgp & P-glycoprotein \\
\hline PI & propidium iodide \\
\hline PLK1 & polo-like kinase-1 \\
\hline Plx1 & polo-like kinase-1 (Xenopus laevis) \\
\hline $\mathrm{pRB}$ & retinoblastoma protein \\
\hline PS & phosphatidylserine \\
\hline PTM & post-translational modification \\
\hline Ptx & paclitaxel/Taxol $®$ \\
\hline PVDF & polyvinylidene fluoride \\
\hline rDNA & relaxed DNA \\
\hline RIPA & radioimmunoprecipitation assay \\
\hline RMS & root mean squared \\
\hline ROS & reactive oxygen species \\
\hline $\mathrm{RS} / \mathrm{DJ}-1$ & RNA-binding regulatory subunit/DJ-1 \\
\hline RT & room temperature \\
\hline SA- $\beta$-gal & senescence-associated $\beta$-galactosidase \\
\hline SAC & spindle assembly checkpoint \\
\hline SAHF & senescence-associated heterochromatin foci \\
\hline SAR & structure activity relationships \\
\hline SCID & severe combined immunodeficiency \\
\hline SDF & senescence-associated DNA-damage foci \\
\hline
\end{tabular}




\begin{tabular}{|c|c|}
\hline sDNA & supercoiled DNA \\
\hline SDEV & standard deviation \\
\hline SDS & sodium dodecyl sulphate \\
\hline SEM & standard error \\
\hline (Sh)RNA & short-hairpin RNA \\
\hline siRNA & small-interfering RNA \\
\hline SP1 & specificity protein 1 \\
\hline SSC & side scatter \\
\hline TEMED & N,N,N',N'-tetramethylethylenediamine \\
\hline TGF- $\beta$ & transforming growth factor $\beta$ \\
\hline TNF & tumour necrosis factor \\
\hline TNFR & tumour necrosis factor receptor \\
\hline TOPO I & topoisomerase I \\
\hline TRAIL & TNF-related apoptosis inducing ligand \\
\hline TSP-1 & thrombospondin-1 \\
\hline TUNEL & terminal deoxynucleotidyl transferase dUTP nick end labelling \\
\hline $\mathrm{UC}$ & The University of Canterbury \\
\hline Vbl & vinblastine/Velban $® /$ Velsar $®$ \\
\hline Vcr & vincristine/Oncovin $\AA$ \\
\hline VDA & vascular disrupting agent \\
\hline VEGF & vascular endothelial growth factor \\
\hline VHL & von Hippel-Lindau \\
\hline VUW & Victoria University of Wellington \\
\hline X-gal & 5-bromo-4-chloro-3-indolyl $\beta$-D-galactosidase \\
\hline
\end{tabular}




\section{Chapter 1. General Introduction}

\subsection{Anti-cancer drug development}

\subsubsection{Chemotherapeutics}

Cancer is a major public health issue worldwide and, after cardiovascular disease, is the second leading cause of mortality. Statistics compiled by the American Cancer Society predicted that in 2011, in the United States alone, there would be almost 1.6 million new diagnoses of cancer, and over half a million people would die from the disease (Siegel et al., 2011). Nowadays, a diagnosis of cancer is no longer an automatic death sentence; however, these grim statistics highlight the fact that the fight against cancer is far from over. Although there has been a strong shift in the industry towards molecular targeted therapies (Chabner and Roberts, 2005; Hambley, 2009; Hait, 2010), chemotherapy drugs that tend to have a more general cytotoxic effect still have an essential role in the treatment of diverse cancers. These chemotherapies combat the uncontrolled and abnormal cell proliferation that drives human malignancies, but they all have their limitations (Jackson et al., 2007). Resistance to chemotherapy drugs is one of the major difficulties in the clinic and is thought to develop from acquired mutations, which can be selected for as a result of various selection pressures, or the presence of self-renewing cancer stem cells within tumours (Alison et al., 2012). Certain mutations can upregulate the expression of cell survival or anti-apoptotic proteins, thus mitigating the toxic effects of the chemotherapy drugs. As reviewed by Alison et al. (2012) cancer stem cells are typically resistant to conventional chemotherapies or radiotherapies, as they are, for example, prone to quiescence (a state of arrested growth), have increased oncogene activity or cell survival signalling (e.g. activated Wnt/ $\beta$-catenin and Notch pathways), and up-regulated DNA damage response pathways. This raises the possibility that surviving cancer stem cells can eventually repopulate tumours, even after treatment with radiation or anti-cancer drugs. Many cancers also overexpress the P-glycoprotein (P-gp) drug efflux pump, which functions to pump drugs out of the cell, making them less effective. Another consideration in terms of the clinical use of chemotherapeutics is that they inevitably cause some quite undesirable side effects, including allergic reaction, neutropenia and neuropathy. Although most of the current chemotherapeutic drugs preferentially target rapidly dividing cancer cells, their clinical use (i.e. dose and length of treatment) is limited by myelosuppressive effects (reduced production of blood cells by the bone marrow, i.e. red blood cells, white blood cells and 
platelets) and more permanent neurotoxic effects (Gascoigne and Taylor, 2009). There are over 100 different types of cancer, and identifying new treatments that have more desirable therapeutic profiles (e.g. drugs that are more effective and/or have reduced side-effects), or that can work synergistically with current drugs, has been an extremely challenging task.

The use of nitrogen mustard and antifolates in the 1940s (to treat non-Hodgkin's lymphoma and acute lymphoblastic leukaemia, respectively) revolutionized chemotherapy and turned it into the multi-billion dollar industry it is today (Chabner and Roberts, 2005). These compounds were the first to demonstrate that systemic administration of drugs could effectively reduce tumour size, and now chemotherapy is used routinely to treat cancer. Most anti-cancer drugs target processes necessary for cell growth and proliferation, such as DNA synthesis, cell cycle progression, and tumour blood supply. Inhibiting such essential processes often leads to tumour cell death, or apoptosis. Some of the more successful treatments to come out of the past few decades include 5-fluorouracil (inhibits DNA replication) and paclitaxel (Ptx; Taxol®) (targets microtubules), as well as the molecular targeted drugs, imatinib (a tyrosine kinase inhibitor), and bevacizumab (Avastin ${ }^{\circledR}$ ) (inhibits vascular endothelial growth factor). Over the past few decades, pharmaceutical companies have invested vast amounts of money into investigating the cellular and molecular aspects of cancer development and progression. A major obstacle, however, has been the transformation of such research into tangible, therapeutic outcomes (i.e. translational research). It has become evident that the production of novel or improved chemotherapeutics is now lagging behind the rapid pace at which our molecular understanding of cancer is advancing (Hait, 2010). In an attempt to bridge the gap between researchers and clinicians, there has been a strategic shift in the industry towards translational research. It will become apparent over the coming years whether this bench-to-bedside approach has led to a measurable improvement in the long-term survival rates of cancer patients.

\subsubsection{Early phase drug discovery}

Anyone that is involved in the development of novel drugs knows what a costly and time-consuming process it is. Pharmaceutical companies typically have to invest approximately one billion dollars to progress a single drug from the initial discovery stages through to the clinic, a process that takes 12 years on average (Kola and Landis, 2004). Unfortunately, the majority of drugs will inevitably fail at some stage during 
their development. It has been suggested that of the oncology drugs that make it into the clinical pipeline (which accounts for $\sim 20 \%$ of all drugs entering clinical trials) only one in four will be approved for clinical use (DiMasi and Grabowski, 2007). A brief look at some of the statistics released by the US Food and Drug Administration (FDA) (see Fig. 1.1) highlights the low numbers of cancer therapeutics gaining FDA approval between 2000 and 2011 (Mullard, 2011). The number of drugs approved each year, although low, has remained fairly steady over the past decade, averaging 22 approvals per year. Of these, only a small proportion (average 14\%) was developed as treatments for cancer. The predominant causes for drugs failing during development, besides lack of a meaningful response, are poor pharmacokinetic profiles (i.e. absorption, distribution, metabolism and excretion - ADME) or adverse toxicity. Hence, it is extremely important that any undesirable drug effects are identified as early on as possible. In order for a drug to successfully make it into the market it must progress through several stages of development: discovery/characterization, preclinical efficacy, and clinical tolerance and effectiveness i.e. phase I, II and III trials. Generally, it is during the preclinical stage of drug development that pharmacodynamic, pharmacokinetic and toxicity profiles are investigated. This is usually carried out in animal models, and even a favourable profile in these preclinical trials does not guarantee a favourable profile in humans. An equally important task during these early stages of drug development is to comprehensively characterize a drug's mechanism of action and, in particular, identify its primary and secondary targets, and determine whether certain subgroups of tumour types, or patients, will respond better to a particular drug. One of the most valuable anticancer drug targets is the cytoskeletal protein, tubulin, (the subunits that comprise microtubule polymers). Several drugs that target tubulin (microtubule-targeting agents or MTAs) are now clinically used, but each is not without its limitations. Due to the relative ease of identifying MTAs in the laboratory, there has been a large increase in the number of such compounds currently being investigated. Whether many of these drugs will surpass their current counterparts will become apparent over the next few years as they progress (or fail) through the various clinical trial stages. The MTA peloruside A (PelA; discussed in detail later in the chapter) will be the major focus of this thesis. 


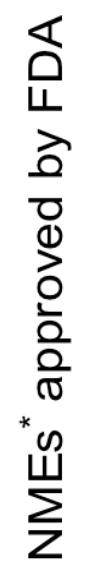

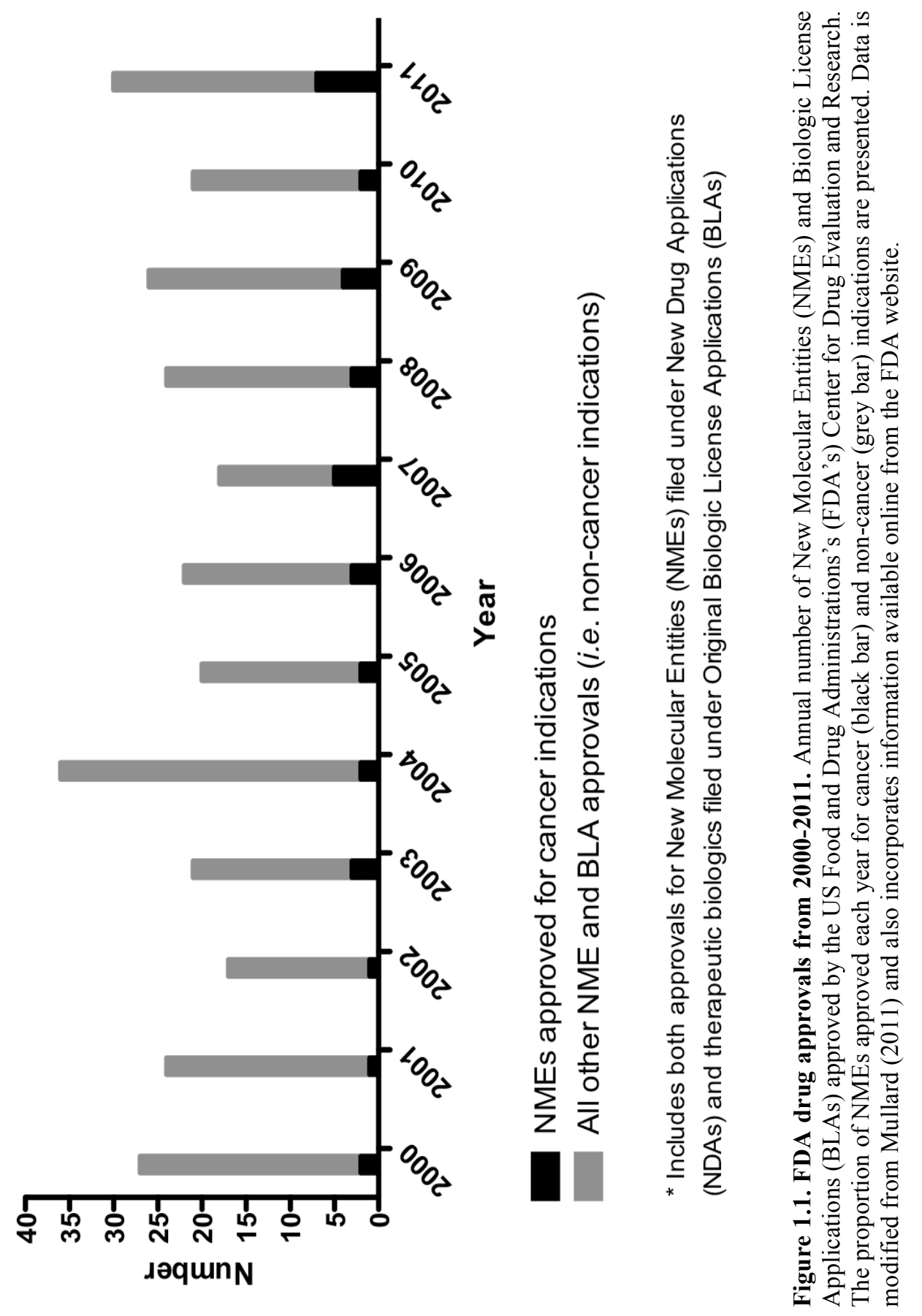




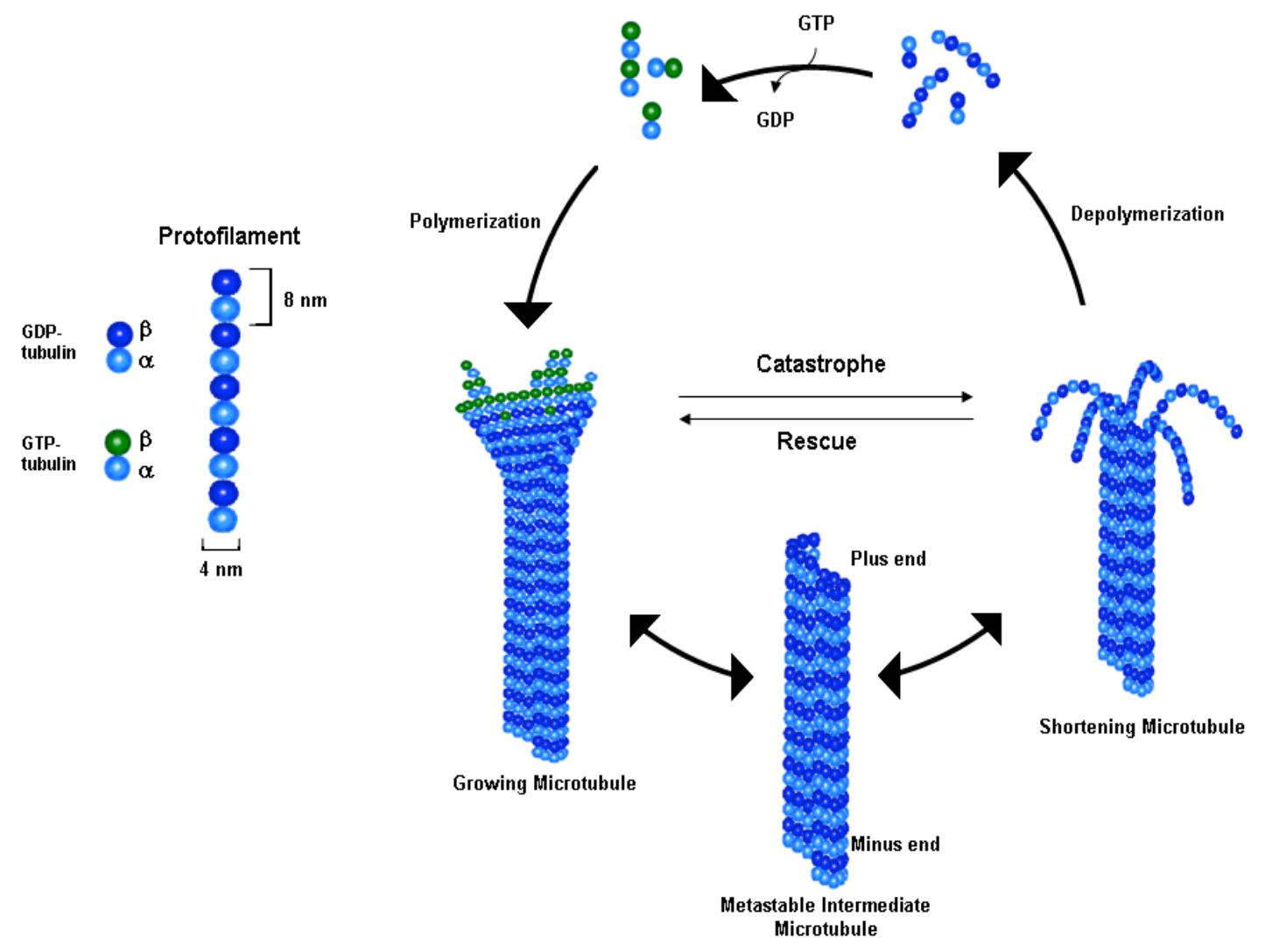

Figure 1.2. Microtubule polymerization and depolymerisation. Microtubule protofilaments are comprised of $\alpha / \beta$-tubulin heterodimers arranged in a head-to-tail fashion. Typically, thirteen of these protofilaments associate with each other to form a microtubule lattice that is $25 \mathrm{~nm}$ in diameter. The microtubule plus end is considerably more dynamic than the minus end. Microtubule polymerization is largely driven by the hydrolysis of GTP upon dimeric $\alpha$-/ $\beta$-tubulin binding to the growing microtubule. It is thought that growing microtubules are stabilised by a "GTP cap", illustrated as the blue $(\alpha-)$ and green $(\beta-)$ tubulin heterodimers on the growing microtubule. Due to the intrinsic curved conformation of GDPtubulin, loss of the GTP cap results in rapid depolymerization as the protofilaments peel away from the microtubule body. An intermediate state can also exist in which there is no observable microtubule growth or shortening. Growing (or paused) microtubules can undergo transitions to shortening, called catastrophe; whereas, shortening microtubules can undergo transitions to growth (or pause), called rescue. Adapted from (Akhmanova and Steinmetz, 2008), and taken from Ariane Chan's BMSC Honours thesis (Chan, 2008). 


\subsection{Structure and function of microtubules}

\subsubsection{Microtubule structure and dynamic instability}

Microtubules are highly dynamic filamentous structures composed of $\alpha$ - and $\beta$-tubulin heterodimers. These heterodimers arrange longitudinally (in a head-to-tail fashion) to form protofilaments, which then associate laterally to form the hollow tube-like structure of the microtubule (see Fig. 1.2). Microtubule polymerisation requires $\gamma$ tubulin, another member of the tubulin family, in order to initiate the nucleationelongation process and is driven by the energy provided by GTP hydrolysis (Nogales, 2001). $\alpha$ - and $\beta$-tubulin can each bind one molecule of GTP. While binding of GTP to the N-site (non-exchangeable) on $\alpha$ - tubulin is irreversible, the binding of GTP to the Esite (exchangeable) on $\beta$-tubulin is reversible and drives polymerisation (the E-site needs to be occupied with GTP before another tubulin dimer can be added). Following the addition of a new tubulin dimer to the growing microtubule, GTP becomes hydrolysed to non-exchangeable GDP (Wade, 2007). Microtubules are able to randomly alternate between phases of growth (polymerisation) and shortening (depolymerisation), behaviour termed "dynamic instability" (Jordan and Wilson, 2004). This stochastic behaviour of microtubules plays a crucial role in their ability to function appropriately in many cell processes. To explain the ability of microtubules to alternate between growth and shortening events, researchers have proposed a "GTP-cap model", where the nucleotide state of $\beta$-tubulin regulates microtubule dynamics. The body of the microtubule is predominantly composed of GDP-tubulin and is considered to be unstable due to the intrinsic curved nature of GDP-tubulin (i.e. the microtubule has a predisposition to depolymerise). It is thought that a "GTP cap" stabilizes the microtubule and arises when the rate of GTP hydrolysis lags behind the rate at which new dimers are added to the microtubule (Horio and Hotani, 1986). If the GTP cap is lost randomly, or the rate of hydrolysis catches up with the rate of polymerization, then the microtubule rapidly depolymerises (Janosi et al., 2002). The transition from polymerization to depolymerization is called "catastrophe", while the transition from a depolymerizing to a polymerizing microtubule is known as "rescue". Microtubules have a more dynamic "plus end" that tends to localize at the cell periphery, and are anchored to the microtubule-organizing center (MTOC) at their "minus ends" (Nogales, 2001; Amos, 2004). Nucleation occurs at the minus end and is dependent on the localization of $\gamma$-tubulin to the MTOC (Nogales, 2001). This polarity is an extremely important feature of microtubules, helping to direct many essential cellular functions. 


\subsubsection{Cellular role of microtubules}

Microtubules are cytoskeletal proteins that are involved in numerous cellular processes. These include relatively simple processes such as maintaining the shape or morphology of the cell, or much more complex functions, as in the case of mitosis. Despite carrying out crucial functions during interphase, it is predominantly the role of microtubules in the mitotic spindle that has generated the widespread interest amongst many cancer researchers. Disrupting mitotic microtubules can have disastrous outcomes for replicating cells, often leading to apoptosis; hence, targeting these polymers has proven to be a highly effective approach to inhibit the proliferative capacity of rapidly dividing cancer cells. In order to maintain accurate chromosome segregation during cell division, sister chromatids must be bilaterally attached to opposing spindle poles. During prometaphase, microtubules grow and shorten in a "search and capture" mechanism until each kinetochore is correctly bound to the mitotic spindle. Although the precise mechanisms are still being elucidated, microtubules are also involved in the intracellular signalling pathways that communicate whether kinetochores are correctly or incorrectly attached and subsequently, whether a cell progresses through mitosis or succumbs to an alternative cell fate.

Many interphase processes also rely on the microtubule cytoskeleton. In conjunction with other proteins, like actin filaments and intermediate filaments, microtubules help to maintain cell shape and polarity as well as regulate cell movement. Rapid reorganization of the cytoskeletal network, for example, is necessary for generating the morphological changes necessary for cell migration and motility. The small family of Rho GTPases (e.g. Rac1, RhoA, and Cdc42) functions alongside microtubules to elicit changes in the actin cytoskeleton that are important for generating cell polarity (e.g. lamellopodia formation at the leading edge of the cell) (Mizuno and Singer, 1994). Additionally, the polymerization and depolymerization of microtubules helps to generate the forces necessary for the appropriate positioning of organelles (TolićNørrelykke, 2008). Cellular transport and intracellular signalling between organelles requires functional, intact microtubules. The microtubule-associated motor proteins, kinesin and dynein are the predominant mediators of cellular transport and use stable microtubules as tracks to facilitate transport to or away from the cell periphery, respectively. Kinesins generally direct movement towards the plus ends of the microtubules (anterograde transport); whereas, dynein directs movement in the opposite direction, towards the minus ends (retrograde transport) (Mallik and Gross, 2004). 
These molecular motors play crucial roles in much of the directional activity that occurs inside the cell. Transport along microtubules is particularly well characterised for neuronal cells, which depend on the extensive movement of proteins and other signalling molecules between the cell body and the axon. In dividing cells, motor proteins are essential for localizing numerous key regulatory proteins. For example, immunodepletion of NuMA (nuclear mitotic apparatus protein) in frog egg extracts highlighted the importance of dynein-mediated localization of NuMA to the spindle poles during mitosis (Merdes et al., 1996). Similarly, antibodies recognizing cytoplasmic dynein also disrupt NuMA localization, prevent mitotic aster assembly and impair the focussing and maintenance of microtubule minus ends at the spindle poles (Gaglio et al., 1997; Hirokawa et al., 1998). Interphase processes mediated by microtubules depend on the rigorous regulation of microtubules, changes to their dynamics and alterations in the activity of their associated proteins. There is no doubt that perturbation of interphase microtubule functions can have just as significant an impact on cellular homeostasis as inhibition of mitotic functions of microtubules.

\subsection{Regulation of microtubules and their dynamics}

\subsubsection{Tubulin isotypes and post-translational modifications}

The functional diversity of microtubules is quite remarkable, but how exactly individual microtubules might be directed to carry out such specific roles has not yet been fully elucidated. The expression of different tubulin isotypes and post-translational modifications (PTMs) are now emerging as crucial regulatory mechanisms that facilitate the generation of different microtubule subtypes. There are multiple genes that encode for seven different tubulin isotypes ( $\beta \mathrm{I}, \beta \mathrm{II}, \beta \mathrm{III}, \beta \mathrm{IVa}, \beta \mathrm{IVb}, \beta \mathrm{V}$, and $\beta \mathrm{VI})$ (Lopata and Cleveland, 1987). Sequences of these isotypes are highly conserved, differing primarily at the carboxy terminus (amino acid substitutions also occur at the amino terminus, but to a lesser extent) (Lopata and Cleveland, 1987). The contribution of isotype expression and localization to the distinct properties of microtubules is thought to be fairly subtle and seems to play more critical roles in highly specialized neuronal cells (Janke and Bulinski, 2011). Mutations in TUBB3, the gene encoding for $\beta$ III tubulin (an isotype that is highly expressed in neurons), for example, has been linked to malformations of cortical development in humans (Poirier et al., 2010). In terms of oncology, there is also increasing evidence that altered expression of various isotypes (in particular, $\beta$ III tubulin) may be a good prognostic biomarker, being 
involved in both drug-sensitivity/resistance and the aggressiveness of disease (Galmarini et al., 2008; Sève and Dumontet, 2008; Kanakkanthara et al., 2012). In vitro studies in our laboratory indicate that $\beta$ III tubulin overexpression contributes to cancer cell resistance to MTAs (Kanakkanthara et al., 2011; Kanakkanthara et al., 2012). These studies support the results of several clinical studies indicating that higher levels of $\beta$ III tubulin are correlated with a poorer response rate to taxane- or vinorelbine-based treatments and poor prognosis in several human tumour types (reviewed in Sève and Dumontet, 2008). Another study published in the same year assessed the usefulness of $\beta$-tubulin isotypes as a predictive marker for taxane-based therapies (Galmarini et al., 2008) Galmarini et al. found that higher levels of $\beta$ III-tubulin improved the probability of response to the MSA docetaxel (Taxotere $\AA$; Dtx) compared to doxorubicin (Adriamycin $\AA$; Doxo) in patients with advanced or metastatic breast cancer (although it should be noted that they did not observe in terms of time to progression or overall survival). There are some suggestions that $\beta$ III tubulin may be a cell survival factor, as it is induced under stressful conditions such as hypoxia and glucose deprivation (Kavallaris, 2010). Obviously, there is still much to be learned regarding the respective roles of each of the tubulin isotypes, not only in terms of microtubule function but also in the wider context of cellular responses to cytotoxic drugs.

In addition to the differences in expression of tubulin isotypes, there are numerous PTMs that occur on microtubules that regulate their function. These PTMs are reversible and tend to localize at the carboxy terminal of microtubules, a region that is highly interactive with other proteins (e.g. microtubule-associated proteins) (Westermann and Weber, 2003). Some of the more common PTMs include acetylation and deacetylation, tyrosination and detyrosination, polyglutamylation, polyglycylation, phosphorylation, and palmitoylation (Westermann and Weber, 2003; Janke and Bulinski, 2011). There are several suggestions as to the possible functional roles of such modified microtubules, including organization of microtubules into supramolecular structures, signalling and transport, cell motility, and the regulation of microtubule dynamics. For example, earlier studies have suggested that the motor protein, kinesin-1 (KIF5), preferentially binds to detyrosinated microtubules (Dunn et al., 2008). Acetylation of the tubulin residue Lys40 has also been proposed to influence the binding of the motor proteins, KIF5 and dynein, to microtubules, and it has been 
suggested that PTMs of tubulin may "mark" particular subsets of microtubules for specialized functions (Reed et al., 2006; Janke and Bulinski, 2011).

\subsubsection{Microtubule-associated proteins}

Microtubule dynamics are regulated by various proteins, which associate with the microtubule lattice and are aptly termed microtubule-associated proteins (MAPs). MAPs can be generally classified into two groups - those that stabilize the microtubule and those that destabilize it. Although quite diverse, MAPs are similar in that they all contain at least one microtubule-binding domain (Bhat and Setaluri, 2007). This is particularly useful for regulating microtubule structure and dynamics, as it allows a single MAP to bind to multiple tubulin dimers simultaneously (Amos et al., 2005). MAPs themselves are regulated during the cell cycle by kinases and phosphatases that control the binding of MAPs to microtubules by altering their phosphorylation states (Cassimeris et al., 2001). MAPs play a crucial role in influencing the behaviour of microtubules, particularly during mitosis. The microtubule polymer level and turnover varies considerably during prophase (specifically at nuclear envelope breakdown (NEB)), and corresponds with an increase in microtubule dynamics (Zhai et al., 1996). This rapid turnover and altered dynamics is likely to be heavily regulated by the interactions of microtubules with numerous MAPs. The activation of microtubule severing proteins (e.g. katanin), for example, has been suggested to contribute to the decrease in microtubule polymer levels observed at NEB (Zhai et al., 1996; Cassimeris, 1999).

Stathmin, also known as oncoprotein 18 (Op18) is one of the better-characterized microtubule-destabilizers (also discussed in Chapter 7). Decreased expression of stathmin, in vitro, using either anti-Op18 antibodies or antisense oligonucleotides, increases the amount of microtubule polymer and decreases the frequency of catastrophes (Howell et al., 1999a). Stathmin induces microtubule destabilization through two independent mechanisms. To inhibit polymerization, stathmin decreases the concentration of tubulin that is available for incorporation into the microtubule by sequestering soluble tubulin dimers (Howell et al., 1999a; Etienne-Manneville, 2004). Additionally, in conditions where stathmin binds weakly to tubulin (i.e. at $\mathrm{pH} 7.5$ or after a C-terminus deletion), it is thought that free stathmin is able to promote catastrophe at the microtubule plus-ends, possibly by perturbing the stabilizing effects of the GTP cap through an increase in GTP hydrolysis (Howell et al., 1999b). Other 
microtubule destabilizers act to regulate microtubules via other mechanisms, for example, by enzymatically severing the microtubule (i.e. katinin), by inducing conformational changes in the tubulin dimers that favour microtubule destabilization (i.e. kin-1 family of kinesin-like proteins), or by inhibiting the nucleation process (i.e. microtubule nucleation suppressor (MINUS)) (Fanara et al., 1999; Nogales, 2001).

Another group of proteins, the MAP2/Tau family (Tau, MAP2, and MAP4) also regulate microtubule dynamics. These vertebrate proteins have increasingly become the focus of intense research, largely due to their implications in the pathogenesis of neurodegenerative disorders like Alzheimer's disease. The MAP2/Tau family members bind to microtubules, promoting stabilization of the microtubule protofilaments and preventing depolymerisation. All of these proteins, along with their splice isoforms, share a conserved carboxy terminal region that contains 3-5 microtubule-binding repeats (Himmler et al., 1989; Lewis et al., 1989; Doll et al., 1993). While MAP2 and Tau are primarily expressed in neurons, MAP4 expression is limited to non-neuronal cells and is found in several organs, including the brain, the liver and the lungs (Dehmelt and Halpain, 2004). MAP2 and Tau play a significant role in regulating axonal microtubule networks. Altered expression of these proteins can lead to a range of neuronal disorders known as tauopathies (Bhat and Setaluri, 2007). The expression levels of many MAPs are altered in tumourigenic cells and have been shown to correlate both with the development of drug resistance in tumour cells and increased tumour sensitivity to MTAs (Jordan and Wilson, 2004). Increased expression of Tau or MAP2, is associated with Ptx-resistance in breast cancer and improved docetaxelsensitivity in pancreatic cancer, respectively (Veitia et al., 2000; Rouzier et al., 2005). Hence, understanding the contributions of some of these proteins to drug sensitivity is an important goal and will impact directly on drug efficacy for particular tumour types.

\subsection{Targeting mitosis as a chemotherapeutic strategy}

\subsubsection{Regulating cell cycle and mitotic progression}

Mitosis is a highly intricate process that allows eukaryotic cells to duplicate their DNA and pass it on to two identical daughter cells. Errors in this process can be extremely detrimental to cell division; thus, cells have evolved several cell cycle checkpoints that must be satisfied before mitotic progression can occur. These checkpoints are necessary to maintain genomic integrity, and if cells cannot satisfy these checkpoints, cell cycle delay (to allow time for the cell to repair), cell growth arrest and/or apoptosis occurs. 
The cell cycle is divided into four phases $-G_{1}$ phase (Gap 1) in which the cells grow and prepare for DNA replication, $\mathrm{S}$ phase (DNA synthesis), in which the DNA is replicated, $\mathrm{G}_{2}$ phase (Gap 2), in which the cell prepares for mitosis, and $\mathrm{M}$ phase (mitosis), in which the cell segregates its duplicated DNA into two identical daughter cells. In mammalian cells there are several key checkpoints that control progression through the cell cycle, including one at the $\mathrm{G}_{1}-\mathrm{S}$ transition (restriction checkpoint), one at the $\mathrm{G}_{2}-\mathrm{M}$ transition (DNA damage checkpoint) and another one during mitosis (spindle-assembly checkpoint). These checkpoints are activated in response to various factors, such as DNA damage, stress signals, and incorrect chromosome attachment to the mitotic spindle (Giono and Manfredi, 2006). Many chemotherapeutic agents on the market today target some aspect of cell cycle regulation and generally exploit intrinsic cell cycle checkpoints to trigger cell cycle arrest or apoptosis. Examples of drugs that activate the DNA-damage response are the two topoisomerase inhibitors, camptothecin and doxorubicin, which arrest cells at $\mathrm{S}$-phase and $\mathrm{G}_{2}$-phase, respectively (Xiao et al., 2003).

\subsubsection{CDKs and pRb}

A family of proteins called cyclin-dependent kinases (CDKs) essentially control cell cycle progression. In turn, CDKs themselves are regulated by cyclins (e.g. cyclins A, B, $\mathrm{D}$ and $\mathrm{E}$ ) and cyclin-dependent kinase inhibitors (CKIs) (Schwartz, 2002). CDKs are serine-threonine kinases that require binding to cyclins for their activation (Blomen and Boonstra, 2007). The expression levels of each of the cyclins are cell cycle dependent and act in conjunction with different CDKs (see Fig. 1.3). For example, cyclin $\mathrm{D} / \mathrm{CDK} 4 / 6$ and cyclin $\mathrm{E} / \mathrm{CDK} 2$ complexes are both active during $\mathrm{G}_{1}$, cyclin $\mathrm{A} / \mathrm{CDK} 1$ is active during $\mathrm{S}$ phase, and both cyclin $\mathrm{A} / \mathrm{CDK} 1$ and cyclin $\mathrm{B} / \mathrm{CDK} 1$ are active during $\mathrm{G}_{2}$ and $\mathrm{M}$ phases (Blomen and Boonstra, 2007). To control cell cycle progression, the phosphatases Cdc25A (targets cyclin E/CDK2 during S-phase) and Cdc25C (targets cyclin $\mathrm{B} / \mathrm{CDK} 1$ during $\mathrm{G}_{2}-\mathrm{M}$ progression) dephosphorylate, and hence activate, their respective cyclin/CDK complexes. Once activated, cyclin/CDK complexes can phosphorylate downstream targets to drive cell cycle progression. During early $G_{1}$, for example, the increase in cyclin D expression (in response to mitogenic signals) and subsequent interaction with CDKs induces hyperphosphorlyation and deactivation of the tumour suppressor retinoblastoma protein (pRb) (Kato et al., 1993; Weinberg, 1995). This releases the sequestered E2F family of transcription factors, resulting in the activation of genes necessary for DNA replication (Giono and Manfredi, 2006). It is this 
interaction between $\mathrm{pRb}$ and cyclin $\mathrm{D} / \mathrm{CDK}$ that is often referred to as the restriction checkpoint. The activity of $\mathrm{pRb}$ is negatively regulated by two families of CKIs, the INK4 (inhibitor of kinase 4) family (i.e. $\mathrm{p} 16^{\mathrm{INK} 4 \mathrm{a}}, \mathrm{p} 15^{\mathrm{INK} 4 \mathrm{~b}}, \mathrm{p} 18^{\mathrm{INK} 4 \mathrm{c}}$, and $\mathrm{p} 19^{\mathrm{INK} 4 \mathrm{~d}}$ ) and the Cip/Kip family (i.e. p2 $7^{\mathrm{Kip} 1}, \mathrm{p} 21^{\mathrm{Cip} 1}$ and $\mathrm{p} 57^{\mathrm{Kip} 2}$ ) (Blomen and Boonstra, 2007). During the induction of cell cycle arrest, CKIs become activated and inhibit the ability of CDKs to phosphorylate $\mathrm{pRb}$, leading to its activation and tumour suppressive effects. Once past the restriction checkpoint the cell commits itself to mitosis and will progress even in the absence of mitogenic stimulation.

\subsubsection{CDKs and p53}

Another key regulator of cell cycle progression is the tumour suppressor protein, p53. Mutations in the p53 gene or the p53 signalling pathway are one of the most frequently observed changes in human cancers, and previous studies have highlighted the importance of p53-mediated cell cycle regulation in preventing tumourigenesis. It has been shown that p53-deficient mice are highly susceptible to the development of numerous tumour types, with $74 \%$ developing cancers by six months (compared to $0 \%$ and $2 \%$ developing tumours in wild-type and heterozygotes, respectively, after 9 months) (Donehower et al., 1992). It is thought that p53 is not necessary for initiating cell cycle arrest but may play more of a role in the maintenance of it (Bunz et al., 1998; Giono and Manfredi, 2006). p53 is activated in response to various stimuli, such as DNA damage, hypoxia, oncogenic activation, $\mathrm{pH}$ changes and heat shock. Activation triggers signalling pathways that will ultimately determine cell fate (i.e. cell cycle arrest, senescence, DNA repair, quiescence, or apoptosis). Activation of either the $G_{1}$ or $G_{2}$ DNA damage checkpoints can drive the p53-mediated transcriptional activation of p $21^{\mathrm{CIP} 1 / \mathrm{WAF} 1}$ (p21), a CKI. Activated p21 translocates to the nucleus where it further inhibits cyclin/CDK complexes and leads to a subsequent cell cycle arrest (see Fig. 1.3). The cyclin $\mathrm{B} / \mathrm{Cdc} 2$ complex is important in the onset of mitosis and can be regulated by the phosphorylation state of $\mathrm{Cdc} 2$. Phosphorylation of $\mathrm{Cdc} 2$ renders the complex inactive; whereas, dephosphorylation by Cdc25C triggers its activation (Strausfeld et al., 1991; Hoffmann et al., 1993). It has been suggested that during $\mathrm{G}_{2}$, activation of the DNA damage checkpoint causes inactivation of $\mathrm{Cdc} 2$ via at least two independent pathways involving p21 (Smits et al., 2000). Other pathways that are prominent in the $\mathrm{G}_{2}-\mathrm{M}$ checkpoint include the p53-activating ATR/CHK1 and ATM/CHK2 pathways, which are also activated in response to DNA damage (Zhou and Elledge, 2000; Giono and Manfredi, 2006). CHK1 and CHK2 phosphorylate Cdc25C, targeting it for 
degradation and inhibiting its activation of cyclin B/CDK1 complexes. The interactions of these cell cycle regulators are highly complex and are still in the process of being fully elucidated. 


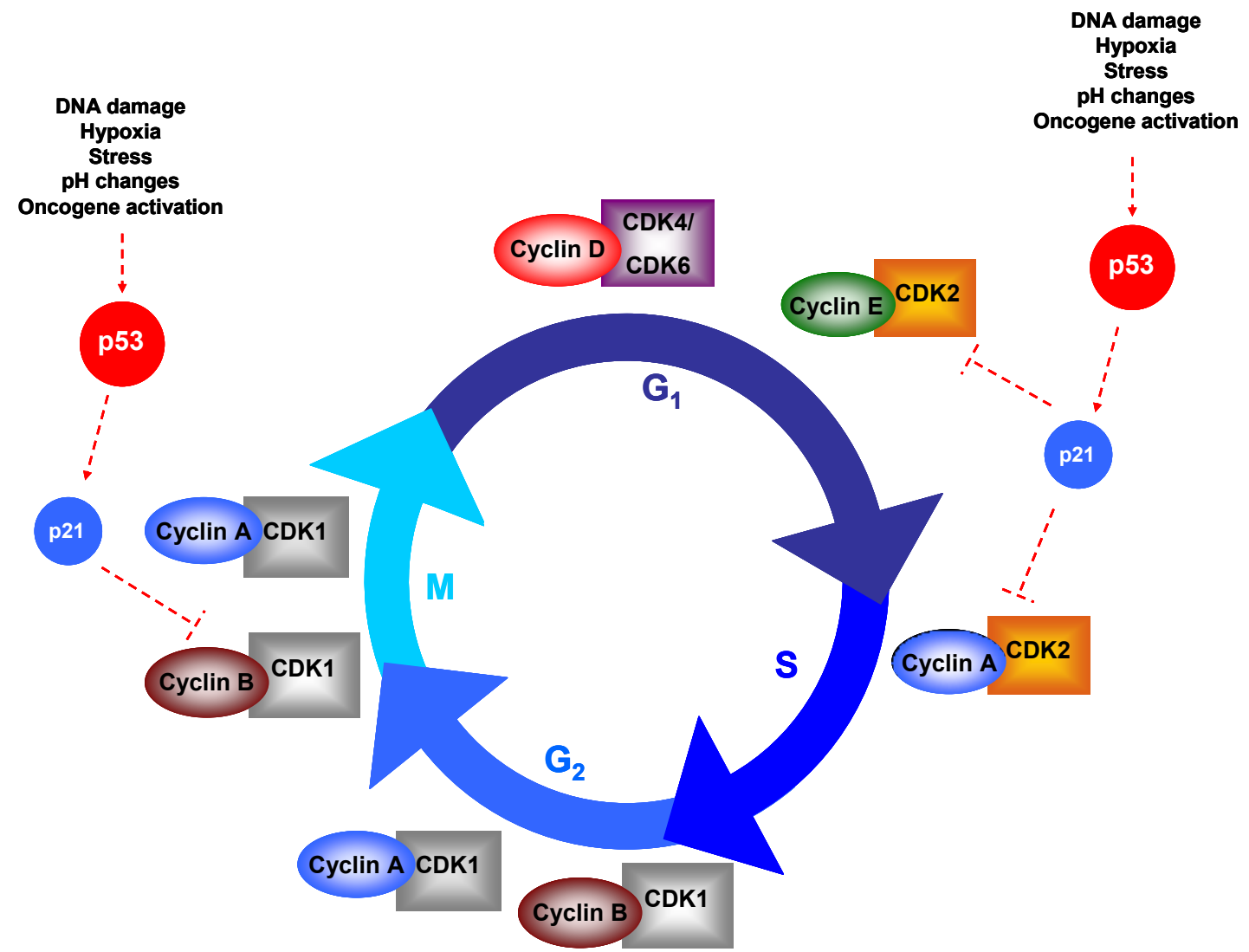

Figure 1.3. Cell cycle regulation by cyclins. A highly simplified diagram showing the cell cycledependent expression of cyclin/cyclin dependent kinase (CDK) complexes. Various stimuli trigger activation of the tumour suppressor protein $\mathrm{p} 53$, leading to activation of the CKI p21 and subsequent inhibition of cyclin/CDK complexes that are necessary for cell cycle progression. 


\subsubsection{The spindle-assembly checkpoint}

Surveillance mechanisms or checkpoints during mitosis offer opportunities to correct errors that are potentially damaging to cell division. Mitosis is divided into 5 distinct phases - prophase, prometaphase, metaphase, anaphase, and telophase. During prophase, chromatin (DNA and its associated proteins) begins to condense into chromosomes, and the nuclear envelope begins to disintegrate. At this stage, molecular motors aid the movement of centrosomes to opposite sides of the cell to begin formation of the mitotic spindle. Prometaphase is described as the stage at which kinetochore microtubules begin their 'search and capture' actions, so that each chromosome is attached to opposite spindle poles. At metaphase, the opposing forces that are generated on each chromosome cause them to align on the metaphase plate. During anaphase, the proteins binding sister chromatids together are degraded, and each chromatid is pulled towards opposite spindle poles. Non-kinetochore microtubules elongate to help drive each set of chromatids apart, and then the initial steps are reversed during telophase (i.e. chromosomes decondense, the nuclear envelope reforms, and the mitotic spindles are lost) (Pines, 2006). Cytokinesis, which involves actin more than microtubules and is not technically part of mitosis, completes cell division by cleaving the cell into its two daughter cells.

It is critically important that both anaphase and cytokinesis do not occur prematurely (i.e. so that chromosomes that are not bi-orientated to the spindle do not segregate early, and that cytokinesis does not take place before chromatid segregation is complete. If mitosis is not properly regulated, chromosomes can be lost or gained, resulting in aneuploidy. The spindle-assembly checkpoint (SAC) is extremely important in ensuring the fidelity of chromosome segregation, acting to delay anaphase until each chromosome is correctly attached to the spindle (the mechanisms underlying the SAC are discussed in more detail in Chapter 4). This checkpoint senses the interactions between kinetochores and the plus ends of kinetochore microtubules (kMTs). The numbers of kMTs that bind to a single kinetochore varies during mitosis, with bundles of approximately 20-30 kMTs forming the subsequent K-fibers (McEwen et al., 1997). Once the SAC has detected incorrect attachments, a number of proteins localize to the kinetochores, triggering various signalling cascades that transfer this information to effector proteins to regulate anaphase progression (Musacchio and Hardwick, 2002). During the "search and capture" phase, chromosomes can undergo several different types of chromosome orientation as they try to correctly attach to the spindles, including 
amphitelic (each sister chromatid attaches to opposite spindle poles), monotelic (only one chromatid attaches to a spindle pole), syntelic (both chromatids attach to K-fibers emanating from the same spindle pole) and merotelic (one chromatid simultaneously attaches to both spindle poles) attachments. Aurora B kinase functions in the chromosomal passenger complex (CPC) along with inner-centromere protein (INCENP), Survivin and Borealin, to destabilize microtubules with erroneous attachments (i.e. syntelic and merotelic attachments). It is thought that the ability of Aurora B to sense these particular attachments is linked to the generation of tension between sister chromatids. One model proposes that a lack of tension brings Aurora B in close enough proximity to phosphorylate kinetochore substrates (e.g. Ncd80, kinesin13 and Dam1) that destabilize microtubule attachments (Tanaka et al., 2002; Liu et al., 2009; Nezi and Musacchio, 2009). Once bi-polar attachment is achieved, the interkinetochore tension physically draws Aurora B away from its targets. However, a somewhat contentious issue that is not yet resolved, is whether the SAC only detects unattached kinetochores or whether it detects both a lack of attachments and a lack of tension. Arguments for and against the SAC sensing both signals have recently been reviewed (Nezi and Musacchio, 2009; Khodjakov and Pines, 2010). It has become increasingly important to understand the underlying mechanisms of the SAC, not only from a cell biology perspective, but also because of the implications for current chemotherapeutics, such as the MTAs and Aurora kinase inhibitors that perturb this checkpoint.

\subsection{Microtubule-targeting agents}

Given the diverse cellular functions of microtubules, it is not surprising that these polymers have become such successful chemotherapeutic targets. MTAs are highly effective anti-proliferative agents, particularly against rapidly dividing cells, because they interfere with the mitotic spindle, leading to mitotic arrest and subsequent cell death. MTAs, however, are not without their drawbacks, and this has led to an ongoing search for novel MTAs with improved therapeutic outcomes. Natural products, in particular, have become a rich source of new tubulin-binding compounds. As a survival mechanism, many organisms evolve protective mechanisms against potential predators, which quite often involve the generation of toxic chemical compounds. Natural products have proven to be a goldmine source for new MTAs, due in part to their impressive diversity and structural complexity, which cannot be easily replicated through synthetic approaches involving targeted drug design (Miller et al., 2010). The 
chirality that tends to occur in these natural compounds facilitates binding to complex biological targets, such as proteins and receptors, a property that drives their success in the pharmaceutical industry (Kingston, 2009). Over the last few decades approximately two-thirds of all oncology drugs have either been isolated from natural products or are derived from natural products (Kingston, 2009). Table 1 illustrates a range of MTAs at various stages of development and lists the diverse natural sources from which they originate (with the exception of 2-methoxyestradiol (2-ME), which is an endogenous metabolite in humans). Intriguingly, these organisms have all evolved to produce compounds against the same primary target (i.e. tubulin), although they have been shown to bind at several different distinct sites. 


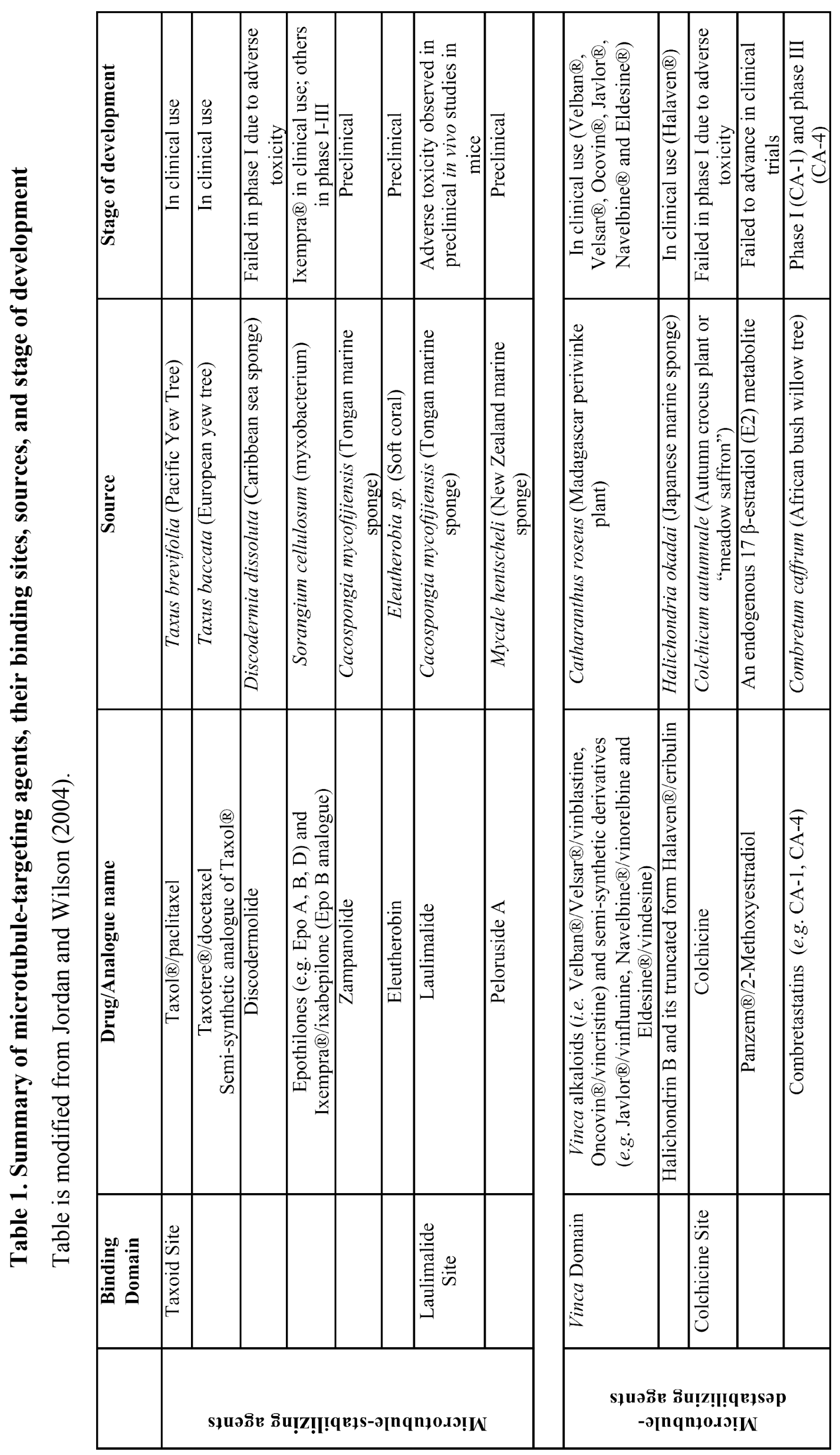


Several binding sites on $\beta$-tubulin have now been identified, including the Taxane site, Vinca domain, colchicine site, and the more recently discovered laulimalide/peloruside site. The microtubule-stabilizing agent (MSA) Ptx is probably the best characterised of the MTAs and has become the gold standard for studying microtubule-interfering compounds. Ptx was first isolated from the Pacific Yew tree, Taxus brevifolia (see Table 1), and has been approved for treatment against several solid tumours, (e.g. breast, ovary, and lung) and Kaposi's sarcoma, and is currently undergoing single and combination trials for various other tumours (Jordan and Wilson, 2004). Ptx and its semi-synthetic analogue Dtx, bind at the taxoid binding site, which is located on the microtubule lumen (Díaz et al., 1998). Competitive inhibition studies using ${ }^{3} \mathrm{H}-\mathrm{Ptx}$ have indicated that the MSAs discodermolide (Disco), eleutherobin, and epothilones A and B (EpoA and EpoB) also compete for the taxoid binding site with ${ }^{3} \mathrm{H}-\mathrm{Ptx}$ (Bollag et al., 1995; Kowalski et al., 1997; Long et al., 1998). Currently, the only other MSAs that have been shown to bind to a site distinct from the taxoid site are laulimalide and PelA (discussed in more detail later in this chapter) (Pryor et al., 2002; Gaitanos et al., 2004). Their binding site has been more contentious, with earlier computer modelling studies suggesting that these drugs bind preferentially to a unique site on $\alpha$-tubulin (Pineda et al., 2004; Jiménez-Barbero et al., 2006). More recent experiments using data-directed molecular docking simulations, however, suggest that PelA binds to a site on the exterior of $\beta$-tubulin (Huzil et al., 2008). Mutational studies from our laboratory group using two PelA- and laulimalide-resistant cell lines, 1A9-R1 and 1A9-L4, further support the site originally proposed by Huzil et al. (Huzil et al., 2008; Kanakkanthara et al., 2011). Sequencing of the predominant $\alpha$ - and $\beta$-tubulin isotypes (K1 $\alpha$ and $\beta I)$ in 1A9-L4 and 1A9-R1 cells identified a heterozygous single point mutation in $\beta I$-tubulin (R306 to either H306 or C306) and single point mutation in $\beta$ I-tubulin (A296T), respectively in the two cell lines. Using several different methods (data-directed molecular docking simulations, ${ }^{3} \mathrm{H}-\mathrm{Pel}$ A competition assays, and mutational analyses), other independent research groups have also proposed a similar region on $\beta$-tubulin for the site of laulimalide and PelA binding (Bennett et al., 2010; Nguyen et al., 2010; Begaye et al., 2011). It is possible that there are two sites for PelA and laulimalide, one on $\beta$-tubulin and a second, lower affinity site on a-tubulin. Although it is still contentious, there is some evidence pointing to a third, lower affinity site, the pore type I site, which can transiently or permanently (this is still under investigation) accommodate drugs that bind to the taxoid site (Díaz et al., 2003; Calvo et al., 2012). 
The pore site is proposed to be an opening in the microtubule wall that assists in the transport of these ligands to the final luminal binding site (Díaz et al., 2003), and work is ongoing to further characterize this potential binding site.

Only two sites are known to exist for microtubule-destabilizing agents (MDAs), one on $\beta$-tubulin (the Vinca alkaloid binding site) and the other at the $\alpha / \beta$-tubulin interface (the colchicine binding site) (Bhattacharyya et al., 2008). Vinblastine (Vbl) and vincristine (Vcr) are plant-derived compounds (see Table 1) that belong to a class of MDAs called the Vinca alkaloids. These two drugs are now used to treat various neoplasms, including leukemias and lymphomas (Vcr), and Hodgkin's disease or testicular cancer (Vbl) (Jordan and Wilson, 2004; Kingston, 2009). Several compounds that bind to the colchicine-site (see Table 1) are currently being investigated as potential therapeutics. Colchicine itself is unlikely to be used as an anti-cancer treatment due to its severe toxicity against non-cancerous cells; however it has been used successfully as an antiinflammatory agent to treat other pathologies like gout, familial Mediterranean fever and liver cirrhosis (Bhattacharyya et al., 2008). 2-ME (an endogenous steroid metabolite) and the combretastatins (derived from the African willow tree, Combretum caffrum) are both undergoing investigation as chemotherapeutics because of their antiproliferative and vascular-disrupting properties, respectively (Kamath et al., 2006; Tozer et al., 2008).

The existence of several binding sites on tubulin has opened up numerous possibilities for combination therapies using MTAs that are able to work synergistically with each other, and also with other classes of anti-cancer drugs. The benefits of combining two or more drugs are two-fold. Lower doses of each drug can be given with improved efficacy, while reducing the severity of toxic side effects. Studies have now challenged a long-standing view that drugs that bind to the same site do not act synergistically. For example, Ptx and Disco have been shown to exhibit synergistic interactions in vitro, indicating that despite similar or overlapping binding sites, MTAs trigger complex signalling pathways that are not necessarily identical (Honore et al., 2004). Additionally, although they have opposing effects on microtubule polymer mass, MSAs and MDAs can also act synergistically together. It has been reported previously that laulimalide, synthetic laulimalide analogues, and Ptx all act synergistically with 2-ME to inhibit proliferation (Clark et al., 2006). Synergy between PelA or laulimalide and taxoid site drugs, have also been reported (Clark et al., 2006; Wilmes et al., 2007; 
Wilmes et al., 2011a). Clinically, Ptx is used in combination chemotherapy to improve progression-free survival and/or overall survival of breast cancer patients, in particular. Examples include the clinical use of Ptx with carboplatin (Paraplatin ${ }^{\circledR}$ ), gemcitabine $\left(\right.$ Gemzar $\left.{ }^{\circledR}\right)$, trastuzumab (Herceptin $\left.{ }^{\circledR}\right)$, or bevacizumab; however, the FDA recently withdrew approval for bevacizumab in combination with Ptx to treat metastatic, HER2negative breast cancer not yet treated with chemotherapy, due to failures at phase III clinical trials (Robert et al., 2011). Many additional combination therapies involving microtubule-targeting drugs are currently being evaluated in ongoing clinical trials. 


\subsection{Tumour suppressive mechanisms of microtubule-targeting agents}

\subsubsection{Programmed cell death}

Programmed cell death (PCD) is a critical control mechanism of physiological development and cellular homeostasis, leading to the destruction and removal of damaged or potentially dangerous cells. Dysfunction in any of the processes regulating PCD can lead to disease pathogenesis through abnormal cell proliferation (e.g. tumourigenesis) or abnormal cell death (e.g. Alzheimer's disease). PCD, a term that was once used synonymously with apoptosis, has now expanded to encompass other forms of cell death that do not necessarily share the morphological and biochemical characteristics of classical apoptosis (Wouters and Chiu, 2007). The Nomenclature Committee on Cell Death have given their recommendations regarding the use of the term 'apoptosis', as well as tentative definitions for alternative cell death modalities that can occur, including mitotic catastrophe, autophagy and necrosis (Kroemer et al., 2009).

The anti-cancer effects of MTAs can be attributed to their ability to exploit multiple pathways leading to cell death, regardless of whether this is via apoptosis or alternative forms of cell death. As this thesis is primarily concerned with apoptosis (rather than alternative forms of PCD), the term "cell death" will be used synonymously with either caspase-dependent or caspase-independent apoptosis (described below), unless specifically stated otherwise. Many chemotherapeutic drugs selectively target malignant cells and rely on the fact that cancer cells tend to grow far more rapidly compared to most normal, healthy cells. Cancer cells are, therefore, more sensitized to treatments that target processes involved in cellular proliferation, including those that target mitosis and induce cellular death. Some non-cancerous cells, however, like intestinal villi cells and hair follicles, also rapidly proliferate and are acutely sensitive to anticancer drugs, giving rise to unwanted side effects of these drugs. Further elucidation of the mechanisms behind PCD is important not only in terms of understanding disease progression and resistance to anti-cancer drugs, but also for identifying novel therapeutic targets or novel methods of sensitizing malignant cells to cell death.

Chemotherapeutic drugs generally trigger some form of PCD (usually apoptosis) in their target cells. Apoptosis is a highly regulated form of PCD, involving a chain of biochemical reactions that terminates in characteristic morphological changes (e.g. blebbing, cell shrinkage, chromatin condensation, and nuclear fragmentation). Enormous effort has gone into characterising, in detail, the signalling pathways that lead 
to apoptosis, although there is still much left to be discovered. The biochemical and molecular mechanisms underlying apoptosis are discussed in more detail in Chapter 3. In short, apoptosis is driven largely by a series of signalling cascades involving caspases (cystein-dependent aspartate specific proteases). Apoptosis is triggered by both intraand extra-cellular cues that lead to the activation of initiator caspases (usually caspase- 8 or caspase-9). This leads to further cleavage and activation of downstream effector or executioner caspases (typically caspase-3). Over 280 caspase substrates have so far been identified that can be either activated or inhibited upon cleavage (Fischer et al., 2003). Substrates include proteins that, once cleaved, promote the morphological changes associated with apoptosis. For instance, cleavage of proteins that are involved in DNA repair (e.g. PARP (poly (ADP) ribose polymerase)) and maintenance of the cytoskeleton (e.g. vimentin) can facilitate DNA fragmentation and cytoskeletal reorganization, respectively (Nicholson et al., 1995; Byun et al., 2001; Fischer et al., 2003). There are two distinct apoptotic pathways, the extrinsic or death receptor pathway (mediated by caspase-8) and the intrinsic or mitochondrial pathway (mediated by caspase-9) (see chapter 3 for more detail). While it is generally accepted that MTAs induce apoptosis via the intrinsic pathway, the molecular links between microtubule binding (and ensuing SAC activation) with the apoptotic machinery are still not well understood (Bhalla, 2003).

MTAs trigger similar apoptotic signalling cascades despite differences in their binding sites and regardless of whether they polymerize or depolymerize microtubules (Estève et al., 2007). Perturbation of microtubule dynamics leads to activation of the SAC, sustained cyclin B1 levels (degradation of cyclin B1 is necessary for mitotic progression), and subsequently, mitotic arrest (Choi et al., 2011). Initially it was thought that prolonged mitotic arrest was primarily responsible for MTA-induced apoptosis; however, it has become apparent that the activation of apoptotic signal transduction pathways by this particular class of anti-cancer drugs is much more complex. Apoptosis can occur (albeit at a lower level) even in the absence of a significant mitotic block, indicating that mitotic arrest is not necessarily essential to cell death induction (Jordan et al., 1996). While some cells ultimately die in mitosis, it has also become apparent that cells can undergo mitotic slippage, in which they exit mitosis (even if they do not satisfy the SAC) without undergoing anaphase/cytokinesis and enter an interphase-like state (Jordan et al., 1996; Brito et al., 2008; Bekier et al., 2009). A relatively recent study has proposed that the duration of mitotic arrest may impact on cell fate. Bekier et 
al. (2009) found that HeLa cells blocked in mitosis for longer than $15 \mathrm{~h}$ died rapidly after exiting mitosis; whereas, cells blocked for less than $15 \mathrm{~h}$ succumbed to various fates with some cells surviving additional cycles of replication to become large and multinucleated (Bekier et al., 2009). In Bekier's study, the Aurora kinase inhibitor, ZM447439, was used to artificially drive cells out of mitosis by abrogation of the SAC. An earlier study used high-content screening (automated microscopy) to follow the natural mitotic progression of a panel of 11 cell lines in the presence of three antimitotic drugs (Ptx, nocodazole and K5I (a kinesin-5 inhibitor)) (Shi et al., 2008). In contrast to the apoptotic response, which was highly variable, the levels of mitotic arrest showed little variation between cell lines; thus, Shi and colleagues found no correlation between these two parameters. Additionally, this study was one of many that have reported a classical, caspase-mediated form of apoptosis in response to MTAs. Since it has been proposed that MTAs trigger apoptosis through the intrinsic pathway (Bhalla, 2003), numerous papers have identified key signalling players of the intrinsic pathway that are involved in MTA-induced apoptosis, most notably, the BCL-2 family of proteins. Inactivation of anti-apoptotic factors (e.g. phosphorylation of BCL-2 and BCL-xL) and activation of pro-apoptotic molecules (e.g. Bad and Bax), have all occurred after treatment with MTAs (Haldar et al., 1995; Poruchynsky et al., 1998; Du et al., 2004; Kutuk and Letai, 2010). While some studies have suggested an alternative caspase-independent mechanism of MTA-induced cell death, this does not appear to be typical of these drugs and may reflect mechanisms specific for particular drugs in particular cell lines (Ofir et al., 2002; Bröker et al., 2004). Despite the progress that has been made, the fact that so many factors can influence the induction of apoptosis (e.g. drug type and drug concentration, treatment duration, and cell type) has hindered drawing firm conclusions about the direct links between microtubule disruption and apoptosis induction. Another complicating factor of many of these studies has been the use of unsynchronized, heterogeneous populations of cells, which may be less informative than single cell studies (Gascoigne and Taylor, 2008). As better probes become available, apoptosis studies may move towards, for example, single cell timelapse studies that will help identify the exact contributions of different signalling players.

\subsubsection{Cellular senescence}

Depending on the severity of the signal, cells respond to damage or stress signals via several distinct means, including apoptosis and cellular senescence (Campisi and 
d'Adda di Fagagna, 2007). Induction of cellular senescence by chemotherapeutic drugs is a well-established mechanism for tumour suppression whereby proliferating cells are induced to adopt a permanent state of arrested cell growth. Two forms of cellular senescence have currently been described - replicative senescence and premature (or accelerated) senescence. Replicative senescence arises from the cumulative shortening of telomeres during successive cell division cycles and was initially proposed by Hayflick and his colleagues, to occur as part of the natural ageing process (Hayflick and Moorhead, 1961). Like apoptosis, replicative senescence acts as an intrinsic homeostatic mechanism to reduce the risk of tumourigenesis. In normal dividing cells, errors in DNA replication and repair, as well as somatic mutations, can contribute to cancer progression. As proliferating cells are more likely to acquire mutations or genomic damage, limiting the number of cell divisions through replicative senescence effectively acts as a tumour suppressive mechanism (Campisi and d'Adda di Fagagna, 2007). Premature senescence (discussed in more detail in Chapter 6) can occur independently of telomere length in response to stressful stimuli (e.g. DNA damage, oxidative stress, irradiation, oncogene activation and overexpression of mitogenic signals) (Klein and Horwitz, 2007). Initially it was thought that the predominant effect of many chemotherapeutic drugs was their cytotoxic effect, although it has now been shown that some cells can evade apoptosis to continue proliferating, transiently stop proliferating $\left(G_{1}\right.$ or $G_{2}$ cell cycle arrest), or adopt a state of arrested cell growth (senescence) (Dimri, 2005; Klein and Horwitz, 2007).

At its most basic level, senescence describes an irreversible state of cell cycle arrest that persists even in the presence of mitogenic stimuli (Shay and Roninson, 2004). Regardless of the initial trigger (i.e. shortened telomeres or cellular stressors) or the precise signal transduction pathway(s) (described below), cells that undergo replicative and premature senescence share similar phenotypical characteristics. To date, no individual marker specific to senescence has been identified; thus, distinguishing senescent cells from, for example, quiescent cells $\left(\mathrm{G}_{0}\right.$ arrested cells that can re-enter the cell cycle), relies on the collective presentation of several phenotypes (although it should be noted that some senescent cells do not display all of the possible senescence features) (Rodier and Campisi, 2011). The morphological and biochemical features that are used to define a senescent phenotype (discussed in more detail in Chapter 6) have been summarized in several reviews, and include: (1) a permanent cell cycle arrest that is essentially irreversible (i.e. cells are metabolically active but no longer synthesize 
DNA), (2) an enlarged, flattened morphology with increased granularity and a vacuolerich cytoplasm, (3) an increase in lysosomal mass that results in increased senescenceassociated $\beta$-galactosidase (SA- $\beta$-gal), (4) the formation of senescence-associated heterochromatin foci (SAHF) (i.e. chromatin that has re-organized into compact foci of DNA), (5) senescence-associated DNA-damage foci (SDF) that can be detected via markers of DNA damage (e.g. phosphorylated histone H2AX $(\gamma-\mathrm{H} 2 \mathrm{AX}))$, and (6) the activation of signalling mediators involved in the p53 and $\mathrm{pRb}$ tumour suppressor pathways (Campisi and d'Adda di Fagagna, 2007; Schmitt, 2007; Gewirtz et al., 2008; Rodier and Campisi, 2011). Surprisingly, relatively few studies have addressed the ultimate fate of these cells although, in theory, senescent cells should be able to survive indefinitely under appropriate environmental conditions. Senescent cells have been known to survive for months (or even over a year) in culture, and it has been suggested that some senescent cells may be resistant to apoptosis due to increased levels of antiapoptotic proteins (d'Adda di Fagagna et al., 2003; Roninson, 2003). However, more research must be done to determine why certain subsets of cells are induced to undergo such varied outcomes, as other cell fates have been observed concurrently with the induction of senescence, including apoptosis and mitotic catastrophe (Klein et al., 2005; Gewirtz et al., 2008; Litwiniec et al., 2010).

Numerous studies have revealed a significant number of senescence-inducing stimuli, which have in some way or another, all been linked to DNA damage. Shortened telomeres, for example, can elicit a persistent DNA damage response (DDR), similar to those induced by DNA double-strand breaks (DSBs) (d'Adda di Fagagna et al., 2003). Unsurprisingly, triggers that directly induce DSBs, such as particular chemotherapy drugs (e.g. Doxo and actinomycin D) and $\gamma$-irradiation, are also very good inducers of cellular senescence (Di Leonardo et al., 1994; Robles et al., 1999). Activation of oncogenes and excessive mitogenic signals can trigger DDR signalling as a result of stalled replication forks, an increased number of DNA replication origins and reactive oxygen species (ROS)-induced DNA damage (Lee et al., 1999; Bartkova et al., 2006; Di Micco et al., 2006). Activation of DDR pathways effectively act to repress genes that are involved in cell cycle progression and stimulate those involved in inhibiting cell proliferation (Shay and Roninson, 2004). The pRb and p53 tumour suppressor pathways (described earlier in this chapter) regulate cell cycle progression and numerous studies have illustrated the involvement of both of these pathways in the induction of cellular senescence (Gewirtz et al., 2008; Rodier and Campisi, 2011). DNA damage can trigger 
activation of the ATR/CHK1 and ATM/CHK2 pathways, both of which lead to activation of p53 (and downstream p21), as well activation of the CKI p16 $6^{\mathrm{INK} 4 \mathrm{a}}$, which subsequently leads to pRb activation (Alcorta et al., 1996; Lin et al., 1998; Schmitt et al., 2002; Bartkova et al., 2005; Rodier and Campisi, 2011).

The induction of cellular senescence by chemotherapeutic drugs acts as an effective mechanism for tumour suppression. Doxo is an anthracycline antibiotic, used widely as an anticancer agent, that stabilizes cleavable DNA-topoisomerase II intermediate complexes (Tewey et al., 1984). While a high dose of chemotherapeutic drug treatment typically induces cell death, it was observed that lower doses (often given over a longer period of time) triggered a cytostatic response. Doxo and other cytotoxic, DNAdamaging compounds, including cisplatin, etoposide and aphidicolin, have been shown to powerfully induce a senescence-like phenotype (Chang et al., 1999a). In comparison, most of the MTAs that have been assessed for their ability to induce accelerated senescence (e.g. Ptx and Vcr) are much less effective at inducing premature senescence (Chang et al., 1999a; Klein and Horwitz, 2007). To date, the only MTA that has been shown to induce premature senescence comparably to Doxo is the taxoid binding site drug, Disco (Klein et al., 2005).

\subsubsection{Tumour-induced angiogenesis and anti-angiogenic drugs}

Although largely studied for their effects on mitosis, many MTAs are also being investigated for their anti-angiogenic or vascular disrupting properties. Angiogenesis is the formation of new blood vessels from pre-existing vessels (as opposed to vasculogenesis - blood vessel formation from newly differentiated endothelial progenitor cells), and it is involved in numerous physiological processes, including embryonic development, wound healing, and reproduction. It is, however, also associated with several pathologies, such as infantile haemangioma, rheumatoid arthritis, atherosclerosis, and cancer (Folkman, 2006). One of the primary concerns when the initial diagnosis of cancer is made is whether or not the cancer has metastasized (spread to other locations distinct from the primary tumour). Metastatic cancers severely impact on a patient's prognosis and also affect the treatment outcomes and treatment options that are available. The processes that lead to metastasis are complex and poorly understood, although it has long been evident that angiogenesis plays a predominant role. Tumour cells that obtain nutrients from their environment through diffusion are limited to sizes of approximately 1-3 $\mathrm{mm}^{3}$ and need an additional 
blood supply for continued growth (Folkman, 1971). The angiogenic process that occurs in some tumours provides these cells with a sufficient supply of nutrients and oxygen, and also increases their metastatic potential by allowing cancer cells to enter the circulating blood stream (see Fig 1.4). It is still unclear, however, why certain tumours are induced to become angiogenic, and hence metastatic, while others are not, and whether new and improved therapies could be developed that target some of the processes that lead to angiogenesis.

Endothelial cells (EC) progress through several well-defined steps during tumour angiogenesis. These cells must first penetrate the existing vasculature by degrading the basement membrane and surrounding extracellular matrix (ECM) to allow migration into the perivascular space (Eichhorn et al., 2007). These cells then proliferate and migrate in a chemotactic manner towards angiogenic stimuli to form new capillaries. A widely accepted theory as to what may trigger angiogenesis, and hence drive tumour progression, is the presence of an angiogenic switch. It has been suggested that angiogenesis induction can be triggered by changes in the balance between pro- and anti-angiogenic factors, some of which are briefly described below (Eichhorn et al., 2007). Various triggers are thought to promote the release of angiogenic stimuli from tumours that act on nearby endothelial cells. Oncogene activation, and cellular stressors resulting from increases in tumour size (such as reduced nutrients, hypoxia, and $\mathrm{pH}$ changes) are all involved in inducing angiogenesis. 


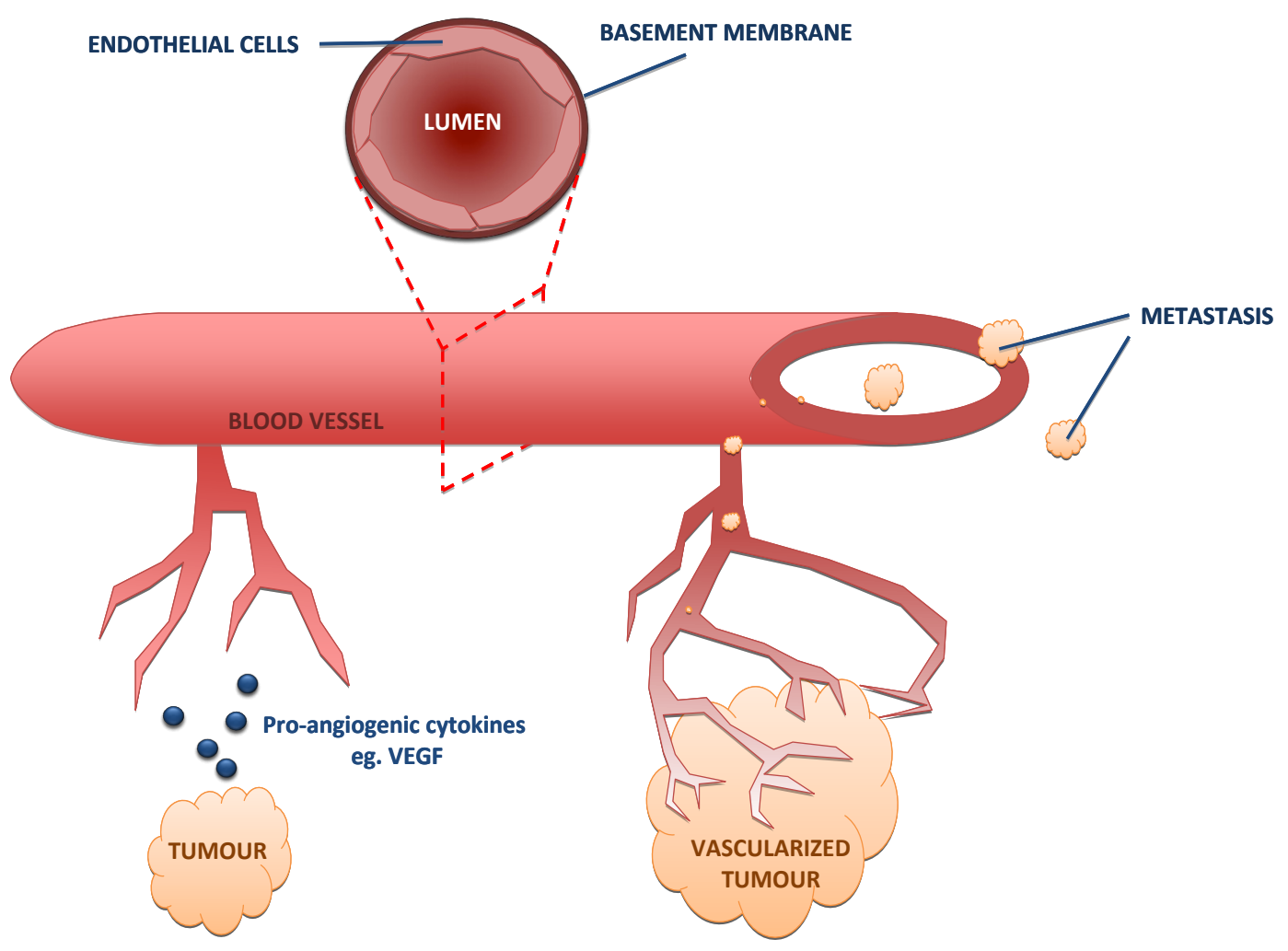

Figure 1.4. Angiogenesis can lead to tumour growth and metastasis. Under certain conditions, tumours release pro-angiogenic cytokines that stimulate the growth of new blood vessels from preexisting capillaries (angiogenesis). Tumour vascularization provides nutrients and oxygen to tumour cells, and removes wastes products, leading to tumour expansion. The new blood vessels also provide a route by which tumour cells can enter the blood stream and travel to other sites (metastasis). A simplified cross-section of a blood vessel and the inner lumen is also shown to illustrate the arrangement of endothelial cells surrounded by the basement membrane. During angiogenesis, this basement membrane is proteolytically degraded to allow endothelial cells to escape into the perivascular space. 
Due to limitations associated with passive diffusion from surrounding tissues, tumours that reach a certain size tend to form quite distinct regions within the tumour itself. Cells towards the periphery of the tumour are better positioned for the diffusion of substances from the surrounding tissue. This creates a viable rim of rapidly proliferating cells that tends to be more resistant to chemotherapies and, after chemotherapy, leads to repopulation of the tumours (Plank and Sleeman, 2003; Tozer et al., 2005). As a stressresponse mechanism, cells that have a reduced nutrient or oxygen supply, especially at the very centre of the tumour, can stop proliferating and become quiescent (Plank and Sleeman, 2003). In the absence of tumour vascularization, continued deprivation of nutrients/oxygen at the centre of the tumour often leads to a necrotic core comprised of dead cells. These low-oxygen or hypoxic regions play an extremely important regulatory role in tumour-induced angiogenesis, a process mediated primarily by the transcription factor, hypoxia inducible factor-1 (HIF-1) (Maxwell et al., 1997; Liao and Johnson, 2007). HIF-1 regulates the physiological responses to changes in oxygen levels, and its overexpression is a poor prognostic factor in many cancers ( $\mathrm{Lu}$ and Kang, 2010). HIF-1 is a heterodimer comprised of the constitutively expressed HIF-1 $\beta$ subunit, and an HIF-1 $\alpha$ subunit whose expression is oxygen-dependent (Wang et al., 1995). Under normoxic conditions, hydroxylation of HIF-1 $\alpha$ facilitates binding of von Hippel-Lindau (VHL) (a tumour suppressor protein that forms part of a complex possessing E3 ubiquitin ligase activity), targeting it for 26S proteosomal degradation (Lu and Kang, 2010). HIF-1 $\alpha$ is, therefore, stabilized in hypoxic conditions, and in conjunction with HIF- $\beta$, can regulate the transcription of a number of genes, some of which are involved in angiogenesis. One of these genes, vascular endothelial growth factor (VEGF), plays a central role in promoting tumour-induced angiogenesis (Forsythe et al., 1996; Maxwell et al., 1997).

Numerous pro-angiogenic soluble factors have now been identified, including the VEGF family, angiopoietins, various growth factors (e.g. basic fibroblast-like growth factor (bFGF), epidermal growth factor (EGF), platelet-derived growth factor (PDGF) and transforming growth factor $\beta$ (TGF- $\beta$ ), interleukin-8 (IL-8) and tumour necrosis factor- $\alpha($ TNF- $\alpha)$ ) (Gupta and Qin, 2003; Liao and Johnson, 2007). VEGF is probably the best characterised of the pro-angiogenic factors and plays a key role in angiogenesis induction. In the early 1990's, it was discovered that inhibition of VEGF, using a VEGF-specific monoclonal antibody, inhibited tumour growth in vivo (Kim et al., 
1993). This $45 \mathrm{kDa}$ protein, of which there are four isoforms, binds to two receptor tyrosine kinases (VEGFR-1 and VEGFR-2) that are expressed by endothelial cells and bone-marrow cells, and a family of coreceptors called the neuropilins (Ferrara et al., 2003). Activation of these receptors triggers similar downstream signalling pathways that regulate angiogenesis. The role of VEGFR-1 in angiogenesis is poorly understood, although there are suggestions that binding of the VEGFR-1 ligand, placenta growth factor (P1GF), can affect multiple pathways, including the phosphatidylinositol 3kinase/Akt, extracellular regulated kinase/mitogen-activated protein kinase and Janus kinase/signal transducer and activator of transcription 3 pathways (Cao, 2009). How these pathways modulate angiogenesis is not clear, but studies using VEGFR-1 knockout mice suggest that VEGFR-1 may negatively regulate angiogenesis (Hoeben et al., 2004; Cao, 2009). VEGF binding to VEGFR-2 leads to its dimerization and autophosphorylation, and can activate similar signalling pathways to VEGFR-1. These pathways can elicit changes that facilitate endothelial cell proliferation, survival, permeability and migration, all of which are important in driving angiogenesis (Gupta and Qin, 2003). Various endogenous anti-angiogenic factors have also been identified, including thrombospondin-1 (TSP-1), endostatin, and angiostatin (Good et al., 1990; O'Reilly et al., 1994; O'Reilly et al., 1997). These factors negatively regulate angiogenesis by inducing EC apoptosis or cell cycle arrest, and/or by inhibiting EC proliferation and migration.

A functional vascular network is necessary for both tumour growth and metastasis and is dependent on the formation of new blood vessels; thus, angiogenic processes offer an attractive chemotherapeutic target. The angiogenesis inhibitor, interferon- $\alpha$, was first reported in the 1980's; however, it wasn't until over two decades later, in 2004, that the first angiogenesis inhibitor was approved by the U.S. FDA for clinical use against advanced colon cancer (in conjunction with traditional chemotherapeutic drugs) (Eichhorn et al., 2007). Bevacizumab is a humanized, recombinant antibody against VEGF that was shown to significantly increase overall survival, progression-free survival, and response rate of patients with metastatic colorectal cancer when included as part of fluorouracil-based combination chemotherapy (Hurwitz et al., 2004). Bevacizumab is currently indicated for use in metastatic colorectal cancer (in combination with 5-fluorouracil-based chemotherapy), non-squamous non-small cell lung cancer (in combination with carboplatin and Ptx), glioblastoma, and metastatic renal cell carcinoma (FDA Label Information for Avastin ${ }^{\circledR}$; retrieved from 
http://www.accessdata.fda.gov/drugsatfda_docs/label/2012/125085s0238lbl.pdf). Other angiogenesis inhibitors that can target ECs directly are at various stages of clinical development. Vitaxin, caplostatin and angiostatin, for example, inhibit EC proliferation and migration; whereas, indirect inhibitors, such as trastuzumab and interferon- $\alpha$, target proteins expressed by tumours that induce angiogenesis or block expression of their receptors on ECs (Bocci et al., 2002; Kerbel and Folkman, 2002; Folkman, 2007). One of the advantages of direct angiogenesis inhibitors is that they target genetically stable ECs, so there is a reduced likelihood of acquired resistance by the cells.

There is now increasing evidence that MTAs can interfere with processes involved in the induction of angiogenesis. Ptx, Dtx, Vbl, and EpoB all modulate endothelial cell function and inhibit angiogenesis at subtoxic concentrations or concentrations that do not inhibit EC proliferation (Belotti et al., 1996; Bijman et al., 2006). Not all cytotoxic drugs, however, inhibit angiogenesis. Comparative studies have shown that cisplatin and Doxo do not significantly alter EC function; whereas, EpoB, Vbl, and Dtx, in the same assays, do (Bijman et al., 2006). Thus, Bijman et al. (2006) found that these three MTAs disrupt tubulin integrity, which in turn prevents proper actin organization and functioning. They also found that MTAs reduced the activity of two Rho GTPases (see Chapter 5 for more detail) essential for endothelial cell motility, Rac1 and Cdc42. Laulimalide is another MTA that has been shown to inhibit human umbilical vein endothelial cell (HUVEC) tubule formation and VEGF-induced HUVEC migration (Lu et al., 2006). Lu et al. (2006) also demonstrated that laulimalide and Dtx, in combination, synergistically inhibited both tubule formation and migration. The ability to simultaneously target the tumour cells themselves as well as mitigate their metastatic potential is an appealing feature for new generation MTAs. MTAs are also being investigated for their ability to directly target and disrupt the already established tumour vasculature (vascular disrupting agents or VDAs). Several VDAs are currently in clinical trials, the most noteworthy being the combretastatins (structurally related to the MDA colchicine), which were initially isolated from the Cape Bushwillow tree (Combretum caffrum) (Pettit et al., 1987). The soluble phosphate salts of combretastatin A-1 (CA-1-P) and combretastatin A-4 (CA-4-P) are currently being investigated in preclinical and clinical trials (Tozer et al., 2008). VDAs differ from anti-angiogenic compounds by causing reduced tumour blood flow and vascular shutdown as opposed to preventing the growth of new blood vessels. The ability to target multiple aspects of tumour blood vessel supply and essentially starve tumour cells of nutrients and oxygen 
is showing great clinical promise, in particular, when used in combination with more conventional chemotherapeutic treatments (Murata et al., 2001; Pedley et al., 2001; Ciric and Sersa, 2010).

\subsection{Peloruside A: a history}

In 2000, Lyndon West (under the supervision of Associate Professor Peter Northcote in the School of Chemical and Physical Sciences, Victoria University of Wellington (VUW)) isolated a novel secondary metabolite from the New Zealand marine sponge, Mycale hentscheli - PelA, along with two previously described metabolites, pateamine and mycalamide A (MycA) (West et al., 2000). Although all three were highly cytotoxic in several mammalian cell lines (e.g. $\mathrm{IC}_{50} \mathrm{~S}$ in P388 murine leukemia cells were 18, 0.3 and $6 \mathrm{nM}$, respectively) (Perry et al., 1990; Northcote et al., 1991; West et al., 2000), PelA has generated the most interest as a potential therapeutic. PelA is a polyoxygenated, 16-membered macrolide (see Fig. 1.5) that was found to have potent microtubule-stabilizing activity, similar to that of the billion dollar drug, Ptx (Hood et al., 2002). In 2004, PelA was patented in the United States (US) by Victoria Link Ltd, the commercial arm of VUW (Northcote et al., 2004). To progress its pre-clinical development, the compound was licensed in 2005 to Reata Pharmaceuticals Inc. (a Texas based company), in collaboration with the University of Texas Southwestern. Initial in vivo studies were very promising, with PelA demonstrating improved efficacy and decreased toxicity, compared to both Ptx and Dtx, in non-small cell lung xenografts in mice (Fig. 1.6) (Meyer et al., 2006). With these results in mind, VUW continued to investigate the cellular mechanisms underlying PelA's actions in cancer cell lines and have since determined that PelA exhibits many favourable characteristics for development into a potential chemotherapeutic.

Much work has been done over the years to better characterize the biological activity profile of PelA. It was initially intended that Reata Pharmaceuticals Inc. would progress the drug through to pre-clinical and clinical trials, and it was recognized early on that a sufficient supply of PelA would be needed. The M. hentscheli species of sponge is found throughout New Zealand; however, it is only a small population of these sponges located in Pelorus Sound (in the Marlborough Sound area of New Zealand) that actually produces PelA (Page et al., 2011). This restricted natural supply and the scientific challenge to synthetic chemists for a total synthesis of PelA, encouraged elucidation of a synthetic route to manufacture the compound, as well as projects to aquaculture large 
amounts of the sponge. The first total synthesis of PelA and determination of its absolute configuration was reported by De Brabender and colleagues (Liao et al., 2003). Since then, several other total synthetic routes have also been published to try and identify a more convergent, efficient approach that would be compatible with industrial scale-up (Jin and Taylor, 2005; Ghosh et al., 2008; Evans et al., 2009). At present, the large-scale synthesis of PelA has yet to be undertaken and harvesting the natural source of PelA is no longer sustainable; thus, generating sufficient quantities is a limiting factor in terms of its clinical development. Another problem with further development is the limited time left on the US patent on PelA. 


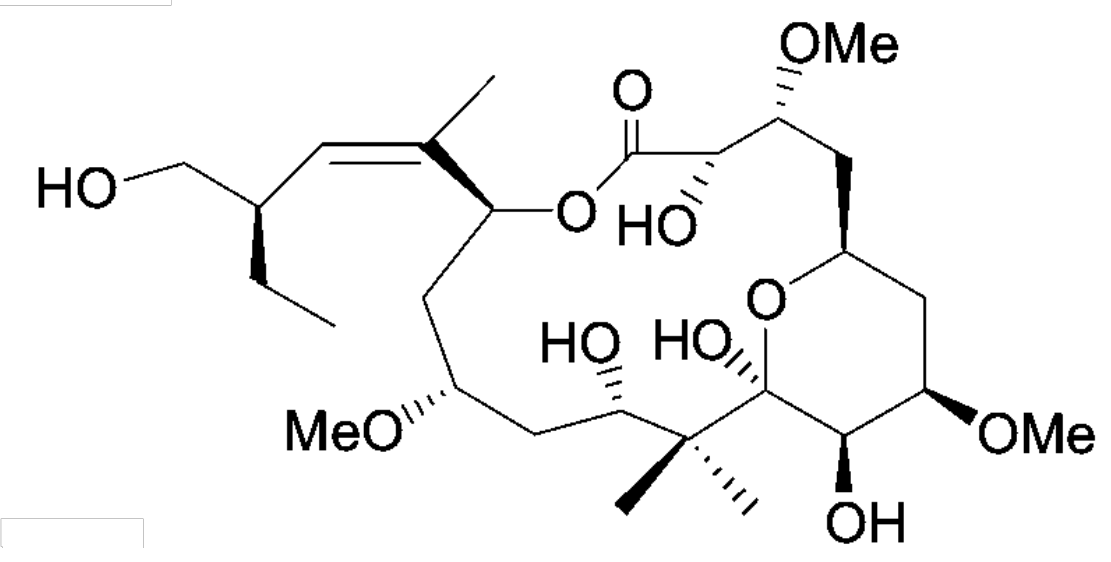

Figure 1.5. Structure of the microtubule-stabilizing agent PelA.

\section{RTA 301, Docetaxel, and Paclitaxel in H460 NSCLC Xenografts}

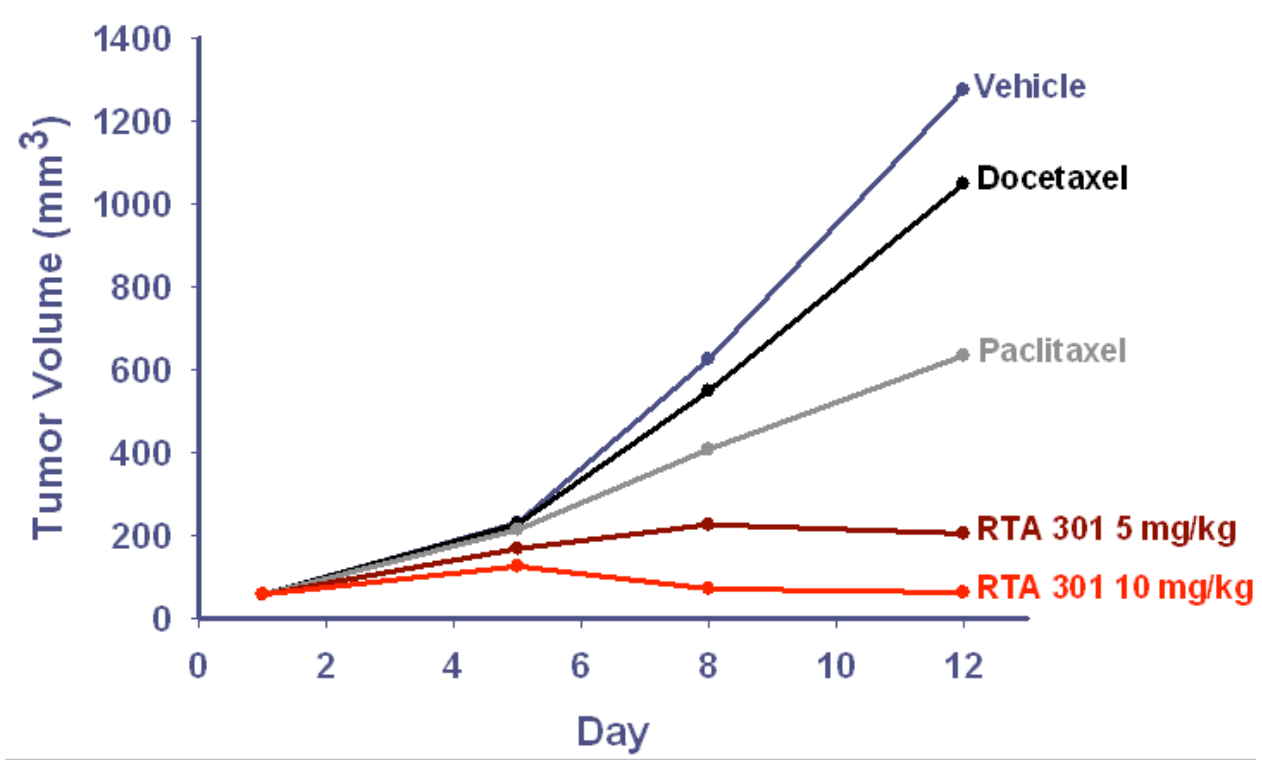

Figure 1.6. In vivo activity of PelA (RT 301). PelA was compared to two clinically approved MSAs for its effects on tumour volume in NCI-H460 non small cell lung cancer xenografts, in athymic $n u / n u$ mice. PelA (RTA301), at either $5 \mathrm{mg} / \mathrm{kg}$ or $10 \mathrm{mg} / \mathrm{kg}$, was administered daily for 5 days by intraperitoneal (i.p.) injection. Ptx and Dtx were administered at $8 \mathrm{mg} / \mathrm{kg}$ i.p daily and $6.25 \mathrm{mg} / \mathrm{kg}$ intravenous (i.v.) every second day, respectively. Compared to vehicle-treated controls, high and low doses of PelA reduced tumour volumes to $5 \%$ and $16 \%$ of controls, respectively. Dtx and Ptx were less effective than PelA, giving tumour volumes that were $82 \%$ and $50 \%$ of controls, respectively. Data taken from Meyer et al., (2006). Figure courtesy of Reata Pharmaceuticals, Inc. 
As a temporary, short-term supply, VUW teamed up with the National Institute of Water and Atmospheric Research Ltd (NIWA) and attempted to generate in-sea cultures of the $M$. hentscheli sponge. Pilot experiments using explants from wild populations of M. hentscheli were seeded at the initial harvest site (Pelorus Sound) and a second site in Wellington harbour (Page et al., 2005). Explants from both sites grew rapidly (4688$4912 \%$ over a year) and exhibited good survival (90-100\%); however, despite being genetically identical only the explants grown at the primary site produced PelA, suggesting environmental factors at Pelorus Sound positively influenced the production of this compound. One interesting finding of the aquaculture was that the PelA levels in the seed sponges correlated well with the yields from the aquaculture. Thus, an explant with low PelA content gave a poor yield after aquaculture, and vice versa. Page and his colleagues then investigated the potential for commercial scale-up of the aquaculture process. From 2004-2007, successive generations of PelA-positive M. hentscheli sponges were cultivated (Page et al., 2011). From the F0, F1 and F2 generations of sponges, 350, 359 and $750 \mathrm{mg}$ of PelA were recovered, respectively, which was earmarked for the pre-clinical studies by Reata Pharmaceuticals Inc. This study, however, highlighted some potential problems for future aquaculture projects. The successive increase in the starting weight of seeded explants coincided with an exponential decrease in biomass yield. This was attributed to severe predation from a nudibranch species, Hoplodoris nodulosa, which was detected at greater levels on farmed sponges compared to wild sponges. The nudibranchs proved to be hugely detrimental to the biomass of $M$. hentscheli that could be recovered and has further highlighted some of the ecological hurdles that will need to be overcome for successful semi-commercial aquaculture of marine species. Attempts to collect predatory nudibranchs with concentrated levels of PelA were unsuccessful (personal communication with Associate Professor Peter Northcote). Regardless, sufficient quantities of PelA were obtained for further biological evaluation studies.

Despite their huge success, one of the major drawbacks in the clinical use of Ptx and Dtx, are their low aqueous solubilities. To overcome their hydrophobicity, these drugs are typically formulated in Cremophor ${ }^{\mathrm{TM}}$ EL (polyethoxylated castor oil), which is, unfortunately, responsible for some of the hypersensitivity reactions associated with these drugs (Gelderblom et al., 2001). Since its FDA approval in 1992, less toxic formulations of Ptx have been investigated, with albumin-bound Ptx (Abraxane ${ }^{\mathrm{TM}}$ ) gaining FDA approval in 2005. Using thin-layer silica gel chromatography with a 5\% 
methanol/dichloromethane solvent, PelA was found to be more polar than Ptx (Rf values of 0.54 and 0.84 , respectively) (personal communication with Dr Peter Northcote and Dr Jonathan Singh, School of Chemical and Physical Sciences, VUW). Hence, due to its greater hydrophilicity, PelA should not require Cremophor ${ }^{\mathrm{TM}}$ to aid drug delivery. In vivo studies in mice investigating the potential of PelA as a treatment for multiple sclerosis, have successfully used PelA without Cremophor ${ }^{\mathrm{TM}}$ (i.e. 100\% ethanol stocks of PelA were diluted in Dulbecco's phosphate buffered saline) (Crume et al., 2009). PelA's reduced hydrophobicity (along with EpoB and Disco) is likely to be a contributing factor underlying its ability to retain its activity in cell lines overexpressing the P-gp efflux pumps (Gaitanos et al., 2004).

An additional feature of PelA that has the potential to be exploited clinically is its distinct binding site on $\beta$-tubulin compared to the taxoid-site drugs. Determining which drug combinations can act synergistically in vitro has important clinical relevance, as these combinations are likely to give some indication of which drug combinations may be successful in human clinical trials. Initial in vitro studies demonstrated that PelA could act synergistically with several of the taxane drugs (e.g. Ptx, EpoA. EpoB, Disco, and eleutherobin), but not with laulimalide, to promote the assembly of purified tubulin (Hamel et al., 2006). There is a general assumption that drugs that bind to different sites on tubulin should act synergistically, while those that bind to the same site should not. However, while this rule tends to hold true in studies looking at the polymerization of isolated, purified tubulin, the complex nature of cultured cells and whole animal models makes it difficult to predict potential synergy. Further synergy investigations carried out in our research group using PelA have highlighted this difficulty. Wilmes et al. demonstrated that PelA, in combination with either Ptx or EpoA, could act synergistically to both inhibit cell proliferation and induce $\mathrm{G}_{2} / \mathrm{M}$ arrest (Wilmes et al., 2007). Not surprisingly, PelA and laulimalide in the same study were unable to act synergistically. More recently, our laboratory group has looked at whether PelA could synergize with MDAs, despite their opposing actions on microtubule polymer mass. While other combinations of MSAs and MDAs have exhibited synergistic interactions previously (e.g. Ptx or Dtx with vinorelbine, Ptx with 2-ME, and laulimalide with 2-ME (Photiou et al., 1997; Budman and Calabro, 2002; Han et al., 2005; Clark et al., 2006), synergy was not observed between PelA and the two MDAs, Vbl and 2-ME (Wilmes et al., 2011a). Interestingly, PelA did not synergise either Dtx or Disco, even though synergy was observed with Ptx, EpoA, and the EpoB analogue, ixabepilone 
(Ixempra $\left.{ }^{\circledR}\right)$. There are numerous potential explanations for these inconsistencies, including differences in the stabilization/destabilization effects on the microtubule, the activation of diverse signalling pathways downstream of tubulin-binding or cell cycle perturbation, and the possibility that each of these drugs may have additional, unique secondary targets. PelA has been shown to inhibit microtubule dynamics, similarly to other MTAs (Chan et al., 2011), but studies indicate that its mode of microtubulestabilization diverges from some of the taxoid site drugs (Huzil et al., 2008). MSAs in general all appear to promote stabilization of the microtubule interdimer interface but exhibit slight differences depending on the site of ligand binding on tubulin. The taxanes used in the study, Dtx and EpoA, improve the longitudinal stabilization around the interdimer interface of the microtubules that does not involve lateral interactions. PelA also improves longitudinal stability, but this is accompanied by relaxation at the intradimer interface and reduced lateral interactions between the protofilaments of $\beta$ tubulin. It is possible that differences in the mode of stabilization between these MSAs may affect their downstream effects, and hence, any potential synergistic interactions.

To summarize, PelA has so far showed many promising features for further development as an anti-cancer chemotherapeutic. Unfortunately, its development has been hampered, mainly due to limitations in supply. Reata pharmaceuticals Inc. has yet to release any additional pre-clinical, in vivo data, and the patent is due to expire in 2022. Although PelA is now available for license, with limited time on the patent there is less economic incentive for companies to further invest time and money into its development. Despite the seemingly impossible task of progressing a chemotherapeutic through to the market, two current FDA approved drugs, Ptx and the halichondrin B analogue, eribulin $\left(\right.$ Halaven $\left.^{\circledR}\right)$ are excellent examples of natural products beating the odds. In addition to the serious toxicity concerns surrounding Ptx during initial phase I trials, issues of supply arose during the development of both of these MTAs, (Cragg, 1998). Although Ptx was originally isolated from the bark of Taxus brevifolia, an alternative source was sought to generate commercial quantities of the drug, so as not to deplete the Pacific Yew tree. The needles of the closely related T. baccata were found to contain a key synthetic precursor (10-desacetylbaccatin III) that has now led to the sustainable semi-synthetic production of Ptx (Denis et al., 1988). Halichondrin B is a macrocyclic polyether that was first isolated from the marine sponge Halichondria okadai (Hirata and Uemura, 1986). While this compound showed great anti-cancer activity, its clinical development, like that of Ptx, was hindered by its limited natural 
supply. An alternative sponge source, Lissodendoryx sp. was published by researchers at the University of Canterbury (UC) almost a decade after the original article by the Japanese group (Litaudon et al., 1994; Litaudon et al., 1997). The UC researchers were able to supply enough compound for further in vivo xenograft studies in mice, and the results were promising enough to stimulate further interest in the halichondrins. Synthetic efforts by the pharmaceutical company Eisai led to the development of several halichondrin B analogues, including E7389 (eribulin mesylate), which were compared in head-to-head studies (Alley et al., 2005). Eribulin proved to be a better drug candidate than halichondrin $\mathrm{B}$, due to its success in clinical trials, simplified structure, and improved water solubility and stability. This led to its FDA approval in 2010 for metastatic breast cancer (Huyck et al., 2011). All in all, it took approximately 30 and 24 years from initial isolation/elucidation to clinical approval, for Ptx and eribulin, respectively. If these drugs are anything to go by, then there is still hope for PelA or novel PelA analogues, to eventually join the elite group of natural productderived oncology drugs in the clinic. 


\subsection{Project Goals}

The overall goals of this project were to evaluate PelA as a potential chemotherapeutic agent and to identify additional cellular mechanisms that may contribute to its tumour suppressive effects.

\subsection{Project Aims}

To this end, this research project had three major aims:

1) To characterize the mechanism by which PelA induces apoptosis

The cell death pathways activated by particular anti-cancer drugs can significantly impact on their clinical effectiveness, especially given that many tumours harbour mutations in many mediators/activators of apoptosis. An earlier study from our research group observed that neither caspase- 8 nor caspase- 9 were activated in response to PelA treatment (Gaitanos et al., 2004). Cathepsin B has been demonstrated to mediate a caspase-independent mechanism of cell death in non-small cell lung cancer cells, induced by several MSAs, such as Ptx, EpoB and Disco (Bröker et al., 2004). The first objective of my research was, therefore, to investigate whether cathepsin B (or other possible proteases) is involved in inducing a caspase-independent mechanism of cell death and to re-examine the contribution of caspases-8, -9 and $-3 / 7$ in PelA-induced apoptosis.

2) To investigate the concentration-dependent effects of PelA on aneuploidy induction and to elucidate the possible underlying mechanisms

Previous work in our laboratory has suggested that at certain concentrations, PelA induces populations of aneuploid cells (Wilmes et al., 2011b). Aneuploidy is known to be involved in tumourigeneis; thus, drug-induced aneuploidy may have further implications for the use of PelA and other MTAs in the clinic. As part of the second objective of the present thesis, the effects of different concentrations of PelA on the induction of aneuploidy were assessed using flow cytometry. Additional studies using co-immunoprecipitation were carried out to investigate the possible roles of the spindlecheckpoint proteins, namely MAD2, BUBR1 and p55CDC, in the induction of aneuploidy at low concentrations of PelA. Premature dissociation of MAD2 and 
BUBR1 from p55CDC has previously been proposed as a mechanism underlying Ptxinduced aneuploidy (Ikui et al., 2005). Earlier chemical genetics studies using a mad2 $\Delta$ (mitotic arrest deficiency-2) deletion yeast strain also indicated a possible involvement of spindle checkpoint proteins in PelA's mechanism of action (Wilmes et al., 2012b).

3) To investigate additional tumour suppressive mechanisms of PelA

Induction of cellular senescence by chemotherapeutic drugs is a well-established mechanism for tumour suppression whereby proliferating cells are induced to adopt a state of arrested cell growth. Although not as effective as the DNA-damaging agent, Doxo, Disco and Ptx have previously been shown to induce accelerated cellular senescence in the A549 non-small cell lung cancer cell line (Klein et al., 2005). Another objective of my research was to determine whether the induction of a senescent phenotype is an alternative mechanism by which PelA may be able to inhibit tumour growth. SA- $\beta$-gal activity and activation of proteins known to be involved in driving a senescent phenotype were assessed in response to several drugs (i.e. PelA, Doxo, Disco and Ptx).

Determining whether PelA also exhibits anti-angiogenic activity will enhance its potential as an anti-cancer drug. The dual ability of PelA to directly target cancer cells (i.e. via prevention of proliferation and induction of apoptosis) as well as tumour blood supply (inhibition of angiogenesis), would improve its attractiveness as a chemotherapeutic. The ability to inhibit tumour growth via multiple mechanisms may help to circumvent some of the issues surrounding drug-resistance in the clinic. Our aim was to investigate PelA's inhibitory effects on the ability of endothelial cells to carry out functions necessary for new blood vessel growth (i.e. using assays to look at EC proliferation, migration and tube formation). 
This project also had one minor aim:

4) To validate the changes in stathmin and c-Myc abundance levels in response to PelA and determine whether topoisomerase I is a secondary target of PelA.

Secondary drug effects are an important aspect of therapeutic efficacy, affecting the therapeutic index and the tolerability to the drug; thus, proteomic and chemical genetics studies have been carried out at VUW to identify secondary targets of PelA, or identify potential mediators that are involved in its mechanism of action (Wilmes et al., 2011b; Wilmes et al., 2012a; Wilmes et al., 2012b). Stathmin and c-Myc were identified as possible signalling mediators of PelA's mechanism (proteomics) and topoisomerase I (TOPO I) was identified as a potential secondary target (chemical genetics). The final aim of my thesis was to independently validate these three hits. Western blotting was used to validate the changes in expression levels of c-Myc and stathmin in the human promyeloid leukaemia cell line (HL-60) following treatment with PelA. A cell-free TOPO I relaxation assay was used to assess whether PelA affects the ability of TOPO I to relax supercoiled plasmid DNA. 


\section{Chapter 2. General Methods}

\subsection{Chemical compounds}

Peloruside A (PelA) and mycalamide A (MycA) were isolated and purified from the marine sponge, Mycale hentscheli, by Dr Jonathan Singh in the School of Chemical and Physical Sciences (under the supervision of Associate Professor Peter Northcote). Paclitaxel (Ptx), docetaxel (Dtx) and doxorubicin (Doxo) were purchased from LC labs (Woburn, MA, USA), and 2-methoxyestradiol (2-ME) was purchased from Merck. Discodermolide (Disco) was a gift from Dr Ian Paterson (Cambridge University, UK). PelA, MycA, Ptx, Dtx, 2-ME and Disco were reconstituted in absolute ethanol to make either $1 \mathrm{mM}$ or $10 \mathrm{mM}$ stocks, which were stored at $-80{ }^{\circ} \mathrm{C}$. Doxo was reconstituted in sterile, deionized Milli-Q (MQ) water as a $1 \mathrm{mM}$ stock, and stored at $-80^{\circ} \mathrm{C}$. Working stocks $(10 \mu \mathrm{M})$ were prepared in complete RPMI 1640 medium and stored at $-20{ }^{\circ} \mathrm{C}$. Due to a significant loss of activity upon multiple freeze-thaws, MycA and Disco working stocks were stored as single use aliquots.

\subsection{Cell culture}

Reagents and equipment used for tissue culture experiments were prepared in a sterile environment. All cell culture work was carried out using aseptic technique in a Purifier Logic Class II, Type A2 Biological Safety Cabinet (Labconco $\left.{ }^{\circledR}\right)$. Both primary cell lines and established cancer cell lines were cultured at $37^{\circ} \mathrm{C}$ in a $5 \% \mathrm{CO}_{2}$-in-air atmosphere (Panasonic $\mathrm{CO}_{2}$ cell culture incubator; Model no. MCO-20AIC; SANYO North American Corporation). HL-60 human myeloid leukemia cells (a gift from Dr Michael Berridge at the Malaghan Institute) were maintained in RPMI-1640 medium supplemented with $10 \%$ fetal calf serum (Gibco) and 1\% Penstrep (i.e. 100 units $/ \mathrm{mL}$ penicillin and 100 units/mL streptomycin final concentration, Invitrogen). HL-60 cells were cultured in tissue culture flasks from Greiner (Raylab, NZ). Cells that were in the $\log$ phase of growth (i.e. at approximately $70-75 \%$ confluency) were either used for experiments, or passaged into fresh medium at a seeding density of $1 \times 10^{6}$ cells per 25 $\mathrm{cm}^{2}$ flask. All other cell lines were cultured in tissue culture plasticware from Becton Dickinson (Becton Dickinson, Franklin Lakes, NJ). 1A9 human ovarian carcinoma cells (a clone of A2780 cells) and MCF7 human breast adenocarcinoma cells (stably transfected with GFP- $\alpha$-tubulin) were grown in RPMI-1640 medium supplemented with $10 \%$ FCS, $1 \%$ Penstrep, and 0.25 units $/ \mathrm{mL}$ insulin (Sigma). Both the 1A9 and MCF7 
cell lines were a gift from Dr Pareskevi Giannakakou at Cornell University (NY, USA) and were previously determined to have doubling times of 18.8 and $26 \mathrm{~h}$, respectively (Chan, 2008). 1A9 and MCF7 cells were passaged at least twice a week (when they were in log-phase growth) and only used to passage 30 . The optimal seeding density for passaging 1A9 and MCF7 cells was approximately $1 \times 10^{4}$ cells $/ \mathrm{cm}^{2}$. To detach adherent cell lines, the trypsin alternative, TrypLE ${ }^{\mathrm{TM}}$ express (Invitrogen) was used. To remove the trypsin, detached cells were pelleted and resuspended in fresh medium. Frozen stocks of all cell lines were stored in liquid nitrogen at a density of 1 x $10^{6}$ cells in complete RPMI-1640 medium containing 10\% DMSO. To culture cells from liquid nitrogen stocks, frozen vials were thawed rapidly in a $37{ }^{\circ} \mathrm{C}$ waterbath, then diluted 10 fold into complete medium. Cells were then centrifuged at $300 \mathrm{~g}$ to pellet the cells. Subsequently, the cells were resuspended in fresh medium and then transferred to a culture flask. HL-60, 1A9 and MCF7 cells were tested regularly for mycoplasma contamination. The methods used for culturing primary human umbilical vein endothelial cells (HUVECs) is described in Chapter 5.

\subsection{MTT cell proliferation assay}

To assess the effects of various drugs on cell proliferation, the MTT (2-(4,5-dimethyl2thiazolyl)-3,5-diphenyl-2H-tetrazolium bromide) cell proliferation assay was used. Using the cofactor NADH, living cells are able to reduce the yellow coloured MTT tetrazolium salt to insoluble purple formazan crystals. Solubilization of these crystals produces a purple coloured solution (detected at a wavelength of $570 \mathrm{~nm}$ ) that is directly proportional to the number of viable cells. To generate concentration-response curves using adherent cells (i.e. 1A9, MCF7 or HUVEC), $100 \mu \mathrm{L}$ of cells at $1 \times 10^{5}$ cells/mL were seeded into each well of a 96-well plate (except for blanks which contained only $100 \mu \mathrm{L}$ medium) and allowed to adhere overnight. The following day, cells were treated with drug (each concentration done in triplicate) using a serial dilution down the plate (except no drug was added to control wells or blanks). After incubation of the cells with drug for 24-120 h (specific incubation times are described in each chapter), $20 \mu \mathrm{L}$ of MTT solution ( $5 \mathrm{mg} / \mathrm{mL}$ in PBS) was added to each well and the plates incubated at $37{ }^{\circ} \mathrm{C}$ in a $5 \% \mathrm{CO}_{2}$ incubator for $2 \mathrm{~h}$. To solubilize the formazan crystals, $100 \mu \mathrm{L}$ of solubilizer (45\% N, N-dimethylformamide, 10\% SDS, pH 4.5 (adjusted using glacial acetic acid)) was added to each well and the plate incubated overnight. Plates were scanned the following day at $570 \mathrm{~nm}$ using a microplate reader 
(VERSAmax ${ }^{\mathrm{TM}}$, Molecular Devices, Sunnyvale, CA or EnSpire ${ }^{\circledR}$ Mulitmode Plate Reader, PerkinElmer, Waltham, MA).

To calculate inhibitory concentration values (e.g. the $\mathrm{IC}_{50}$ ), the absorbance of the solution was first calculated as a percentage of controls after correcting for the blank (i.e. average absorbance for drug concentration/average absorbance of controls x 100). The concentration-response curves and $\mathrm{IC}_{50}$ values (the half-maximal inhibitory concentration) were generated using a four-parameter logistic curve (equation (2.1)) in SigmaPlot $^{\circledR}$ software (Systat Software Inc., Chicago, IL; Version 11.0). The plot equation function was used to determine additional inhibitor concentration values (e.g. $\mathrm{IC}_{25}, \mathrm{IC}_{80}$ etc.).

$$
y=\min +\frac{\max -\min }{1+\left(\frac{x}{E C 50}\right)^{- \text {Hillsiope }}}
$$

\subsection{Cell cycle analysis by flow cytometry}

Cell cycle analysis was carried out using the DNA intercalator propidium iodide (PI). Seeding of cells for cell cycle analysis varied depending on the experiment and specific methods are described in the individual chapters (i.e. seeding densities, drug treatments and incubation times); however, the basic harvesting/staining protocol is as described below. Adherent cells were detached using TrypLE express, and washed off the dishes/plates using $1 \mathrm{x}$ PBS. Cells were centrifuged at $300 \mathrm{~g}$ for $5 \mathrm{~min}$ and washed again in PBS. Cells were either fixed and treated with RNase, or stained directly with the PI staining buffer (see below). Pelleted cells were resuspended in $1.5 \mathrm{~mL}$ ice-cold PBS, and then fixed by adding $3.5 \mathrm{~mL}$ ice-cold absolut ethanol drop-wise, while vortexing. Cells were then incubated at $4{ }^{\circ} \mathrm{C}$ overnight and stored at $4{ }^{\circ} \mathrm{C}$ or $-20^{\circ} \mathrm{C}$. Fixed cells were washed 3 times with PBS, with care taken not to disturb the flocculent pellet. The cell pellets were resuspended in $500 \mu \mathrm{L}$ of PBS containing $100 \mu \mathrm{g} / \mathrm{mL}$ RNase (Invitrogen) and incubated for $30 \mathrm{~min}$ at $37{ }^{\circ} \mathrm{C}$. Cells were centrifuged and the cell pellet resuspended in 250-500 $\mu \mathrm{L}$ PI staining buffer $(0.05 \mathrm{mg} / \mathrm{ml}$ PI, $0.1 \%$ sodium citrate, $0.1 \%$ Triton $\mathrm{X}-100$ ). Cells were incubated for $15-30 \mathrm{~min}$ at room temperature (RT) before analysing by flow cytometry (BD FACScan or BD FACSCanto II, Becton Dickinson, Franklin Lakes, NJ). By gating on cells in the forward scatter (FSC) versus side scatter (SSC) plot, debris was excluded in the analysis. Doublets were excluded by 
gating out the appropriate cells in a FSC-height versus FSC-area plot. Histogram plots of PI intensity versus cell counts were used to determine the percentage of cells in each cell cycle phase. These were either calculated by manually drawing gates around the sub- $\mathrm{G}_{1}, \mathrm{G}_{1}, \mathrm{~S}$, or $\mathrm{G}_{2} / \mathrm{M}$ peaks, or by using FlowJo software (Treestar, Inc. Ashland, OR; Version 7.6.1) to determine the percentage of cells in each of the cell cycle phases using mathematical models. Either the Watson Pragmatic Model or the Dean-Jett-Fox (DJF) algorithms were used, and constraints were placed on the peaks to give the lowest root mean squared (RMS) values (which assesses how good a fit the model is).

\subsection{Western blotting}

The following standard protocol was used for Western blotting, and recipes for all the buffers, gels and reagents are given in Appendix A. The specific antibody concentrations, incubation times, and protein amounts are detailed in individual chapters. For preparation of the cell lysates, cell pellets (either fresh or frozen) were lysed using RIPA buffer containing $10 \mu \mathrm{L} / \mathrm{mL}$ Protease Inhibitor Cocktail I (Sigma) (approximately $30 \mu \mathrm{L}$ of lysis buffer per $1 \times 10^{6}$ cells). Lysates were kept on an orbital shaker at $4{ }^{\circ} \mathrm{C}$ for $30 \mathrm{~min}$, then centrifuged at $16,100 \mathrm{~g}$ for $20 \mathrm{~min}$ at $4{ }^{\circ} \mathrm{C}$. Supernatant was transferred to fresh Eppendorf tubes (leaving behind the pelleted DNA), and the tubes were kept on ice for all subsequent steps. Protein concentration was determined using protein quantification kits (Bio-rad Protein Assay kit or Thermofisher BCA kit), following the manufacturers' instructions. Leftover lysates were stored at $-20{ }^{\circ} \mathrm{C}$ (or $80{ }^{\circ} \mathrm{C}$ if the protein was unstable). If the protein of interest was susceptible to degradation, lysates were prepared from fresh pellets for single use and kept on ice at 4 ${ }^{\circ} \mathrm{C}$ during all handling steps. Protein lysate was diluted in MQ water and boiled for 5 min at $95{ }^{\circ} \mathrm{C}$ in a sample buffer containing $10 \% \beta$-mercaptoethanol. Depending on the abundance of the proteins of interest, $20 \mu \mathrm{L}$ samples containing 30-60 $\mu \mathrm{g}$ of protein were loaded into each lane of either a $10 \%$ or $12 \%$ polyacrylamide separating gel $(1.5$ mm thick) with a 6\% stacking gel. A molecular weight ladder (Dual Colour Precision Plus, Bio-Rad Laboratories) was also loaded ( $3 \mu \mathrm{L} /$ lane) for size comparison. The gel was run at $120 \mathrm{~V}$ for $1.5 \mathrm{~h}$ (or until the blue dye front had reached the end). Proteins were electrophoretically transferred to a low background fluorescence PVDF membrane (Immobilon FL, Millipore) by running at $20 \mathrm{~V}$ for $17 \mathrm{~h}$ in a $4{ }^{\circ} \mathrm{C}$ cold room. Membranes were then blocked for $1 \mathrm{~h}$ using 5\% BSA in PBST (1 x PBS containing 0.1\% Tween20). Primary and secondary antibody concentrations and incubations, as well as loading 
controls are described in the individual chapters. After each addition of antibody, membranes were washed three times (10 min per wash) with PBST, except for the last wash prior to scanning of the membrane, which was carried out using PBS. Membranes were scanned using a Fujifilm FLA-5100 Fluorescent Image Analyzer (Fuji Photo Film Co. Ltd, Tokyo, Japan). Densitometry was carried out using the opensource MBFImageJ software (Rasband, W.S., ImageJ, National Institute of Health, Bethesda, Maryland, USA). A rectangle was manually drawn around each individual band, and the integrated density was measured for the area defined by the rectangle. Using the same sized box (area-wise), ten integrated densities were obtained of the membrane background. The background was measured as randomly as possible, with care taken not to measure the background readings over non-specific bands. Background values were subtracted from both the protein of interest bands and the loading control bands. Protein integrated densities were then normalized to the corresponding loading control, and the values were expressed relative to controls (i.e. as a ratio or percentage of controls).

\subsection{Staining protein gels with Coomassie blue}

After electrophoresis, acrylamide gels were immediately immersed in a fixative $(50 \%$ EtOH, 3\% ortho-phosphoric acid) at RT for $1 \mathrm{~h}$ or overnight. Gels that had been fixed overnight required an additional rehydration step in MQ water. Gels were washed at least three times with MQ water (30 min per wash) and then enough Coomassie staining solution was added to cover the gel (see Appendix A for recipe). Gels were left in stain until the protein bands were visible and darkly stained (the gel also stained blue). Gels were washed in MQ water until most of the blue staining had disappeared from the gel. Gels were scanned using a Molecular Dynamics Personal Densitometer SI. 


\section{Chapter 3. Mechanisms underlying the induction of apoptosis by peloruside A}

\subsection{Introduction}

\subsubsection{The extrinsic (death receptor) and intrinsic (mitochondrial) pathways}

Impaired cellular apoptosis is one of the hallmarks of cancer, and is also implicated in the development of resistance to chemotherapeutic drugs. As mentioned in the General Introduction, two distinct biochemical pathways involving a family of caspases leads to the cellular and morphological changes that are characteristic of apoptosis. Physiological and chemical stimuli (e.g. heat, oxidative stress, UV light, and chemical drugs, etc.) can activate apoptosis through either the extrinsic death receptor pathway or the intrinsic mitochondrial pathway, although both pathways seem to share common signalling mediators. The activation of either of two initiator caspases, caspase-8 (for the extrinsic death receptor pathway) or caspase-9 (for the intrinsic pathway), by proteolytic cleavage, leads to the subsequent cleavage of downstream cellular substrates by effector or executioner caspases, such as caspase-3, -6 or -7 (Kaufmann et al., 2001; Kopper and Peták, 2008).

The mechanisms underlying the extrinsic pathway were elucidated primarily through work carried out on the TNF family of ligands and the corresponding death receptors members of the TNF receptor (TNFR) family. Some of the more prominent ligands of this 19-membered family of cytokines include TNF- $\alpha$, TRAIL (TNF-related apoptosis inducing ligand), and FasL (Fas ligand), which can elicit responses that are involved in a range of physiological functions, including proliferation, inflammation and apoptosis (Ricci and El-Deiry, 2007). Death receptors contain an intracellular region, approximately 80 amino acids long, called the "death domain", that is important for signal transduction (Fulda and Debatin, 2006). Binding of ligands to the death receptors typically causes their aggregation, followed by recruitment of adaptor proteins, such as FADD (Fas associated death domain), to the death domain regions (Walczak and Krammer, 2000). These adaptor proteins then recruit initiator caspases, such as procaspase- 8 (or procaspase-10), forming what is known as the death inducing signalling complex (DISC). Accumulation of procaspase- 8 drives its autoproteolytic cleavage and activation, allowing it to directly cleave downstream effector caspases (Fulda and Debatin, 2006). Some crosstalk exists between the extrinsic pathway and the 
intrinsic pathway (described below), as caspase- 8 activation can also indirectly lead to activation of caspase- 3 through cleavage of BID (a proapoptotic BCL-2 family member) and release of cytochrome c from the mitochondria (Li et al., 1998).

Mitochondria are key organelles in the induction of apoptosis via the intrinsic pathway, acting as a reservoir for pro-apoptotic factors. Mitochondrial outer membrane permeabilization (MOMP), which leads to the release of proteins such as cytochrome c and Smac, is regulated by the BCL-2 (B cell lymphoma 2) family of proteins (Frenzel et al., 2009). The BCL-2 proteins are categorized based on the number of BCL-2 homology domains (BH1-4) they contain, which are important for the interactions between family members (reviewed by Ola et al., (2011)). There are the pro-apoptotic multidomain proteins (e.g. BAK, BAX and BOK), the pro-apoptotic BH3 only proteins (e.g. BID, BIM, PUMA, BAD, NOXA, BMF, and BIK) and the anti-apoptotic multidomain proteins (e.g. BCL-2, BCL-X $\mathrm{L}_{\mathrm{L}}, \mathrm{Mc11}$, BCL-W and BFL1/A1, and BOO) (Frenzel et al., 2009; Ola et al., 2011). The BCL-2 proteins can function through interactions with each other, although some require PTMs, such as cleavage or phosphorylation, for their activation. For example, BIM, which is sequestered to microtubules and released upon stimulation by apoptotic factors, has been shown to interact with both pro-apoptotic (i.e. BAX) and anti-apoptotic (i.e. BCL-2 and BCL-X $\mathrm{X}_{\text {) }}$ members of this family (Marani et al., 2002). It is thought that it is primarily the relative level of these proteins that determines cell fate (Estève et al., 2007). These proteins have multi-compartmental cellular localizations in the cytosol and in nuclear, outer mitochondrial and endoplasmic reticular membranes (Ola et al., 2011). Activation of some of these BCL-2 members leads to their translocation from the cytosol to the mitochondria, as was previously demonstrated using Cos-7 kidney epithelial and L929 fibroblast cells transiently transfected with GFP-tagged BAX. It was also determined that this re-localization was important for the ability of BAX to promote apoptosis (Wolter et al., 1997). There is still no clear consensus as to how exactly interactions between BCL-2 family members leads to MOMP, although one suggestion is that BAXBAK complexes form pores at the outer mitochondrial membrane, allowing the release of apoptotic mediators (Zhou and Chang, 2008). Released cytochrome c, for example, forms part of a multimeric complex called the apoptosome, along with APAF-1, procaspase-9 and the cofactor dATP (Li and Yuan, 2008). APAF-1 is the primary component of the apoptosome and contains an N-terminal CARD domain (caspase recruitment domain) and a C-terminal WD40 domain. The WD40 domain is important 
for binding to cytochrome c, while, the CARD domain facilitates dimerization and activation of caspase-9 (Cain et al., 2000; Renatus et al., 2001; Li and Yuan, 2008).

\subsubsection{The link between microtubules and apoptosis}

The correlation between the induction of mitotic arrest by MTAs and cell death is wellestablished; however, the precise signal transduction pathways linking the binding of these compounds to microtubules and apoptosis still remains unclear (GanansiaLeymarie et al., 2003). There is also uncertainty as to why cells treated with MTAs can undergo such varied cell fates. As mentioned in the General Introduction, cells can escape from mitosis with double the normal DNA (mitotic slippage), or become apoptotic during mitosis after prolonged mitotic arrest and after mitotic slippage, or they can even undergo an alternative form of mitotic cell death that is characterized by multiple micronuclei (mitotic catastrophe) (Galàn-Malo et al., 2012).

MTA-induced cell death generally activates the intrinsic mitochondrial apoptotic pathway (Estève et al., 2007). MTAs alter the expression and activation states of BCL-2 family members to overcome the threshold for apoptosis, leading to loss of mitochondrial membrane integrity and subsequent caspase activation (Estève et al., 2007). Up-regulation of the pro-apoptotic proteins PUMA, BAD, BAX and BAK have been observed in response to both MSAs and MDAs, including Ptx, an EpoB analogue, vinflunine and Vbl (Yamaguchi et al., 2004). This is often associated with the concurrent phosphorylation and/or downregulation of anti-apoptotic proteins in response to MTAs, such as BCL-2 and BCL-xL, leading to their loss of function and the unhindered progression of apoptosis (Haldar et al., 1995; Poruchynsky et al., 1998). BIM, which is sequestered to microtubules via dynein, has also been implicated in MTA-induced apoptosis (Puthalakath et al., 1999). Upon apoptotic stimulation, BIM and the dynein component LC8 are released from microtubules to bind and inhibit antiapoptotic proteins, including BCL-2 (Puthalakath et al., 1999). It is not surprising then, that drugs that perturb microtubule structure and dynamics influence BIM activation. BIM has been shown to play a role in mediating apoptosis via the mitochondrial pathway in cells treated with Ptx and the MDA, combretastatin-A4, both through the release of BIM from microtubules, as well as regulation at the transcriptional level (Li et al., 2005; Mendez et al., 2011; Rovini et al., 2011). 
Another key player in MTA-induced apoptosis is the transcription factor, p53. Activation of $\mathrm{p} 53$, in response to vinorelbine, up- and downregulates the expression levels of BAX and BCL-2, respectively (Giannakakou et al., 2001; Bourgarel-Rey et al., 2009). p53 can mediate apoptosis by binding to the BCL-2 promoter, as well as interacting directly with BCL-2 family members to promote MOMP (Bourgarel-Rey et al., 2009; Rovini et al., 2011). There has also been some speculation that the microtubule cytoskeleton may directly influence mitochondrial integrity. This has stemmed from experiments demonstrating that MTAs (but not other classes of anticancer drugs) can trigger cytochrome c release from mitochondria isolated from tumour cells (André et al., 2000). The regulation of mitochondrial motility, which is mediated by microtubules, changes in their dynamics and associated motor proteins, may also play a role in MTA-induced cell death (Rovini et al., 2011). 


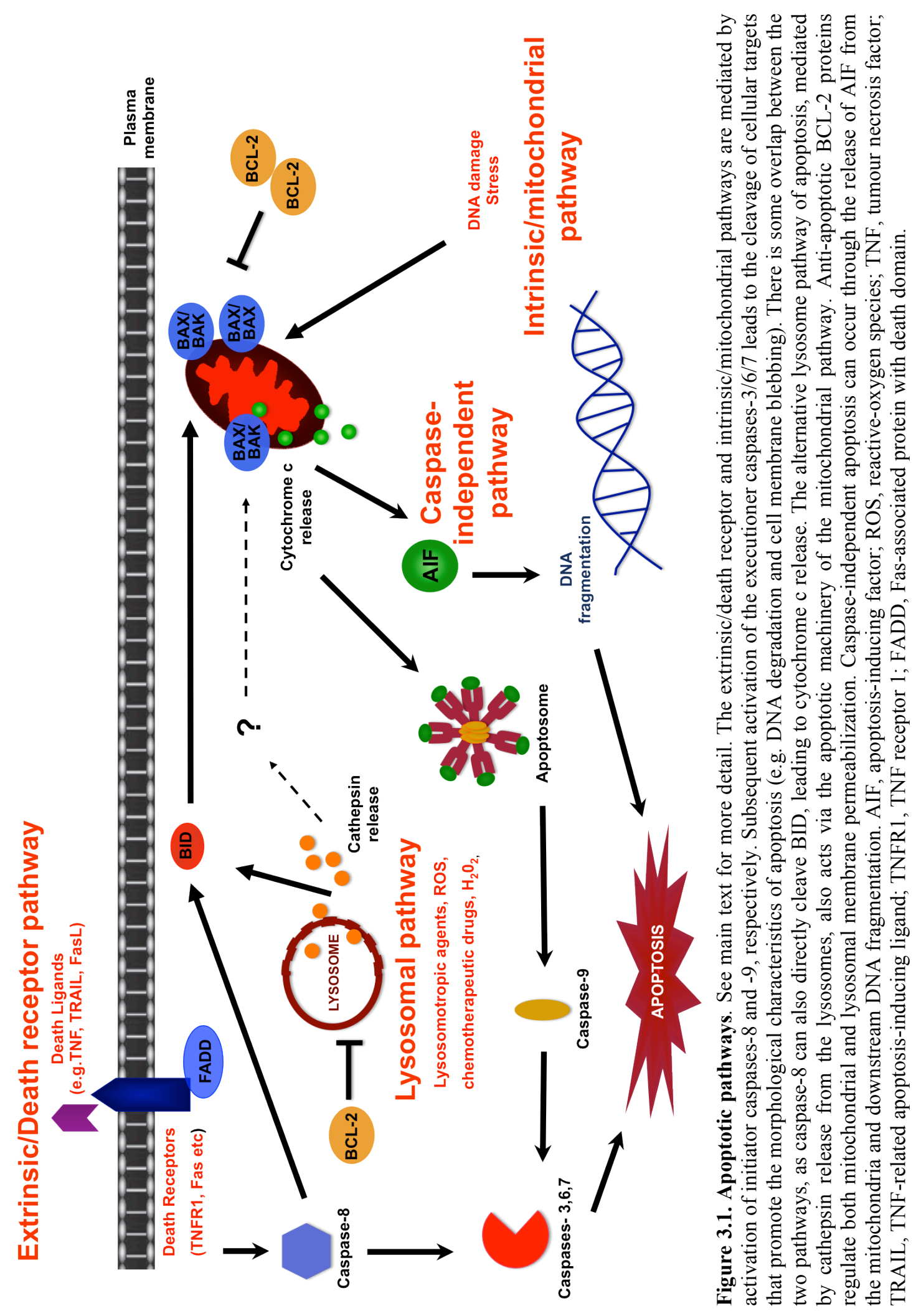




\subsubsection{Apoptosis induced by secondary metabolites of Mycale hentscheli}

In a previous study, the apoptosis-inducing effects of PelA were compared to two bioactive secondary metabolites, MycA and pateamine. All three of these compounds have been isolated from the same M. hentscheli sponge species (West et al., 2000; Miller et al., 2004). MycA is structurally related to the pederin group of compounds (e.g. pederin, the onnamides, and the theopederins) and was first isolated by a group at the University of Canterbury in 1988 (Perry et al., 1988; Perry et al., 1990). It is a potent protein synthesis inhibitor and crystal structures that have been presented show MycA binding to the E-site of the large ribosomal unit in H. marismortui. (Gürel et al., 2009). The first isolation of pateamine was reported by Northcote et al. in 1991, and this compound was subsequently found to demonstrate potent cytotoxicity against the murine leukemia cell line P388 $\left(\mathrm{IC}_{50}=0.27 \mathrm{nM}\right.$ ) (Northcote et al., 1991). It is proposed that pateamine inhibits eukaryotic translation by stimulating the activity of eukaryotic initiation factor 4A (eIF4A) family members, which are ATP-dependent RNA helicases that are important for the recruitment of ribosomes to mRNA (Bordeleau et al., 2005). All three compounds typically exhibit cytotoxicity in the low nanomolar range, and induce biochemical changes characteristic of apoptosis (i.e. DNA laddering, positive staining for annexin V, membrane blebbing, and nuclear fragmentation) (Hood et al., 2001b; Miller et al., 2004). In the study led by Dr Miller, both MycA and pateamine triggered activation of initiator caspases within 12 hours in HL-60 cells. The activation of caspase- 8 occurred within the first hour of exposure to MycA, which was followed by later activation of caspase-9; whereas, pateamine exposure led to caspase-9 activation within 2 hours, followed by relatively mild caspase- 8 activation (Miller et al., 2004). In the same study, however, PelA did not activate either caspase- 8 or -9 , even up to 24 hours of exposure in HL-60 cells. An alternative form of cell death by PelA was suggested due to the lack of caspase- 8 and -9 activation despite the induction of apoptotic events (i.e. annexin $\mathrm{V}$ binding, micronuclei and apoptotic body formation, and DNA laddering) (Hood et al., 2001a; Hood et al., 2002; Miller et al., 2004).

\subsubsection{Lysosomal pathway of apoptosis}

Caspases are by far the most prominent mediators of apoptosis; however, it is now widely accepted that an additional lysosomal pathway of cell death exists. Lysosomes are membrane-bound cellular organelles, which contain hydrolytic enzymes (hydrolases) that are involved in the enzymatic degradation of macromolecules (Luzio et al., 2007). The initial step in this cell death pathway involves the release of these 
enzymes after lysosomal membrane permeabilization (LMP), which can be triggered by both exogenous and endogenous stimuli (Česen et al., 2012). Of these hydrolases, proteases known as cathepsins play an important role in lysosomal-mediated cell death (Repnik et al., 2012). Cathepsins D and E, and cathepsin G are aspartic and serine proteases, respectively. In addition there are 11 known human cysteine proteases, including cathepsins B, C, F, H, K, L, O, S, V, W and X. Research over the last decade or so (reviewed in Repnik et al., 2010) has now provided ample evidence that the induction of apoptosis by the release of cathepsin after LMP, involves mediators of the mitochondrial signal transduction pathway (Fig. 3.1).

An anti-sense cDNA library approach in HeLa cells was used by Deiss et al. (1996) to screen for genes that are important for cytokine-induced apoptosis. Inhibition of cathepsin D protected HeLa cells from cell death induced by TNF- $\alpha$, interferon- $\gamma$, or Fas/Apo-1, and represented one of the first studies to link cathepsins with the induction of apoptosis (Deiss et al., 1996). A few years later another study demonstrated that recombinant active caspase- 8 can release cathepsin $\mathrm{B}$ from lysosomes into the cytosol and implicated cathepsin B in TNF- $\alpha$-mediated hepatocyte apoptosis (Guicciardi et al., 2000). Guicciardi et al. (2000) further showed that cytochrome c release, caspase-9 activation, and cleavage of known effector caspase substrates were diminished in cathepsin B knockout mice. Cathepsins are not known to directly cleave executioner caspases- 3 and -7 , and research into the identification of possible cathepsin substrates that can mediate cytochrome $\mathrm{c}$ release is still ongoing. Lysosomal extracts in the absence of the BCL-2 family member, BID, are unable to cause cytochrome c release in isolated mitochondria (Stoka et al., 2001), and additional studies have now reported cleavage of BID and degradation of anti-apoptotic family members, by specific cathepsins (e.g. cathepsins B, K, L, H and S) (Stoka et al., 2001; Cirman et al., 2004; Droga-Mazovec et al., 2008). However, an in vivo study found that BID cleavage was not detected in cathepsin-B-mediated cerebellar granule cell apoptosis, suggesting a role for other cathepsin substrates that are still to be identified. Additionally, it has been shown that phosphorylated (i.e. active) BCL-2 can protect against oxidative stressinduced LMP, suggesting an upstream modulatory role of the BCL-2 family of proteins prior to the release of lysosomal proteases (Zhao et al., 2001). As this is a relatively new field, much work still needs to be done to elucidate the factors that regulate LMP, as well as the downstream mediators that lead to cell death. 


\subsubsection{Caspase-independent cell death by MSAs}

The release of lysosomal proteases generally leads to cytochrome c release from the mitochondria and the ultimate activation of executioner caspases; however, several studies have indicated that a caspase-independent mechanism of cell death can occur in response to chemotherapeutic agents (reviewed in Bröker et al., 2005). For example, non-small cell lung carcinomas (NSCLCs), which are notoriously resistant towards traditional chemotherapy and radiation treatment, have been shown to undergo apoptosis in response to staurosporine, in a caspase-independent manner (Gallego et al., 2004). Gallego et al., (2004) demonstrated that inhibition of the apoptosis-inducing factor (AIF) in U18010 cells (via microinjected anti-AIF antibodies) prevented staurosporine induced chromatin condensation. The authors further showed that overexpression of AIF could enhance staurosporine-induced apoptosis even in the presence of the pan-caspase inhibitor, zVAD-fmk; whereas, ectopically introduced cytochrome $\mathrm{c}$ or recombinant active caspase- 3 were unable to induce similar nuclear changes associated with apoptosis.

Similarly to cytochrome c, AIF resides in the mitochondria and in response to apoptotic stimuli, is released into the cytosol, whereby it translocates to the nucleus to initiate chromatin condensation and DNA fragmentation (Candé et al., 2002). The notion that AIF is able to mediate a caspase-independent form of apoptosis has mainly come about from studies where caspase activity has been abrogated (either through the use of chemical caspase inhibitors or caspase-9 and -3 knockout cell lines); however, as seems to be the case with all of the apoptotic pathways identified to date, some crosstalk exists between AIF and the apoptotic machinery of the caspase signalling cascades (Candé et al., 2002). Caspase-independent forms of cell death have been been observed after treatment with MSAs although the exact pathways are still being elucidated (Bröker et al., 2002; Huisman et al., 2002; Ahn et al., 2004; Bröker et al., 2004). In one study, Ptx treatment of SKOV3 ovarian carcinoma cells, which caused phosphatidyl serine (PS) externalization and DNA fragmentation, did not activate caspase-3 but did result in translocation of AIF to the nucleus (Ahn et al., 2004). It should be noted, however, that in this case the authors showed that AIF release from the mitochondria and Ptxmediated cell death occurred concurrently, but did not demonstrate the absolute requirement for AIF in the induction of apoptosis. Other studies have indicated that mitochondrial mediators of apoptosis (i.e. anti-apoptotic BCL-2 and BCL- $\mathrm{x}_{\mathrm{L}}$, and cytochrome c release) and caspases (i.e. caspase-8 and -9) play a limited role in MSA- 
induced cell death in several NSCLC cell lines (Bröker et al., 2002; Huisman et al., 2002). A subsequent study used a panel of protease inhibitors against caspases, cathepsins $\mathrm{B}$ and $\mathrm{D}$, and calpain, to assess the mechanism by which Ptx, EpoB, and Disco induced cell death in NSCLC cells (Bröker et al., 2004). Only the cathepsin B inhibitor provided protection against the appearance of morphological changes associated with apoptosis, suggesting a predominant role for this lysosomal protease in MSA-induced apoptosis in NSCLC cells. The exact mechanistic pathway leading to LMP and release of cathepsin B, as well as the subsequent downstream 57 signalling cascades, are yet to be elucidated. The mechanisms linking cathepsin B release and the induction of morphological features associated with apoptosis were not identified in these studies. Future identification of potential cathepsin substrates is an important aspect of characterizing caspase-independent pathways of apoptosis and, in particular, whether cathepsins themselves are acting as the executors of cell death or whether they just instigate and amplify apoptotic signals. It is apparent that the particular 57signalling pathways that are triggered are dependent on the cell line, as well as the strength and type of stimulus used to induce apoptosis. Still, the use of drugs that activate alternative forms of cell death could be beneficial in complementing conventional apoptosisinducing agents, as well as in overcoming resistance to particular chemotherapies. 


\subsubsection{Aims}

Since PelA did not appear to activate either caspase-8 or -9 in HL-60 cells, we wanted to investigate alternative mechanisms of programmed cell death (Miller et al., 2004). The induction of cathepsin B by the MSAs Ptx, EpoB, and Disco, has previously been demonstrated to mediate a caspase-independent mechanism of cell death in non-small cell lung cancer cells (Bröker et al., 2004). Cathepsin D has also been implicated in the cytotoxic response of the ovarian carcinoma cell line, A2780, to both Ptx and etoposide. Given that previous results have demonstrated that MSAs can trigger apoptosis via cathepsins B and D (Bröker et al., 2004; Castino et al., 2009), we wanted to test whether these proteases were responsible for PelA-induced cell death. Our first aim was, therefore:

1) To investigate whether a panel of protease inhibitors, zVAD-fmk (a pan-caspase inhibitor), CA-074Me (a cathepsin B inhibitor), pepstatin A (a cathepsin D inhibitor) and calpeptin (a calpain inhibitor), could provide protection against PelAinduced apoptosis in 1A9 human ovarian carcinoma cells. Firstly, an MTT assay was used to titrate the effects of each of the protease inhibitors in combination with PelA. To assess the protective effects against actual markers of apoptosis, we also ran an annexin $\mathrm{V}$ assay using flow cytometry.

The results from the protease inhibitor screen indicated that, rather than cathepsin B, caspases were the primary mediators of PelA-induced apoptosis. This led us to our second and third aims:

2) To confirm the activation of executioner caspases in response to PelA using an ApoLive-Glo ${ }^{\mathrm{TM}}$ assay. This assay measures caspase 3/7 activation and viability simultaneously, giving an indication of whether cell death is occurring via apoptosis or necrosis.

3) To re-examine the activation of initiator caspases, caspase- 8 or caspase- 9 , using FLICA $^{\mathrm{TM}}$ (Fluorescent Labelled Inhibitor of Caspases) probes and analysis by flow cytometry. 


\subsection{Methods}

\subsubsection{MTT cell proliferation assay}

A colorimetric MTT assay was carried out as described in the General Methods (Chapter 2). 1A9 cells were treated for $48 \mathrm{~h}$ with either PelA, Disco, or MycA, to determine the appropriate concentrations for the apoptosis and caspase activation assays. Concentration-response curves were generated using Sigma Plot (Systat Software Inc.), and the concentrations that inhibited $50 \%$ and $80 \%$ of cell growth $\left(\mathrm{IC}_{50}\right.$ and $\left.\mathrm{IC}_{80}\right)$ were determined for each drug.

\subsubsection{Titration of protease inhibitors}

An MTT assay was used to titrate the protective effects of several protease inhibitors (i.e. zVAD-fmk (a pan-caspase inhibitor; BD biosciences), CA-074Me (a cathepsin B inhibitor), pepstatin A (a cathepsin D inhibitor) and calpeptin (a calpain inhibitor)) against PelA, Disco and MycA. 1A9 cells were plated at $1 \times 10^{4}$ cells/well in a 96-well plate and allowed to attach overnight. Initially, cells were pre-treated with a 2-fold serial dilution of each inhibitor for $1 \mathrm{~h}$ at $37^{\circ} \mathrm{C}$, starting at $200 \mu \mathrm{M}$ for each inhibitor. Subsequent replicates of the MTT assay started with the highest non-toxic dose of each inhibitor seen in the preliminary experiments $(200 \mu \mathrm{M}$ for zVAD-fmk, $100 \mu \mathrm{M}$ for pepstatin $\mathrm{A}$, and $6.25 \mu \mathrm{M}$ for $\mathrm{CA}-074 \mathrm{Me}$ and calpeptin). After pre-treatment with the protease inhibitors, the cells were then treated for $48 \mathrm{~h}$ with the $\mathrm{IC}_{80}$ concentration of PelA, Disco or MycA.

\subsubsection{Annexin $V$ apoptosis assay}

To determine whether any of the protease inhibitors conferred protection against apoptosis, an annexin $\mathrm{V}$ flow cytometry assay was used. 1A9 cells were plated at $2 \mathrm{x}$ $10^{5}$ cells/well in a 12-well plate and left to attach overnight (extra wells were plated for compensation, as described in the next section). Cells were pre-treated for $1 \mathrm{~h}$ with 200 $\mu \mathrm{M}$ zVAD-fmk, $100 \mu \mathrm{M}$ pepstatin A, $6.25 \mu \mathrm{M}$ CA-074Me or $6.25 \mu \mathrm{M}$ calpeptin (in a total volume of $500 \mu \mathrm{L}$ ). The approximate $\mathrm{IC}_{80}$ concentrations of PelA, Disco or MycA were then added to the wells and cells were incubated for $0 \mathrm{~h}, 24 \mathrm{~h}$ or $48 \mathrm{~h}$. At each timepoint, the supernatant was transferred to an Eppendorf tube so that cells that had lifted off during the drug treatment were included in the analyses. Attached cells were then washed in KREBS buffer and detached using $100 \mu \mathrm{L} \operatorname{TrypLE}^{\mathrm{TM}}$ express per well. The detached cells were washed off the plate with KREBS buffer and transferred to the 
Eppendorf tubes containing the supernatant. Cells were then centrifuged at $300 \mathrm{~g}$ for 5 min, and the supernatant was aspirated off. Cells were resuspended in a total volume of $250 \mu \mathrm{L}$ KREBS buffer containing $0.5 \mu \mathrm{g}$ annexin V-FITC and $20 \mu \mathrm{L}$ of propidium iodide (PI) $(100 \mu \mathrm{g} / \mathrm{mL}$ in KREBS buffer). The FITC/PI stained samples were incubated for $15 \mathrm{~min}$ at RT and then analyzed by flow cytometry using the FL1- and FL2-channels, respectively. To correct for the spectral overlap between the FITC and PI fluorophores, additional samples were prepared and fluorescence compensation was carried out as described below.

\subsubsection{Flow cytometry compensation}

For compensating for fluorescent signal overlap on the flow cytometer, unstained and single-stained samples for each fluorophore were used. Compensation samples were prepared by plating $2 \times 10^{6}$ cells for the $0 \mathrm{~h}$ timepoint and $1 \times 10^{6}$ cells for the $24 \mathrm{~h}$ and $48 \mathrm{~h}$ timepoints into $60 \mathrm{~mm}$ culture dishes. To correct for the spectral spillover, each of the single-stained samples must contain both negatively (i.e. no apoptosis or cell death) and positively (i.e. cells that are apoptotic/necrotic) stained cells. The untreated cells were harvested using $300 \mu \mathrm{L}$ of TrypLE ${ }^{\mathrm{TM}}$ express and split into two $15 \mathrm{~mL}$ Falcon tubes. One tube was microwaved in three or four $2 \mathrm{~s}$ bursts (until the tube was warm to the touch) and once cooled, was pooled with the viable cells in the other $15 \mathrm{~mL}$ Falcon tube. These cells were divided into 8 Eppendorf tubes and centrifuged at $300 \mathrm{~g}$ for 5 min. Cells were then resuspended in KREBS buffer containing either no stain, $0.5 \mu \mathrm{g}$ annexin V-FITC, $50 \mu \mathrm{g}$ PI, or both annexin V-FITC and PI, in duplicate. Additional drug-treated and drug/protease inhibitor-treated cells were plated for each timepoint (in the 12-well plates) and harvested as in the previous section. Cells were resuspended in a total volume of $250 \mu \mathrm{L}$ KREBS buffer containing no stain, $0.5 \mu \mathrm{g}$ annexin V-FITC or $50 \mu \mathrm{g}$ PI, for comparison with the microwaved cells.

Unlabelled cells were first analyzed using a BD FACScan analytic flow cytometer (Becton Dickinson, Franklin Lakes, NJ). Debris was excluded by gating on the main population of cells using forward scatter (FSC) versus side scatter (SSC). To adjust for spectral overlap, a 2-parameter scatter plot was used (FL2 versus FL1) and the PMT voltages were adjusted until the unlabelled population was sitting in the left-hand bottom corner of the plot (i.e. negative for both annexin V-FITC and PI). Using the quadrant-statistics function, colour compensation of the FL1 and FL2 channels was carried out so that the $\mathrm{x}$-axis means were similar for the $\mathrm{PI}^{-\mathrm{ve}} /$ annexin ${ }^{-\mathrm{ve}}$ (unstained) 
population and the $\mathrm{PI}^{+\mathrm{ve}} /$ annexin ${ }^{-\mathrm{ve}}$ population, and the $\mathrm{y}$-axis means were similar for

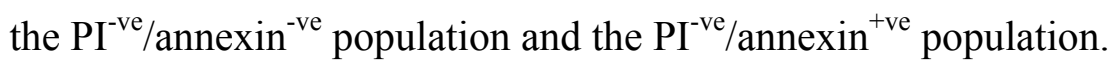

\subsubsection{ApoLive-Glo ${ }^{\text {TM }}$ multiplex assay}

To distinguish between necrotic and apoptotic cell death, 1A9 cells were simultaneously assessed for viability and caspase-3/7 activation using an ApoLive-Glo ${ }^{\mathrm{TM}}$ multiplex assay (Promega Corporation, WI, USA). Intact, viable cells contain a live cell protease that can be detected by using a cell-permeant, fluorogenic substrate (glycylphenylalanyl-amino fluorocoumarin; GF-AFC). Cleavage of GF-AFC generates a fluorescent signal that is proportional to the number of viable cells. Apoptosis can also be detected using the luminogenic substrate, Z-DEVD-aminoluciferin, which contains the caspase-3/7 specific tetrapepitide sequence DEVD (Asp-Glu-Val-Asp). Cleavage by caspase-3/7 releases aminoluciferin, which upon reaction with UltraGlo ${ }^{\mathrm{TM}}$ Luciferase, generates light. Cells were seeded at 1 x $10^{5}$ into each well of a black, glass bottom 96well plate and allowed to attach overnight. To analyze caspase-3/7 activation over time, cells were treated with the $\mathrm{IC}_{50}$ or $\mathrm{IC}_{80}$ concentrations of PelA, Disco, or MycA and then analyzed at various timepoints $(4,8,24$ or $48 \mathrm{~h})$, as per the manufacturer's instructions. Briefly, $20 \mu \mathrm{L}$ of supernatant was removed from each well and replaced with $20 \mu \mathrm{L}$ of the fluorescent viability reagent. Plates were vortexed for $30 \mathrm{~s}$ and then incubated at $37{ }^{\circ} \mathrm{C}$ for $1 \mathrm{~h}$. The fluorescence was then read at 400/505 (Ex/Em) on a Spectramax multiwell platereader (Molecular Devices, Inc., CA, USA). Caspase-glo reagent $(100 \mu \mathrm{L})$ was then added to each well and incubated for a further $1 \mathrm{~h}$ at RT. Luminescence was subsequently read on the Spectramax plate reader.

\subsubsection{FLICA-8 and FLICA-9 assays}

To determine whether PelA induced apoptosis primarily through the extrinsic or intrinsic apoptotic pathway, FLICA ${ }^{\mathrm{TM}}$ (fluorescently labelled inhibitors of caspases) probes were used that specifically targeted either caspase- 8 or caspase-9 (i.e. FAMLETD-FMK and FAM-LEHD-FMK, respectively). FLICA ${ }^{\mathrm{TM}}$ probes are comprised of a green fluorescent carboxyfluorescein label (i.e. fluorescein amidite (FAM) group), a specific tetra-peptide amino acid sequence containing an aspartic residue (i.e. Leu-GluThr-Asp (LETD) and Leu-Glu-His-Asp (LEHD) for caspase-8 and -9 binding, respectively), and a fluoromethyl ketone (FMK) moiety that permits the irreversible, covalent binding of the inhibitor probe to the reactive cysteine residue on active caspase enzymes. The FLICA ${ }^{\mathrm{TM}}$ reagents are non-toxic and cell permeable; thus, covalently 
bound reagent remains inside the cell, while unbound reagent diffuses out of the cell and can be washed away. 1A9 cells were plated into $60 \mathrm{~mm}$ dishes at a density of $4 \mathrm{x}$ $10^{5}$ cells/dish and left to attach overnight. At $\mathrm{t}=0 \mathrm{~h}$, the medium in each dish was replaced with either $4 \mathrm{~mL}$ fresh medium without drug, or fresh medium containing 40 $\mathrm{nM}$ or $100 \mathrm{nM}$ PelA. At various timepoints (i.e. 0, 12, 24, 32, 40, 48, 56, 64 or $72 \mathrm{~h}$ ), the supernatant from each dish was collected (into $15 \mathrm{~mL}$ Falcon tubes) and the remaining cells detached using $300 \mu \mathrm{L}$ TrypLE ${ }^{\mathrm{TM}}$ express. Cells were mechanically washed from the dish using the corresponding supernatant and transferred back into the Falcon tubes. Cells were pelleted at $300 \mathrm{~g}$ for $5 \mathrm{~min}$ and resuspended in $300 \mu \mathrm{L}$ fresh medium. Samples were diluted to approximately $2 \times 10^{6}$ cells $/ \mathrm{mL}$ (in a total volume of $300 \mu \mathrm{L}$ ), except for drug-treated samples in which significant cell death had occurred (these samples were not diluted). A 30 x working stock $(10 \mu \mathrm{L})$ of FLICA ${ }^{\mathrm{TM}}-8$ or FLICA $^{\mathrm{TM}}-9$ (ImmunoChemistry Technologies, LLC, Bloomington, MN, USA) was added to the appropriate samples and incubated for $1 \mathrm{~h}$ at $37{ }^{\circ} \mathrm{C}$ in the dark (the lids of the tubes were loosened, and the tubes were mixed once or twice during the incubation). Cells were washed by adding $3 \mathrm{~mL}$ of $1 \mathrm{x}$ FLICA $^{\mathrm{TM}}$ wash buffer, pelleting the cells and washing once more with $3 \mathrm{~mL}$ wash buffer. Cells were then fixed in $100 \mu \mathrm{L}$ of a paraformaldehyde (PFA)-based fixative (from the Click-iT EdU flow cytometry kit; Invitrogen) or $100 \mu \mathrm{L}$ of 1 x PFA based fixative, diluted in $1 \times$ PBS (from the FLICA ${ }^{\mathrm{TM}}$ kit) and incubated for $15 \mathrm{~min}$ at RT in the dark. Cells were washed once with $3 \mathrm{~mL} \mathrm{1 \%}$ BSA in PBS and then resuspended in $500 \mu \mathrm{L} 1 \%$ BSA in PBS. Cells were stored for up to $24 \mathrm{~h}$ at $4{ }^{\circ} \mathrm{C}$, so that three timepoints could be run together on the flow cytometer. Samples were divided so that unlabelled controls could also be run. These unlabelled controls were resuspended in $250 \mu \mathrm{L}$ of cell cycle buffer $(0.1 \%$ sodium citrate and $0.1 \%$ triton X-100 in PBS), and all FLICA ${ }^{\mathrm{TM}}$ stained samples were resuspended in $500 \mu \mathrm{L}$ of cell cycle buffer. Samples were analyzed using a BD FACSCanto ${ }^{\mathrm{TM}}$ II flow cytometer (BD Biosciences). Live cells were first gated using forward scatter and side scatter and then doublets excluded using forward scatter (height) versus forward scatter (area) gating. FLICA ${ }^{\mathrm{TM}}$-stained samples were analyzed using the green fluorescence channel, excited by a 488 laser. 10, 000 gated events were collected for each sample. Data were analyzed by manually gating using FlowJo software (version 9.4.10; Tree Star, Inc., Ashland, OR). Due to a shift in fluorescence of MSA-treated samples, the negative FLICA $^{\mathrm{TM}}$ gates were set quite wide to take this into account (see Appendix B, Fig. B.6). 


\subsection{Results}

\subsubsection{MTT assays to titrate the effects of different protease inhibitors}

The concentrations of drug that inhibited $50 \%\left(\mathrm{IC}_{50}\right)$ or $80 \%\left(\mathrm{IC}_{80}\right)$ of cell proliferation were determined using an MTT assay. 1A9 cells were treated with different drugs for 48 h. The $\mathrm{IC}_{50}$ or $\mathrm{IC}_{80}$ values are presented in Table 3.1. We chose to assess the apoptotic effects of PelA, MycA and Disco using the $\mathrm{IC}_{80}$ concentrations of these drugs.

Table 3.1. $\mathrm{IC}_{50}$ or $\mathrm{IC}_{80}$ values for $1 \mathrm{A9}$ cells treated with various three different drugs

\begin{tabular}{ccc}
\hline & & 48 h \\
& IC $_{\mathbf{5 0}}(\mathbf{n M})$ & $\mathbf{I C}_{\mathbf{8 0}}(\mathbf{n M})$ \\
\hline PelA & $15.0 \pm 1.6$ & $28.8 \pm 1.8$ \\
MycA & $1.4 \pm 0.2$ & $3.6 \pm 0.3$ \\
Disco & $47.1 \pm 7.9$ & $111.0 \pm 19.2$
\end{tabular}

Data presented are the mean \pm SEM, $n=8-11$

Dr Tom Gaitanos had previously shown that $100 \mathrm{nM}$ PelA did not activate caspase- 8 or -9 in a human myeloid leukemic HL-60 cell line over a 24 h time-course (Miller et al., 2004; Gaitanos, 2005). To investigate whether PelA involved other proteases in the induction of apoptosis, several protease inhibitors were used to determine if they could confer protection against PelA in an ovarian carcinoma 1A9 cell line. An MTT assay was also used to titrate the protective effects of the protease inhibitors, specifically, zVAD-fmk (a pan-caspase inhibitor), CA-074Me (a cathepsin B inhibitor), pepstatin A (a cathepsin D inhibitor) and calpeptin (a calpain inhibitor)), against PelA, Disco and MycA, and also to determine the highest non-toxic concentration to use in subsequent assays. The effects of the individual inhibitors at a series of concentrations (serial dilution starting at either $200 \mu \mathrm{M}$ or $6.25 \mu \mathrm{M}$ ) are shown in Figure 3.2. The highest non-toxic concentrations for each of the drugs were determined to be $200 \mu \mathrm{M}$ for zVAD-fmk, $100 \mu \mathrm{M}$ for pepstatin A, and 6.25 $\mu \mathrm{M}$ for both CA-074Me and calpeptin. There appeared to be a distinct effect on cell proliferation at $200 \mu \mathrm{M}$ pepstatin $\mathrm{A}$ compared to vehicle-treated cells. Even though we did not find a statistically significant inhibition of cell growth $(\mathrm{p}$ value $=0.1709)$ at this concentration (possibly due to the large variation in response) we decided to avoid further use of this concentration. 
Starting at the above concentrations, the effects of each protease inhibitor was then assessed in combination with PelA, Disco, or MycA (Fig. 3.3) to see whether protease inhibition could protect against the drugs. The same data set is also presented in an alternative format (as percent control values rather than as a protective index) in Appendix Fig. B.2. The MTT assay was used to determine cell proliferation responses. The pan-caspase inhibitor zVAD-fmk gave the greatest level of protection against PelA (75\% protection compared to PelA-only controls (minus inhibitor)) (Fig. 3.3 A); however, the lesser but still significant protection conferred by both the cathepsin $\mathrm{D}$ inhibitor (pepstatin A) (Fig. 3.3 B) and the cathepsin B inhibitor (CA074-Me) (Fig. 3.3, C) indicate that cathepsins also play a more minor role in PelA's mode of action. The calpain inhibitor, calpeptin, did not appreciably alter the effects of PelA except at 3.1 $\mu \mathrm{M}$ (Fig. 3.3 D). Interestingly, the anti-proliferative effects of $\mathrm{MycA}$, which was previously shown to activate both caspase- 8 and -9 , were not rescued by the addition of the pan-caspase inhibitor zVAD-fmk. The greatest effect was seen with $6.25 \mu \mathrm{M} \mathrm{CA}$ 074Me, which conferred approximately 38\% protection against MycA compared to untreated cells. Similarly to PelA, zVAD-fmk gave the greatest protection against Disco (38\% protection compared to Disco-only controls), with only minor protective effects seen with the other three protease inhibitors.

The combinations of zVAD-fmk/pepstatin A/CA-074Me, or zVAD-fmk/pepstatin A were also briefly assessed for protective effects against PelA, Disco and MycA (see Appendix B, Figure B.1). In the absence of drug, the combination of zVAD$\mathrm{fmk} /$ pepstatin A caused an increase in cell proliferation at the two highest concentrations tested $(100 \mu \mathrm{M} / 100 \mu \mathrm{M}$, and $50 \mu \mathrm{M} / 50 \mu \mathrm{M})$ compared to untreated controls. In contrast, the triple combination of zVAD-fmk/pepstatin A/CA-074Me was toxic at the highest concentration tested $(50 \mu \mathrm{M} / 50 \mu \mathrm{M} / 6.25 \mu \mathrm{M})$. Taking into account the stimulatory and inhibitory effects of the inhibitors on their own at the higher concentrations, the combination of two or more of these inhibitors did not seem to have any greater protective effects against PelA, Disco or MycA, than any of the inhibitors alone. In light of this preliminary screen, combinations of inhibitors were not further assessed. 


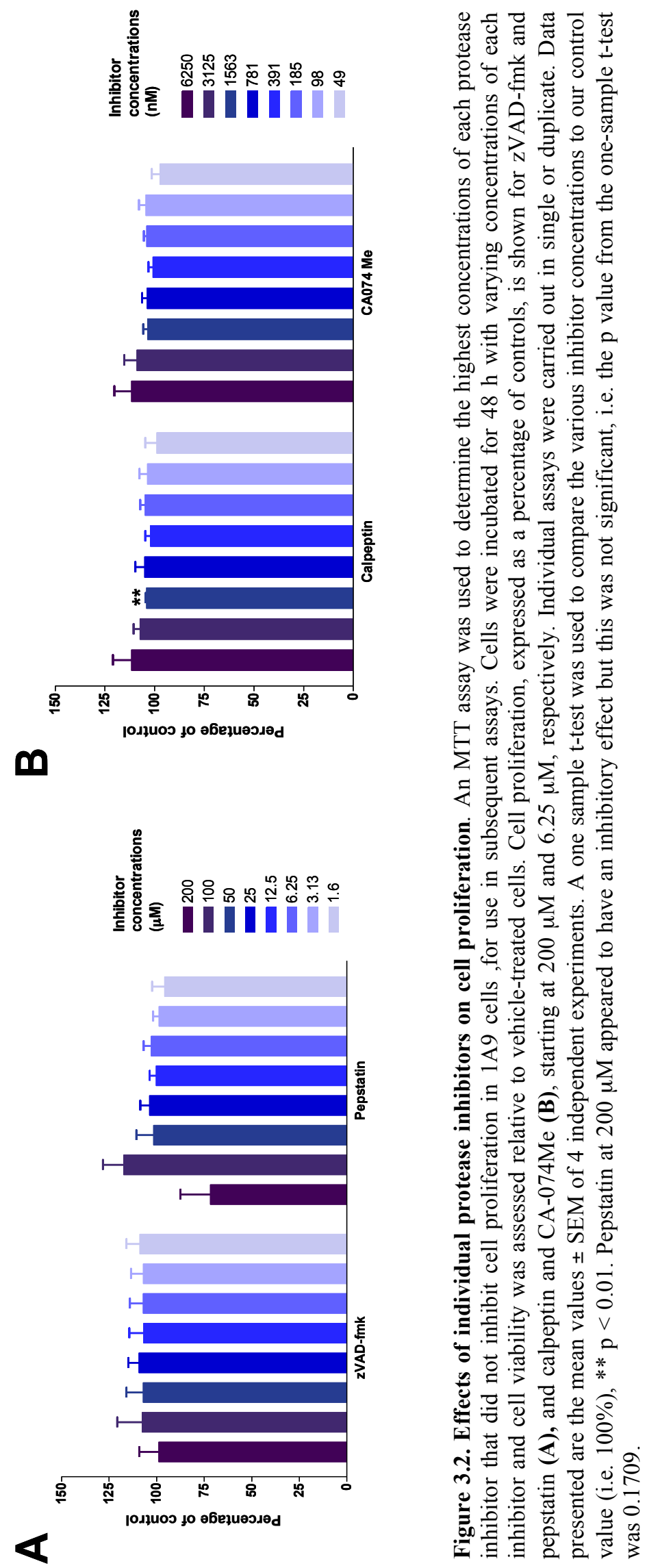




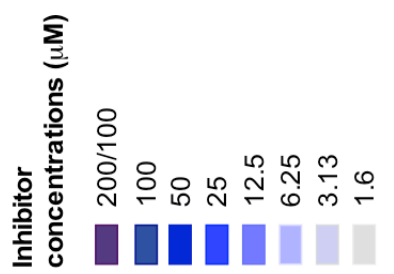

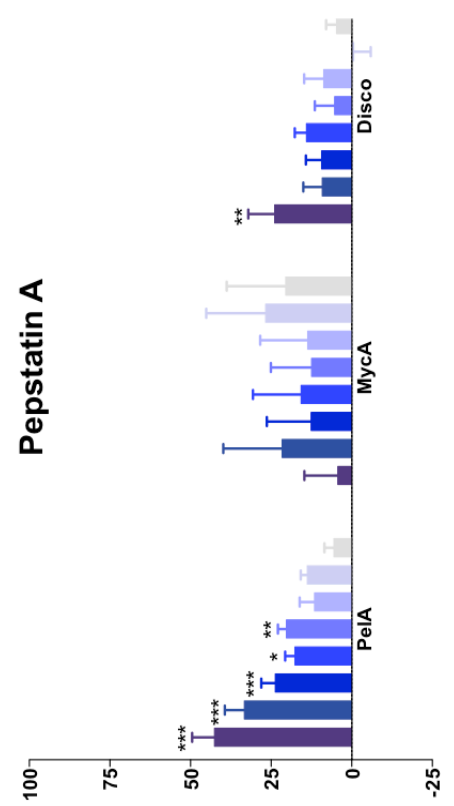

(\%) әбиечо әбеұиәэдәд

$\boldsymbol{m}$

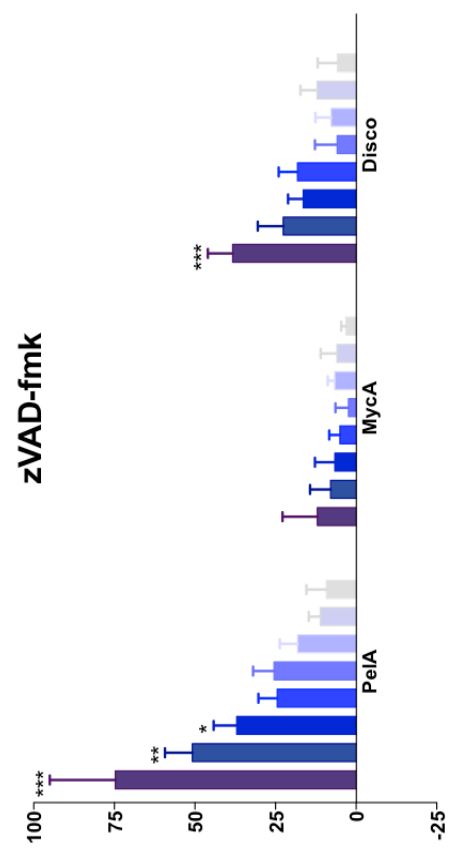

(\%) әбиечว әбеұиәэәд

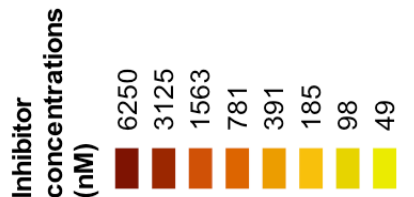

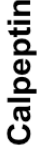

(\%) әбиечว әбеұนәวมәd

口

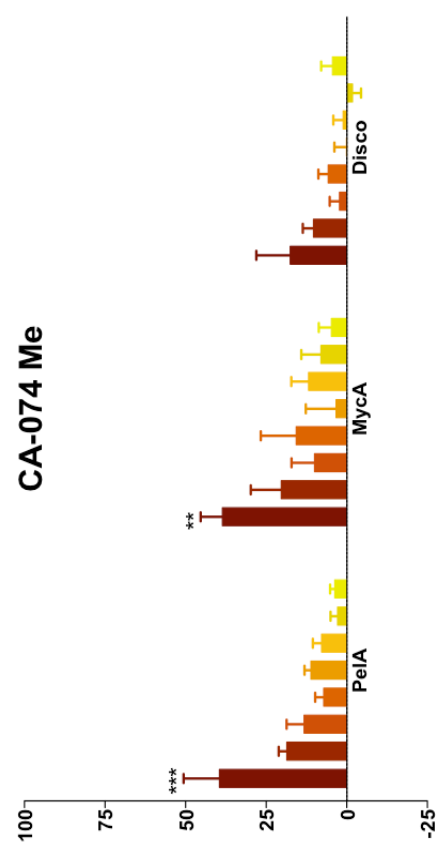

(\%) әбиечว әбеұนәэдә

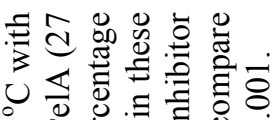

in 0 .

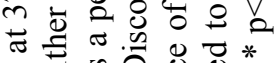

$=$ 的讨

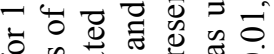

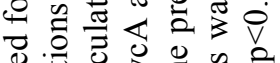

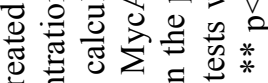

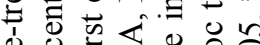

迸

o 0 这

$3 \cup 3$ 至

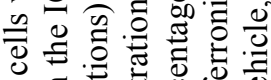

बे.

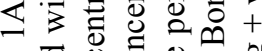

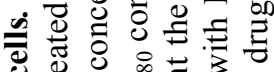

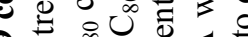

ใे $00_{0}^{\infty} 0$ \&

I

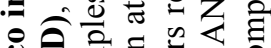

¿ ڤ

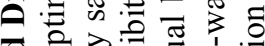

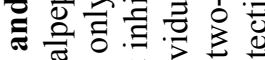

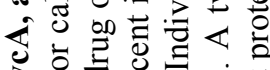

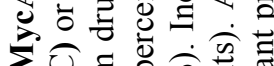

$\sum \circlearrowright \approx$ 巳

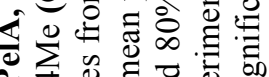

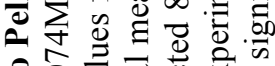

옳 元苍这

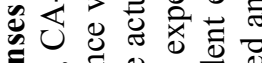

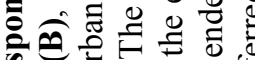

¿

$\lambda \Xi \tilde{\sigma}: 0$

岕跑 表

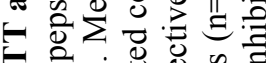

$=$ 元

ङ

$\checkmark$ ᄒ웡

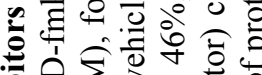

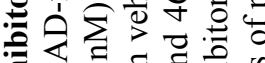

灵令

०

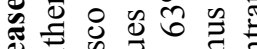

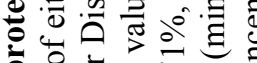

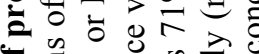

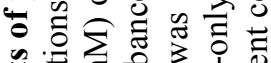

뉼

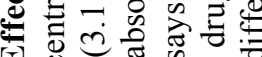

ஸे

ஸे ठ ठ छ

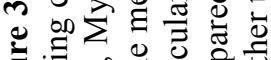

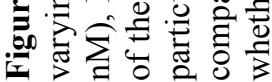




\subsubsection{Protective effects of different protease inhibitors on PelA-, MycA- or Disco- induced apoptosis}

The protective effects of each of the protease inhibitors were again assessed against PelA, MycA and Disco, but this time using an actual biomarker of apoptosis. One of the initial events during apoptosis is the externalization of the membrane phospholipid, PS, from the inner leaflet to the outer leaflet of the plasma membrane. This step precedes the complete loss of membrane integrity that occurs later during apoptosis. Annexin V (a protein that binds with high affinity to PS) and PI (which is excluded by viable cells with intact membrane integrity) were used to detect cells in the earlier (annexin $\mathrm{V}$ staining) and later (annexin V and PI staining) stages of apoptosis using flow cytometry. We found that treatment with the pan-caspase inhibitor, zVAD-fmk, significantly protected against both the early and late stages of PelA-induced apoptosis (i.e. the treatment of cells with solvent alone or PelA/zVAD-fmk led to very similar levels of apoptosis) (see Fig. 3.4 A). Likewise, zVAD-fmk also conferred almost complete protective affects against MycA (Fig. 3.4 B). Interestingly, none of the protease inhibitors that we screened offered significant protective affects against Disco, although there was a small, but non-significant, protective effect of zVAD-fmk against the early apoptotic response to Disco (see Fig. 3.4 C, F, and I). Additionally, the cathepsin D (Fig. 3.4 D, E, and F), cathepsin B (Fig. 3.4 G, H, and I) and calpain inhibitors (Fig. 3.4 $\mathrm{J}, \mathrm{K}$, and L) did not alter the percentage of apoptotic cells in response to any of the drugs, with the exception of a slight protective effect by CA-074Me against the early apoptotic effects of PelA (Fig. $3.4 \mathrm{G}$ ). As zVAD-fmk was the only inhibitor to provide any significant protective effects, representative flow cytometry scatter plots for the zVAD-fmk inhibitor are presented in Appendix B, Figure B.2, but no plots for the other three caspase inhibitors are presented. 


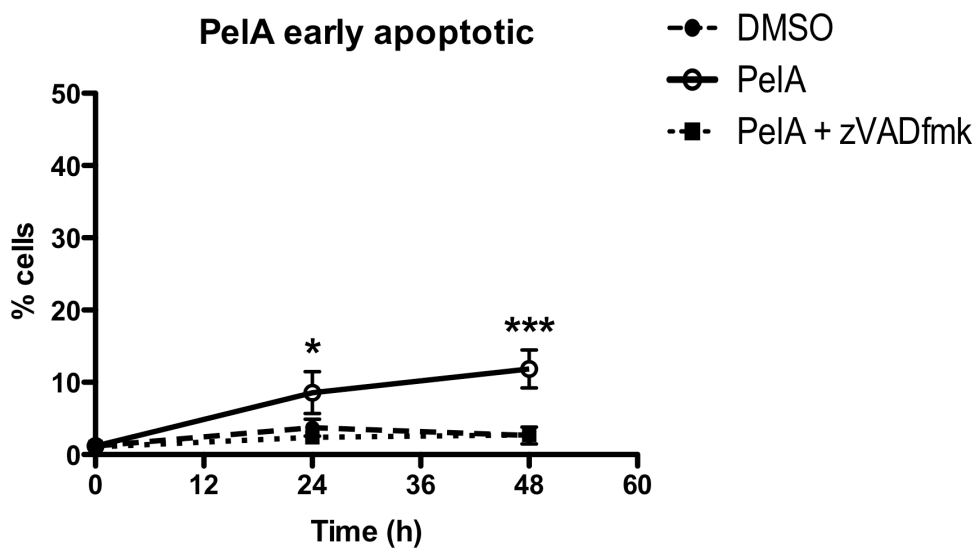

PelA late apoptotic

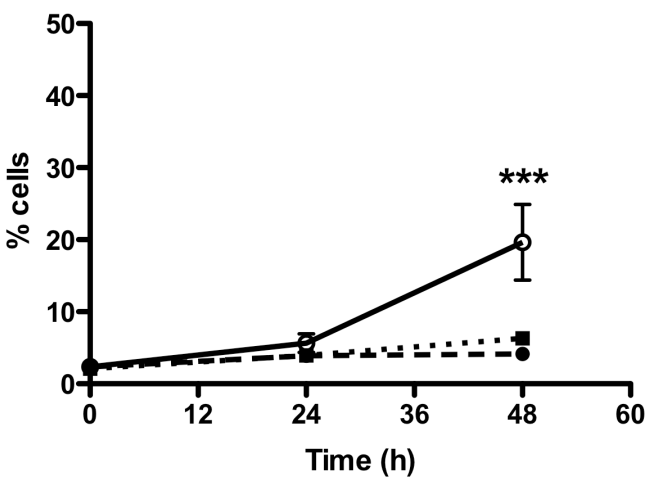

PelA early/late apoptotic

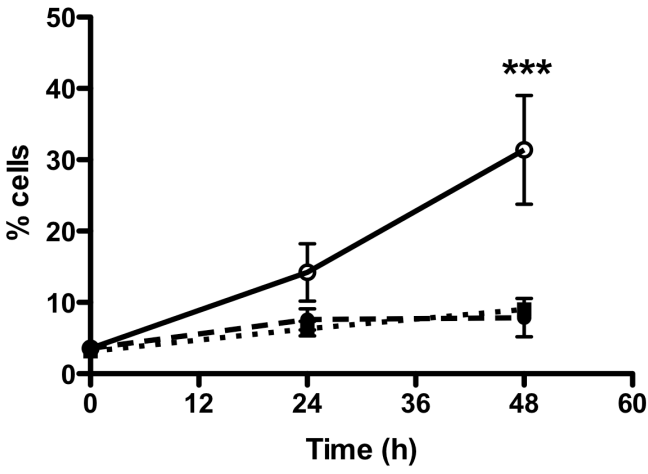

Figure 3.4. Protective effects of protease inhibitors on PelA-, MycA- and Disco-induced apoptosis.

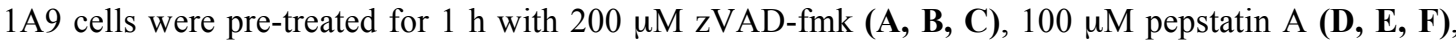
$6.25 \mu \mathrm{M}$ CA-074Me $(\mathbf{G}, \mathbf{H}, \mathbf{I})$, or $6.25 \mu \mathrm{M}$ calpeptin $(\mathbf{J}, \mathbf{K}, \mathbf{L})$ before being treated with the $\mathrm{IC}_{80}$ concentrations of PelA, MycA or Disco. Cells were harvested at several timepoints $(0,24$ and $48 \mathrm{~h})$, stained with annexin $\mathrm{V}$ and propidium iodide (PI) and 10, 000 gated events collected by flow cytometry. Graphs indicate the percentage of cells that are early apoptotic (annexin $\mathrm{V}^{+} / \mathrm{PI}^{-}$), late apoptotic (annexin $\mathrm{V}^{+} / \mathrm{PI}^{+}$), or early and late apoptotic (annexin $\mathrm{V}^{+}$) after treatment with vehicle (DMSO), drug alone, or drug + protease inhibitor. Data points represent the mean value \pm SEM of 3-5 independent experiments. A two-way ANOVA with a Bonferroni post-hoc test was used to compare whether the drug alone and drug + protease inhibitor data points were significantly different at each of the timepoints, $* \mathrm{p}<0.05, * *$ $\mathrm{p}<0.01, * * \mathrm{p}<0.001$. 
B

\section{zVAD-fmk}

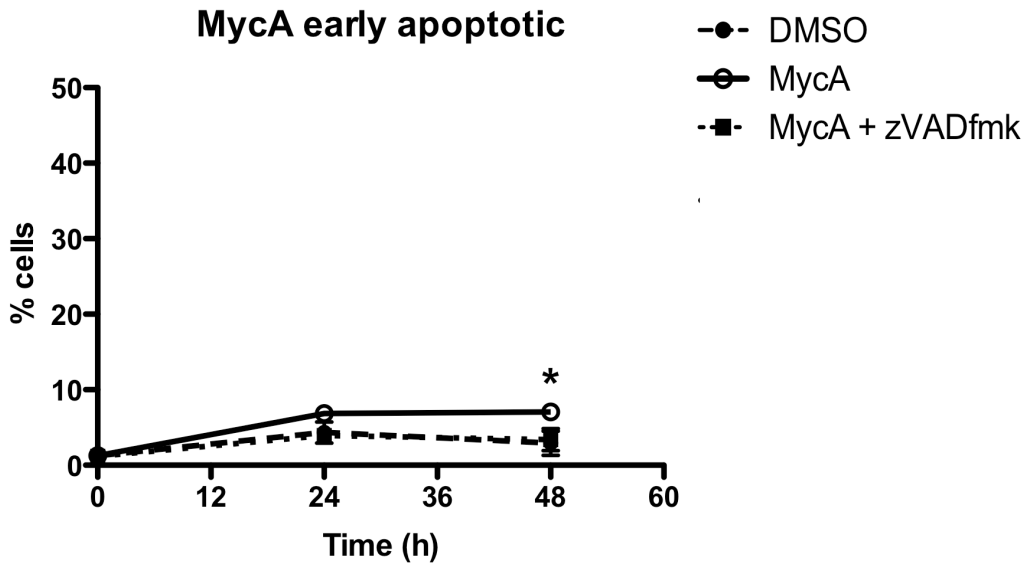

MycA late apoptotic

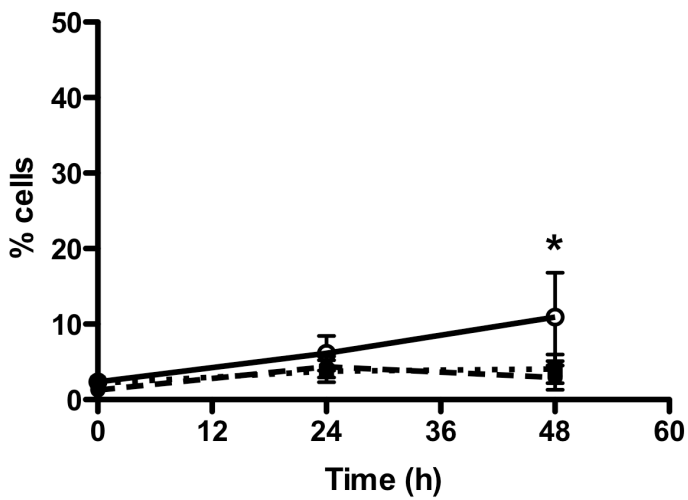

MycA early/late apoptotic

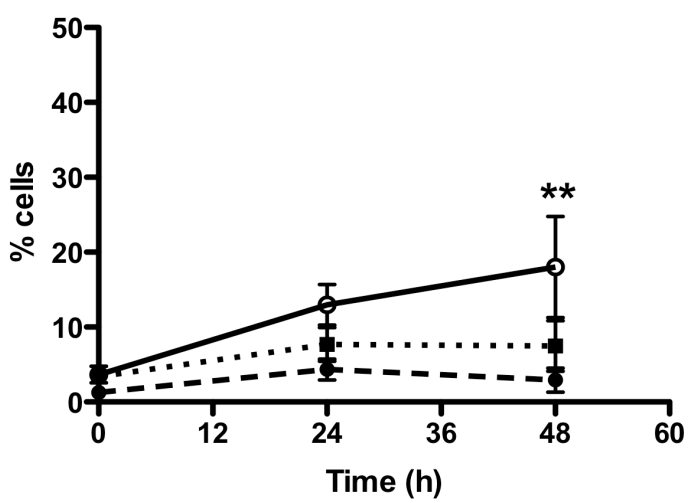

Figure 3.4 (continued) 
C

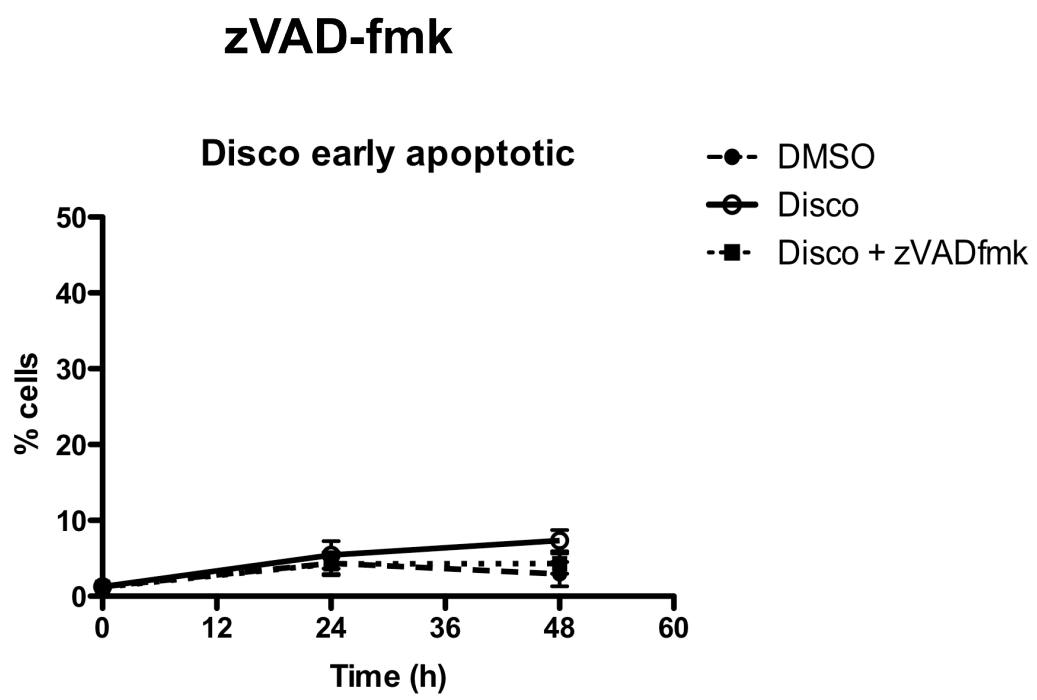

Disco late apoptotic

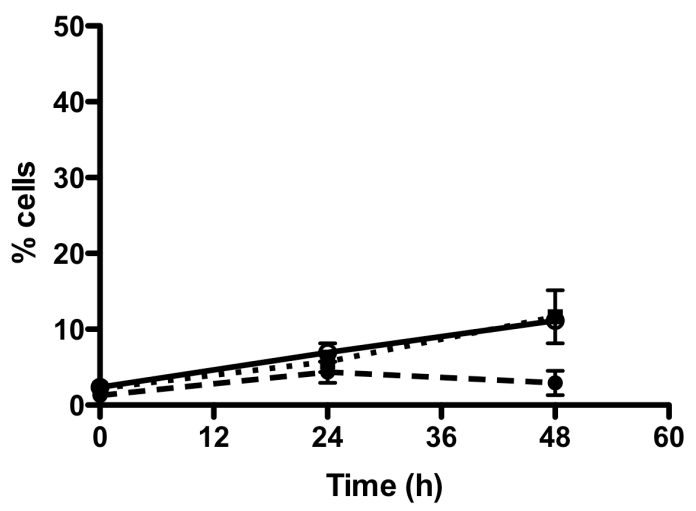

Disco early/late apoptotic

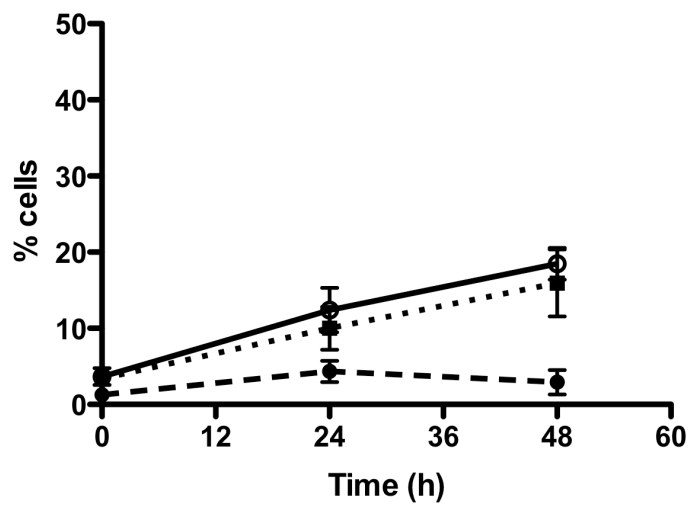

Figure 3.4 (continued) 
D

Pepstatin A

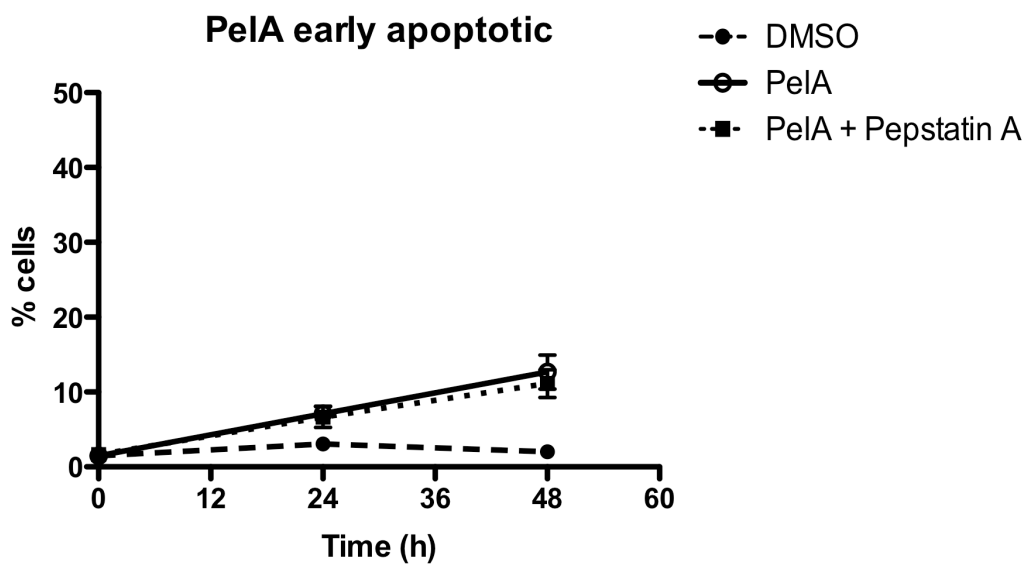

PelA late apoptotic

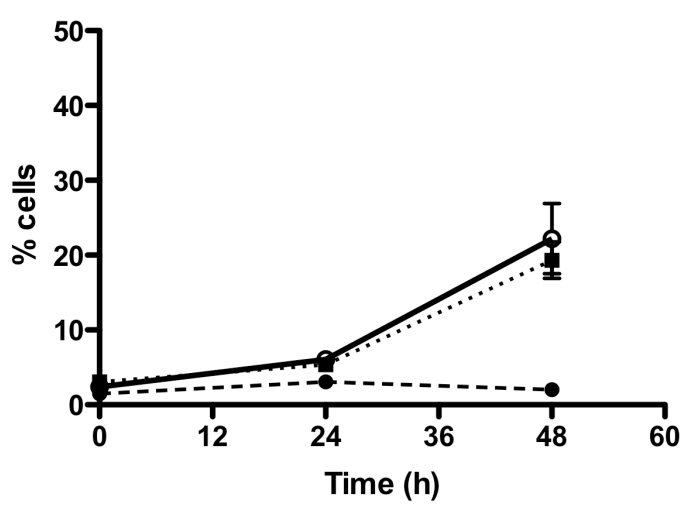

PelA early/late apoptotic

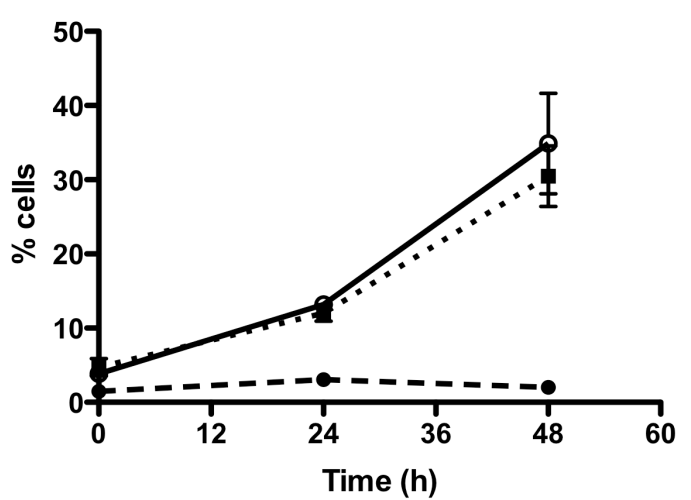

Figure 3.4 (continued) 
E

Pepstatin A

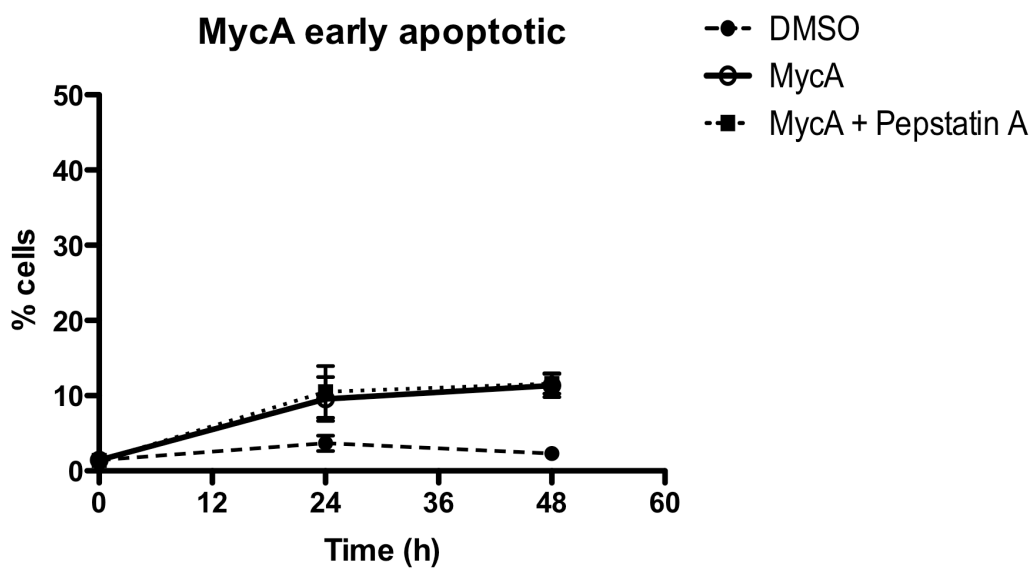

MycA late apoptotic

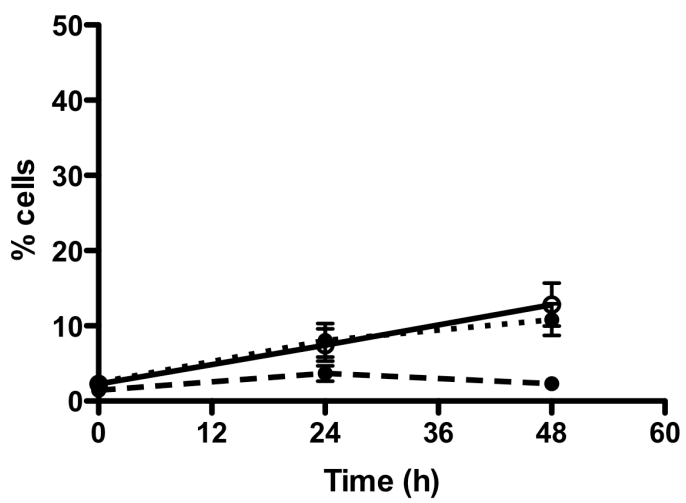

MycA early/late apoptotic

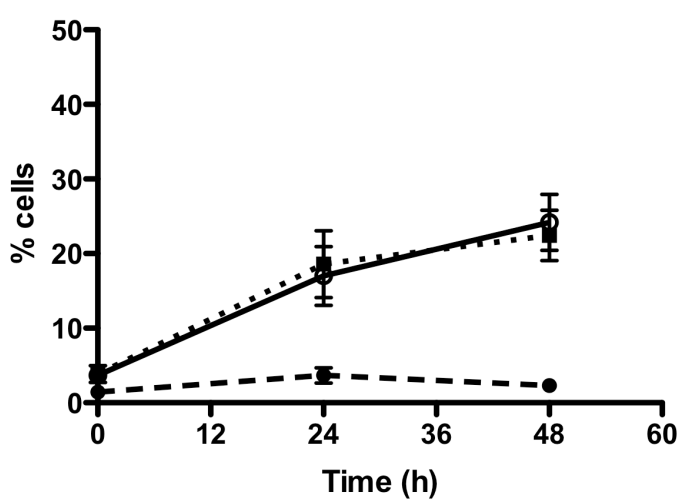

Figure 3.4 (continued) 
$\mathbf{F}$

Pepstatin A

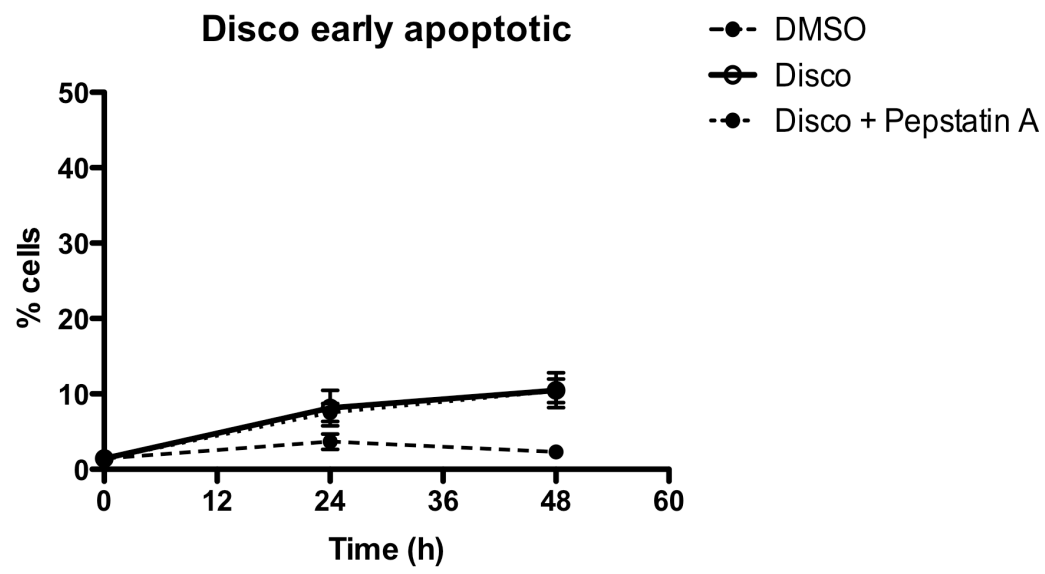

Disco late apoptotic

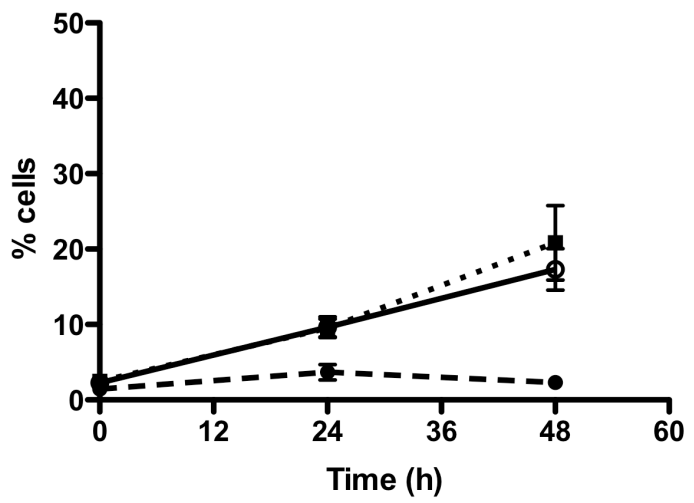

Disco early/late apoptotic

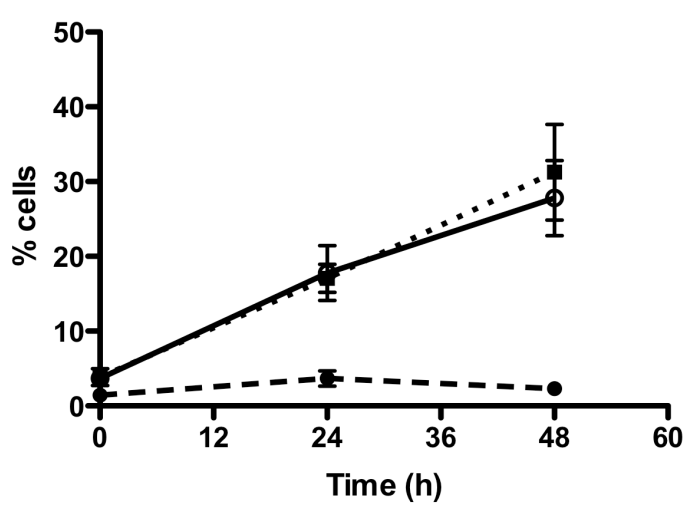

Figure 3.4 (continued) 


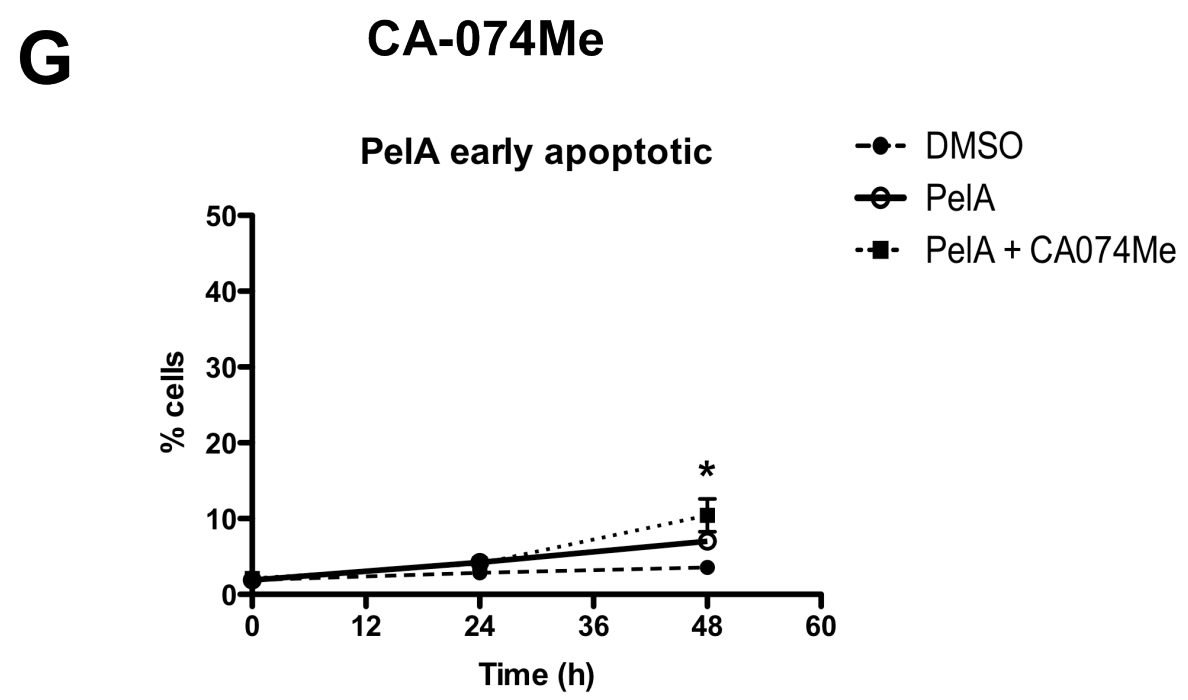

PelA late apoptotic

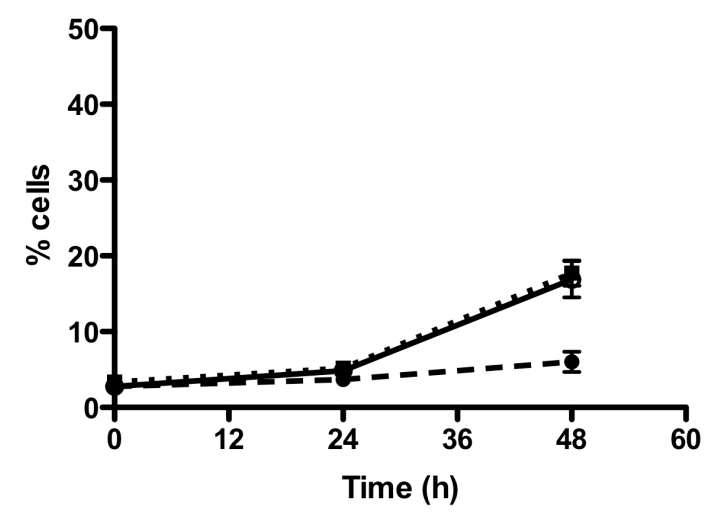

PelA early/late apoptotic

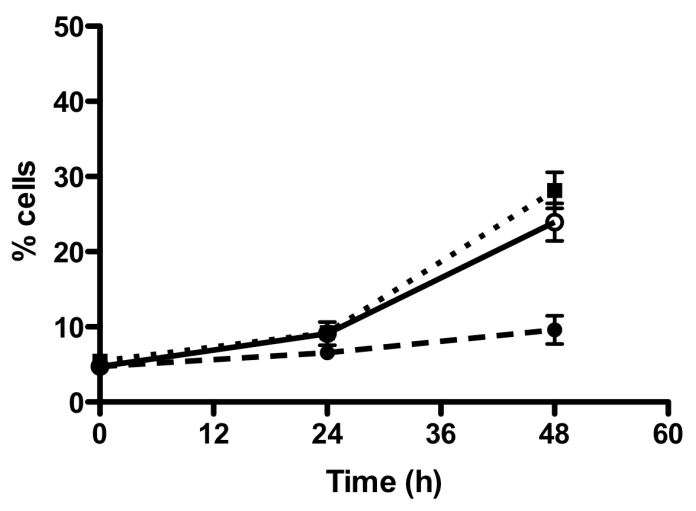

Figure 3.4 (continued) 
H

CA-074Me

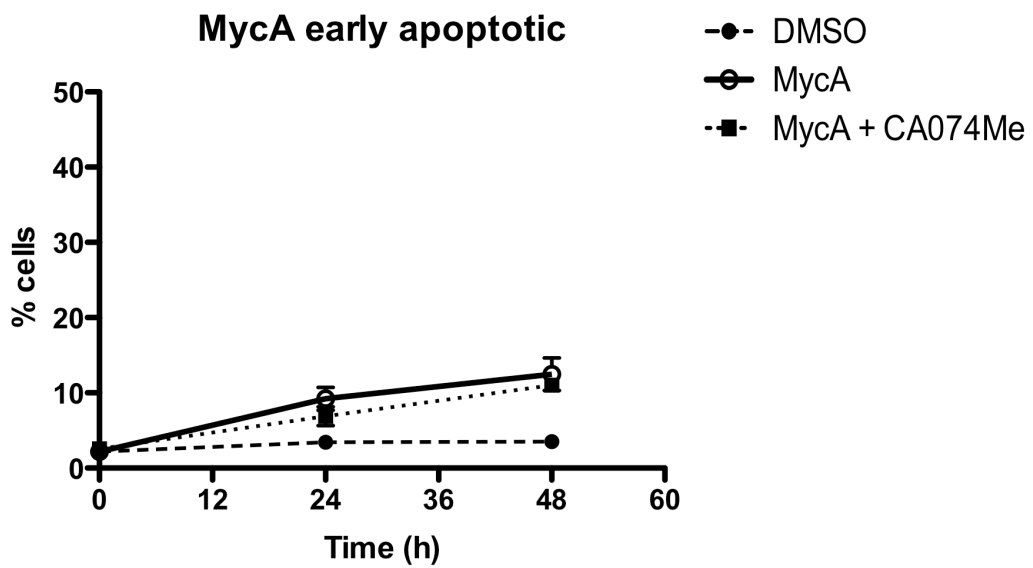

MycA late apoptotic

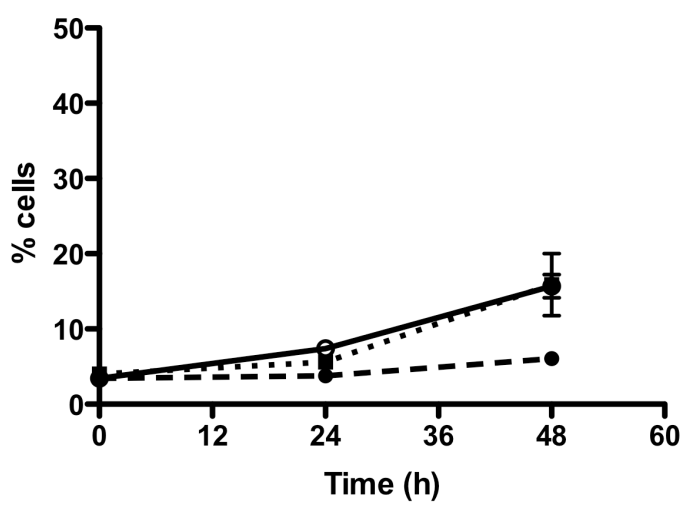

MycA early/late apoptotic

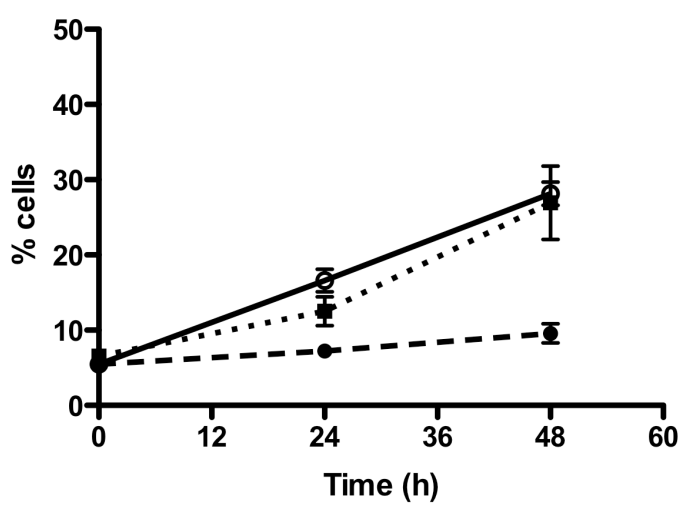

Figure 3.4 (continued) 


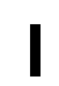

CA-074Me

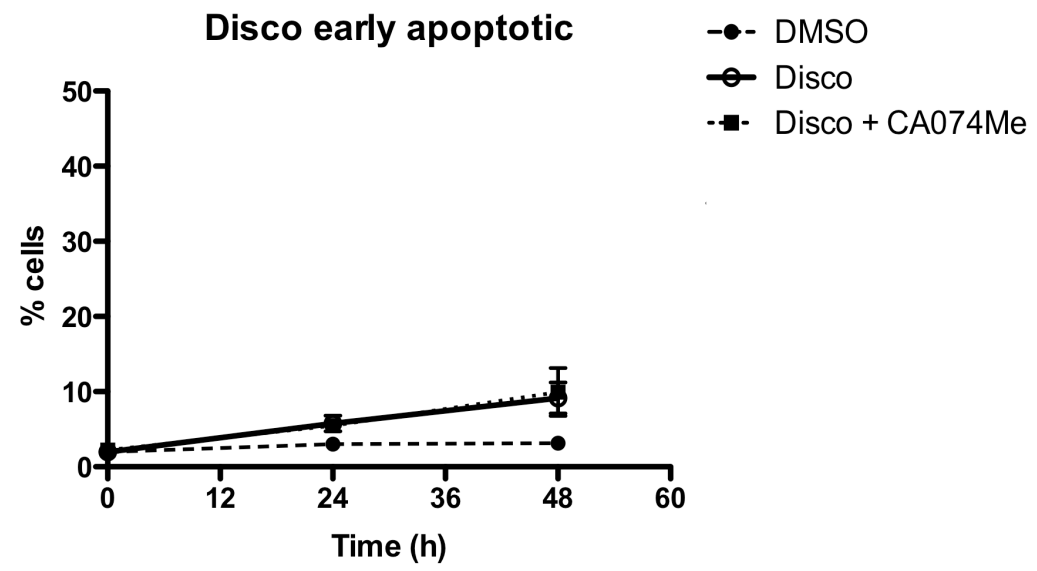

Disco late apoptotic

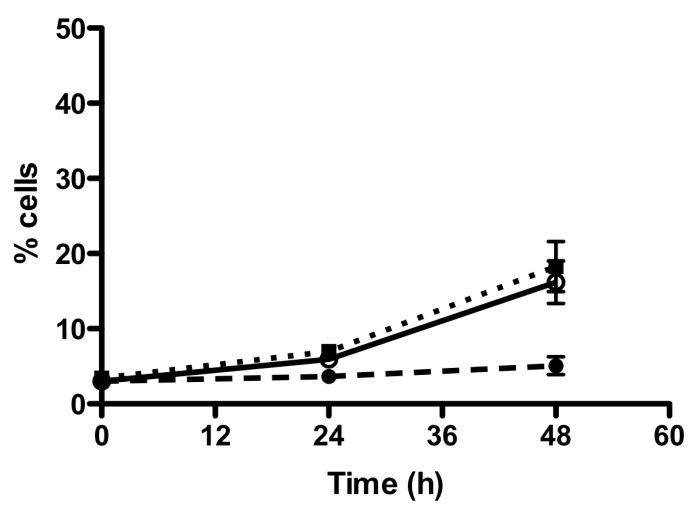

Disco early/late apoptotic

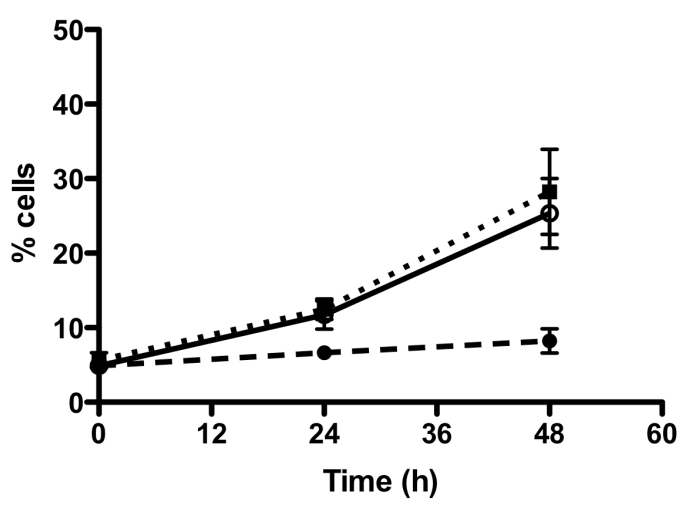

Figure 3.4 (continued) 
J

\section{Calpeptin}

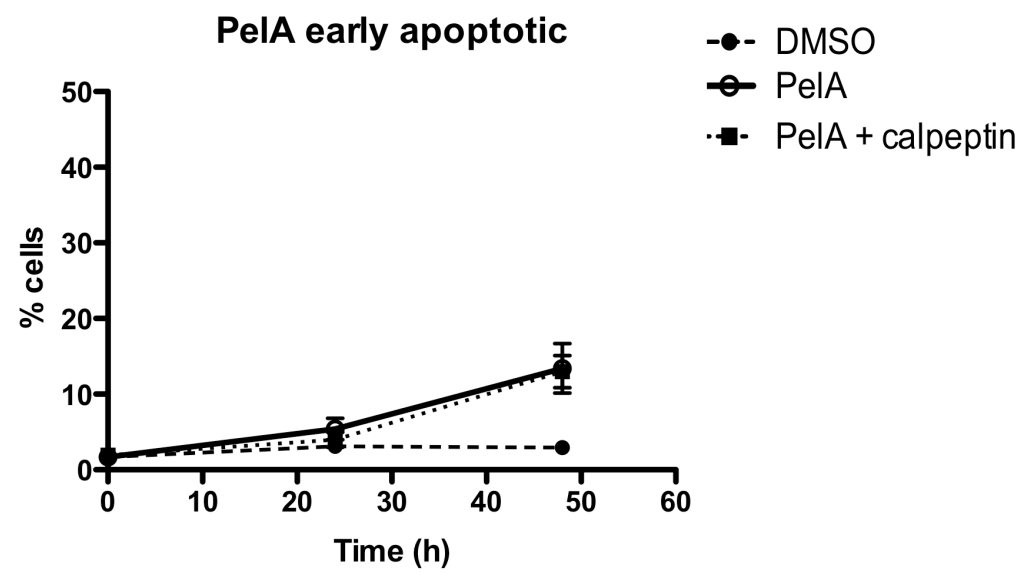

PelA late apoptotic

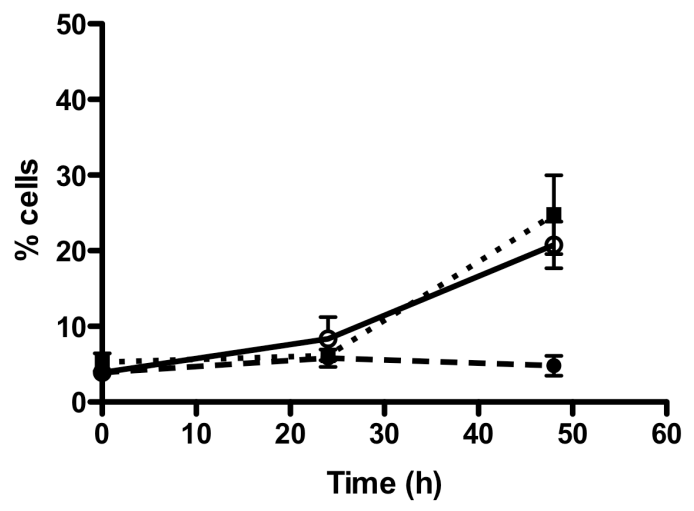

PelA early/late apoptotic

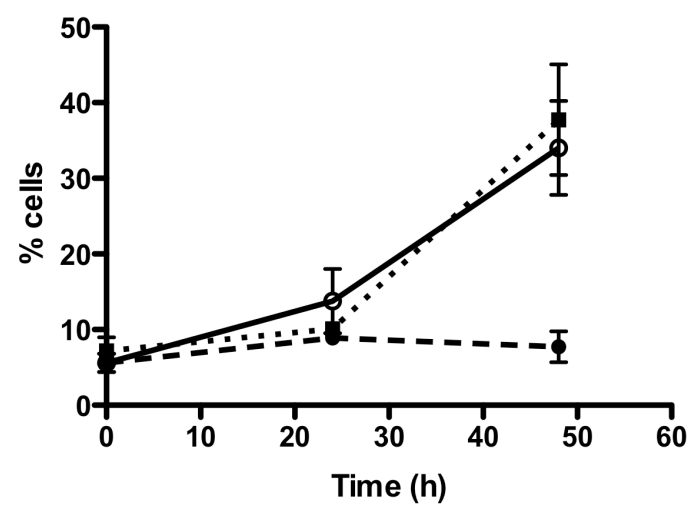

Figure 3.4 (continued) 


\section{Calpeptin}

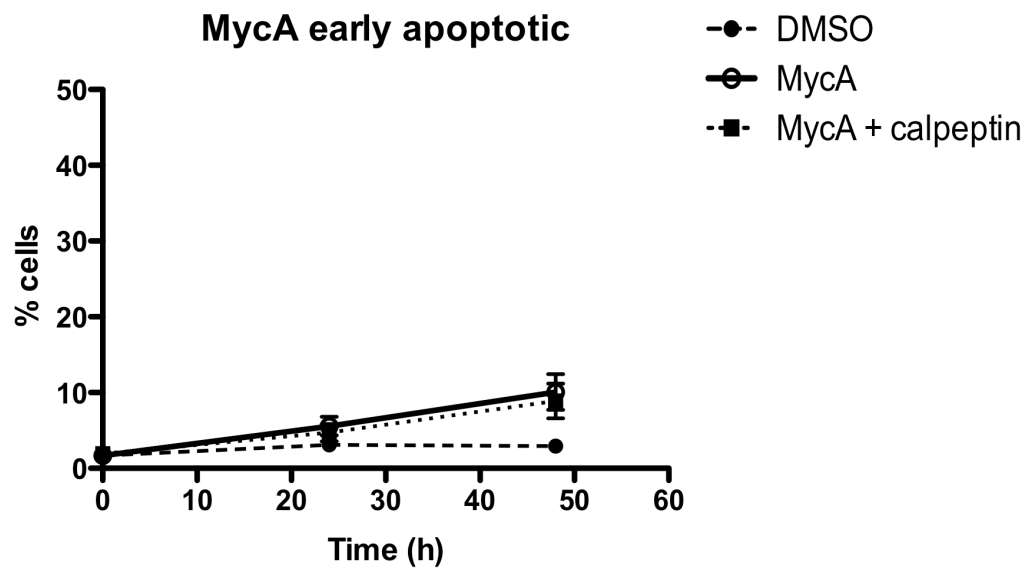

MycA late apoptotic

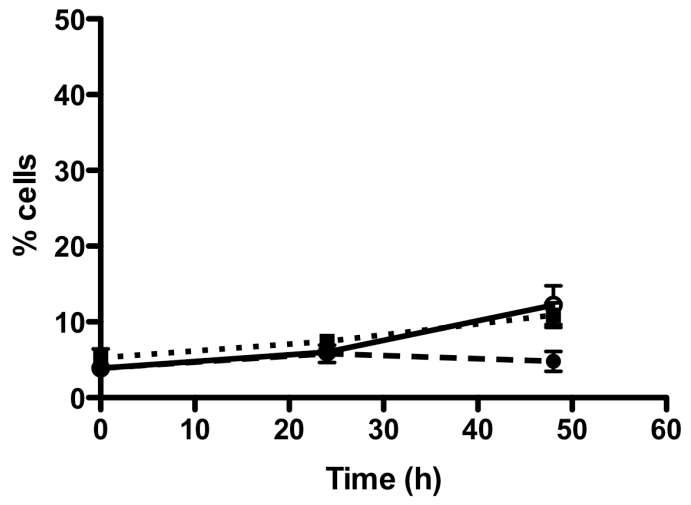

MycA early/late apoptotic

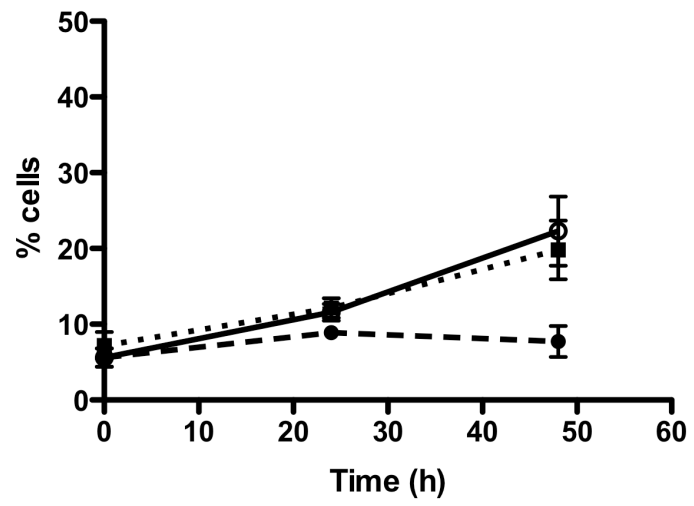

Figure 3.4 (continued) 


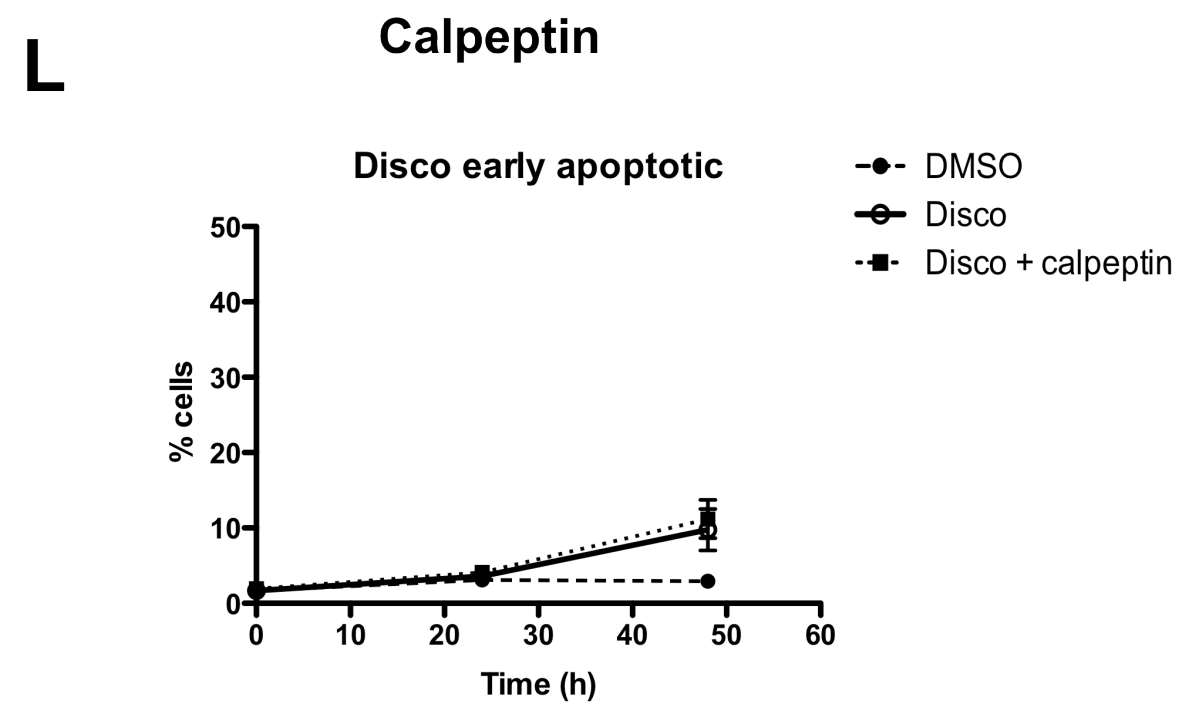

Disco late apoptotic

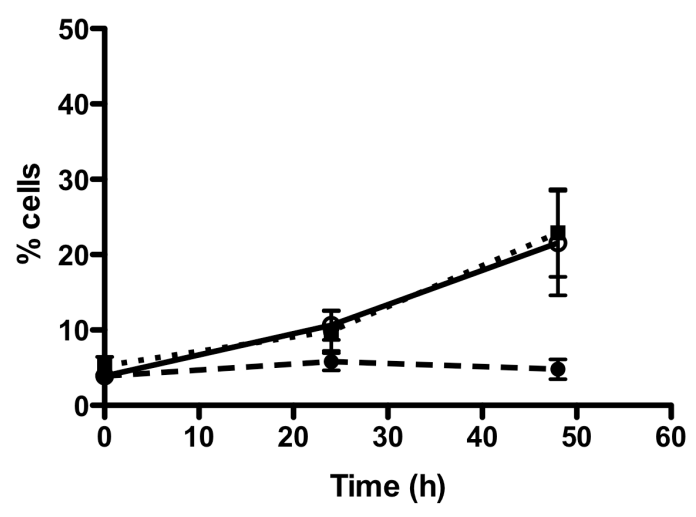

Disco early/late apoptotic

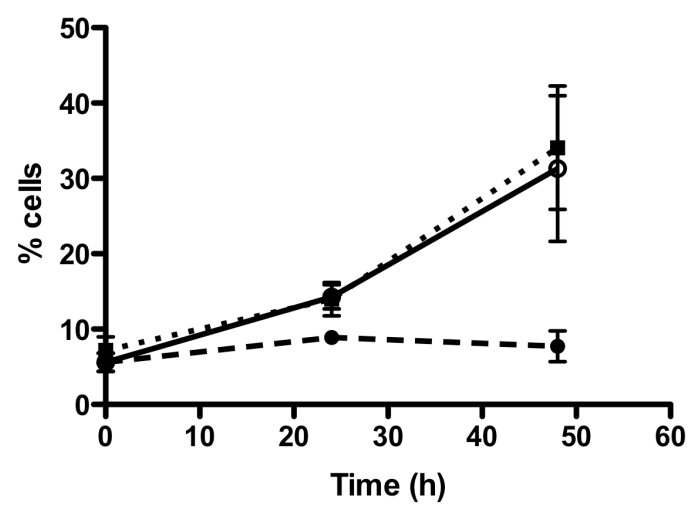

Figure 3.4 (continued) 


\subsubsection{PelA activates caspase-3/7}

Using the panel of protease inhibitors the annexin V assay suggested that PelA- and MycA-induced apoptosis was primarily mediated through the activation of caspases. To confirm this, we decided to look at the activation of executioner caspases using an ApoLive-Glo ${ }^{\mathrm{TM}}$ multiplex assay that simultaneously assesses cell viability (by detecting the activity of a cellular protease only present in live cells) and caspase-3/7 activation. A preliminary, single experiment was run to find the optimal timepoint for caspase-3/7 activation in response to PelA, MycA and Disco (Figure 3.5). The reduction in cell viability, observed in response to both PelA and Disco, was accompanied by an increase in caspase-3/7 activation. The degree of caspase-3/7 activation by PelA and Disco was notably greater than that induced by MycA, despite a significant reduction in cell viability by MycA. The greatest effect was seen at $48 \mathrm{~h}$ for all three drugs, so this timepoint was chosen for further assays. It should also be noted that cell viability was greatly reduced at the $\mathrm{IC}_{80}$ concentrations of both PelA and $\mathrm{MycA}$, but a lesser cytotoxic effect was seen at the $\mathrm{IC}_{80}$ for Disco.

We then looked at the concentration-response effect of the three drugs after $48 \mathrm{~h}$, using the same assay since (according to the manufacturer) a decrease in cell viability and simultaneous increase in caspase-3/7 activity is indicative of apoptosis, while a decrease in cell viability with no caspase-3/7 activity is indicative of primary necrosis. However, if caspase-independent mechanisms of cell death (described in the introduction to this chapter) were being utilized, decreased cell viability without activation of caspase-3/7 and could give similar result to necrosis. Although the same trend was observed between replicate experiments, unfortunately, the ApoLive-Glo ${ }^{\mathrm{TM}}$ assay was not very reproducible. Due to the large variation between experiments, we could not combine replicate data; thus, each replicate is presented in individual graphs (Fig. 3.6). As expected, we observed a concentration-dependent decrease in cell viability with PelA, MycA and Disco. We also observed caspase-3/7 activation with all three drugs, although to different extents, which was fairly consistent with our results from the protease inhibitor screens. zVAD-fmk conferred significant protection against PelA and Disco in the MTT assays, and PelA and MycA in the annexin V flow cytometry assays, implying the involvement of caspases in the mode of actions of all three drugs. We generally saw a 2-4-fold increase in caspase-3/7 activation with Disco, and a much lesser degree of activation in response to MycA (an approximate 1.5-3-fold increase in activation). At the $\mathrm{IC}_{80}$ concentration, PelA exhibited the greatest caspase-3/7 activation 
(typically 3-5-fold) compared to the other two drugs. The simultaneous decrease in cell viability and caspase-3/7 activation that occurred with all three drugs suggests an apoptotic form of cell death (rather than necrotic), although the MycA results were not as clear-cut. Despite a simultaneous decrease in viability and increase in caspase-3/7 activity, the degree of caspase activation is not as obvious compared to PelA and Disco. A possible interpretation from the MycA results is that there may be some necrotic cell death occurring. Caspase-3/7 acts as the executioner caspase in both typical apoptosis and lysosome-mediated apoptosis. Treatment of cells with protease inhibitors prior to drug treatment also confirmed the activation of caspase-3/7 in response to all three drugs regardless of whether cathepsins or calpain were inhibited, suggesting classical caspase-mediated apoptosis rather than a lysosomal pathway of apoptosis (Fig. 3.7). zVAD-fmk inhibited the induction of caspase-3/7 in response to PelA and Disco, but no protection was seen by the other protease inhibitors (with the exception of CA-074Me against PelA). In the absence of protease inhibitor, MycA did not activate caspases-3/7 above control levels (untreated cells); however, the little caspase-induction that was induced was also reduced by zVAD-fmk. MycA exerts its effects very quickly (within hours) since it is a protein synthesis inhibitor and not an anti-mitotic drug like PelA and Disco. Hence, caspase-3/7 activation may have peaked well before the activity was measured (see Discussion). 
A

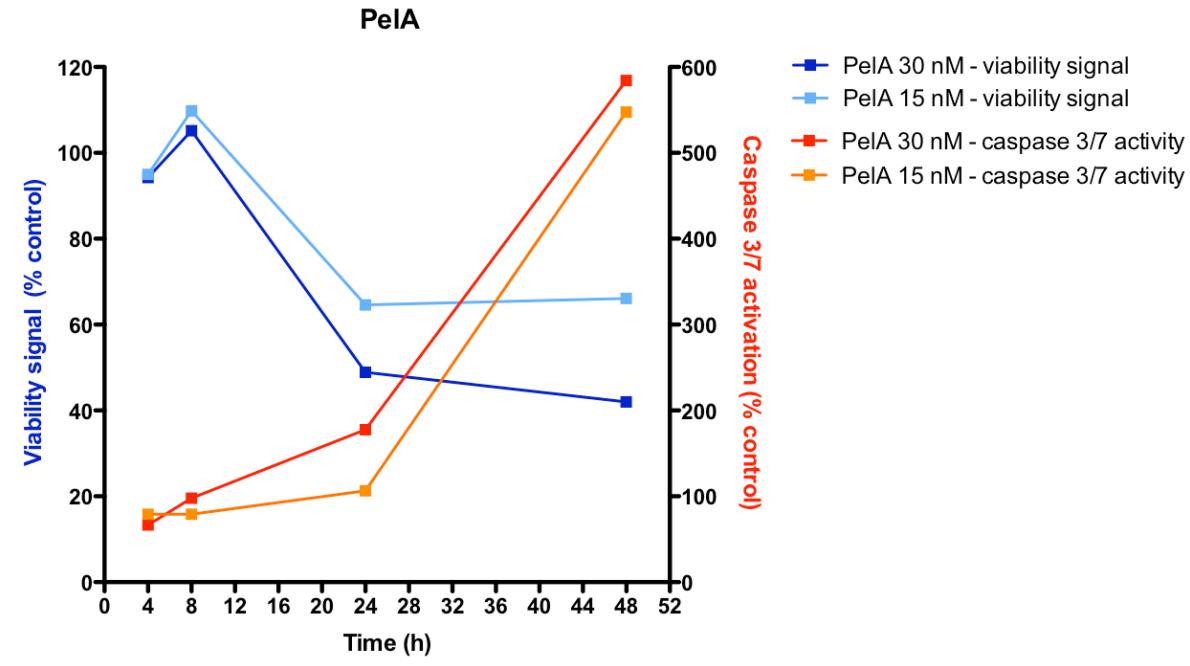

B

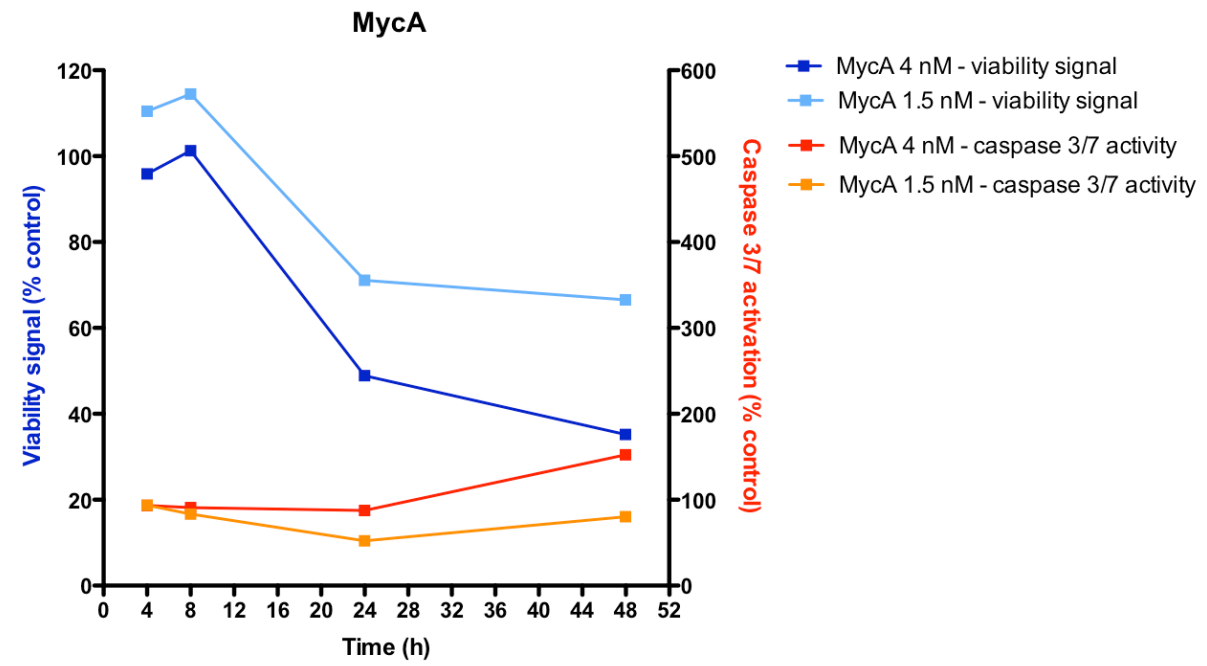

C

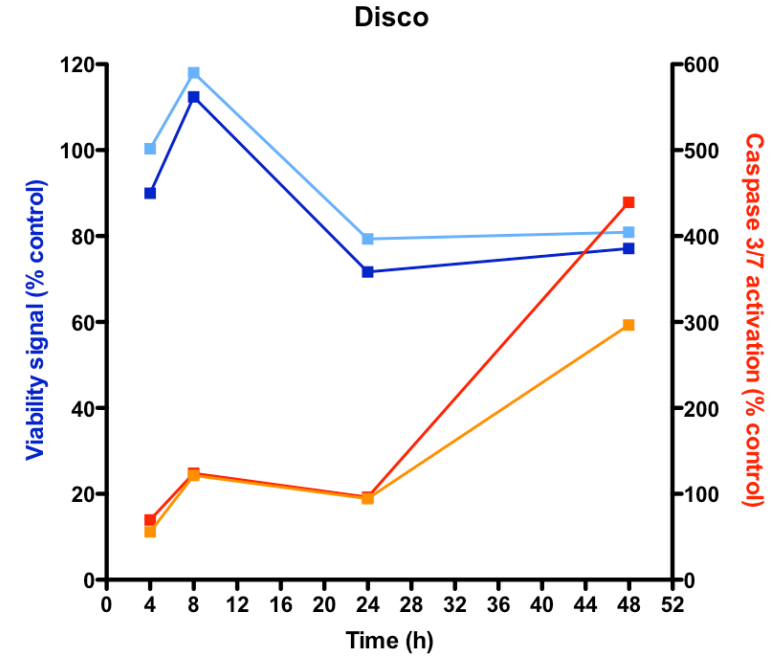

$\rightarrow$ Disco $111 \mathrm{nM}$ - viability signal

-Disco $50 \mathrm{nM}$ - viability signal

- Disco $111 \mathrm{nM}$ - caspase $3 / 7$ activity

- Disco $50 \mathrm{nM}$ - caspase $3 / 7$ activity

Figure 3.5. ApoLive-Glo ${ }^{\mathrm{TM}}$ multiplex assay - time course. To simultaneously assess caspase-3/7 activation and cell viability signal (activity of a live cell protease), $1 \mathrm{~A} 9$ cells were treated with the $\mathrm{IC}_{80}$ or $\mathrm{IC}_{50}$ concentrations of PelA (A), MycA (B) or Disco (C). At various timepoints, cells were incubated with the fluorescent $(\mathrm{Fl})$ viability and luminescent (Lum) caspase-3/7 substrates and read on a multiwall plate reader. Data indicate the changes in viability signal (left axis, blue curves (Fl)) and caspase-3/7 activation (right axis, orange curves (Lum)) (expressed as a percentage of control values), from a single experiment. 


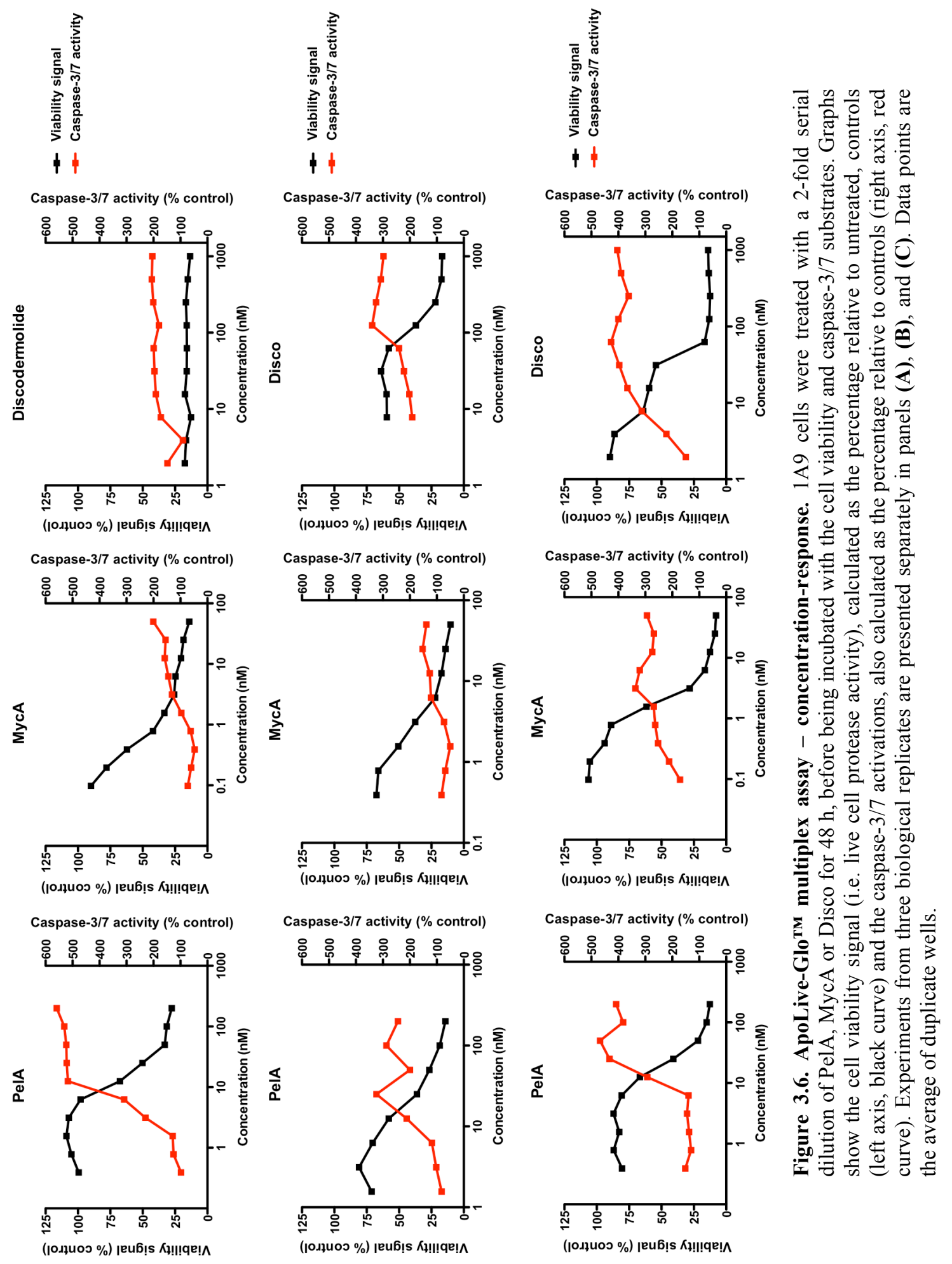



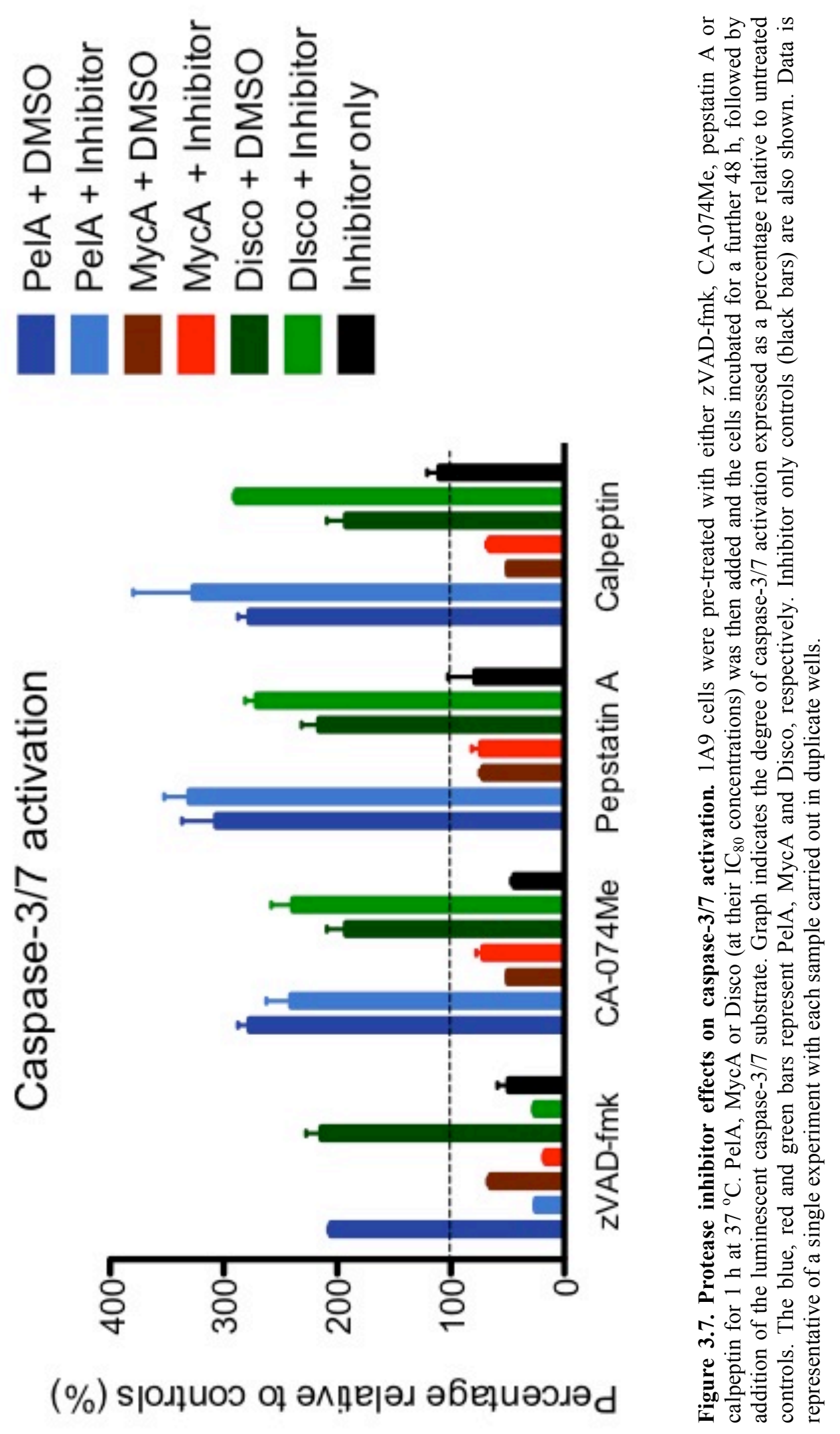


\subsubsection{PelA activates caspases-8 and -9}

We then wanted to determine whether PelA-induced apoptosis was occurring via the extrinsic or intrinsic pathways (i.e. whether apoptosis was mediated primarily by caspase-8 or -9). FLICA ${ }^{\mathrm{TM}}$ probes, which act as fluorescent indicators of caspase activation, were used to evaluate the timing of activation of these initiator caspases. Due to the limited amount of reagent, we did not assess caspase- 8 or -9 activation in response to MycA and Disco. Activation of caspase- 8 or -9 is upstream of caspase-3/7 activation; thus, based on the ApoLive-Glo ${ }^{\mathrm{TM}}$ results for PelA (Fig. 3.5), we expected the initiator caspase(s) to peak somewhere between 24 and 48 h (we saw very little caspase-3/7 activation by PelA prior to the $24 \mathrm{~h}$ timepoint). Using the FLICA ${ }^{\mathrm{TM}}$ probes, we found very similar activation patterns for both caspase- 8 and caspase-9 (Figure 3.8 $A$ and $B$, respectively). An increase in autofluorescence was observed in response to PelA treatment (Appendix B, Fig. B.3); therefore, wider gates for the FLICA ${ }^{\mathrm{TM}}$ negative populations were set than are typically used. Camptothecin was chosen as a positive control for both caspase- 8 and caspase- 9 activation, as this drug has been shown previously to activate both these caspases in A2780 cells (the 1 A9 cell line is derived from a single-cell clone of the A2780 cell line) (Milner et al., 2002). Representative histograms for camptothecin are presented in Appendix B, Fig. B.4; however, camptothecin was disappointing as a positive control because only minimal activation at $10 \mu \mathrm{M}$ camptothecin was observed (possibly because we did not use an optimal timepoint to observe caspase activation). As expected, there were only very minor changes in caspase activation until $32 \mathrm{~h}$ after the addition of PelA. At $48 \mathrm{~h}$, treatment with $40 \mathrm{nM}$ PelA resulted in $15 \%$ and $14 \%$ of cells being positive for caspase8 and -9 , respectively (Figure $3.9 \mathrm{~A}$ and $\mathrm{B}$, respectively). Interestingly, this occurs despite a lack in prolonged $\mathrm{G}_{2} / \mathrm{M}$ arrest in cells treated at this concentration (see Chapter 4). At $100 \mathrm{nM}$ PelA, $34 \%$ and $36 \%$ of cells stained positive for caspase- 8 and 9, respectively, after $48 \mathrm{~h}$ of drug treatment (Figure 3.9, A and B, respectively). We also checked the progression of caspase- 8 and -9 activation for up to $72 \mathrm{~h}$ using the FLICA $^{\mathrm{TM}}$ probes (see Appendix B, B.5). Instead of the typical increase in a single FLICA $^{\text {TM }}$-positive peak (as was observed up to $48 \mathrm{~h}$ ), longer incubations with PelA resulted in what appears to be multiple positive peaks. This unusual effect as well as a significant degree of cell death (and hence low cell recovery), replicate experiments were only continued for up to $48 \mathrm{~h}$. 
A

Caspase-8
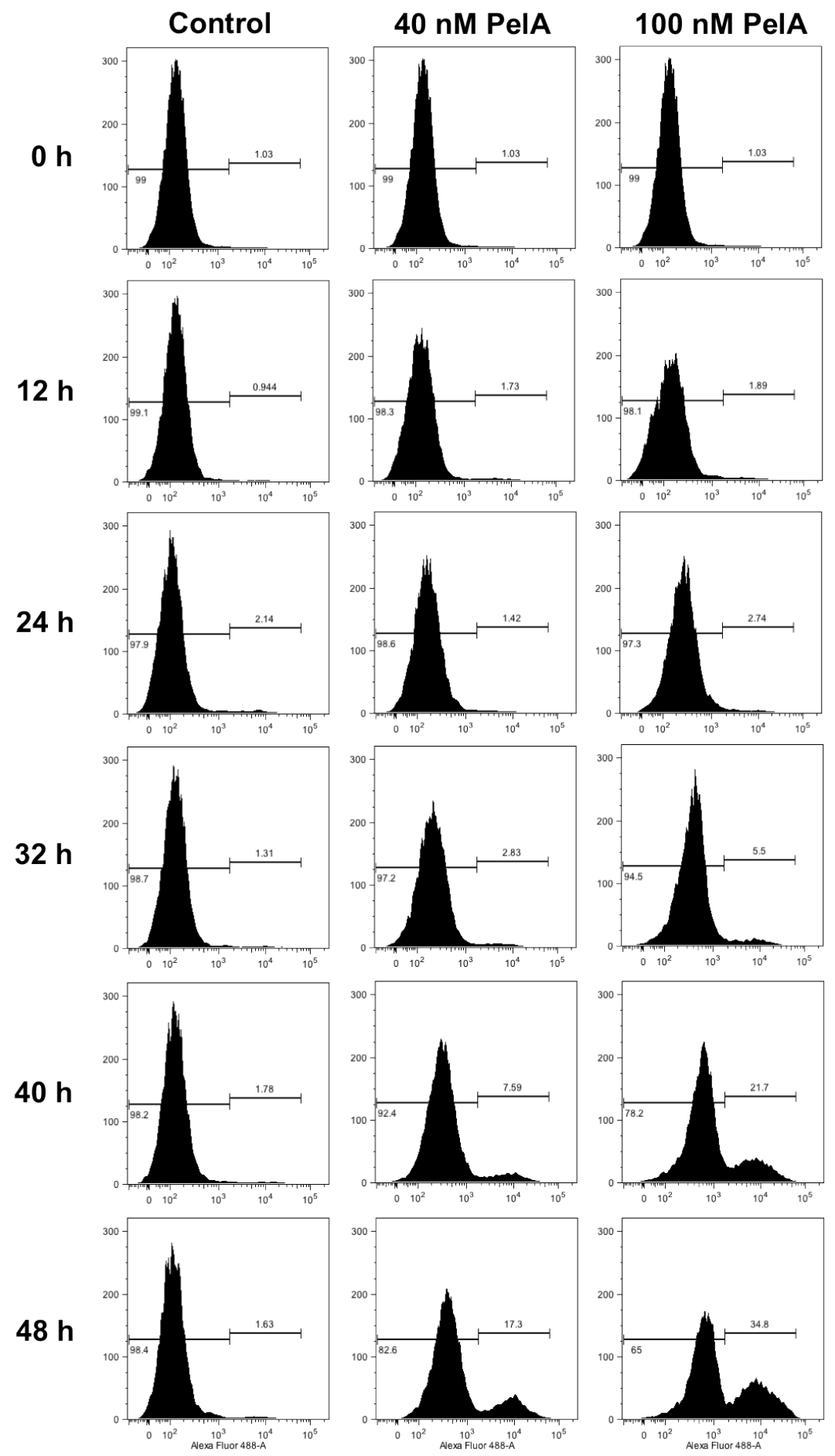

Figure 3.8. FLICA assay for detecting caspase-8 and $\mathbf{- 9}$ activation by PelA. 1A9 cells were treated with PelA and harvested at various times between 0 and $48 \mathrm{~h}$. Cells were either stained for caspase- 8 (A) or -9 (B) using FLICA probes, fixed in PFA and then analyzed by flow cytometry. Gates indicate cells that were negative or positive for FLICA (left and right gates, respectively), with the percentage of gated cells also given. The histograms are representative of three independent experiments. 
B Caspase-9

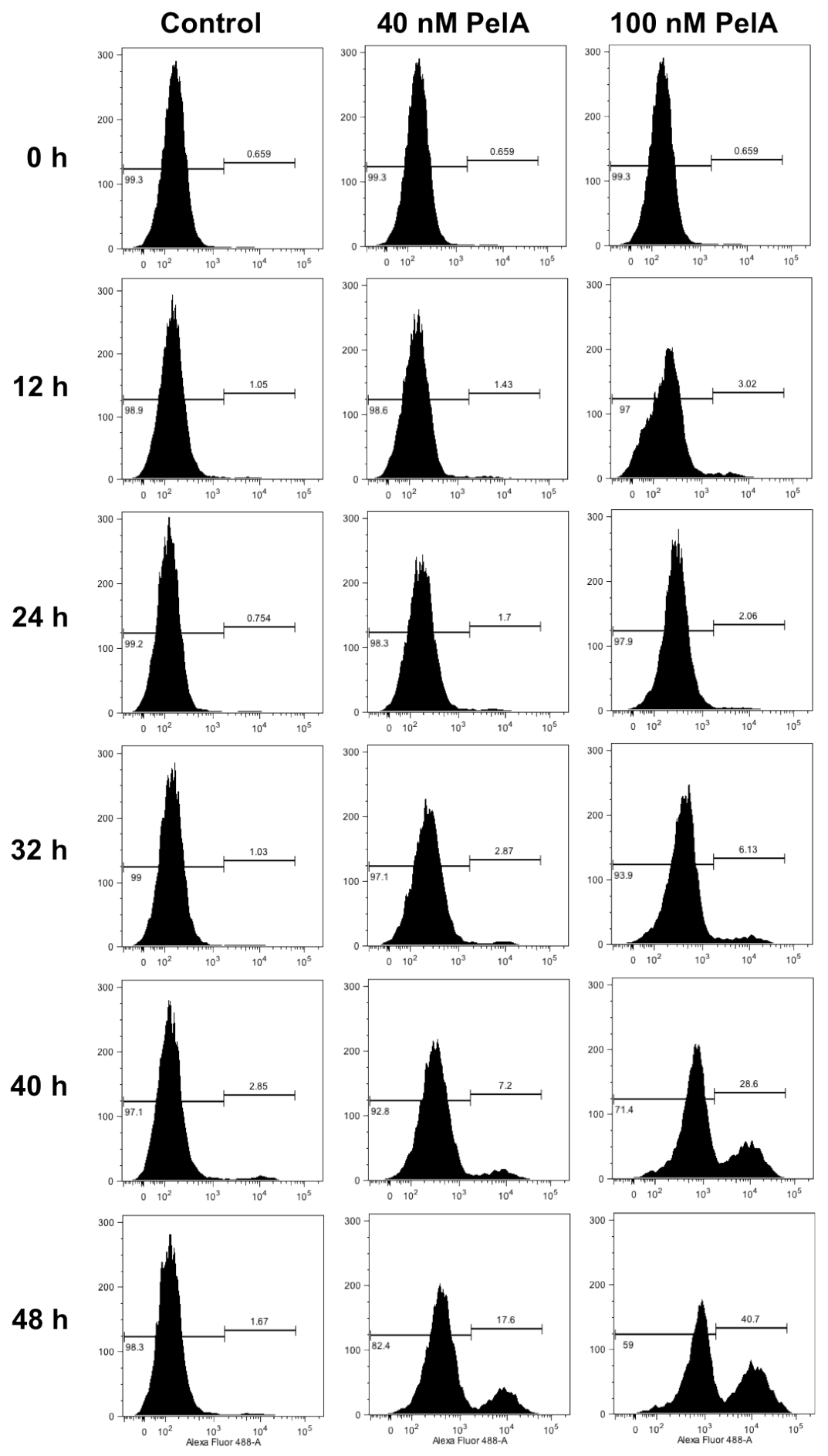

Figure 3.8 (continued) 


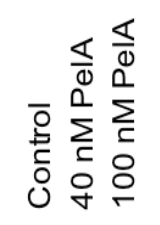

\section{III}

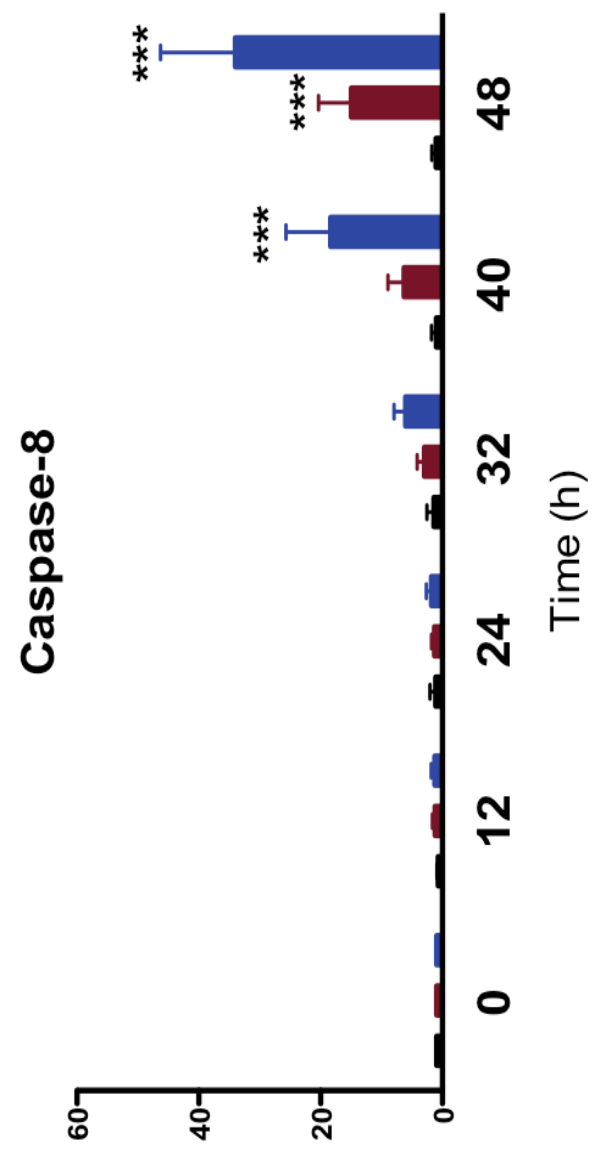

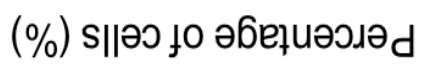

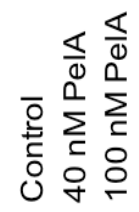

III

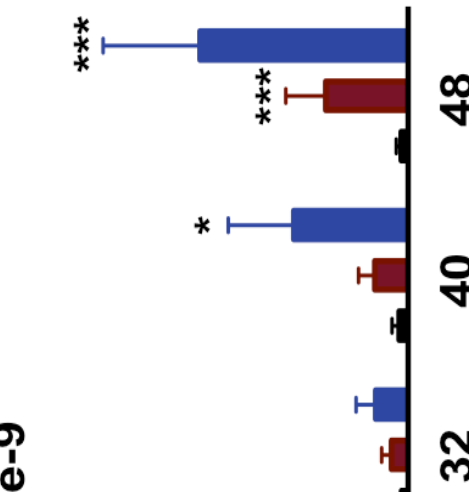

d

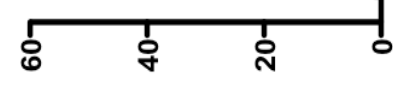

(\%) s||әว јо әбеұиәэәә

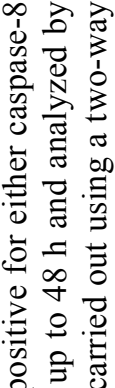

过

要

흘 흘

$\exists$ 흘

业需

8 政

荡 专

要运语

氙焉

过

(ᄃ)

(1) 000 ठ

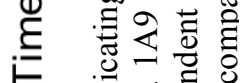

导范。

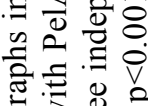

03

过若艺草

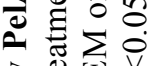

홉

三

政

过

西吉

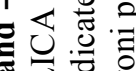

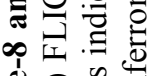

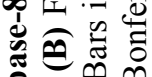

on

ชั

$\circ$ 政

ले है

ㅎํ워

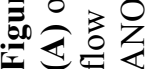




\subsection{Discussion}

\subsubsection{Protective effects of a panel of protease inhibitors against PelA, MycA and}

\section{Disco}

Using a panel of protease inhibitors, we sought to elucidate the signalling mediators underlying the apoptotic effects of several cytotoxic drugs, including PelA, MycA and Disco. As summarized in Table 3.2, we found that the pan-caspase inhibitor (zVADfmk) provided the greatest level of protection against the MSAs PelA and Disco, using a $48 \mathrm{~h}$ MTT cell proliferation assay. Additionally, the cathepsin D (pepstatin A), cathepsin B (CA-074Me) and calpain (calpeptin) inhibitors conferred some protection against the cytotoxic effects of PelA (cathepsins D and B, and calpain) and Disco (cathepsin D only). In terms of the protein synthesis inhibitor MycA, only the cathepsin B inhibitor was able to confer any significant protection against the cytotoxic effects of the drug in the MTT assay. An annexin V/PI assay using the same panel of protease inhibitors gave results that did not completely align with the MTT assay results (Table 3.2) and reasons for these differences will be discussed. Consistent with the MTT results, caspase inhibition did provide a significant protective effect against the PelAinduced apoptosis (both earlier and later stages). However, zVAD-fmk also provided protection against the effects of MycA but not Disco, which contrasted with the MTT assay results. A small reduction in the percentage of cells exhibiting annexin $\mathrm{V}^{+\mathrm{ve}} / \mathrm{PI}^{-\mathrm{ve}}$ staining (i.e. early apoptosis) was observed when Disco- or MycA-treated cells were pre-treated with zVAD-fmk or CA-074Me, respectively, but these effects were not statistically significant. The only other inhibitor to demonstrate any protective effects in this assay was CA-074Me, but only against the early apoptotic effects of PelA; whereas, in the MTT assay, the cathepsins and calpain also seemed to be involved in PelA's mechanism. Taken together, these results suggest the involvement of multiple mediators in the induction of apoptosis by theses drugs. The effects of PelA appear to be primarily mediated by caspases, and to a lesser extent by other proteases. Similarly, our results suggest that MycA- and Disco-induced apoptosis involves caspases and cathepsin D, although the results for these drugs were not as clear-cut. One confounding factor is that despite pre-determination of the $\mathrm{IC}_{80}$ values, PelA, MycA and Disco inhibited cell viability by $71 \%, 63 \%$ and $46 \%$, respectively (not the expected $80 \%$ ) (Figure 3.3), and may have contributed to some of the inconsistencies between the various assays. 
Table 3.2. Summary of findings from the protease inhibitor screen and caspase activation assays for PelA, MycA and Disco

\begin{tabular}{|c|c|c|c|c|c|}
\hline & & & \multicolumn{3}{|c|}{ TREATMENT } \\
\hline & & & PelA & MycA & Disco \\
\hline & \multirow{4}{*}{48 h MTT assay } & zVAD-fmk ${ }^{a}$ & + & 0 & 0 \\
\hline & & Pepstatin A & + & 0 & + \\
\hline & & CA-074Me ${ }^{c}$ & + & + & 0 \\
\hline & & Calpeptin $^{\mathrm{d}}$ & + & 0 & 0 \\
\hline \multirow{15}{*}{ 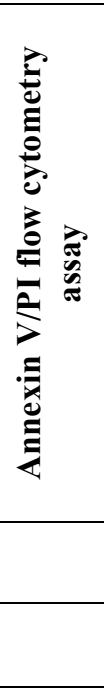 } & \multirow{4}{*}{$\begin{array}{l}\text { Early apoptosis } \\
\left(\text { Annexin } V^{-v e}\right)\end{array}$} & zVAD-fmk & + & + & $0^{\mathbf{e}}$ \\
\hline & & Pepstatin A & 0 & 0 & 0 \\
\hline & & CA-074Me & + & $0^{\mathbf{e}}$ & 0 \\
\hline & & Calpetpin & 0 & 0 & 0 \\
\hline & \multirow{4}{*}{$\begin{array}{l}\text { Late apoptosis } \\
\left(\text { Annexin } \mathbf{V}^{-v e} / \mathbf{P I}^{+v e}\right)\end{array}$} & zVAD-fmk & + & + & 0 \\
\hline & & Pepstatin A & 0 & 0 & 0 \\
\hline & & CA-074Me & 0 & 0 & 0 \\
\hline & & Calpetpin & 0 & 0 & 0 \\
\hline & \multirow{4}{*}{$\begin{array}{l}\text { Early/late apoptotic } \\
\left(\text { Annexin } \mathbf{V}^{+v e} / \mathbf{P I}^{+v e}\right)\end{array}$} & zVAD-fmk & + & + & 0 \\
\hline & & Pepstatin A & 0 & 0 & 0 \\
\hline & & CA-074Me & 0 & 0 & 0 \\
\hline & & Calpetpin & 0 & 0 & 0 \\
\hline & $\begin{array}{l}\text { ApoLive-Glo } \\
\text { assay }\end{array}$ & Caspases-3/7 & + & + & + \\
\hline & \multirow{2}{*}{ FLICA $^{\mathrm{TM}}$ assay } & Caspase-8 & + & $n d$ & $n d$ \\
\hline & & Caspase-9 & + & $n d$ & $n d$ \\
\hline
\end{tabular}

${ }^{\mathbf{a}}$ pan-caspase inhibitor; ${ }^{\mathbf{b}}$ cathepsin D inhibitor; ${ }^{\mathbf{c}}$ cathepsin B inhibitor; ${ }^{\mathbf{d}}$ calpain inhibitor

+ indicates a positive effect i.e. protection by protease inhibitors against apoptosis/growth inhibition, or positive caspase activation

$\mathbf{0}$ indicates no effect i.e. no protection by protease inhibitors against apoptosis/growth inhibition

$\mathbf{0}^{\mathbf{e}}$ indicates some positive protective effects were observed but were not statistically significant $n d=$ not determined 
Based on previous results in our laboratory in which PelA did not induce caspase- 8 and -9 activation in an HL-60 cell line (Miller et al., 2004), the results from the panel of protease inhibitors were a little unexpected. MycA and Disco were assessed alongside PelA because they had been shown previously to activate caspases and cathepsin B, respectively (Bröker et al., 2004; Miller et al., 2004). In comparison to the MTT assay, which measures cell viability, the annexin $\mathrm{V}$ assay detects an early stage of apoptosis. It is not surprising, then, that the protective effects of the inhibitors did not completely align with the annexin $\mathrm{V}$ assay results given the differences in the timing of druginduced effects. The effects of the protease inhibitors were assessed at $48 \mathrm{~h}$ in both assays; however, unlike the externalization of PS, the more general cytotoxic effects of the drugs may not become apparent until later timepoints and could be missed in the MTT assay. Also, it has been suggested that rather than initiating apoptosis, cathepsins may play more of a role in amplifying the apoptotic signal (Oberle et al., 2010; Schrader et al., 2010). Hence, a more noticeable protective effect by the cathepsin inhibitors in the MTT assays, but not the annexin V/PI assays, may be due to a dampened apoptotic response. This reduced signal amplification, which may not affect the appearance of initial apoptotic events (e.g. PS externalization), could delay the induction of apoptosis. This could lead to delayed effects on cell viability, which become detectable only towards the end (or outside of) the $48 \mathrm{~h}$ window of our MTT assay, leading to a somewhat false impression of protective effects. An apoptotic delay has been shown previously in mouse embryonic fibroblasts (MEFs) isolated from mice deficient in cathepsin B or L (Oberle et al., 2010). In this study, the authors showed that MEFs treated with the topoisomerase II inhibitor, etoposide, exhibited a significant delay in both annexin V/PI staining and caspase-3 activation.

PelA gave the most consistent and conclusive results from the protease inhibitor screens. There are, however, several caveats associated with the use of these inhibitors that must be taken into consideration. There is evidence that the pan-caspase inhibitor, zVAD-fmk, does not inhibit all caspases equally, even at the high concentrations that are commonly used (Chauvier et al., 2007). Additionally, zVAD-fmk has been shown to exhibit cross-reactivity with proteases other than the caspases (e.g. cathepsins and calpain) and similarly, CA-074Me can also inhibit proteases other than its specific target, cathepsin B (Schotte et al., 1999; Bogyo et al., 2000; Repnik et al., 2012). There is also some evidence that, in vivo, the caspase inhibitors themselves have toxic effects, possibly due to the metabolic conversion of the "fmk" (fluoromethylketone) moiety to 
fluoroacetate (Van Noorden, 2001; Chauvier et al., 2007). Thus, caution must be taken when interpreting results from experiments using these caspase inhibitor probes.

\subsubsection{Activation of caspase-3/7 by PelA}

Most microtubule-stabilizing agents engage components of the caspase signalling cascades (usually the mitochondrial pathway), and from the above results it would appear that this may also the case for PelA (Bhalla, 2003). The ApoLive-Glo ${ }^{\mathrm{TM}}$ assay kit uses a luminescent substrate containing the tetrapeptide sequence, DEVD, which is recognized by caspase-3/7. The ApoLive-Glo ${ }^{\mathrm{TM}}$ assay results confirmed that both PelA and Disco induced the activation of one (or both) of these executioner caspases, and the simultaneous decrease in cell viability indicated that these drugs acted primarily through apoptosis (Table 3.2). This also tends to exclude a predominant necrotic pathway of cell death (characterized by the widespread swelling of cells and organelles), which occurs independently of caspases (Golstein and Kroemer, 2005). It is not clear why zVAD-fmk could not protect against Disco in the annexin V assay, since caspase-3/7 is clearly activated in this particular assay. It is possible that Disco triggers multiple cell death pathways that are caspase-independent and/or mediated by proteases that were not included in our inhibitor screen. Disco has been shown to trigger caspase-independent apoptosis via cathepsin B in NSCLC cells, so it is possible that other non-caspase proteases are involved in its cytotoxicity in other cell lines (Bröker et al., 2004). Although clearly activated, the increase in caspase-3/7 in response to MycA was not as pronounced compared to the MSAs. A clear inhibition of cell viability as the concentration of MycA was increased, combined with a relatively weak caspase-3/7 activity, suggests that some of the cytotoxic effects of MycA may be due to primary necrotic mechanisms as a result of the inhibition of protein synthesis. Cellular necrosis has been observed after treatment of squamous carcinoma cells with the synthetic analogue 18-O-methyl mycalamide $\mathrm{B}$ and is a cell fate that could be shared by cells treated with MycA (Richter et al., 1997). Additionally, a previous study by our laboratory group found that MycA activated caspase- 8 and -9 within a few hours $(1.5$ and 6 h, respectively) (Miller et al., 2004), so by only looking at later time points, we may have missed the peak caspase activity and/or reduced cell numbers to such an extent that caspase activation was below the detection threshold of the kit.

Casapse-3/7 acts as the executioner caspase during both classical apoptotic pathways (i.e. death receptor and mitochondrial) and lysosomal pathways of apoptosis (acting 
downstream of cytochrome c release from the mitochondria; see Fig. 3.1). Pre-treatment of $1 \mathrm{~A} 9$ cells with pepstatin A, CA-074Me or calpeptin prior to incubation with PelA, MycA or Disco, was unable to prevent the activation of caspase-3/7. Protease release from the lysosomes results in apoptotic signalling via the apoptotic machinery of the mitochondrial pathway, culminating in the activation of caspases. If a lysosomal pathway was solely responsible for the induction of cell death, then the cathepsin inhibitors should have reduced caspase-3/7 activation by PelA, MycA and Disco; thus, although only carried out as a single experiment, these results are inconsistent with a lysosomal pathway of apoptosis mediated by cathepsins B/D (or calpain). Conversely, zVAD-fmk reduced caspase-3/7 activation to below that of the controls for all three drugs further implicating a classical caspase-mediated pathway of cell death. It would be interesting to see whether PelA can trigger an alternative form of cell death in caspase-3 knock out cell lines, as this will help to elucidate possible compensatory mechanisms of MSA-induced cell death. The MCF7 breast cancer cell line is reported to lack detectable procaspase-3 and upon Ptx treatment can induce the activation of caspases-9 and -7 (Kottke et al., 2002). Both MycA and Disco proved to be inappropriate positive controls (for caspases- and cathepsin B-mediated apoptosis, respectively) under our experimental conditions. What has become apparent from these assays is that the cell lines, timepoints and drug concentrations that are used greatly influence the apoptotic signalling pathways; thus much consideration must be given to the experimental conditions.

\subsubsection{PelA activates caspases-8 and -9}

To determine whether the mitochondrial or death receptor pathways primarily mediate PelA-mediated apoptosis, two FLICA ${ }^{\mathrm{TM}}$ probes were used to assess caspase- 8 versus caspase-9 activation in 1A9 cells. Due to limitations in the quantity of probes we had available, we decided to only focus on initiator caspase activation by PelA. Many of the commonly used synthetic caspase inhibitor probes bind irreversibly through a fluoromethylketone (fmk) moiety and contain a peptide sequence that determines their specificity. For example, VAD (Val-Ala-Asp) confers broad caspase specificity; whereas, DEVD (Asp-Glu-Val-Asp), I/LETD (Ile/Leu-Glu-Thr-Asp), and LEHD (LeuGlu-His-Asp) are designed to target caspase-3/7, caspase- 8 and caspase-9, respectively. As mentioned earlier, the gates for the FLICA ${ }^{\mathrm{TM}}$-negative populations were set wider than normal, due to a positive shift in the negative populations of PelA-treated cells compared to negative control populations. Unlabelled PelA-treated cells revealed a 
concentration- and time- dependent increase in the autofluorescence of FLICA ${ }^{\mathrm{TM}_{-}}$ negative populations (Appendix B, Figure B.3). All mammalian cells exhibit some degree of inherent autofluorescence, which is influenced by physiological factors, including NADH and flavins (Aubin, 1979; Benson et al., 1979). An increase in green flavoprotein fluorescence has been shown previously to correlate with increased mitochondrial stress and apoptosis (in human retinal pigment epithelial cells) and may explain why we see this positive shift in the FLICA assay (this positive shift was also observed in the annexin V assay, but this was not shown) (Field et al., 2011). Despite cells becoming blocked in $\mathrm{G}_{2} / \mathrm{M}$ as early as $12 \mathrm{~h}$ (possibly earlier as we did not look at any timepoints between 0 and $12 \mathrm{~h}$; data not shown) at both 40 and $100 \mathrm{nM}$ PelA, we did not begin to see an increase in caspase- 8 or -9 activation until $32 \mathrm{~h}$. This timing of activation correlates well with the activation of caspase-3/7, which peaked at $48 \mathrm{~h}$ (although we did not test beyond this timepoint). This suggests that Bekier et al. may be correct in their conclusion that a prolonged mitotic arrest promotes an apoptotic cell fate (Bekier et al., 2009). This would also explain why previous studies in our laboratory that looked at PelA treatment for up to $24 \mathrm{~h}$ failed to detect activation of either of the initiator caspases (Miller et al., 2004; Gaitanos, 2005).

It is unusual to see a similar activation pattern, both in terms of the timing and the degree of activation, for both of the initiator caspases. Although both the mitochondrial and death receptor pathways can cross-react and activate each other, a sequential activation pattern is expected, not a simultaneous activation as seen in the present study. It is possible that the timepoints that we chose were not optimal for either of the initiator caspases, and that we missed the window of peak caspase activation. Another possibility is that the high similarity in the peptide sequences recognized by caspase- 8 and -9 leads to cross-reactivity by the FLICA ${ }^{\text {TM }}$ probes. Previous studies investigating the specificity of such caspase inhibitors have suggested that they are not very specific, and caution against their use to monitor the activity of individual caspases (Pereira and Song, 2008). For example, all of the 6 "specific" caspase inhibitors that Pereira et al. tested completely inhibited caspases-1, $-6,-8$, and -9 at $1 \mu \mathrm{M}$, and caspases $-2,-3$, and 7 at just $10 \mu \mathrm{M}$ (Pereira and Song, 2008). The lack in complete specificity of the caspase- 8 and -9 probes in the FLICA assay may explain why we observed, at the later timepoints ( $>48 \mathrm{~h}$ ), multiple positive peaks (see Appendix B, Figure B.5). Once the initiator caspases have triggered late-stage apoptosis, the probes may be detecting the more abundant caspase-3/7 activity, despite the FLICA ${ }^{\mathrm{TM}}$ probes containing the 
preferred tetrapeptide recognition sequences (personal communication with Brian Lee, Vice President of Research, ImmunoChemistry Technologies, LLC (the company that owns the FLICA ${ }^{\text {TM }}$ trademark)). It is possible that the peaks at the higher fluorescence intensities represent the final stages of apoptosis and predominantly contain caspase-3, which is detected non-specifically by the caspase- $8 / 9$ probes. Ptx has been shown by different research groups to activate several caspases, including caspases-6, -8, -9, and 10 (Park et al., 2004; Wang et al., 2004b; Janssen et al., 2007; Mielgo et al., 2009). It is highly likely that PelA activates different or multiple caspases as well, depending on the cell lines used.

Considering that tumour cell resistance is often correlated with impaired apoptotic signalling (particularly the BCL-2 family of proteins) (Kaufmann and Vaux, 2003), the ability of particular drugs to induce cell death via multiple mechanisms may be a useful property to circumvent some of the issues surrounding resistance to chemotherapy. Depending on the particular conditions of the system that is used (e.g. cell type or apoptotic stimuli), abrogation of the primary cell death pathway can lead to varied results (Golstein and Kroemer, 2005). One study showed that Jurkat cells deficient of caspase- 8 were resistant to Fas-mediated apoptosis (i.e. via activation of endogenous Fas or oligomerization of the Fas death domain); whereas, in the same cell line, FADD oligomerization triggered caspase-independent necrotic cell death (Kawahara et al., 1998). In studies like ours, pharmacological inhibitors of proteases are used to probe signalling mediators by screening for protection against apoptosis. It is interesting that necrotic cell death was not observed when caspase activation was inhibited in PelAtreated cells. Perhaps the strength or type of our apoptotic signal (e.g. drug concentration and mitotic arrest) was not enough to switch from the apoptotic to the necrotic pathways. Another study found that treatment of caspase- $9^{-/-}$and caspase $-3^{-/-}$ mice with Jo2 (a Fas agonist that induces apoptosis via a BID-mediated mitochondrial pathway) activated alternative caspases (i.e. caspases- 6 and -2 , and caspases- 6 and -7 , respectively) to induce apoptosis, highlighting the redundancies in apoptotic signalling (Zheng et al., 2000).

\subsubsection{Conclusions and future directions}

While not as conclusive as we had hoped, our data suggests that PelA at least works primarily through caspases. We have also demonstrated that other proteases, such as the cathepsins and calpain, participate (but are not necessary) in the apoptotic signalling of 
PelA, MycA and Disco. It is now evident that the use of caspase inhibitors is useful only to a limited extent. In terms of identifying the contribution of individual caspases, knockout cell lines or animal models would provide more definitive results. Caspase- $8^{-/-}$ or $-9^{-/-}$cell lines, for example, could tell us whether one, both, or neither of these initiator caspases are absolutely required for PelA-induced apoptosis. We could not determine for sure whether PelA triggered apoptosis via the mitochondrial (caspase-9) or death receptor pathway (caspase-8), although the FLICA ${ }^{\text {TM }}$ results suggest that components of both are simultaneously being activated. To give us more complete answers, we need to use caspase-specific antibodies to monitor their specific cleavage products. If we had more time, we could also have used flow cytometry to look at changes associated with the mitochondria, such as MOMP and cytochrome c release, as these changes are indicative of the mitochondrial apoptotic pathway. Apoptosis is a complex event, and it is difficult to make comparisons between studies that use different cell lines and different drug concentrations. Given the importance of apoptosis in cellular homeostasis, cell lines that are impaired in particular signalling pathways, as is often the case with cancer cell lines, will often compensate for this by making use of additional pathways. This further complicates attempts to elucidate apoptotic signalling pathways for individual drugs. 


\section{Chapter 4. Chromosome mis-segregation induced by low concentrations of peloruside $A$}

\subsection{Introduction}

\subsubsection{The spindle-assembly checkpoint}

In eukaryotic cells, the spindle-assembly checkpoint (SAC) has evolved as a surveillance mechanism to ensure the proper segregation of chromosomes during mitosis. Accurate chromosome segregation is essential for normal cell function, embryonic development and tissue homeostasis. Defects in the SAC are thought to contribute to chromosomal instability, a driving factor behind the induction of aneuploidy (an abnormal number of chromosomes arising from the loss or gain of one or more chromosomes during cell division) and tumourigenesis (Bharadwaj and $\mathrm{Yu}$, 2004). The SAC is tightly regulated by numerous checkpoint proteins and functions to prevent chromosome mis-segregation and aneuploidy by delaying anaphase onset until all chromosomes are correctly attached to the mitotic spindle (Musacchio and Salmon, 2007). Although the precise mechanisms are still being elucidated, the SAC senses unattached kinetochores (and/or the lack of tension at kinetochores) and generates a "wait anaphase" signal at prometaphase, providing the cell with time to repair any erroneous attachments. The E3 ubiquitin ligase, anaphase-promoting complex/cyclosome $(\mathrm{APC} / \mathrm{C})$, is a multiprotein complex that targets many key cell cycle regulators for degradation (e.g. cyclin B) (Zachariae and Nasmyth, 1999). SAC proteins, including MAD2 and BUBR1 (described below) inhibit the APC/C coactivator, CDC20 (cell division cycle 20 (yeast); p55CDC (mammalian)), thereby preventing the APC/C-mediated ubiquitinylation of cyclin B and securin (Kallio et al., 1998; Zachariae and Nasmyth, 1999; Sudakin et al., 2001) (see Fig. 4.1). The separation of sister chromatids requires proteolysis of one of the subunits of cohesin (a protein complex that hold sister chromatids together) by a protease called separase (Nasmyth, 2002; Bharadwaj and $\mathrm{Yu}, 2004)$. Once the SAC is satisfied, the APC/C targets the separase inhibitor, securin, for degradation by the $26 \mathrm{~S}$ proteasome, allowing chromosome separation and the onset of anaphase. The degradation of cyclin B, another ubiquitin target of the $\mathrm{APC} / \mathrm{C}$, promotes mitotic exit via inactivation of CDK1 (cyclindependent kinase-1) (Peters, 2006).

Previous screens in Saccharomyces cerevisiae have identified several highly conserved genes that are now well established as being involved in the SAC. Two independent 
studies found that mads (Li and Murray, 1991) and bubs (Hoyt et al., 1991) mutants were not arrested in mitosis in response to the MDA benzimidizole, implicating a role for these genes in the feedback control mechanism of cell cycle progression. Critical genes that regulate mitosis include the $M A D$ (mitotic-arrest deficient) genes, $M A D 1$, $M A D 2$ and MAD3 (BUBR1 in humans), the $B U B$ (budding uninhibited by benzimidizole) genes, $B U B 1, B U B 2$ and $B U B 3$, and the MPS1 (multipolar spindle-1) gene (Wu et al., 2000; Musacchio and Salmon, 2007). Several products of these genes form the mitotic checkpoint complex (MCC), which plays a predominant role in restraining the $\mathrm{APC} / \mathrm{C}$ through inhibition of CDC20 (Sudakin et al., 2001). An earlier study by Rieder et al. (1995) used live cell imaging to follow mitosis in PtK (rat kangaroo kidney epithelial) cells, and showed that a single unattached kinetochore was sufficient to delay anaphase onset (Rieder et al., 1995). They also showed that elimination of the unattached kinetochore (through laser ablation) allowed mitosis to continue despite the aberrant microtubule attachment, suggesting that it was signals generated by the kinetochore that were inhibitory to mitotic progression. Key components of the MCC that have since been identified (i.e. MAD2, BUBR1 (Mad3 in S. cerevisiae), BUB3 and CDC20) concentrate and localize at unattached kinetochores during prophase/prometaphase (Waters et al., 1998; Sudakin et al., 2001). In addition to the MCC, MAD1, MPS1, BUB1, and Aurora B kinases (Ipl1 in S. cerevisiae) are also thought to play a role in SAC signal amplification and the MCC formation rate (Musacchio and Salmon, 2007). Numerous other proteins help to regulate the SAC, including various protein kinases (e.g. mitogen activated protein kinase (MAPK), CDK1-cyclin B1, NEK2, and polo-like kinase-1 (PLK1)) and microtubule-associated transporter proteins (e.g. kinesin-7 and dynein). The SAC becomes satisfied/silenced once bipolar chromosome attachments have been achieved. Much intense effort has gone into trying to elucidate the exact temporal and spatial mechanisms behind the activation, sustainment, and silencing of the SAC (Cleveland et al., 2003; Maiato et al., 2004).

\subsubsection{Function of MAD2 and BUBR1 in the MCC}

Understanding the precise mechanisms driving the SAC has been an extremely complex and challenging task. It is still uncertain how the MCC is formed and what drives its disassembly during SAC activation and silencing. It is well established that the MCC interacts dynamically with kinetochores, cycling on and off with a high turnover rate (Howell et al., 2000). The overexpression of MAD2, one of the first characterised MCC 
proteins, leads to mitotic arrest in both yeast and mammalian cells (likely due to an increase in inhibitory associations with Cdc20) (Hwang et al., 1998; Bharadwaj and Yu, 2004). MAD2 is thought to act as a sensor for the attachment of spindle microtubules to kinetochores, as it is present on unattached kinetochores and dissociates upon kinetochore-microtubule binding (Waters et al., 1998). It also seems to play more of direct role, given that it has been shown, in vitro, to inhibit the ligase activity of purified APC (Li et al., 1997; Fang et al., 1998). Later studies, however, determined this in vitro inhibition to be over 3000-fold less than the inhibitory effects of the MCC; therefore, it is not likely that this particular effect is physiologically relevant (Sudakin et al., 2001). The association of MAD2 with CDC20 is dependent on both MD1 and MPS1 (Hwang et al., 1998; Musacchio and Salmon, 2007). Two somewhat overlapping models (the "two-state MAD2" and "MAD2 template" models) have emerged to try to rationalize the MAD1-mediated activation of MAD2 (De Antoni et al., 2005; Yu, 2006). In both models, MAD2 exists in either an open (active) conformational state (O-MAD2) or in a closed conformational state (C-MAD2). O-MAD2 in the cytosol is recruited to kinetochore-localized Mad1 where it undergoes a conformational change to form a MAD1-C-MAD2 complex that can further recruit more O-MAD2 (see Fig. 4.1) (Yu, 2006). One of the key differences between the two models is the active MAD2 species. In the two-state model, the O-MAD2 that is recruited to the MAD1-C-MAd2 heterodimer undergoes a further conformational change to C-MAD2, which can then be directly passed on to $\mathrm{CDC} 20$ or released from the kinetochores ( $\mathrm{Yu}, 2006)$. The template model suggests that it is the additional recruited O-MAD2 that is active, and binds CDC20 to form C-MAD2-CDC20. This would allow signal amplification away from kinetochores, as the C-MAD2-CDC20 can act as a template (similarly to the MAD1-C-MAD2 heterodimer) to recruit and activate more O-MAD2 (De Antoni et al., 2005; $\mathrm{Yu}, 2006)$. There is still some debate as to which of these models is more plausible, and the system is currently under further investigation.

Similarly to MAD2, the protein kinase, BUBR1, plays a crucial role in negatively regulating the APC-promoting activity of CDC20, and it can also localize to kinetochores during spindle checkpoint activation (Chan et al., 1998; Wu et al., 2000). BUBR1 depletion impairs the localization of BUB1, MAD1, MAD2 and CENP-E (centromere-associated protein E), suggesting it also plays an important role in the recruitment of other spindle checkpoint proteins to the kinetochores (Chen, 2002). It has been suggested that BUBR1 senses the tension between kinetochores (Logarinho et al., 
2004; Elowe et al., 2007). Phosphorylation of kinetochore proteins is likely to be involved in regulating anaphase progression, as tensionless kinetochores have long been known to recognize a 3F3/2 phospho-specific antibody (Gorbsky and Ricketts, 1993; Logarinho et al., 2004). More recently it was discovered that BubR1 was the antigen responsible for this phospoepitope (at least in Xenopus laevis), and its spindle checkpoint kinase activity was enhanced by prior phosphorylation by Cdk1 and Plx1 (Xenopus laevis polo-like kinase-1) (Wong and Fang, 2007). BUBR1 is consistently hyperphosphorylated during mitosis (e.g. by CDK1, PLK1 and MAD1), although the significance of this has not yet been fully elucidated (Chen, 2002; Elowe et al., 2007; Wong and Fang, 2007). Wong et al. speculated that the kinase activity of BubR1 is likely to be important in facilitating correct microtubule-kinetochore attachments or regulating kinetochore structures (Wong and Fang, 2007).

\subsubsection{Erroneous microtubule-kinetochore attachments that are not detected by the SAC}

Due to the stochastic nature of microtubule dynamics during prometaphase, microtubule-kinetochore attachments can undergo different orientations as they try to achieve amphitelic attachments. As mentioned in the General Introduction, these can include amphitelic, syntelic, monotelic and merotelic orientations. Unattached kinetochores and tensionless kinetochores can activate the SAC to delay anaphase progression; however, despite the potential for causing chromosome mis-segregation, merotelic chromosomes seemingly avoid detection by the SAC (Cimini et al., 2001) (see Fig. 4.2). This particular type of attachment satisfies the SAC requirement that both kinetochore sites be occupied by microtubules, as well as the need for tension between the two sister chromatids. This has been demonstrated previously, where merotelically oriented chromosomes exhibit similar staining patterns of MAD2 and the 3F3/2 phosphoepitope compared to normal bi-oriented kinetochores (Cimini et al., 2001). As the sister chromatids begin to separate during anaphase, merotelically oriented chromosomes are subjected to equal forces from opposing spindle poles; therefore, these "lagging chromosomes" remain at the spindle equator and, if eventually segregated into the wrong daughter cell, can lead to aneuploidy. In mammalian cells, merotelic chromosomes occur at a relatively high frequency during early mitosis and are likely to occur simply as a consequence of the 'search and capture' behaviour of spindle microtubules (Cimini et al., 2003). There seem to be two surveillance mechanisms in place to detect merotelic chromosomes, one that acts before anaphase 
and one that acts during anaphase (Salmon et al., 2005). Delaying cells in metaphase (i.e. before anaphase onset), does not alter the frequency of merotelically oriented kinetochores, but does reduce the number of lagging chromosomes during chromosome segregation, suggesting the presence of error-correcting mechanisms prior to anaphase (Cimini et al., 2003). The chromosome passenger complex (CPC), comprised of proteins including Aurora B, INCENP (inner centromere protein), Survivin and Borealin, has been implicated in correcting such merotelic attachments (Lampson and Cheeseman, 2011). Aurora B, in particular, is thought to play a primary role in destabilizing microtubules at incorrectly attached kinetochores (i.e. those that lack proper tension) (Lampson et al., 2004). Syntelic and monotelic attachments, if not corrected, can also result in aneuploidy, but the SAC more readily detects these particular types of orientation. 


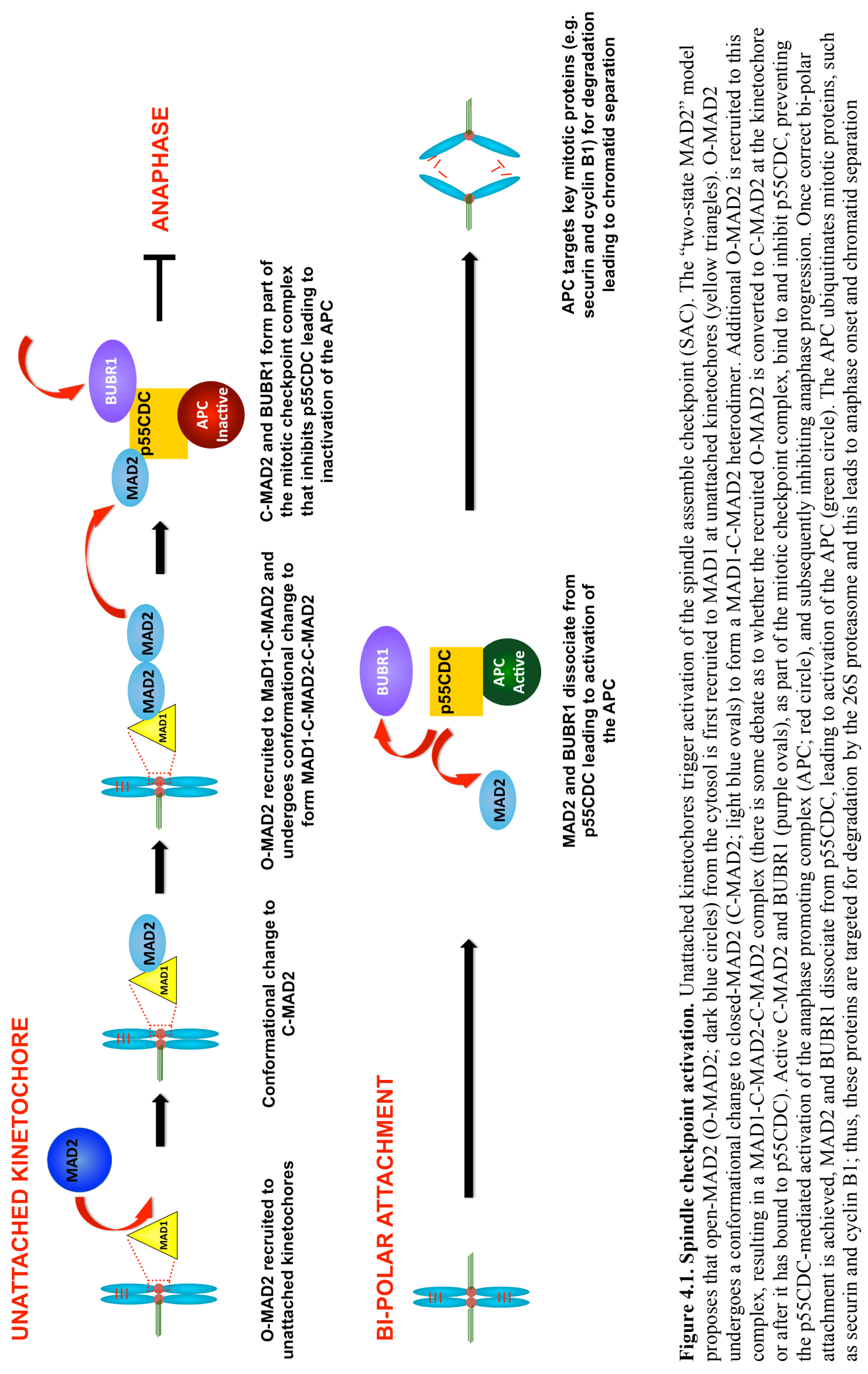




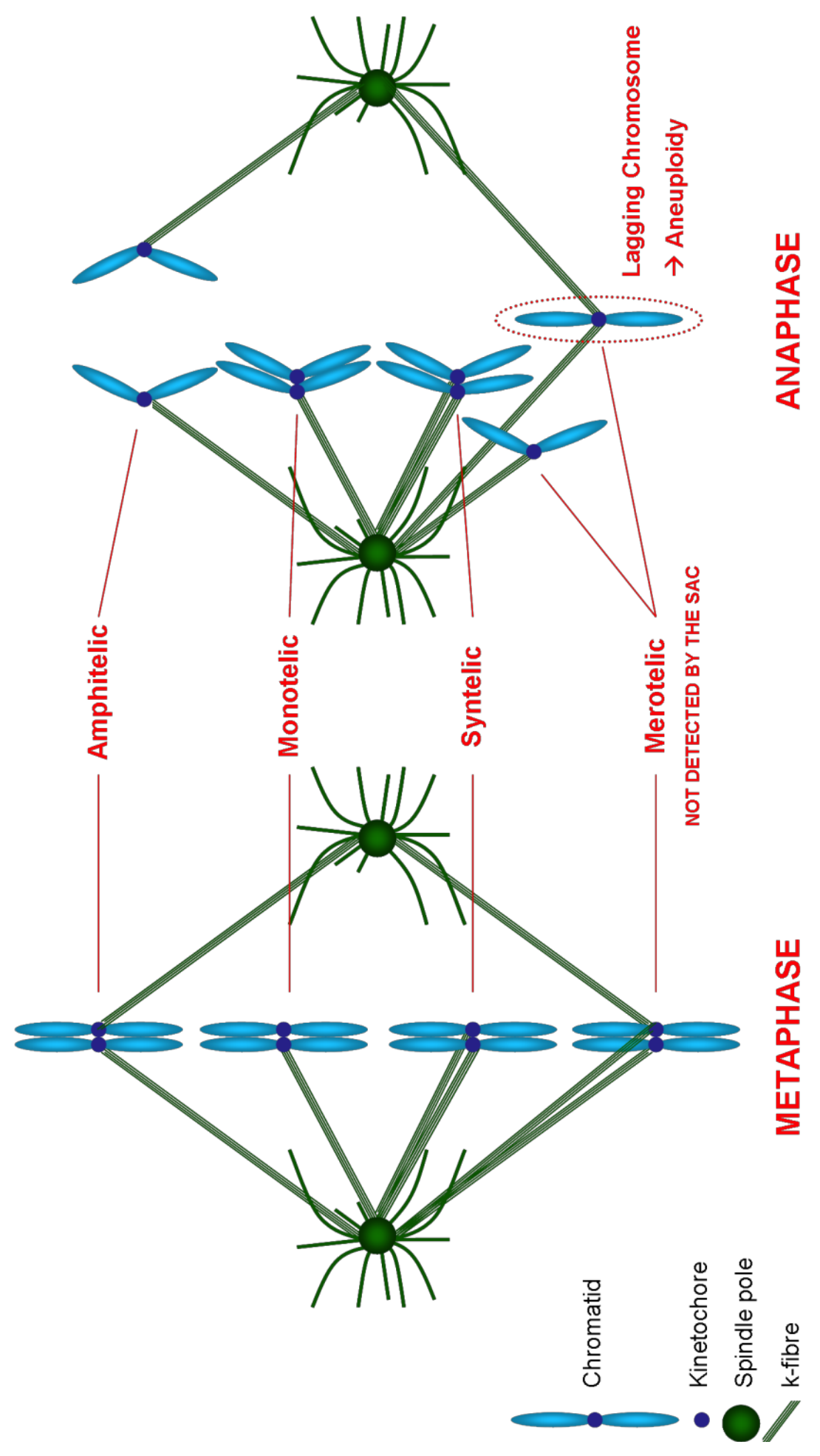

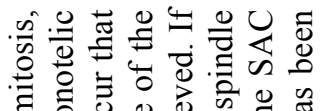
on

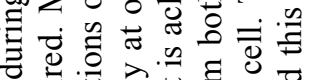

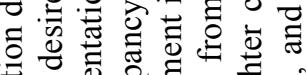

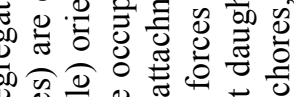

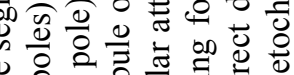

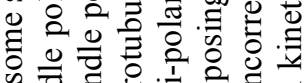
等 की 은

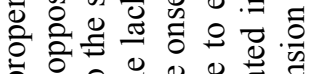

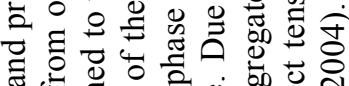

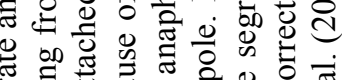

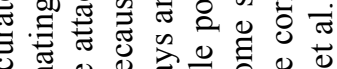
政

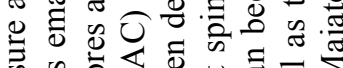

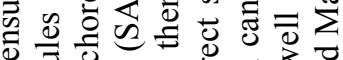

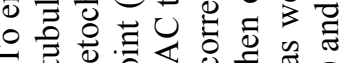

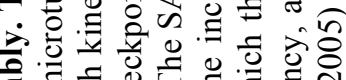

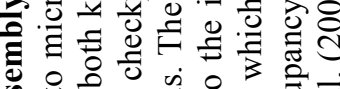

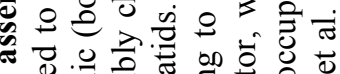

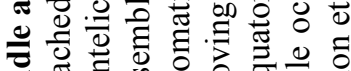

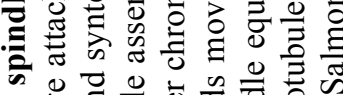

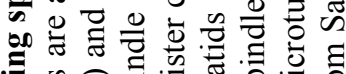
के क्षे

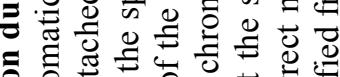

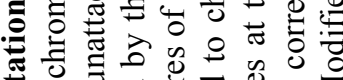

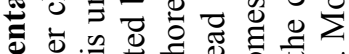
部它

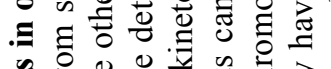

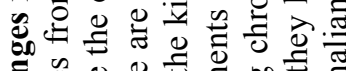

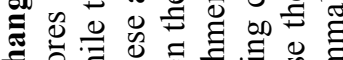

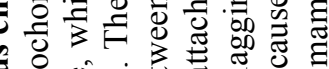

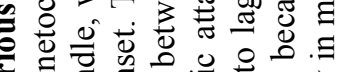

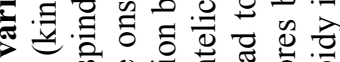
品 के

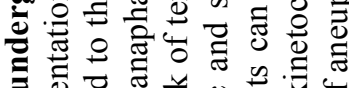

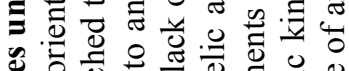

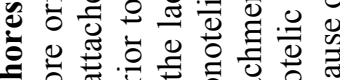

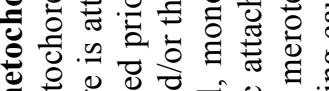

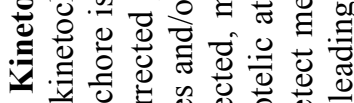

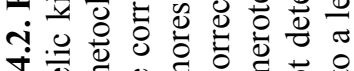

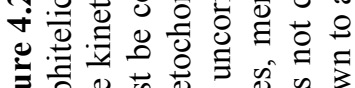

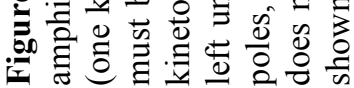




\subsubsection{Aims}

Previous work in our laboratory used a chemical genetics screening approach in yeast to identify potential targets or protein interactions of PelA (Wilmes, 2008; Wilmes et al., 2012b). A HIP (haploid insufficiency profiling) microarray was carried out in a yeast deletion set (Wilmes et al., 2012b). Compared to the Saccharomyces cerevisiae haploid wildtype strain (BY4741), a mad2s haploid mutant strain exhibited greater sensitivity (synthetic lethality) to PelA, indicating that MAD2 might be involved in the mode of action of PelA (Wilmes et al., 2012b). The mad2A mutant was also identified in a HOP (homozygous deletion profiling) microarray, which identifies functionally related genes of the primary target that reduce survival against a given drug when knocked out (Wilmes et al., 2012b). Deletion of genes encoding various spindle checkpoint proteins, such as $M A D 2$ and $B U B R 1$, have previously demonstrated sensitivity to another MTA, benzimidizole, due to the inability of these cells to activate the SAC (Hoyt et al., 1991; Li and Murray, 1991).

In order to test for a role of MAD2 in the mode of action of PelA in mammalian cells, we looked for a possible role of MAD2 in PelA-induced aneuploidy. We have previously seen in our laboratory that low, but not high, concentrations of PelA in HL60 cells induces a sub-G $G_{1}$ population of cells, which is indicative of aneuploidy (Wilmes, 2008; Wilmes et al., 2011b). Additionally, Ikui et al. (2005) proposed that premature dissociation of MAD2 and BUBR1 from $\mathrm{p} 55 \mathrm{CDC}$ leads to aneuploidy at low concentrations of Ptx. Thus, the initial aim for the current study was:

1) To characterize the concentration-dependent effects of PelA-induced aneuploidy in 1A9 human ovarian carcinoma cells, and to look at the induction of aneuploidy in the context of cell cycle progression. This involved using flow cytometry to follow the cell cycle histograms of synchronized cells released from cell cycle block into low or high concentrations of PelA.

We also wanted to investigate whether MAD2 was involved in the induction of aneuploidy by looking at its functional role as part of the SAC. To this end, we proposed the following experiments: 
2) To use Western blotting to determine whether PelA altered the expression levels of proteins associated with cell cycle regulation, including MAD2, BUBR1, p55CDC and cyclin B1.

3) To assess whether there are differences in the cell cycle kinetics of MAD2 and BUBR1 dissociation from $\mathrm{p} 55 \mathrm{CDC}$ in the presence of low versus high PelA concentrations, and to determine whether premature dissociation may be a possible mechanism underlying PelA-induced aneuploidy. In order to look at temporal changes in association, co-immunoprecipitation (Co-IP) and Western blotting were carried out on synchronized cells released from cell cycle block into medium containing PelA, which had been harvested at various timepoints.

Based on the results from the Co-IP assay, we decided to carry out a pilot study to investigate the possibility that an additional kinetochore-attachment mechanism may be involved in PelA-induced aneuploidy.

4) Merotelic attachments are known to contribute to aneuploidy in mammalian cells (Cimini et al., 2001). To explore this as a potential mechanism, we looked at the possibility that the frequency of merotelic attachments were increased at low but not high concentrations of PelA. Using confocal microscopy, images of cells in anaphase were used to quantify the frequency of lagging chromosomes, which indirectly suggest the presence of merotelically-oriented chromosomes. 


\subsection{Methods}

\subsubsection{Cell cycle analysis using flow cytometry}

To assess the concentration-dependent effects on the induction of aneuploidy, 1A9 cells were seeded into a 24 -well plate at $1 \times 10^{5}$ cells/well and allowed to attach overnight. Cells were then treated with varying concentrations of PelA $(0-100 \mathrm{nM})$ for $16 \mathrm{~h}$. Cells were harvested using TrypLETM Express (Life Technologies), transferred to $15 \mathrm{~mL}$ Falcon tubes and then washed three times with 1 x PBS. Harvested cells were stained for DNA content and analyzed as described in the General Methods (Chapter 2). DNA content was analyzed by flow cytometry using a BD FACScan analytic flow cytometer (Becton Dickinson, Franklin Lakes, NJ), and the percentage of cells in each cell cycle phase was determined using Cellquest Pro software (Becton Dickinson, Franklin Lakes, NJ). The concentrations of PelA that gave the maximal percentage of aneuploidy cells $(40 \mathrm{nM})$ or $\mathrm{G}_{2} / \mathrm{M}$ arrest $(100 \mathrm{nM})$ were chosen for the subsequent time-course assay. This initial assay to look at concentration-dependent effects of PelA was carried out by Dora Leahy (Research Assistant for Prof. John Miller, VUW, 2010), as part of her training on the flow cytometer.

To look at the induction of aneuploidy as cells progressed through the cell cycle, 1A9 cells were first synchronized at the $\mathrm{G}_{1} / \mathrm{S}$ border by a double thymidine block. Cells were seeded at $4.25 \times 10^{5}$ cells into a $60 \mathrm{~mm}$ tissue culture dish. After attaching overnight, the cells were incubated for $12 \mathrm{~h}$ in complete medium containing $2 \mathrm{mM}$ thymidine. Cells were washed three times with 1 x PBS and incubated in fresh thymidine-free medium for $18 \mathrm{~h}$. Cells were incubated for a further $12 \mathrm{~h}$ in medium containing $2 \mathrm{mM}$ thymidine (i.e. double thymidine block). Cells were then washed 3 times in 1 x PBS and released into complete medium, medium containing $40 \mathrm{nM}$ PelA, or medium containing $100 \mathrm{nM}$ PelA (taken as time $=0 \mathrm{~h})$. Cells were harvested at various timepoints $(0,2,4$, $5,6,7,8,9,10,11,12,13,14,15 \mathrm{~h}$ ) as described above, with an additional fixation step after the third PBS wash. Cells were resuspended in $1.5 \mathrm{~mL}$ of $1 \mathrm{x}$ PBS and then fixed by adding $3.5 \mathrm{~mL}$ ice-cold absolute ethanol drop-wise while vortexing (final ethanol concentration was $70 \%$ ). Cells were fixed overnight at $4{ }^{\circ} \mathrm{C}$ and then transferred to -20 ${ }^{\circ} \mathrm{C}$ for longer-term storage. Fixed cells were centrifuged at $700 \mathrm{~g}$ for $5 \mathrm{~min}$ to pellet the cells. The supernatant was removed carefully as the fixed cells were very flocculent. Cells were washed 3 times with PBS, and subsequently RNase treated and stained and analyzed for DNA content as described in the General Methods (Chapter 2). 


\subsubsection{Western blot analysis of cell cycle proteins}

For Western blot analysis, the cells were synchronized and released into medium or PelA as described above for the time-course assay. Cells were harvested at the various timepoints (0-15 h) using PBS, transferred to Eppendorf tubes and centrifuged at $300 \mathrm{~g}$ for $5 \mathrm{~min}$ at $4{ }^{\circ} \mathrm{C}$. Cells were washed 3 times in ice-cold PBS, and then the total number of cells in each sample was approximated by counting cells on a haemocytometer. The final cell pellet was then kept frozen at $-20{ }^{\circ} \mathrm{C}$. Cells were lysed in RIPA buffer containing $1 \%$ protease inhibitor ( $35 \mu \mathrm{L}$ lysis buffer per $1 \times 10^{6}$ cells) by vortexing for $30 \mathrm{~min}$ at $4{ }^{\circ} \mathrm{C}$. DNA was removed by centrifuging the lysate at $16,100 \mathrm{~g}$ for $20 \mathrm{~min}$ at $4{ }^{\circ} \mathrm{C}$ and transferring the supernatant to a fresh tube. Proteins were resolved by $10 \%$ SDS-PAGE (30 $\mu$ g protein/lane) and transferred by electrophoresis ( $20 \mathrm{~V}$ for $17 \mathrm{~h}$, at 4 ${ }^{\circ} \mathrm{C}$ ) to a low background fluorescence PVDF membrane (Immobilon FL, Millipore). Membranes were probed with the following primary antibodies for $1 \mathrm{~h}$ at RT: antip55CDC (Santa Cruz Biotechnology, sc-13162, 1:1000), anti-MAD2 (BD Biosciences, cat. No. 610679, 1:2000), anti-BUBR1 (BD Biosciences, cat. No 612503 1:500), and anti-cyclin B1 (BD Biosciences, cat. No. 554177; 1:1000). Membranes were then stained with mouse-Ig-Cy5, for $1 \mathrm{~h}$ at RT (GE-Healthcare, 1:2000). Membranes were probed for the loading control, actin, by incubating with anti-actin (Sigma mouse monoclonal anti- $\beta$-actin, A2228, 1:4000) for $30 \mathrm{~min}$ at RT, followed by an additional incubation with mouse-Ig-Cy5, as above. Relative density was calculated by normalizing to the actin loading control then dividing by the control normalized density.

\subsubsection{Harvesting cells for co-immunoprecipitation}

For the Co-IP, cells were synchronized as above using the double thymidine block, but with some minor adjustments due to a need for larger amounts of protein. 1A9 cells were plated out at $1.25 \times 10^{6}$ cells per $\mathrm{T} 75 \mathrm{~cm}^{2}$ tissue culture flasks. The double thymidine block was carried out as above, except for a shorter thymidine release $(15 \mathrm{~h})$. Cells were released into medium and drug (as above), and harvested at the same timepoints. To prevent dissociation of protein complexes, once cells had begun lifting off after treatment with TrypLe Express, the flask was placed on ice and subsequently kept on ice (or at $4{ }^{\circ} \mathrm{C}$ ) where possible during all subsequent steps. Cells were washed off the flask surface with ice-cold PBS and then transferred to a $15 \mathrm{~mL}$ Falcon tube. Cells were centrifuged at $300 \mathrm{~g}$ for $5 \mathrm{~min}$ at $4{ }^{\circ} \mathrm{C}$, resuspended in $1 \mathrm{~mL}$ of ice-cold PBS and then transferred to Eppendorf tubes. Cells were washed another two times, and total cell counts of each sample were made. Cell pellets were then frozen at $-20{ }^{\circ} \mathrm{C}$ until later 
analysis. Several optimization steps that were carried out prior to the full Co-IP analysis can be found in Appendix C, Figure C.1.

\subsubsection{Crosslinking of protein $G$ Dynabeads ${ }^{\circledR}$ to anti-p55CDC antibody}

Protein G Dynabeads ${ }^{\circledR}$ were crosslinked to $4 \mu \mathrm{g}$ anti-p55CDC antibody. The following steps describe the crosslinking of beads to antibody for the immunoprecipitation of one sample (this was repeated so that there were enough crosslinked beads for all timepoints/drug treatments). Dynabeads ${ }^{\circledR}(50 \mu \mathrm{L})$ were transferred to an Eppendorf tube and collected with a magnet. The supernatant was removed, and the beads were resuspended in $200 \mu \mathrm{L}$ of PBST $(0.02 \%$ Tween 20 in PBS) containing anti-p55CDC. The beads and antibody were incubated with rotation on a 12-tube mixing wheel (Dynabeads ${ }^{\circledR}$ MX1 Mixer) for $15 \mathrm{~min}$ at RT. The Eppendorf tube was placed on the magnet again and the supernatant removed. The bead-antibody complexes were washed with $200 \mu \mathrm{L}$ PBST. The Eppendorf tube was again placed on the magnet, and the supernatant was removed. The bead-antibody complexes were washed three times with $1 \mathrm{~mL} 0.2 \mathrm{M}$ triethanolamine ( $\mathrm{pH} 8.2$ ), then resuspended in $1 \mathrm{~mL} 0.2 \mathrm{M}$ triethanolamine containing $5.4 \mathrm{mg}$ dimethyl pimelimidate (DMP), which was freshly added before use. The bead-antibody complexes were incubated with rotation for $30 \mathrm{~min}$ at RT. The Eppendorf tube was then placed on the magnet and the supernatant removed. The reaction was stopped by adding $1 \mathrm{~mL}$ of $50 \mathrm{mM}$ Tris (pH 7.5) and incubating with rotation for $15 \mathrm{~min}$ at $\mathrm{RT}$. The Eppendorf tube was placed on the magnet and the supernatant discarded. The crosslinked bead-antibody complexes were washed $3 \mathrm{x}$ in $200 \mu \mathrm{L}$ PBST and then stored in PBST at $4{ }^{\circ} \mathrm{C}$.

\subsubsection{Co-immunoprecipitation}

Cells were lysed in non-reducing lysis buffer containing $1 \%$ protease inhibitor $(30 \mu \mathrm{L}$ per $1 \times 10^{6}$ cells) by incubating for $30 \mathrm{~min}$ at $4{ }^{\circ} \mathrm{C}$ with gentle shaking. Lysates were centrifuged at $16,100 \mathrm{~g}$ for $20 \mathrm{~min}$ at $4{ }^{\circ} \mathrm{C}$ and the supernatant transferred to fresh Eppendorf tubes. The following steps were carried out at $4{ }^{\circ} \mathrm{C}$ to prevent dissociation of protein complexes. Eppendorf tubes containing crosslinked beads-antibody complexes were placed on a magnet. The supernatant was removed, and the complexes were resuspended in $900 \mu \mathrm{g}$ of protein diluted to a final volume of $200 \mu \mathrm{L}$ in ice-cold PBST $(0.02 \%$ Tween 20$)$. Complexes were incubated with lysate for $2 \mathrm{~h}$ at $4{ }^{\circ} \mathrm{C}$ with rotation on the mixing wheel. Eppendorf tubes were placed on a magnet and the supernatant was removed and kept aside for further analysis. Bead-antibody-protein complexes were 
washed three times with $200 \mu \mathrm{L}$ ice-cold PBST. Complexes were resuspended in 100 $\mu \mathrm{L}$ ice-cold PBS and transferred to a fresh tube. Tubes were placed on a magnet and complexes were resuspended in $16 \mu \mathrm{L}$ of $\mathrm{dH}_{2} 0$. Reducing buffer $(4 \mu \mathrm{L}$ of $5 \mathrm{x}$ concentrate, containing $10 \% \quad \beta$-mercaptoethanol) was added to the beads. The complexes were then stored at $-20{ }^{\circ} \mathrm{C}$ until later analysis. Protein complexes were dissociated from the Dynabeads by boiling at $90{ }^{\circ} \mathrm{C}$ for $5 \mathrm{~min}$. Eppendorf tubes were placed on a magnet and the denatured protein was resolved on a 10\% SDS-PAGE gel. Protein was then transferred by electrophoresis to PVDF membranes $(20 \mathrm{~V}$ for $17 \mathrm{~h}$ at 4 $\left.{ }^{\circ} \mathrm{C}\right)$. The membranes from the Co-IP were incubated with anti-p55CDC (1:1000), antiMAD2 (1:2000), and anti-BUBR1 (1:500) for $1 \mathrm{~h}$ at RT and then stained with secondary anti-mouse IgG-Cy5 (1:2000) for a further $1 \mathrm{~h}$ at RT. The supernatant samples were stained with anti-p55CDC (1:1000), anti-MAD2 (1:2000), anti-BUBR1 (1:500), and anti-actin (1:4000) for $1 \mathrm{~h}$ at RT and then stained with secondary mouseIg-Cy5, as above.

\subsubsection{Immunostaining to detect possible merotelic attachments}

Sterile glass coverslips (No. 1.5, $22 \times 22 \mathrm{~mm}$ ) were placed into the wells of a 6-well plate. To promote cell attachment, coverslips were covered with a sterile solution (50 $\mu \mathrm{g} / \mathrm{mL}$ ) of poly-D-lysine (PDL) and incubated at $37{ }^{\circ} \mathrm{C}$ overnight. The PDL was aspirated off and the coverslips were washed three times with sterile MQ water. Coverslips were either used immediately or air-dried and stored at $-20{ }^{\circ} \mathrm{C}$. 1A9 cells were plated at $1 \times 10^{5}$ cells/coverslip in a small volume of $400 \mu \mathrm{L}$, so that only the coverslips were covered with cells. Cells were allowed to attach to the coverslip $(\sim 1-2 \mathrm{~h}$ at $37^{\circ} \mathrm{C}$ ) before carefully adding $1 \mathrm{~mL}$ medium to each well. After allowing the cells to adhere further overnight, the medium was aspirated off and the cells incubated with medium or medium containing PelA (40 or $100 \mathrm{nM}$ ) for $6 \mathrm{~h}$ at $37^{\circ} \mathrm{C}$. Cells were washed rapidly with PHEM buffer (see Appendix A for buffer recipes) and then extracted using PHEM buffer containing 0.5\% Triton X-100 (PHEMT) for $5 \mathrm{~min}$ at RT. Cells were fixed in ice-cold methanol:acetone (1:1) for $6 \mathrm{~min}$ at RT before being washed three times with PBS. Cells were blocked using 5\% boiled donkey serum in PHEMT for $1 \mathrm{~h}$ at RT. The cells were probed overnight at $4{ }^{\circ} \mathrm{C}$ using the following primary antibodies diluted in blocking buffer: human anti-centromere antibody diluted 1:1000 (ImmunoVision, Inc. Springdale, AR); rabbit anti- $\alpha$-tubulin diluted 1:1000 (ab18251, Abcam). Cells were washed three times with PBST (PBS containing 0.05\% Tween 20) and then incubated for $1 \mathrm{~h}$ at RT with the following secondary antibodies diluted in 
PBST: donkey anti-human IgG Rhodamine Red-X diluted 1:2000 (Jackson ImmunoResearch Laboratories, Inc.); goat anti-rabbit IgG Alexa Fluor ${ }^{\circledR} 488$ diluted 1:1000 (Invitrogen). After washing three times with PBS, coverslips were mounted using ProLong Gold ${ }^{\circledR}$ antifade with DAPI ( $8 \mu$ L per coverslip).

\subsubsection{Confocal microscopy and image acquisition}

Confocal microscopy was used to image cells at various stages during mitosis. The presence of lagging chromosomes during anaphase is indicative of merotelic microtubule-kinetochore attachments. We carried out a small pilot study to determine whether we could detect such attachments. In order to verify the type of attachments at each kinetochore, images with enhanced resolution are required. Images can be processed using 3-D deconvolution, which effectively removes some of the inherent optical distortions in the image. Although deconvolution was not carried as part of this preliminary study, all images were acquired in accordance with the Nyquist sampling rate so that 3-D deconvolution could be applied at a later date. The microscope used was an Olympus FluoView FV1000 laser-scanning confocal microscope (model IX81) equipped with a $100 \mathrm{X}$ (1.4 NA) oil immersion objective and a CCD camera. Image acquisition was performed using FV10-ASW software. Z-series (i.e. stacks of optical sections) were collected for cells in prometaphase, metaphase or anaphase. To align with the Nyquist criteria as determined for our particular microscopic parameters, sample scan sizes in the $\mathrm{X}, \mathrm{Y}$, and $\mathrm{Z}$ dimensions were 34, 34, and $90 \mathrm{~nm}$, respectively, or smaller. The pixel dimensions (X x Y) were determined by the zoom magnification and image pixel format (set to $1024 \times 1024$ pixels). To ensure that all of the light, including that from the point spread functions, was included in the images, the voltage was increased, and the top and bottom positions of the z-stack were set $2 \mu \mathrm{m}$ above where the light disappeared. To collect the maximum dynamic range of fluorescence intensity without data clipping, the oversaturation and undersaturation colour range indicator (HiLo LUT) was used to set the appropriate gain and offset settings, respectively (i.e. so that the image contained no zero or 4095 pixel intensities (12-bit), in order to avoid low-intensity data clipping and oversaturation, respectively). Images were acquired in the sequential scanning mode using LD $405 \mathrm{~nm}$, LD $559 \mathrm{~nm}$ and multi-line argon-ion $488 \mathrm{~nm}$ lasers. Kalman averaging was set to two, and pixel dwell time was set to $2 \mu \mathrm{s} /$ pixel to try to minimize photobleaching. 


\subsubsection{Scoring of mitotic morphologies}

In addition to the image acquisition, mitotic abnormalities of cells in prometaphase, metaphase or anaphase were very roughly scored. This was done manually by looking down the microscope (i.e. scoring was not done on the acquired cell images) and assigning mitotic cells into the following different categories: a) normal mitosis; b) cells with multiple and/or asymmetric asters; c) cells in 'pseudo-metaphase' (a morphology previously described by Weaver et al. (2003), in which the majority of chromosomes are aligned except for one to several chromosome pairs that localize at or near the spindle poles (polar chromosomes)); d) cells with grossly misaligned chromosomes and a few polar chromosomes; and e) lagging chromosomes at the metaphase plate during anaphase. 


\subsection{Results}

\subsubsection{Induction of aneuploidy by PelA in unsynchronized $1 \mathrm{A9}$ cells}

Flow cytometry was used to quantify the percentage of aneuploid cells (sub- $G_{1}$ peak) in response to various concentrations of PelA. In 1A9 cells, PelA induced aneuploidy at concentrations lower than those needed to block cells in $\mathrm{G}_{2} / \mathrm{M}$ phase of the cell cycle (Fig. 4.3). The induction of aneuploidy was concentration-dependent, and the greatest aneuploid population (14\%) was seen at $40 \mathrm{nM}$ PelA. This concentration of PelA coincided with a small, but significant, population of cells becoming blocked in $\mathrm{G}_{2} / \mathrm{M}$ (i.e. $51.8 \%$ of cells in $\mathrm{G}_{2} / \mathrm{M}$ at $40 \mathrm{nM}$ compared to $30.6 \%$ in untreated controls). As the percentage of cells arresting in $\mathrm{G}_{2} / \mathrm{M}$ increased (with increasing concentrations of PelA), however, the frequency of aneuploidy decreased.

\subsubsection{Induction of aneuploidy at low concentrations of PelA in synchronized cells}

$1 \mathrm{~A} 9$ cells were synchronized at the $\mathrm{G}_{1} / \mathrm{S}$ border by a double thymidine block, so that cells could be followed through the cell cycle in the presence of low (40 nM) versus high (100 nM) PelA concentrations. Cells were harvested at different times over a $15 \mathrm{~h}$ period after release from the thymidine block, and their DNA content was analyzed by flow cytometry. In control cells and cells treated with $40 \mathrm{nM}$ PelA, the majority of cells (56.6. \% and $60.6 \%$, respectively) peaked in $\mathrm{G}_{2} / \mathrm{M}$ at $6 \mathrm{~h}$ and progressed through to $\mathrm{G}_{1}$ (Fig. $4.4 \mathrm{~A}$ and B). In contrast, $100 \mathrm{nM}$ PelA caused a $2 \mathrm{~h}$ mitotic delay (74.1\% of cells peaked in $\mathrm{G}_{2} / \mathrm{M}$ at $8 \mathrm{~h}$ ) and a sustained block in $\mathrm{G}_{2} / \mathrm{M}$ phase (Fig. $4.4 \mathrm{C}$ ). The frequency of aneuploidy in untreated control cells was very low, and never increased above $1 \%$ of the total cells. Lower concentrations of PelA $(\leq 40 \mathrm{nM})$ were the most conducive towards the formation of aneuploid cells. At $40 \mathrm{nM}$, as the majority of cells progressed through mitosis $(6-13 \mathrm{~h})$, the frequency of aneuploidy increased from 1.9 to $10.4 \%$ (Fig. 4.4 B and Fig. 4.5). In response to $100 \mathrm{nM}$ PelA, a small degree of aneuploidy arose from $11 \mathrm{~h}$ onwards (1.9 to $3.2 \%$ ) but in comparison was much less pronounced. From the cell cycle histograms, it can also be seen that $100 \mathrm{nM}$ results in some tetraploid populations of cells after about $6 \mathrm{~h}$ (Fig. 4.5). These tetraploid populations are also seen in controls and at $40 \mathrm{nM}$ PelA, but to a lesser extent. 


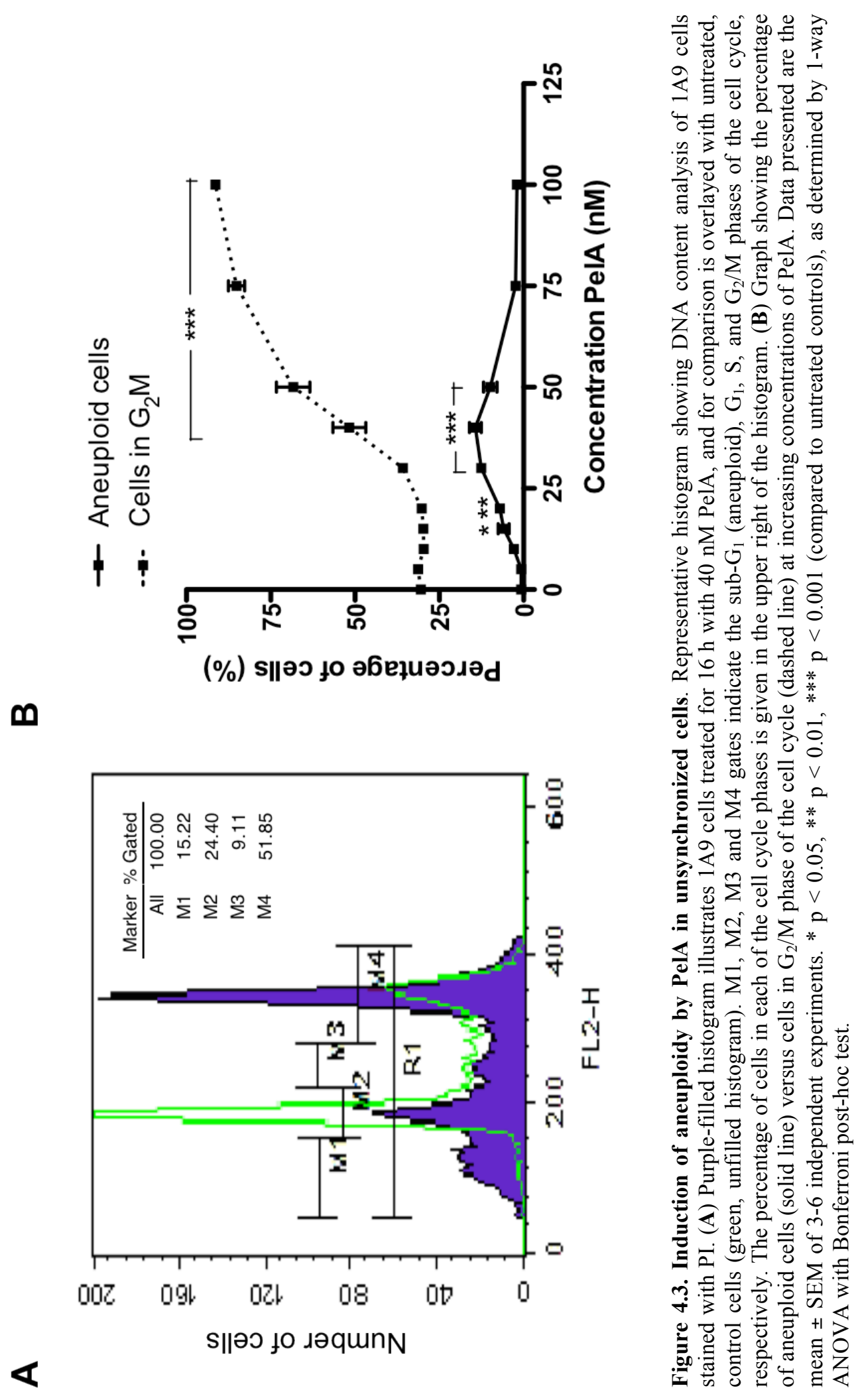




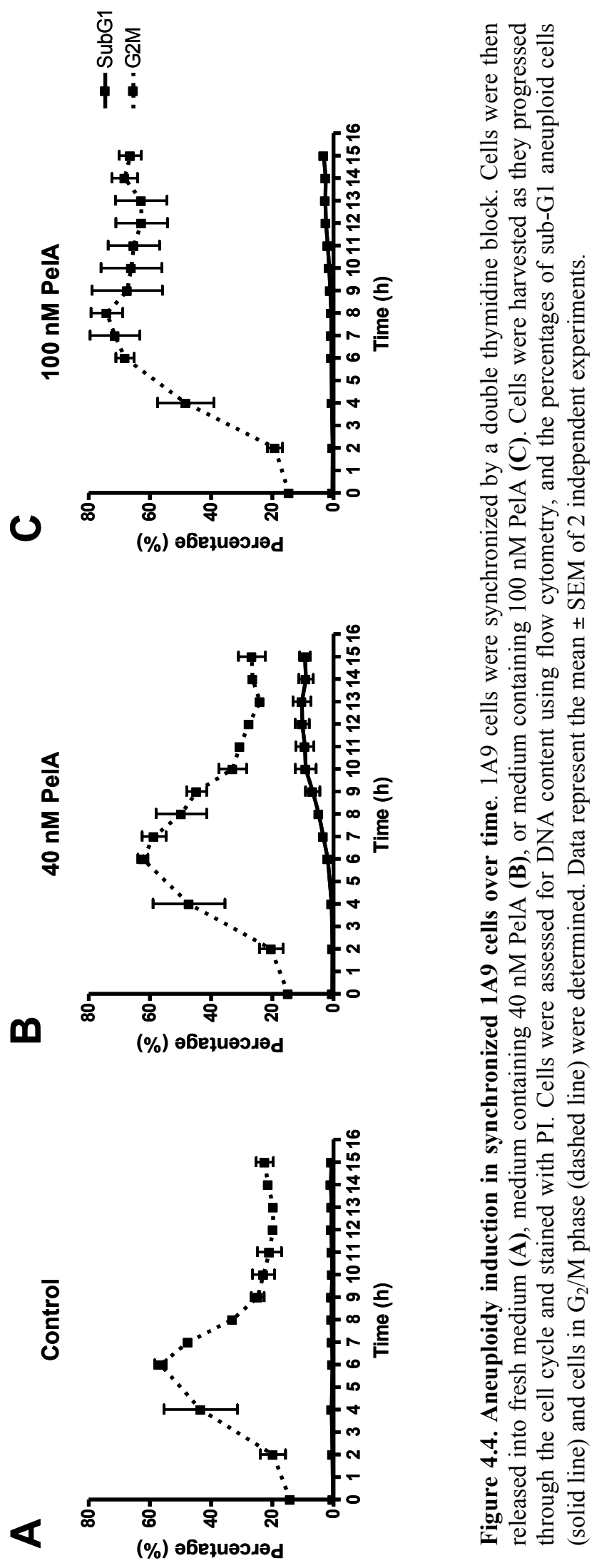



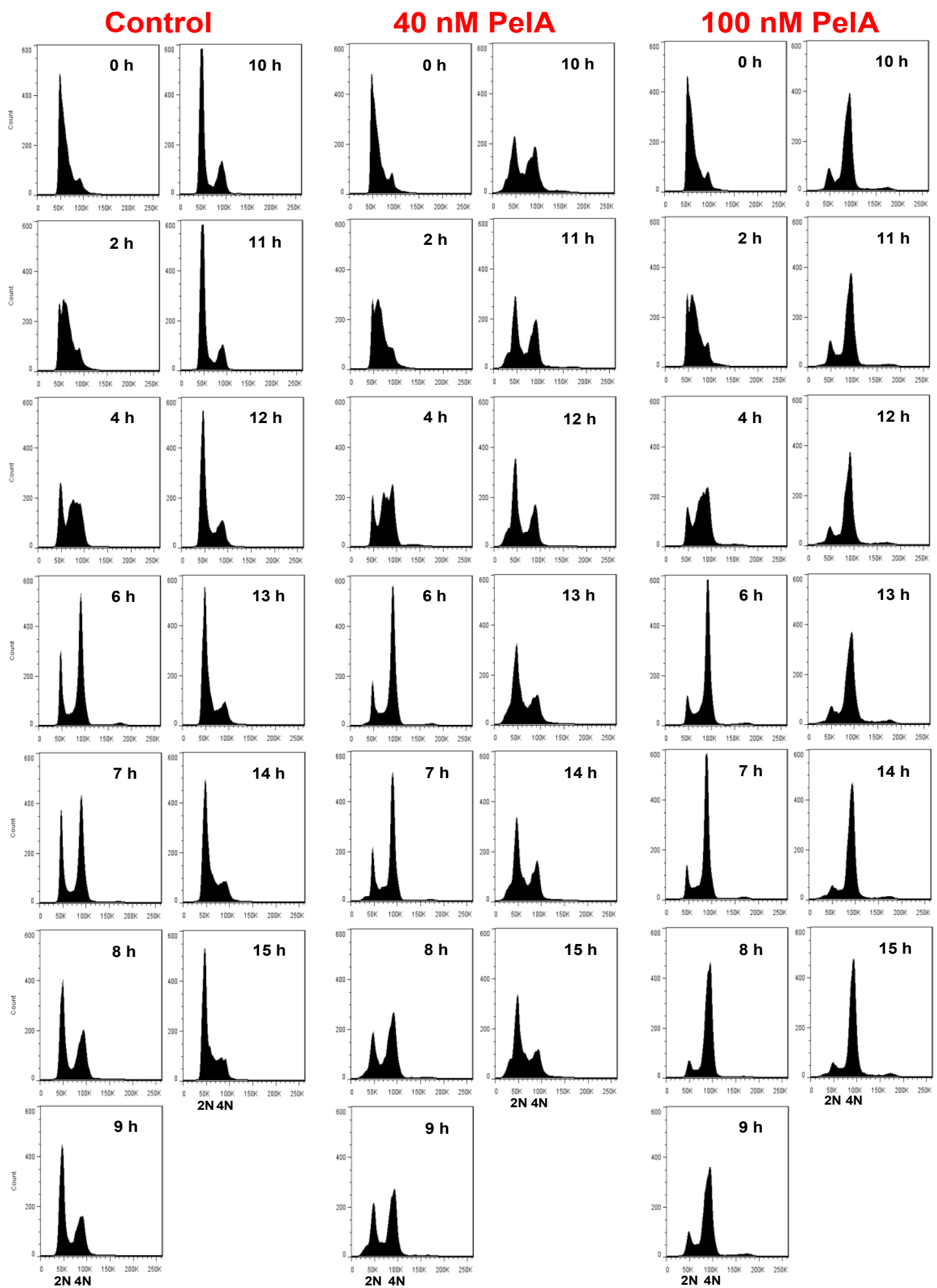

Figure 4.5. Cell cycle progression at low and high concentrations of PelA. 1A9 cells were synchronized by a double thymidine block and released into low (40 nM) or high (100 nM) concentrations of PelA. This method of synchronization achieved approximately $80-85 \%$ of cells synchronized at the $\mathrm{G}_{1}-\mathrm{S}$ border. Cells were harvested at various timepoints after release into drug, fixed in $70 \% \mathrm{EtOH}$ and then stained for DNA content using PI. The position of cells with $2 \mathrm{~N}$ and $4 \mathrm{~N}$ DNA content is indicated at the bottom of each column of histograms, with counts along the y-axis. Aneuploidy cells can be seen in the $\mathrm{subG}_{1}$ region as a shoulder off the $\mathrm{G}_{1}$ peak. Histograms were analyzed using FlowJo flow cytometry analysis software (TreeStar, Inc., Ashland, OR) and are representative of two independent experiments. 


\subsubsection{Differences in cell cycle protein expression in response to PelA}

Our next aim was to look at several cell cycle proteins that are involved in mitotic progression, to determine whether changes in these proteins at different PelA concentrations correlate with the cell cycle. Again, cells were synchronized by a double thymidine block and harvested at various timepoints $(0-15 \mathrm{~h})$. Differences in the expression of, p55CDC, MAD2, BUBR1 and cyclin B1 in response to $40 \mathrm{nM}$ or 100 nM PelA, were assessed over time (Fig. 4.6 and 4.7). In both untreated control cells and PelA-treated cells (40 $\mathrm{nM}$ and $100 \mathrm{nM}$ ), the expression of the cell cycle regulator, p55CDC, increased steadily as cells progressed through $\mathrm{G}_{1}, \mathrm{~S}$ and $\mathrm{G}_{2}$ phases, and peaked at mitosis (Fig. 4.6 A and B, and 4.7, A). In control cells and cells treated with $40 \mathrm{nM}$ PelA, p55CDC levels then decreased fairly rapidly as cells cycled back through to $G_{1}$. Despite the majority of cells arresting in $G_{2} / M$, a similar (although slightly delayed) decrease in p55CDC levels occurred in cells treated with $100 \mathrm{nM}$ PelA (Fig. 4.6 $\mathrm{C}$ and $4.7 \mathrm{~A}$ ). Additionally, there did not appear to be any differences in the expression levels of BubR1 between controls and PelA-treated cells (Fig. 4.6 and 4.7, B). Similarly, for MAD2, PelA appeared to have little or no effect on its relative expression levels (Fig. 4.6 and 4.7 C). Slower migrating BUBR1 bands could be seen in mitotic cells, when released into both $40 \mathrm{nM}$ PelA and $100 \mathrm{nM}$ PelA (Fig $4.7 \mathrm{~B}$ and C). These bands are most likely indicative of BUBR1 phosphorylation, which occurs during spindle checkpoint activation. The degree of phosphorylation appears to be more pronounced at the higher PelA concentration. PelA had the most noticeable effects on cyclin B1 levels (Fig. 4.6 and 4.7 D). Cyclin B1 expression steadily increased until about $6 \mathrm{~h}$ in both controls and $40 \mathrm{nM}$ PelA, before being rapidly degraded, thus allowing cells to progress through mitosis. When treated with $100 \mathrm{nM}$ PelA, there was a similar increase in cyclin B1 levels in cells over the first $7 \mathrm{~h}$; however, this particular drug concentration resulted in prolonged, elevated levels of cyclin B1 that corresponded with mitotic arrest at this PelA concentration. Cyclin B1 levels in untreated cells and cells treated with $40 \mathrm{nM}$ PelA were rapidly degraded over a period of approximately 2-3 $\mathrm{h}$; however, at $100 \mathrm{nM}$ PelA, cyclin B1 was degraded gradually over a longer period of time (i.e. approximately 5-6 h).

\subsubsection{Cell cycle kinetics of MAD2 and BUBR1 association with p55CDC}

The association of the SAC proteins, MAD2 and BUBR1, were followed by Co-IP. Synchronized cells were released into fresh medium or PelA-medium (40 or $100 \mathrm{nM}$ ) and subsequently harvested at various timepoints from $0 \mathrm{~h}-15 \mathrm{~h}$. Firstly, p55CDC was 
immunoprecipitated and the associated proteins resolved by SDS-PAGE. While there were no significant effects of PelA on the relative expression levels of p55CDC (Fig. 4.8 and 4.9), the drug did elicit different responses in terms of the cell cycle kinetics of association between p55CDC and MAD2 or BUBR1. Since p55CDC itself has altered expression levels at different stages of the cell cycle (Fig 4.9 A), both the total relative density of co-immunoprecipitated MAD2 and BUBR1 (normalized to time $0 \mathrm{~h}$ ) (Fig. $4.9 \mathrm{~B}$ and $\mathrm{C}$, respectively), as well as the ratios of co-immunoprecipitated MAD2 and BUBR1 to p55CDC were determined (Fig. 4.9 D and E, respectively). Across all three treatment groups, p55CDC-associated MAD2 levels increased steadily and peaked at 7 $\mathrm{h}$ (in controls and cells treated with $40 \mathrm{nM}$ PelA) and $8 \mathrm{~h}$ (for cells treated with $100 \mathrm{nM}$ PelA) after synchrony release (Fig 4.9). This timing corresponded with the majority of cells being in $\mathrm{G}_{2} / \mathrm{M}$ phase (Fig. 4.5). However, despite the similar timing of MAD2 association, there appeared to be a concentration-dependent increase in the amount of MAD2 associating with p55CDC after the release of synchronized cells into PelA. In control cells, for example, the increase in MAD2 association with p55CDC was 6-fold greater compared to cells just released from the synchrony $(0 \mathrm{~h})$. At $40 \mathrm{nM}$ PelA and $100 \mathrm{nM}$ PelA, the increase in MAD2 association peaked at levels 8-fold and 14-fold greater, respectively (compared to $0 \mathrm{~h}$ ). The association/dissociation pattern of MAD2 with p55CDC was very similar between untreated cells and cells treated with $40 \mathrm{nM}$ PelA ( 1 h delay at $40 \mathrm{nM}$ PelA). However, although MAD2 began to dissociate from p55CDC from about $8 \mathrm{~h}$ onwards in cells treated with $100 \mathrm{nM}$ PelA, there was quite a distinct, sustained association of these two proteins.

In untreated cells, there was an increase in p55CDC-associated BubR1 as cells progressed through $\mathrm{G}_{1}$ and $\mathrm{S}$ phases. These complexes peaked earlier (at $6 \mathrm{~h}$ ) compared to p55CDC-MAD2 $(7 \mathrm{~h})$, before dissociating as the cells passed through $\mathrm{G}_{2} / \mathrm{M}$. PelA treatment affected the degree of association between BUBR1 and p55CDC. $100 \mathrm{nM}$ PelA resulted in a slight overall decrease in the peak amount of complexed BUBR1/p55CDC (approximately 6-fold for controls and 4-fold for $100 \mathrm{nM}$ PelA, compared to time $0 \mathrm{~h}$ ), and $40 \mathrm{nM}$ PelA resulted in an even more pronounced decrease in the peak p55CDC-BUBR1 association (approximately 3-fold compared to time $0 \mathrm{~h}$ ). Furthermore, both concentrations of PelA caused a slight delay in the timing of BUBR1 dissociation from p55CDC. In control cells, BUBR1 began to dissociate from $6 \mathrm{~h}$ onwards, while $40 \mathrm{nM}$ PelA and $100 \mathrm{nM}$ PelA caused dissociation from $7 \mathrm{~h}$ and $8 \mathrm{~h}$, respectively. Parallel to the effects of $100 \mathrm{nM}$ PelA on MAD2/p55CDC association, this 
higher concentration of PelA caused a similar sustained association of BUBR1 with p55CDC. 
A

CONTROL

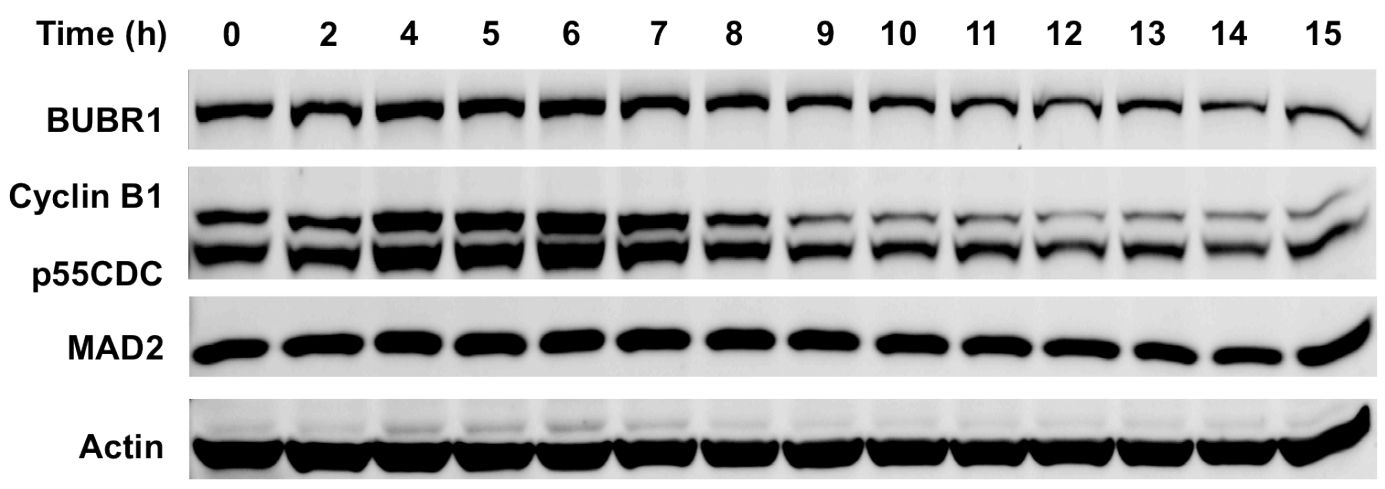

B

40 nM PelA

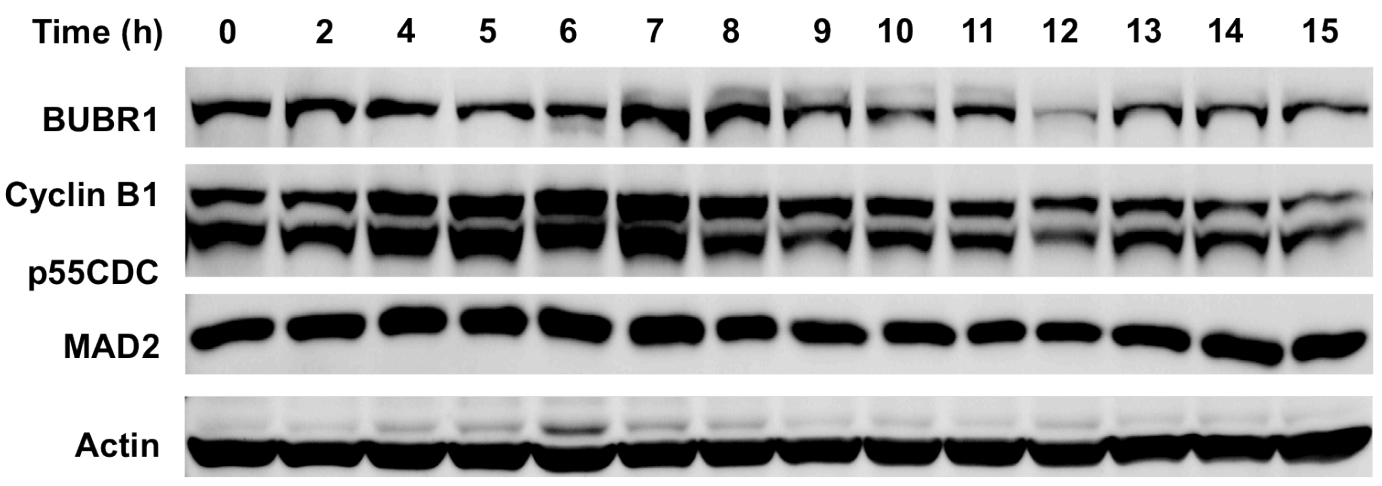

C

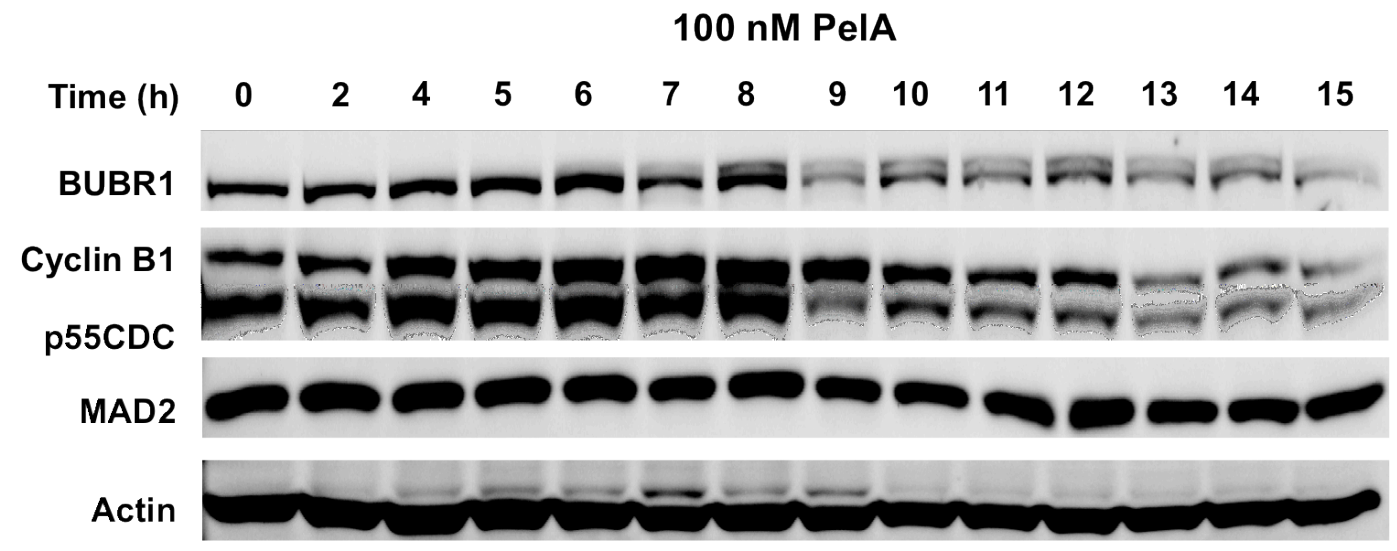

Figure 4.6. Representative Western blots showing changes in total protein expression of cell cycle proteins during cell cycle progression. $1 \mathrm{~A} 9$ cells were synchronized by a double thymidine block and released into drug-free medium (A), $40 \mathrm{nM}$ PelA (B) or $100 \mathrm{nM}$ PelA (C). Cells were harvested at various timepoints after release, and the protein expression levels of the cell cycle proteins BUBR1, Cyclin B1, p55CDC, and MAD2 were assessed by Western blotting. Actin expression was used as a loading control. 

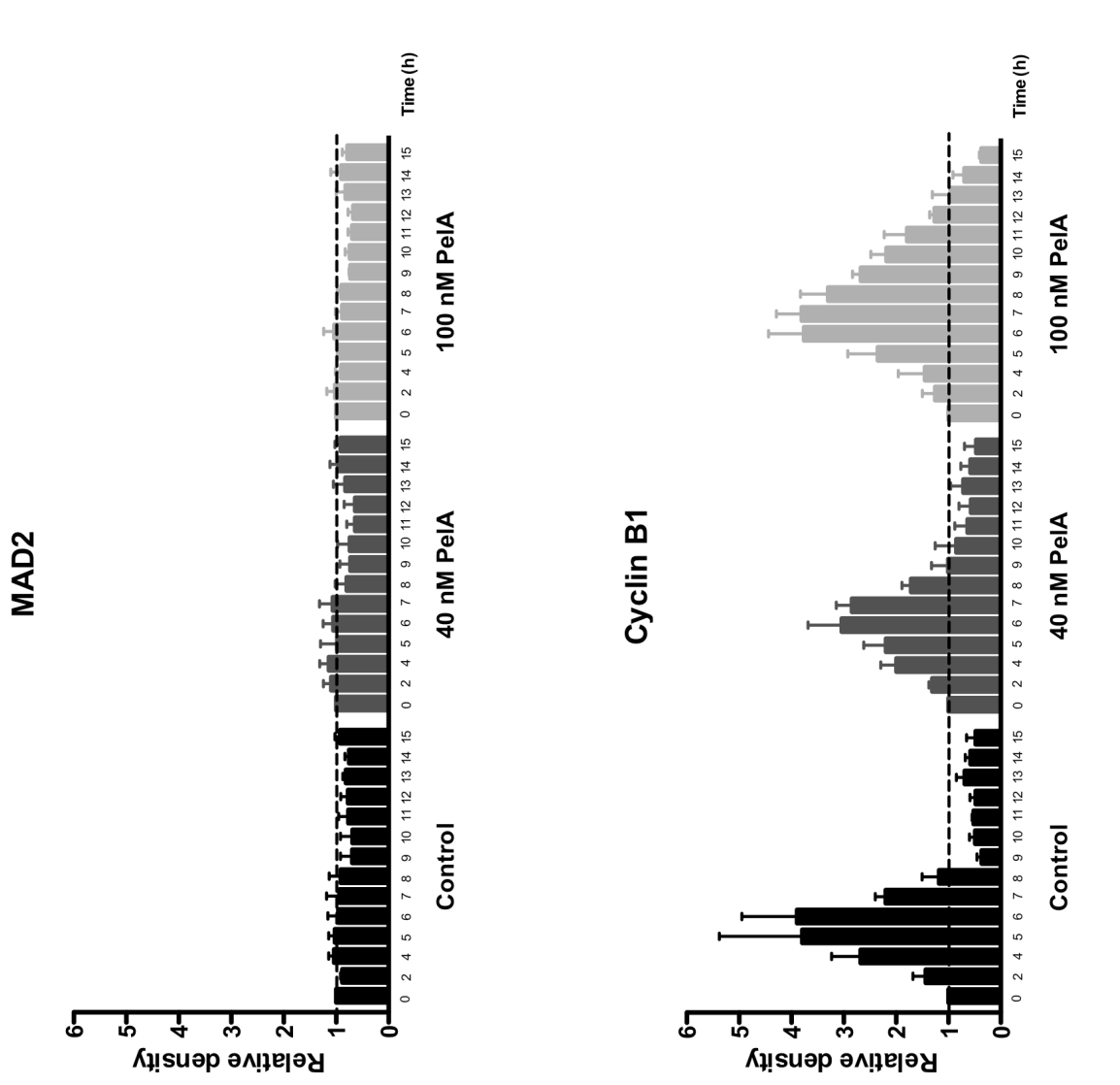

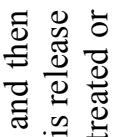

兑全

응

要

궁

홍

讨

흥

$\pi$ क

승 항

ठ্

就

?

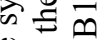

ธัँ

330

正

से

.

至是

응

口

잉

0

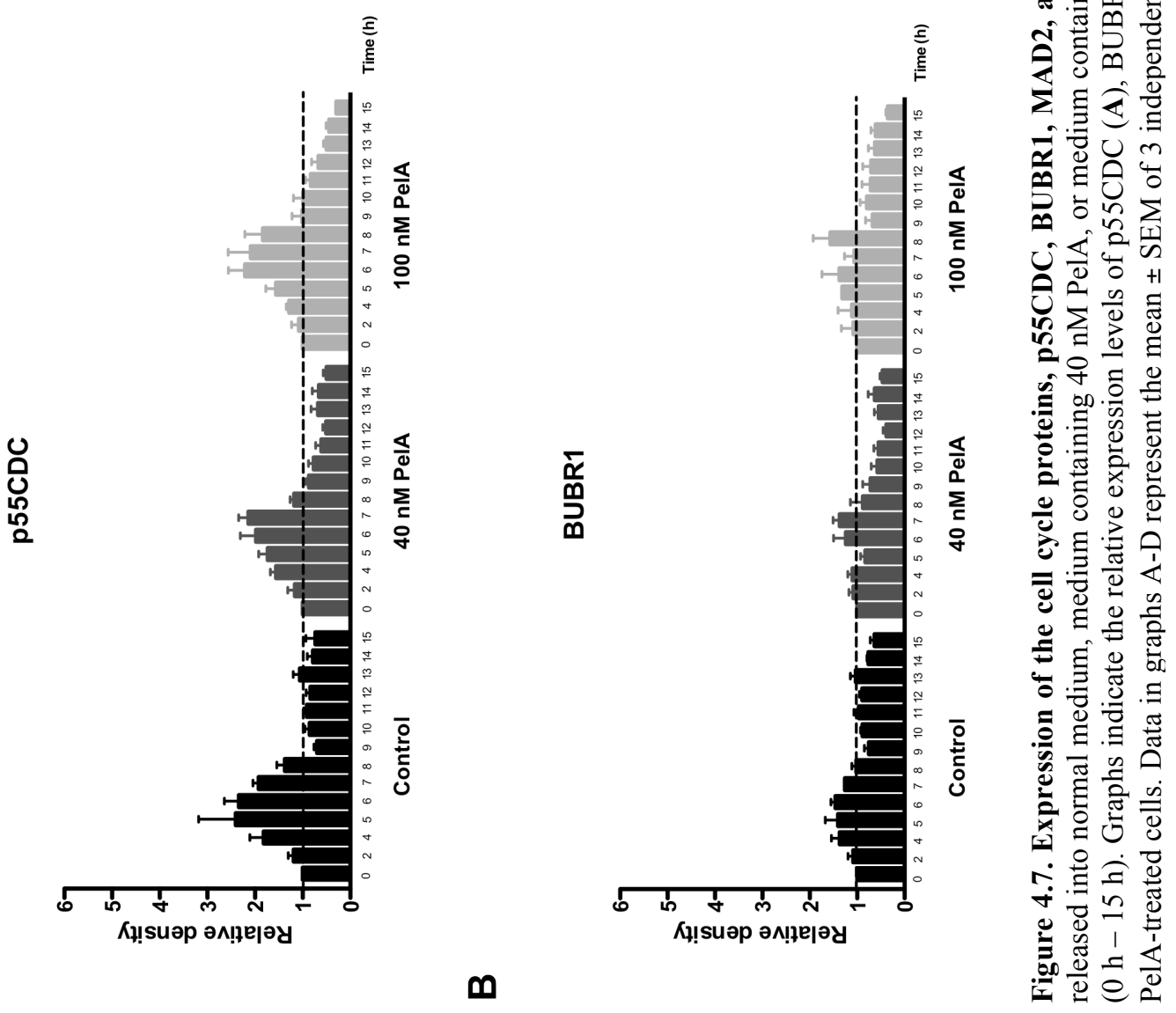



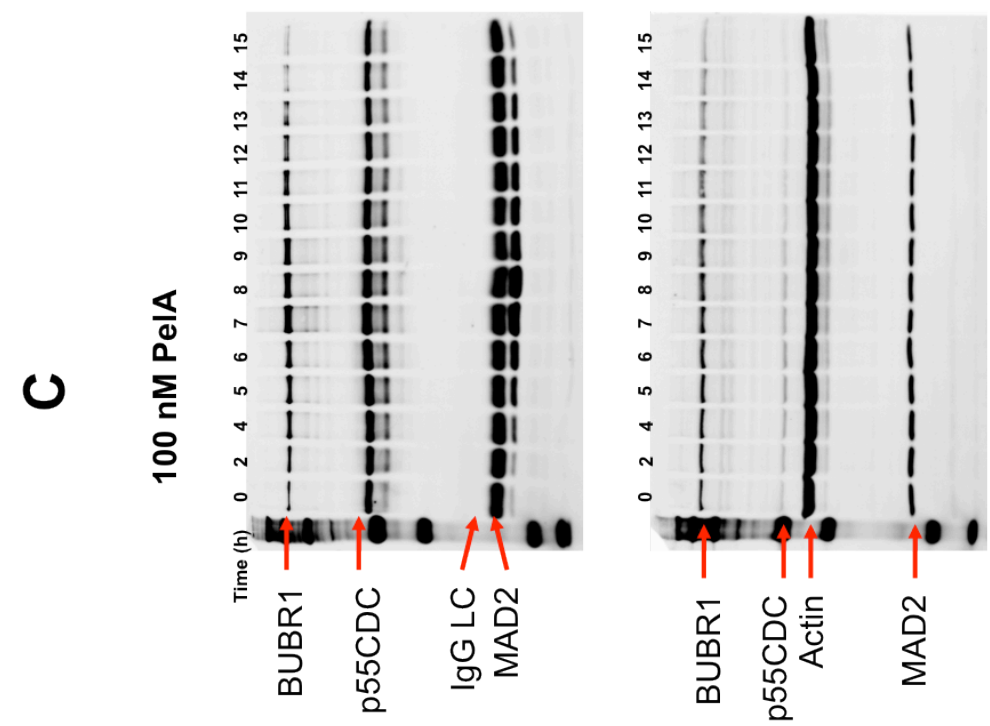

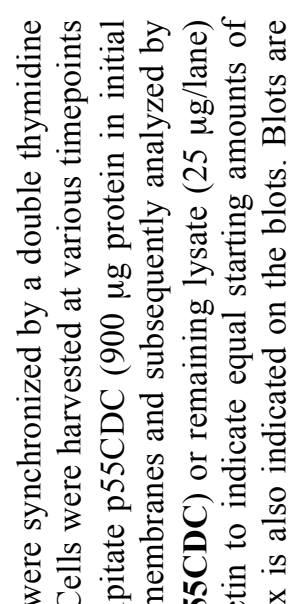

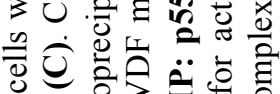

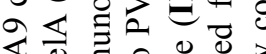
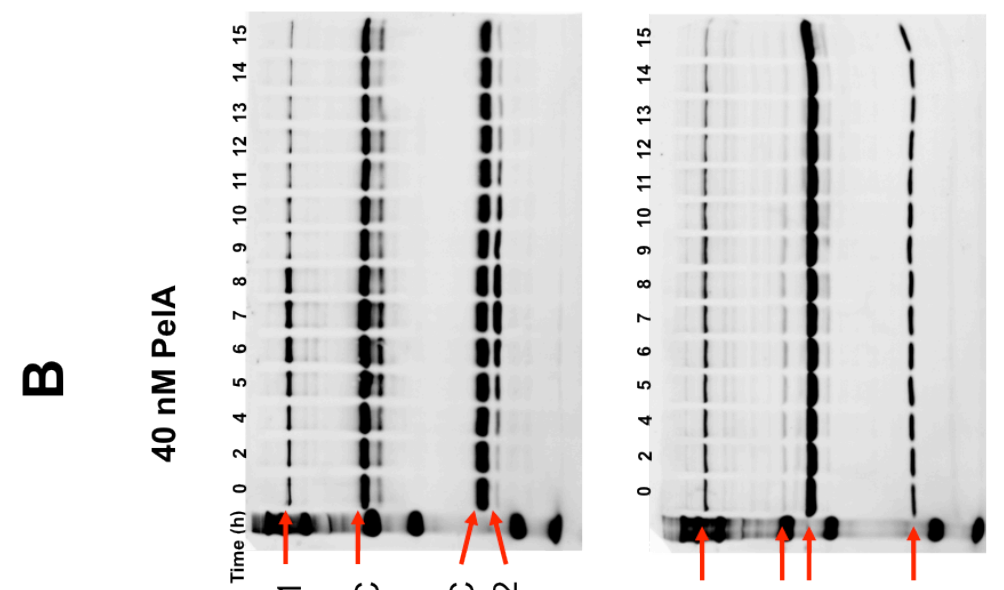

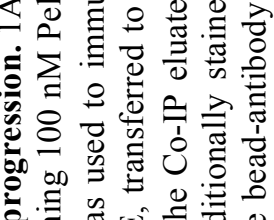

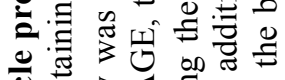

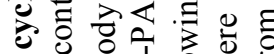

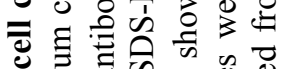

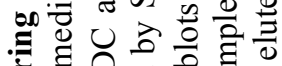

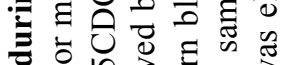
สิ

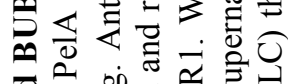

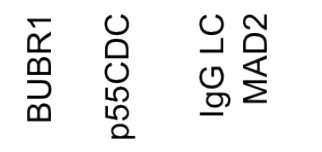

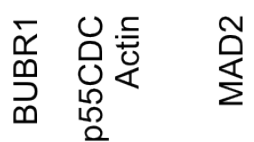

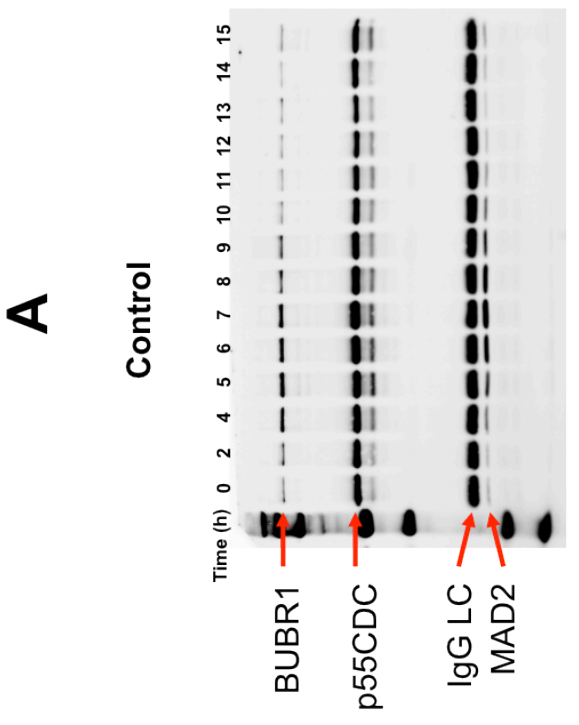

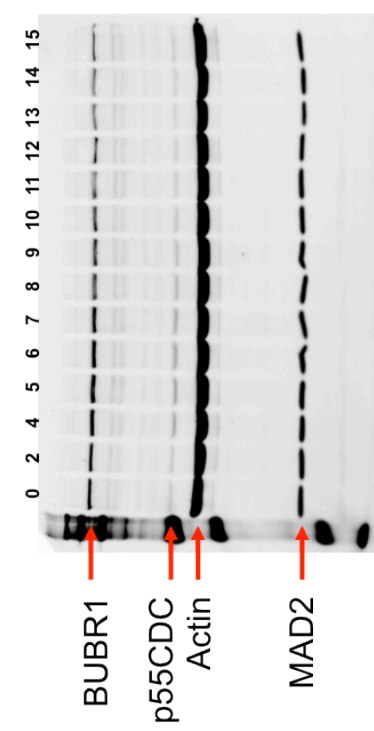

ұuejeusədns

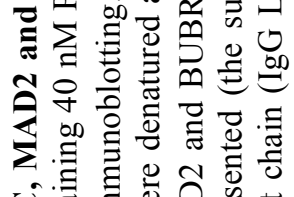

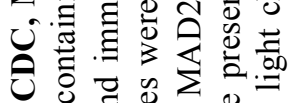

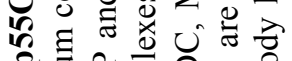

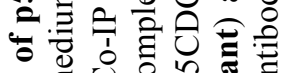

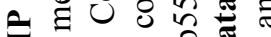

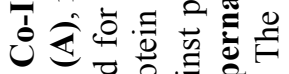

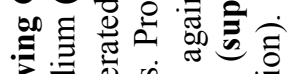

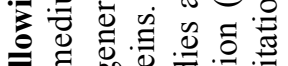

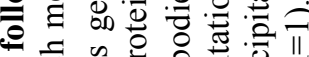

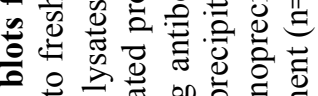

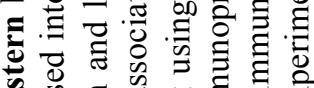
क्षे

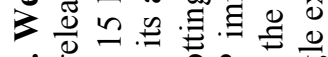

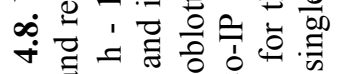
和用目U

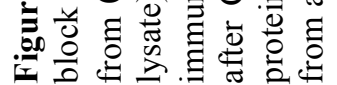




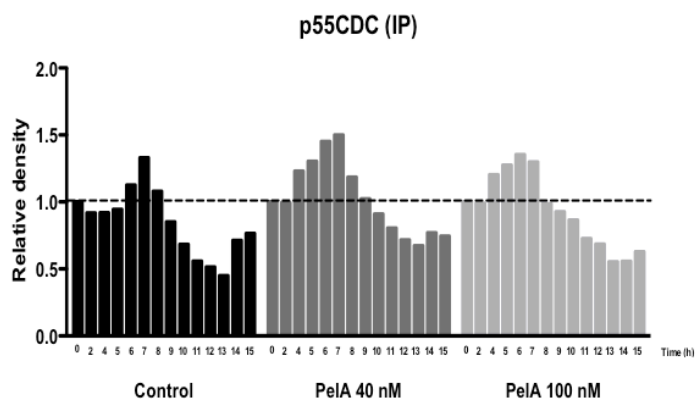

B

D
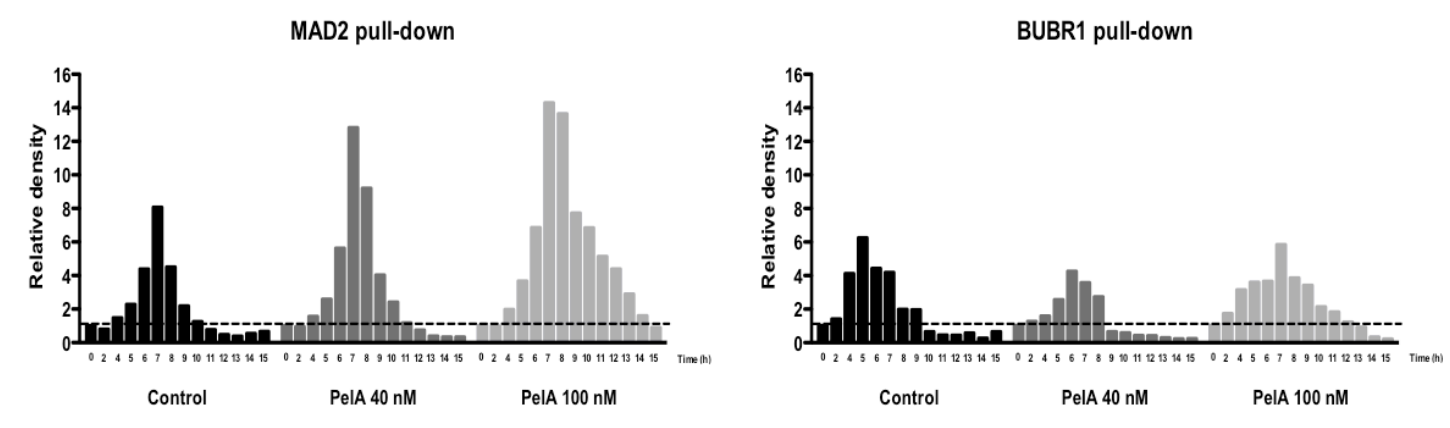

C

E
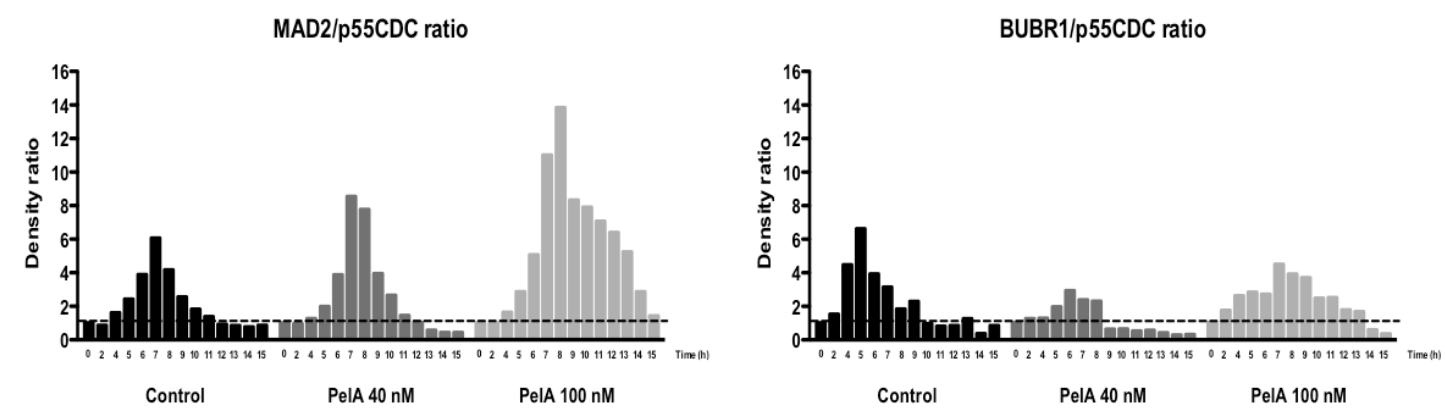

Figure 4.9. Changes in association of MAD2 and BUBR1 with p55CDC as determined by Co-IP and Western blotting. To determine the cell cycle-influenced association of MAD2 and BUBR1 to p55CDC, densitometry was carried out on the Western blots following Co-IP (see Fig. 4.8). The relative changes (normalized to time $0 \mathrm{~h}$ ) in expression levels of the immunoprecipitated protein, p55CDC, are shown in (A). The total expression levels of MAD2 and BUBR1 (relative to $0 \mathrm{~h}$, as indicated by the dashed lines) are also graphed ( $\mathbf{B}$ and $\mathbf{C}$, respectively), as well as the ratio of each protein to p55CDC (i.e. MAD2/p55CDC (D) and BUBR1/p55CDC (E)). Graphs represent data from a single experiment (n $=1$ ). 


\subsubsection{PelA treatment does not increase the frequency of lagging chromosomes but does impair chromosome alignment}

To assess whether an increase in the frequency of merotelic attachments was occurring at low concentrations of PelA, we carried out a preliminary experiment to look for lagging chromosomes at the metaphase plate during anaphase. Untreated cells or cells treated with PelA (40 or $100 \mathrm{nM}$ ) were stained simultaneously for centromeres, chromosomes and microtubules and assessed for obvious mitotic abnormalities. No lagging chromosomes were observed in anaphase cells from untreated or PelA-treated cells; however, we did observe differences in the congression of chromosomes at the metaphase plate. In the control samples, metaphase and anaphase cells all appeared to be normal (i.e. all cells aligned at the metaphase plate and no lagging chromosomes were observed) (Fig. 4.10). Although we observed cells with normal mitotic features at $40 \mathrm{nM}$ PelA, the majority of mitotic cells either presented with multiple asters or appeared to be in pseudo-metaphase (i.e. in which the majority of chromosomes appear to align properly at the metaphase plate except for a few polar chromosomes that localize at or very close to the spindle poles) (Fig. 4.11). These polar chromosomes are likely to be attached through monotelic attachments (see inset in Fig. 4.11, a). Polar chromosomes were also observed in cells treated with $100 \mathrm{nM}$ PelA; however, the majority of the remaining chromosomes in these cells failed to align at the metaphase plate (Fig. 4.12). Similarly to what was seen at the lower PelA concentration, many mitotic cells at $100 \mathrm{nM}$ PelA presented with multiple asters.

Mitotic cells in the different treatment groups were manually scored by eye for different mitotic morphologies (Table 4.1). It should be noted that this was a single experiment and the counts were made while looking down the microscope (rather than from images); thus, these counts represent a very rough approximation. As already mentioned, all mitotic cells in the untreated control samples exhibited normal mitotic features. At 40 and $100 \mathrm{nM}$ PelA, only $10 \%$ and $0 \%$ of mitotic cells appeared normal, respectively. PelA also caused a concentration-dependent increase in the percentage of cells with multiple or asymmetric asters, from $48 \%(40 \mathrm{nM})$ to $78 \%(100 \mathrm{nM})$. Inversely, a greater percentage of pseudo-metaphase cells (41\%) were observed at 40 nM PelA, compared to $100 \mathrm{nM}$ PelA (6\%). At $100 \mathrm{nM}$ PelA, approximately $17 \%$ of cells contained grossly misaligned chromosomes with some polar chromosomes, a morphology not seen at $40 \mathrm{nM}$ PelA. Additionally, no lagging chromosomes were observed at either PelA concentration. 


\section{CONTROL}
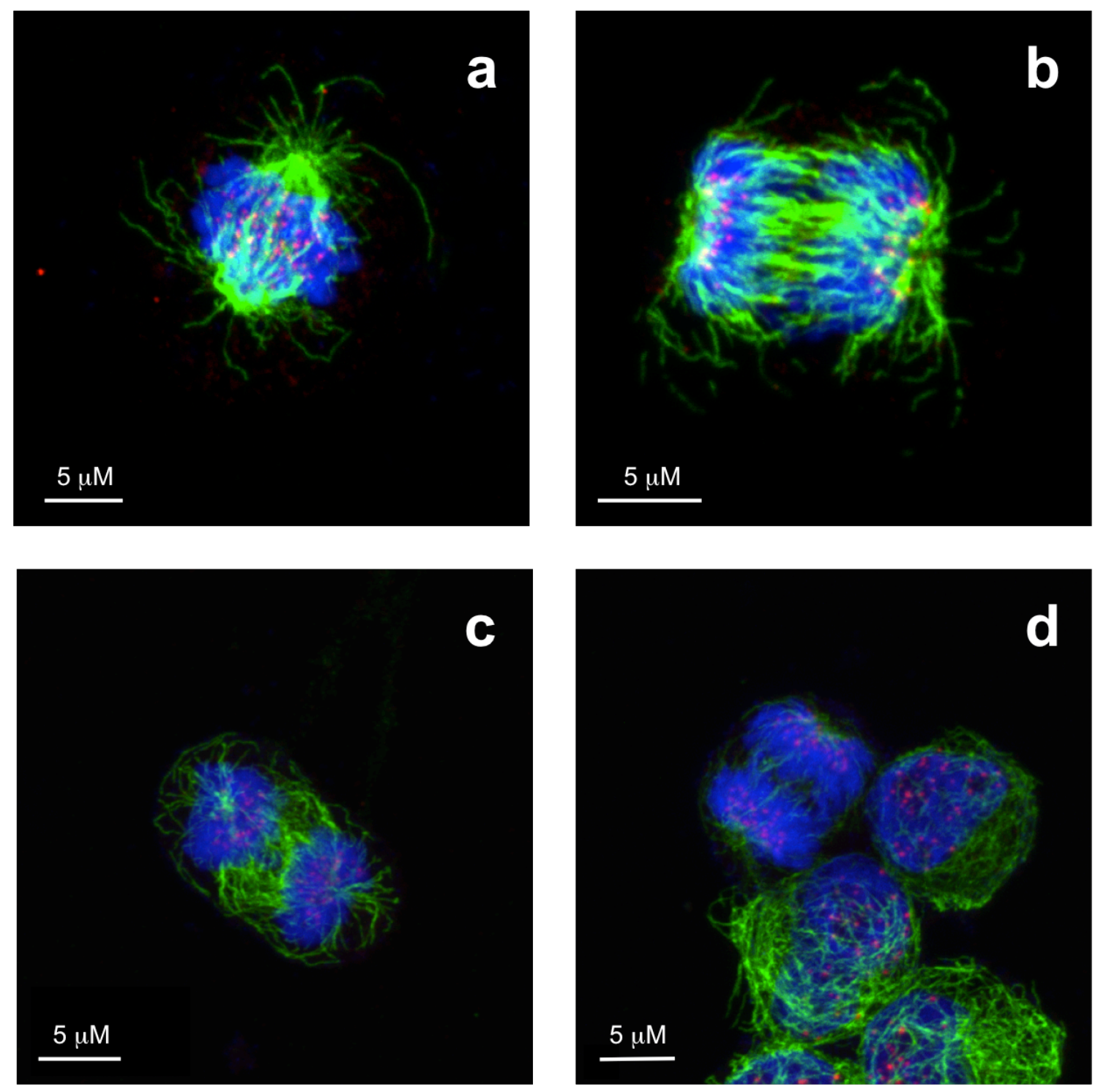

Figure 4.10. Untreated cells undergo normal mitosis. 1A9 cells were stained for $\alpha$-tubulin (green), centromeres (red), and chromosomes (blue), and imaged using confocal microscopy. A representative image of a cell in metaphase is presented (a), and shows the typical alignment of all chromosomes at the metaphase plate, prior to anaphase (note that occasional bright red spots could be seen that were not associated with chromosomes but these were considered artifacts). No lagging chromosomes at the metaphase plate were observed in cells undergoing anaphase (b-d). 


\section{PelA 40 nM}
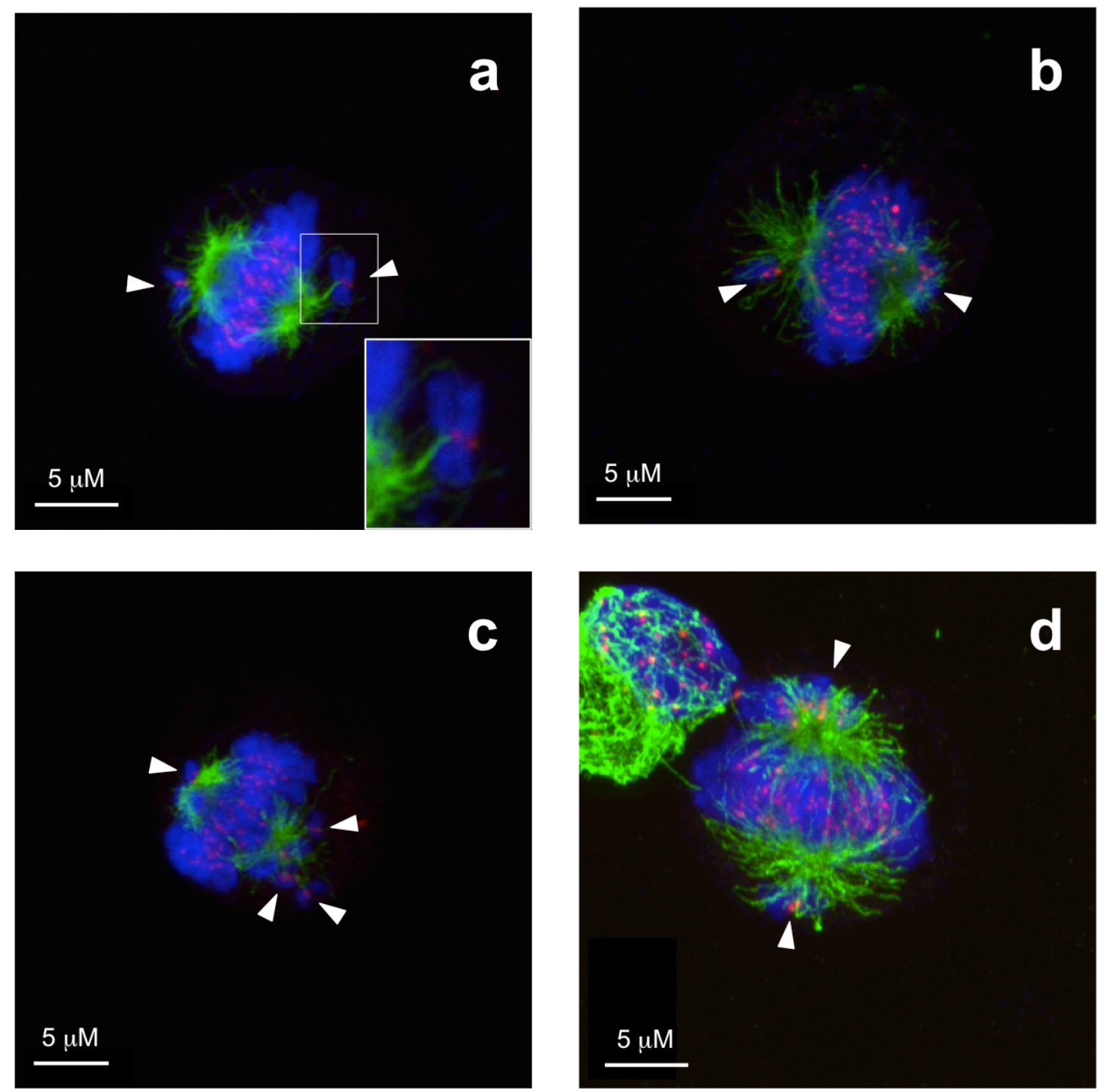

Figure 4.11. Examples of pseudo-metaphase cells after treatment with low concentration PelA. 1A9 cells that had been treated for $6 \mathrm{~h}$ with $40 \mathrm{nM}$ PelA were stained for $\alpha$-tubulin (green), centromeres (red), and chromosomes (blue), and imaged using confocal microscopy. Examples of cells are presented (a-d) that appeared to be in a "pseudo-metaphase" stage (in which the majority of chromosomes are aligned at the metaphase plate, except for one to several chromosomes that are juxtaposed to the spindle poles). White arrowheads indicate polar chromosomes. One such chromosome (possibly attached monotelically to the spindle pole) is enlarged in the inset in (a). 


\section{PelA 100 nM}
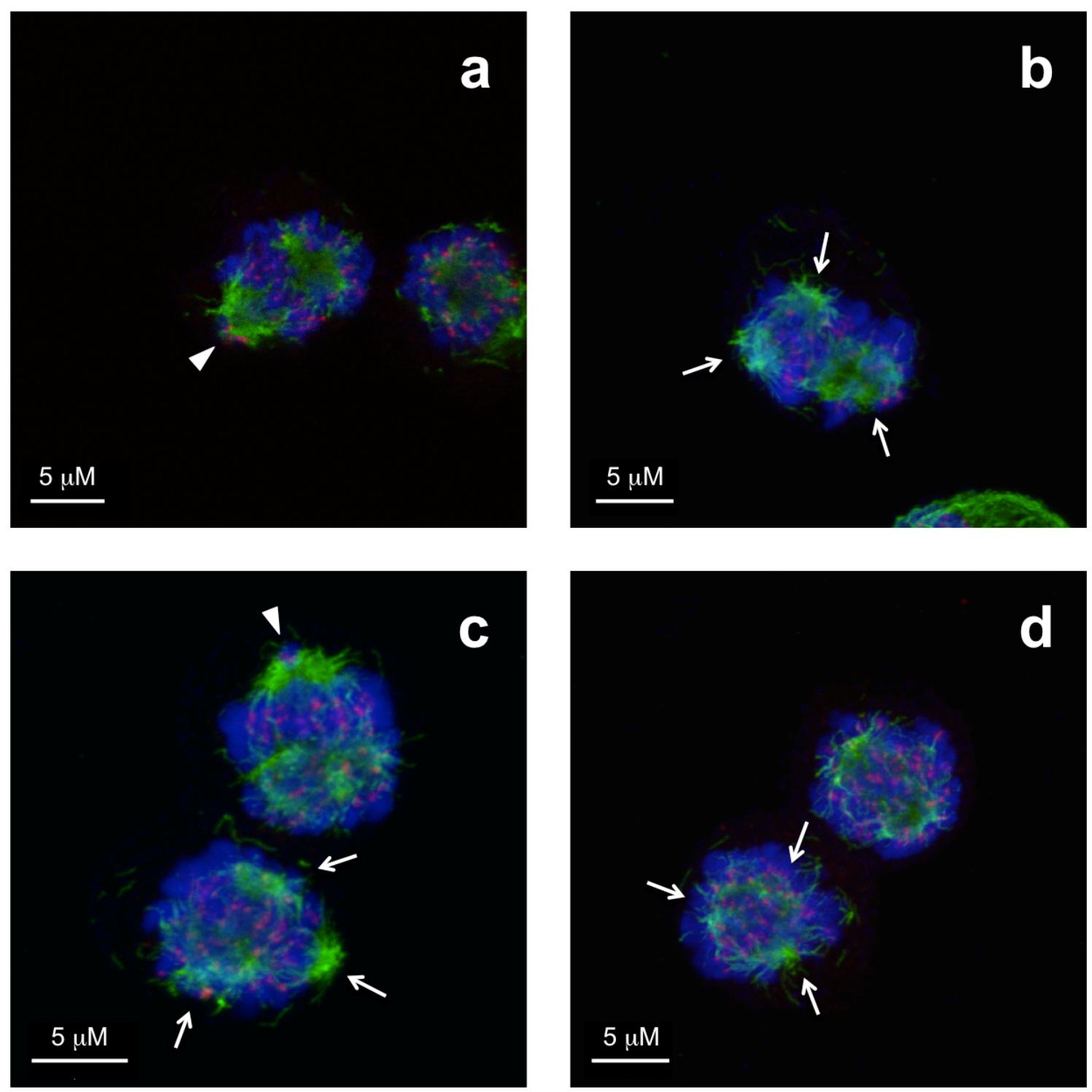

Figure 4.12. Abnormal mitosis at high concentrations of PelA. 1A9 cells that had been treated for $6 \mathrm{~h}$ with $100 \mathrm{nM}$ PelA were stained for $\alpha$-tubulin (green), centromeres (red), and chromosomes (blue), and imaged using confocal microscopy (a-d). Many cells treated at this concentration appeared to be blocked in prometaphase. Similarly to cells treated with $40 \mathrm{nM}$ PelA, polar chromosomes were also observed (white arrowheads in $\mathbf{a}$ and $\mathbf{c}$ ); however, in contrast to the pseudo-metaphase cells, the remaining chromosomes were not aligned at the metaphase plate. Another feature that was often observed at 100 nM PelA was the presence of multiple asters (white arrows in b-d), which often appeared to be asymmetric. 
Table 4.1. Frequency of cells exhibiting different mitotic morphologies in response to PelA.

\begin{tabular}{|c|c|c|c|c|c|c|}
\hline & \multicolumn{6}{|c|}{ TREATMENT } \\
\hline & \multicolumn{2}{|c|}{ Control } & \multicolumn{2}{|c|}{ PelA 40 nM } & \multicolumn{2}{|c|}{ PelA $100 \mathrm{nM}$} \\
\hline & \# cells & $\%$ & \# cells & $\%$ & \# cells & $\%$ \\
\hline Normal mitosis & 8 & 100 & 5 & 11.4 & 0 & 0 \\
\hline $\begin{array}{c}\text { Multiple and/or } \\
\text { asymmetric asters }\end{array}$ & 0 & 0 & 21 & 47.7 & 28 & 77.8 \\
\hline Pseudo-metaphase & 0 & 0 & 18 & 40.9 & 2 & 5.6 \\
\hline $\begin{array}{c}\text { Polar chromosomes + } \\
\text { grossly misaligned } \\
\text { chromosomes }\end{array}$ & 0 & 0 & 0 & 0 & 6 & 16.7 \\
\hline $\begin{array}{c}\text { Lagging anaphase } \\
\text { chromosomes }\end{array}$ & 0 & 0 & 0 & 0 & 0 & 0 \\
\hline Total cells counted & 8 & & 44 & & 36 & \\
\hline
\end{tabular}

Results are from a single preparation for each group. 


\subsection{Discussion}

\subsubsection{PelA induces aneuploidy at low concentrations in the absence of mitotic}

arrest

The results from the cell cycle analysis of asynchronous and synchronized 1A9 cells demonstrate that aneuploidy is induced at lower concentrations of PelA ( $<40 \mathrm{nM}$ PelA) that do not induce a significant $\mathrm{G}_{2} / \mathrm{M}$ block. Temporally, there was little difference in mitotic progression between controls and $40 \mathrm{nM}$ PelA (in the synchronized cells), indicating either an impairment in the ability of the SAC to detect spindle defects, or mechanisms that are independent of the SAC. These results are similar to those found for low concentrations of Ptx, EpoB, and Disco, indicating that this induction of aneuploidy is likely a general consequence of MSAs (Chen and Horwitz, 2002; Ikui et al., 2005). Interestingly, low concentrations of MDAs (e.g. colchicine, nocodazole and Vbl) do not have the same effect (Chen and Horwitz, 2002). A potential mechanism that was proposed by Chen and Horwitz (2002) was that the MSAs generated multiple spindle poles in response to low concentrations of MSAs, leading to aberrant chromosome segregation (Chen and Horwitz, 2002). Chen and Horwitz found that MDAs did not trigger the formation of multipolar spindles, which would explain why they failed to see aneuploidy in response to these drugs. We have previously quantified the induction of multiple asters in response to increasing concentrations of PelA in 1A9 cells (Kanakkanthara et al., 2011). At $40 \mathrm{nM}$, there was a dramatic increase in mitotic cells exhibiting multiple asters compared to lower PelA concentrations (53\% of mitotic cells had mitotic asters at $40 \mathrm{nM}$ ), and this may contribute to the frequency of aneuploidy that was observed in the current study. However, $100 \mathrm{nM}$ PelA, a concentration at which we do not observe significant aneuploidy, resulted in $65 \%$ of mitotic cells containing multiple asters (Kanakkanthara et al., 2011). If the formation of multiple asters is the primary mechanism of aneuploidy induction, then it is unusual that we only see aneuploidy at lower concentrations of PelA.

\subsubsection{PelA does not affect the expression of proteins involved in the SAC, but high concentrations lead to prolonged, elevated cyclin B1 expression levels}

Premature dissociation of MAD2 and BUBR1 from the APC activator, p55CDC, has been proposed as a possible mechanism underlying aneuploidy induction at low concentrations of Ptx (Ikui et al., 2005). Thus, an aim of this study was to explore the potential role of the SAC in PelA-induced aneuploidy. In particular, we wanted to focus on MAD2, because had been previously identified through a chemical genetics HOP 
microarray as being involved in the mode of action of PelA (Wilmes et al., 2012b). We did not observe altered cell-cycle dependent expression levels of MAD2, BUBR1 or p55CDC in response to either 40 and $100 \mathrm{nM}$ PelA (Fig. 4.7), although this is not surprising given that their functional roles as part of the SAC are predominantly regulated through interactions with each other and other mitotic proteins. Cyclin B1 expression on the other hand was degraded much slower in response to $100 \mathrm{nM}$ PelA, correlating with the mitotic arrest seen at this concentration (Fig. 4.4, 4.5 and 4.7). Although there was a slow degradation of cyclin B1, it was not reduced to the likely threshold level for anaphase progression. Others have shown that very low levels of non-degradable cyclin B1 ( $<30 \%$ compared to endogenous cyclin B1), is sufficient to prevent the onset of anaphase (Chang et al., 2003a). The arrest of cells in $G_{2} / M$ at 100 $\mathrm{nM}$ would suggest that the SAC is being activated to delay anaphase. Persistent cyclin B1 degradation despite an active SAC allows some cells to undergo mitotic slippage (Brito and Rieder, 2006; Brito et al., 2008). This mechanism is likely to be responsible for the small population of tetraploid cells that we see in the cell cycle histograms (Fig. 4.5). The timing of cyclin B1 degradation at $40 \mathrm{nM}$ PelA compared to controls was delayed only slightly (7-8 $\mathrm{h}$ versus 6-7 $\mathrm{h}$, respectively), indicating that low concentrations of PelA are activating the SAC for slightly longer. The presence of multiple centrosomes has been shown to prolong the duration of mitosis (doubling the number of centrosomes increases the duration of mitosis by 3-fold), and could explain this difference (Yang et al., 2008). A slower migrating BUBR1 band in the Western blotting (Fig. 4.6 C), which is indicative of phosphorylation, was also observed at 100 $\mathrm{nM}$ PelA. Hyperphosphorylation of BUBR1 is known to occur during mitosis, and in particular in response to the lack of tension between sister kinetochores (Taylor et al., 2001; Elowe et al., 2007).

\subsubsection{MAD2 and BUBR1 dissociation from p55CDC differs at low versus high concentrations of PelA}

Ikui et al. (2005) proposed that premature dissociation of MAD2 and BUBR1 from the SAC protein, p55CDC, was responsible for aneuploidy induction at low concentrations of Ptx; hence, we decided to look at the interactions between the two MCC proteins and p55CDC as cells progressed through the cell cycle. Our PelA supply comes from the natural sponge source and is in very limited supply. Due to the large amount of protein that was needed for the Co-IP, and hence the large amount of drug, the immunoprecipitation of $\mathrm{p} 55 \mathrm{CDC}$ was only carried out once $(n=1)$. The number of 
samples also meant that we needed a large amount of Dynabeads ${ }^{\mathrm{TM}}$ and antibody for the immunoprecipitation assay. Initially we tried to optimize a non-denaturing protocol that would have allowed us to re-use the Dynabeads ${ }^{\mathrm{TM}}$. Unfortunately, the non-denaturing methods that we tried did not efficiently elute all of the bound p55CDC; thus, an SDS eluting buffer was used instead (data not shown). Antibody heavy and light chains (HC and LC) typically migrate at approximately $50 \mathrm{kDa}$ and $25 \mathrm{kDa}$, respectively. These, unfortunately, were very close to the expected sizes of both p55CDC (55 kDa) and MAD2 $(24 \mathrm{kDa})$. Preliminary experiments also indicated that the $\mathrm{HC}$ and LC were detected to some extent by our secondary antibody on its own, and this would interfere with our ability to distinguish the IgG subunits from the SAC proteins. Crosslinking of the p55CDC antibody to the protein G Dynabeads ${ }^{\mathrm{TM}}$ prevented elution of the HC (see Appendix C, Figure C.1). The light chain eluted despite the crosslinking but migrated slightly slower than the MAD2 band.

Our results are very similar to those obtained previously for Ptx (Ikui et al., 2005). There were two different aspects of the association that we had to consider: (1) the differences in the timing of the dissociation at different drug concentrations, and (2) differences in the amounts of MAD2 and BUBR1 that were binding to p55CDC. With regard to MAD2 and $\mathrm{p} 55 \mathrm{CDC}$, it appeared that low concentrations of PelA did not affect the timing of their association, as the amount of MAD2 bound was at its maximum at $7 \mathrm{~h}$ for both untreated and $40 \mathrm{nM}$ PelA treated cells; however, at $40 \mathrm{nM}$ PelA, it took slightly longer (approximately $1 \mathrm{~h}$ ) for MAD2 to dissociate from p55CDC, indicating prolonged activation of the SAC and delayed mitosis. At $100 \mathrm{nM}$ PelA, the $\mathrm{MAD} 2 / \mathrm{p} 55 \mathrm{CDC}$ association was persistent due to the inability of the cells to satisfy the SAC at high concentrations. Although MAD2/p55CDC complexes did begin to dissociate at $9 \mathrm{~h}$, MAD2 protein levels in the complex remained high (up to the $13 \mathrm{~h}$ timepoint), which is consistent with the mitotic arrest observed at this concentration. We also observed a concentration-dependent increase in the ratio of MAD2/p55CDC as PelA was increased, particularly as cells were entering mitosis. Greater levels of p55CDC association with the MCC proteins, MAD2, BUBR1 and BUB1, have been observed previously in response to spindle disruption (Chen, 2002). Increased MAD2/p55CDC complex formation could be explained by the MAD2 template model, which supports the concept of a positive feedback loop to generate MAD2/CDC20 complexes in the presence of unattached kinetochores (De Antoni et al., 2005; Yu, 2006; Musacchio and Salmon, 2007). 
We did observe differences in the temporal association/dissociation kinetics between BUBR1 and p55CDC. In control cells, levels of BUBR1/p55CDC complexes peaked 5 $\mathrm{h}$ after synchronization release, corresponding with the majority of cells entering mitosis. PelA treatment (40 or $100 \mathrm{nM}$ ) caused a delay in the peak BUBR1/p55CDC association ( $\sim 6$ and $7 \mathrm{~h}$, respectively), which is slightly earlier than the peak MAD2/p55CDC complex levels. This could be due to the role that BUBR1 plays in recruiting other SAC proteins to kinetochores, as well as its potential role in facilitating $\mathrm{MAD} 2 / \mathrm{p} 55 \mathrm{CDC}$ association (although it should be noted that MAD2 is also thought to facilitate the binding of BUBR1 to p55CDC) (Chen, 2002). Low concentrations of PelA $(40 \mathrm{nM})$ caused a delay $(\sim 2 \mathrm{~h})$ in BUBR1/p55CDC dissociation, while high concentrations $(100 \mathrm{nM})$ caused a prolonged association of BUBR1 with p55CDC, presumably due to the inability to silence the SAC. Unexpectedly, $40 \mathrm{nM}$ PelA resulted in the lowest ratio of BUBR1 to p55CDC, and this ratio was also lower at higher PelA concentrations compared to controls. Several checkpoint proteins (e.g. Bub1 and Aurora B) are important in stabilizing the BUBR1/p55CDC interaction during SAC activation (Morrow et al., 2005). There is a possibility that these proteins may be affected by PelA, perhaps due to drug effects on the tension requirements of the SAC. Whether this reduction in BUBR1 levels is a true effect in response to PelA would, however, require additional replicates of this assay.

\subsubsection{Increased frequency of pseudo-metaphase cells as a possible mechanism underlying the induction of aneuploidy at low PelA concentrations.}

Ikui et al. (2005) suggested that low concentrations of Ptx perturb the SAC (possibly through premature dissociation of MAD2 from p55CDC), allowing cells to escape from mitotic block and become aneuploid. In order to come to this conclusion, concurrent assays need to be carried out to determine whether there is reduced kinetochore staining of MAD2 and/or BUBR1 in the presence of erroneous microtubule-kinetochore attachments. It is also unlikely that perturbation of the SAC at low MSA concentrations would only result in a small percentage of aneuploid cells, considering how crucial a role the SAC plays in regulating anaphase progression. How MSAs could cause a premature dissociation of MAD2 or BUBR1 from p55CDC is also unclear. Additionally, if the SAC were in fact a target of PelA, as Ikui et al. (2005) seem to suggest, one would expect a concentration-dependent effect on the SAC that would result in aneuploidy being observed at both low and high concentrations. It seems more likely that the induction of aneuploidy by low concentrations of MSAs is likely to be 
due to direct effects on microtubules, their dynamics, or the ability of microtubules to correctly associate with kinetochores or kinetochore-associated proteins. Merotelic kinetochore attachments are a leading cause of aneuploidy in mammalian cells, and it has been observed that the frequency of merotely is increased in cells recovering from a nocodazole block (possibly due to morphological affects of nocodazole on the kinetochores that promote merotely) (Cimini et al., 2001). Hence, we hypothesized that low concentrations of an MSA like PelA would increase the frequency of merotelickinetochore attachments. These particular attachments are not detected by the SAC (see Fig. 4.2), and this could explain why MAD2 and BUBR1 dissociation was fairly normal at $40 \mathrm{nM}$ PelA.

The presence of lagging chromosomes during anaphase can be used as an indirect indicator of uncorrected merotelic attachments (Cimini et al., 2001; Cimini et al., 2003). In the present study, cells were simultaneously immunostained for microtubules, centromeres, and chromosomes, and anaphase cells were imaged by confocal microscopy to detect lagging chromosomes. The actual orientation of chromosome attachments to the mitotic spindles can also be assessed but requires high-resolution images that have been enhanced by deconvolution. Deconvolution is a post-processing technique that uses image analysis software to remove some of the inherent blur in fluorescent images. This method, however, was beyond the scope of the current set of experiments. Hence, our analysis of mitotic morphologies was carried out on unprocessed images. From both acquired images and manual observations, we noted an absence of lagging chromosomes in anaphase cells incubated with or without PelA; however, due to the limited resolution of our images, we cannot rule out the possibility that merotelic attachments were present in prometaphase or metaphase cells. Additionally, this pilot confocal study was conducted as a single experiment, and we were limited in the number of mitotic cells (especially those in anaphase) that we could find. This problem was exacerbated because we used unsynchronized cells (which will enter mitosis at different times) and also because the durations of the different mitotic phases in mammalian cells are very short (i.e. approximately 22, 19 and $<10$ mins for prometaphase, metaphase and anaphase, respectively, in HeLa cells) (Giodini et al., 2002).

Interestingly, we did observe aberrant mitotic morphologies in our PelA-treated cells that may account for the induction of aneuploidy at low concentrations of MSAs. In 
agreement with previous findings, PelA treatment led to a concentration-dependent increase in the numbers of cells with multiple asters ((Kanakkanthara et al., 2011), but, as explained above, this is unlikely to be the cause of aneuploidy at lower drug concentrations. We observed an increase in the number of 'pseudo-metaphase' cells (Weaver et al., 2003) at $40 \mathrm{nM}$ PelA, but not $100 \mathrm{nM}$ PelA. Regardless of the type of chromosome orientation of these polar chromosomes, if they are not corrected, then they are a potential source of aneuploidy. Although we also observed polar chromosomes in cells treated with $100 \mathrm{nM}$ PelA, we did not see a significant induction of aneuploidy at this concentration (Chapter 4). At $100 \mathrm{nM}$ PelA, the mitotic cells had grossly misaligned chromosomes regardless of whether polar chromosomes were present or not. The failure of these chromosomes to congress at the metaphase plate is likely to lead to these cells arresting in prometaphase due to prolonged activation of the SAC (thus preventing aneuploidy).

Unlike merotelic attachments, unattached or monotelically-oriented chromosomes should trigger the SAC (see Fig. 4.2). It has been suggested that a single chromosome is sufficient to activate the SAC (Rieder et al., 1995); thus, if this is the case, then pseudometaphase cells should not progress through to anaphase, and would instead become blocked in metaphase. Pseudo-metaphase cells have also been observed previously at low concentrations of Ptx (Jordan et al., 1993); however, the authors suggested that cells exhibiting this particular type of aberrant spindle formation contributed to the mitotic arrest at the metaphase/anaphase transition, but did not actually demonstrate whether these pseudo-metaphase cells were in fact arrested (nor did they link these morphologies to aneuploidy). From our preliminary observations, which will need to be confirmed in a more comprehensive and quantitative study, we suggest that the ability of most of the chromosomes to congress normally in pseudo-metaphase cells is sufficient to either silence or bypass (through an unknown mechanism) the SAC. Pseudo-metaphase cells have been observed in fibroblast cells depleted of CENP-E, and despite the localization of several SAC proteins (BUB1, MAD1 and MAD2) to the kinetochores of the polar chromosomes, these cells still progressed through anaphase (Weaver et al., 2003). Weaver et al. (2003) also demonstrated that in the absence of CENP-E, SAC signals generated by one or a few of these polar chromosomes are weakened, such that they cannot continue to delay anaphase onset. CENP-E is required for BUBR1 recruitment to unattached kinetochores and is also involved in regulating BUBR1 kinase activity (Weaver et al., 2003). It is possible that PelA either reduces 
CENP-E expression or impairs its localization to kinetochores. If this is the case, the weakened SAC signalling of polar chromosomes at low concentrations of PelA could permit anaphase progression. Even if CENP-E expression/activity was also reduced at higher PelA concentrations, the cumulative SAC signals (despite being weakened) of a larger number of unattached kinetochores, is probably enough to delay anaphase onset. Decreased CENP-E expression/activity by PelA (and the concentration-dependent effects of PelA on mitotic morphologies) may also explain why we observed decreased BUBR1/p55CDC association at $40 \mathrm{nM}$ PelA but not $100 \mathrm{nM}$ PelA.

\subsubsection{Conclusions and future directions}

Our results from the cell cycle kinetic study of MAD2 and BUBR1 association and dissociation from p55CDC were very similar to those observed previously by Ikui et al. (2005). We observed a delayed, but otherwise fairly normal dissociation profile for these SAC proteins at $40 \mathrm{nM}$ PelA, suggesting that the induction of aneuploidy may be due to the improper silencing or bypassing of the SAC, despite the presence of incorrectly attached chromosomes to the mitotic spindle. A preliminary assessment of mitotic morphologies suggests that the induction of aneuploidy at low concentrations may be due to an increase in the frequency of pseudo-metaphase cells at low concentrations of PelA that are unable to generate enough SAC signal to delay anaphase onset. To confirm our observations from the confocal study, we would need to repeat our results and accurately quantify the different mitotic morphologies at low and high concentrations of PelA. Additionally, to confirm our observation that PelA did not alter the frequencies of merotelic chromosomes, our images would need to be deconvoluted to improve the resolution. It would also be useful to assess differences in the recruitment of SAC proteins to the kinetochores of polar chromosomes, as well as the recruitment and expression levels of CENP-E in response to different concentrations of PelA. 


\section{Chapter 5. Potential anti-angiogenic activity of peloruside A}

\subsection{Introduction}

Novel anti-angiogenic agents are highly sought after in the clinic because of their huge potential for preventing both tumour growth and metastasis. Endothelial cells (EC) play a central role in tumour angiogenesis and must transition through several key steps in order to form a functional vascular network (see General Introduction and Fig. 1.4). Initial steps involve degradation of the basement membrane and subsequent invasion into the perivascular space. Cell migration and proliferation follow, terminating in endothelial cell rearrangement into three-dimensional (3D) capillary structures. Research into some of the non-mitotic functions of microtubules, such as EC migration, has led to an increased understanding of the biological and molecular aspects behind tumour angiogenesis, as well as highlighted alternative clinical uses of some of the more traditional anti-cancer agents, such as the MSAs. MSAs have a profound effect on the actions of microtubules during angiogenic processes, in particular on microtubulemediated actions during cell migration (described below).

\subsubsection{Cytoskeletal involvement in cell migration}

The directional cell migration that occurs when ECs form new blood vessels is dependent on the ability of these cells to become highly polarized. Migrating cells develop a leading and lagging edge, with the microtubule-organizing center (MTOC) and Golgi apparatus localized towards the front of the cell (Watanabe et al., 2005). This polarization process is complex and largely involves the actin cytoskeleton, which must reorganize itself into an asymmetric array to facilitate cell migration. It also involves the stabilization of microtubule plus-ends towards the leading edge, and co-ordination between the actin filament, microtubule and intermediate filament networks (EtienneManneville, 2004; Watanabe et al., 2005). During migration, a protrusion of the cytoplasm (lamellipodium) and finger-like projections (filopodia) form at the front of the cell (Small et al., 2002). The plasticity of the actin cytoskeleton drives lamellipodium formation, and its assembly into stress fibers is important for cell adhesion and contractility (Small et al., 2002; Naumanen et al., 2008). As a cell migrates it must be able to regulate its attachments to its substrate, by extending at the leading edge and contracting at the rear. To provide a link between the actin cytoskeleton and the ECM, and also to provide traction points during motility, transient complexes called focal adhesions are formed (Ezratty et al., 2005). These complexes 
consist of numerous proteins that play an important role in signal transduction, including focal adhesion kinases (FAKs) and the transmembrane receptor family of integrins (Ezratty et al., 2005; Ezratty et al., 2009). The activation of myosin II by Rho A (Rho GTPases are described below) plays an important role in the contractility of the actin cytoskeleton, which, in turn, is important for the formation and maintenance of stress fibers and focal adhesions (Chrzanowska-Wodnicka and Burridge, 1996; Small et al., 2002). Specifically, the myosin IIA isoform has been implicated in regulating the crosstalk between the acto-myosin and microtubule cytoskeletons, as well promoting microtubule dynamics (Even-Ram et al., 2007).

Actin is often perceived as the predominant mediator of cell migration, and many of its actions can occur independently of microtubules; however, microtubules are also gaining recognition as key regulators of cell motility. Treatment of fibroblasts with the depolymerizing drugs vinblastine (Vbl) or nocodazole, for example, quickly induces the formation of actin stress fibers and focal adhesions (Enomoto, 1996). Additionally, similar treatment with the MDA, colcemid, inhibits lamellipodia formation (Bershadsky et al., 1991) in migrating fibroblasts. Focal adhesions can capture and stabilize microtubules to regulate the turnover of these sites. Earlier studies using time-lapse video microscopy observed the repetitive targeting of microtubules to focal adhesions, and the authors of this study suggested a possible function of microtubules in delivering signal molecules to these sites (Kaverina et al., 1998). Through a mechanism that has not yet been fully elucidated, microtubule interactions with focal adhesions promotes their disassembly (Kaverina et al., 1999). This process is mediated by FAKs and dynamin and leads to the downstream clathrin-mediated endocytosis of integrins (Ezratty et al., 2005; Ezratty et al., 2009). Increasing evidence is emerging that suggests feedback loops may exist between the various interacting partners, since stress fibers, focal adhesions, and the actin and microtubule cytoskeletons can influence the actions of each other (Etienne-Manneville, 2004).

Rho GTPases are a family of proteins that also play a central role in cell migration processes. These ubiquitously expressed proteins regulate signal transduction pathways by cycling between their active (GTP-bound) and inactive (GDP-bound) states. One of the more prominent effects of active Rho GTPases (e.g. Rho, Rac and Cdc42) in terms of cell migration is the regulation of actin functions (Wittmann and Waterman-Storer, 2001). Rho GTPases can be activated by GTPase activating proteins (GAPs) or 
following the release of guanine nucleotide exchange factors (GEFs) from depolymerizing microtubules, and they are inhibited by Rho-GDP-dissociation inhibitors (GDIs) (Raftopoulou and Hall, 2004; Watanabe et al., 2005). Microtubule dynamics can also influence Rho GTPase activities; therefore, drugs that affect the polymerization or depolymerization of microtubules can profoundly affect these molecular switches. GEFs are inactive when sequestered to microtubules but can be released and activated by microtubule-depolymerization, thus promoting the conversion of inactive GDP-bound Rho GTPases to their active GTP-bound state (Watanabe et al., 2005). RhoA signal transduction is important in stress fiber and focal adhesion formation (and therefore acto-myosin contractility) and is activated by non microtubulebound GEF-H1 (Krendel et al., 2002; Nalbant et al., 2009). Actin polymerization, particularly at the leading edge, is also controlled by Rho GTPases, whereby Rac1 and Cdc42 can induce lamellipodia and filopodia formation, respectively (Watanabe et al., 2005). Microtubule polymerization induced by MDA washout or Ptx treatment promotes Rac1-mediated lamellipodia formation, and it has been suggested that Rac1GTP, which only binds to soluble tubulin dimers, may get released upon tubulin polymerization (Best et al., 1996; Waterman-Storer et al., 1999). While regulators of Rho GTPases, such as GEFs, are regulated by dynamic instability, there is evidence to suggest that Rho GTPases may, in turn, regulate microtubule dynamics. Both Rac1 and Cdc42 target the serine/threonine kinase p65PAK, which is known to phosphorylate stathmin (an inducer of microtubule-destabilization); therefore, Rac1 and Cdc42 activities at the leading edge act to regulate both the actin and microtubule cytoskeletons to facilitate cell migration (Daub et al., 2001).

\subsubsection{In vitro assays to screen for anti-angiogenic compounds}

Angiogenesis is a highly complex, multistep process, and there is currently no single, in vitro assay that can replicate each of the steps involved. Several assays are commonly used to assess anti-angiogenic potential. Two of these assays that are used to assess the ability of a drug to inhibit the migratory capacity of ECs are the wound scratch healing assay and the transwell migration assay (modified Boyden chamber assay). In the wound scratch assay, a scratch is made in a monolayer of ECs, and the ability of the cells to migrate and cover the wound is quantitatively determined. The transwell migration assay consists of ECs plated on top of a porous membrane. The insert containing the cells is then placed inside another chamber containing VEGF (usually a multi-well plate), as well as other factors or drugs that can stimulate or inhibit migration 
of the ECs through the pores of the membrane. There are a couple of caveats to this particular assay. One is that quantifying the degree of migration can be quite a tedious process. The membranes must be fixed, stained, cut out, photographed and then the cells counted, which can all be quite time-consuming. Additionally, the inserts themselves are expensive, especially the newer versions that are more high-throughput and automated, and allow scanning of the membranes using a multi-well plate reader. To look at cell invasion, rather than migration, the transwell inserts are additionally coated with a gel that resembles the ECM, such as Matrigel ${ }^{\mathrm{TM}}$. This matrix must be enzymatically degraded before cells can to migrate through the pores of the membranes.

Matrigel $^{\mathrm{TM}}$ is a reconstituted, soluble extract that is secreted from Engelbreth-HolmSwarm (EHS) mouse sarcoma and resembles the basement membrane surrounding many tissues and tumours (McGonigle and Shifrin, 2008). It contains many proteins and growth factors that stimulate ECs to behave similarly to how they would in vivo. The main components in Matrigel $^{\mathrm{TM}}$ are laminin and collagen IV, but it also contains heparin sulfate proteoglycans, entactin and growth factors that naturally occur in EHS sarcomas (e.g. TGF- $\beta$, EGF, bFGF, PDGF) (Kleinman et al., 1982; Kleinman et al., 1986; Vukicevic et al., 1992). One of the most widely used assays, particularly for screening compounds with anti- or pro-angiogenic activity, is the Matrigel ${ }^{\mathrm{TM}}$ tube formation assay. HUVECs rapidly form capillary-like structures containing lumens, with cell alignment occurring within the first few hours (Kubota et al., 1988). Two components of the ECM, laminin and collagen IV, are thought to be key factors promoting this endothelial differentiation (Kubota et al., 1988). The advantages of this assay are that Matrigel ${ }^{\mathrm{TM}}$ is commercially available, the assay can be high throughput, is reliable, and is relatively easy to quantitate. Additionally, it incorporates many of the steps involved in angiogenesis, including proteolytic degradation of the basement membrane, cell adhesion, cell motility and polarization, cell-to-cell signalling, alignment, and formation into three-dimensional (3D) structures (McGonigle and Shifrin, 2008; Arnaoutova et al., 2009). ECs plated on Matrigel ${ }^{\mathrm{TM}}$ are rapidly induced to form capillary-like structures, making it ideal for screening angiogenic inhibitors.

\subsubsection{Aims}

Many EC functions that are important for tumour-induced angiogenesis are dependent on an intact, functional cytoskeleton. The disruption of microtubules, in addition to its cytotoxic effects on primary tumours, is currently being investigated as a therapeutic 
strategy to prevent tumour growth and metastases. Several MSAs have previously displayed anti-angiogenic activity including, Ptx, EpoB, Dtx, and laulimalide (Belotti et al., 1996; Woltering et al., 2003; Lu et al., 2006). Anti-angiogenic screens have not previously been carried out at VUW; thus, we optimized several in vitro assays using primary HUVECs that could be used to assess PelA's ability to inhibit specific EC functions. To this end, our aims for this chapter were as follows:

1) To assess the ability of PelA to inhibit HUVEC proliferation. The MTT cell proliferation assay, a commonly used method in our laboratory, was used to determine the $\mathrm{IC}_{50}$ concentration for this MSA.

2) To investigate PelA's effects on cell migration. We initially wanted to use the transwell migration assay, but as explained in the discussion, we were unable to get the assay to work; thus, a wound scratch healing assay was used as a nonchemotactic alternative.

3) To assess whether PelA can inhibit the ability of HUVECs to form capillary-like structures using a Matrigel ${ }^{\mathrm{TM}}$ tube formation assay. 


\subsection{Methods}

\subsubsection{HUVEC culturing conditions}

HUVECs were a generous gift from Dr Sarah Gunningham (Mackenzie Cancer Research Group, University of Canterbury). HUVECs were supplied at passage 2 and only used up to passage 6 for all experiments. Cells were cultured at $37^{\circ} \mathrm{C}$ in a $5 \% \mathrm{CO}_{2}$ in-air atmosphere (Panasonic $\mathrm{CO}_{2}$ cell culture incubator; Model no. MCO-20AIC; SANYO North American Corporation). Culture medium consisted of MCDB131 medium (Invitrogen) supplemented with 2\% fetal calf serum (Gibco), 1\% PenStrep (i.e. 100 units/mL penicillin and 100 units/mL streptomycin final concentration, Invitrogen), $10 \mathrm{ng} / \mathrm{mL}$ epidermal growth factor (Invitrogen), $12 \mu \mathrm{g} / \mathrm{mL}$ endothelial cell growth supplement (Sigma), $1 \mu \mathrm{g} / \mathrm{mL}$ hydrocortisone (Sigma), $10 \mathrm{U} / \mathrm{mL}$ heparin (Sigma), 2 mM L-glutamine (Sigma). HUVECs were cultured in tissue culture treated polystyrene flasks from Becton Dickinson (Becton Dickinson, Franklin Lakes, NJ). When HUVECs were approximately $90-95 \%$ confluent, cells were either passaged into fresh medium (at a seeding density of approximately $1 \times 10^{4}$ cells $/ \mathrm{cm}^{2}$ ) or used for experimental assays. HUVECs were trypsinized (as described previously in Chapter 2 for 1A9 and MCF7 cell lines) except cells were rinsed three times with sterlie PBS prior to the addition of trypsin.

\subsubsection{MTT cell proliferation assay}

The MTT assay is described in Chapter 2. HUVECs were treated with either PelA or Dtx for $120 \mathrm{~h}$ or $16 \mathrm{~h}$.

\subsubsection{Wound scratch healing assay}

Two lines were drawn approximately $5 \mathrm{~mm}$ apart through the centre of each well on the underside of 24-well plate (see Appendix D, Figure D.1. for diagram). Another single line, perpendicular to the previous lines, was drawn through the centre of the well. Cells were plated into each well at a seeding density of $5 \times 10^{4}$ cells/well and grown for two days until confluent (fresh medium was given after $24 \mathrm{~h}$ ). Cells were pre-treated for $5 \mathrm{~h}$ with either PelA or Dtx (each condition was run in triplicate wells) diluted in MCDB131 medium supplemented with $1 \mu \mathrm{g} / \mathrm{mL}$ hydrocortisone, 10 units/mL heparin, 2 $\mathrm{mM}$ L-glutamine, 100 units/mL penicillin, and 100 units/mL streptomycin (serum-free and growth factor-free). A plate insert with a tip holder (see Appendix D, Figure D.2. for photos; made by Craig Doney, School of Biological Sciences, VUW) was used as a 
guide for mechanically creating a wound down the centre of each well with a $200 \mu \mathrm{L}$ sterile pipette tip. Cells were washed 3 times with 1 x PBS and complete MCDB131 medium containing drug was added to the cells. Photos were taken on an Olympus IX51 inverted microscope, using the $4 \mathrm{x}$ objective lens (so that the wound was captured in a single field of view) at $0 \mathrm{~h}$ and again after $18 \mathrm{~h}$, using the marker lines to orientate the sequential images. Wound area was calculated by drawing around the wound using the segmented line tool in the opensource MBF-ImageJ software (Rasband, W.S., ImageJ, National Institutes of Health, Bethesda, Maryland, USA). Inhibition of cell migration was determined by calculating the percentage wound area at $18 \mathrm{~h}$ compared to the initial wound area at $0 \mathrm{~h}$. The extent of wound closure was determined by measuring the wound area at $0 \mathrm{~h}$ and $18 \mathrm{~h}$ and calculating the percentage of wound recovery after $18 \mathrm{~h}$ (i.e. $100-[($ wound area at $18 \mathrm{~h}) /($ wound area at $0 \mathrm{~h}) * 100])$.

\subsubsection{Tube formation assay}

The glass slides were removed from the polystyrene vessels of 8-well chamber slides $\left(\mathrm{BD}\right.$ Falcon ${ }^{\mathrm{TM}}$ ) and replaced with glass coverslips before sterilizing (wiped-down with $70 \%$ ethanol and UV-sterilized for $1 \mathrm{~h}$ ). Each well was coated as evenly as possible with $105 \mu \mathrm{L}$ phenol-red free Matrigel ${ }^{\mathrm{TM}}$ matrix (BD Biosciences) on ice (Matrigel was thawed overnight at $4{ }^{\circ} \mathrm{C}$ in an ice bucket). The gel was then polymerized by incubation at $37{ }^{\circ} \mathrm{C}$ for $30 \mathrm{~min}$. Drug diluted in complete MCDB131 medium was added to each chamber before seeding $4.5 \times 10^{5}$ cells/well (in a total volume of $200 \mu \mathrm{L}$ ). After $16 \mathrm{~h}$, cells were washed once gently in pre-warmed KREBS buffer. Cells were stained in 4 $\mu \mathrm{M}$ calcein (Becton Dickinson) diluted in KREBS buffer for $30 \mathrm{~min}$ at $37^{\circ} \mathrm{C}$. Images were taken on an FV1000 confocal laser scanning microscope (inverted model IX81) using the $4 \mathrm{X}$ and $10 \mathrm{X}$ objective lenses (Olympus). For the images taken at $4 \mathrm{X}$, the multi-area time lapse function was used to stitch together one larger image composed of $2 \times 2$ fields of view. This $2 \times 2$, stitched image is what was used for both the manual and automated quantification. 


\subsection{Results}

\subsubsection{Inhibition of EC proliferation by the microtubule-stabilizing drugs PelA and}

Dtx.

Angiogenesis is dependent on the ability of ECs to proliferate. A colorimetric MTT cell proliferation assay was used to determine the effects of PelA and Dtx on EC proliferation. Both of these microtubule-stabilizing agents (MSAs) showed similar antiproliferative activity towards human umbilical vein ECs (HUVECs). The $\mathrm{IC}_{50}$ values of PelA and Dtx were $1.4 \mathrm{nM}$ and $1.7 \mathrm{nM}$, respectively (Fig. 5.1).

\subsubsection{Inhibition of HUVEC migration by PelA and Dtx.}

During angiogenesis, ECs proliferate and migrate towards angiogenic signals, such as those secreted by tumour cells. To determine whether PelA inhibited HUVEC migration, a wound scratch healing assay was used. To minimize the effects of promigratory effects of components in the cell culture medium, confluent cells were pretreated in drug diluted in serum-free and growth factor-free medium for $5 \mathrm{~h}$ prior to mechanically creating a "wound". After wound induction, the degree of wound closure in the presence or absence of drug was assessed after $18 \mathrm{~h}$ and compared to time $0 \mathrm{~h}$. A concentration-dependent inhibitory effect was seen with both PelA and Dtx, as shown by the images taken at each timepoint (Fig. 5.2 $\mathrm{A}$ and $\mathrm{B}$, respectively) and by determining the percentage wound area at $18 \mathrm{~h}$ compared to the wound area at time $0 \mathrm{~h}$ (controls) (Fig. 5.2 C). During the $18 \mathrm{~h}$ incubation period almost the entire wound (98.6 $\pm 0.7 \%$ ) was closed by migrating HUVECs in untreated cells. Both PelA and Dtx significantly inhibited the ability of HUVECs to migrate into the lesion, with a $71.9 \pm$ $3.4 \%$ and $84.7 \pm 2.0 \%$ inhibition of wound closure at the highest concentrations of PelA and Dtx, respectively. The lowest concentration of PelA tested $(2.5 \mathrm{nM})$ was still able to inhibit migration by $36 \pm 3.5 \%$, although the effects of Dtx on HUVEC migration were more potent. Both $2.5 \mathrm{nM}$ and $5 \mathrm{nM}$ Dtx inhibited wound closure to a greater extent than PelA at $15 \mathrm{nM}(76.2 \pm 3.8 \%$ and $84.7 \pm 2 \%$ compared with $71.9 \pm 3.4 \%)$. Consistent scratch wounds were important for this particular assay; therefore, a method was used such that the average error in the initial wound area for a triplicate was $4.1 \%$ or less. 

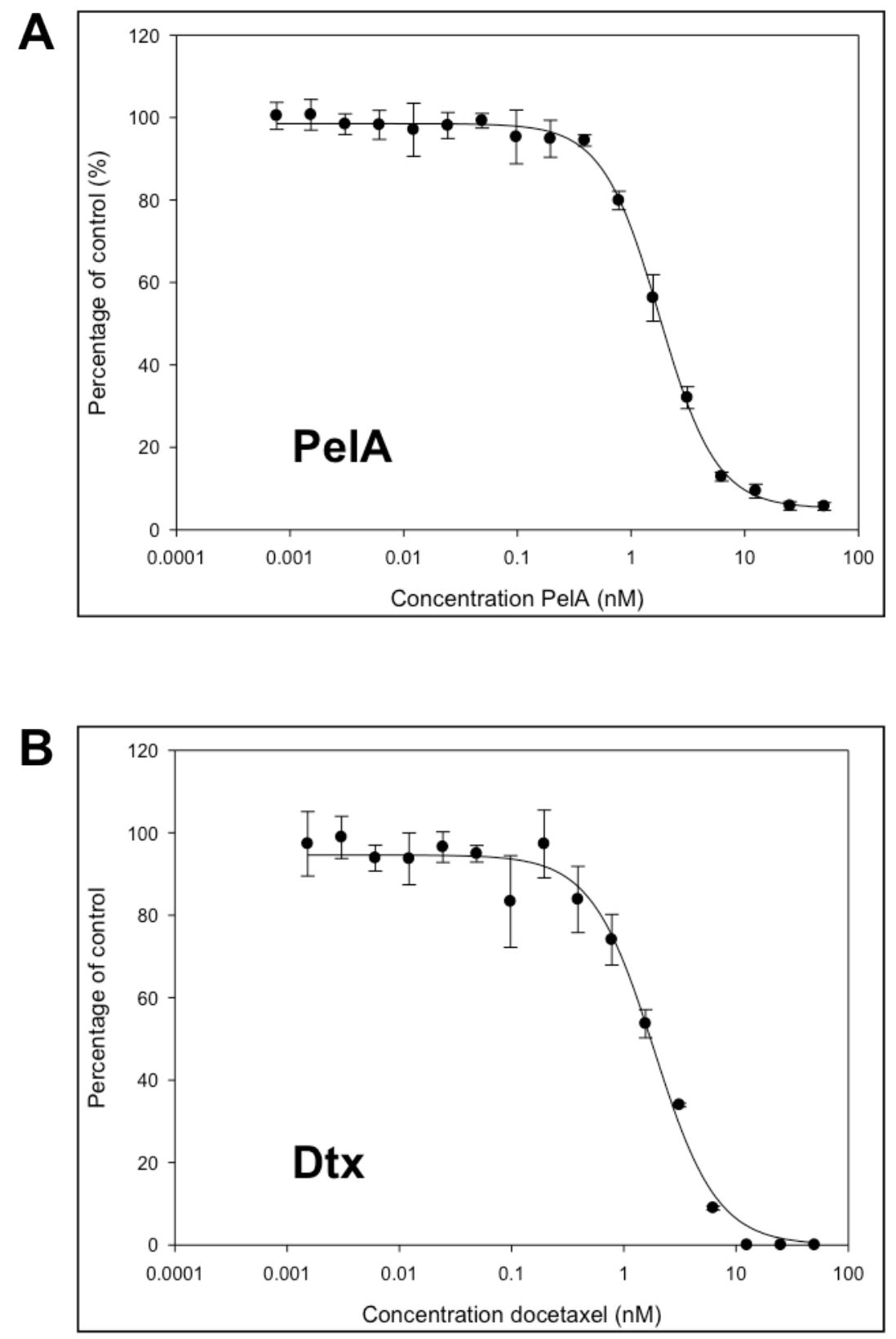

Figure 5.1. Inhibition of HUVEC proliferation by PelA and Dtx. Representative concentrationresponse curves of HUVECs treated with PelA (A) or Dtx (B) for $120 \mathrm{~h}$. Both PelA and Dtx showed a similar inhibition of cell proliferation with $\mathrm{IC}_{50}$ values of $1.4 \pm 0.3 \mathrm{nM}$ and $1.7 \pm 0.3 \mathrm{nM}$, respectively (values are the mean \pm SEM, $n=5($ PelA) or $n=3($ Dtx $)$ ). . A Mann-Whitney U test to comparing these $\mathrm{IC}_{50}$ values gave a $\mathrm{p}$ value of 0.4506$)$. 

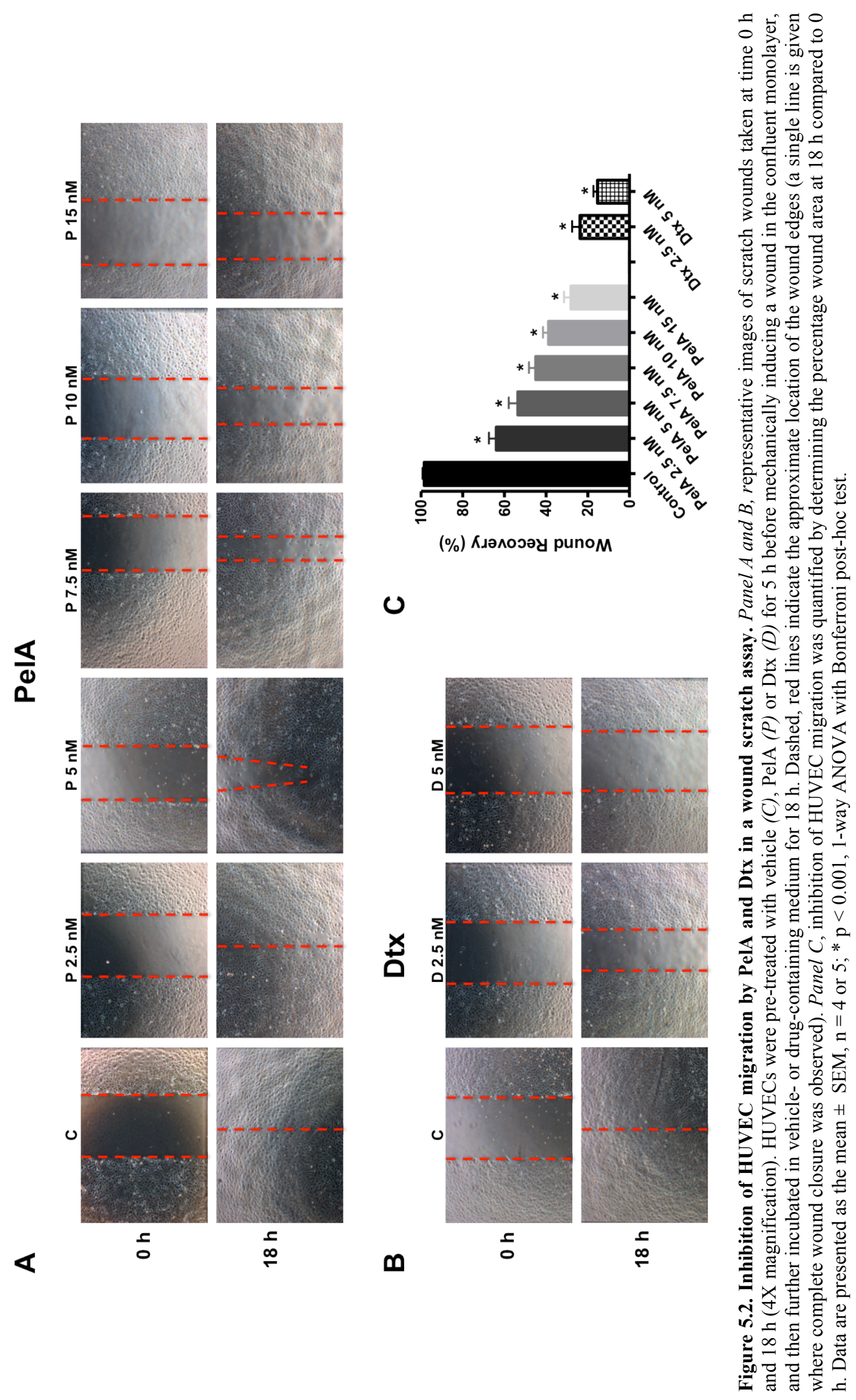


\subsubsection{PelA and Dtx inhibit HUVEC capillary-like tube formation}

One of the final steps in the growth of new blood vessels is the organization of ECs into three-dimensional capillary structures with a lumen. The ability of PelA and Dtx to inhibit this particular step in the neo-vascularization process was determined using a Matrigel $^{\mathrm{TM}}$ tube formation assay. HUVECs were plated onto Matrigel ${ }^{\mathrm{TM}}$-coated chamber slides, and incubated with or without drug for $16 \mathrm{~h}$. Images of the fluorescently stained capillary-like structures were taken using a confocal microscope (Fig. 5.3). The effects of both PelA and Dtx on capillary tubule morphology and organization can be seen in Figure 5.3 A and 5.3 B, respectively. In the presence of MSAs, the ability to form complete networks of branched tubules was significantly inhibited. PelA-treated HUVECs failed to form complete networks, and the branches that did form were much shorter than those in control cells. To quantify these effects, the number of tubules (with branching at both ends) was manually counted (Fig. $5.3 \mathrm{C}$ ). PelA reduced the total number of tubules in a concentration-dependent manner, with a $60 \%$ reduction at $10 \mathrm{nM}$ PelA, and a $5 \%$ reduction at $1 \mathrm{nM}$ PelA. The effects of Dtx were more potent, with a $63 \%$ and $81 \%$ reduction in tube number at $5 \mathrm{nM}$ and $10 \mathrm{nM}$ Dtx, respectively. Initially, a more automated method for quantifying drug effects on capillary tube formation was also carried out. Using a computer macro that is compatible with Image-Pro Plus software (created by Pan Xiaotao, Media Cybernetics), both total tube length and total tube area (Fig. 5.4 A and B, respectively) were determined. There was a concentrationdependent effect on total tube length, but not on total tube area in HUVECs treated with PelA; however, the results from the macro did not correlate as well with the confocal images as they did with the manual measurements. This is possibly due to the fact that the macro cannot distinguish between properly formed capillary tubes (those with branching points) and malformed tubes or clumped cells. Still, it is evident that PelA and Dtx both potently inhibit EC processes that are crucial for angiogenesis. At the highest drug concentrations tested $(10 \mathrm{nM})$, HUVECs that were incubated for the same length of time as the Matrigel ${ }^{\mathrm{TM}}$ assay showed minimal cytotoxicity in an MTT assay (Fig. 5.5), indicating that the effects on tube formation were more likely due to effects on angiogenic pathways than on cytotoxic activity. 
A

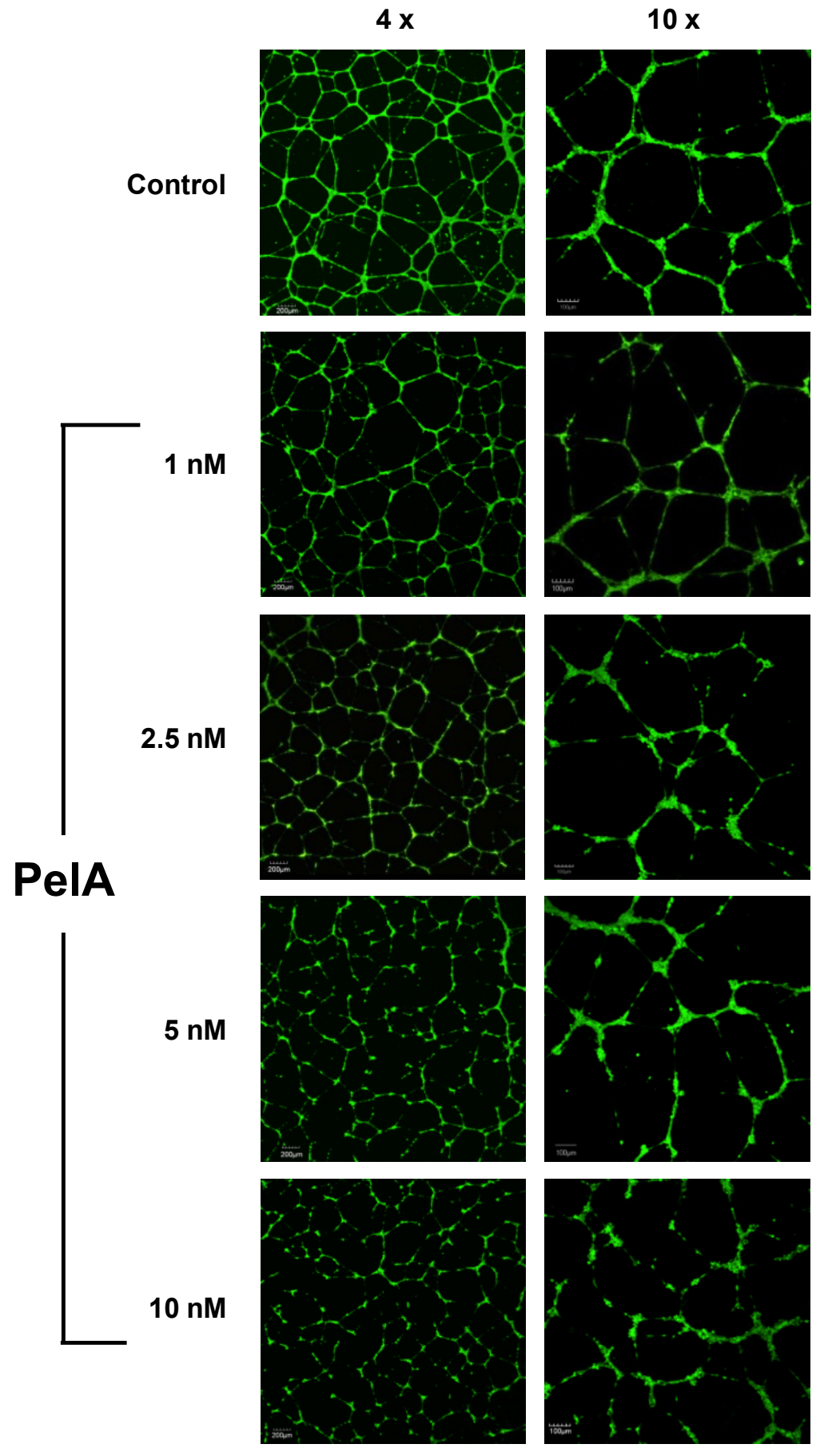

Figure 5.3. Inhibition of capillary tube formation by PelA and Dtx. A tube formation assay was used to assess the ability of PelA and Dtx to inhibit the ability of HUVECs to form tube-like structures on Matrigel $^{\mathrm{TM}}$. HUVECs were plated onto Matrigel ${ }^{\mathrm{TM}}$ in the presence of vehicle (Control), PelA or Dtx and incubated at $37^{\circ} \mathrm{C}$ for $16 \mathrm{~h}$. Cells were stained with calcein AM and images taken using both the $4 \mathrm{X}$ and 10X objectives on a laser scanning confocal microscope. The effects of PelA and Dtx on the formation of capillary-like structures are shown in $\mathbf{A}$ and $\mathbf{B}$, respectively. The total number of tubes with branch points at both ends was manually counted and is presented relative to controls (values are the mean \pm SEM; $\mathrm{n}=3$ ) (C). ${ }^{*} \mathrm{p}<0.01,{ }^{*} \mathrm{p}<0.001,1$-way ANOVA with Bonferroni post-hoc test. 
B

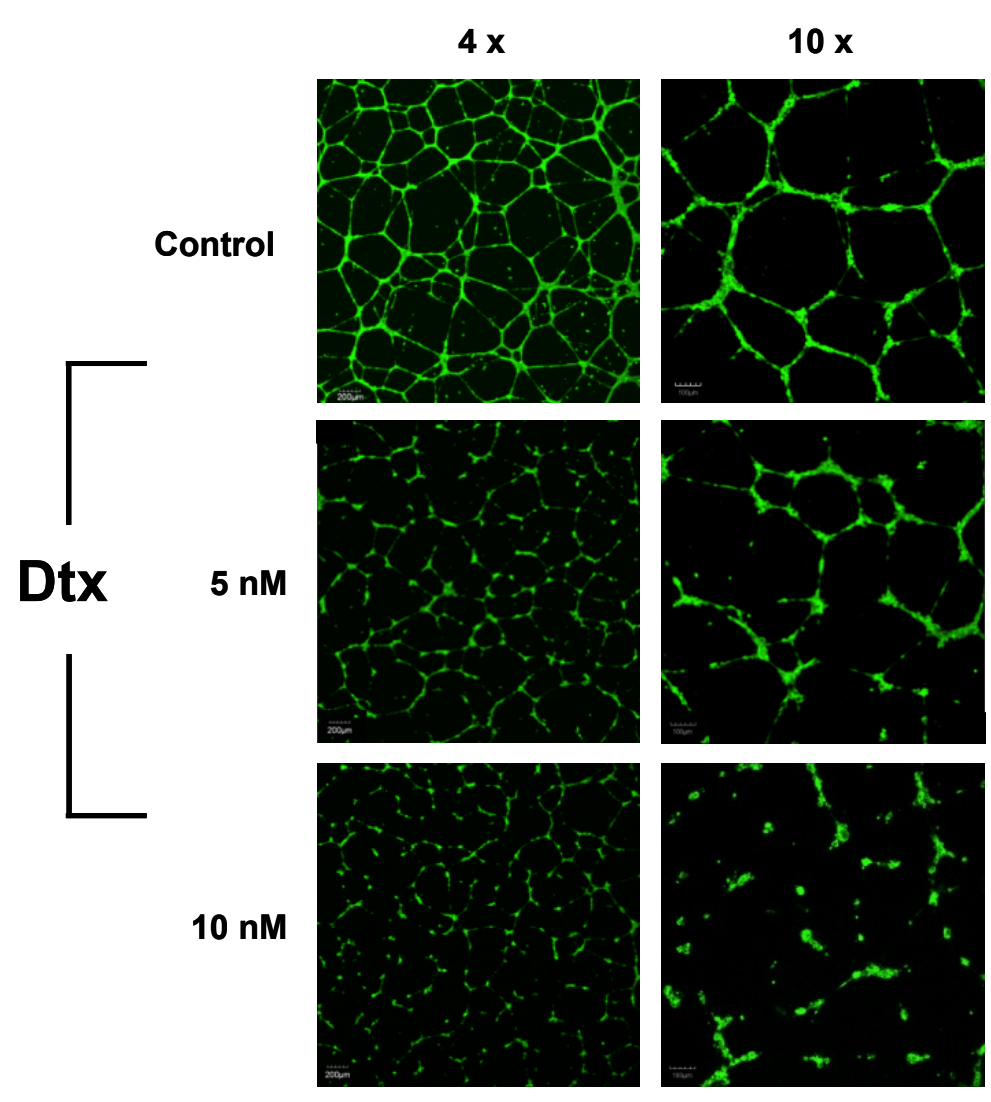

C

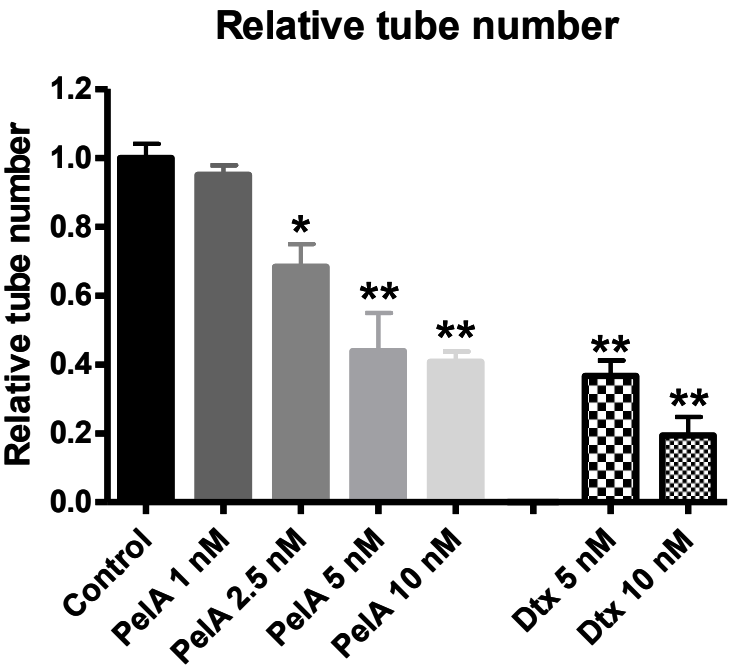

Figure 5.3 (continued) 
A

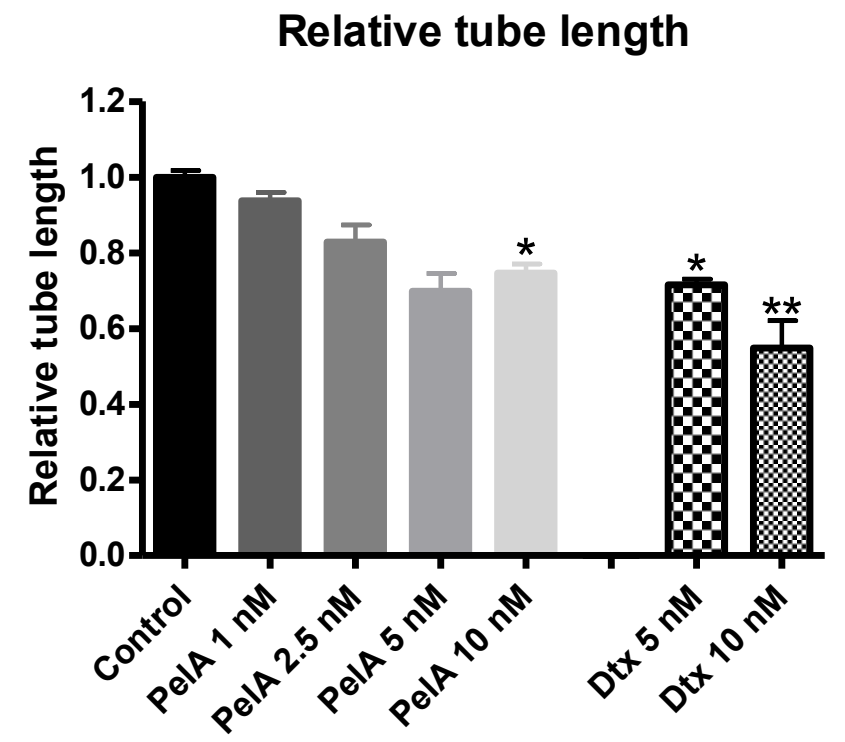

B

\section{Relative tube area}

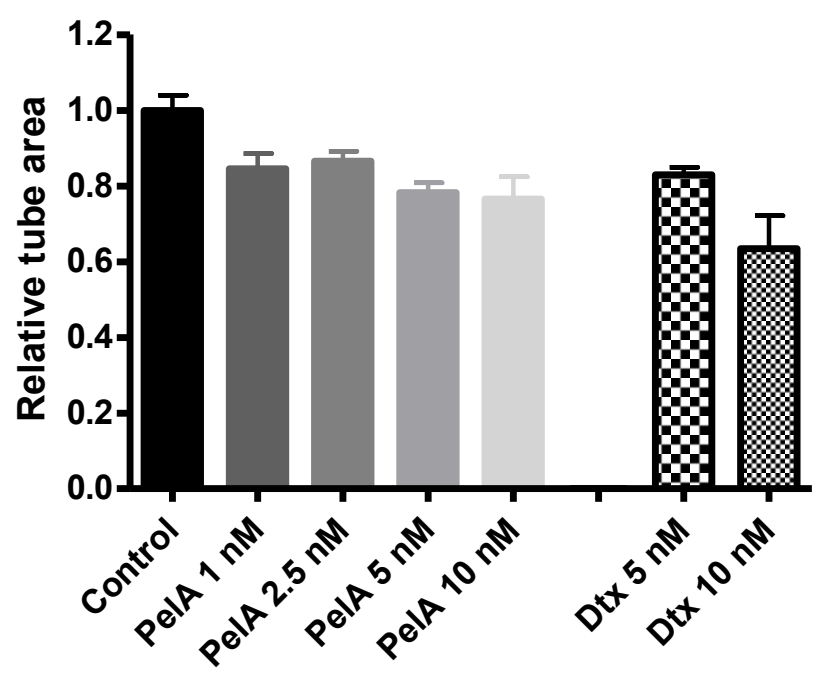

Figure 5.4. Effects of PelA and Dtx on total tube length and total tube area. HUVECs were plated onto Matrigel $^{\mathrm{TM}}$ in the presence of vehicle, PelA or Dtx and incubated at $37{ }^{\circ} \mathrm{C}$ for $16 \mathrm{~h}$. Cells were stained with calcein AM and $2 \times 2$ images, taken using the $4 \mathrm{X}$ objective, were stitched together using Fluoview software (Olympus). An Image-Pro Plus macro was used to determine the total tube length (A) and total tube area (B). Data are the mean \pm SEM, normalized to controls $(n=3) . * p<0.05$, **p $<0.01,1$-way ANOVA with Bonferroni post-hoc test. 

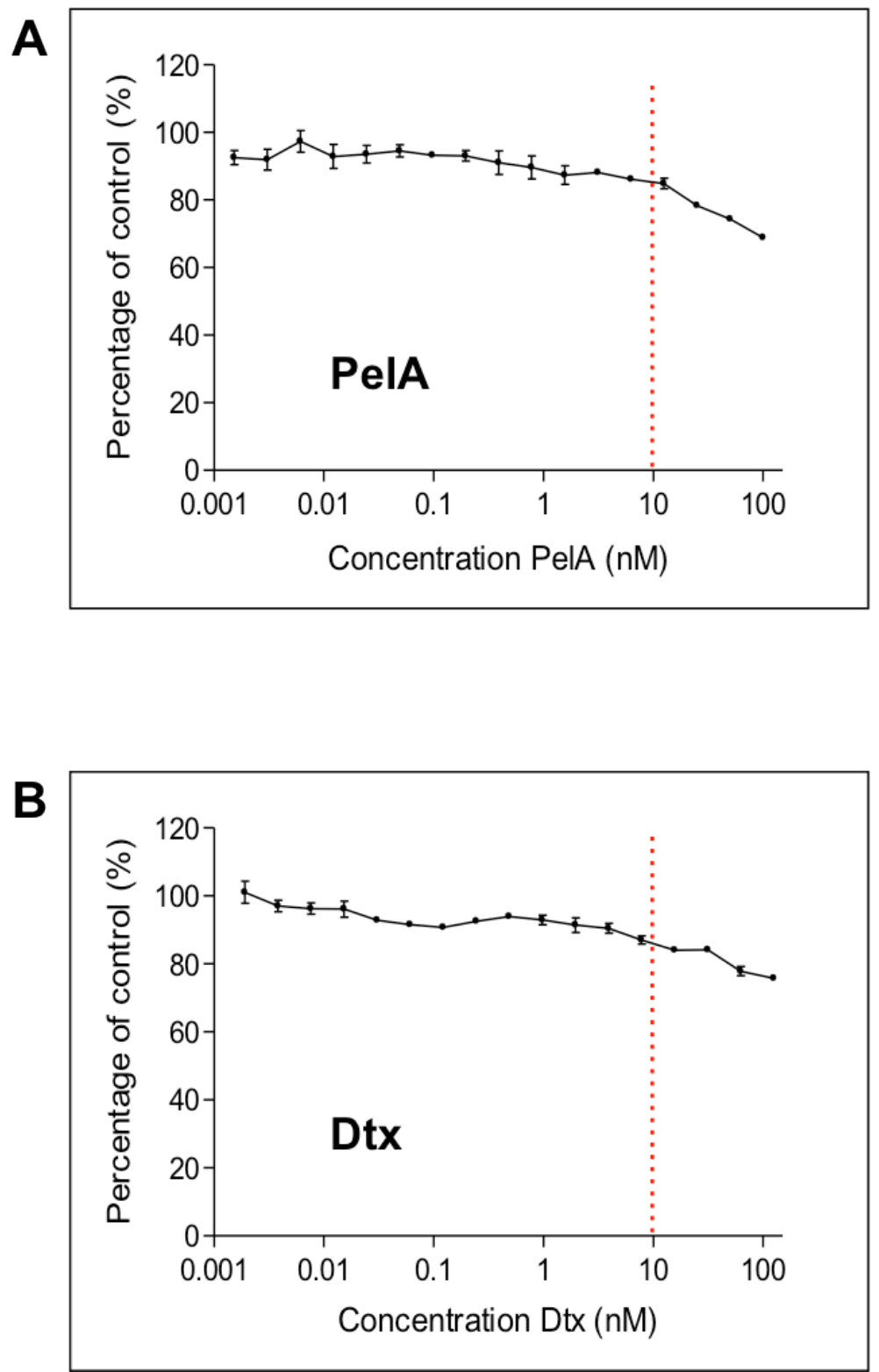

Figure 5.5. Inhibition of HUVEC proliferation over $16 \mathbf{h}$. HUVECs were treated for $16 \mathrm{~h}$ (the same incubation period as the Matrigel ${ }^{\mathrm{TM}}$ tube formation assay) with either PelA (A) or Dtx (B). Effects of these drugs on cell proliferation were assessed using a colourimetric MTT assay. Both drugs inhibited HUVEC proliferation by approximately $15 \%$ compared to controls (values are the mean of triplicate wells $\pm \mathrm{SEM}, \mathrm{n}=$ a single experiment). 


\subsection{Discussion}

\subsubsection{PelA inhibits HUVEC proliferation}

During angiogenesis, one of the key steps after degradation of the basement membrane is EC proliferation. The MTT results indicated that both PelA and Dtx are able to inhibit HUVEC proliferation at relatively low concentrations. Due to the slower growing nature of this EC line, a longer incubation period was required (120 h) compared to the incubation periods for previous work on cancer cell lines (typically 48-72 h). There was very little difference in $\mathrm{IC}_{50}$ values between the two MSAs (1.4 and $1.7 \mathrm{nM}$ for PelA and Dtx, respectively). These values are comparable to previous studies where HUVEC proliferation was inhibited by MSAs in the low nanomolar range. Dtx and laulimalide, which binds to the same site as PelA on tubulin, had $\mathrm{IC}_{50}$ values of 1 and $4 \mathrm{nM}$, respectively (6 day incubation) (Lu et al., 2006). In another study also using HUVECs, the MTAs, EpoB, Dtx and Vbl, exhibited $\mathrm{IC}_{50}$ values ranging from $1.9-71.5 \mathrm{nM}(1 \mathrm{~h}$ drug incubation followed by $96 \mathrm{~h}$ release) (Bijman et al., 2006). These MTAs were much more potent than two classic chemotherapeutic drugs, cisplatin and doxorubicin, which had $\mathrm{IC}_{50}$ values of 58.5 and $1.5 \mu \mathrm{M}$, respectively. These MTT results indicate that PelA may be more effective at inhibiting EC proliferation than cancer cell proliferation $\left(\mathrm{IC}_{50}\right.$ in cancer cell lines typically ranges from $\sim 12-25 \mathrm{nM}$ ), although we cannot directly compare the MTT results, given the differences in drug incubation times.

\subsubsection{PelA inhibits EC migration}

During the vascularization process of tumours, ECs migrate toward pro-angiogenic signalling factors (Eichhorn et al., 2007). Compared to the wound scratch healing assay, transwell migration assays are better at encapsulating this event as they monitor the migration of ECs towards a stimulating chemokine, such as VEGF. Unfortunately, our attempts at making our own transwell inserts and even using manufactured inserts, were unsuccessful (see Appendix D, Figure D.3). We observed significant migration of HUVECs through the membrane pores, even in the absence of VEGF. Titration of the VEGF effects were also inconsistent, and it would have been difficult to confirm any drug-induced effects using this assay. Hence, we decided to use the wound scratch assay, although it should be noted that in this particular assay, the EC migratory response is not dependent on chemotaxis. 
At each of the drug concentrations of PelA that were tested $(2.5-15 \mathrm{nM})$, we observed a significant impairment of HUVEC migration. At $2.5 \mathrm{nM}$, HUVEC migration was inhibited by $36 \%$, indicating that migration could be disrupted in the low nanomolar range of PelA. Despite similar anti-proliferative effects in the MTT assay, Dtx, which was used as a positive control in this assay, was much more effective at inhibiting migration (wound recovery was inhibited by $76 \%$ at $2.5 \mathrm{nM}$ Dtx). Dtx has previously been shown to be more potent at inhibiting migration compared to other MTAs (i.e. EpoB and Vbl (Bijman et al., 2006). It is highly likely that the difference in binding sites between these MTAs influences the signalling pathways involved in cell migration to different extents. Dtx and laulimalide, for example, differentially affected targets downstream of VEGF-receptor (VEGFR) activation (Lu et al., 2006). Dtx was better at inhibiting the association of VEGFR-2 with $\alpha_{5} \beta_{1}$ integrin in response to VEGF; whereas, laulimalide was more potent in its impairment of RhoA and $\alpha_{5} \beta_{1}$ integrin association. It would be interesting to determine whether these differences were drugspecific or binding site-specific (i.e. whether PelA and laulimalide have similar effects on the VEGFR-2 signalling cascade). The family of GTPases have also proven to be key targets of MTA-inhibited EC migration. Rac1 and Cdc42 activities were both reduced in HUVECs treated with Dtx, EpoB and Vbl, but not with non-MTA drugs (Bijman et al., 2006). Given the important role that Rho GTPases play in cytoskeletal reorganization during cell migration, it would not be surprising if these were also downstream targets of PelA. Interestingly, no Rho GTP family members were identified as hits in a proteomics screen comparing differences in response to PelA and Ptx; however, this particular study was carried out in HL-60 cells as opposed to ECs (Wilmes et al., 2011b; Wilmes et al., 2012a). Furthermore, the proteomics screens would not pick up altered interactions of Rho GTPases with other proteins or changes in protein activity, as it only detects increases or decreases in protein abundance levels.

It is still unclear as to how exactly binding of MTAs to microtubules affects the signalling pathways involved in cell migration. A mechanism that has been identified for Ptx and vinflunine that may be common to all MTAs is their ability to increase the dynamic instability of microtubules in ECs specifically (microtubule dynamics are inhibited in cancer cell lines) (Pasquier et al., 2005; Pourroy et al., 2006). This dynamic instability is an important regulatory mechanism for the Rho family of GTPases. For example, microtubule depolymerization in response to colchicine has been reported to activate Rho (Ren et al., 2009); whereas, microtubule polymerization has been shown to 
trigger Rac1 activation (Waterman-Storer et al., 1999; Bijman et al., 2006). This has not always been the case, however, as Rac1 activity was decreased upon Ptx treatment in one particular study (Bijman et al., 2006). These discrepancies could be due to differences in cell lines (the Bijman et al. study were carried out in ECs rather than fibroblasts), or perhaps that impaired microtubule dynamics in ECs, may be enough to perturb the regulation of Rho family GTPases. Bijman et al. proposed that decreased Rac1 activation could be due to inhibited microtubule dynamics; however, they did not directly measure dynamic instability and cannot verify whether microtubule dynamics were increased or decreased in their study.

\subsubsection{PelA inhibits capillary-like tube formation}

During the angiogenesis process, ECs undergo morphological differentiation into 3dimesional (3D) capillary structures (Schwartz, 2009). In vitro, this process is often mimicked by plating HUVECs onto the basement membrane matrix, Matrigel ${ }^{\mathrm{TM}}$. To further evaluate the potential for PelA to inhibit angiogenic processes, we plated HUVECs onto Matrigel ${ }^{\mathrm{TM}}$ in the presence of varying concentrations of drug and assessed the effects on tubule formation. It was evident from the confocal images of the capillary-like structures that both PelA and Dtx had significant effects on the quality of the tubes that were formed. Although Dtx was more effective at equimolar concentrations, both drugs inhibited the total number of tubules that were formed. The low concentrations that were used in this study are not likely to cause cytotoxicity, although attempts to verify this using an annexin $\mathrm{V}$ apoptosis assay were unsuccessful, because it was difficult to detach the HUVECs once they had differentiated on the Matrigel $^{\mathrm{TM}}$. In addition, the dispase enzyme that was needed to dissolve the matrix may have caused non-specific flipping of PS to the outside of the membrane, generating false positives in the assay. At the highest drug concentrations used (10 nM), there was less than $15 \%$ inhibition of cell proliferation using the MTT assay, indicating that this is unlikely to be the predominant factor in the inhibition of capillary tube formation.

\subsubsection{Possible mechanisms linking microtubule-binding to anti-angiogenic effects}

It is difficult to pinpoint exactly which aspect of EC function is being affected by MSAs like PelA, as tubulin-binding is likely to affect multiple points in the signalling cascades. Some of the downstream effects of MSAs that are known to contribute to the inhibition of angiogenesis are illustrated in Figure 5.6. These include, impaired repositioning of the MTOC in migrating cells, decreased signalling from focal 
adhesions and VEGFR-2 (via proteasomal degradation of heat shock protein 90 (Hsp90)), impaired integrin formation and activation, decreased cell polarization, altered Rho-GTPase signalling and impaired EC dynamic instability (Waterman-Storer et al., 1999; Hotchkiss et al., 2002; Pasquier et al., 2005; Bijman et al., 2006; Lu et al., 2006; Murtagh et al., 2006; Pourroy et al., 2006; Schwartz, 2009). The specific effects of PelA on the VEGF signalling cascade have yet to be determined, but it is likely that PelA will elicit many of the same effects as other MSAs that have already been investigated for their anti-angiogenic potential. It is interesting to note that despite both Ptx and laulimalide causing a substantial decrease in the expression of Hsp90, this protein was not picked up in earlier proteomics screens using Ptx-treated (or PelAtreated) HL-60 cells (Murtagh et al., 2006; Wilmes et al., 2011b; Wilmes et al., 2012a). Although Murtagh et al. suggested possible mechanisms by which Dtx binding could lead to Hsp90 degradation in HUVECs (i.e. displacement of Hsp90 from microtubules and direct binding of Dtx to Hsp90), it has not yet been reported that Hsp90 is degraded in response to MTAs in cancer cell lines. Exploiting these cell line-dependent differences in the downstream targets of tubulin-binding may lead to improved specificity of anti-angiogenic compounds.

It is now becoming apparent that the anti-angiogenic effects of many MTAs (e.g. Ptx, Dtx, and EpoB) typically occur at concentrations that are non-cytotoxic (Hayot et al., 2002; Bijman et al., 2006). There have been some exceptions to this, with two MDAs, $\mathrm{Vbl}$ and vinorelbine, exerting their anti-angiogenic effects through cytotoxic effects (in the Bijman et al. study, however, Vbl was found to inhibit angiogenesis at subtoxic doses) (Hayot et al., 2002). PelA's inhibitory effects on cell migration and capillary network formation are also largely due to non-cytotoxic mechanisms; thus, PelA has the potential ability to inhibit tumour vasculogenesis in vivo at relatively low concentrations. There has been some controversy around the most effective treatment regimen for reducing tumour growth. Conventionally, the maximum tolerated dose (MTD) is given as a bolus dose that requires resting periods between each treatment cycle. This can lead to tumour re-growth (relapse) and the expansion of resistant tumour cells, as well as exacerbate any side effects of the drug (Scharovsky et al., 2009). Lowdose metronomic therapy (lower doses that are frequently spaced and given continuously with no resting periods) is now being explored in the clinic as an antiangiogenic strategy. What is becoming increasingly evident is that metronomic scheduling of anti-angiogenic agents and conventional chemotherapeutics used in 
combination may prove to be more effective at inhibiting tumour growth. Promising results have been observed in a phase I clinical trial using metronomic dosing of Dtx in combination with zoledronic acid (a biphosphonate used to treat bone conditions like osteoporosis and bone metastases) against hormone resistant prostate cancer. A preclinical study using a mouse model of ovarian cancer, demonstrated greater tumour reduction using low-dose metronomic scheduling of Dtx and AEE788 (a dual epidermal growth factor receptor and VEGFR inhibitor), compared to either of the drugs alone, or when Dtx at its MTD was used with AEE788. Should PelA progress into clinical trials, metronomic dosing of the drug is an option that should be evaluated.

\subsubsection{Conclusion}

The current study shows that PelA exhibits anti-angiogenic features that would be beneficial for treatment of solid tumours to prevent vascularization. PelA is able to impair several EC functions that are crucial to angiogenesis - cell proliferation, migration and EC ability to form 3D tubules. Previous work using PelA has highlighted the significant inhibitory effects on the growth of tumour cells, both in vitro and in vivo; however, we have now shown that PelA also has the potential to simultaneously inhibit the tumour vascularization process. It is significant that the observed effects on EC functions occur at lower concentrations than those needed to affect tumour cells. For example, inhibitory effects on EC functions in the present chapter are evident at $<10$ $\mathrm{nM}$, while concentrations $>40 \mathrm{nM}$ are needed to cause a $\mathrm{G}_{2} \mathrm{M}$ arrest in 1 A9 cells (see Chapter 4). This would suggest that at concentrations that are therapeutically relevant for inhibiting tumour growth, drugs like PelA are also likely to impair angiogenic processes. The next step would be to look at the effects of PelA on the VEGF signalling cascade in order to further elucidate its mechanism of action in ECs. Ideally, if there was a large enough supply of the compound, PelA could also be tested for antiangiogenic effects in more clinically relevant, in vivo mouse models. 


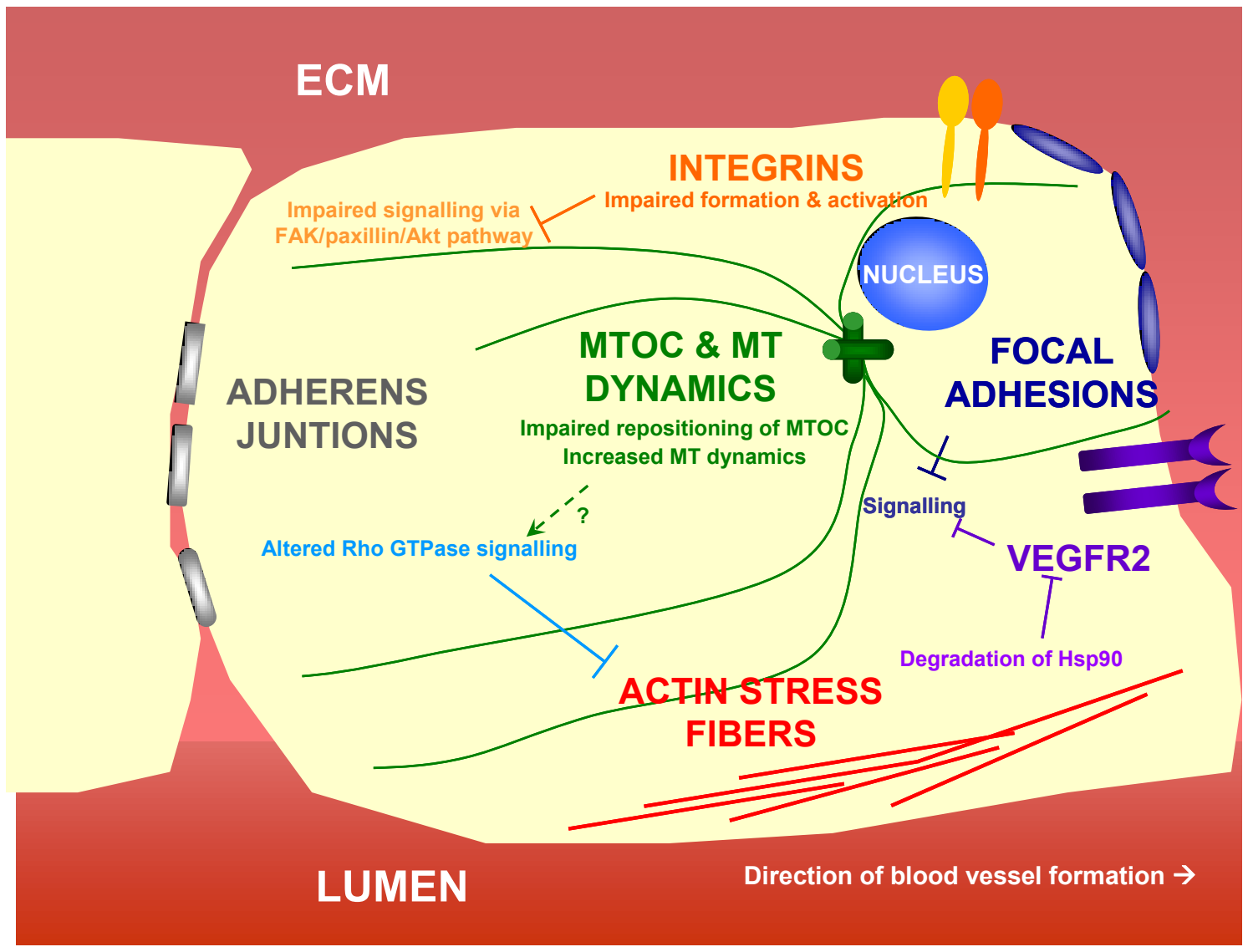

Figure 5.6. MSA effects on endothelial cell functions. MSAs have numerous downstream effects on endothelial cells that may contribute to their anti-angiogenic effects. Microtubule-stabilization or impaired dynamic instability can affect the re-organization of the MTOC, impair the formation of adherens junctions, integrins, focal adhesions, and actin stress fibers, and disrupt signalling pathways (e.g. VEGFR2, integrin and Rho GTPase signalling). Modified from Schwartz (2009). 


\section{Chapter 6. Induction of premature senescence by peloruside A}

\subsection{Introduction}

\subsubsection{Replicative and premature senescence}

Cellular senescence is defined as a state of permanent cell cycle arrest and, in addition to apoptosis, functions as an effective tumour suppressor mechanism. As mentioned in the General Introduction, two different types of cellular senescence have so far been characterized: (1) replicative senescence, which occurs as part of normal cellular ageing in response to telomere shortening, and (2) premature or accelerated senescence, which occurs irrespective of telomere length and can be triggered by several mechanisms, including oncogenic stress, DNA damage, hypoxia, pharmacological agents, and radiation (Schmitt, 2007; Larsson, 2011). Oncogene-induced senescence (OIS) can arise from the inappropriate activation of mitogenic oncogenes such as Ras and Myc, or conversely, from the inactivation of tumour suppressor genes that restrain oncogenic activity (Larsson, 2011). Deregulated oncogene expression is potentially tumourigenic; thus, senescence is one way of counteracting the excessive proliferative signals that can drive tumour progression (Rodier and Campisi, 2011).

\subsubsection{Characteristics of senescent cells}

Cellular senescence is described as an irreversible cell cycle arrest, in contrast to a transient form of cell cycle arrest termed quiescence. While senescent cells no longer actively synthesize DNA, they still remain metabolically active. Quiescent cells also undergo a prolonged cell cycle arrest but in contrast to senescent cells they can resume proliferation with the appropriate mitogenic stimuli (Campisi and d'Adda di Fagagna, 2007). As outlined in the General Introduction and described below, senescent cells are characterized by several morphological and biochemical features, which are also depicted in Figure 6.1, A (Schmitt, 2007). Senescent cells tend to exhibit an enlarged, flattened, granular appearance and have a vacuole-rich cytoplasm. These arrested cells can usually be distinguished from quiescent and terminally differentiated cells (which can also adopt a similar large, flattened morphology) by their characteristic staining for senescence-associated $\beta$-galactosidase (SA- $\beta$-gal) activity (generally in the perinuclear region). In most mammalian cells lysosomal galactosidase activity can be detected by incubating with the chromogenic substrate X-gal (5-bromo-4-chloro-3-indolyl $\beta$-Dgalactoside), an assay usually carried out at the optimal pH 4.0 (Kurz et al., 2000). In senescent cells, an increase in cellular lysosomal content (the physiological relevance of 
this lysosomal expansion in senescent cells is unknown) rather than up-regulated enzyme activity, allows detection of galactosidase activity at the suboptimal $\mathrm{pH} 6.0$ (Dimri et al., 1995; Kurz et al., 2000; Schmitt, 2007). Unfortunately, these morphological characteristics and positive staining for SA- $\beta$-gal are not limited to senescence and cannot be used as exclusive markers. SA- $\beta$-gal activity, for example, can also be triggered by the cellular stress induced by confluent culture conditions and highlights the need to assess additional features of cellular senescence (Severino et al., 2000; Campisi and d'Adda di Fagagna, 2007).

Senescent cells can also be probed for characteristics associated with a state of arrested growth, including a lack of DNA synthesis (typically detected using a BrdU incorporation assay) and activation of DDR and tumour suppressor pathways (Figure 6.1 B; these pathways are described in more detail below) (Schmitt, 2007). Activation of p53 and pRb signalling pathways play a crucial role in promoting senescence. As explained later in this chapter, the diverse stress signals that lead to the induction of senescence often culminates in the activation of the DDR. Phosphorylated histone $\operatorname{H} 2 \mathrm{AX}(\gamma-\mathrm{H} 2 \mathrm{AX})$ is a marker for DNA double-strand breaks (DSBs) that can be detected in senescent cells (d'Adda di Fagagna et al., 2003). Another feature often exhibited in response to OIS is the re-organization of chromatin into distinct, punctate foci that express particular heterochromatin-associated histone modifications (termed senescence-associated heterochromatin foci (SAHFs)) (Narita et al., 2003; Campisi and d'Adda di Fagagna, 2007). Due to the lack of a single, definitive marker for cellular senescence, positive determination of a senescent phenotype requires the assessment of many of these features concurrently. 


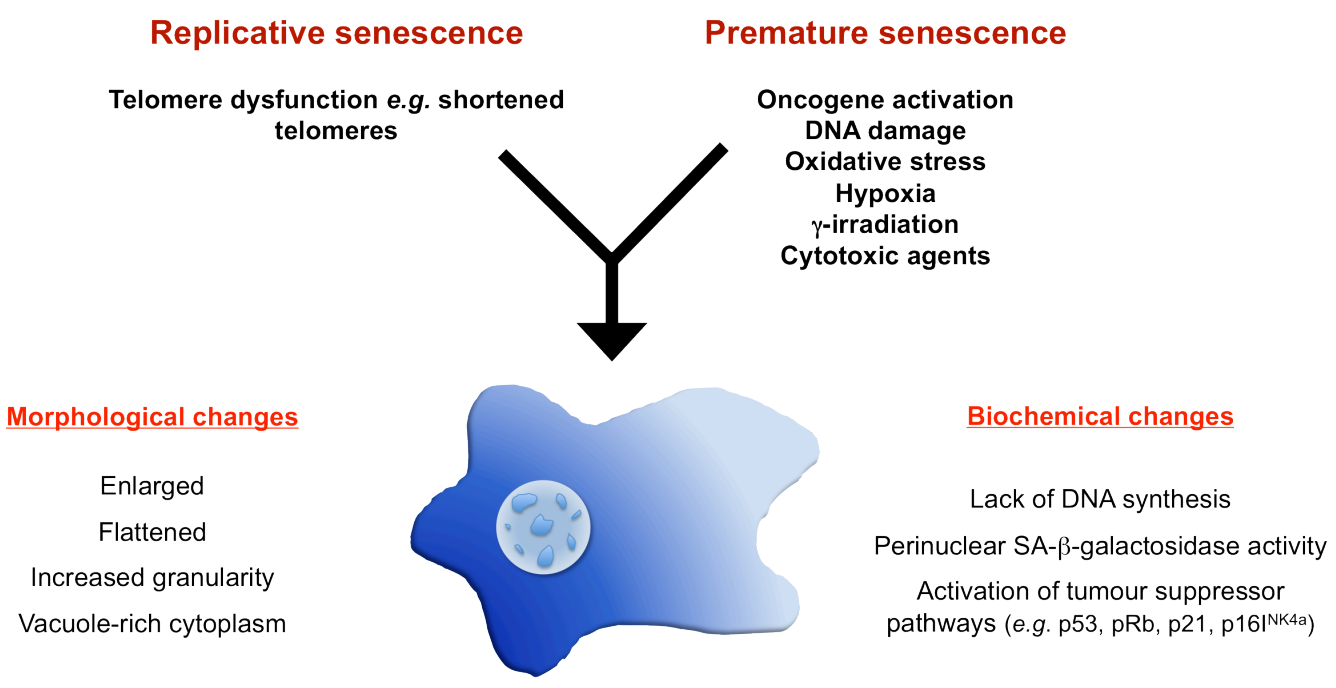

DNA and chromatin changes

DNA damage (detected by expression
of $\gamma \mathrm{H} 2 \mathrm{AX}$ )

Formation of senescence-associated heterochromatin foci (SAHFs)

B

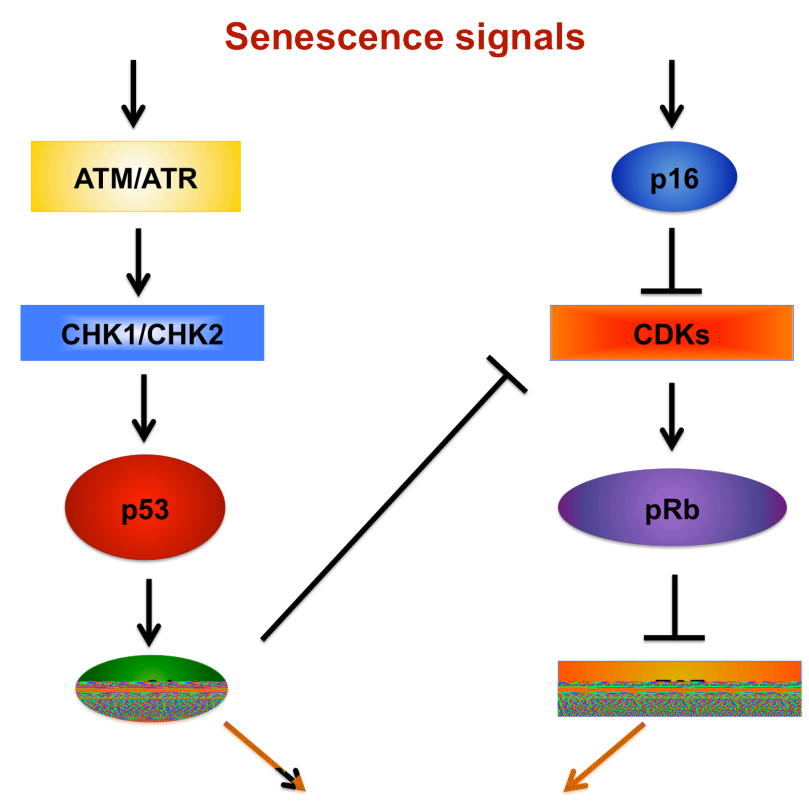

SFNECRENRE

Figure 6.1. Features of cellular senescence and typical signalling pathways. (A) The general triggers of both replicative and premature senescence are shown, as well as the morphological, biochemical, and DNA/chromatin changes that are associated with a senescent phenotype. (B) Activation of the $\mathrm{p} 53$ and $\mathrm{pRb}$ tumour suppressor pathways function to establish and maintain cell cycle arrest. The ATM-CHK2 and ATR-CHK1 signalling cascades are triggered in response to DNAdamage and play prominent roles in the cellular response to senescence triggers. Acting downstream of $\mathrm{p} 53$, the cyclin-dependent kinase (CDK) inhibitor p21 inhibits the phosphorylation of $\mathrm{pRb}$, thus, keeping it in its active state. Active $\mathrm{pRb}$ sequesters E2F to prevent transcription of genes involved in cell cycle progression. The p16 CDK inhibitor also regulates pRB activity, and can function independently of p53-p21 signalling. Modified from Campisi and d'Adda di Fagagna (2007) and Schmitt (2007). 


\subsubsection{Mechanisms underlying cellular senescence induction}

The induction and maintenance of cellular senescence involves a highly complex signalling network that can be initiated by multiple stress signals. However, irrespective of the initial trigger, the senescence response consistently involves activation of either the p53-p21 or p16-pRb tumour suppressor pathways (in some cases both pathways are activated; see Figure 6.1) (Campisi and d'Adda di Fagagna, 2007). This is not surprising, given that many of the known senescence triggers can directly lead to DNA damage. The DDR is typically sensed by the ATM-CHK2 and ATR-CHK1 pathways, acting upstream of p53 to promote cell cycle arrest (described in the General Introduction). At least two models have been proposed to account for the DNA damage observed in response to deregulated oncogene activation (Courtois-Cox et al., 2008). Double-strand breaks, stalled replication fork progression, and an increased number of DNA replication origins are signs of DNA replication stress that have been correlated with DDR and OIS induction (Bartkova et al., 2006; Di Micco et al., 2006). Reactive oxygen species (ROS)-induced DNA damage can also lead to cell growth arrest. Activation of Ras, for example, and the subsequent increase in intracellular ROS levels of, is thought to mediate the generation of the senescence phenotype (Lee et al., 1999). Very recently, Weyemi et al., assessed the changes in mRNA levels of several NADPH oxidases from the NOX/DUOX family in response to increased ROS levels following the induction of H-RasV12 in a human non-tumoral thyroid cell line (Hthy-ori) (Weyemi et al., 2012). NOX4 mRNA was specifically up-regulated under these conditions, and siRNA inhibition decreased ROS production, implicating this enzyme in regulating ROS levels. Additionally, H-RasV12 expression led to histone $\gamma-\mathrm{H} 2 \mathrm{AX}$ phosphorylation (a marker for DSBs) and a positive senescence response (determined by a senescence-associated heterochromatin foci (SAHF) assay), which were accompanied by activation of the CDKI, p21.

In human fibroblast cells, chronic exposure to chemotherapeutic drugs that induce double-strand DNA breaks, (e.g. Doxo, etoposide, cisplatin, and actinomycin D) can induce permanent cell cycle arrest and increased expression of SA- $\beta$-gal (Robles et al., 1999). This type of drug-induced, telomere-independent cellular senescence has been termed premature or accelerated senescence, and seems to involve the activation of p53 and its downstream effector, p21 (Chang et al., 1999a; Chang et al., 1999b; Gewirtz et al., 2008). Homozygous knockout of p53 and p21 in HCT116 colon carcinoma cells decreased, but did not completely abolish, induction of a senescent phenotype, 
indicating that additional signalling mediators are involved (Chang et al., 1999b). It is a particularly difficult task to decipher the relative contributions of all the factors that lead to an induction of senescence (compared to other cell fates such as apoptosis and quiescence). Cellular age, cell type and genetic differences (e.g. expression levels of p53, Ras, etc.) can all affect how cells respond to different stressors (Campisi and d'Adda di Fagagna, 2007). Additionally, the degree of p53 activation (high p53 levels inhibit mTOR (mammalian target of rapamycin) and push cells towards a quiescent phenotype) can influence the induction of senescence and may help to explain why senescence is only induced by low concentrations of DNA-damaging agents (Leontieva et al., 2010). Attempts to determine the signal transduction pathway driving DDRinduced senescence suggest that a positive feedback loop, involving p21 signalling (i.e. p53-p21-GADD45-p38-GRB2-TGFBR2-TGF $\beta$ ) and the subsequent mitochondrial dysfunction and ROS production is important in maintaining the senescent phenotype after the initial growth arrest (Passos et al., 2010).

DNA-damaging drugs appear to be one of the few classes of chemotherapeutic drugs that can induce premature senescence. Few studies have looked at MTA-induced premature senescence, but given their prominence in the oncology clinic, further investigation is warranted. Following a four-day exposure (using $\mathrm{IC}_{85}$ concentrations), 23-79\% of human fibrosarcoma cells (HT-1080 3'SS6) exhibited positive staining for SA- $\beta$-gal activity in response to several DNA-damaging agents (i.e. cisplatin, Doxo, aphidicolin, and cytarabine); whereas, the MTAs, Ptx and vincristine, only triggered senescence in less than $10 \%$ of cells (Chang et al., 1999a). MTAs that trigger premature senescence to levels that are comparable to the DNA-damaging agents (i.e. Doxo and adriamycin) include the MSA Disco and the MDAs, disorazole $\mathrm{C}_{1}$ and JG-03-14 (Klein et al., 2005; Arthur et al., 2007; Tierno et al., 2009). Premature senescence was observed in tumour specimens from two of three patients receiving carboplatin and Ptx neoadjuvant therapy (compared to none in the patients receiving no chemotherapy) (Roberson et al., 2005). It is unclear whether the DNA-alkylating agent carboplatin predominantly drives the induction of senescence in these patients or whether Ptx also contributes to the effect. Given that senescence may be as effective a tumour suppressive mechanism as other cell fates, additional in vivo studies looking specifically at MTA-induced premature senescence are needed. 


\subsubsection{Senescence as a tumour suppressive mechanism}

Research in the last decade has confirmed the link between senescence and tumourigenesis, in vivo. Tissue sections of premalignant lung adenomas, taken from mice with inducible K-Ras, are positive for several markers of cellular senescence that are predominantly absent in malignant adenocarcinomas (Collado et al., 2005). This suggests that OIS acts as an effective barrier to tumour progression, and that progression to a more malignant stage involves overcoming or bypassing senescence mechanisms. Similarly, a more recent study using a transgenic mouse model with inducible Ras found that high levels of Ras could induce an irreversible cellular senescence in mammary epithelial cells (Sarkisian et al., 2007). Sustained low levels of Ras stimulated cell proliferation but, in order to trigger mammary tumourigenesis, cells required the spontaneous up-regulation of activated Ras and evasion of senescence checkpoints. Whether these studies using mouse models reflect a physiologically relevant anti-tumour response in humans is still under investigation. Activation of DDR pathways in premalignant human colon adenomas, and the subsequent decrease in proliferative capacity, suggests that DDR is an important anti-cancer mechanism (Bartkova et al., 2005). In a later paper by the same authors using a tumour mouse model, short-hairpin (sh)RNA inhibition of ATM, a component of the DDR, was shown to reduce senescence and thereby promote tumour growth (Bartkova et al., 2006). In another study using human tissue, BRAF, an oncogene signalling mediator commonly mutated in human naevi (moles or benign tumours of melanocytes) was shown to be involved in cell cycle arrest and the induction of a senescent phenotype (Michaloglou et al., 2005). Thus, cellular senescence is a likely mechanism preventing the progression of human premalignant tumours into more aggressive cancers. Currently under investigation is the possibility that senescent markers could be exploited as prognostic tools to help predict the malignant potential of certain cancers (Larsson, 2011).

\subsubsection{Fate of senescent cells}

The final fate of senescent cells is still somewhat controversial and this poses intriguing questions about their effectiveness in the clinic. Several studies have shown that senescent cells can in fact resume proliferating but only after experimental manipulation of DDR checkpoint pathways (e.g. ATM/ATR and Cdc2/Cdk1) (d'Adda di Fagagna et al., 2003; Roberson et al., 2005). Wang et al. suggested that glioblastoma cells with functional p53 can re-enter the cell cycle after replating in drug-free medium; however, 
they failed to demonstrate definitively that the resulting regrowth was not due to a subset of non-senescent cells in their cultures (Wang et al., 2004a). Thus, DDR seems to be important in maintaining the senescent phenotype, but whether this is relevant in a clinical setting remains to be seen.

If senescent cells do persist in an in vivo setting, what happens to them? Once cultured cells have entered a senescent state, different cell lines exhibit variable susceptibilities to apoptotic stimuli (Campisi and d'Adda di Fagagna, 2007). Exploiting mechanisms that can push senescent cells toward apoptosis is obviously of interest in terms of anticancer therapy, as the evidence suggests that senescent tumour cells may not completely lose their proliferative capacity. Additionally, the immune system may be able to promote tumour regression via the direct clearance of senescent cells (Campisi and d'Adda di Fagagna, 2007). Senescent cells can up-regulate inflammatory cytokines or alter the gene expression profiles of immuno-modulatory factors to recruit phagocytic members of the innate immune system (i.e. macrophages, neutrophils and natural killer cells) and promote the clearance of senescent cells (Xue et al., 2007). The elucidation of factors that can promote the detection and clearance of senescent cells by the immune system will improve the usefulness of senescence-inducing agents in the clinic. Thus, a better understanding of how to guide senescent cells towards apoptosis or immunemediated clearance is of great clinical interest. 


\subsubsection{Aims}

Studies looking at the induction of premature senescence by administration of MTAs are currently lacking. Of the MTAs that have been assessed, only ones that are not in clinical use (i.e. Disco, disorazole $\mathrm{C}_{1}$, and JG-03-14) demonstrated any encouraging senescence-inducing activity (Chang et al., 1999a; Klein et al., 2005; Arthur et al., 2007; Tierno et al., 2009). The primary aim of the study described in this chapter was to assess the ability of the MSA PelA to induce premature senescence in a breast cancer cell line (MCF7). To investigate senescence, we used the following assays:

1) The SA- $\beta$-gal assay, to assess the morphological changes that characterize senescence and to screen for cells expressing increased galactosidase enzyme activity.

2) An EdU (5-ethynyl-2'-deoxyuridine) incorporation assay, to determine whether drug-treated cells are still carrying out DNA synthesis.

3) A clonogenic assay, to assess the proliferative capacity of drug-treated cells at concentrations that induce senescent features.

Since senescence often leads to activation of two main pathways - the p53 and $\mathrm{pRb}$ tumour suppressor pathways, Western blotting was also carried out to assess activation of these two proteins during the induction of premature senescence. 


\subsection{Methods}

\subsubsection{Cell culture and MTT assay}

The MTT assay was carried out as described in the General Methods (Chapter 2). Briefly, MCF7 cells were plated in RPMI medium with $10 \%$ FCS and $1 \%$ Penstrep at 1 x $10^{4}$ cells/well (into each well of a 96-well plate) and allowed to attach overnight. Cells were then treated for $72 \mathrm{~h}$ with PelA, Disco, Ptx or Doxo before the addition of MTT and solubilizer. Concentration-response curves were generated using SigmaPlot software, and from these curves, the $\mathrm{IC}_{5}, \mathrm{IC}_{10}, \mathrm{IC}_{25}, \mathrm{IC}_{50}$ and/or $\mathrm{IC}_{70}$ values were determined.

\subsubsection{Senescence-associated- $\beta$-galactosidase assay}

All recipes for the solutions/buffers for the senescence-associated- $\beta$-galactosidase (SA$\beta$-gal) assay are given in the Appendix E. For the drug-treated samples, MCF7 cells were plated into 6-well plates at a density of $1 \times 10^{5}$ cells/well. Due to their greater proliferative capacity, untreated, control cells were plated at $1 \times 10^{4}$ cells per $60 \mathrm{~mm}$ dish. All treatment groups were prepared in duplicate for each individual experiment. During preliminary experiments, it was determined that using the equivalent $\mathrm{IC}_{50}$ values (as calculated from the $72 \mathrm{~h}$ MTT assays) produced quite variable results in terms of the degree of cell death after a 6 day incubation with drug; hence, it was decided that MCF7 cells would be treated with a range of concentrations that gave similar degrees of cell death (determined by a comparison of the confluency (cell density) at day 6 , by eye). MCF7 cells were allowed to attach overnight, and the medium was then aspirated off and replaced with $4 \mathrm{~mL}$ of medium (for control dishes), or $2 \mathrm{~mL}$ of PelA medium $\left(\mathrm{IC}_{25}\right.$, $\mathrm{IC}_{50}$ and $\mathrm{IC}_{70}$ ), Disco medium $\left(\mathrm{IC}_{10}, \mathrm{IC}_{25}\right.$ and $\mathrm{IC}_{50}$ ), Doxo medium ( $\mathrm{IC}_{10}, \mathrm{IC}_{25}$ and $\mathrm{IC}_{50}$ ), or Ptx medium $\left(\mathrm{IC}_{5}, \mathrm{IC}_{10}\right.$ and $\left.\mathrm{IC}_{25}\right)$. Cells were cultured in medium/drug for 6 days, then washed 2 times with PBS and fixed in $2 \%$ formaldehyde $/ 0.2 \%$ glutaraldehyde for 5 min at RT. Cells were washed again with PBS, and then incubated with $2 \mathrm{~mL}$ (per well for a 6 -well plate) or $4 \mathrm{~mL}$ (for a $60 \mathrm{~mm}$ dish) of the freshly made SA- $\beta$-gal staining solution (40 $\mathrm{mM}$ citric acid/sodium phosphate buffer at $\mathrm{pH} 6.0,5 \mathrm{mM}$ potassium ferrocyanide, 5 $\mathrm{mM}$ ferricyanide, $150 \mathrm{mM} \mathrm{NaCl}, 2 \mathrm{mM} \mathrm{MgCl} 2$, and $1 \mathrm{mg} / \mathrm{mL} \mathrm{X-Gal} \mathrm{(from} \mathrm{a} 20 \mathrm{mg} / \mathrm{mL}$ stock in dimethylformamide) for $16 \mathrm{~h}$ at $37{ }^{\circ} \mathrm{C}$, in a non- $\mathrm{CO}_{2}$ incubator. Cells were washed with PBS, as previously described, and then rinsed once with methanol. Plates were kept in the dark wherever possible. 


\subsubsection{Scoring of cells for SA- $\beta$-gal activity}

Plates containing the stained cells were viewed using bright-field microscopy (Olympus IX51 inverted microscope and CC-12 digital colour camera) and 15 random fields of view per well (using the 10X objective) were imaged (fields of view with no or very few cells, or much too many cells were discarded). Images were viewed using Image J software and the cell counting tool was used to score each cell as positive or negative for galactosidase. Cells were considered positive if they exhibited blue staining and had a flattened, enlarged morphology. Cells that had blue staining but did not have the flattened/enlarged morphology were considered negative. The initial cell scoring was carried out by Nikki Templeton and Connie Gilfillan, (Undergraduate students volunteering for Prof. John Miller, VUW, summer 2011-2012) but was reviewed by myself to ensure consistent scoring.

\subsubsection{Click-iT EdU incorporation assay}

To assess the degree of DNA synthesis after drug treatment, an EdU incorporation assay, similar to the commonly used BrdU incorporation assay, was used. The Click$\mathrm{iT}^{\circledR}$ EdU Flow Cytometry Assay (Invitrogen) is based on the incorporation of a nucleoside analogue to thymidine, EdU (5-ethynyl-2'-deoxyuridine), into actively synthesized DNA. The alkyne group on EdU is readily detected by a "click" reaction (a copper catalyzed reaction between an alkyne group and a fluorophore-conjugated azide). The small size of the azide group allows the use of milder conditions to permit its access to DNA-incorporated EdU (i.e. compared to the detection of DNAincorporated BrdU, which requires harsh denaturing conditions, e.g. heat and $\mathrm{HCl}$ ); thus, the use of EdU offers a faster, easier alternative to BrdU incorporation protocols (Li et al., 2010). Thus, increased incorporation of EdU into DNA is indicative of active DNA synthesis, and can be detected by a positive shift in fluorescence intensity by flow cytometry. MCF7 cells were plated into 6-well plates for drug-treated and $60 \mathrm{~mm}$ dishes for control cells. Cells were treated with the concentration of drug that would allow the recovery of a sufficient number of cells i.e. Doxo $\left(\mathrm{IC}_{25}\right)$, Disco $\left(\mathrm{IC}_{25}\right)$, PelA $\left(\mathrm{IC}_{50}\right)$ or Ptx $\left(\mathrm{IC}_{10}\right)$. For each treatment group, 4 wells or dishes were pooled for the double staining of EdU and PI, and the remaining wells or dishes used for single-stained and unlabelled controls. To assess DNA synthesis, the Click-iT EdU Pacific Blue ${ }^{\mathrm{TM}}$ Flow Cytometry kit (Life Technologies) was used as per the manufacturer's instructions. After 6 days, EdU was added to the medium to a final concentration of $10 \mu \mathrm{M}$, and the cells were incubated for $30 \mathrm{~min}$ at $37{ }^{\circ} \mathrm{C}$. Cells were then harvested by trypsinization 
and collected in $15 \mathrm{~mL}$ Falcon centrifuge tubes. Cells were washed twice with 1\% BSA in PBS, and a final cell pellet containing $1 \times 10^{6}$ cells was prepared. Cells were fixed by adding $100 \mu \mathrm{L}$ of the kit's PFA-based fixative directly to the cell pellet (cells were vortexed to prevent clumping) and incubated for $15 \mathrm{~min}$ at RT. Cells were washed twice with $1 \% \mathrm{BSA}$ in PBS, and then permeabilized by incubating the cells in $100 \mu \mathrm{L}$ of saponin buffer on ice for $10 \mathrm{~min}$. Cells were centrifuged and then resuspended in 500 $\mu \mathrm{L}$ of Click-iT kit reaction buffer for $30 \mathrm{~min}$ at RT in the dark. After washing twice in the saponin buffer, cells were treated with RNase and then incubated with PI staining buffer for $30 \mathrm{~min}$ at RT (as described in the General Methods). Unlabelled and singlestained controls were also prepared in order to set up the compensation corrections for the flow cytometer. Samples were run on a BD FACSCanto II flow cytometer (BD Biosciences) using the Pacific Blue ${ }^{\mathrm{TM}}$ and PI channels, and 10, 000 events were collected for each sample.

\subsubsection{Clonogenic Assay}

MCF7 cells were plated and treated with drugs as described above for the Click-iT EdU assay. After the 6 day incubation with drug, cells were harvested by trypsinization (see General Methods). A single-cell suspension was generated by passing cells through a 25 gauge $(0.5 \mathrm{~mm})$ needle 10 times, and then 5 times through a $70 \mu \mathrm{m}$ cell strainer. Cells were then plated at 700 cells per $10 \mathrm{~cm}$ petri dish, in a total volume of $10 \mathrm{~mL}$ of drugfree medium and then incubated for a further 10 days. After the 10-day incubation, the plates were rinsed two times with PBS, fixed with $5 \mathrm{~mL}$ methanol at RT for $5 \mathrm{~min}$, then rinsed again with $5 \mathrm{~mL}$ methanol. Cells were subsequently stained using $5 \mathrm{~mL}$ of a $1 \%$ crystal violet solution, then washed in distilled water until the excess dye was removed. Images of the plates were taken using a Canon PowerShot S3 IS digital camera (using Zoom Browser EX software). The number of colonies (clusters comprising greater than 50 cells) in each plate were manually scored on the plates with the aid of an inverted microscope to assess colony sizes.

\subsubsection{Western blotting}

MCF7 cells were plated and treated with either PelA ( $\left.\mathrm{IC}_{50}\right)$, Disco $\left(\mathrm{IC}_{25}\right)$, Doxo $\left(\mathrm{IC}_{25}\right)$ or Ptx $\left(\mathrm{IC}_{10}\right)$ for 1, 2, 3, 4, 5 or 6 days; however, due to the low recovery of cells after 6 days, the cells from each treatment condition were pooled from up to 6 wells or dishes (6-well plate or $60 \mathrm{~mm}$ dishes). Western blotting was carried out as described in the General Methods with minor amendments. Phosphatase inhibitor (Sigma) was also 
added to the RIPA buffer ( $10 \mu \mathrm{L}$ per $1 \mathrm{~mL}$ lysis buffer). For detecting $\mathrm{p} 53$ and $\mathrm{pRb}, 30$ $\mu \mathrm{g}$ of protein was loaded into each well of a $10 \%$ and $8 \%$ gel, respectively, and the proteins separated by electrophoresis. After electrophoretic transfer of the proteins to PVDF membranes, the membranes were probed using an anti-mouse p53 (Abcam, \#Ab28; diluted 4:3000 in 5\% BSA/PBST) incubated for $3 \mathrm{~h}$ at RT or anti-mouse $\mathrm{pRb}$ (BD Biosciences, \#554136; 6:1000 dilution in 5\% BSA/PBST) incubated overnight at 4 ${ }^{\circ} \mathrm{C}$. The primary antibodies were stained with anti-mouse IgG-Cy5 (GE Healthcare; 1:2000 in PBST) for $1 \mathrm{~h}$ at RT. Membranes were probed for the actin loading control using anti-actin (Sigma; 1:4000 in 5\% BSA/PBST) incubated for $15 \mathrm{~min}$ at RT, followed by anti-mouse IgG-Cy5 (1:2000) for $15 \mathrm{~min}$ at RT. In addition to the SA- $\beta$-gal scoring, Nikki Templeton and Connie Gilfillan (working under my supervision) also aided with some of the protein preparation, and antibody staining and washing steps. All other Western blotting steps were carried out by myself. 


\subsection{Results}

\subsubsection{MTT assay}

Table 6.1. Growth inhibitory concentration values for MCF7 cells treated with various drugs

\begin{tabular}{|c|c|c|c|c|c|}
\hline & \multicolumn{5}{|c|}{$72 \mathrm{~h}$} \\
\hline & $\mathrm{IC}_{5}(\mathrm{nM})$ & $\mathrm{IC}_{10}(\mathrm{nM})$ & $I_{25}(n M)$ & $\mathrm{IC}_{50}(\mathrm{nM})$ & $\mathrm{IC}_{70}(\mathrm{nM})$ \\
\hline PelA & nd & nd & $4.2 \pm 1.0$ & $9.6 \pm 1.9$ & $18.5 \pm 3.1$ \\
\hline Disco & nd & $10.2 \pm 2.1$ & $27.0 \pm 3.5$ & $72.2 \pm 4.4$ & nd \\
\hline Ptx & $1.2 \pm 0.1$ & $1.8^{*}$ & $3.3 \pm 0.5$ & $5.8 \pm 1.0$ & $n d$ \\
\hline Doxo & nd & $29.5 \pm 15.3$ & $58.1 \pm 21.8$ & $278.8 \pm 74.6$ & nd \\
\hline
\end{tabular}

* $\mathrm{n}=2$; thus, SEM could not be calculated

All other data presented are the mean \pm SEM, $n=3$

$n d$ not determined

MTT assays for PelA, Disco, Ptx, and Doxo were carried out to determine the different inhibitory concentrations for each drug. MCF7 cells were treated for $72 \mathrm{~h}$ with each drug, and the results are presented in Table 6.1. The $\mathrm{IC}_{50}$ values for both PelA and Ptx were in the low nanomolar range (10 and $6 \mathrm{nM}$, respectively). Disco and Doxo were much less effective in this cell line, with $\mathrm{IC}_{50}$ values of 72 and $279 \mathrm{nM}$, respectively. The effects of Doxo were also the most variable between replicate MTT assays, as indicated by the fairly high SEM values.

\subsubsection{Senescence-associated $\beta$-galactosidase assay}

An SA- $\beta$-gal assay was carried out to determine the extent to which each of the four drugs induced premature senescence. From preliminary tests, it was determined that longer incubation times (greater than 3-4 days) were preferable for the observation of a senescence response; thus, we chose 6 days as our endpoint, which has also been used previously (Klein et al., 2005). Initially, the $\mathrm{IC}_{50}$ values (determined from $72 \mathrm{~h}$ MTT assays) of each drug were used, but we observed a significant difference in the cytotoxic effects of each drug after 6 days. We thus decided to use a range of concentrations for each drug that gave similar cytotoxicity responses (as determined by rough assessment by eye, i.e. the amount of dead cells that had lifted off the surface of the wells) after the 6-day incubation period. The decision to plate control MCF7 cells in larger $60 \mathrm{~cm}$ dishes was to reduce the degree of positive SA- $\beta$-gal staining as a result of the cells 
reaching confluency. After 6 days in culture the control cells were more confluent than was desired, and this was reflected in a high positive staining for SA- $\beta$-gal activity; however, many of these positive-stained cells lacked the morphological criteria for senescence (flattened and enlarged cytoplasm), and thus were scored as negative. The mean number of cells positive for premature senescence in untreated control samples was below $2 \%$. Images of the entire field of view for each drug concentration are presented in Figure 6.2. It is difficult to see the senescent cells in detail in these low power images; therefore, examples of enlarged images of individual senescent cells are also provided (Fig. 6.3). It should be noted that there were many cells that stained blue with the SA- $\beta$-gal assay, but did not exhibit the enlarged flattened morphology as well as cells that were enlarged but did not stain positive. Both of these phenotypes were scored as negative for senescence.

Doxo is one of the gold-standard drugs used to induce premature senescence and was used as a positive control for comparison to MSA-treated cultures (see Fig. 6.4 A). In this particular assay, the greatest level of senescence (62\%) was induced by treatment with the $\mathrm{IC}_{50}$ concentration of Doxo. The lowest concentration of Doxo that was tested resulted in approximately $40 \%$ of the cells undergoing premature senescence. A very similar level of senescence induction was obtained with Disco and Ptx, but only at the highest concentrations that were tested $\left(\mathrm{IC}_{50}\right.$ and $\mathrm{IC}_{25}$, respectively). Lower concentrations of both these MSAs induced relatively little premature senescence (9$23 \%)$. The two highest concentrations of PelA that were tested $\left(\mathrm{IC}_{50}\right.$ and $\left.\mathrm{IC}_{70}\right)$ induced premature senescence in approximately $31 \%$ of cells; thus, PelA appeared to be the least potent of the MSAs at inducing premature senescence.

This particular assay determines the percentage of cells that stain positive for SA- $\beta$-gal, but only includes cells that remain attached (i.e. that have survived drug treatment) by the end of the incubation period. It does not, however, take into account any differences in cytotoxicity, and/or proliferation levels, two culture variables that may affect the absolute number of cells that are scored. Figure $6.4 \mathrm{~B}$ indicates the mean total number of cells (both positive and negative for SA- $\beta$-gal) that were scored for each replicate. Doxo $\left(\mathrm{IC}_{10}\right)$, Disco $\left(\mathrm{IC}_{50}\right)$ and Ptx $\left(\mathrm{IC}_{25}\right)$ all induced approximately $40 \%$ senescence, but for Doxo this was determined from a total of 743 cells, compared to only 342 and 284 cells for Disco and Ptx, respectively. As discussed later, this is an important consideration when trying to quantify the extent of induction of premature senescence. 
The total number of cells is also presented for controls, but because these were plated at a lower seeding density into larger tissue culture dishes (to allow for the greater level of proliferation in the absence of drugs), these counts are not directly comparable to the drug-treated values. 

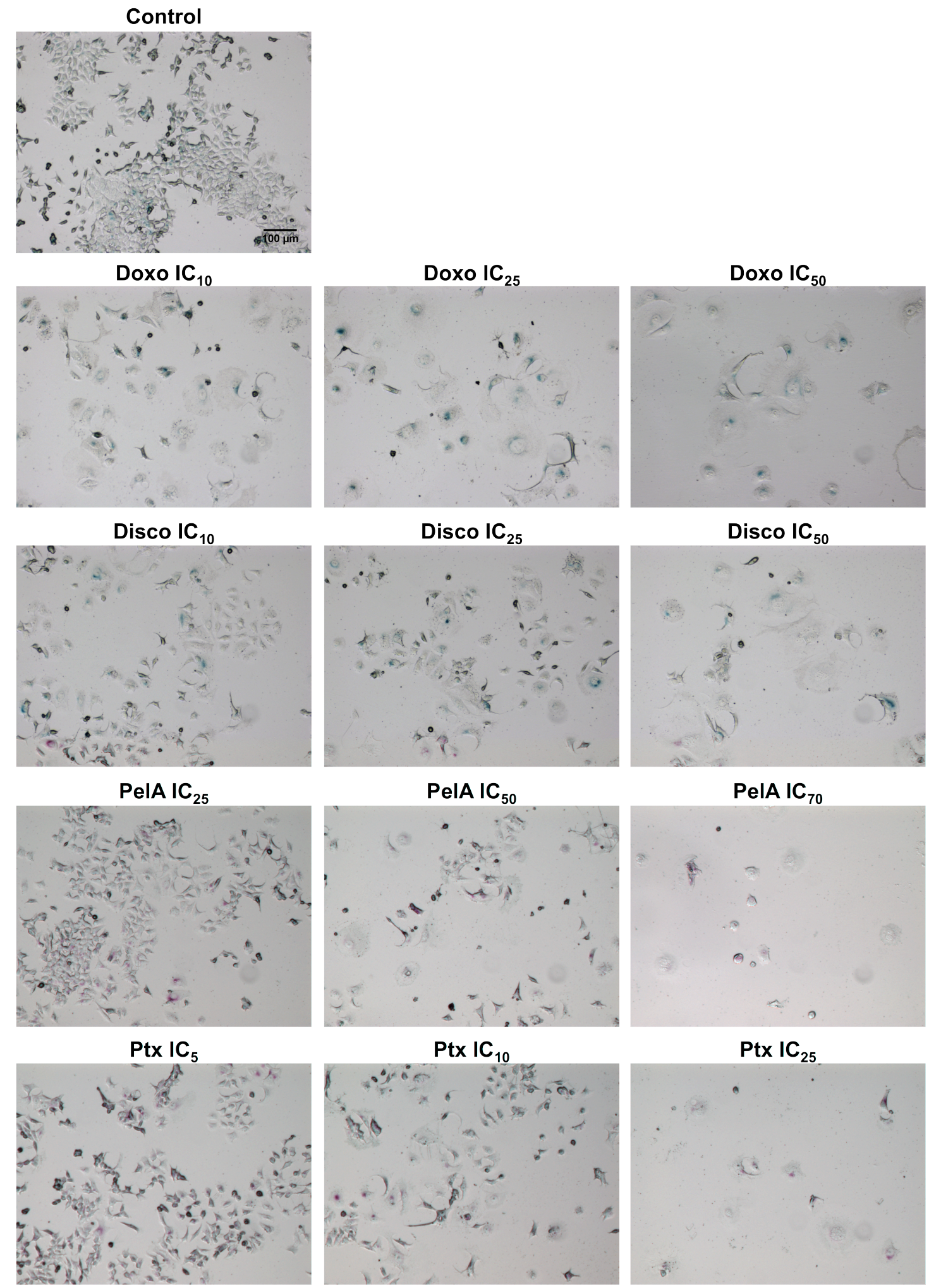

Figure 6.2. Photomicrographs of premature senescence induction. MCF7 cells were treated for 6 days with different concentrations of Doxo, Disco, PelA or Ptx (see text). Cells were then fixed and stained for SA- $\beta$-gal activity, and then photos of cells at each condition were taken, using the $10 \mathrm{X}$ objective lens. A $100 \mu \mathrm{m}$ scale bar is presented in the control image at the top left. 


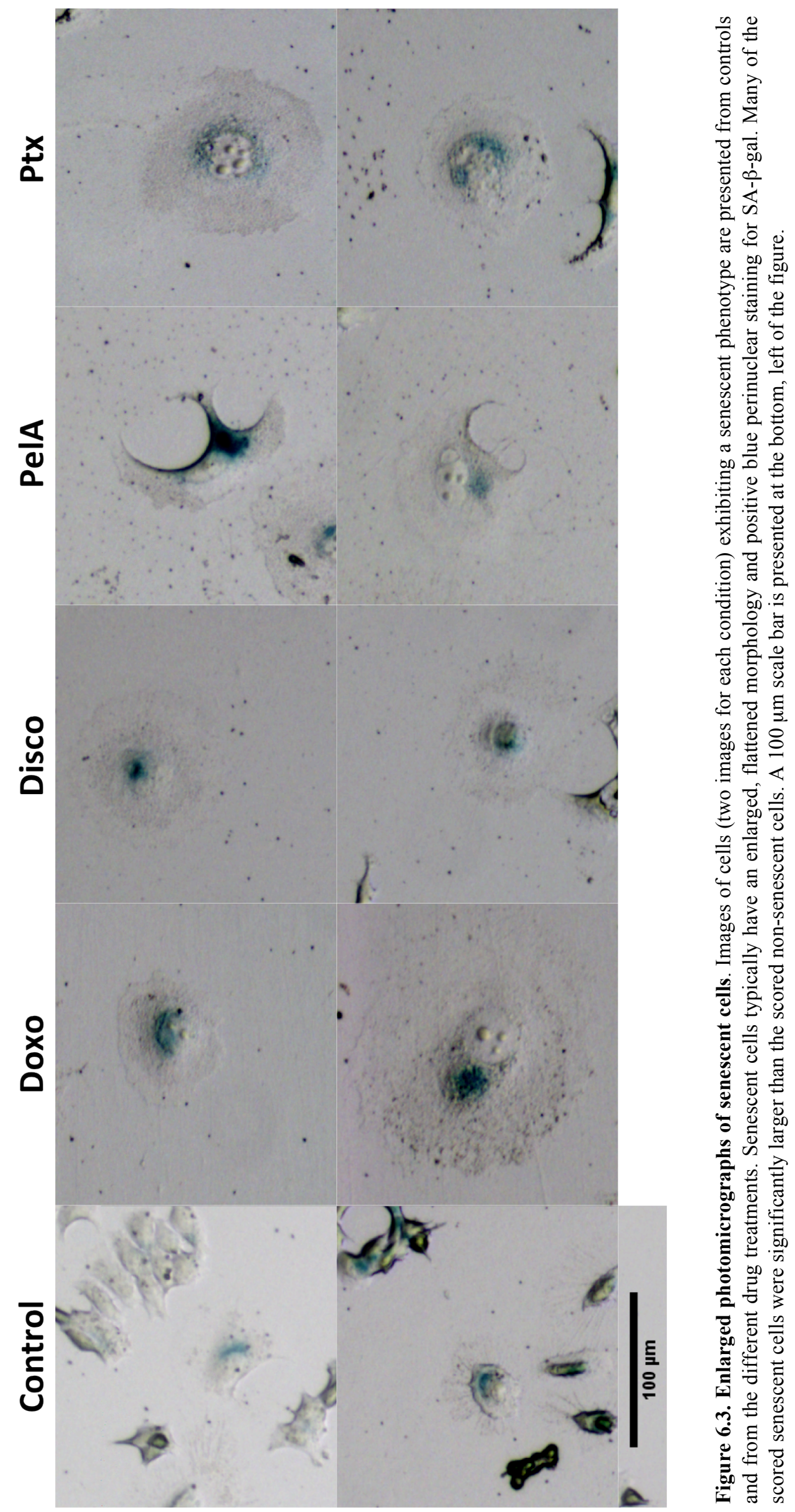




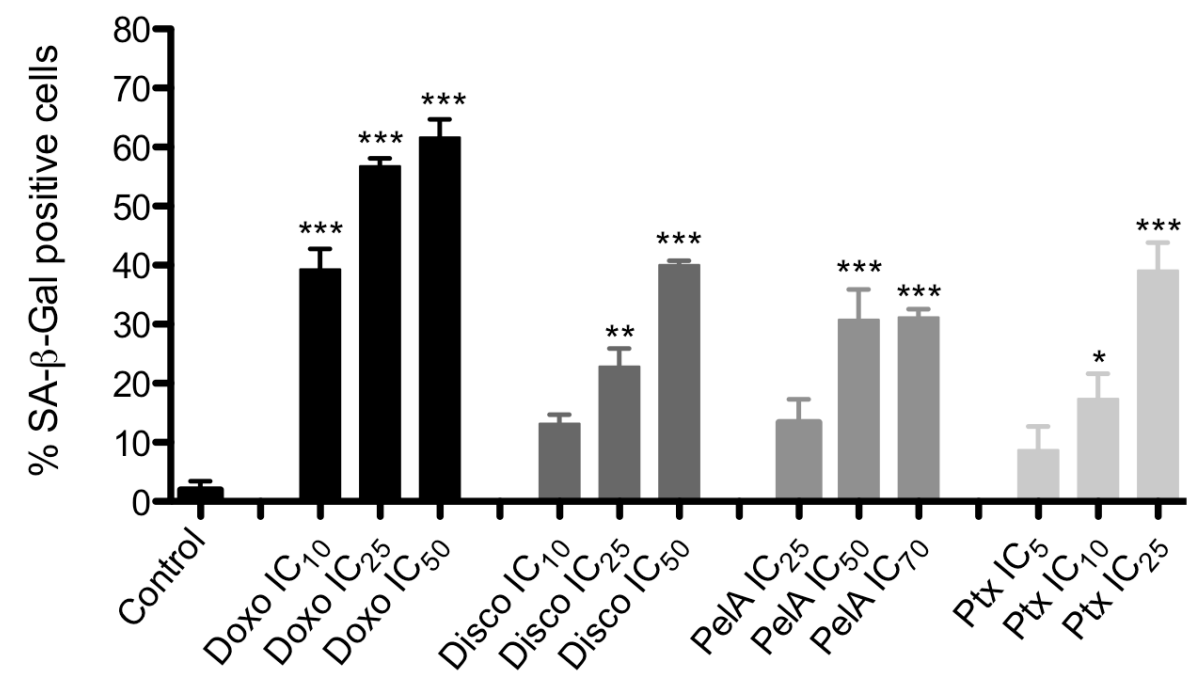

B

\section{Cell number}

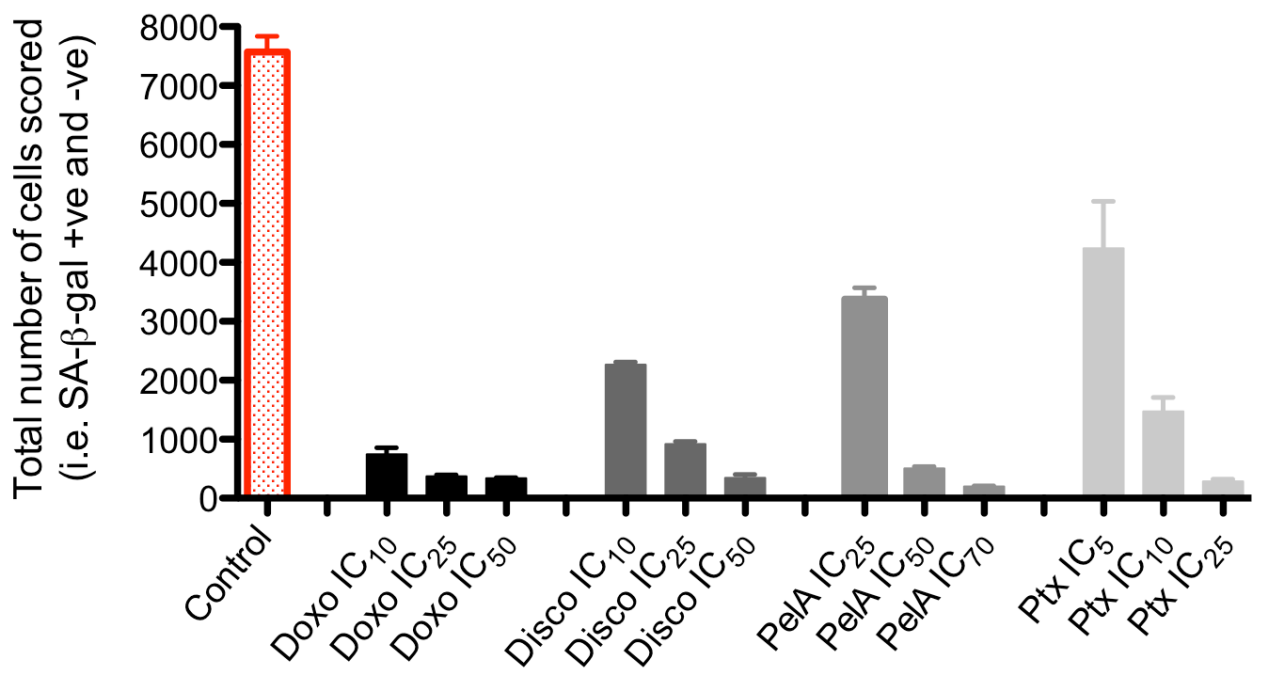

Figure 6.4. Induction of premature senescence. MCF7 cells were incubated for 6 days with medium or medium containing different concentrations of Doxo, Disco, PelA and Ptx (refer to the text for concentrations used). Cells were then fixed and stained for SA- $\beta$-gal activity. Images of stained cells were scored for premature senescence, as determined by positive SA- $\beta$-gal staining and flattened/enlarged morphology. (A) Graph indicates the mean percentage of cells positive for SA- $\beta$-gal activity $\left( \pm\right.$ SEM) from 3 independent experiments. ${ }^{*} \mathrm{p}<0.05, * * \mathrm{p}<0.01, * * * \mathrm{p}<0.001$ compared to untreated controls, as determined by one-way ANOVA and Bonferroni post-hoc test. (B) After the 6day incubation with drug, the mean total number of cells ( \pm SEM) from each condition was also recorded (i.e. the total number of SA- $\beta$-gal positive and negative cells that were scored from a full set of 15 images). For completion's sake the mean total number of cells is also presented for controls (red bar). It should be noted, however, that these cells were grown in a larger $60 \mathrm{~mm}$ tissue culture dishes and plated at a lower seeding density compared to drug-treated cells, to account for the increased proliferation in the absence of drug; thus, the mean total number of cells in controls is not directly comparable to the mean total number for drug-treated samples, which were plated in 6-well plates (see main text). 


\subsubsection{EdU incorporation assay}

To determine the effects of PelA, Doxo, Disco and Ptx on DNA synthesis and cell cycle arrest, we assessed DNA synthesis by looking at incorporation of the nucleoside analogue EdU. Cells were harvested after a 6-day incubation with drug (i.e. the same endpoint as for the SA- $\beta$-gal assay), and then double-stained for EdU and PI (see Fig. 6.5). A single concentration of each drug was chosen that induced a significant degree of premature senescence, but allowed for a sufficient number of cells to be recovered for the assay. Even in control cells (which should contain proliferating cells), the fluorescent staining intensity between what should be the negative $\left(G_{1}\right.$ and $G_{2} / M$ phases) and positive populations (S-phase) were not distinct (i.e. S-phase cells appeared to have a similar staining intensity to the $G_{1}$ and $G_{2} / M$ phases). This was also seen for the drug-treated samples. There was also a slight positive shift in fluorescence intensity in the Pacific Blue ${ }^{\mathrm{TM}}$ channel. This poor cell cycle phase separation and fluorescence shift was consistent between replicate experiments and made the results difficult to interpret. Thus, no accurate conclusions regarding DNA synthesis could be made from the EdU assay and only the cell cycle histograms are presented here (Fig. 6.5) with typical EdU results presented in Appendix Fig. E.2).

The PI histograms highlighted differences in the cell cycle distribution following drug treatment. The $\mathrm{G}_{2} / \mathrm{M}$ region in the control cells after 6 days in culture was not as distinct as normally seen in proliferating cultures, indicating that the control cells were approaching confluency. Doxo gave very distinct $\mathrm{G}_{1}$ and $\mathrm{G}_{2} / \mathrm{M}$ peaks (with very few Sphase cells). This is in stark contrast to the cell cycle histograms for PelA and Ptx. Although distinct $G_{1}$ and $G_{2} / M$ peaks could be seen with Disco, treatment for 6 days with PelA and Ptx resulted in a predominant $G_{1}$ peak that broadly tapered into the Sphase and $\mathrm{G}_{2} / \mathrm{M}$ regions. All four drugs also caused an increase in the number of cells in the sub- $\mathrm{G}_{1}$ and supra- $\mathrm{G}_{2} / \mathrm{M}$ regions. 

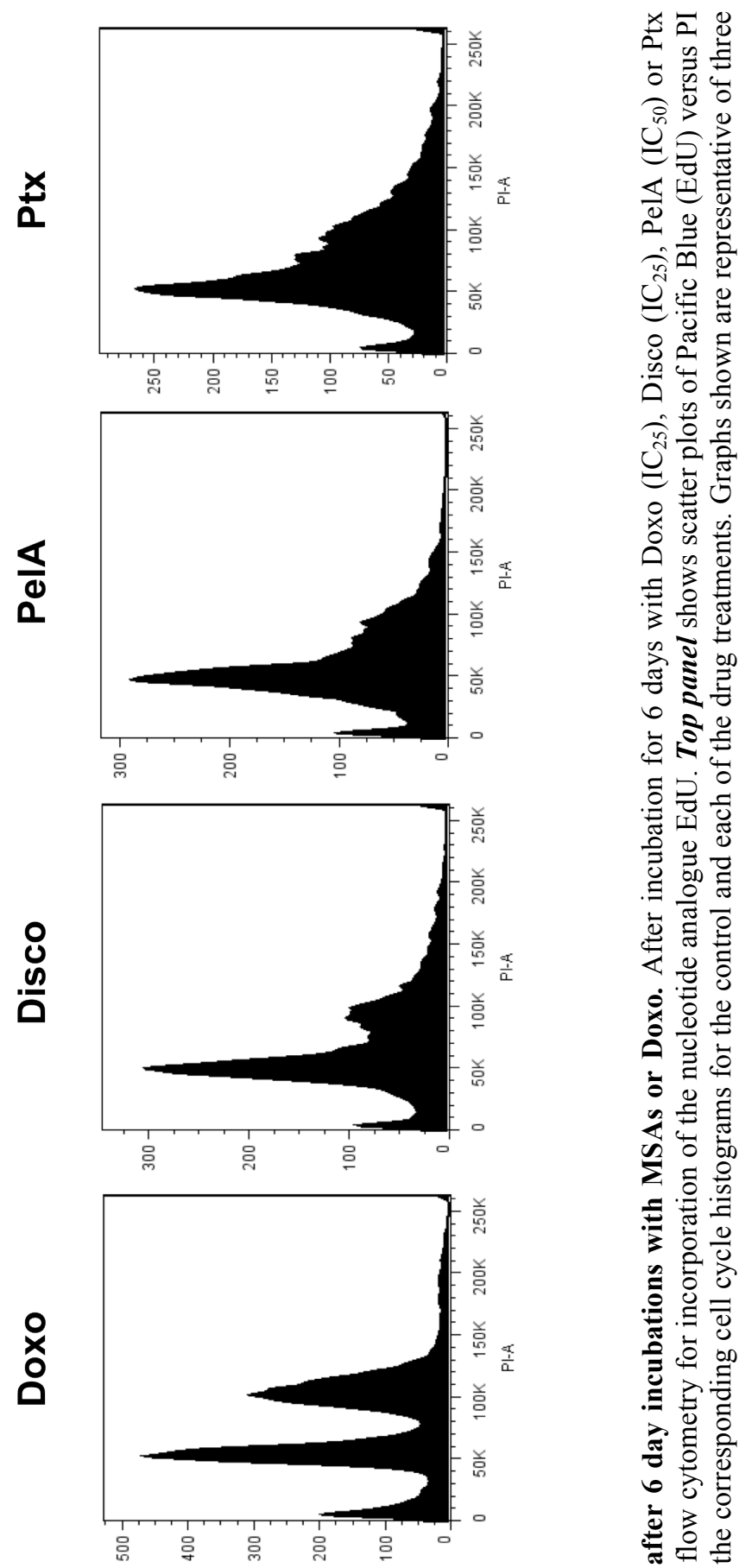

홍

ธิ

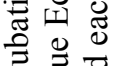

总

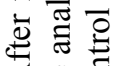

《

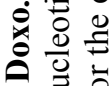

ว

造芒

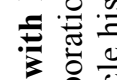

응

.0气

은

.

홍

6 ठ

司令

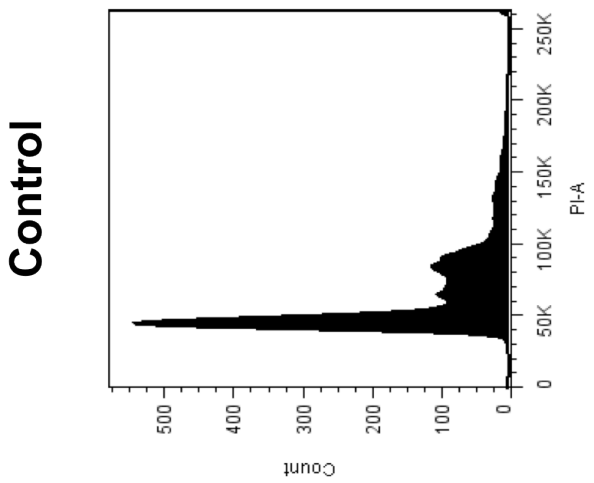

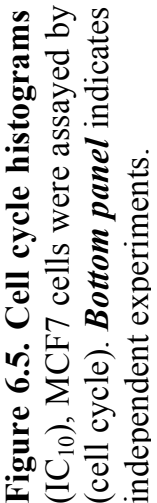




\subsubsection{Clonogenic assay}

A clonogenic survival assay was used to assess the ability of individual cells to survive and continue replicating after treatment with various drugs (see Fig 6.6 and Appendix E, Figure E.2 for photographs). This would provide information on whether senescent cells could recover and continue growth after drug washout. Cells that had been treated for 6 days were harvested, and subsequently re-plated at 700 cells per $10 \mathrm{~cm}$ dish into drugfree medium for a further 10 days. Doxo and Disco, which were the most effective at inducing a senescent phenotype, significantly impaired the ability of drug-treated cells to continue proliferating once released from the drug. Out of the 700 cells that were replated, only 10-13 colonies survived at the lowest concentrations of Doxo or Disco that were used $\left(\mathrm{IC}_{10}\right)$, compared to 151 surviving colonies in untreated controls. Higher concentrations of either of these drugs led to very few or no colonies forming. The lowest concentration of PelA that was used $\left(\mathrm{IC}_{10}\right)$ only had a minor inhibitory effect on colony formation; whereas, at the $\mathrm{IC}_{50}$ and $\mathrm{IC}_{70}$, PelA significantly impaired clonogenic survival. The two lowest concentrations of Ptx $\left(\mathrm{IC}_{5}\right.$ and $\left.\mathrm{IC}_{10}\right)$ did not inhibit colony formation compared to controls. The $\mathrm{IC}_{5}$ concentration actually seemed to increase the numbers of colonies, though this was not statistically significant. At the $\mathrm{IC}_{25}$, there was a sharp increase in clonogenic inhibition compared to the two lower concentrations, as indicated by the very few colonies that were present after the 10-day culturing period.

A brief assessment of whether the induction of senescence correlated with the inhibition of clonogenic survival was also carried out, irrespective of the drug treatment. For each drug (at each of the varying concentrations), the mean percentage of SA- $\beta$-gal positive cells was plotted against the mean number of colonies that survived after the additional 10-day incubation (Fig. 6.7). As the degree of senescence increased, there was a reduction in the number of colonies that survived in this particular assay. From the scatter plot, it appears that drug concentrations that achieve a particular threshold of senescence induction (i.e. $>20 \%$ ) drastically reduced the clonogenic potential of those cells. 


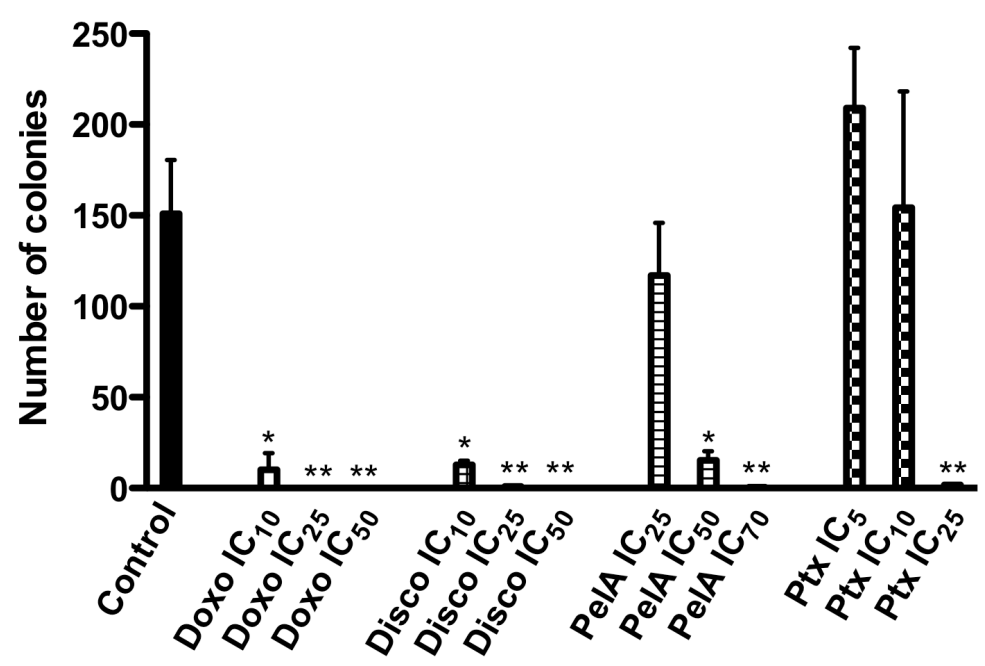

Figure 6.6. Effects of chemotherapeutic drugs on clonogenic survival. MCF7 cells were treated for 6 days with several concentrations of Doxo, Disco, PelA or Ptx (Taxol). Cells were then harvested and then re-plated at 700 cells $/ 10 \mathrm{~cm}$ dish, as a single-cell suspension, and cultured for a further 10 days in drug-free medium. Graph indicates the number of crystal violetstained colonies containing $\geq 50$ cells. after staining with crystal violet $(1 \%)$. Data represent the mean \pm SEM of four independent experiments. ${ }^{*} \mathrm{p}<0.01, * * \mathrm{p}<0.001$ as determined by a oneway ANOVA and Bonferroni post-hoc test.

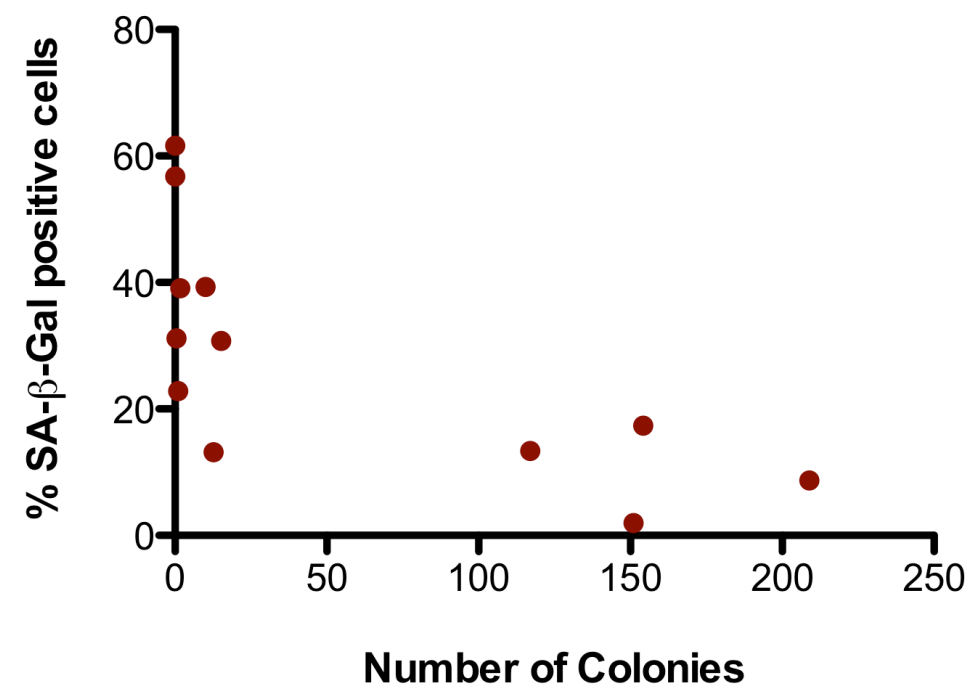

Figure 6.7. Relationship between induction of senescence and clonogenic survival. For each drug treatment group, the mean values for the percentage of SA- $\beta$-gal-positive cells were plotted against the mean number of surviving colonies. 


\subsubsection{Changes in $p 53$ and $p R b$ expression levels}

The $\mathrm{p} 53$ and $\mathrm{pRb}$ tumour suppressor pathways are often implicated in the induction of senescence. Western blotting was carried out to observe the time-dependent changes in expression levels of p53 and pRb after treatment with the various chemotherapeutic agents. MCF7 cells were harvested at various times from 1-6 days to assess whether there were any time-dependent effects on these proteins (i.e. days 1-6 for p53, and days 3 and 6 for $\mathrm{pRB}$ ). After 6 days of drug treatment there were very few cells remaining in the dishes. Due to the amount of protein that was required for the Western blotting the cells from an entire 6-well plate (or multiple $60 \mathrm{~mm}$ dishes for non-drug treated samples) had to be pooled for each condition, at each timepoint. PelA and Disco come from limited natural supplies and it was not feasible to carry out the full-time course for both $\mathrm{p} 53$ and $\mathrm{pRb}$, hence the reduced number of timepoints for the latter. Representative Western blots showing changes in p53 and pRb expression are shown in Figure $6.8 \mathrm{~A}$ and $\mathrm{B}$, respectively, with the corresponding summary graphs indicating the relative densitometry presented in Figure 6.9 A and B, respectively. p53 expression was increased 2-3 fold in response to PelA, Disco and Doxo; whereas, only a very minor increase was seen after Ptx treatment. Although the changes in p53 expression levels in response to all four chemotherapeutic drugs (Doxo, Disco, PelA and Ptx) were not statistically significant, there did appear to be slight differences in the timing of peak p53 activation (Fig. 6.9 A). Doxo and Disco, which were the most potent inducers of premature senescence, peaked slightly later ( days 3-5) compared to PelA ( days 2-3). p53 activation in response to Ptx was very similar to untreated cells, and did not follow the same pattern of activation as the other three drugs.

To conserve drug and $\mathrm{pRb}$ antibody, a full time-course for $\mathrm{pRb}$ expression was not carried out. After treatment for 3 days with all four drugs, $\mathrm{pRb}$ expression levels were significantly decreased compared to controls (Fig. 6.8 B and 6.9 B). PelA treatment cause the greatest reduction in $\mathrm{pRb}$ levels (60\% reduction compared to controls), followed by Doxo and Ptx (both reducing pRb levels by 50\%) and then Disco (reduced $\mathrm{pRb}$ by $30 \%)$. pRb expression levels were still less than controls after 6 days of drug treatment, but much more variable compared to day 3 results and hence, not statistically significant. The phosphorylation state of $\mathrm{pRb}$ regulates its cell cycle activities (see General Introduction). Lower molecular weight bands are indicative of hypophosphorylated $\mathrm{pRb}$. The drug-treated bands tended to run on the gel at a slightly lower molecular weight than the primary band in controls (this was more evident at day 3 than 
day 6; see Fig. 6.9, B), indicating that all four drugs may decrease the levels of hyperphosphorylated $\mathrm{pRb}$. Despite this mobility shift in the $\mathrm{pRb}$ bands, a phospho-specific antibody was not tested against $\mathrm{pRb}$ (the antibody used detects both phosphorylated and unphosphorylated forms of the protein). 
A

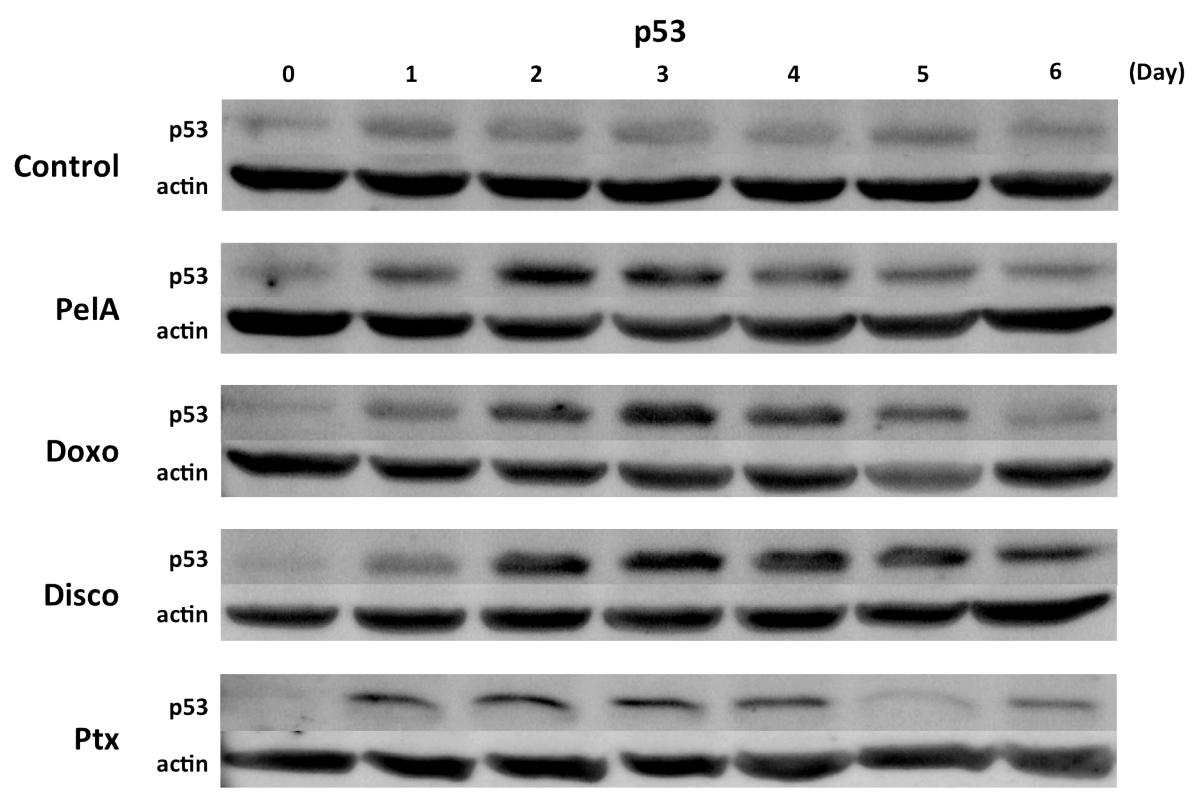

B

pRb

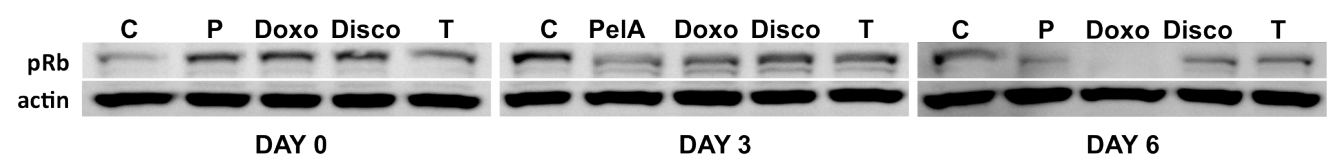

Figure 6.8. Changes in p53 and pRb expression. MCF7 cells were treated with Doxo $\left(\mathrm{IC}_{25}\right)$, Disco $\left(\mathrm{IC}_{25}\right)$, PelA $\left(\mathrm{IC}_{50}\right)$ or Ptx $\left(\mathrm{IC}_{10}\right)$ and harvested at various times (1-6 days for p53 expression and days 3 and 6 for $\mathrm{pRb}$ expression). Cell lysate $(30 \mu \mathrm{g}$ protein) was separated by gel electrophoresis $(10 \%$ and $8 \%$ gels for $\mathrm{p} 53$ and $\mathrm{pRb}$, respectively) and then transferred electrophoretically onto PVDF membranes. Membranes were probed for p53 (A), pRb (B) or actin (loading control). Membranes are representative of three independent experiments. Abbreviations: C, control; P, PelA; T, Ptx. 
A

P53 expression

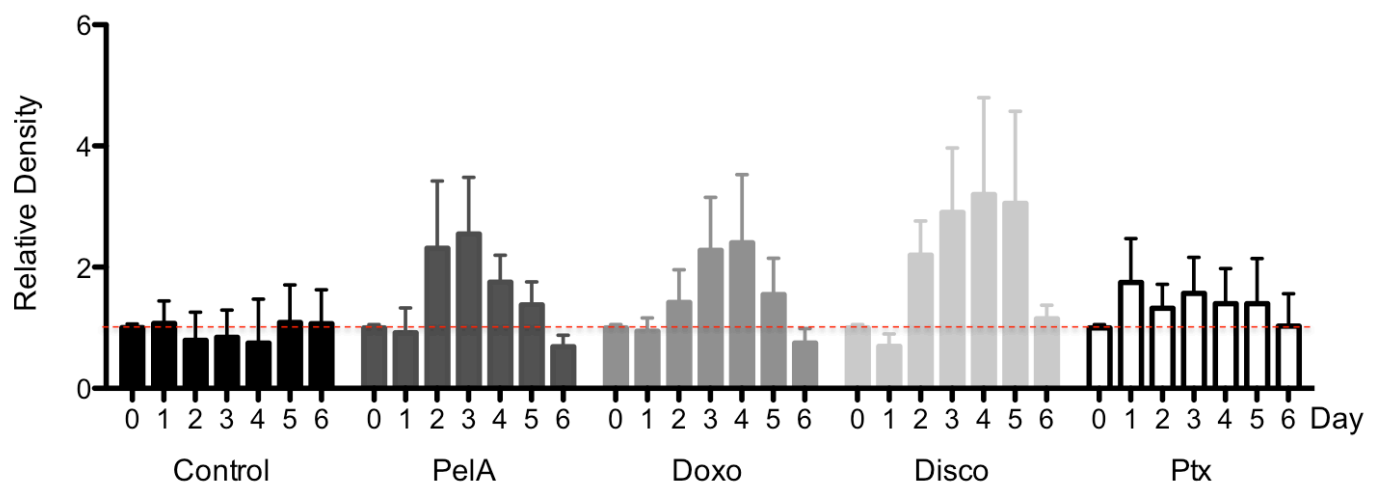

B

Rb expression

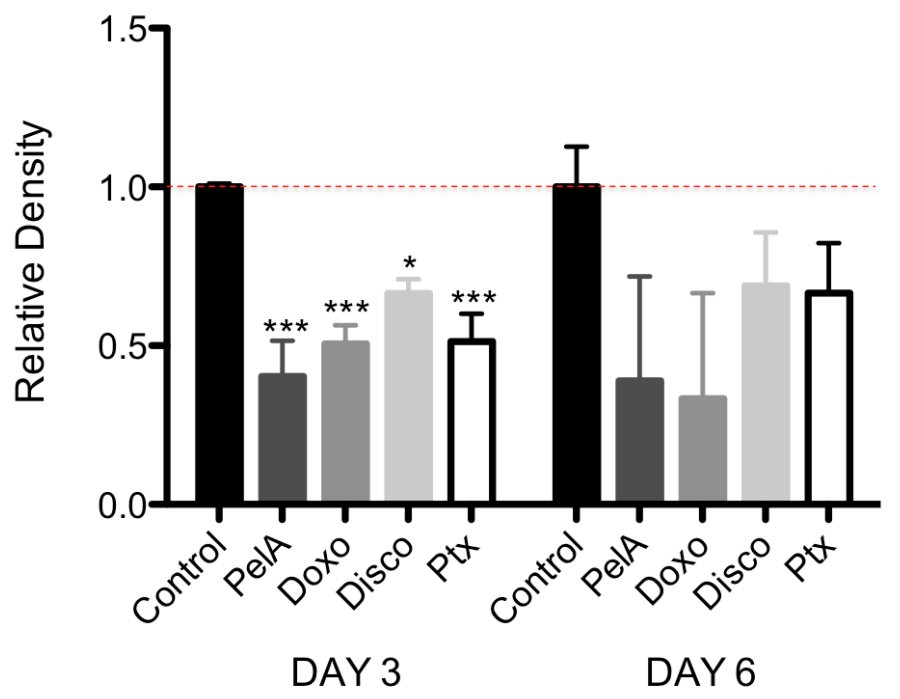

Figure 6.9. Graphs showing relative changes in p53 and pRb expression. MCF7 cells were treated with Doxo $\left(\mathrm{IC}_{25}\right)$, Disco $\left(\mathrm{IC}_{25}\right)$, PelA $\left(\mathrm{IC}_{50}\right)$ or Ptx $\left(\mathrm{IC}_{10}\right)$ and harvested at various times (1-6 days for $\mathrm{p} 53$ expression and days 3 and 6 for $\mathrm{pRb}$ expression). Western blotting was carried out to assess the changes in expression levels of p53 (A) and pRb (B). The relative density of each band compared to the loading control, actin, was determined and then normalized to the untreated, control treatment group for each timepoint. $* \mathrm{p}<0.05, * * * \mathrm{p}<0.001$ as determined by a one-way ANOVA and Bonferroni post-hoc test. 


\subsection{Discussion}

\subsubsection{Increased SA- $\beta$-gal activity in response to MSAs}

Previous work has demonstrated that cells treated with low concentrations of chemotherapy drugs (e.g. DNA-damaging agents) can induce features of premature senescence, such as positive SA- $\beta$-gal staining and flattened/enlarged morphology (Chang et al., 1999a; Elmore et al., 2002; Klein et al., 2005; Litwiniec et al., 2010). In light of an earlier study by Klein et al. (2005) that demonstrated the induction of premature senescence by the MSA Disco we decided to explore a similar mechanism by PelA that may potentially contribute to its tumour suppressive effects. Our study demonstrated similar results to Klein et al. (2005) in which Doxo induced the greatest level of premature senescence of the three drugs screened. In the A549 NSCLC cell line, Disco showed significantly greater senescence-inducing activity compared to Ptx, but this was not the case in our study using the breast cancer cell line, MCF7. All three of the MSAs we tested were able to induce premature senescence (although not to the same extent as the positive control Doxo), and Disco and Ptx were only marginally more effective than PelA. Our results may differ for several reasons, one of which is the fact that the use of different cell lines will of course influence the experimental outcome, as will the passage number of the cell line used. Additionally, the decision to progress towards particular cell fates (e.g. apoptosis versus senescence) is a highly complex process, and it is difficult to pinpoint exactly why cells in a particular population, although treated exactly the same, can have such a varied response to different drugs. The endogenous levels of pro- and anti-apoptotic factors, as well as the propensity to utilize particular signalling pathways (or the threshold of activation), may drive cells towards different cell fates (Leontieva et al., 2010; Galàn-Malo et al., 2012).

Initially we wanted to screen the senescence-inducing effects of PelA in the 1A9 cell line; however, due to the rapid growth of these cells, the long drug treatment times that are needed to induce senescence can lead to over-confluent cells and differentiation in some cultures. One caveat associated with the widely used SA- $\beta$-gal assay is its lack of specificity to senescence. Culture stress (e.g. serum withdrawal, contact inhibition or differentiation at confluence) and conditions that increase lysosomal expansion, such as autophagy, can result in increased lysosomal galactosidase activity that can be detected at suboptimal $\mathrm{pH}$ 6.0, irrespective of the fact that these cells are not senescent (Severino et al., 2000; Arthur et al., 2007; Kuilman et al., 2010). Thus, to reduce false-positives in the SA- $\beta$-gal assay due to culture conditions, we used the slower growing MCF7 cell 
line. In highly-dense areas of cells, even in controls, we did observe greater levels of blue staining in the SA- $\beta$-gal assay; however, many of these cells lacked the morphological characteristics (i.e. flattened and enlarged morphology) of senescence and were scored as negative. An increased lysosomal content was recently observed in high-density and serum starved cultures, although not to the same extent as the increase observed in senescent cells (Cho and Hwang, 2012). Earlier studies that followed the differentiation of mammary or renal epithelial cells in culture, observed a decrease in the number of lysosomes (Russo et al., 1976; Pfaller et al., 1990), which suggest that the positive staining in contact-inhibited MCF7 cells is probably not due to differentiation. While useful as a tool to identify senescent cells, the SA- $\beta$-gal assay must be used with caution. Fundamentally, the SA- $\beta$-gal assay detects increased lysosomal mass and it is important that more research is done to probe its relevance to the senescence phenotype. Generally, other senescent features are assessed alongside the use of the SA- $\beta$-gal assay and, as described below, changes in proliferative ability and altered expression of tumour suppressor proteins were also investigated in the present study to confirm the induction of senescence.

\subsubsection{Cell cycle changes after prolonged exposure to chemotherapeutic agents}

Senescent cells remain metabolically active but do not replicate; hence, it was expected that the number of cells actively synthesizing DNA in response to concentrations of drugs that induced significant premature senescence, would be decreased. Unfortunately, although the EdU incorporation assay results look somewhat similar to previously published work using BrdU (Klein et al., 2005), the data were difficult to interpret. A good separation was not seen between the positive S-phase cells (which should be incorporating the EdU into their DNA) and the negative $G_{1}$ and $G_{2} / M$ phase cells, even in the controls. The Pacific Blue ${ }^{\mathrm{TM}}$ fluorescent dye is known to be dim, and this may have contributed to the poor separation observed in the S-phase cells. A shift in drug-treated cells into the region where positive EdU cells should be was also seen. This was probably not due to incorporation of the nucleotide by the $G_{1}$ and $G_{2} / M$ populations, but rather due to an increased autofluorescence in response to the drugs (this had been seen previously in our apoptotic assays using 1A9 cells- see Chapter 3). In hindsight we should have also run drug-treated unlabelled controls to demonstrate a drug-dependent increase in autofluorescence in MCF7 cells. 
Although the EdU results were inconclusive, from the cell cycle histograms it was seen that Doxo, at least, was behaving as expected for a DNA-damaging agent. Doxo-treated cells became blocked in $\mathrm{G}_{1}$ and $\mathrm{G}_{2} / \mathrm{M}$ (with a noticeable absence of S-phase cells), as expected after triggering the DNA-damage checkpoints at both $\mathrm{G}_{1}$ and $\mathrm{G}_{2} / \mathrm{M}$ (the DDR and pathways that trigger cell cycle arrest are reviewed in Zhou and Elledge (2000)). The cell cycle profile of the surviving cells after a 6-day treatment with MSAs looked significantly different to Doxo, as there was no distinct cell cycle block. Some of these cells (as may also be the case for the $\mathrm{G}_{2} / \mathrm{M}$ arrested Doxo-treated cells) could in fact be $\mathrm{G}_{1}$ cells that are aneuploid, or have multiple nuclei (a phenomenon that was observed but not quantified in this study, that has also been seen by others (Klein et al., 2005; Litwiniec et al., 2010)). Additionally, cells can escape from mitotic arrest and enter into $\mathrm{G}_{1}$ (mitotic slippage), after which they typically either arrest at $\mathrm{G}_{0} / \mathrm{G}_{1}$ or undergo what is known as endoreduplication (DNA synthesis in the absence of cell division) (Vogel et al., 2004; Galàn-Malo et al., 2012). At the concentrations used for each of the drugs in our study, only $17-57 \%$ of cells exhibited a senescent phenotype. If a distinct fluorescent marker of senescence were available, we could then determine, using flow cytometry, where exactly in the cell cycle these MSA-induced senescence cells become growth-arrested.

\subsubsection{Activation of $\mathrm{p53}$ and down-regulation of $\mathrm{pRb}$}

Western blot analyses were carried out to investigate some of the possible signalling pathways underlying premature senescence. Knockout cell lines and cell lines overexpressing p53 and pRb (as well as their up- and downstream signalling partners) have, for a long time, implicated the p53-p21 and p16 ${ }^{\mathrm{INK} 4 \mathrm{a}}-\mathrm{pRb}$ tumour suppressor pathways in the induction of the senescent phenotype (Chang et al., 1999a; Wang et al., 1999; Elmore et al., 2002; Te Poele et al., 2002). Unfortunately, it was too costly and time-consuming to look at the same range of drug concentrations and timepoints that were used in the SA- $\beta$-gal assay (and also would have used up a significant amount of our limited drug supplies); thus, a single concentration was chosen for each drug. A series of timepoints was initially carried out to assess changes in p53 expression (i.e. every day up to day 6). However, the low cell recovery at some of the drug concentrations meant that fresh lysate had to be prepared in order to probe for $\mathrm{pRb}$, and to conserve on drug and consumables, $\mathrm{pRb}$ expression was only assessed every third day (i.e. days 0,3 and 6 ). Ptx, at its $\mathrm{IC}_{10}$ concentration, induced $\mathrm{p} 53$ expression to slightly above control levels, and there was no clear peak in expression. At this 
concentration, Ptx did not significantly reduce clonogenic survival compared to untreated controls, suggesting that it was not overly effective at inducing cell death or senescence. In contrast, p53 levels peaked with PelA at days 2-3, Doxo at days 3-4, and Disco at days 3-5, possibly reflecting differences in the timing of induction of the senescent phenotype.

In response to all drugs, except for Ptx a peak in p53 was seen followed by a subsequent decrease back to control levels. Highlighting their involvement in the induction of senescence, studies using p53 -/- and p21 -/- homozygous knockout mice have shown that the absence of either of these signalling mediators can diminish, but not completely abrogate, the senescence response, or facilitate an apoptotic rather than a senescent response (Chang et al., 1999b; Chang et al., 2002; Elmore et al., 2002). It has been suggested that while $\mathrm{p} 21$, the downstream effector of $\mathrm{p} 53$, is transiently elevated during the initial stages of cell cycle arrest, the sustained up-regulation of the CKI p16 ${ }^{\mathrm{INK} 4 \mathrm{a}}$ which occurs after senescence induction, is important in maintaining the prolonged cell cycle arrest (Alcorta et al., 1996; Stein et al., 1999; Te Poele et al., 2002). pRb appeared to be hypophosphorylated (and thus activated) in response to all four chemotherapeutic drugs. This activation of $\mathrm{pRb}$ is typical of cells undergoing cell cycle arrest or cellular senescence (see Fig. 6.1), since activation of CKIs (which were not assessed in our study) prevents the CDK-mediated phosphorylation of $\mathrm{pRb}$ (resulting in the $\mathrm{pRb}$ mediated sequestration of E2F transcription factors, which are necessary for cell cycle progression) (Alcorta et al., 1996; Stein et al., 1999; Narita et al., 2003). The significant decrease in $\mathrm{pRb}$ that was observed at day 3 (in response to all four drugs) has been observed previously, suggesting that, like p53 and $\mathrm{p} 21, \mathrm{pRb}$ is not involved in maintaining the senescent phenotype (Stein et al., 1999; Klein et al., 2005). Whether $\mathrm{pRb}$ was transiently increased following drug treatment was not clear in the present study due to the lack of additional timepoints. It is important to stress the fact that changes in many of these tumour suppressors and cell cycle regulating proteins are activated in response to numerous cell fates (i.e. activation of p53 during apoptosis and hypophosphorylation of $\mathrm{pRb}$ during quiescence); therefore, changes in their expression levels must be interpreted with caution.

The delayed peak activation of p53 in response to Doxo and Disco, compared to PelA, highlight the fact that these drugs are triggering distinct signalling responses. Proteomic screens (see Chapter 7) have shown that protein expression changes in response to 
MTAs are not identical, despite a common primary target (MacKeigan et al., 2003; Verrills et al., 2003; Lee et al., 2005; Yang et al., 2006; Wilmes et al., 2012a). It has been suggested that senescence can be induced by p53-independent mechanisms or by signalling primarily via the $\mathrm{p} 16^{\mathrm{INK} 4 \mathrm{a}}-\mathrm{pRb}$ pathway, which could explain why $\mathrm{p} 53$ signalling was mostly absent after Ptx treatment (Campisi and d'Adda di Fagagna, 2007; Larsson, 2011). The p53 signalling pathways are also critical in apoptotic signalling, and research is currently underway to determine why some cells enter a senescent state rather than undergoing other cell fates (such as apoptosis and quiescence). The reversibility of cell cycle arrest in response to activated tumour suppressor pathways has important clinical relevance, as arrested cells that re-enter the cell cycle can act as a source for tumour re-growth. As demonstrated in this study, PelA, Disco, Ptx and Doxo concentrations that increased SA- $\beta$-gal activity also significantly correlated with a reduction in clonogenic survival, but whether this was causative or co-incidental was not clear.

\subsubsection{Premature senescence versus other cell fates}

Initially, $\mathrm{IC}_{50}$ values (as determined by a 3-day MTT assay) were chosen to run preliminary tests to assess the induction of premature senescence after incubation with each drug; however, it became apparent that the 3-day $\mathrm{IC}_{50}$ values did not accurately reflect the degree of cytotoxicity after 6 days. Ptx, in particular, caused considerable cell death compared to the other drugs despite the use of equitoxic doses (based on 3-day $\mathrm{IC}_{50}$ values). A range of concentrations for each drug was chosen to account for the variation in responses and to ensure that there were still cells remaining after incubation with each drug. Low concentrations of drugs seem to be more effective than higher concentrations at inducing premature senescence (Eom et al., 2005), and this is likely due to a preferential push towards apoptosis at higher concentrations. An induction of apoptosis in response to PelA and Disco (in 1A9 cells) was presented in Chapter 3. Although we did not quantify apoptosis in this study, an indirect approximation of cell death was made, by quantifying the total number of cells that were scored (both positive and negative) in the SA- $\beta$-gal assay. Apoptotic cells detach from their substrate, so this gives us some idea of how many surviving cells were still attached to the culture plates or dishes. It should be noted though that this was a crude method and cannot provide information on whether the reduced number of cells remaining in drug-treated samples was due to apoptotic or cytostatic responses. Also, if cells retain their proliferative capacity particularly at the lower concentrations of drug, then they can quickly outgrow 
senescent cells in the population, and this makes interpretation more difficult. The degree of apoptosis is an important aspect to consider when interpreting the SA- $\beta$-gal assay results. For example, Doxo at the $\mathrm{IC}_{10}$ resulted in a similar level of premature senescence compared to Disco at the $\mathrm{IC}_{50}$ (Fig. 6.4 A). However, Disco treatment resulted in just fewer than half the number of cells compared to Doxo (Fig. 6.4 B), suggesting additional cytotoxic or cytostatic responses. Thus, quantifying senescence at concentrations that also trigger significant apoptosis could overstate the therapeutic relevance of premature senescence.

Many cell fates, such as senescence, quiescence, mitotic catastrophe, and apoptosis share similar characteristics and are not necessarily mutually exclusive. While not carried out in the present study, another recent study has suggested that apoptosis and senescence should be evaluated concurrently (Litwiniec et al., 2010). Studies by Litwiniec et al. (2010) and Klein et al. (2005) investigated chemotherapy-induced premature senescence and assessed both senescence and apoptosis (e.g. via caspase activation, mitotic catastrophe and/or DNA fragmentation). Although there is no clear definition of this particular form of cell death, mitotic catastrophe can either cause cell death directly during mitosis or result in giant $G_{1}$ cells that have multiple nuclei and undergo a delayed form of cell death (Castedo et al., 2004; Klein et al., 2005). Multinucleate cells that also stain positive for SA- $\beta$-gal have been observed in the present study (not shown) and that of others (Eom et al., 2005; Klein et al., 2005). Eom et al. suggested that a senescence-like phenotype preceded mitotic catastrophe in response to low concentrations of Doxo, implying that cells expressing SA- $\beta$-gal underwent eventual cell death. An earlier study by another group suggested that because the probability of SA- $\beta$-gal positive and SA- $\beta$-gal negative cells undergoing cell death was the same, senescence and mitotic cell death were independent events (Chang et al., 1999a). Two recent studies have suggested that the induction of senescence is regulated at least in part by mTOR activity levels (i.e. high levels of mTOR correlate with senescence; whereas, low concentrations correlate with quiescence) (Litwiniec et al., 2010; Cho and Hwang, 2012). mTOR itself can be inhibited by high p53 levels and can explain the concentration-dependent effect on senescence induction, where higher concentrations either push cells towards quiescence or cell death (rather than senescence) depending on the strength of the p53 signal (Litwiniec et al., 2010). It is highly likely that the threshold levels of other signalling mediators (e.g. anti- and proapoptotic BCL-2 proteins) also play a role in deciding the ultimate cell response to 
particular stimuli. Clearly, further investigations on the molecular determinants underlying the different responses to chemotherapeutic drugs are needed, to better understand whether these cell fates are distinct or overlapping.

\subsubsection{Mechanisms underlying MSA-induced senescence}

Doxo, and the majority of drugs that induce senescence, do so by activating one of several DNA damage checkpoints (Robles et al., 1999). It is possible that MSAs, in addition to their microtubule-stabilizing effects (and hence effects on mitotic progression), may also inflict some degree of DNA damage. Indeed, Ptx and BPROY007 (a microtubule-depolymerizing agent), have both been shown previously to inhibit topoisomerase II $\alpha$ and topoisomerase I, respectively, in cell-free systems (Dhawan and Swaffar, 1999; Chang et al., 2003b). The ability of MTAs to target both microtubules, and to a lesser extent, topoisomerases offers an attractive explanation for the observed induction of premature senescence. A chemical genetics screen in Saccharomyces cerevisiae identified topoisomerase I as a potential secondary target of PelA (Wilmes et al., 2012b) and studies are currently underway in our laboratory to screen some of these MSAs for topoisomerase inhibiting activity (see Chapter 7). Additional experiments could also be carried out to assess DNA damage by MSAs, for example via the COMET assay or by probing for $\gamma$-H2AX (phosphorylated-H2AX), a histone variant that is rapidly phosphorylated upon DSBs (Burma et al., 2001). If we had been able to use the 1A9 cell line rather than the MCF7 cell line, it would have been interesting to compare the ability of the MSAs to induce senescence in our 1A9-derived MSA-resistant cell lines (i.e.1A9-R1 (PelA resistant by 6-fold) and 1A9-L4 cells (PelA and laulimalide resistant by 40 -fold)), which both contain mutations in the $\beta I$-tubulin isotype (Kanakkanthara et al., 2011). If MSA-induced senescence were triggered by microtubule disruption then we would expect to see a diminished senescence response in the resistant cell lines. This would give us some clues as to whether there is an alternative microtubule-independent mechanism triggering senescence.

\subsubsection{Conclusion}

According to the SA- $\beta$-gal assay, of the four drugs that we tested Doxo was the most effective at inducing a senescent phenotype, followed by the MSAs, Disco $>$ Ptx $>$ and PelA. However, this assay does not provide us with useful information about the degree of apoptosis at each drug concentration. In terms of clinical effectiveness, drugs that are better at inducing senescence, such as Doxo, are not necessarily better than drugs that 
preferentially induce apoptosis, as may be the case for the MSAs. In fact, given the in vitro studies demonstrating that senescence can be reversed (after manipulation of DDR pathways) (d'Adda di Fagagna et al., 2003; Roberson et al., 2005), apoptosis is probably a more robust tumour suppressive mechanism. A better assessment of the relative contributions of different cell fates (i.e. senescence versus apoptosis) are needed before we can make any definitive conclusions regarding the effectiveness of PelA compared to other MSAs. After assessing the treatment of cells with relatively low concentrations of PelA, it appeared that premature senescence played an important, albeit not predominant, role in it's mechanism. Further experiments using PelA are needed to fully characterize the range of short-term (i.e. $<72$ h) and long-term $(>6$ days) responses by concurrently assessing the morphological and biochemical changes of apoptosis (e.g. annexin V/PI staining), senescence (e.g. SA- $\beta$-gal activity, flattened and enlarged morphology, activation of tumour suppressor pathways etc.), and mitotic catastrophe (e.g. multiple nuclei).

Drug discovery and mode of action studies on anti-cancer drugs generally focus on the ability of drugs to cause tumour cell death. Often neglected are the additional contributions of other cellular fates in preventing tumour growth and/or causing tumour regression. A better understanding of the induction of chemotherapy-induced premature senescence, particularly in a clinical setting, is required. It is important that the mechanisms underlying a cell's decision to follow one particular cell fate over another are determined so that therapeutics can be designed to promote specific outcomes. Another important aspect that needs further investigation is to answer the question of whether senescent cells remain in their state of arrested growth, re-enter the cell cycle, or eventually undergo cell death, as the permanence of senescence is currently under debate. The lack of distinct senescence markers is, however, hindering progress in this area. The ability of drugs to induce a state of arrested growth or premature senescence plays almost as effective a role as apoptosis; thus, understanding the full range of clinical efficacies presented by anti-neoplastic agents is important for their development. Regardless of the underlying trigger, the ability of MSAs to induce premature senescence undoubtedly contributes to their potential tumour suppressive effects. 


\section{Chapter 7. Stathmin, c-Myc and topoisomerase I role in the action of peloruside A}

\subsection{Introduction}

\subsubsection{Identifying drug targets and elucidating mode of action}

Drug discovery is a multi-step process that can prove to be very costly for pharmaceutical companies, industry partners and academia, alike. Prior to assessing the efficacy and safety in pre-clinical animal models, drug targets and effectors must be identified and validated in vitro. Ideally, a drug should be highly specific and highly active against a single target; however, it is not uncommon for drugs to affect multiple pathways through secondary target interactions (Marton et al., 1998). In fact, drugs are being marketed that have two distinct modes of action, for example, the Auckland Cancer Society Research Centre is developing a compound called PWT33597, with dual inhibitory activities against phosphoinositide-3-kinase (PI3K) and mTOR. The identification of additional secondary drug targets and elucidation of mediators underlying cellular mechanisms can be used to predict potential side effects or toxicities of a drug. Relatively recent technological advances have paved the way for more accurate large-scale and higher-throughput techniques that are now incorporated into many drug discovery programs. Global genetic and protein expression analyses via DNA microarrays and proteomics screens have not only allowed for a direct comparison of the genetic differences between malignant and healthy cells, but are also useful tools for mapping out the complex signalling networks triggered by different drugs.

\subsubsection{Effects of PelA on the cellular proteome}

Cells must be able to adapt rapidly to changes in their environment and can do so by regulating protein expression levels, or through post-translational modifications. While highly informative, one of the limitations of using DNA microarrays is that mRNA expression does not always directly correlate with protein expression (for example, as a result of differences in translation, alternative splicing or protein degradation), as cellular localization and protein-protein interactions can also affect activity (Lockhart and Winzeler, 2000; Kraljevic et al., 2006). Pioneering work carried out at the University of Sydney in the mid-90's opened up the field of proteomics, a welcome complementary approach to the huge advances that were being made in genomic sequencing capabilities (Wasinger et al., 1995; Wilkins, 1996). Proteomics refers to the 
analysis of all the proteins expressed by a genome, a somewhat more complex system than the study of a relatively unchanging genome. Proteomics traditionally relies on the ability to reproducibly, and with high-resolution, separate out thousands of proteins simultaneously using 2-dimensional gel electrophoresis (2-DE) (i.e. the separation of proteins first by isoelectric point, followed by separation by molecular weight in the second dimension) (Wilkins et al., 1995). Several techniques are available for protein quantification and identification, but it seems that the current method of choice is mass spectrometry (MS) (e.g. matrix assisted laser desorption ionization- time of flight (MALDI-TOF) or tandem MS (MS/MS)). This allows the determination of protein mass either by measuring the time it takes for a peptide to accelerate through an electric field to a detector, or by measuring the parent peptide and its daughter ions after fragmentation. Such data can subsequently be applied to comprehensive databases to identify protein matches.

Very few proteomics studies have been carried out on MTAs, possibly because tubulin is already such a well-validated target. The side effects of these drugs arising from potential secondary interactions, however, warrant further investigation. Proteomic profiling of non-small cell lung cancer H157 cells treated with Ptx, the MEK inhibitor U0126, or a combination of the two, was carried out to investigate possible mediators underlying the synergistic interactions of Ptx and U0126 in inducing apoptosis (MacKeigan et al., 2003). In this case, the authors were primarily interested in the protein changes that were unique to the combined treatment (i.e. decreased expression of two proteins involved in the apoptotic response, RS/DJ-1 (an RNA-binding regulatory subunit/DJ-1) and Rho GDI $\alpha$ (Rho GDP-dissociation inhibitor $\alpha$ )). In the paper, the proteins that changed in response to Ptx alone were not presented. Another study used a proteomics approach to carry out a comprehensive analysis of protein expression in HeLa cervical carcinoma cells (Lee et al., 2005). 2-DE identified 23 upregulated and 24 down-regulated proteins that were differentially expressed after treatment with PelA, and these proteins were subsequently identified using MALDITOF-MS. Many of the proteins that were significantly altered were involved in general cell processes such as apoptosis, cell cycle, DNA repair, immune responses, stress responses and transcription regulation. Western blotting confirmed the increased expression of BUB3 (involved in the mitotic checkpoint) and the apoptotic mediators, caspase- 8 and -5 and TRAIL, and the decreased expression of the anti-apoptotic factor BCL-2 and the transcription factor c-Myc. Several other studies, which will not be 
described in detail, have used similar methods to look for protein changes between parental cell lines and their Ptx-, vincristine-, or Vbl-resistant derivatives (Verrills et al., 2003; Yang et al., 2006; Chuthapisith et al., 2007; Dowling et al., 2007)

A comparative proteomic analysis was carried out in our laboratory by Dr Anja Wilmes to identify differences in the proteome of HL-60 cells (a human promyeloid leukemia cell line) treated with either PelA or Ptx (Wilmes et al., 2011b; Wilmes et al., 2012a). This study used 2-DE combined with differential in-gel electrophoresis (DIGE), which offers an additional advantage over standard 2-DE since complex mixtures can be separately labelled with different fluorescent dyes (e.g. Cy2, Cy3 and Cy5 dyes), combined, and then separated on the same gel (reducing the inter-gel variability). Proteome changes were assessed in response to $100 \mathrm{nM}$ PelA and $100 \mathrm{nM}$ Ptx (for 24 h). Changes that were increased or decreased $\geq 2$-fold were then identified by MALDITOF (or LC MS/MS in some cases) (Wilmes et al., 2011b; Wilmes et al., 2012a). A direct comparison of the HL-60 proteomes after treatment with either of the MSAs identified similar effects on, for example, cytoskeletal proteins (e.g. $\alpha$-tubulin IC and myosin heavy chain), and apoptotic- and stress-related proteins (e.g. nucleic acid binding proteins (hnRNPs), ATP synthase and BCL-2-associated anthanogene 2 (BAG2)).

In the studies by Wilmes et al. (2011, 2012a), there were changes in protein expression that were unique to each of the MSAs. For example, Ptx up-regulated $\alpha$-tubulin 1A, $\beta$ tubulin $2 \mathrm{C}$, eukaryotic translation elongation factor-1 and ribosomal proteins SA and $\mathrm{S} 31$, and down-regulated lamin B1, fuse binding protein, heat shock protein 105, KRAB associated protein (KAP-1), thymopoietin alpha, and upstream of NRAS. Changes unique to PelA treatment included an increased abundance of $\beta$-actin (a minor fragment), $\beta$-tubulin $\mathrm{V}$, hnRNP, succinate dehydrogenase, and tumour rejection antigen1 , and a decrease in the expression of stathmin, proteasome subunit alpha type II and proteasome activator complex subunit 3. As part of the proteomics screen of PelA, ingenuity pathway analysis (IPA) was used to map out potential interacting proteomic networks underlying the mechanism of PelA. Several interaction "hubs" were identified, including c-Myc, retinoic acid and tumour necrosis factor (TNF). Differences in microtubule stabilization (and downstream signalling) or differences in secondary targets likely underlie the altered protein expressions observed in response to PelA and Ptx. 


\subsubsection{HIP and HOP microarray analyses of PelA}

Chemical genetics is a powerful approach to probe for secondary targets and drug interactions (Cong et al., 2012). A library of gene deletion mutants can be used to probe for increased drug sensitivity, indicating possible targets or mechanistic pathways (Cong et al., 2012). In our laboratory, Dr Anja Wilmes carried out a chemical genetic screen of PelA in the yeast Saccharomyces cerevisiae, using both haploinsufficiency profiling (HIP) and homozygous deletion profiling (HOP) (Wilmes, 2008; Wilmes et al., 2012b). The HIP microarray involves heterozygous deletion of a diploid strain, in which one copy of a gene is functional and one copy is deleted. Drugs that interact with the product of a particular gene will sensitize the cell to the drug. HIP is, therefore, a valuable tool for identifying direct targets of a drug (Giaever et al., 1999; Giaever et al., 2004; Lum et al., 2004). HOP profiling uses either haploid deletion sets (no copy of a gene is present) or diploid homozygous deletion sets (both copies of a gene are deleted). Only non-essential genes can be tested using this method; thus, while HOP microarrays can also identify direct drug targets, they most often reveal proteins that are involved in buffering a cell against the cytotoxic or antiproliferative effects of a drug (i.e. "friends of the target") (Wilmes et al., 2012b). Each deleted gene is flanked by unique 20-base oligomer sequences or "barcodes" (i.e. an UP-TAG (UP) and a DOWN-TAG (DN)). PCR amplification of these barcodes and subsequent hybridization to a microarray containing complementary sequences to all tags allows individual strains to be assayed for growth from within a mixed pool (Winzeler et al., 1999).

This approach was carried out in our laboratory by Dr Anja Wilmes to identify potential targets or mechanistic pathways underlying the mode of action of PelA (Wilmes et al., 2012b). A HIP microarray using the $S$. cerevisiae diploid (BY4743) mutant library identified 13 genes that caused increased drug sensitivity (z-scores $\leq-3$ ) to $10 \mu \mathrm{M}$ PelA (cells were initially treated for $15 \mathrm{~h}$ (approximately 10 doublings), then re-diluted into $10 \mu \mathrm{M}$ PelA for an additional $15 \mathrm{~h}$ ). Topoisomerase 1 (TOPl) and Pho85 cyclin 1 (PCL1) were interesting hits because of their roles in DNA replication (Rothenberg, 1997) and cell cycle progression (Espinoza et al., 1994; Keniry et al., 2004), respectively. However, these microarray hits were unable to be validated when individual mutant clones were independently grown in PelA. Using the BY4743 homozygous deletion set, the HOP microarray generated 33 hits that had significant zscores (i.e. hits that were common between two biological replicates; z-scores $\leq-3$ ). Ignoring the seven genes that are involved in multiple-drug resistance, five genes were 
identified (RTS1, SAC1, MAD1, MAD2 and LSM1) that exhibited significant scores in both the UP and DN tags. The functions of these gene products varied, with LSM1 is involved in mRNA degradation, SAC1 encoding a lipid phosphatase, RTS1 encoding a subunit of protein phosphatase 2A, and both MAD1 and MAD2 involved in the mitotic spindle checkpoint. Other gene hits were also identified that only had the UP or DN tag amplified, but not both. These genes were involved in mitosis, and included BIKI (orthologous to the human plus-end tracking protein CLIP-170), SLI15 (the mammalian equivalent of INCENP, which complexes with Aurora B as part of the chromosomal passenger complex) and CTF19 (involved in regulating the interaction of kinetochores with the mitotic spindle in budding yeast) (Hyland et al., 1999; Tanaka et al., 2002; Tanenbaum et al., 2006; Wilmes et al., 2012b). These mitosis-related hits are particularly interesting because they promote correct microtubule-kinetochore attachments and function to protect the cell against chromosome mis-segregation, which has been observed upon low-concentration PelA treatment (see Chapter 4). 


\subsubsection{Aims}

As with other large-scale assays, the results from the proteomics screen need to be independently validated. BAG-2 and myosin $2 \mathrm{~A}$, which were identified as changing in abundance in response to both PelA and Ptx, had been previously validated by Western blot analyses (Wilmes et al., 2012a). Although we did not have the resources to validate all of the remaining protein expression changes that had been identified in the proteomics screen, two additional proteins, stathmin and c-Myc, were chosen to independently assess changes in expression levels in response to PelA.

Stathmin acts as a microtubule-destabilizing protein, and the binding of Ptx and Vbl are decreased and increased, respectively, in cells overexpressing stathmin (Alli et al., 2002). Despite these opposing effects, the inhibitory effects of stathmin on drug-binding (Ptx only) and progression through to mitosis (Ptx and Vbl) decreased the sensitivity of several breast cancer cell lines to these drugs (Alli et al., 2002; Alli et al., 2007). Stathmin is often overexpressed in breast cancer and is involved in some mechanisms of resistance to MTAs (Curmi et al., 2000; Alli et al., 2002; Alli et al., 2007); thus, it is important to understand any effects PelA might have on stathmin in a clinical setting. This led to the first aim of this chapter:

1) To validate the changes in expression of stathmin in the HL-60 cell line, using Western blotting.

The MYC family of proto-oncogenes comprises four family members; c-, N-, L- and SMyc. Myc proteins are overexpressed in many cancer types and can also promote tumourigenesis. These proteins act as transcription factors, regulating up to $15 \%$ of all genes, and seem to be involved in many aspects of cellular function (e.g. cell proliferation, differentiation, growth, apoptosis, cell cycle progression, and tumourigenesis) (Patel et al., 2004; Adhikary and Eilers, 2005). c-Myc, which was identified as a hub in PelA and Ptx action in HL-60 cells by IPA analysis (Wilmes et al., 2011b; Wilmes et al., 2012a), acts in complex with its binding partner Max (Blackwood et al., 1992). Previous studies have demonstrated a reduced expression of c-Myc upon treatment with Ptx, 2-ME and Disco (Klein et al., 2005; Lee et al., 2005; Chow et al., 2008), although it should be noted that other classes of drugs (e.g. DNA intercalators) have triggered a similar inhibition (Villamarín et al., 2002). Activation of MAD2 and 
BubR1 genes (involved in the SAC; see Chapter 4) by c-Myc (Menssen et al., 2007), as well as its role in the induction of senescence (Wu et al., 2007; Zhang et al., 2012), were particularly interesting in terms of the potential mediators underlying the cellular response to PelA. A second aim of this chapter was, therefore:

2) To investigate the effect of PelA on c-Myc expression over time, using Western blot analyses of HL-60 cells.

Chemical genetics assays have the potential to identify novel secondary targets or mediators of a drug (Smith et al., 2010). TOP1 and MAD2 deletion mutants (top-I $\Delta$ and $\operatorname{mad} 2 \Delta$, respectively) were found to be sensitized to PelA, suggesting that these proteins are involved in mediating the drug's effects (Wilmes, 2008; Wilmes et al., 2012b). $M A D 2$ was also identified as a hit in the HOP assay, and its potential role in aneuploidy induction by PelA was investigated in the present study (see Chapter 4). DNA topoisomerase (TOPO) enzymes are crucial for DNA replication. They act to relieve the tensional strain generated from DNA supercoiling by transiently introducing single(type I TOPOs) or double-stranded (type II TOPOs) nicks into DNA. This allows the newly free DNA strand to unwind before being reannealed, permitting procession of the replication fork (Topcu, 2001). TOPO inhibitors can act as "TOPO poisons" that bind the intermediate TOPO-DNA cleavage complex, or as "TOPO inhibitors" that interfere with the catalytical activity of the enzyme (Rothenberg, 1997). Since TOPO I, was identified in a HIP microarray as a possible secondary target of PelA (Wilmes et al., 2012b), DNA damage could offer a plausible mechanistic explanation for the induction of premature senescence by PelA (see Chapter 6). A third aim of this chapter was therefore:

3) To validate TOPO I as a secondary target of PelA using a relaxation assay. This particular assay is an in vitro, cell-free system that assesses the ability of test compounds to inhibit the relaxation of supercoiled DNA by TOPO I. Due to its more compact structure, supercoiled DNA can be distinguished from relaxed topoisomers by agarose gel electrophoresis (i.e. supercoiled DNA migrates faster).

Unfortunately, the TOPO I results were unsuccessful and we could not make any definitive conclusions from our data; thus, the methods, results and brief conclusions for this particular aim are presented in Appendix G. 


\subsection{Methods}

\subsubsection{Western blotting for c-Myc}

Western blotting for c-Myc was carried out as described in the General Methods. Briefly, HL-60 cells were seeded into 6-well plates at a density of $3 \times 10^{6}$ cells per well. The following day, cells were treated with either $30 \mathrm{nM}$ Pel, $30 \mathrm{nM}$ Ptx or vehicle (ethanol). At various timepoints $(6,18$ and 24 h) HL-60 cells were harvested for Western blot analysis. Proteins were resolved by 10\% SDS-PAGE (40 $\mu \mathrm{g} / \mathrm{lane})$ and transferred onto a low fluorescence background PVDF membrane (Immobilon FL, Millipore). Membranes were probed overnight at $4{ }^{\circ} \mathrm{C}$ with the primary anti-c-Myc antibody (Abcam Inc., ab32, 1:500). Membranes were incubated with the secondary antibody, anti-mouse IgG-Cy5, for $1 \mathrm{~h}$ at RT (GE-Healthcare, 1:2500). Actin was probed as a loading control, by incubation with antibody (Sigma, anti-actin, 1:50000) for $15 \mathrm{~min}$ at $\mathrm{RT}$, followed by a $1 \mathrm{~h}$ incubation at RT with anti-mouse IgG-Cy5 secondary antibody (GE-Healthcare, 1:4000) for.

\subsubsection{Western blotting for stathmin}

HL-60 cells were used to validate changes of stathmin in response to PelA. Cells that were approximately $70-80 \%$ confluent (i.e. still in the exponential phase of cell growth) were seeded at $3 \times 10^{6}$ cells per T25 $\mathrm{cm}^{2}$ tissue culture flask. In a total volume of $10 \mathrm{~mL}$ of medium, cells were treated with $40 \mathrm{nM}$ or $100 \mathrm{nM}$ PelA or Ptx (or the EtOH equivalent of the $100 \mathrm{nM}$ treatment). A sufficient number of flasks were set up so that one could be harvested at each timepoint $(0,6,18$ or $24 \mathrm{~h})$. Protein was isolated and quantified as described in the General Methods. Protein (25 $\mu \mathrm{g}$ per lane) was resolved by $12 \%$ SDS-PAGE and transferred electrophoretically onto PVDF membranes. Membranes were blocked in 5\% BSA/PBST $(0.1 \%$ Tween-20) for $1 \mathrm{~h}$ at RT before probing overnight at $4{ }^{\circ} \mathrm{C}$ with the primary rabbit anti-stathmin antibody (Abcam, Inc., ab28; diluted 1:10, 000 in blocking buffer). Protein was detected by incubating membranes with anti-rabbit IgG-Cy5 secondary antibody (GE healthcare; 1:2000 dilution in PBST) for $1 \mathrm{~h}$ at RT. Membranes were the probed for actin $(1: 4000$ in $\mathrm{BSA} / \mathrm{PBST}$ ) for $15 \mathrm{~min}$ at RT followed by another $15 \mathrm{~min}$ incubation with the same secondary antibody at RT. 


\subsection{Results}

\subsection{1 c-Myc expression is decreased by both PelA and Ptx}

A previous study by our laboratory group had looked at c-Myc expression at 6 and $24 \mathrm{~h}$ after treatment with $100 \mathrm{nM}$ PelA (Wilmes et al., 2011b). The possible link between cMyc and aneuploidy led us to extend this study to investigate the expression of c-Myc at concentrations that induced aneuploidy in the HL-60 cell line (i.e. $30 \mathrm{nM}$ ). This timecourse study used Western blot analyses to assess changes in c-Myc expression at 6, 18 and $24 \mathrm{~h}$ (Fig. 7.1) and was published as part of a follow up proteomics paper comparing these two MSAs (Fig. 4 from Wilmes et al., 2012a). At 6 hours, although we observed no change in c-Myc expression after PelA treatment, we did observe a 34\% reduction (not statistically significant) in expression in response to Ptx (Fig. 7.1, A). By 18 hours, the effects on c-Myc expression were more comparable (i.e. a $46 \%$ and $51 \%$ reduction in response to PelA and Ptx, respectively). At the latest time point (24 h), Ptx treatment triggered a further reduction (69\% compared to controls) in c-Myc expression. In contrast, in the presence of PelA, c-Myc protein levels appeared to recover slightly compared to the $18 \mathrm{~h}$ levels.

\subsubsection{PelA and Ptx reduce stathmin expression levels}

To look at changes in stathmin expression, HL-60 cells were treated with high (100 nM) or low $(40 \mathrm{nM})$ concentrations of PelA or Ptx for 6,18 or $24 \mathrm{~h}$ and the cell lysates analysed by Western blotting (Fig. 7.2). Both MSAs decreased stathmin expression, although there were differences in the extent to which each drug had an effect. For example, PelA at $40 \mathrm{nM}$ triggered a similar reduction in stathmin expression to Ptx, regardless of the incubation time (i.e. $32-40 \%$ decrease; see Fig. 7.2 A and C). As expected, a higher concentration of PelA (100 nM) reduced stathmin levels even further, but again, there was not a large difference over time (i.e. a 50-60\% decrease). After 6 h, Ptx gave a similar response to PelA at both concentrations (a 40\% decrease), but at 18 and $24 \mathrm{~h}$, stathmin was decreased more by Ptx than PelA at both concentrations (Fig. 7.2 B and D). A point of difference between PelA and Ptx was that the high concentrations of Ptx resulted in very similar levels of stathmin expression as seen for the lower concentration. At each of the timepoints tested, differences between high and low Ptx were never greater than 10\%; whereas, with PelA, differences of 18 $24 \%$ were observed. 
A

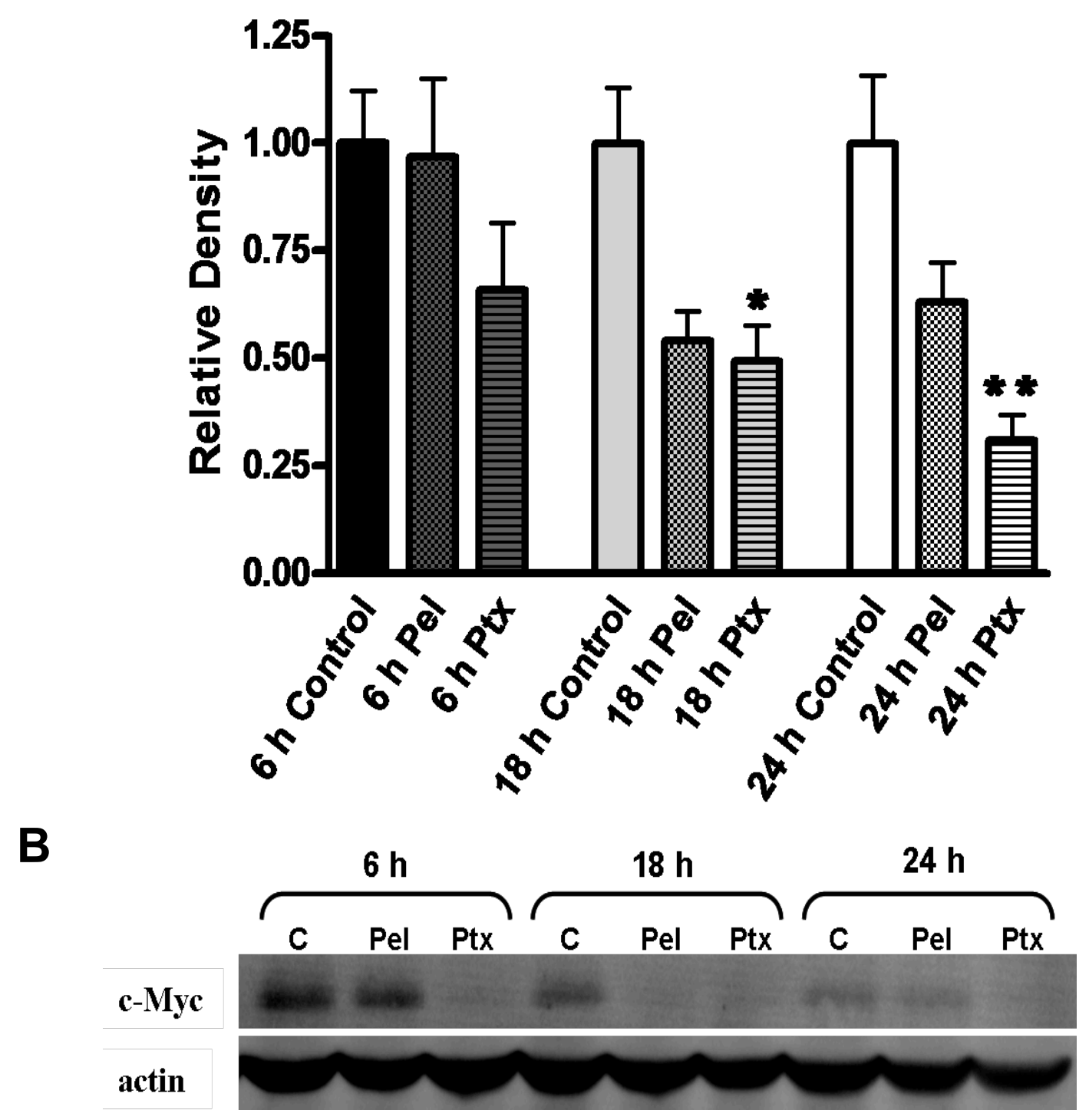

Figure 7.1. PelA and Ptx reduce c-Myc expression levels. HL-60 cells were treated with $30 \mathrm{nM}$ PelA (Pel), 30 nM Ptx (Ptx) or vehicle (C) for 6 h, 18 h or 24 h. Protein from whole cell lysates was resolved on 10\% SDS-PAGE gels $(40 \mu \mathrm{g})$ and probed for c-Myc and actin. (A) Relative densities of c-Myc from the Western blot analyses are presented, normalized to each time-point vehicle control (A). Bars represent the mean and SEM of 8 independent experiments. ${ }^{*} \mathrm{P}<0.05 ; * * \mathrm{P}<0.01$ as determined by a one-way ANOVA, with Bonferroni post-hoc test. (B) A representative Western blot showing the effects of Pel and Ptx on c-Myc over time. Reproduced from Wilmes et al. (2012a). 
A

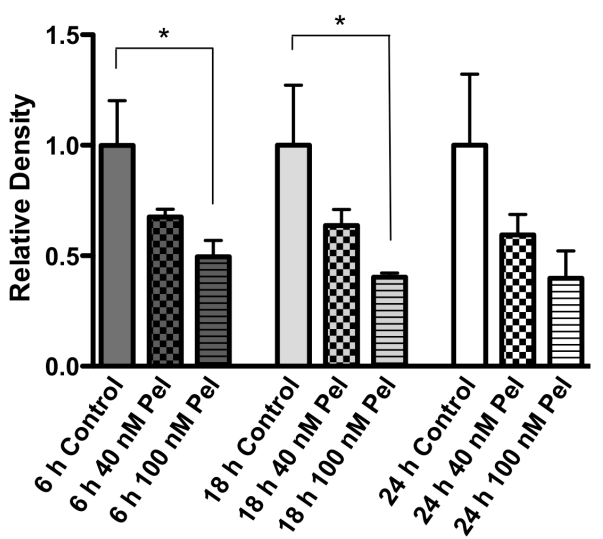

B

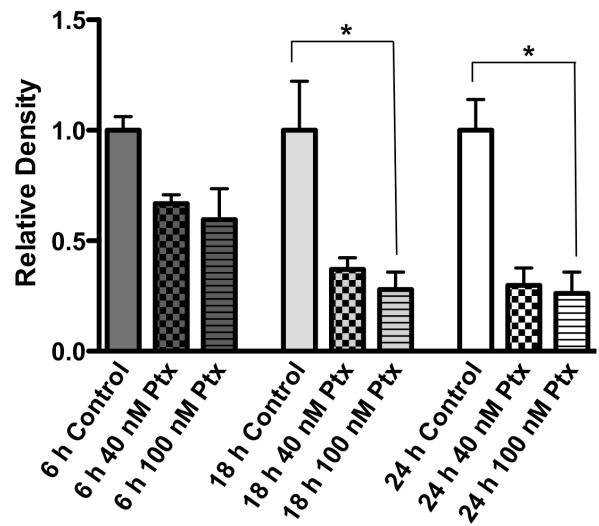

C Peloruside A

$\mathbf{O h}$

$6 \mathrm{~h}$ $18 \mathrm{~h}$

$24 \mathrm{~h}$

$\begin{array}{llll}C & C & 40 & 100\end{array}$

C $\quad 40 \quad 100$

C $\quad 40 \quad 100$

Stathmin

Actin

D

Paclitaxel

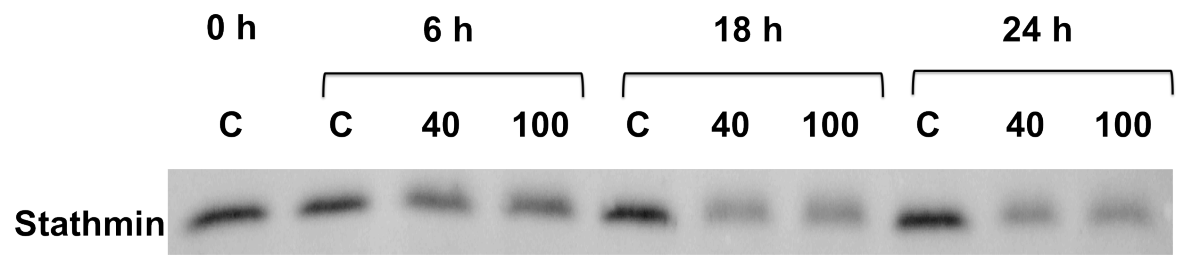

Actin

Figure 7.2. PelA and Ptx reduce stathmin expression levels. HL-60 cells were treated with vehicle (C), $40 \mathrm{nM}$ or $100 \mathrm{nM}$ PelA (Pel) or paclitaxel (Ptx) for $6 \mathrm{~h}, 18 \mathrm{~h}$ or $24 \mathrm{~h}$. Protein from whole cell lysates was resolved on $12 \%$ SDS-PAGE gels $(25 \mu \mathrm{g} / \mathrm{lane})$ and probed for stathmin and actin. Graphs in (A) and (B) indicate the relative densities of stathmin after treatment with PelA and Ptx, respectively. Bars represent the mean and SEM of 3 independent experiments. $* \mathrm{P}<0.05$, as determined by a one-way ANOVA with Bonferroni post-hoc test. Representative Western blots showing the effects of PelA and Ptx on stathmin expression levels over time are presented in (C) and (D), respectively. The densities of the bands were calculated relative to the appropriate vehicle controls and normalized to the actin loading control. 


\subsection{Discussion}

\subsubsection{Inhibition of c-Myc expression by PelA and Ptx}

c-Myc expression is deregulated in many cancers and has been implicated in malignant transformation and tumourigenesis (Pelengaris et al., 2002). Through Western blot analysis, it was confirmed that the expression of c-Myc (which was identified in the IPA analysis) was altered by treatment with PelA. We observed a time-dependent decrease in c-Myc expression in response to PelA and Ptx, although the effects of the latter were slightly more potent. Myc proteins are estimated to bind to $\sim 25,000$ sites on the human genome, and hence, regulate a large proportion of genes, some of which are likely to be directly involved in the mechanism of PelA action (Adhikary and Eilers, 2005). c-Myc can act to transcriptionally activate or suppress a range of downstream target genes including those involved in cell cycle progression (e.g. CDK4 and cyclin B1) (Hermeking et al., 2000; Yin et al., 2001) and apoptosis (e.g. BCL-2, BAG-2 and ARF) (Zindy et al., 1998; Eischen et al., 2001; Zhang et al., 2012). That c-Myc is affected by MTAs is not surprising, although whether c-Myc expression is downregulated or up-regulated appears to be largely context dependent. In some studies, cMyc expression has been shown to decrease in response to an MDA like 2-ME (in human acute myeloid leukemia cells and human myeloma cells) and an MSA like Ptx (in cervical carcinoma cells) (Lee et al., 2005; Zhou et al., 2007; Chow et al., 2008); whereas, in another study, c-Myc protein levels were unchanged in response to Ptx and the two MDAs, Vbl and podophyllotoxin (in the human B-cell line p493-6) (Frenzel et al., 2011). An earlier study demonstrated the differential effects that cell-line, MTA concentration, and the type of microtubule-disruption (i.e. stabilization or destabilization via Ptx, or $\mathrm{Vbl}$ and nocodazole, respectively) can have on c-Myc transcription (Bourgarel-Rey et al., 2000). The cellular role of c-Myc often seems paradoxical and is proving difficult to elucidate, as c-Myc overexpression can promote unrestrained cell proliferation, as well as sensitize certain cells to apoptosis (Nilsson and Cleveland, 2003). It is not clear why we see a plateau in the degree of c-Myc expression in response to PelA over time, compared to a continuous reduction as seen with Ptx. Despite having the same primary target, a number of proteins were differentially up- or down-regulated in the proteomics screen, highlighting the distinct signalling pathways induced by Ptx and PelA, which are likely to affect different regulatory proteins upstream of c-Myc. 
Additional functional studies are needed to further characterize the extent to which the decrease in c-Myc induced by both PelA and Ptx in our study mediates the effects of these drugs. As we have shown, PelA has quite distinct cellular responses (e.g. premature senescence, inhibition of angiogenesis and aneuploidy induction) that have all in some way or another been linked to c-Myc and/or its downstream signalling mediators. BAG-2, for example, was up-regulated 3.7- and 4-fold in response to PelA and Ptx in the proteomics screens from our laboratory (Wilmes et al., 2011b; Wilmes et al., 2012a). A recent paper demonstrated that c-Myc, in complex with the transcription factor specificity protein 1 (SP1), acts to negatively regulate BAG-2 transcription (Zhang et al., 2012). Using a proteomics approach, Zhang et al. showed that downregulation of c-Myc consistently led to an increase in BAG-2 protein abundance, which was correlated with the p21-mediated induction of cellular senescence. Senescence and tumour regression following c-Myc inactivation may require the simultaneous increased expression or activation of other tumour suppressor proteins, such as p16 ${ }^{\text {Ink4a }}, \mathrm{p} 53$ and $\mathrm{pRb}$ (Wu et al., 2007). Down-regulated c-Myc is likely to influence the induction of premature senescence, as observed in the MCF7 cell line in the present study (see Chapter 6). Similarly, the anti-angiogenic effects of MSAs such as PelA (see Chapter 5) are likely to be influenced by c-Myc levels in an in vivo setting. It has been shown that c-Myc can increase mRNA expression of the pro-angiogenic cytokine VEGF as well as increase mRNA turnover of the angiogenesis inhibitor thrombopsondin-1 (TSP-1) (Janz et al., 2000; Knies-Bamforth et al., 2004).

Previously, it has been shown that the activation of Mad2 and BubRl gene expression by c-Myc delayed the duration of prometaphase (Menssen et al., 2007). We chose to follow the expression of c-Myc using $30 \mathrm{nM}$ PelA or Ptx because there may be a correlation between c-Myc and aneuploidy induction at low concentrations of these drugs (Ikui et al., 2005; Wilmes et al., 2011b; Wilmes et al., 2012a). However, Menssen et al. did not find a link between increased MAD2 and BUBR1 protein abundance and the induction of chromosomal instability (which can cause chromosome missegregation) (Menssen et al., 2007), and similarly we did not observe any changes in MAD2 or BUBR1 expression in response to PelA (see Chapter 4; note that this study was done in 1A9 cells and we did not measure c-Myc expression in this cell line). This suggests that if c-Myc is involved in aneuploidy induction, it is not likely to be through regulation of MAD2 or BUBR1 expression. 


\subsubsection{Inhibition of stathmin by PelA and Ptx}

In the proteomics screen, stathmin was identified as a protein that changed in response to PelA but not Ptx (Wilmes et al., 2011b; Wilmes et al., 2012a). The results from the Western blot analysis in the present study, however, indicate that Ptx induces a similar (although slightly more potent) decrease in stathmin protein abundance to PelA. One of the caveats of global protein analysis is that protein changes are scored based on a predetermined threshold level of change. For example, stathmin did actually decrease in response to Ptx but, unlike with PelA, did not meet the threshold cut-off level $(\geq 2$ fold). Decreased stathmin expression in response to MSAs is interesting because of the dual role of this protein as a microtubule-destabilizing protein and cell cycle regulator. At the $G_{2} / M$ transition, stathmin becomes inactivated via phosphorylation at four serine residues (see Appendix F, Figure F.1), allowing progression through to mitosis (Larsson et al., 1995). This is likely to be important in allowing the formation of the mitotic spindle (Alli et al., 2007). Furthermore, stathmin overexpression is correlated with a poor prognosis and disease aggressiveness in several cancer types (e.g. breast, endometrial and oral squamous carcinomas) (Curmi et al., 2000; Kouzu et al., 2006; Trovik et al., 2011) as well as a reduced sensitivity to the MTAs Ptx and Vbl (Alli et al., 2002). Attempts to reverse the effects of stathmin overexpression and increase cancer cell susceptibility to MTAs have so far worked well. For example, a recent study found that silencing either stathmin or Wee-1 protein kinase (involved in delaying $G_{2} / M$ progression) increased the susceptibility of cancer cells to both Ptx and Vbl (Alli et al., 2007). These results were similar to a previous study in erythroleukemic cells (K562) (Iancu et al., 2001). Iancu et al. (2001) had shown that antisense inhibition of stathmin increased sensitivity to Ptx; however, in contrast to the results from Alli et al. (2007), this study found that sensitivity to Vbl was decreased. More recently, down-regulation of stathmin using an siRNA approach in the retinoblastoma cell line Y79 indicated that these cells were 10- and 2-fold more sensitive to Ptx and vincristine treatment, respectively (Mitra et al., 2011). The authors suggested that targeting stathmin in combination therapy with MTAs could generate synergistic effects that would allow the use of lower concentrations of drug and potentially reduce drug side effects. If cells are also sensitized to PelA by stathmin inhibition, then PelA's effects on stathmin abundance could act in a similar fashion to a positive-feedback mechanism to enhance its chemotherapeutic effectiveness. 


\section{Chapter 8. Final Discussion}

\subsection{Significance}

Compounds that target microtubules are currently recognized as one of the most successful classes of anti-cancer agents; however, as with all drugs, MTAs are not without their limitations, and there will always be a need for novel, improved therapeutics. Microtubules play a crucial role in the formation of the mitotic spindle, as well as the accurate segregation of chromosomes during cell division. Minor disruption to this tightly regulated process can be hugely detrimental to cells, leading to a prolonged mitotic arrest that typically results in apoptosis (Jordan and Wilson, 2004). It has become apparent that apoptosis only represents one of several different cell fates after microtubule perturbation. Cellular senescence, mitotic slippage (exit from mitosis into $\mathrm{G}_{1}$ without cell division), mitotic catastrophe (a form of cell death occurring during mitosis) and aneuploidy, have all been observed in response to MTAs and highlight their complex cellular actions (Ikui et al., 2005; Klein et al., 2005; Brito and Rieder, 2006; Gascoigne and Taylor, 2008; Wilmes et al., 2011b; Galàn-Malo et al., 2012).

Despite their widespread use in the clinic, the precise mechanisms underlying such varied responses has continued to perplex both research scientists and clinicians. Tumour cells are highly heterogeneous, in part due to their inherent genomic instability and predisposition towards genetic mutations. This feature contributes to a high rate of acquired resistance in tumours, one of the main reasons that drugs often fail in the clinic. Elucidating the signalling pathways linking microtubule-disruption to such varied cellular outcomes can help to identify potential markers that can pre-determine sensitivity or resistance towards MTAs. This will also enable us to use these drugs to their full potential and improve on the use of highly targeted combination therapies. In an effort to provide continued encouragement for its clinical development, we have investigated some of the potential tumour suppressive effects of the novel MSA PelA. We have also proposed potential mechanisms underlying the induction of alternative cell fates, as summarized in Figure 8.1.

As indicated in the diagram, PelA concentration and/or the duration of drug exposure can elicit quite distinct cellular mechanisms, which can make these studies difficult to interpret. Some of these cell fates can occur simultaneously or sequentially. In a given 
population of cells, for example, aneuploidy and apoptosis are likely to occur simultaneously, but in different subsets of cells. However, cells that become aneuploid may also eventually be triggered to undergo apoptosis. Similarly, p53 is known to be involved in the induction of senescence, as well as apoptosis, suggesting that depending on the context, some cells are induced to undergo cell death or to follow an alternative cell fate. These distinct cellular pathways were observed over a range of drug concentrations (i.e. at lower drug concentrations $\leq 40 \mathrm{nM}$, or higher drug concentrations $>100 \mathrm{nM}$ ). It is likely that PelA must reach a threshold level of signalling in order to trigger specific pathways. Which threshold is crossed first may ultimately decide the cell fate pathway that is induced. From a therapeutic perspective, understanding the factors that influence these cell fate decisions is an important aspect of drug optimization, as some of these signalling pathways could be manipulated to control treatment outcomes. 


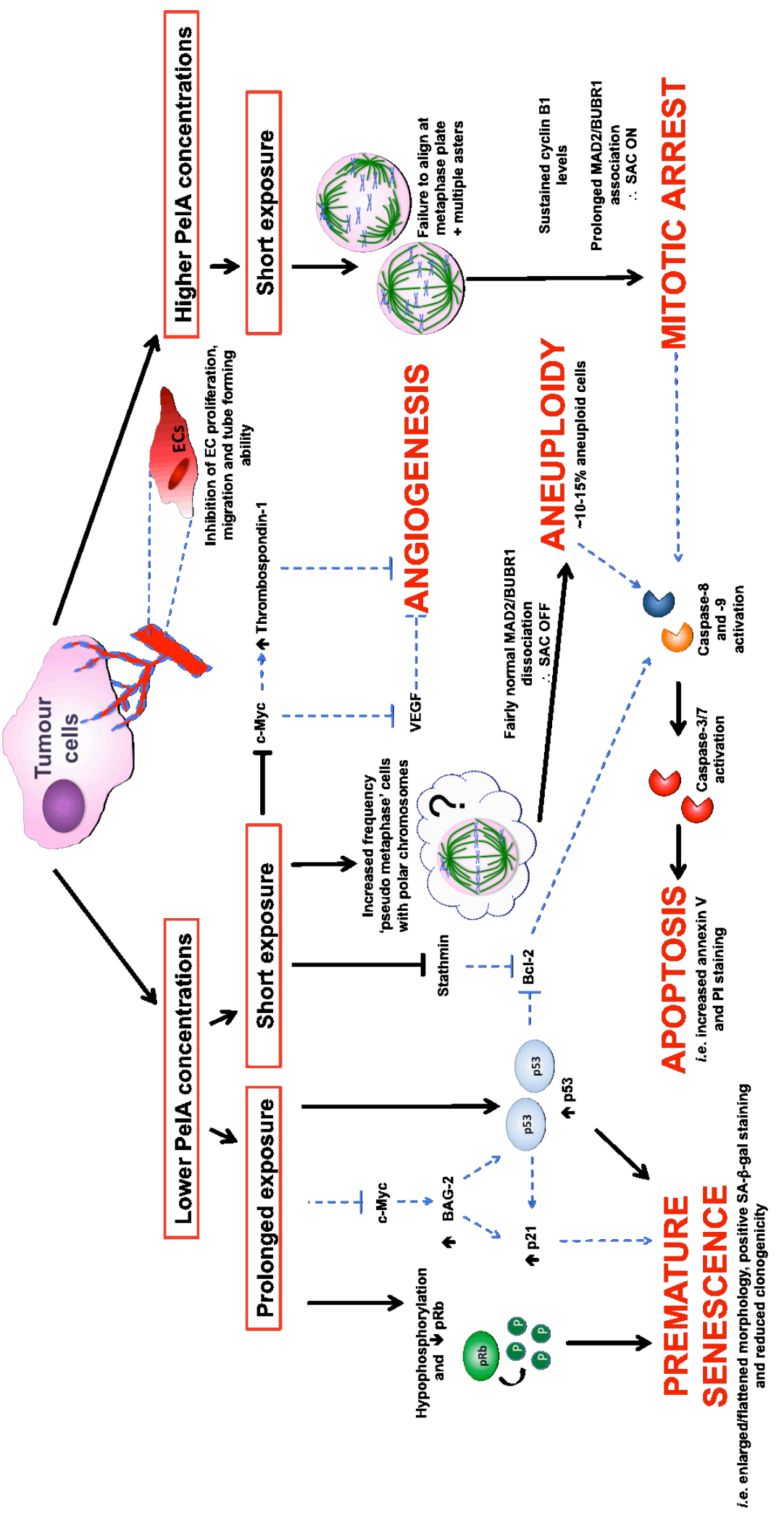

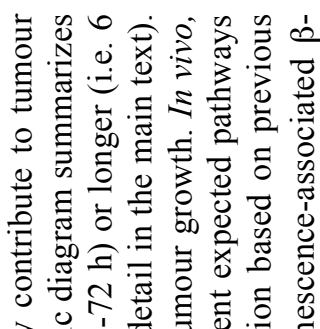

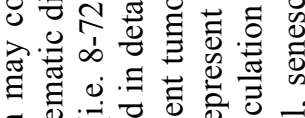

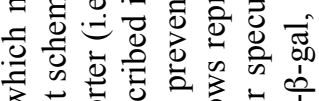

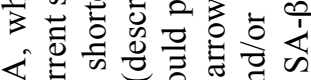

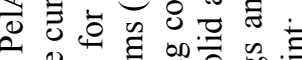

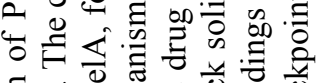

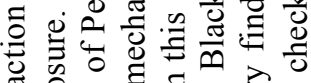

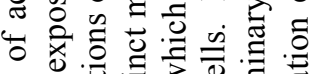

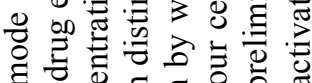

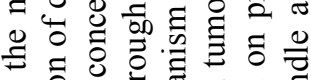

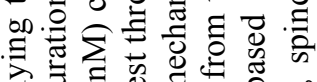

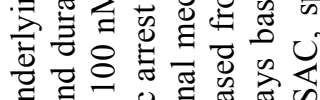
要

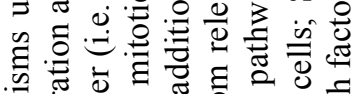

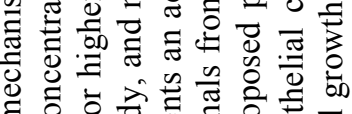

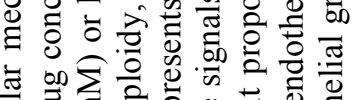

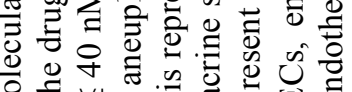

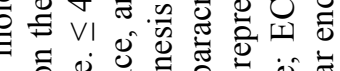

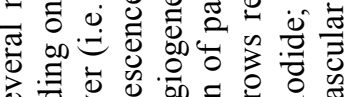

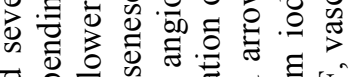

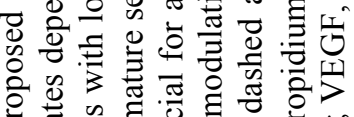

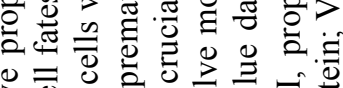

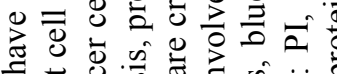

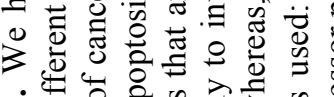

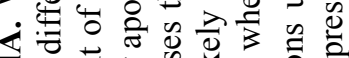
ब

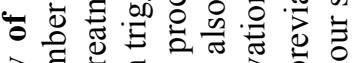

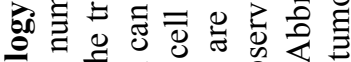

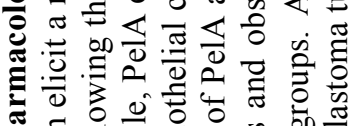

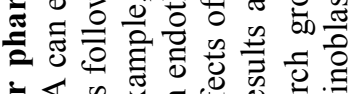

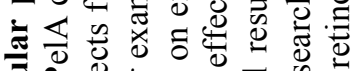

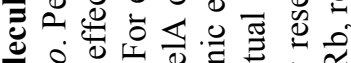

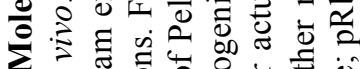

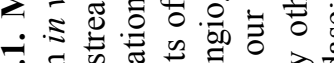
o

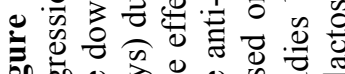

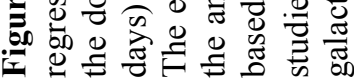




\subsection{Summary of findings}

\subsubsection{PelA induces apoptosis via a caspase-dependent mechanism in $1 \mathrm{A9}$ cells}

Experiments carried out by Dr Tom Gaitanos suggested that PelA could induce apoptosis by a caspase-independent mechanism (Gaitanos et al., 2004). Another study identified a cathepsin B-mediated mechanism of apoptosis in response to Disco, but not Ptx, in NSCLC cells (Bröker et al., 2004), and we hypothesized that this might also be the case for PelA. This led us on a somewhat convoluted approach to identify the mediators of PelA-induced apoptosis (see Chapter 3). We tested PelA in the presence or absence of a panel of protease inhibitors. Only the pan-caspase inhibitor zVAD-fmk conferred any significant protective affects against the anti-proliferative actions of PelA (as determined by MTT assays) or known markers of apoptosis (i.e. positive annexin-V and PI staining). The cathepsin B inhibitor CA-074Me conferred protective effects against PelA during the initial stages of apoptosis (annexin V staining) but was clearly not as effective as zVAD-fmk. Previous work in the HL-60 cell line followed caspase-8 or -9 activation up to $24 \mathrm{~h}$ after treatment with $100 \mathrm{nM}$ PelA, with no distinct activation of either of these proteases (Miller et al., 2004; Gaitanos, 2005). Despite these previous results, $1 \mathrm{~A} 9$ cells treated with $38 \mathrm{nM}$ PelA (the $\mathrm{IC}_{80}$ ) triggered caspase- 8 , -9 and $-3 / 7$ activation, which tended to peak between 24 and $48 \mathrm{~h}$ (determined using FLICA probes or an ApoLive-Glo ${ }^{\mathrm{TM}}$ assay). These results strongly suggest a caspase-dependent mechanism of cell death by PelA that requires prolonged drug treatment (presumably to allow cells to enter mitosis). Due to the lack of specificity in our probes, we could not definitively conclude whether PelA acts via the death receptor (caspase-8) or mitochondrial pathways (caspase-9). Previous studies suggest that MTAs primarily trigger apoptosis via the latter, although this remains to be shown for PelA (Estève et al., 2007). In Chapter 7, we also followed up hits from a proteomics study to identify possible mediators of PelA's effects in HL-60 cells (Wilmes et al., 2011b; Wilmes et al., 2012a) and found that stathmin was decreased in abundance after PelA treatment. Stathmin has been linked to the the anti-apoptotic protein BCL-2 and also influences sensitivity to other MTAs (Iancu et al., 2001; Alli et al., 2002; Alli et al., 2007; Wang et al., 2011); thus, as suggested in Fig 8.1 stathmin is likely to contribute to PelA-induced apoptosis. 


\subsubsection{Low concentrations of PelA induce aneuploidy and may involve incorrect microtubule-kinetochore attachments}

In addition to triggering apoptosis, low concentrations of MSAs lead to a concentrationdependent induction of aneuploidy in the absence of a significant $\mathrm{G}_{2} / \mathrm{M}$ arrest (Ikui et al., 2005). In Chapter 4 we observed peak levels of aneuploidy (10-14\% of cells) at low concentrations of PelA $(\sim 40 \mathrm{nM})$ and sought to identify a possible underlying mechanism. Using Co-IP and Western blotting we assessed the association and dissociation profile of two SAC proteins MAD2 and BUBR1, with the mitotic regulator p55CDC, as cells progressed through mitosis. Consistent with the observed mitotic arrest, a high concentration of PelA $(100 \mathrm{nM})$ triggered prolonged expression of cyclin B1 (a protein that must be degraded during at the end of metaphase for anaphase to occur (Peters, 2006)) and sustained association of MAD2 and BUBR1 with p55CDC. Although entry into mitosis was delayed by $\sim 1 \mathrm{~h}$, cells treated with low concentrations of PelA exhibited a similar cyclin B1 expression and degradation profile compared to controls. Association and disassociation of the SAC proteins was also fairly normal. From these results it can be concluded that the SAC is inappropriately being silenced, despite the presence of erroneous microtubule-kinetochore attachments at low concentrations.

Merotelically attached chromosomes are a common mechanism underlying aneuploidy in mammalian cells (Cimini et al., 2001; Cimini et al., 2003) and have been observed after release from nocodazole blocks (Cimini et al., 2001). Additionally, merotelic attachments are not detected as faulty by the SAC, because they provide the necessary kinetochore tension and microtubule occupancy to satisfy the SAC (Maiato et al., 2004; Salmon et al., 2005). An increase in the frequency of merotelic attachments at low, but not high concentrations of PelA, offered an attractive explanation for the increase in aneuploidy. Preliminary confocal experiments, however, hinted at a possible alternative defective mechanism that will need further investigation. No lagging chromosomes (which would be indicative of merotelic attachments) were observed at either of the PelA concentrations tested. At low concentrations, however, we did observe 'pseudometaphase' cells where approximately 1-6 chromosome pairs failed to align at the metaphase plate (Fig. 4.11), despite alignment of the remaining chromosomes. These polar chromosomes were positioned close to (or sometimes behind) the mitotic spindle poles, possibly through monotelic attachments. If not corrected, monotelically attached chromosomes will lead to chromosome mis-segregation (Maiato et al., 2004). 
Monotelic attachments, however, activate the SAC so if this were the mechanism underlying aneuploidy these cells would need to somehow bypass the SAC. At high concentrations of PelA we observed a similar phenomenon where chromosomes were located close to the centrosomes (Fig. 4.12); however, the failure of the remaining chromosomes in these cells to align at the metaphase plate is likely to generate a strong SAC signal that subsequently leads to mitotic arrest. This may account for some of the concentration differences in PelA-induced aneuploidy. This induction of aneuploidy raised some interesting questions: Do these cells ultimately become apoptotic? Can we increase or decrease the frequency of chromosome mis-segregation? If low concentrations of MSAs can induce aneuploidy, could this actually act to promote tumourigenesis in a clinical context? Aneuploidy has long been known to promote tumourigenesis (Weaver and Cleveland, 2007); thus, the ultimate fate of these MSAinduced aneuploid cells and the clinical relevance of drug-induced aneuploidy still needs to be investigated.

\subsubsection{Potential tumour suppressive mechanism of PelA: Induction of premature senescence and inhibition of angiogenesis}

One of the broader goals of this project was to identify PelA-induced tumour suppressive mechanisms that would further evaluate PelA as a potential drug candidate. Effective tumour regression does not rely solely on the ability of drugs to elicit an apoptotic or necrotic response in he tumour, and Chapters 5 and 6 demonstrate an alternative mechanism. Drugs that can trigger a permanent, cytostatic effect in tumour cells can be as effective in controlling disease as drugs that act primarily through cytotoxic mechanisms. Previous reports identified Disco, but not Ptx as a potent inducer of premature senescence (Klein et al., 2005). Our study was the first to assess the induction of senescence in response to MSAs that bind to the distinct, non-taxoid PelA/laulimalide binding site on tubulin (Gaitanos et al., 2004). We treated MCF7 cells for 6 days with several MSAs and compared the actions of these drugs with a powerful inducer of senescence, Doxo. We considered a senescent phenotype to be an enlarged/flattened morphology and the positive staining for SA- $\beta$-gal activity, a widely accepted method for assaying for cellular senescence (Dimri et al., 1995). Although the MSAs were not as effective as Doxo, which induced premature senescence in up to $62 \%$ cells, Disco, PelA and Ptx triggered senescence (approximately 31-40 \% senescence) at the highest concentrations tested ( $\mathrm{IC}_{50}, \mathrm{IC}_{70}$ and $\mathrm{IC}_{25}$, respectively). A typical feature of senescent cells is that they are metabolically active but do not replicate their DNA. Our 
EdU cell proliferation assay was unable to demonstrate whether or not treatment with PelA led to decreased DNA synthesis. However, we were able to show that the induction of premature senescence correlated with a decreased replicative ability of these cells once replated in a clonogenic assay.

This activation of premature senescence appeared to involve both a transient upregulation of the tumour suppressor p53 and the decreased expression of another tumour suppressor, pRb (the majority of which was in a hypophosphorylated, active state). These signalling pathways are thought to be important in the initial senescence trigger (p53) and maintenance of the senescent phenotype (pRb) (Alcorta et al., 1996; Chang et al., 1999a; Stein et al., 1999; Te Poele et al., 2002; Klein et al., 2005). pRb is important in binding to and inhibiting the E2F-family of transcription factors, which control the expression of proteins necessary for DNA replication (Kato et al., 1993; Weinberg, 1995; Giono and Manfredi, 2006). Activation of $\mathrm{pRb}$ through hypophosphorylation facilitates senescence by preventing progression through the $G_{1} / S$ checkpoint. Many of the drugs that are known to induce senescence activate these tumour suppressor pathways in response to DNA damage. Our attempts at linking this induction of senescence with potential DNA-damage by PelA were inconclusive (Chapter 7). Topoisomerase I (TOPO I), an enzyme involved in DNA replication, was identified as a potential secondary target of PelA in a chemical genetics HIP microarray (Wilmes et al., 2012b). Unfortunately, inconsistencies in our TOPO I relaxation assay failed to provide us with a clear answer to this question. Even so, the fact that PelA can at least induce some cells in a population to undergo premature senescence is an attractive property for an anti-cancer drug.

Most of the effects the effects that we have observed after PelA treatment are related to its effects on mitotic progression; however, interphase microtubules, as we demonstrated in Chapter 5, also presented a valuable target for PelA. MSAs have been shown to inhibit microtubule-dependent processes in ECs that are crucial to particular stages of angiogenesis (for review, see Schwartz (2009)). We carried out a series of assays using HUVECs to screen PelA for anti-angiogenic properties. Several key steps were assessed, namely, EC proliferation, migration and the ability of cells in culture to form 3D networks on Matrigel ${ }^{\mathrm{TM}}$. Summarized below, the anti-angiogenic effects of PelA were comparable to other MSAs that have been reported previously to inhibit angiogenesis (Bijman et al., 2006; Lu et al., 2006; Belletti et al., 2008). 
HUVECs were particularly sensitive to the anti-proliferative effects of PelA and Dtx, with $\mathrm{IC}_{50}$ values of $1.4 \mathrm{nM}$ for PelA and $1.7 \mathrm{nM}$ for Ptx in a 5-day MTT assay using HUVECs. A wound scratch healing assay demonstrated a significant ability of the MSAs to inhibit HUVEC migration, a process that is highly dependent on microtubule functions (Etienne-Manneville, 2004; Watanabe et al., 2005). This inhibitory effect of PelA on migration was concentration-dependent, with inhibition from 2.5-15 nM PelA ranging from $36-72 \%$ compared to controls. One of the last steps in the formation of new blood vessels is the re-organization of ECs into 3D tubule structures. ECs spontaneously form 3D capillary-like structures when grown on the basement membrane matrix Matrigel ${ }^{\mathrm{TM}}$, and we observed a concentration-dependent decrease in the relative numbers of tubules that formed in the presence of PelA compared to controls. This decrease was in the order of $60-63 \%$ at 5-10 nM. In Fig. 8.1 we have suggested a possible mechanistic link between c-Myc expression and inhibition of angiogenesis. c-Myc regulates the pro-angiogenic cytokine VEGF and the angiogenesis inhibitor thrombospondin-1 (Janz et al., 2000; Knies-Bamforth et al., 2004). In response to PelA treatment, c-Myc protein levels were decreased in the HL-60 cell line (Chapter 7). It will be interesting to be determine whether PelA and Ptx inhibit c-Myc expression in other cancer cell lines. 


\subsection{Future Directions}

There are several additional experiments that will help to consolidate the current findings, and these are summarized briefly below:

\subsubsection{Confirmation of the primary apoptotic signalling pathway induced by PelA}

It was demonstrated that caspases play a significant role in PelA-induced apoptosis in 1A9 cells. The lack of specificity of the FLICA probes, however, meant that it was not possible to conclude for certain whether the mitochondrial or death receptor pathways were activated (our results suggested activation of both pathways simultaneously). A similar time course experiment could be carried out using Western blotting instead of flow cytometry and this would offer a more reliable method for distinguishing between activation of these two initiator caspases. Antibodies can also be purchased, for example from Cell Signalling Technologies, Inc. (Danvers, MA) that binds to procaspase-9 (47 $\mathrm{kDa}$ ) and two fragments (37 and $10 \mathrm{kDa}$ ), or to full-length caspase-8 (57 kDa), its cleaved intermediates ( 41 and $43 \mathrm{kDa})$ and its active fragment $(18 \mathrm{kDa})$. Additionally, probing for cytochrome $\mathrm{c}$ release from the mitochondria into the cytosol would also indicate whether the mitochondrial pathway was playing a prominent role during PelAinduced apoptosis. Experimental options for this include separating out the cytosolic and mitochondrial cellular fractions, followed by Western blotting for cytochrome $\mathrm{c}$, or alternatively, an immunocytochemistry approach could be used to dual stain fixed cells with mitochondria selective dyes (e.g. MitoTracker ${ }^{\circledR}$ dyes) and antibodies against cytochrome $\mathrm{c}$. In terms of the BCL-2 family of proteins, if the mitochondrial pathway is involved, what is expected is activation of pro-apoptotic proteins (e.g. BAD, BAK and BAX) and decreased expression and phosphorlyation of anti-apoptotic proteins (e.g. BCL-2 and BCL-xL) (Haldar et al., 1995; Poruchynsky et al., 1998; Yamaguchi et al., 2004). It should be noted that mitochondrial damage and cytochrome c release can also triggered by caspase-8-mediated BID cleavage (Li et al., 1998). Thus, several different approaches would need to be assessed concurrently to obtain a definitive answer. Another aspect of apoptosis that should be assessed is whether stathmin inhibition (e.g. using siRNA methods) can increase the sensitivity of cells to PelA (as has been shown previously for Ptx (Iancu et al., 2001; Mistry et al., 2007)), and how this relates to the polymerization state of microtubules and/or the role of stathmin in cell cycle regulation. 


\subsubsection{Quantification of the frequencies of erroneous microtubule-kinetochore attachments at low PelA concentrations}

It would be interesting to carry out quantitative analyses of abnormal microtubulekinetochore attachments in response to PelA by dual staining of the centromeres and microtubules. In order to see these attachments in detail we would need to deconvolute our confocal images, following procedures described in a previous study that measured the frequency of merotelic attachments in mammalian cells (Cimini et al., 2001; Cimini et al., 2003). Our preliminary experiment described in Chapter 4 suggests an increase in the number of polar chromosomes (with possible monotelic attachments), as opposed to an increase in merotelic chromosomes, as initially hypothesized. These chromosomes were present regardless of the PelA concentration; however, at low concentrations the majority of the chromosomes were aligned tightly at the metaphase plate. This contrasted with the failure of most of the chromosomes to properly congress at the metaphase plate at high concentrations of PelA. Unlike merotelic attachments, monotelic attachments activate the spindle checkpoint (Maiato et al., 2004). If these chromosomes did contribute to the generation of aneuploid cells, then we should be able to show that the aneuploid cells induced at low concentrations of PelA are able to somehow bypass the spindle checkpoint; whereas, the gross failure of chromosomes to align at the metaphase plate at high PelA concentrations should activate the SAC. One way to assess SAC activation in these cells is to simultaneously stain cells for microtubules, centromeres and either MAD2 or BUBR1. Recruitment of these spindle checkpoint proteins to kinetochores signals an active spindle checkpoint and should be able to give us some indication of whether cells possessing polar chromosomes are likely to progress through to anaphase.

Depletion of centromere-associated proteins A and E (CENP-A and CENP-E), for example by siRNA or by using cell lines derived from knockout mice, has previously been shown to generate a similar phenotype to what we observed in response to low PelA concentrations (i.e. 'pseudo-metaphase') (Weaver et al., 2003; Tanudji et al., 2004; Régnier et al., 2005). Weaver et al. suggested that one or a few incorrectly attached chromosomes, in the absence of CENP-E, weakens the checkpoint signal generated by these chromosomes such that it does not meet the threshold level for anaphase inhibition (Weaver et al., 2003). These cells continue to progress through anaphase, leading to chromosome mis-segregation. It would, therefore, be of interest to assess the effects of PelA on CENP-E and/or CENP-A expression levels and their 
recruitment to kinetochores, to determine whether these proteins play a role in PelAinduced aneuploidy.

\subsubsection{Confirmation of whether PelA decreases c-Myc expression in additional cancer cell lines and potential implications for angiogenesis}

The initial proteomics screen using PelA was carried out in HL-60 cells (Wilmes et al., 2011b; Wilmes et al., 2012a); hence, validation of some of the hits in the present study was similarly performed in this cell line. It would be interesting to determine whether the c-Myc abundance levels were also decreased in cells lines derived from solid tumours, and how exactly regulation of this transcription factor might influence the antiangiogenic effects of PelA. Gain or loss of function studies on c-Myc and some of its known angiogenic target such as VEGF and thrombospondin-1, could be used to assess the role of these proteins in angiogenic signalling pathways (Janz et al., 2000; KniesBamforth et al., 2004). Several methods are available to probe angiogenesis in an environment that allows paracrine signalling pathways between tumour cells and ECs, including culturing of ECs in conditioned medium harvested from drug-treated versus drug-free cancer cell lines (which should contain pro- and anti-inhibitory factors secreted by these cells), co-culturing of ECs and tumour cells on Matrigel ${ }^{\mathrm{TM}}$, and using a SCID (severe combined immunodeficiency) mouse model of tumour angiogenesis (Zeng et al., 2005).

\subsubsection{Confirming a lack of DNA replication in response to senescence-inducing conditions}

We were unable to confirm the lack of DNA replication in our premature senescence study using the EdU incorporation assay. It may be better to use the traditional BrdU incorporation assay with a brighter fluorophore (i.e. Alexa Fluor ${ }^{\circledR} 488$ ) or probe for Sphase markers, such as PCNA (proliferating cell nuclear antigen) or cyclin A. TOPO I inhibition by PelA also needs to be confirmed, as this may provide a mechanistic link between PelA and senescence. Although we were unable to get the TOPO I relaxation assay working, we could still look for possible DNA-damaging effects of MTAs (i.e. through $\mathrm{H} 2 \mathrm{AX}$ phosphorylation or a COMET assay). It has not yet been shown whether the induction of senescence by MTAs is dependent on the ability of these drugs to perturb microtubules or whether there is another underlying mechanism. The expression of kinase-inactive forms of DDR mediators (e.g. ATM, ATR, CHK1 and CHK2) could 
be used to determine whether PelA-induced senescence requires intact DNA damage signalling pathways (d'Adda di Fagagna et al., 2003).

\subsubsection{Future in vivo experiments}

In order to further develop PelA as a potential chemotherapeutic drug, pre-clinical pharmacokinetic tests need to be carried out. The ability to perform in vivo pharmacological studies, however, will largely depend on obtaining sufficient quantities of the drug. The labelling of PelA with tritium $\left({ }^{3} \mathrm{H}\right)$, although costly, would make pharmacokinetic studies significantly easier because PelA lacks a UV chromophore for HPLC monitoring of degradation. The complex structure of PelA has hindered efforts to attach a fluorescent or radioactive label to its structure; however, Dr Ernest Hamel (NCI/NIH) and Victor Sidorov (AmBios Labs, Inc.) successfully prepared ${ }^{3} \mathrm{H}-\mathrm{PelA}$, and if sufficient quantities of labelled drug were available then this would be a possible avenue that could be pursued (Miller et al., 2010). Generating bioavailability, efficacy and toxicity profiles for PelA to identify optimal dosing schedules for PelA in clinical trials is an important drug development goal. Labelled drug would also allow us to assess whether the drug is transported across the blood brain barrier, another important pharmacokinetic parameter that must be determined.

\subsection{Final conclusion}

The road to progressing a new compound into clinical trials is often fraught with obstacles, and PelA has been no exception. A significant aquaculture effort by Victoria University and NIWA to generate a sufficient supply of PelA for preclinical studies in animals was unsuccessful and has hampered our efforts to progress PelA to the clinic (Page et al., 2005; Page et al., 2011). With the PelA US patent expiration date approaching, it is becoming less and less economically viable for a pharmaceutical company to invest in PelA's development as a new chemotherapeutic agent. However, taken together, the results from this thesis highlight the fact that in addition to its primary apoptosis-inducing effects, PelA triggers multiple tumour suppressive mechanisms that may be beneficial in the clinic. These complementary effects (i.e. apoptosis, induction of senescence and inhibition of angiogenesis) are likely to have significantly contributed to the striking tumour regression results that were observed in xenograft mouse models treated with PelA (Meyer et al., 2006). Furthermore, this study by Reata Pharmaceuticals and more recent work investigating the ability of PelA to protect against experimental autoimmune encephalomyelitis (a mouse model of multiple 
sclerosis) (Crume et al., 2009), have demonstrated that PelA is better tolerated in vivo (in mice at least) than the MSA Taxol®). In addition to providing some mechanistic insights into the cellular actions of PelA, we hope that the results presented here further enhance the prospects of PelA one day making it into the clinic as an anti-cancer drug. 


\section{References}

Adhikary, S. and Eilers, M. Transcriptional regulation and transformation by Myc proteins. Nat Rev Mol Cell Biol 6 (2005), pp. 635-645.

Ahn, H.J., Kim, Y.S., Kim, J.U., Han, S.M., Shin, J.W. and Yang, H.O. Mechanism of Taxol-induced apoptosis in human SKOV3 ovarian carcinoma cells. $J$ Cell Biochem 91 (2004), pp. 1043-1052.

Akhmanova, A. and Steinmetz, M.O. Tracking the ends: a dynamic protein network controls the fate of microtubule tips. Nature Rev Mol Cell Biol 9 (2008), pp. 309-322.

Alcorta, D.A., Xiong, Y., Phelps, D., Hannon, G., Beach, D. and Barrett, J.C. Involvement of the cyclin-dependent kinase inhibitor p16 (INK4a) in replicative senescence of normal human fibroblasts. PNAS 93 (1996), pp. 13742-13747.

Alison, M.R., Lin, W.R., Lim, S.M.L. and Nicholson, L.J. Cancer stem cells: In the line of fire. Cancer Treat Rev 38 (2012), pp. 589-598.

Alley, M.C., Smith, A.C., Donohoe, S.J., Schweikart, K.M., Newman, D.J. and Tomaszewski, J.E.: Comparison of the relative efficacies and toxicities of halichondrin B analogues, Proceedings of the AACR-NCI-EORTC Conference on Molecular Targets and Cancer Therapeutics; 2005 Nov 14-18; Philadelphia, $P A$. American Association for Cancer Research, Philadelphia, PA (2005), pp. Abstract C230, 257.

Alli, E., Bash-Babula, J., Yang, J.-M. and Hait, W.N. Effect of stathmin on the sensitivity to antimicrotubule drugs in human breast cancer. Cancer Res 62 (2002), pp. 6864-6869.

Alli, E., Yang, J.-M., Ford, J.M. and Hait, W.N. Reversal of stathmin-mediated resistance to paclitaxel and vinblastine in human breast carcinoma cells. Mol Pharmacol 71 (2007), pp. 1233-1240.

Amos, L.A. Microtubule structure and its stabilisation. Org Biomol Chem 2 (2004), pp. 2153-2160.

Amos, L.A., Schlieper, D., Squire, J.M. and Parry, D.A.D.: Microtubules and maps, Advances in Protein Chemistry. Academic Press (2005), pp. 257-298.

André, N., Braguer, D., Brasseur, G., Gonçalves, A., Lemesle-Meunier, D., Guise, S., Jordan, M.A. and Briand, C. Paclitaxel induces release of cytochrome c from mitochondria isolated from human neuroblastoma cells. Cancer Res 60 (2000), pp. 5349-5353.

Arnaoutova, I., George, J., Kleinman, H.K. and Benton, G. The endothelial cell tube formation assay on basement membrane turns 20: State of the science and the art. Angiogenesis 12 (2009), pp. 267-274.

Arthur, C.R., Gupton, J.T., Kellogg, G.E., Yeudall, W.A., Cabot, M.C., Newsham, I.F. and Gewirtz, D.A. Autophagic cell death, polyploidy and senescence induced in breast tumor cells by the substituted pyrrole JG-03-14, a novel microtubule poison. Biochem Pharmacol 74 (2007), pp. 981-991.

Aubin, J.E. Autofluorescence of viable cultured mammalian cells. J Histochem Cytochem 27 (1979), pp. 36-43.

Bartkova, J., Hořejší, Z., Koed, K., Krämer, A., Tort, F., Zleger, K., Guldberg, P., Sehested, M., Nesland, J.M., Lukas, C., Orntoft, T., Lukas, J. and Bartek, J. DNA damage response as a candidate anti-cancer barrier in early human tumorigenesis. Nature 434 (2005), pp. 864-870.

Bartkova, J., Rezaei, N., Liontos, M., Karakaidos, P., Kletsas, D., Issaeva, N., Vassiliou, L.V.F., Kolettas, E., Niforou, K., Zoumpourlis, V.C., Takaoka, M., Nakagawa, H., Tort, F., Fugger, K., Johansson, F., Sehested, M., Andersen, C.L., Dyrskjot, L., Ørntoft, T., Lukas, J., Kittas, C., Helleday, T., Halazonetis, 
T.D., Bartek, J. and Gorgoulis, V.G. Oncogene-induced senescence is part of the tumorigenesis barrier imposed by DNA damage checkpoints. Nature 444 (2006), pp. 633-637.

Begaye, A., Trostel, S., Zhao, Z., Taylor, R.E., Schriemer, D.C. and Sackett, D.L. Mutations in the $\beta$-tubulin binding site for peloruside A confer resistance by targeting a cleft significant in side chain binding. Cell Cycle 10 (2011), pp. 3387-3396.

Bekier, M.E., Fischbach, R., Lee, J. and Taylor, W.R. Length of mitotic arrest induced by microtubule-stabilizing drugs determines cell death after mitotic exit. $\mathrm{Mol}$ Cancer Ther 8 (2009), pp. 1646-1654.

Belletti, B., Nicoloso, M.S., Schiappacassi, M., Berton, S., Lovat, F., Wolf, K., Canzonieri, V., D'Andrea, S., Zucchetto, A., Friedl, P., Colombatti, A. and Baldassarre, G. Stathmin activity influences sarcoma cell shape, motility, and metastatic potential. Mol Biol Cell 19 (2008), pp. 2003-2013.

Belotti, D., Vergani, V., Drudis, T., Borsotti, P., Pitelli, M.R., Viale, G., Giavazzi, R. and Taraboletti, G. The microtubule-affecting drug paclitaxel has antiangiogenic activity. Clin Cancer Res 2 (1996), pp. 1843-1849.

Bennett, M.J., Barakat, K., Huzil, J.T., Tuszynski, J. and Schriemer, D.C. Discovery and characterization of the laulimalide-microtubule binding mode by mass shift perturbation mapping. Chem Biol 17 (2010), pp. 725-734.

Benson, R.C., Meyer, R.A., Zaruba, M.E. and McKhann, G.M. Cellular autofluorescence. Is it due to flavins? J Histochem Cytochem 27 (1979), pp. 4448.

Bershadsky, A.D., Vaisberg, E.A. and Vasiliev, J.M. Pseudopodial activity at the active edge of migrating fibroblast is decreased after drug-induced microtubule depolymerization. Cell Motil Cytoskeleton 19 (1991), pp. 152-158.

Best, A., Ahmed, S., Kozma, R. and Lim, L. The Ras-related GTPase Rac1 binds tubulin. J Biol Chem 271 (1996), pp. 3756-3762.

Bhalla, K.N. Microtubule-targeted anticancer agents and apoptosis. Oncogene 22 (2003), pp. 9075-9086.

Bharadwaj, R. and Yu, H. The spindle checkpoint, aneuploidy, and cancer. Oncogene 23 (2004), pp. 2016-2027.

Bhat, K.M.R. and Setaluri, V. Microtubule-associated proteins as targets in cancer chemotherapy. Clin Cancer Res 13 (2007), pp. 2849-2854.

Bhattacharyya, B., Panda, D., Gupta, S. and Banerjee, M. Anti-mitotic activity of colchicine and the structural basis for its interaction with tubulin. Med Res Rev 28 (2008), pp. 155-183.

Bijman, M.N.A., van Nieuw Amerongen, G.P., Laurens, N., van Hinsbergh, V.W.M. and Boven, E. Microtubule-targeting agents inhibit angiogenesis at subtoxic concentrations, a process associated with inhibition of Rac1 and Cdc42 activity and changes in the endothelial cytoskeleton. Mol Cancer Ther 5 (2006), pp. 2348-2357.

Blackwood, E.M., Luscher, B. and Eisenman, R.N. Myc and Max associate in vivo. Genes Dev 6 (1992), pp. 71-80.

Blomen, V. and Boonstra, J. Cell fate determination during G1 phase progression. Cell Mol Life Sci 64 (2007), pp. 3084-3104.

Bocci, G., Nicolaou, K.C. and Kerbel, R.S. Protracted low-dose effects on human endothelial cell proliferation and survival in vitro reveal a selective antiangiogenic window for various chemotherapeutic drugs. Cancer Res 62 (2002), pp. 6938-6943. 
Bogyo, M., Verhelst, S., Bellingard-Dubouchaud, V., Toba, S. and Greenbaum, D. Selective targeting of lysosomal cysteine proteases with radiolabeled electrophilic substrate analogs. Chem Biol 7 (2000), pp. 27-38.

Bollag, D.M., McQueney, P.A., Zhu, J., Hensens, O., Koupal, L., Liesch, J., Goetz, M., Lazarides, E. and Woods, C.M. Epothilones, a new class of microtubulestabilizing agents with a Taxol-like mechanism of action. Cancer Res 55 (1995), pp. 2325-2333.

Bordeleau, M.E., Matthews, J., Wojnar, J.M., Lindqvist, L., Novac, O., Jankowsky, E., Sonenberg, N., Northcote, P., Teesdale-Spittle, P. and Pelletier, J. Stimulation of mammalian translation initiation factor eIF4A activity by a small molecule inhibitor of eukaryotic translation. PNAS 102 (2005), pp. 10460-10465.

Bourgarel-Rey, V., El Khyari, S., Rimet, O., Bordas, B., Guigal, N., Braguer, D., Seree, E., Barra, Y. and Briand, C. Opposite effects of antimicrotubule agents on c-myc oncogene expression depending on the cell lines used. Eur J Cancer 36 (2000), pp. 1043-1049.

Bourgarel-Rey, V., Savry, A., Hua, G., Carré, M., Bressin, C., Chacon, C., Imbert, J., Braguer, D. and Barra, Y. Transcriptional down-regulation of Bcl-2 by vinorelbine: Identification of a novel binding site of p53 on Bcl-2 promoter. Biochem Pharmacol 78 (2009), pp. 1148-1156.

Brito, D.A. and Rieder, C.L. Mitotic checkpoint slippage in humans occurs via cyclin B destruction in the presence of an active checkpoint. Curr Biol 16 (2006), pp. 1194-1200.

Brito, D.A., Yang, Z. and Rieder, C.L. Microtubules do not promote mitotic slippage when the spindle assembly checkpoint cannot be satisfied. Cancer Res 182 (2008), pp. 623-629.

Bröker, L.E., Huisman, C., Ferreira, C.G., Rodriguez, J.A., Kruyt, F.A.E. and Giaccone, G. Late activation of apoptotic pathways plays a negligible role in mediating the cytotoxic effects of discodermolide and epothilone B in non-small cell lung cancer cells. Cancer Res 62 (2002), pp. 4081-4088.

Bröker, L.E., Huisman, C., Span, S.W., Rodriguez, J.A., Kruyt, F.A.E. and Giaccone, G. Cathepsin B mediates caspase-independent cell death induced by microtubule stabilizing agents in non-small cell lung cancer eclls. Cancer Res 64 (2004), pp. 27-30.

Budman, D.R. and Calabro, A. In vitro search for synergy and antagonism: evaluation of docetaxel combinations in breast cancer cell lines. Breast Cancer Res Treat 74 (2002), pp. 41-46.

Bunz, F., Dutriaux, A., Lengauer, C., Waldman, T., Zhou, S., Brown, J.P., Sedivy, J.M., Kinzler, K.W. and Vogelstein, B. Requirement for p53 and p21 to Sustain G2 Arrest After DNA Damage. Science 282 (1998), pp. 1497-1501.

Burma, S., Chen, B.P., Murphy, M., Kurimasa, A. and Chen, D.J. ATM phosphorylates histone $\mathrm{H} 2 \mathrm{AX}$ in response to DNA double-strand breaks. J Biol Chem 276 (2001), pp. 42462-42467.

Byun, Y., Chen, F., Chang, R., Trivedi, M., Green, K.J. and Cryns, V.L. Caspase cleavage of vimentin disrupts intermediate filaments and promotes apoptosis. Cell Death Diff 8 (2001), p. 443.

Cain, K., Bratton, S.B., Langlais, C., Walker, G., Brown, D.G., Sun, X.M. and Cohen, G.M. Apaf-1 oligomerizes into biologically active $\sim 700-\mathrm{kDa}$ and inactive $\sim 1.4-$ MDa apoptosome complexes. J Biol Chem 275 (2000), pp. 6067-6070.

Calvo, E., Barasoain, I., Matesanz, R., Pera, B., Camafeita, E., Pineda, O., Hamel, E., Vanderwal, C.D., Andreu, J.M., Loppez, J.A. and Díaz, J.F. Cyclostreptin derivatives specifically target cellular tubulin and further map the paclitaxel site. Biochem 51 (2012), pp. 329-341. 
Campisi, J. and d'Adda di Fagagna, F. Cellular senescence: when bad things happen to good cells. Nat Rev Mol Cell Biol 8 (2007), pp. 729-740.

Candé, C.1., Cecconi, F., Dessen, P. and Kroemer, G. Apoptosis-inducing factor (AIF): key to the conserved caspase-independent pathways of cell death? J Cell Sci 115 (2002), pp. 4727-4734.

Cao, Y. Positive and negative modulation of angiogenesis by VEGFR1 ligands. Sci Signal 2 (2009), p. re1.

Cassimeris, L. Accessory protein regulation of microtubule dynamics throughout the cell cycle. Curr Opin Cell Biol 11 (1999), pp. 134-141.

Cassimeris, L., Spittle, C. and Kwang, W.J. Regulation of microtubule-associated proteins. Int Rev Cytol Volume 210 (2001), pp. 163-226.

Castedo, M., Perfettini, J.L., Roumier, T., Andreau, K., Medema, R. and Kroemer, G. Cell death by mitotic catastrophe: A molecular definition. Oncogene 23 (2004), pp. 2825-2837.

Castino, R., Peracchio, C., Salini, A., Nicotra, G., Trincheri, N.F., Demoz, M., Valente, G. and Isidora, C. Chemotherapy drug response in ovarian cancer cells strictly depends on a cathepsin D-Bax activation loop. J Cell Mol Med 13 (2009), pp. 1096-1109.

Česen, M.H., Pegan, K., Špes, A. and Turk, B. Lysosomal pathways to cell death and their therapeutic applications. Exp Cell Res 318 (2012), pp. 1245-1251.

Chabner, B.A. and Roberts, T.G. Chemotherapy and the war on cancer. Nat Rev Cancer 5 (2005), pp. 65-72.

Chan, A.: Investigating the action of peloruside A on microtubule dynamics. BBmedSc Honours Thesis. School of Biological Sciences, Victoria University of Wellington (2008).

Chan, A., Andreae, P., Northcote, P. and Miller, J. Peloruside A inhibits microtubule dynamics in a breast cancer cell line MCF7. Invest New Drugs 29 (2011), pp. 615-626.

Chan, G.K.T., Schaar, B.T. and Yen, T.J. Characterization of the kinetochore binding domain of CENP-E reveals interactions with the kinetochore proteins CENP-F and hBUBR1. J Cell Biol 143 (1998), pp. 49-63.

Chang, B.-D., Broude, E.V., Dokmanovic, M., Zhu, H., Ruth, A., Xuan, Y., Kandel, E.S., Lausch, E., Christov, K. and Roninson, I.B. A senescence-like phenotype distinguishes tumor cells that undergo terminal proliferation arrest after exposure to anticancer agents. Cancer Res 59 (1999a), pp. 3761-3767.

Chang, B.D., Swift, M.E., Shen, M., Fang, J., Broude, E.V. and Roninson, I.B. Molecular determinants of terminal growth arrest induced in tumor cells by a chemotherapeutic agent. PNAS 99 (2002), pp. 389-394.

Chang, B.D., Xuan, Y., Broude, E.V., Zhu, H., Schott, B., Fang, J. and Roninson, I.B. Role of p53 and p21(waf1/cip1) in senescence-like terminal proliferation arrest induced in human tumor cells by chemotherapeutic drugs. Oncogene 18 (1999b), pp. 4808-4818.

Chang, D.C., Xu, N. and Luo, K.Q. Degradation of cyclin B is required for the onset of anaphase in mammalian cells. J Biol Chem 278 (2003a), pp. 37865-37873.

Chang, J.-Y., Hsieh, H.-P., Pan, W.-Y., Liou, J.-P., Bey, S.-J., Chen, L.-T., Liu, J.-F. and Song, J.-S. Dual inhibition of topoisomerase I and tubulin polymerization by BPR0Y007, a novel cytotoxic agent. Biochem Pharmacol 65 (2003b), pp. 20092019.

Chauvier, D., Ankri, S., Charriaut-Marlangue, C., Casimir, R. and Jacotot, E. Broadspectrum caspase inhibitors: From myth to reality? Cell Death Diff 14 (2007), pp. 387-391. 
Chen, J.-G. and Horwitz, S.B. Differential mitotic responses to microtubule-stabilizing and -destabilizing drugs. Cancer Res 62 (2002), pp. 1935-1938.

Chen, R.H. BubR1 is essential for kinetochore localization of other spindle checkpoint proteins and its phosphorylation requires Mad1. J Cell Biol 158 (2002), pp. 487496.

Cho, S. and Hwang, E.S. Status of mTOR activity may phenotypically differentiate senescence and quiescence. Mol Cells (2012), pp. 1-8.

Choi, H.J., Fukui, M. and Zhu, B.T. Role of cyclin B1/Cdc2 up-regulation in the development of mitotic prometaphase arrest in human breast cancer cells treated with nocodazole. PLoS ONE 6 (2011), p. e24312.

Chow, J.M., Liu, C.R., Lin, C.P., Lee, C.N., Cheng, Y.C., Lin, S. and Liu, H.E. Downregulation of c-Myc determines sensitivity to 2-methoxyestradiol-induced apoptosis in human acute myeloid leukemia. Exp Hematol 36 (2008), pp. 140148.

Chrzanowska-Wodnicka, M. and Burridge, K. Rho-stimulated contractility drives the formation of stress fibers and focal adhesions. J Cell Biol 133 (1996), pp. 14031415.

Chuthapisith, S., Layfield, R., Kerr, I.D., Hughes, C. and Eremin, O. Proteomic profiling of MCF-7 breast cancer cells with chemoresistance to different types of anti-cancer drugs. Int J Oncol 30 (2007), pp. 1545-1551.

Cimini, D., Howell, B., Maddox, P., Khodjakov, A., Degrassi, F. and Salmon, E.D. Merotelic kinetochore orientation is a major mechanism of aneuploidy in mitotic mammalian tissue cells. J Cell Biol 152 (2001), pp. 517-527.

Cimini, D., Moree, B., Canman, J.C. and Salmon, E.D. Merotelic kinetochore orientation occurs frequently during early mitosis in mammalian tissue cells and error correction is achieved by two different mechanisms. J Cell Sci 116 (2003), pp. 4213-4225.

Ciric, E. and Sersa, G. Radiotherapy in combination with vascular-targeted therapies. Radiol Oncol 44 (2010), pp. 67-78.

Cirman, T., Orešic', K., Mazovec, G.D., Turk, V., Reed, J.C., Myers, R.M., Salvesen, G.S. and Turk, B. Selective disruption of lysosomes in HeLa cells triggers apoptosis mediated by cleavage of Bid by multiple papain-like lysosomal cathepsins. J Biol Chem 279 (2004), pp. 3578-3587.

Clark, E.A., Hills, P.M., Davidson, B.S., Wender, P.A. and Mooberry, S.L. Laulimalide and synthetic laulimalide analogues are synergistic with paclitaxel and 2methoxyestradiol. Mol Pharm 3 (2006), pp. 457-467.

Cleveland, D.W., Mao, Y. and Sullivan, K.F. Centromeres and kinetochores: From epigenetics to mitotic checkpoint signaling. Cell 112 (2003), pp. 407-421.

Collado, M., Gil, J., Efeyan, A., Guerra, C., Schuhmacher, A.J., Barradas, M., Benguría, A., Zaballos, A., Flores, J.M., Barbacid, M., Beach, D. and Serrano, M. Tumour biology: Senescence in premalignant tumours. Nature 436 (2005), p. 642.

Cong, F., Cheung, A.K. and Huang, S.M.A. Chemical genetics based target identification in drug discovery. Annu Rev Pharmacol Toxicol 52 (2012), pp. 5778.

Courtois-Cox, S., Jones, S.L. and Cichowski, K. Many roads lead to oncogene-induced senescence. Oncogene 27 (2008), pp. 2801-2809.

Cragg, G.M. Paclitaxel (Taxol $\left.{ }^{\circledR}\right)$ : A success story with valuable lessons for natural product drug discovery and development. Med Res Rev 18 (1998), pp. 315-331.

Crume, K.P., O'Sullivan, D., Miller, J.H., Northcote, P.T. and La Flamme, A.C. Delaying the onset of experimental autoimmune encephalomyelitis with the microtubule-stabilizing compounds, paclitaxel and Peloruside A. J Leukoc Biol 86 (2009), pp. 949-958. 
Curmi, P.A., Noguès, C., Lachkar, S., Carelle, N., Gonthier, M.P., Sobel, A., Lidereau, R. and Bièche, I. Overexpression of stathmin in breast carcinomas points out to highly proliferative tumours. Br J Cancer 82 (2000), pp. 142-150.

d'Adda di Fagagna, F., Reaper, P.M., Clay-Farrace, L., Fiegler, H., Carr, P., Von Zglinicki, T., Saretzki, G., Carter, N.P. and Jackson, S.P. A DNA damage checkpoint response in telomere-initiated senescence. Nature 426 (2003), pp. 194-198.

Daub, H., Gevaert, K., Vandekerckhove, J., Sobel, A. and Hall, A. Rac/Cdc42 and p65PAK regulate the microtubule-destabilizing protein stathmin through phosphorylation at serine 16. J Biol Chem 276 (2001), pp. 1677-1680.

De Antoni, A., Pearson, C.G., Cimini, D., Canman, J.C., Sala, V., Nezi, L., Mapelli, M., Sironi, L., Faretta, M., Salmon, E.D. and Musacchio, A. The Mad1/Mad2 complex as a template for Mad2 activation in the spindle assembly checkpoint. Curr Biol 15 (2005), pp. 214-225.

Dehmelt, L. and Halpain, S. The MAP2/Tau family of microtubule-associated proteins. Genome Biol 6 (2004), p. 204.

Deiss, L.P., Galinka, H., Berissi, H., Cohen, O. and Kimchi, A. Cathepsin D protease mediates programmed cell death induced by interferon- $\gamma$, Fas/APO-1 and TNFa. EMBO J 15 (1996), pp. 3861-3870.

Denis, J.N., Greene, A.E., Guénard, D., Guéritte-Voegelein, F., Mangatal, L. and Potier, P. A highly efficient, practical approach to natural taxol. J Am Chem Soc 110 (1988), pp. 5917-5919.

Dhawan, V. and Swaffar, D. A unique paclitaxel-mediated modulation of the catalytic activity of topoisomerase II $\alpha$. Anti-Cancer Drugs 10 (1999), pp. 397-404.

Di Leonardo, A., Linke, S.P., Clarkin, K. and Wahl, G.M. DNA damage triggers a prolonged p53-dependent G1 arrest and long-term induction of Cip1 in normal human fibroblasts. Genes Dev 8 (1994), pp. 2540-2551.

Di Micco, R., Fumagalli, M., Cicalese, A., Piccinin, S., Gasparini, P., Luise, C., Schurra, C., Garr $\left.{ }^{(}\right)$, M., Giovanni Nuciforo, P., Bensimon, A., Maestro, R., Giuseppe Pelicci, P. and D'Adda Di Fagagna, F. Oncogene-induced senescence is a DNA damage response triggered by DNA hyper-replication. Nature 444 (2006), pp. 638-642.

Díaz, J.F., Barasoain, I. and Andreu, J.M. Fast kinetics of Taxol binding to microtubules: Effects of solution variables and microtubule-associated proteins. J Biol Chem 278 (2003), pp. 8407-8419.

Díaz, J.F., Valpuesta, J.M., Chacón, P., Diakun, G. and Andreu, J.M. Changes in microtubule protofilament number induced by Taxol binding to an easily accessible site. J Biol Chem 273 (1998), pp. 33803-33810.

DiMasi, J.A. and Grabowski, H.G. Economics of new oncology drug development. $J$ Clin Oncol 25 (2007), pp. 209-216.

Dimri, G.P. What has senescence got to do with cancer? Cancer cell 7 (2005), pp. 505512.

Dimri, G.P., Lee, X., Basile, G., Acosta, M., Scott, G., Roskelley, C., Medrano, E.E., Linskens, M., Rubelj, I. and Pereira-Smith, O. A biomarker that identifies senescent human cells in culture and in aging skin in vivo. PNAS 92 (1995), pp. 9363-9367.

Doll, T., Meichsner, M., Riederer, B.M., Honegger, P. and Matus, A. An isoform of microtubule-associated protein 2 (MAP2) containing four repeats of the tubulinbinding motif. J Cell Sci 106 (1993), pp. 633-639.

Donehower, L.A., Harvey, M., Slagle, B.L., McArthur, M.J., Montgomery, C.A., Butel, J.S. and Allan, B. Mice deficient for p53 are developmentally normal but susceptible to spontaneous tumours. Nature 356 (1992), pp. 215-221. 
Dowling, P., Meleady, P., Dowd, A., Henry, M., Glynn, S. and Clynes, M. Proteomic analysis of isolated membrane fractions from superinvasive cancer cells. $B B A$ Prot Proteom 1774 (2007), pp. 93-101.

Droga-Mazovec, G., Bojič, L., Petelin, A., Ivanova, S., Romih, R., Repnik, U., Salvesen, G.S., Stoka, V., Turk, V. and Turk, B. Cysteine cathepsins trigger caspase-dependent cell death through cleavage of bid and antiapoptotic Bcl-2 homologues. J Biol Chem 283 (2008), pp. 19140-19150.

Du, L., Lyle, C.S. and Chambers, T.C. Characterization of vinblastine-induced Bcl-xL and Bcl-2 phosphorylation: evidence for a novel protein kinase and a coordinated phosphorylation//dephosphorylation cycle associated with apoptosis induction. Oncogene 24 (2004), pp. 107-117.

Dunn, S., Morrison, E.E., Liverpool, T.B., Molina-París, C., Cross, R.A., Alonso, M.C. and Peckham, M. Differential trafficking of Kif5c on tyrosinated and detyrosinated microtubules in live cells. J Cell Sci 121 (2008), pp. 1085-1095.

Eichhorn, M., Kleespies, A., Angele, M., Jauch, K.W. and Bruns, C. Angiogenesis in cancer: molecular mechanisms, clinical impact. Langenbecks Arch of Surg 392 (2007), pp. 371-379.

Eischen, C.M., Packham, G., Nip, J., Fee, B.E., Hiebert, S.W., Zambetti, G.P. and Cleveland, J.L. Bcl-2 is an apoptotic target suppressed by both c-Myc and E2F1. Oncogene 20 (2001), pp. 6983-6993.

Elmore, L.W., Rehder, C.W., Di, X., McChesney, P.A., Jackson-Cook, C.K., Gewirtz, D.A. and Holt, S.E. Adriamycin-induced senescence in breast tumor cells involves functional p53 and telomere dysfunction. J Biol Chem 277 (2002), pp. 35509-35515.

Elowe, S., Hümmer, S., Uldschmid, A., Li, X. and Nigg, E.A. Tension-sensitive Plk1 phosphorylation on BubR1 regulates the stability of kinetochore-microtubule interactions. Genes Dev 21 (2007), pp. 2205-2219.

Enomoto, T. Microtubule disruption induces the formation of actin stress fibers and focal adhesions in cultured cells: Possible involvement of the rho signal cascade. Cell Struct Funct 21 (1996), pp. 317-326.

Eom, Y.W., Kim, M.A., Park, S.S., Goo, M.J., Kwon, H.J., Sohn, S., Kim, W.H., Yoon, G. and Choi, K.S. Two distinct modes of cell death induced by doxorubicin: Apoptosis and cell death through mitotic catastrophe accompanied by senescence-like phenotype. Oncogene 24 (2005), pp. 4765-4777.

Espinoza, F.H., Ogas, J., Herskowitz, I. and Morgan, D.O. Cell cycle control by a complex of the cyclin HCS26 (PCL1) and the kinase PHO85. Science 266 (1994), pp. 1388-1391.

Estève, M.A., Carré, M. and Braguer, D. Microtubules in apoptosis induction: Are they necessary? Curr Cancer Drug Targets 7 (2007), pp. 713-729.

Etienne-Manneville, S. Actin and microtubules in cell motility: which one is in control? Traffic 5 (2004), pp. 470-477.

Evans, D.A., Welch, D.S., Speed, A.W.H., Moniz, G.A., Reichelt, A. and Ho, S. An aldol-based synthesis of (+)-peloruside a, a potent microtubule stabilizing agent. J Am Chem Soc 131 (2009), pp. 3840-3841.

Even-Ram, S., Doyle, A.D., Conti, M.A., Matsumoto, K., Adelstein, R.S. and Yamada, K.M. Myosin IIA regulates cell motility and actomyosin-microtubule crosstalk. Nat Cell Biol 9 (2007), pp. 299-309.

Ezratty, E.J., Bertaux, C., Marcantonio, E.E. and Gundersen, G.G. Clathrin mediates integrin endocytosis for focal adhesion disassembly in migrating cells. $J$ Cell Biol 187 (2009), pp. 733-747. 
Ezratty, E.J., Partridge, M.A. and Gundersen, G.G. Microtubule-induced focal adhesion disassembly is mediated by dynamin and focal adhesion kinase. Nat Cell Biol 7 (2005), pp. 581-590.

Fanara, P., Oback, B., Ashman, K., Podtelejnikov, A. and Brandt, R. Identification of MINUS, a small polypeptide that functions as a microtubule nucleation suppressor. EMBO J 18 (1999), pp. 565-577.

Fang, G., Yu, H. and Kirschner, M.W. The checkpoint protein MAD2 and the mitotic regulator CDC20 form a ternary complex with the anaphase-promoting complex to control anaphaseâ€\%oinitiation. Genes \& Dev 12 (1998), pp. 1871-1883.

Ferrara, N., Gerber, H.P. and LeCouter, J. The biology of VEGF and its receptors. Nat Med 9 (2003), pp. 669-676.

Field, M.G., Yang, D., Bian, Z.M., Petty, H.R. and Elner, V.M. Retinal flavoprotein fluorescence correlates with mitochondrial stress, apoptosis, and chemokine expression. Exp Eye Res 93 (2011), pp. 548-555.

Fischer, U., Janicke, R.U. and Schulze-Osthoff, K. Many cuts to ruin: a comprehensive update of caspase substrates. Cell Death Diff 10 (2003), pp. 76-100.

Folkman, J. Tumor angiogenesis: therapeutic implications. New Engl J Med 285 (1971), pp. 1182-1186.

Folkman, J. Angiogenesis. Annu Rev Med 57 (2006), pp. 1-18.

Folkman, J. Angiogenesis: An organizing principle for drug discovery? Nat Rev Drug Discov 6 (2007), pp. 273-286.

Forsythe, J.A., Jiang, B.H., Iyer, N.V., Agani, F., Leung, S.W., Koos, R.D. and Semenza, G.L. Activation of vascular endothelial growth factor gene transcription by hypoxia-inducible factor 1. Mol Cell Biol 16 (1996), pp. 46044613.

Frenzel, A., Grespi, F., Chmelewskij, W. and Villunger, A. Bcl2 family proteins in carcinogenesis and the treatment of cancer. Apoptosis 14 (2009), pp. 584-596.

Frenzel, A., Zirath, H., Vita, M., Albihn, A. and Henriksson, M.A. Identification of cytotoxic drugs that selectively target tumor cells with MYC overexpression. PLOS ONE 6 (2011).

Fulda, S. and Debatin, K.M. Extrinsic versus intrinsic apoptosis pathways in anticancer chemotherapy. Oncogene 25 (2006), pp. 4798-4811.

Gaglio, T., Dionne, M.A. and Compton, D.A. Mitotic spindle poles are organized by structural and motor proteins in addition to centrosomes. J Cell Biol 138 (1997), pp. 1055-1066.

Gaitanos, T.: Cellular and molecular actions of peloruside A, a novel anti-cancer agent, PhD Thesis. Victoria University of Wellington, Wellington, NZ (2005).

Gaitanos, T.N., Buey, R.M., Díaz, J.F., Northcote, P.T., Teesdale-Spittle, P., Andreu, J.M. and Miller, J.H. Peloruside A does not bind to the taxoid site on $\beta$-tubulin and retains its activity in multidrug-resistant cell lines. Cancer Res 64 (2004), pp. 5063-5067.

Galàn-Malo, P., Vela, L., Gonzalo, O., Calvo-Sanjuàn, R., Gracia-Fleta, L., Naval, J. and Marzo, I. Cell fate after mitotic arrest in different tumor cells is determined by the balance between slippage and apoptotic threshold. Toxicol App Pharmacol 258 (2012), pp. 384-393.

Gallego, M.A., Joseph, B., Hemström, T.H., Tamiji, S., Mortier, L., Kroemer, G., Formstecher, P., Zhivotovsky, B. and Marchetti, P. Apoptosis-inducing factor determines the chemoresistance of non-small-cell lung carcinomas. Oncogene 23 (2004), pp. 6282-6291.

Galmarini, C.M., Treilleux, I., Cardoso, F., Bernard-Marty, C., Durbecq, V., Gancberg, D., Bissery, M.-C., Paesmans, M., Larsimont, D., Piccart, M.J., Di Leo, A. and Dumontet, C. Class III $\beta$-tubulin isotype predicts response in advanced breast 
cancer patients randomly treated either with single-agent doxorubicin or docetaxel. Clin Cancer Res 14 (2008), pp. 4511-4516.

Ganansia-Leymarie, V., Bischoff, P., Bergerat, J.P. and Holl, V. Signal transduction pathways of taxanes-induced apoptosis. Curr Med Chem - Anti-Cancer Agents 3 (2003), pp. 291-306.

Gascoigne, K.E. and Taylor, S.S. Cancer cells display profound intra- and interline variation following prolonged exposure to antimitotic drugs. Cancer Cell 14 (2008), pp. 111-122.

Gascoigne, K.E. and Taylor, S.S. How do anti-mitotic drugs kill cancer cells? J Cell Sci 122 (2009), pp. 2579-2585.

Gelderblom, H., Verweij, J., Nooter, K. and Sparreboom, A. Cremophor EL: the drawbacks and advantages of vehicle selection for drug formulation. Eur $J$ Cancer 37 (2001), pp. 1590-1598.

Gewirtz, D.A., Holt, S.E. and Elmore, L.W. Accelerated senescence: An emerging role in tumor cell response to chemotherapy and radiation. Biochem Pharmacol 76 (2008), pp. 947-957.

Ghosh, A.K., Xu, X., Kim, J.H. and Xu, C.X. Enantioselective total synthesis of peloruside A: A potent microtubule stabilizer. Org Lett 10 (2008), pp. 10011004.

Giaever, G., Flaherty, P., Kumm, J., Proctor, M., Nislow, C., Jaramillo, D.F., Chu, A.M., Jordan, M.I., Arkin, A.P. and Davis, R.W. Chemogenomic profiling: Identifying the functional interactions of small molecules in yeast. PNAS 101 (2004), pp. 793-798.

Giaever, G., Shoemaker, D.D., Jones, T.W., Liang, H., Winzeler, E.A., Astromoff, A. and Davis, R.W. Genomic profiling of drug sensitivities via induced haploinsufficiency. Nat Genet 21 (1999), pp. 278-283.

Giannakakou, P., Robey, R., Fojo, T. and Blagosklonny, M.V. Low concentrations of paclitaxel induce cell type-dependent p53, p21 and G1/G2 arrest instead of mitotic arrest: Molecular determinants of paclitaxel-induced cytotoxicity. Oncogene 20 (2001), pp. 3806-3813.

Giodini, A., Kallio, M.J., Wall, N.R., Gorbsky, G.J., Tognin, S., Marchisio, P.C., Symons, M. and Altieri, D.C. Regulation of microtubule stability and mitotic progression by survivin. Cancer Res 62 (2002), pp. 2462-2467.

Giono, L.E. and Manfredi, J.J. The p53 tumor suppressor participates in multiple cell cycle checkpoints. J Cell Physiol 209 (2006), pp. 13-20.

Golstein, P. and Kroemer, G. Redundant cell death mechanisms as relics and backups. Cell Death Diff 12 (2005), pp. 1490-1496.

Good, D.J., Polverini, P.J., Rastinejad, F., Le Beau, M.M., Lemons, R.S., Frazier, W.A. and Bouck, N.P. A tumor suppressor-dependent inhibitor of angiogenesis is immunologically and functionally indistinguishable from a fragment of thrombospondin. PNAS 87 (1990), pp. 6624-6628.

Gorbsky, G.J. and Ricketts, W.A. Differential expression of a phosphoepitope at the kinetochores of moving chromosomes. J Cell Biol 122 (1993), pp. 1311-1321.

Guicciardi, M.E., Deussing, J., Miyoshi, H., Bronk, S.F., Svingen, P.A., Peters, C., Kaufmann, S.H. and Gores, G.J. Cathepsin B contributes to TNF- $\alpha$-mediated hepatocyte apoptosis by promoting mitochondrial release of cytochrome c. $J$ Clin Invest 106 (2000), pp. 1127-1137.

Gupta, M.K. and Qin, R.Y. Mechanism and its regulation of tumor-induced angiogenesis. World J Gastroenterol 9 (2003), pp. 1144-1155.

Gürel, G., Blaha, G., Steitz, T.A. and Moore, P.B. Structures of triacetyloleandomycin and mycalamide $\mathrm{A}$ bind to the large ribosomal subunit of Haloarcula marismortui. Antimicrob Agents Chemother 53 (2009), pp. 5010-5014. 
Hait, W.N. Anticancer drug development: the grand challenges. Nat Rev Drug Discov 9 (2010), pp. 253-254.

Haldar, S., Jena, N. and Croce, C.M. Inactivation of Bcl-2 by phosphorylation. PNAS 92 (1995), pp. 4507-4511.

Hambley, T.W. Is anticancer drug development heading in the right direction? Cancer Res 69 (2009), pp. 1259-1262.

Hamel, E., Day, B.W., Miller, J.H., Jung, M.K., Northcote, P.T., Ghosh, A.K., Curran, D.P., Cushman, M., Nicolaou, K.C., Paterson, I. and Sorensen, E.J. Synergistic effects of peloruside A and laulimalide with taxoid site drugs, but not with each other, on tubulin assembly. Mol Pharmacol 70 (2006), pp. 1555-1564.

Han, G.-Z., Liu, Z.-J., Shimoi, K. and Zhu, B.T. Synergism between the anticancer actions of 2-methoxyestradiol and microtubule-disrupting agents in human breast cancer. Cancer Res 65 (2005), pp. 387-393.

Hayflick, L. and Moorhead, P.S. The serial cultivation of human diploid cell strains. Exp Cell Res 25 (1961), pp. 585-621.

Hayot, C., Farinelle, S., De Decker, R., Decaestecker, C., Darro, F., Kiss, R. and Van Damme, M. In vitro pharmacological characterizations of the anti-angiogenic and anti-tumor cell migration properties mediated by microtubule-affecting drugs, with special emphasis on the organization of the actin cytoskeleton. Int $J$ Oncol 21 (2002), pp. 417-425.

Hermeking, H., Rago, C., Schuhmacher, M., Li, Q., Barrett, J.F., Obaya, A.J., O'Connell, B.C., Mateyak, M.K., Tam, W., Kohlhuber, F., Dang, C.V., Sedivy, J.M., Eick, D., Vogelstein, B. and Kinzler, K.W. Identification of CDK4 as a target of c-MYC. PNAS 97 (2000), pp. 2229-2234.

Himmler, A., Drechsel, D., Kirschner, M.W. and Martin, D.W. Tau consists of a set of proteins with repeated C-terminal microtubule-binding domains and variable Nterminal domains. Mol Cell Biol 9 (1989), pp. 1381-1388.

Hirata, Y. and Uemura, D. Halichondrins - antitumor polyether macrolides from a marine sponge. Pure and Appl. Chem 58 (1986), pp. 701-710.

Hirokawa, N., Noda, Y. and Okada, Y. Kinesin and dynein superfamily proteins in organelle transport and cell division. Curr Opin Cell Biol 10 (1998), pp. 60-73.

Hoeben, A., Landuyt, B., Highley, M.S., Wildiers, H., Van Oosterom, A.T. and De Bruijn, E.A. Vascular endothelial growth factor and angiogenesis. Pharmacol Rev 56 (2004), pp. 549-580.

Hoffmann, I., Clarke, P.R., Marcote, M.J., Karsenti, E. and Draetta, G. Phosphorylation and activation of human cdc25-C by cdc2-cyclin B and its involvement in the self-amplification of MPF at mitosis. EMBO J 12 (1993), pp. 53-63.

Honore, S., Kamath, K., Braguer, D., Horwitz, S.B., Wilson, L., Briand, C. and Jordan, M.A. Synergistic suppression of microtubule dynamics by discodermolide and paclitaxel in non-small cell lung carcinoma cells. Cancer Res 64 (2004), pp. 4957-4964.

Hood, K.A., Bäckström, B.T., West, L.M., Northcote, P.T., Berridge, M.V. and Miller, J.H. The novel cytotoxic sponge metabolite peloruside A, structurally similar to bryostatin-1, has unique bioactivity independent of protein kinase C. AntiCancer Drug Design 16 (2001a), pp. 155-166.

Hood, K.A., West, L.M., Northcote, P.T., Berridge, M.V. and Miller, J.H. Induction of apoptosis by the marine sponge (Mycale) metabolites, mycalamide $\mathrm{A}$ and pateamine. Apoptosis 6 (2001b), pp. 207-219.

Hood, K.A., West, L.M., Rouwe, B., Northcote, P.T., Berridge, M.V., Wakefield, S.J. and Miller, J.H. Peloruside A, a novel antimitotic agent with paclitaxel-like microtubule-stabilizing activity. Cancer Res 62 (2002), pp. 3356-3360. 
Horio, T. and Hotani, H. Visualization of the dynamic instability of individual microtubules by dark-field microscopy. Nature 321 (1986), pp. 605-607.

Hotchkiss, K.A., Ashton, A.W., Mahmood, R., Russell, R.G., Sparano, J.A. and Schwartz, E.L. Inhibition of endothelial cell function in vitro and angiogenesis in vivo by docetaxel (Taxotere): Association with impaired repositioning of the microtubule organizing center. Mol Cancer Ther 1 (2002), pp. 1191-1200.

Howell, B., Deacon, H. and Cassimeris, L. Decreasing oncoprotein 18/stathmin levels reduces microtubule catastrophes and increases microtubule polymer in vivo. $J$ Cell Sci 112 (1999a), pp. 3713-3722.

Howell, B., Larsson, N., Gullberg, M. and Cassimeris, L. Dissociation of the tubulinsequestering and microtubule catastrophe-promoting activities of oncoprotein 18/stathmin. Mol Biol Cell 10 (1999b), pp. 105-118.

Howell, B.J., Hoffman, D.B., Fang, G., Murray, A.W. and Salmon, E.D. Visualization of Mad2 dynamics at kinetochores, along spindle fibers, and at spindle poles in living cells. J Cell Biol 150 (2000), pp. 1233-1249.

Hoyt, M.A., Totis, L. and Roberts, B.T. S. cerevisiae genes required for cell cycle arrest in response to loss of microtubule function. Cell 66 (1991), pp. 507-517.

Huisman, C., Ferreira, C.G., Broker, L.E., Rodriguez, J.A., Smit, E.F., Postmus, P.E., Kruyt, F.A.E. and Giaccone, G. Paclitaxel triggers cell death primarily via caspase-independent routes in the non-small cell lung cancer cell line NCIH460. Clin Cancer Res 8 (2002), pp. 596-606.

Hurwitz, H., Fehrenbacher, L., Novotny, W., Cartwright, T., Hainsworth, J., Heim, W., Berlin, J., Baron, A., Griffing, S., Holmgren, E., Ferrara, N., Fyfe, G., Rogers, B., Ross, R. and Kabbinavar, F. Bevacizumab plus irinotecan, fluorouracil, and leucovorin for metastatic colorectal cancer. New Engl J Med 350 (2004), pp. 2335-2342.

Huyck, T.K., Gradishar, W., Manuguid, F. and Kirkpatrick, P. Eribulin mesylate. Nat Rev Drug Discov 10 (2011), pp. 173-174.

Huzil, J.T., Chik, J.K., Slysz, G.W., Freedman, H., Tuszynski, J., Taylor, R.E., Sackett, D.L. and Schriemer, D.C. A unique mode of microtubule stabilization induced by peloruside A. J Mol Biol 378 (2008), pp. 1016-1030.

Hwang, L.H., Lau, L.F., Smith, D.L., Mistrot, C.A., Hardwick, K.G., Hwang, E.S., Amon, A. and Murray, A.W. Budding yeast Cdc20: A target of the spindle checkpoint. Science 279 (1998), pp. 1041-1044.

Hyland, K.M., Kingsbury, J., Koshland, D. and Hieter, P. Ctf19p: A novel kinetochore protein in Saccharomyces cerevisiae and a potential link between the kinetochore and mitotic spindle. J Cell Biol 145 (1999), pp. 15-28.

Iancu, C., Mistry, S.J., Arkin, S., Wallenstein, S. and Atweh, G.F. Effects of stathmin inhibition on the mitotic spindle. J Cell Sci 114 (2001), pp. 909-916.

Ikui, A.E., Yang, C.-P.H., Matsumoto, T. and Horwitz, S.B. Low concentrations of Taxol ${ }^{\circledR}$ cause mitotic delay followed by premature dissociation of $\mathrm{p} 55 \mathrm{CDC}$ from Mad2 and BubR1 and abrogation of the spindle checkpoint, leading to aneuploidy. Cell Cycle 4 (2005), pp. 1385-1388.

Jackson, J.R., Patrick, D.R., Dar, M.M. and Huang, P.S. Targeted anti-mitotic therapies: Can we improve on tubulin agents? Nature Rev Cancer 7 (2007), pp. 107-117.

Janke, C. and Bulinski, J.C. Post-translational regulation of the microtubule cytoskeleton: mechanisms and functions. Nat Rev Mol Cell Biol 12 (2011), pp. 773-786.

Janosi, I.M., Chretien, D. and Flyvbjerg, H. Structural microtubule cap: stability, catastrophe, rescue, and third state. Biophys $J \mathbf{8 3}$ (2002), pp. 1317-1330.

Janssen, K., Pohlmann, S., Jänicke, R.U., Schulze-Osthoff, K. and Fischer, U. Apaf-1 and caspase- 9 deficiency prevents apoptosis in a Bax-controlled pathway and 
promotes clonogenic survival during paclitaxel treatment. Blood 110 (2007), pp. 3662-3672.

Janz, A., Sevignani, C., Kenyon, K., Ngo, C.V. and Thomas-Tikhonenko, A. Activation of the Myc oncoprotein leads to increased turnover of thrombospondin-1 mRNA. Nucleic Acids Res 28 (2000), pp. 2268-2275.

Jaxel, C., Kohn, K.W., Wani, M.C., Wall, M.E. and Pommier, Y. Structure-activity study of the actions of camptothecin derivatives on mammalian topoisomerase I: Evidence for a specific receptor site and a relation to antitumor activity. Cancer Res 49 (1989), pp. 1465-1469.

Jiménez-Barbero, J., Canales, A., Northcote, P.T., Buey, R.M., Andreu, J.M. and Diaz, J.F. NMR determination of the bioactive conformation of peloruside A bound to microtubules. J Am Chem Soc 128 (2006), pp. 8757-8765.

Jin, M. and Taylor, R.E. Total synthesis of (+)-peloruside A. Org Lett 7 (2005), pp. 1303-1305.

Jordan, M.A., Toso, R., Thrower, D. and Wilson, L. Mechanism of mitotic block and inhibition of cell proliferation by Taxol at low concentrations. Proc Natl Acad Sci USA 90 (1993), pp. 9552-9556.

Jordan, M.A., Wendell, K., Gardiner, S., Brent Derry, W., Copp, H. and Wilson, L. Mitotic block induced in HeLa cells by low concentrations of paclitaxel (Taxol) results in abnormal mitotic exit and apoptotic cell death. Cancer Res 56 (1996), pp. 816-825.

Jordan, M.A. and Wilson, L. Microtubules as a target for anticancer drugs. Nat Rev Cancer 4 (2004), pp. 253-265.

Kallio, M., Weinstein, J., Daum, J.R., Burke, D.J. and Gorbsky, G.J. Mammalian p55CDC mediates association of the spindle checkpoint protein Mad2 with the cyclosome/anaphase-promoting complex, and is involved in regulating anaphase onset and late mitotic events. J Cell Biol 141 (1998), pp. 1393-1406.

Kamath, K., Okouneva, T., Larson, G., Panda, D., Wilson, L. and Jordan, M.A. 2Methoxyestradiol suppresses microtubule dynamics and arrests mitosis without depolymerizing microtubules. Mol Cancer Ther 5 (2006), pp. 2225-2233.

Kanakkanthara, A., Northcote, P.T. and Miller, J.H. $\beta$ II-tubulin and $\beta$ III-tubulin mediate sensitivity to peloruside A and laulimalide, but not paclitaxel or vinblastine, in human ovarian carcinoma cells. Mol Cancer Ther 11 (2012), pp. 393-404.

Kanakkanthara, A., Wilmes, A., O'Brate, A., Escuin, D., Chan, A., Gjyrezi, A., Crawford, J., Rawson, P., Kivell, B., Northcote, P.T., Hamel, E., Giannakakou, P. and Miller, J.H. Peloruside- and laulimalide-resistant human ovarian carcinoma cells have $\beta$ I-tubulin mutations and altered expression of BII- and BIII-tubulin isotypes. Mol Cancer Ther 10 (2011), pp. 1419-1429.

Kato, J., Matsushime, H., Hiebert, S.W., Ewen, M.E. and Sherr, C.J. Direct binding of cyclin $\mathrm{D}$ to the retinoblastoma gene product $(\mathrm{pRb})$ and $\mathrm{pRb}$ phosphorylation by the cyclin D-dependent kinase CDK4. Genes Dev 7 (1993), pp. 331-342.

Kaufmann, S., Kottke, T., Miguel, M., Henzing, A. and Earnshaw, W. Analysis of caspase activation during apoptosis. Curr Protoc Cell Biol 11 (2001), pp. 18.2.118.2.29.

Kaufmann, S.H. and Vaux, D.L. Alterations in the apoptotic machinery and their potential role in anticancer drug resistance. Oncogene 22 (2003), pp. 7414-7430.

Kavallaris, M. Microtubules and resistance to tubulin-binding agents. Nat Rev Cancer 10 (2010), pp. 194-204.

Kaverina, I., Krylyshkina, O. and Small, J.V. Microtubule targeting of substrate contacts promotes their relaxation and dissociation. J Cell Biol 146 (1999), pp. 1033-1044. 
Kaverina, I., Rottner, K. and Small, J.V. Targeting, capture, and stabilization of microtubules at early focal adhesions. J Cell Biol 142 (1998), pp. 181-190.

Kawahara, A., Ohsawa, Y., Matsumura, H., Uchiyama, Y. and Nagata, S. Caspaseindependent cell killing by Fas-associated protein with death domain. $J$ Cell Biol 143 (1998), pp. 1353-1360.

Keniry, M.E., Kemp, H.A., Rivers, D.M. and Sprague Jr, G.F. The Identification of Pc11-Interacting Proteins That Genetically Interact with Cla4 May Indicate a Link between G1 Progression and Mitotic Exit. Genetics 166 (2004), pp. 11771186.

Kerbel, R. and Folkman, J. Clinical translation of angiogenesis inhibitors. Nat Rev Cancer 2 (2002), pp. 727-739.

Khodjakov, A. and Pines, J. Centromere tension: A divisive issue. Nat Cell Biol 12 (2010), pp. 919-923.

Kim, K.J., Li, B., Winer, J., Armanini, M., Gillett, N., Phillips, H.S. and Ferrara, N. Inhibition of vascular endothelial growth factor-induced angiogenesis suppresses tumour growth in vivo. Nature 362 (1993), pp. 841-844.

Kingston, D.G.I. Tubulin-interactive natural products as anticancer agents. J Nat Prod 72 (2009), pp. 507-515.

Klein, L. and Horwitz, S.: Contributions of apoptosis and senescence to cytotoxicity produced by microtubule-stabilizing agents. In: Gewirtz, D.A., Holt, S.E. and Grant, S. (Editors), Apoptosis, Senescence, and Cancer. Humana Press (2007), pp. 465-476.

Klein, L.E., Freeze, B.S., Smith, A.B.r. and Horwitz, S.B. The microtubule stabilizing agent discodermolide is a potent inducer of accelerated cellular senescence. Cell Cycle 4 (2005), pp. 501-507.

Kleinman, H.K., McGarvey, M.L., Hassell, J.R., Star, V.L., Cannon, F.B., Laurie, G.W. and Martin, G.R. Basement membrane complexes with biological activity. Biochem 25 (1986), pp. 312-318.

Kleinman, H.K., McGarvey, M.L., Liotta, L.A., Robey, P.G., Tryggvason, K. and Martin, G.R. Isolation and characterization of type IV procollagen, laminin, and heparan sulfate proteoglycan from the EHS sarcoma. Biochem 21 (1982), pp. 6188-6193.

Knies-Bamforth, U.E., Fox, S.B., Poulsom, R., Evan, G.I. and Harris, A.L. c-Myc interacts with hypoxia to induce angiogenesis in vivo by a vascular endothelial growth factor-dependent mechanism. Cancer Res 64 (2004), pp. 6563-6570.

Kola, I. and Landis, J. Can the pharmaceutical industry reduce attrition rates? Nat Rev Drug Discov 3 (2004), pp. 711-716.

Kopper, L. and Peták, I.: Apoptosis and cancer. In: Kaiser, H.E. and Nasir, A. (Editors), Selected aspects of cancer progression: metastasis, apoptosis and immune response. Springer Science (2008), pp. 103-113.

Kottke, T.J., Blajeski, A.L., Meng, X.W., Svingen, P.A., Ruchaud, S., Mesner, P.W., Boerner, S.A., Samejima, K., Henriquez, N.V., Chilcote, T.J., Lord, J., Salmon, M., Earnshaw, W.C. and Kaufmann, S.H. Lack of correlation between caspase activation and caspase activity assays in paclitaxel-treated MCF-7 breast cancer cells. J Biol Chem 277 (2002), pp. 804-815.

Kouzu, Y., Uzawa, K., Koike, H., Saito, K., Nakashima, D., Higo, M., Endo, Y., Kasamatsu, A., Shiiba, M., Bukawa, H., Yokoe, H. and Tanzawa, H. Overexpression of stathmin in oral squamous-cell carcinoma: Correlation with tumour progression and poor prognosis. Br J Cancer 94 (2006), pp. 717-723.

Kowalski, R.J., Giannakakou, P., Gunasekera, S.P., Longley, R.E., Day, B.W. and Hamel, E. The microtubule-stabilizing agent discodermolide competitively inhibits the binding of paclitaxel (Taxol) to tubulin polymers, enhances tubulin 
nucleation reactions more potently than paclitaxel, and inhibits the growth of paclitaxel-resistant cells. Mol Pharmacol 52 (1997), pp. 613-622.

Kraljevic, S., Sedic, M., Scott, M., Gehrig, P., Schlapbach, R. and Pavelic, K. Casting light on molecular events underlying anti-cancer drug treatment: What can be seen from the proteomics point of view? Cancer Treat Rev 32 (2006), pp. 619629.

Krendel, M., Zenke, F.T. and Bokoch, G.M. Nucleotide exchange factor GEF-H1 mediates cross-talk between microtubules and the actin cytoskeleton. Nat Cell Biol 4 (2002), pp. 294-301.

Kroemer, G., Galluzzi, L., Vandenabeele, P., Abrams, J., Alnemri, E.S., Baehrecke, E.H., Blagosklonny, M.V., El-Deiry, W.S., Golstein, P., Green, D.R., Hengartner, M., Knight, R.A., Kumar, S., Lipton, S.A., Malorni, W., Nuñez, G., Peter, M.E., Tschopp, J., Yuan, J., Piacentini, M., Zhivotovsky, B. and Melino, G. Classification of cell death: Recommendations of the Nomenclature Committee on Cell Death 2009. Cell death diff 16 (2009), pp. 3-11.

Kubota, Y., Kleinman, H.K., Martin, G.R. and Lawley, T.J. Role of laminin and basement membrane in the morphological differentiation of human endothelial cells into capillary-like structures. J Cell Biol 107 (1988), pp. 1589-1598.

Kuilman, T., Michaloglou, C., Mooi, W.J. and Peeper, D.S. The essence of senescence. Genes Dev 24 (2010), pp. 2463-2479.

Kurz, D.J., Decary, S., Hong, Y. and Erusalimsky, J.D. Senescence-associated (beta)galactosidase reflects an increase in lysosomal mass during replicative ageing of human endothelial cells. J Cell Sci 113 (2000), pp. 3613-3622.

Kutuk, O. and Letai, A. Displacement of Bim by Bmf and Puma rather than increase in Bim level mediates paclitaxel-induced apoptosis in breast cancer cells. Cell Death Diff 17 (2010), pp. 1624-1635.

Lampson, M.A. and Cheeseman, I.M. Sensing centromere tension: Aurora B and the regulation of kinetochore function. Trends Cell Biol 21 (2011), pp. 133-140.

Lampson, M.A., Renduchitala, K., Khodjakov, A. and Kapoor, T.M. Correcting improper chromosomes-spindle attachments during cell division. Nat Cell Biol 6 (2004), pp. 232-237.

Larsson, L.G. Oncogene- and tumor suppressor gene-mediated suppression of cellular senescence. Sem Cancer Biol 21 (2011), pp. 367-376.

Larsson, N., Melander, H., Marklund, U., Osterman, O. and Gullberg, M. G $/ \mathrm{M}_{2}$ transition requires multisite phosphorylation of oncoprotein 18 by two distinct protein kinase systems. $J$ Biol Chem 270 (1995), pp. 14175-14183.

Lee, A.C., Fenster, B.E., Ito, H., Takeda, K., Bae, N.S., Hirai, T., Yu, Z.X., Ferrans, V.J., Howard, B.H. and Finkel, T. Ras proteins induce senescence by altering the intracellular levels of reactive oxygen species. J Biol Chem 274 (1999), pp. 7936-7940.

Lee, K.H., Yim, E.K., Kim, C.J., Namkoong, S.E., Um, S.J. and Park, J.S. Proteomic analysis of anti-cancer effects by paclitaxel treatment in cervical cancer cells. Gynecol Oncol 98 (2005), pp. 45-53.

Leontieva, O.V., Gudkov, A.V. and Blagosklonny, M.V. Weak p53 permits senescence during cell cycle arrest. Cell Cycle 9 (2010), pp. 4323-4327.

Lewis, S.A., Ivanov, I.E., Lee, G.-H. and Cowan, N.J. Organization of microtubules in dendrites and axons is determined by a short hydrophobic zipper in microtubuleassociated proteins MAP2 and tau. Nature 342 (1989), pp. 498-505.

Li, H., Zhu, H., Xu, C.-j. and Yuan, J. Cleavage of BID by caspase 8 mediates the mitochondrial damage in the Fas pathway of apoptosis. Cell 94 (1998), pp. 491501. 
Li, J. and Yuan, J. Caspases in apoptosis and beyond. Oncogene 27 (2008), pp. 61946206.

Li, K., Lee, L.A., Lu, X. and Wang, Q. Fluorogenic "click" reaction for labeling and detection of DNA in proliferating cells. Biotechniques 49 (2010), pp. 525-527.

Li, R., Moudgil, T., Ross, H.J. and Hu, H.M. Apoptosis of non-small-cell lung cancer cell lines after paclitaxel treatment involves the BH3-only proapoptotic protein Bim. Cell Death Diff 12 (2005), pp. 292-303.

Li, R. and Murray, A.W. Feedback control of mitosis in budding yeast. Cell 66 (1991), pp. 519-531.

Li, Y., Gorbea, C., Mahaffey, D., Rechsteiner, M. and Benezra, R. MAD2 associates with the cyclosome/anaphase-promoting complex and inhibits its activity. PNAS 94 (1997), pp. 12431-12436.

Liao, D. and Johnson, R.S. Hypoxia: A key regulator of angiogenesis in cancer. Cancer Metastasis Rev 26 (2007), pp. 281-290.

Liao, X., Wu, Y. and De Brabander, J.K. Total synthesis and absolute configuration of the novel microtubule-stabilizing agent peloruside A. Angew Chem Int Ed Engl 42 (2003), pp. 1648-1652.

Lin, A.W., Barradas, M., Stone, J.C., Van Aelst, L., Serrano, M. and Lowe, S.W. Premature senescence involving p53 and p16 is activated in response to constitutive MEK/MAPK mitogenic signaling. Genes Dev 12 (1998), pp. 30083019.

Litaudon, M., Hart, J.B., Blunt, J.W., Lake, R.J. and Munro, M.H. Isohomohalichondrin $\mathrm{B}$, a new antitumour polyether macrolide from the new zealand deep-water sponge Lissodendoryx sp. Tetrahedron Lett 35 (1994), pp. 9435-9438.

Litaudon, M., Hickford, S.J.H., Lill, R.E., Lake, R.J., Blunt, J.W. and Munro, M.H.G. Antitumor polyether macrolides: New and hemisynthetic halichondrins from the New Zealand deep-water sponge Lissodendoryx sp. J Org Chem 62 (1997), pp. 1868-1871.

Litwiniec, A., Grzanka, A., Helmin-Basa, A., Gackowska, L. and Grzanka, D. Features of senescence and cell death induced by doxorubicin in A549 cells: organization and level of selected cytoskeletal proteins. J Cancer Res Clin Oncol 136 (2010), pp. 717-736.

Liu, D., Vader, G., Vromans, M.J.M., Lampson, M.A. and Lens, S.M.A. Sensing chromosome bi-orientation by spatial separation of Aurora B kinase from kinetochore substrates. Science 323 (2009), pp. 1350-1353.

Lockhart, D.J. and Winzeler, E.A. Genomics, gene expression and DNA arrays. Nature 405 (2000), pp. 827-836.

Lodish, H.F., Berk, A., Zipursky, S.L., Matsudaira, P., Baltimore, D. and Darnell, J., Chapter 12: DNA Replication, Repair, and Recombination, 4 ed. W. H. Freeman, New York (2000).

Logarinho, E., Bousbaa, H., Dias, J.M., Lopes, C., Amorim, I., Antunes-Martins, A. and Sunkel, C.E. Different spindle checkpoint proteins monitor microtubule attachment and tension at kinetochores in Drosophila cells. J Cell Sci 117 (2004), pp. 1757-1771.

Long, B.H., Carboni, J.M., Wasserman, A.J., Cornell, L.A., Casazza, A.M., Jensen, P.R., Lindel, T., Fenical, W. and Fairchild, C.R. Eleutherobin, a novel cytotoxic agent that induces tubulin polymerization, is similar to paclitaxel (Taxol®). Cancer Res 58 (1998), pp. 1111-1115.

Lopata, M.A. and Cleveland, D.W. In vivo microtubules are copolymers of available beta-tubulin isotypes: localization of each of six vertebrate beta-tubulin isotypes using polyclonal antibodies elicited by synthetic peptide antigens. J Cell Biol 105 (1987), pp. 1707-1720. 
Lu, H., Murtagh, J. and Schwartz, E.L. The microtubule binding drug laulimalide inhibits vascular endothelial growth factor-induced human endothelial cell migration and is synergistic when combined with docetaxel (Taxotere). Mol Pharmacol 69 (2006), pp. 1207-1215.

$\mathrm{Lu}, \mathrm{X}$. and Kang, Y. Hypoxia and hypoxia-inducible factors: Master regulators of metastasis. Clin Cancer Res 16 (2010), pp. 5928-5935.

Lum, P.Y., Armour, C.D., Stepaniants, S.B., Cavet, G., Wolf, M.K., Butler, J.S., Hinshaw, J.C., Garnier, P., Prestwich, G.D., Leonardson, A., Garrett-Engele, P., Rush, C.M., Bard, M., Schimmack, G., Phillips, J.W., Roberts, C.J. and Shoemaker, D.D. Discovering modes of action for therapeutic compounds using a genome-wide screen of yeast heterozygotes. Cell 116 (2004), pp. 121-137.

Luzio, J.P., Pryor, P.R. and Bright, N.A. Lysosomes: Fusion and function. Nature Rev Mol Cell Biol 8 (2007), pp. 622-632.

MacKeigan, J.P., Clements, C.M., Lich, J.D., Pope, R.M., Hod, Y. and Ting, J.P.Y. Proteomic profiling drug-induced apoptosis in non-small cell lung carcinoma: identification of RS/DJ-1 and RhoGDIa. Cancer Res 63 (2003), pp. 6928-6934.

Maiato, H., DeLuca, J., Salmon, E.D. and Earnshaw, W.C. The dynamic kinetochoremicrotubule interface. J Cell Sci 117 (2004), pp. 5461-5477.

Mallik, R. and Gross, S.P. Molecular motors: strategies to get along. Curr Biol 14 (2004), pp. R971-R982.

Marani, M., Tenev, T., Hancock, D., Downward, J. and Lemoine, N.R. Identification of novel isoforms of the $\mathrm{BH} 3$ domain protein bim which directly activate Bax to trigger apoptosis. Mol Cell Biol 22 (2002), pp. 3577-3589.

Marton, M.J., Derisi, J.L., Bennett, H.A., Iyer, V.R., Meyer, M.R., Roberts, C.J., Stoughton, R., Burchard, J., Slade, D., Dai, H., Bassett Jr, D.E., Hartwell, L.H., Brown, P.O. and Friend, S.H. Drug target validation and identification of secondary drug target effects using DNA microarrays. Nat Rev Med 4 (1998), pp. 1293-1301.

Maxwell, P.H., Dachs, G.U., Gleadle, J.M., Nicholls, L.G., Harris, A.L., Stratford, I.J., Hankinson, O., Pugh, C.W. and Ratcliffe, P.J. Hypoxia-inducible factor-1 modulates gene expression in solid tumors and influences both angiogenesis and tumor growth. PNAS 94 (1997), pp. 8104-8109.

McEwen, B.F., Heagle, A.B., Cassels, G.O., Buttle, K.F. and Rieder, C.L. Kinetochore fiber maturation in PtK1 cells and its implications for the mechanisms of chromosome congression and anaphase onset. J Cell Biol 137 (1997), pp. 15671580 .

McGonigle, S. and Shifrin, V. In vitro assay of angiogenesis: Inhibition of capillary tube formation. Curr Protoc Pharmacol Supp 43 (2008).

Mendez, G., Policarpi, C., Cenciarelli, C., Tanzarella, C. and Antoccia, A. Role of Bim in apoptosis induced in $\mathrm{H} 460$ lung tumor cells by the spindle poison Combretastatin-A4. Apoptosis 16 (2011), pp. 940-949.

Menssen, A., Epanchintsev, A., Rezaei, N., Lodygin, D., Jung, P., Verdoodt, B., Diebold, J. and Hermeking, H. c-MYC delays prometaphase by direct transactivation of MAD2 and BubR1: Identification of mechanisms underlying c-MYC-induced DNA damage and chromosomal instability. Cell Cycle 6 (2007), pp. 339-352.

Merdes, A., Ramyar, K., Vechio, J.D. and Cleveland, D.W. A complex of NuMA and cytoplasmic dynein is essential for mitotic spindle assembly. Cell 87 (1996), pp. 447-458.

Meyer, C., Ferguson, D., Krauth, M., Wick, M. and Northcote, P.T. RTA 301 (peloruside): a novel microtubule stabilizer with potent in vivo activity against lung cancer and resistant breast cancer. Eur J Cancer Suppl 4 (2006), p. 192. 
Michaloglou, C., Vredeveld, L.C.W., Soengas, M.S., Denoyelle, C., Kuilman, T., Van Der Horst, C.M.A.M., Majoor, D.M., Shay, J.W., Mooi, W.J. and Peeper, D.S. BRAFE600-associated senescence-like cell cycle arrest of human naevi. Nature 436 (2005), pp. 720-724.

Mielgo, A., Torres, V.A., Clair, K., Barbero, S. and Stupack, D.G. Paclitaxel promotes a caspase 8-mediated apoptosis through death effector domain association with microtubules. Oncogene 28 (2009), pp. 3551-3562.

Miller, J.H., Rouwé, B., Gaitanos, T.N., Hood, K.A., Crume, K.P., Bäckström, B.T., La Flamme, A.C., Berridge, M.V. and Northcote, P.T. Peloruside A enhances apoptosis in H-ras-transformed cells and is cytotoxic to proliferating $\mathrm{T}$ cells. Apoptosis 9 (2004), pp. 785-796.

Miller, J.H., Singh, A.J. and Northcote, P.T. Microtubule-stabilizing drugs from marine sponges: focus on peloruside A and zampanolide. Mar Drugs 8 (2010), pp. 1059-1079.

Milner, A.E., Palmer, D.H., Hodgkin, E.A., Eliopoulos, A.G., Knox, P.G., Poole, C.J., Kerr, D.J. and Young, L.S. Induction of apoptosis by chemotherapeutic drugs: The role of FADD in activation of caspase- 8 and synergy with death receptor ligands in ovarian carcinoma cells. Cell Death Diff 9 (2002), pp. 287-300.

Mistry, S.J., Bank, A. and Atweh, G.F. Synergistic anti-angiogenic effects of stathmin inhibition and Taxol exposure. Mol Cancer Res 5 (2007), pp. 773-782.

Mitra, M., Kandalam, M., Sundaram, C.S., Verma, R.S., Maheswari, U.K., Swaminathan, S. and Krishnakumar, S. Reversal of stathmin-mediated microtubule destabilization sensitizes retinoblastoma cells to a low dose of antimicrotubule agents: A novel synergistic therapeutic intervention. Invest Ophthalmol Vis Sci 52 (2011), pp. 5441-5448.

Mizuno, M. and Singer, S.J. A possible role for stable microtubules in intracellular transport from the endoplasmic reticulum to the Golgi apparatus. J Cell Sci 107 (1994), pp. 1321-1331.

Morrow, C.J., Tighe, A., Johnson, V.L., Scott, M.I.F., Ditchfield, C. and Taylor, S.S. Bub1 and aurora B cooperate to maintain BubR1-mediated inhibition of APC/C ${ }^{\mathrm{Cdc} 20} . J$ Cell Sci 118 (2005), pp. 3639-3652.

Mullard, A. 2010 FDA drug approvals. Nat Rev Drug Discov 10 (2011), pp. 82-85.

Murata, R., Siemann, D.W., Overgaard, J. and Horsman, M.R. Interaction between combretastatin A-4 disodium phosphate and radiation in murine tumors. Radiother Oncol 60 (2001), pp. 155-161.

Murtagh, J., Lu, H. and Schwartz, E.L. Taxotere-induced inhibition of human endothelial cell migration is a result of heat shock protein 90 degradation. Cancer Res 66 (2006), pp. 8192-8199.

Musacchio, A. and Hardwick, K.G. The spindle checkpoint: Structural insights into dynamic signalling. Nat Rev Mol Cell Biol 3 (2002), pp. 731-741.

Musacchio, A. and Salmon, E.D. The spindle-assembly checkpoint in space and time. Nat Rev Mol Cell Biol 8 (2007), pp. 379-393.

Nalbant, P., Chang, Y.C., Birkenfeld, J., Chang, Z.F. and Bokoch, G.M. Guanine nucleotide exchange factor-H1 regulates cell migration via localized activation of RhoA at the leading edge. Mol Biol Cell 20 (2009), pp. 4070-4082.

Narita, M., Nuñez, S., Heard, E., Lin, A.W., Hearn, S.A., Spector, D.L., Hannon, G.J. and Lowe, S.W. Rb-mediated heterochromatin formation and silencing of E2F target genes during cellular senescence. Cell 113 (2003), pp. 703-716.

Nasmyth, K. Segregating sister genomes: The molecular biology of chromosome separation. Science 297 (2002), pp. 559-565.

Naumanen, P., Lappalainen, P. and Hotulainen, P. Mechanisms of actin stress fibre assembly. J Microsc 231 (2008), pp. 446-454. 
Nezi, L. and Musacchio, A. Sister chromatid tension and the spindle assembly checkpoint. Curr Opin Cell Biol 21 (2009), pp. 785-795.

Nguyen, T.L., Xu, X., Gussio, R., Ghosh, A.K. and Hamel, E. The assembly-inducing laulimalide/peloruside a binding site on tubulin: Molecular modeling and biochemical studies with $\left[{ }^{3} \mathrm{H}\right]$ Peloruside A. J Chem Inf Mod 50 (2010), pp. 2019-2028.

Nicholson, D.W., Ali, A., Thornberry, N.A., Vaillancourt, J.P., Ding, C.K., Gallant, M., Gareau, Y., Griffin, P.R., Labelle, M., Lazebnik, Y.A., Munday, N.A., Raju, S.M., Smulson, M.E., Yamin, T.-T., Yu, V.L. and Miller, D.K. Identification and inhibition of the ICE/CED-3 protease necessary for mammalian apoptosis. Nature 376 (1995), pp. 37-43.

Nilsson, J.A. and Cleveland, J.L. Myc pathways provoking cell suicide and cancer. Oncogene 22 (2003), pp. 9007-9021.

Nogales, E. Structural insights into microtubule function. Ann Rev Biophys Biomol Struct 30 (2001), pp. 397-420.

Northcote, P.T., Blunt, J.W. and Munro, M.H.G. Pateamine: a potent cytotoxin from the New Zealand marine sponge, mycale sp. Tetrahedron Lett 32 (1991), pp. 64116414.

Northcote, P.T., Miller, J.H., West, L.M. and Hood, K.A.: Bioactive compound (Peloruside A). US Patent Number US 6,790,862 B2. Victoria Link Limited, Wellington, NZ, US (2004).

O'Reilly, M.S., Boehm, T., Shing, Y., Fukai, N., Vasios, G., Lane, W.S., Flynn, E., Birkhead, J.R., Olsen, B.R. and Folkman, J. Endostatin: An endogenous inhibitor of angiogenesis and tumor growth. Cell 88 (1997), pp. 277-285.

O'Reilly, M.S., Holmgren, L., Shing, Y., Chen, C., Rosenthal, R.A., Moses, M., Lane, W.S., Cao, Y., Helene Sage, E. and Folkman, J. Angiostatin: A novel angiogenesis inhibitor that mediates the suppression of metastases by a Lewis lung carcinoma. Cell 79 (1994), pp. 315-328.

Oberle, C., Huai, J., Reinheckel, T., Tacke, M., Rassner, M., Ekert, P.G., Buellesbach, J. and Borner, C. Lysosomal membrane permeabilization and cathepsin release is a Bax/Bak-dependent, amplifying event of apoptosis in fibroblasts and monocytes. Cell Death Diff 17 (2010), pp. 1167-1178.

Ofir, R., Seidman, R., Rabinski, T., Krup, M., Yavelsky, V., Weinstein, Y. and Wolfson, M. Taxol-induced apoptosis in human SKOV3 ovarian and MCF7 breast carcinoma cells is caspase-3 and caspase-9 independent. Cell Death Diff 9 (2002), pp. 636-642.

Ola, M.S., Nawaz, M. and Ahsan, H. Role of Bcl-2 family proteins and caspases in the regulation of apoptosis. Mol Cell Biochem 351 (2011), pp. 41-58.

Page, M.J., Handley, S.J., Northcote, P.T., Cairney, D. and Willan, R.C. Successes and pitfalls of the aquaculture of the sponge Mycale hentscheli. Aquaculture 312 (2011), pp. 52-61.

Page, M.J., Northcote, P.T., Webb, V.L., Mackey, S. and Handley, S.J. Aquaculture trials for the production of biologically active metabolites in the New Zealand sponge Mycale hentscheli (Demospongiae: Poecilosclerida). Aquaculture 250 (2005), pp. 256-269.

Park, S.J., Wu, C.H., Gordon, J.D., Zhong, X., Emami, A. and Safa, A.R. Taxol induces caspase-10-dependent apoptosis. J Biol Chem 279 (2004), pp. 51057-51067.

Pasquier, E., Honore, S., Pourroy, B., Jordan, M.A., Lehmann, M., Briand, C. and Braguer, D. Antiangiogenic concentrations of paclitaxel induce an increase in microtubule dynamics in endothelial cells but not in cancer cells. Cancer Res $\mathbf{6 5}$ (2005), pp. 2433-2440. 
Passos, J.F., Nelson, G., Wang, C., Richter, T., Simillion, C., Proctor, C.J., Miwa, S., Olijslagers, S., Hallinan, J., Wipat, A., Saretzki, G., Rudolph, K.L., Kirkwood, T.B.L. and Von Zglinicki, T. Feedback between p21 and reactive oxygen production is necessary for cell senescence. Mol Sys Biol 6 (2010).

Patel, J.H., Loboda, A.P., Showe, M.K., Showe, L.C. and McMahon, S.B. Analysis of genomic targets reveals complex functions of MYC. Nat Rev Cancer 4 (2004), pp. 562-568.

Pedley, R.B., Hill, S.A., Boxer, G.M., Flynn, A.A., Boden, R., Watson, R., Dearling, J., Chaplin, D.J. and Begent, R.H.J. Eradication of colorectal xenografts by combined radioimmunotherapy and combretastatin A-4 3-O-phosphate. Cancer Res 61 (2001), pp. 4716-4722.

Pelengaris, S., Khan, M. and Evan, G. c-MYC: More than just a matter of life and death. Nature Rev Cancer 2 (2002), pp. 764-776.

Pereira, N.A. and Song, Z. Some commonly used caspase substrates and inhibitors lack the specificity required to monitor individual caspase activity. Biochem Biophys Res Commun 377 (2008), pp. 873-877.

Perry, N.B., Blunt, J.W., Munro, M.H.G. and Pannell, L.K. Mycalamide A, an antiviral compound from a New Zealand sponge of the genus Mycale. J Am Chem Soc 110 (1988), pp. 4850-4851.

Perry, N.B., Blunt, J.W., Munro, M.H.G. and Thompson, A.M. Antiviral and antitumor agents from a New Zealand sponge, Mycale sp. 2. Structures and solution conformations of mycalamides A and B. J Org Chem 55 (1990), pp. 223-227.

Peters, J.-M. The anaphase promoting complex/cyclosome: a machine designed to destroy. Nat Rev Mol Cell Biol 7 (2006), pp. 644-656.

Pettit, G.R., Cragg, G.M. and Singh, S.B. Antineoplastic agents, 122. Constituents of Combretum caffrum. J Nat Prod 50 (1987), pp. 386-391.

Pfaller, W., Gstraunthaler, G. and Loidl, P. Morphology of the differentiation and maturation of LLK-PK1 epithelia. J Cell Physiol 142 (1990), pp. 247-254.

Photiou, A., Shah, P., Leong, L.K., Moss, J. and Retsas, S. In vitro synergy of paclitaxel (Taxol) and vinorelbine (navelbine) against human melanoma cell lines. Eur $J$ Cancer 33 (1997), pp. 463-470.

Pineda, O., Farras, J., Maccari, L., Manetti, F., Botta, M. and Vilarrasa, J. Computational comparison of microtubule-stabilising agents laulimalide and peloruside with Taxol and colchicine. Bioorg Med Chem Lett 14 (2004), pp. 4825-4829.

Pines, J. Mitosis: A matter of getting rid of the right protein at the right time. Trends Cell Biol 16 (2006), pp. 55-63.

Plank, M.J. and Sleeman, B.D. Tumour-induced angiogenesis: A review. J Theor Med 5 (2003), pp. 137-153.

Poirier, K., Saillour, Y., Bahi-Buisson, N., Jaglin, X.H., Fallet-Bianco, C., Nabbout, R., Castelnau-Ptakhine, L., Roubertie, A., Attie-Bitach, T., Desguerre, I., Genevieve, D., Barnerias, C., Keren, B., Lebrun, N., Boddaert, N., EnchaRazavi, F. and Chelly, J. Mutations in the neuronal $\beta$-tubulin subunit TUBB3 result in malformation of cortical development and neuronal migration defects. Hum Mol Genet 19 (2010), pp. 4462-4473.

Poruchynsky, M.S., Wang, E.E., Rudin, C.M., Blagosklonny, M.V. and Fojo, T. Bcl-xL is phosphorylated in malignant cells following microtubule disruption. Cancer Res 58 (1998), pp. 3331-3338.

Pourroy, B., Honoré, S., Pasquier, E., Bourgarel-Rey, V., Kruczynski, A., Briand, C. and Braguer, D. Antiangiogenic concentrations of vinflunine increase the interphase microtubule dynamics and decrease the motility of endothelial cells. Cancer Res 66 (2006), pp. 3256-3263. 
Pryor, D.E., O'Brate, A., Bilcer, G., Díaz, J.F., Wang, Y., Wang, Y., Kabaki, M., Jung, M.K., Andreu, J.M., Ghosh, A.K., Giannakakou, P. and Hamel, E. The microtubule stabilizing agent laulimalide does not bind in the taxoid site, kills cells resistant to paclitaxel and epothilones, and may not require its epoxide moiety for activity. Biochemistry 41 (2002), pp. 9109-9115.

Puthalakath, H., Huang, D.C.S., O'Reilly, L.A., King, S.M. and Strasser, A. The proapoptotic activity of the Bcl-2 family member Bim is regulated by interaction with the dynein motor complex. Mol Cell 3 (1999), pp. 287-296.

Raftopoulou, M. and Hall, A. Cell migration: Rho GTPases lead the way. Dev Biol 265 (2004), pp. 23-32.

Reed, N.A., Cai, D., Blasius, T.L., Jih, G.T., Meyhofer, E., Gaertig, J. and Verhey, K.J. Microtubule acetylation promotes kinesin-1 binding and transport. Curr Biol 16 (2006), pp. 2166-2172.

Régnier, V., Vagnarelli, P., Fukagawa, T., Zerjal, T., Burns, E., Trouche, D., Earnshaw, W. and Brown, W. CENP-A is required for accurate chromosome segregation and sustained kinetochore association of BubR1. Mol Cell Biol 25 (2005), pp. 3967-3981.

Ren, X., Dai, M., Lin, L.-P., Li, P.-K. and Ding, J. Anti-angiogenic and vascular disrupting effects of $\mathrm{C} 9$, a new microtubule-depolymerizing agent. $\mathrm{Br} J$ Pharmacol 156 (2009), pp. 1228-1238.

Renatus, M., Stennicke, H.R., Scott, F.L., Liddington, R.C. and Salvesen, G.S. Dimer formation drives the activation of the cell death protease caspase 9. PNAS 98 (2001), pp. 14250-14255.

Repnik, U., Stoka, V., Turk, V. and Turk, B. Lysosomes and lysosomal cathepsins in cell death. Biochim Biophys Acta Prot Proteomics 1824 (2012), pp. 22-33.

Ricci, M. and El-Deiry, W.: The extrinsic pathway of apoptosis

Apoptosis, Senescence, and Cancer. In: Gewirtz, D.A., Holt, S.E. and Grant, S. (Editors). Humana Press (2007), pp. 31-54.

Richter, A., Kocienski, P., Raubo, P. and Davies, D.E. The in vitro biological activities of synthetic 18-O-methyl mycalamide B, 10-epi-18-O-methyl mycalamide B and pederin. Anticancer Drug Des 12 (1997), pp. 217-227.

Rieder, C.L., Cole, R.W., Khodjakov, A. and Sluder, G. The checkpoint delaying anaphase in response to chromosome monoorientation is mediated by an inhibitory signal produced by unattached kinetochores. J Cell Biol 130 (1995), pp. 941-948.

Roberson, R.S., Kussick, S.J., Vallieres, E., Chen, S.Y.J. and Wu, D.Y. Escape from therapy-induced accelerated cellular senescence in p53-null lung cancer cells and in human lung cancers. Cancer Res 65 (2005), pp. 2795-2803.

Robert, N.J., Diéras, V., Glaspy, J., Brufsky, A.M., Bondarenko, I., Lipatov, O.N., Perez, E.A., Yardley, D.A., Chan, S.Y.T., Zhou, X., Phan, S.C. and O'Shaughnessy, J. RIBBON-1: Randomized, double-blind, placebo-controlled, phase III trial of chemotherapy with or without bevacizumab for first-line treatment of human epidermal growth factor receptor 2-negative, locally recurrent or metastatic breast cancer. J Clin Oncol 29 (2011), pp. 1252-1260.

Robles, S.J., Buehler, P.W., Negrusz, A. and Adami, G.R. Permanent cell cycle arrest in asynchronously proliferating normal human fibroblasts treated with doxorubicin or etoposide but not camptothecin. Biochem Pharmacol 58 (1999), pp. 675-685.

Rodier, F. and Campisi, J. Four faces of cellular senescence. J Cell Biol 192 (2011), pp. 547-556.

Roninson, I.B. Tumor cell senescence in cancer treatment. Cancer Res 63 (2003), pp. 2705-2715. 
Rothenberg, M.L. Topoisomerase I inhibitors: Review and update. Annals Oncol 8 (1997), pp. 837-855.

Rouzier, R., Rajan, R., Wagner, P., Hess, K.R., Gold, D.L., Stec, J., Ayers, M., Ross, J.S., Zhang, P., Buchholz, T.A., Kuerer, H., Green, M., Arun, B., Hortobagyi, G.N., Symmans, W.F. and Pusztai, L. Microtubule-associated protein tau: A marker of paclitaxel sensitivity in breast cancer. PNAS 102 (2005), pp. 83158320.

Rovini, A., Savry, A., Braguer, D. and Carr $\sqrt{ }(\mathrm{C}$, M. Microtubule-targeted agents: When mitochondria become essential to chemotherapy. Biochimica et Biophysica Acta - Bioenergetics 1807 (2011), pp. 679-688.

Russo, J., Furmanski, P. and Bradley, R. Differentiation of normal human mammary epithelial cells in culture: an ultrastructural study. Am J Anat 145 (1976), pp. 5777.

Salmon, E.D., Cimini, D., Cameron, L.A. and DeLuca, J.G. Merotelic kinetochores in mammalian tissue cells. Phil TransR Soc B 360 (2005), pp. 553-568.

Sarkisian, C.J., Keister, B.A., Stairs, D.B., Boxer, R.B., Moody, S.E. and Chodosh, L.A. Dose-dependent oncogene-induced senescence in vivo and its evasion during mammary tumorigenesis. Nature Cell Biol 9 (2007), pp. 493-505.

Scharovsky, O.G., Mainetti, L.E. and Rozados, V.R. Metronomic chemotherapy: changing the paradigm that more is better. Curr Oncol 16 (2009), pp. 7-15.

Schmitt, C.A. Cellular senescence and cancer treatment. Biochim Biophys Acta Rev Cancer 1775 (2007), pp. 5-20.

Schmitt, C.A., Fridman, J.S., Yang, M., Lee, S., Baranov, E., Hoffman, R.M. and Lowe, S.W. A senescence program controlled by p53 and p16INK4a contributes to the outcome of cancer therapy. Cell 109 (2002), pp. 335-346.

Schotte, P., Declercq, W., Van Huffel, S., Vandenabeele, P. and Beyaert, R. Nonspecific effects of methyl ketone peptide inhibitors of caspases. FEBS Lett 442 (1999), pp. 117-121.

Schrader, K., Huai, J., Jöckel, L., Oberle, C. and Borner, C. Non-caspase proteases: Triggers or amplifiers of apoptosis? Cell Mol Life Sci 67 (2010), pp. 1607-1618.

Schwartz, E.L. Antivascular actions of microtubule-binding drugs. Clin Cancer Res $\mathbf{1 5}$ (2009), pp. 2594-2601.

Schwartz, G.K. CDK inhibitors: cell cycle arrest versus apoptosis. Cell Cycle 1 (2002), pp. 113-114.

Sève, P. and Dumontet, C. Is class III $\beta$-tubulin a predictive factor in patients receiving tubulin-binding agents? Lancet Oncol 9 (2008), pp. 168-175.

Severino, J., Allen, R.G., Balin, S., Balin, A. and Cristofalo, V.J. Is $\beta$-galactosidase staining a marker of senescence in vitro and in vivo? Exp Cell Res 257 (2000), pp. 162-171.

Shay, J.W. and Roninson, I.B. Hallmarks of senescence in carcinogenesis and cancer therapy. Oncogene 23 (2004), pp. 2919-2933.

Shi, J., Orth, J.D. and Mitchison, T. Cell type variation in responses to antimitotic drugs that target microtubules and kinesin-5. Cancer Res 68 (2008), pp. 3269-3276.

Siegel, R., Ward, E., Brawley, O. and Jemal, A. Cancer statistics, 2011. CA Cancer J Clin 61 (2011), pp. 212-236.

Small, J.V., Geiger, B., Kaverina, I. and Bershadsky, A. How do microtubules guide migrating cells? Nat Rev Mol Cell Biol 3 (2002), pp. 957-964.

Smith, A.M., Ammar, R., Nislow, C. and Giaever, G. A survey of yeast genomic assays for drug and target discovery. Pharmacol Ther 127 (2010), pp. 156-164.

Smits, V.A.J., Klompmaker, R., Vallenius, T., Rijksen, G., Mäkelä, T.P. and Medema, R.H. p21 Inhibits Thr161 phosphorylation of Cdc2 to enforce the G2 DNA damage checkpoint. J Biol Chem 275 (2000), pp. 30638-30643. 
Stein, G.H., Drullinger, L.F., Soulard, A. and Dulić, V. Differential roles for cyclindependent kinase inhibitors p21 and p16 in the mechanisms of senescence and differentiation in human fibroblasts. Mol Cell Biol 19 (1999), pp. 2109-2117.

Stoka, V., Turk, B., Schendel, S.L., Kim, T.-H., Cirman, T., Snipas, S.J., Ellerby, L.M., Bredesen, D., Freeze, H., Abrahamson, M., Brل方me, D., Krajewski, S., Reed, J.C., Yin, X.-M., Turk, V. and Salvesen, G.S. Lysosomal protease pathways to apoptosis. J Biol Chem 276 (2001), pp. 3149-3157.

Strausfeld, U., Labbe, J.C., Fesquet, D., Cavadore, J.C., Picard, A., Sadhu, K., Russell, P. and Doree, M. Dephosphorylation and activation of a p34(cdc2)/cyclin B complex in vitro by human CDC25 protein. Nature 351 (1991), pp. 242-245.

Sudakin, V., Chan, G.K.T. and Yen, T.J. Checkpoint inhibition of the APC/C in HeLa cells is mediated by a complex of BUBR1, BUB3, CDC20, and MAD2. J Cell Biol 154 (2001), pp. 925-936.

Tanaka, T.U., Rachidi, N., Janke, C., Pereira, G., Galova, M., Schiebel, E., Stark, M.J.R. and Nasmyth, K. Evidence that the Ipl1-Sli15 (Aurora Kinase-INCENP) complex promotes chromosome bi-orientation by altering kinetochore-spindle pole connections. Cell 108 (2002), pp. 317-329.

Tanenbaum, M.E., Galjart, N., Van Vugt, M.A.T.M. and Medema, R.H. CLIP-170 facilitates the formation of kinetochore-microtubule attachments. EMBO J 25 (2006), pp. 45-57.

Tanudji, M., Shoemaker, J., L'Italien, L., Russell, L., Chin, G. and Schebye, X.M. Gene silencing of CENP-E by small interfering RNA in HeLa cells leads to missegregation of chromosomes after a mitotic delay. Mol Biol Cell 15 (2004), pp. 3771-3781.

Taylor, S.S., Hussein, D., Wang, Y., Elderkin, S. and Morrow, C.J. Kinetochore localisation and phosphorylation of the mitotic checkpoint components Bub1 and BubR1 are differentially regulated by spindle events in human cells. J Cell Sci 114 (2001), pp. 4385-4395.

Te Poele, R.H., Okorokov, A.L., Jardine, L., Cummings, J. and Joel, S.P. DNA damage is able to induce senescence in tumor cells in vitro and in vivo. Cancer Res $\mathbf{6 2}$ (2002), pp. 1876-1883.

Tewey, K.M., Rowe, T.C. and Yang, L. Adriamycin-induced DNA damage mediated by mammalian DNA topoisomerase II. Science 226 (1984), pp. 466-468.

Tierno, M.B., Kitchens, C.A., Petrik, B., Graham, T.H., Wipf, P., Xu, F.L., Saunders, W.S., Raccor, B.S., Balachandran, R., Day, B.W., Stout, J.R., Walczak, C.E., Ducruet, A.P., Reese, C.E. and Lazo, J.S. Microtubule binding and disruption and induction of premature senescence by disorazole C 1. J Pharmacol Exp Ther 328 (2009), pp. 715-722.

Tolić-Nørrelykke, I. Push-me-pull-you: how microtubules organize the cell interior. Eur Biophys J 37 (2008), pp. 1271-1278.

Topcu, Z. DNA topoisomerases as targets for anticancer drugs. J Clin Pharm Ther 26 (2001), pp. 405-416.

Tozer, G.M., Kanthou, C. and Baguley, B.C. Disrupting tumour blood vessels. Nat Rev Cancer 5 (2005), pp. 423-435.

Tozer, G.M., Kanthou, C., Lewis, G., Prise, V.E., Vojnovic, B. and Hill, S.A. Tumour vascular disrupting agents: Combating treatment resistance. $\mathrm{Br} J$ Radiol $\mathbf{8 1}$ (2008), pp. S12-S20.

Trovik, J., Wik, E., Stefansson, I.M., Marcickiewicz, J., Tingulstad, S., Staff, A.C., Njolstad, T.S., Vandenput, I., Amant, F., Akslen, L.A., Salvesen, H.B., Engh, M.E., Oddenes, K., Rokne, J.A., Jugum, J.T. and Lode, M.S. Stathmin overexpression identifies high-risk patients and lymph node metastasis in endometrial cancer. Clin Cancer Res 17 (2011), pp. 3368-3377. 
Van Noorden, C.J.F. Editorial: The history of Z-VAD-FMK, a tool for understanding the significance of caspase inhibition. Acta Histochem 103 (2001), pp. 241-251.

Veitia, R., David, S., Barbier, P., Vantard, M., Gounon, P., Bissery, M.C. and Fellous, A. Proteolysis of microtubule associated protein 2 and sensitivity of pancreatic tumours to docetaxel. Br J Cancer 83 (2000), pp. 544-549.

Verrills, N.M., Walsh, B.J., Cobon, G.S., Hains, P.G. and Kavallaris, M. Proteome analysis of Vinca alkaloid response and resistance in acute lymphoblastic leukemia reveals novel cytoskeletal alterations. J Biol Chem 278 (2003), pp. 45082-45093.

Villamarín, S., Ferrer-Miralles, N., Mansilla, S., Priebe, W. and Portugal, J. Induction of $\mathrm{G} 2 / \mathrm{M}$ arrest and inhibition of c-myc and p53 transcription by WP631 in Jurkat T lymphocytes. Biochem pharmacol 63 (2002), pp. 1251-1258.

Vogel, C., Kienitz, A., Hofmann, I., Müller, R. and Bastians, H. Crosstalk of the mitotic spindle assembly checkpoint with p53 to prevent polyploidy. Oncogene 23 (2004), pp. 6845-6853.

Vukicevic, S., Kleinman, H.K., Luyten, F.P., Roberts, A.B., Roche, N.S. and Reddi, A.H. Identification of multiple active growth factors in basement membrane Matrigel suggests caution in interpretation of cellular activity related to extracellular matrix components. Exp Cell Res 202 (1992), pp. 1-8.

Wade, R.: Microtubules: an overview. In: Zhou, J. (Editor), Microtubule Protocols. Humana Press (2007), pp. 1-16.

Walczak, H. and Krammer, P.H. The CD95 (APO-1/Fas) and the TRAIL (APO-2L) apoptosis systems. Exp Cell Res 256 (2000), pp. 58-66.

Wang, F., Wang, L.X., Li, S.L., Li, K., He, W., Liu, H.T. and Fan, Q.X. Downregulation of stathmin is involved in malignant phenotype reversion and cell apoptosis in esophageal squamous cell carcinoma. J Surg Oncol 103 (2011), pp. 704-715.

Wang, G.L., Jiang, B.H., Rue, E.A. and Semenza, G.L. Hypoxia-inducible factor 1 is a basic-helix-loop-helix-PAS heterodimer regulated by cellular $\mathrm{O}_{2}$ tension. PNAS 92 (1995), pp. 5510-5514.

Wang, Y., Blandino, G. and Givol, D. Induced p21(waf) expression in H1299 cell line promotes cell senescence and protects against cytotoxic effect of radiation and doxorubicin. Oncogene 18 (1999), pp. 2643-2649.

Wang, Y., Zhu, S., Cloughesy, T.F., Liau, L.M. and Mischel, P.S. p53 disruption profoundly alters the response of human glioblastoma cells to DNA topoisomerase I inhibition. Oncogene 23 (2004a), pp. 1283-1290.

Wang, Y.F., Chen, C.Y., Chung, S.F., Chiou, Y.H. and Lo, H.R. Involvement of oxidative stress and caspase activation in paclitaxel-induced apoptosis of primary effusion lymphoma cells. Cancer Chemother Pharmacol 54 (2004b), pp. 322-330.

Wasinger, V.C., Cordwell, S.J., Cerpa-Poljak, A., Yan, J.X., Gooley, A.A., Wilkins, M.R., Duncan, M.W., Harris, R., Williams, K.L. and Humphery-Smith, I. Progress with gene-product mapping of the Mollicutes: Mycoplasma genitalium. Electrophoresis 16 (1995), pp. 1090-1094.

Watanabe, T., Noritake, J. and Kaibuchi, K. Regulation of microtubules in cell migration. Trends Cell Biol 15 (2005), pp. 76-83.

Waterman-Storer, C.M., Worthylake, R.A., Liu, B.P., Burridget, K. and Salmon, E.D. Microtubule growth activates Rac1 to promote lamellipodial protrusion in fibroblasts. Nat Cell Biol 1 (1999), pp. 45-50.

Waters, J.C., Chen, R.H., Murray, A.W. and Salmon, E.D. Localization of Mad2 to kinetochores depends on microtubule attachment, not tension. J Cell Biol 141 (1998), pp. 1181-1191. 
Weaver, B.A.A., Bonday, Z.Q., Putkey, F.R., Kops, G.J.P.L., Silk, A.D. and Cleveland, D.W. Centromere-associated protein-E is essential for the mammalian mitotic checkpoint to prevent aneuploidy due to single chromosome loss. J Cell Biol 162 (2003), pp. 551-563.

Weaver, B.A.A. and Cleveland, D.W. Aneuploidy: Instigator and inhibitor of tumorigenesis. Cancer Res 67 (2007), pp. 10103-10105.

Weinberg, R.A. The retinoblastoma protein and cell cycle control. Cell 81 (1995), pp. 323-330.

West, L.M., Northcote, P.T. and Battershill, C.N. Peloruside A: a potent cytotoxic macrolide isolated from the New Zealand marine sponge Mycale sp. J Org Chem 65 (2000), pp. 445-449.

Westermann, S. and Weber, K. Post-translational modifications regulate microtubule function. Nat Rev Mol Cell Biol 4 (2003), pp. 938-948.

Weyemi, U., Lagente-Chevallier, O., Boufraqech, M., Prenois, F., Courtin, F., Caillou, B., Talbot, M., Dardalhon, M., Al Ghuzlan, A., Bidart, J.M., Schlumberger, M. and Dupuy, C. ROS-generating NADPH oxidase NOX4 is a critical mediator in oncogenic H-Ras-induced DNA damage and subsequent senescence. Oncogene 31 (2012), pp. 1117-1129.

Wilkins, M.R. From proteins to proteomes: large scale protein identification by twodimensional electrophoresis and amino acid analysis. Bio/Technol 14 (1996), pp. 61-65.

Wilkins, M.R., Sanchez, J.C., Gooley, A.A., Appel, R.D., Humphery-Smith, I., Hochstrasser, D.F. and Williams, K.l. Progress with proteome projects: why all proteins expressed by a genome should be identified and how to do it. Biotechnol Genet Eng Rev 13 (1995), pp. 19-50.

Wilmes, A.: Differences in mode of action between peloruside A and paclitaxel, two microtubule-stabilizing agents, PhD Thesis. Victoria University of Wellington, Wellington, NZ (2008).

Wilmes, A., Bargh, K., Kelly, C., Northcote, P.T. and Miller, J.H. Peloruside A synergizes with other microtubule stabilizing agents in cultured cancer cell lines. Mol Pharmaceut 4 (2007), pp. 269-280.

Wilmes, A., Chan, A., Rawson, P., Jordan, T.W. and Miller, J.H. Paclitaxel effects on the proteome of HL-60 promyelocytic leukemic cells: Comparison to peloruside A. Invest New Drugs 30 (2012a), pp. 121-129.

Wilmes, A., Hanna, R., Heathcott, R.W., Northcote, P.T., Atkinson, P.H., Bellows, D.S. and Miller, J.H. Chemical genetic profiling of the microtubule-targeting agent peloruside A in budding yeast Saccharomyces cerevisiae. Gene 497 (2012b), pp. 140-146.

Wilmes, A., O’Sullivan, D., Chan, A., Chandrahasen, C., Paterson, I., Northcote, P., Flamme, A. and Miller, J. Synergistic interactions between peloruside A and other microtubule-stabilizing and destabilizing agents in cultured human ovarian carcinoma cells and murine T cells. Cancer Chemother Pharmacol 68 (2011a), pp. 117-126.

Wilmes, A., Rawson, P., Peng, L., McLauchlan, D., Northcote, P., Jordan, T. and Miller, J. Effects of the microtubule stabilizing agent peloruside A on the proteome of HL-60 cells. Invest New Drugs 29 (2011b), pp. 544-553.

Winzeler, E.A., Shoemaker, D.D., Astromoff, A., Liang, H., Anderson, K., Andre, B., Bangham, R., Benito, R., Boeke, J.D., Bussey, H., Chu, A.M., Connelly, C., Davis, K., Dietrich, F., Dow, S.W., El Bakkoury, M., Foury, F., Friend, S.H., Gentalen, E., Giaever, G., Hegemann, J.H., Jones, T., Laub, M., Liao, H., Liebundguth, N., Lockhart, D.J., Lucau-Danila, A., Lussier, M., M'Rabet, N., Menard, P., Mittmann, M., Pai, C., Rebischung, C., Revuelta, J.L., Riles, L., 
Roberts, C.J., Ross-MacDonald, P., Scherens, B., Snyder, M., SookhaiMahadeo, S., Storms, R.K., Véronneau, S., Voet, M., Volckaert, G., Ward, T.R., Wysocki, R., Yen, G.S., Yu, K., Zimmermann, K., Philippsen, P., Johnston, M. and Davis, R.W. Functional characterization of the $S$. cerevisiae genome by gene deletion and parallel analysis. Science 285 (1999), pp. 901-906.

Wittmann, T. and Waterman-Storer, C.M. Cell motility: can Rho GTPases and microtubules point the way? J Cell Sci 114 (2001), pp. 3795-3803.

Wolter, K.G., Hsu, Y.-T., Smith, C.L., Nechushtan, A., Xi, X.-G. and Youle, R.J. Movement of Bax from the cytosol to mitochondria during apoptosis. $J$ Cell Biol 139 (1997), pp. 1281-1292.

Woltering, E.A., Lewis, J.M., Maxwell Iv, P.J., Frey, D.J., Wang, Y.Z., Rothermel, J., Anthony, C.T., Balster, D.A., O'Leary, J.P., Harrison, L.H., Thompson, J.C., Pinson, C.W. and Beenken, S.W. Development of a novel in vitro human tissuebased angiogenesis assay to evaluate the effect of antiangiogenic drugs. Ann Surg 237 (2003), pp. 790-800.

Wong, O.K. and Fang, G. Cdk1 phosphorylation of BubR1 controls spindle checkpoint arrest and Plk1-mediated formation of the 3F3/2 epitope. J Cell Biol 179 (2007), pp. 611-617.

Wouters, B.G. and Chiu, R.K.: Evaluating the importance of apoptosis and other determinants of cell death and survival. In: Gewirtz, D.A., Holt, S.E. and Grant, S. (Editors), Apoptosis, senescence, and cancer. Humana Press (2007), pp. 5572.

Wu, C.H., Van Riggelen, J., Yetil, A., Fan, A.C., Bachireddy, P. and Felsher, D.W. Cellular senescence is an important mechanism of tumor regression upon c-Myc inactivation. PNAS 104 (2007), pp. 13028-13033.

Wu, H., Lan, Z., Li, W., Wu, S., Weinstein, J., Sakamoto, K.M. and Dai, W. $\mathrm{p} 55 \mathrm{CDC} / \mathrm{hCDC} 20$ is associated with BUBR1 and may be a downstream target of the spindle checkpoint kinase. Oncogene 19 (2000), pp. 4557-4562.

Xiao, Z., Chen, Z., Gunasekera, A.H., Sowin, T.J., Rosenberg, S.H., Fesik, S. and Zhang, H. Chk1 mediates S and G2 arrests through Cdc25A degradation in response to DNA-damaging agents. J Biol Chem 278 (2003), pp. 21767-21773.

Xue, W., Zender, L., Miething, C., Dickins, R.A., Hernando, E., Krizhanovsky, V., Cordon-Cardo, C. and Lowe, S.W. Senescence and tumour clearance is triggered by p53 restoration in murine liver carcinomas. Nature 445 (2007), pp. 656-660.

Yamaguchi, H., Chen, J., Bhalla, K. and Wang, H.-G. Regulation of Bax activation and apoptotic response to microtubule-damaging agents by $\mathrm{p} 53$ transcriptiondependent and -independent pathways. J Biol Chem 279 (2004), pp. 3943139437.

Yang, Y.X., Xiao, Z.Q., Chen, Z.C., Zhang, G.Y., Yi, H., Zhang, P.F., Ii, J.L. and Zhu, G. Proteome analysis of multidrug resistance in vincristine-resistant human gastric cancer cell line SGC7901/VCR. Proteomics 6 (2006), pp. 2009-2021.

Yang, Z., Lončarek, J., Khodjakov, A. and Rieder, C.L.: Extra centrosomes and/or chromosomes prolong mitosis in human cells, Nat Cell Biol (2008), pp. 748751.

Yin, X.Y., Grove, L., Datta, N.S., Katula, K., Long, M.W. and Prochownik, E.V. Inverse regulation of cyclin B1 by c-Myc and p53 and induction of tetraploidy by cyclin B1 overexpression. Cancer Res 61 (2001), pp. 6487-6493.

$\mathrm{Yu}, \mathrm{H}$. Structural activation of Mad2 in the mitotic spindle checkpoint: The two-state Mad2 model versus the Mad2 template model. J Cell Biol 173 (2006), pp. 153157.

Zachariae, W. and Nasmyth, K. Whose end is destruction: Cell division and the anaphase-promoting complex. Genes Dev 13 (1999), pp. 2039-2058. 
Zeng, Q., Li, S., Chepeha, D.B., Giordano, T.J., Li, J., Zhang, H., Polverini, P.J., Nor, J., Kitajewski, J. and Wang, C.Y. Crosstalk between tumor and endothelial cells promotes tumor angiogenesis by MAPK activation of Notch signaling. Cancer cell 8 (2005), pp. 13-23.

Zhai, Y., Kronebusch, P.J., Simon, P.M. and Borisy, G.G. Microtubule dynamics at the G2/M transition: abrupt breakdown of cytoplasmic microtubules at nuclear envelope breakdown and implications for spindle morphogenesis. J Cell Biol 135 (1996), pp. 201-214.

Zhang, J., Lou, X., Yang, S., He, S., Yang, L., Liu, M., Zhu, H., Shan, Q., Su, S., Zhan, Q., $\mathrm{Xu}, \mathrm{N}$. and Liu, S. BAG2 is a target of the c-Myc gene and is involved in cellular senescence via the p21 ${ }^{\text {CIP1 }}$ pathway. Cancer Lett 318 (2012), pp. 34-41.

Zhao, M., Eaton, J.W. and Brunk, U.T. Bcl-2 phosphorylation is required for inhibition of oxidative stress-induced lysosomal leak and ensuing apoptosis. FEBS Lett 509 (2001), pp. 405-412.

Zheng, T.S., Hunot, S., Kuida, K., Momoi, T., Srinivasan, A., Nicholson, D.W., Lazebnik, Y. and Flavell, R.A. Deficiency in caspase-9 or caspase-3 induces compensatory caspase activation. Nat Med 6 (2000), pp. 1241-1247.

Zhou, B.B.S. and Elledge, S.J. The DNA damage response: Putting checkpoints in perspective. Nature 408 (2000), pp. 433-439.

Zhou, L. and Chang, D.C. Dynamics and structure of the Bax-Bak complex responsible for releasing mitochondrial proteins during apoptosis. J Cell Sci 121 (2008), pp. 2186-2196.

Zhou, X., Jiang, H. and Hou, J. Coordination of upregulated XBP-1 and downregulated c-myc during myeloma cell differentiation induced by 2-methoxyestradiol. Leuk res 31 (2007), pp. 1259-1265.

Zindy, F., Eischen, C.M., Randle, D.H., Kamijo, T., Cleveland, J.L., Sherr, C.J. and Roussel, M.F. Myc signaling via the ARF tumor suppressor regulates p53dependent apoptosis and immortalization. Genes Dev 12 (1998), pp. 2424-2433. 


\section{Appendices}

\section{Appendix A: General Recipes}

\section{Acrylamide Gels}

$10 \%$ separating gel - enough for two gels

$\begin{array}{ll}\text { MQ } & 8 \mathrm{~mL} \\ 1.5 \mathrm{M} \text { Tris }(\mathrm{pH} 8.8) & 5 \mathrm{~mL} \\ 10 \% \text { SDS } & 200 \mu \mathrm{L} \\ 30 \% \text { bis-acrylamide } & 6.66 \mathrm{~mL} \\ 10 \% \text { APS } & 100 \mu \mathrm{L} \\ \text { TEMED } & 10 \mu \mathrm{L}\end{array}$

(100\% isopropanol layered on top while gel is setting)

$12 \%$ separating gel - enough for two gels

$\begin{array}{ll}\text { MQ } & 5.3 \mathrm{~mL} \\ 1.5 \mathrm{M} \text { Tris }(\mathrm{pH} 8.8) & 4 \mathrm{~mL} \\ 10 \% \text { SDS } & 160 \mu \mathrm{L} \\ 30 \% \text { bis-acrylamide } & 6.4 \mathrm{~mL} \\ 10 \% \text { APS } & 160 \mu \mathrm{L} \\ \text { TEMED } & 16 \mu \mathrm{L}\end{array}$

(100\% isopropanol layered on top while gel is setting)

4\% stacking gel - enough for two gels

$\begin{array}{ll}\text { MQ } & 6.1 \mathrm{~mL} \\ 0.5 \mathrm{M} \text { Tris }(\mathrm{pH} 6.8) & 2.5 \mathrm{~mL} \\ 10 \% \text { SDS } & 100 \mathrm{~mL} \\ 30 \% \text { bis-acrylamide } & 1.33 \mathrm{~mL} \\ 10 \% \text { APS } & 50 \mathrm{~mL} \\ \text { TEMED } & 10 \mathrm{~mL}\end{array}$

Agarose gel (1\%) - enough for one large gel
Agarose
$800 \mathrm{mg}$
$1 \times$ TAE buffer $80 \mathrm{~mL}$

\section{$10 x$ Annexin V binding buffer (1L)}

$\begin{array}{ll}\operatorname{HEPES}(0.1 \mathrm{M}) & 23.82 \mathrm{~g} \\ \mathrm{NaCl}(1.4 \mathrm{M}) & 81.816 \mathrm{~g} \\ \mathrm{CaCl}_{2}(25 \mathrm{mM}) & 2.775 \mathrm{~g}\end{array}$

pH 7.4 


\section{$5 \%$ Boiled Donkey Serum for blocking $(100 \mathrm{~mL})$}

$5 \mathrm{~mL}$ donkey serum and $45 \mathrm{~mL}$ MQ boiled together for $10 \mathrm{~min}$. Watch the boiling carefully! Let solution cool.

Add $50 \mathrm{~mL}$ PBS containing $50 \mathrm{mg}$ sodium azide (final conc $0.05 \% \mathrm{Na}$ Azide)

Spin at $18000 \mathrm{rpm}$ for $1 \mathrm{~h}$

Filter and store at $4{ }^{\circ} \mathrm{C}$

\section{Cell lysis buffers}

\section{RIPA buffer (1L)}

$\begin{array}{ll}\text { Tris-HCl } & 1.211 \mathrm{~g} \\ \mathrm{NaCl} & 8.766 \mathrm{~g} \\ \text { EDTA } & 0.3722 \mathrm{~g} \\ \mathrm{SDS} & 1.0 \mathrm{~g} \\ \text { Triton X-100 } & 10 \mathrm{~mL} \\ \text { Sodium deoxycholate } & 10.0 \mathrm{~g}\end{array}$

$\mathrm{pH}$ to 7.5

Non-denaturing lysis buffer

$\begin{array}{ll}\text { Tris- } \mathrm{HCl} & 0.047 \mathrm{~g} \\ \mathrm{NaCl} & 0.087 \mathrm{~g} \\ \mathrm{Mg}-\text { Acetate } & 0.043 \mathrm{~g} \\ \mathrm{CHAPS}(1 \%) & 0.1 \mathrm{~g}\end{array}$

$\mathrm{pH}$ to 7.5

\section{Colloidal coomassie fixative $(500 \mathrm{~mL})$}

Ethanol (50\%) $250 \mathrm{~mL}$

Ortho-phosphoric acid (3\%) $\quad 15 \mathrm{~mL}$

\section{Colloidal coomassie staining solution $(500 \mathrm{~mL})$}

Coomassie brilliant blue G250 $\quad 0.5 \mathrm{~g}$

Acetic acid (10\%) $\quad 50 \mathrm{~mL}$

Methanol (50\%) $250 \mathrm{~mL}$

\subsection{EDTA $(500 \mathrm{~mL})$}

$\mathrm{Na}_{2}$.EDTA. $2 \mathrm{H}_{2} \mathrm{O}$

$\mathrm{pH}$ to 8.0 (need to get $\mathrm{pH}$ close to 8.0 to get EDTA to dissolve, then adjust volume) 
KREBS buffer (1L)

$\begin{array}{ll}\mathrm{NaCl}(130 \mathrm{mM}) & 7.5972 \mathrm{~g} \\ \mathrm{KCl}(1.3 \mathrm{mM}) & 0.0976 \mathrm{~g} \\ \mathrm{CaCl}_{2} .2 \mathrm{H}_{2} \mathrm{O}(1.8 \mathrm{mM}) & 0.2646 \mathrm{~g} \\ \mathrm{MgSO}_{4} \cdot 6 \mathrm{H}_{2} \mathrm{O}(1.2 \mathrm{mM}) & 0.2742 \mathrm{~g} \\ \mathrm{KH}_{2} \mathrm{PO} 4(1.2 \mathrm{mM}) & 0.163308 \mathrm{~g} \\ \mathrm{HEPES}(10 \mathrm{mM}) & 2.603 \mathrm{~g} \\ \text { D-glucose }(10 \mathrm{mM}) & 1.8016 \mathrm{~g}\end{array}$

$\mathrm{pH}$ to 7.4

\section{MTT solution}

$5 \mathrm{mg} / \mathrm{mL}$ MTT (3-(4,5-dimethylthiazol-2-yl)-2,5-diphenyltetrazolium bromide) (Sigma) $1 \times$ PBS

Filter sterilized and kept sterile

\section{MTT solubilizer (500 mL)}

Sodium dodecyl sulfate $(10 \%) \quad 50 \mathrm{~g}$

$\mathrm{N}, \mathrm{N}$-dimethylformamide (45\%) $225 \mathrm{~mL}$

$\mathrm{pH}$ to 4.5 using glacial acetic acid

\section{4\% paraformaldehyde $(25 \mathrm{~mL})$}

1 g paraformaldehyde

Add $\sim 20 \mathrm{~mL} 1 \times$ PBS and a few drops of $10 \mathrm{M} \mathrm{NaOH}$

Heat in $60{ }^{\circ} \mathrm{C}$ waterbath until dissolved

$\mathrm{pH}$ to 7.4 and then adjuts volume to $25 \mathrm{~mL}$

\section{2 x PHEM buffer $(500 \mathrm{~mL})$}

$\begin{array}{ll}\text { PIPES } & 18.14 \mathrm{~g} \\ \text { HEPES } & 6.5 \mathrm{~g} \\ \text { EGTA } & 3.8 \mathrm{~g} \\ \mathrm{MgSO}_{4} & 0.99 \mathrm{~g}\end{array}$

$\mathrm{pH}$ to 7.0 with $\mathrm{KOH}$

Filter and store as frozen aliquots or at $4{ }^{\circ} \mathrm{C}$

For PHEM with $0.5 \%$ triton $\mathrm{X}-100$ - add $50 \mu \mathrm{L}$ per $10 \mathrm{~mL}$ buffer and make fresh

\section{Propidium iodide staining solution (for cell cycle)}

$0.05 \mathrm{mg} / \mathrm{mL}$ propidium iodide (Sigma)

$0.1 \%$ sodium citrate

$0.1 \%$ triton $\mathrm{X}-100$ 


\section{$10 \times$ PBS (1L)}

$\mathrm{NaCl}(137 \mathrm{mM})$

$80 \mathrm{~g}$

$\mathrm{KCl}(1.6 \mathrm{mM})$

$2 \mathrm{~g}$

$\mathrm{Na}_{2} \mathrm{HPO}_{4}(10.1 \mathrm{mM})$

$14.4 \mathrm{~g}$

$\mathrm{KH}_{2} \mathrm{PO}_{4}(1.8 \mathrm{mM})$

$2.4 \mathrm{~g}$

pH 7.4

5 x sample buffer

SDS

$4 \mathrm{M}$

Glycerol

$6.1 \mathrm{M}$

Tris-HCl (pH 6.8)

$0.22 \mathrm{M}$

Bromophenol blue

$0.75 \mathrm{mM}$

$10 \% \beta$-mercaptoethanol added immediately before use

$10 \times$ SDS running buffer (1L)

SDS

Tris-base

Glycine
$10 \mathrm{~g}$

$30.3 \mathrm{~g}$

$144.1 \mathrm{~g}$

Transfer buffer for Western blot (1L)

Tris-base

Glycine

$3.03 \mathrm{~g}$

Methanol

$14.4 \mathrm{~g}$

$200 \mathrm{~mL}$

Prepared fresh and used chilled at $4{ }^{\circ} \mathrm{C}$

\section{$50 \times$ TAE buffer $(500 \mathrm{~mL})$}

Tris-base

Acetic acid

0.5 M EDTA (pH 8.0)
$121 \mathrm{~g}$ $28.6 \mathrm{~mL}$

$50 \mathrm{~mL}$ 
Appendix B: Chapter 3

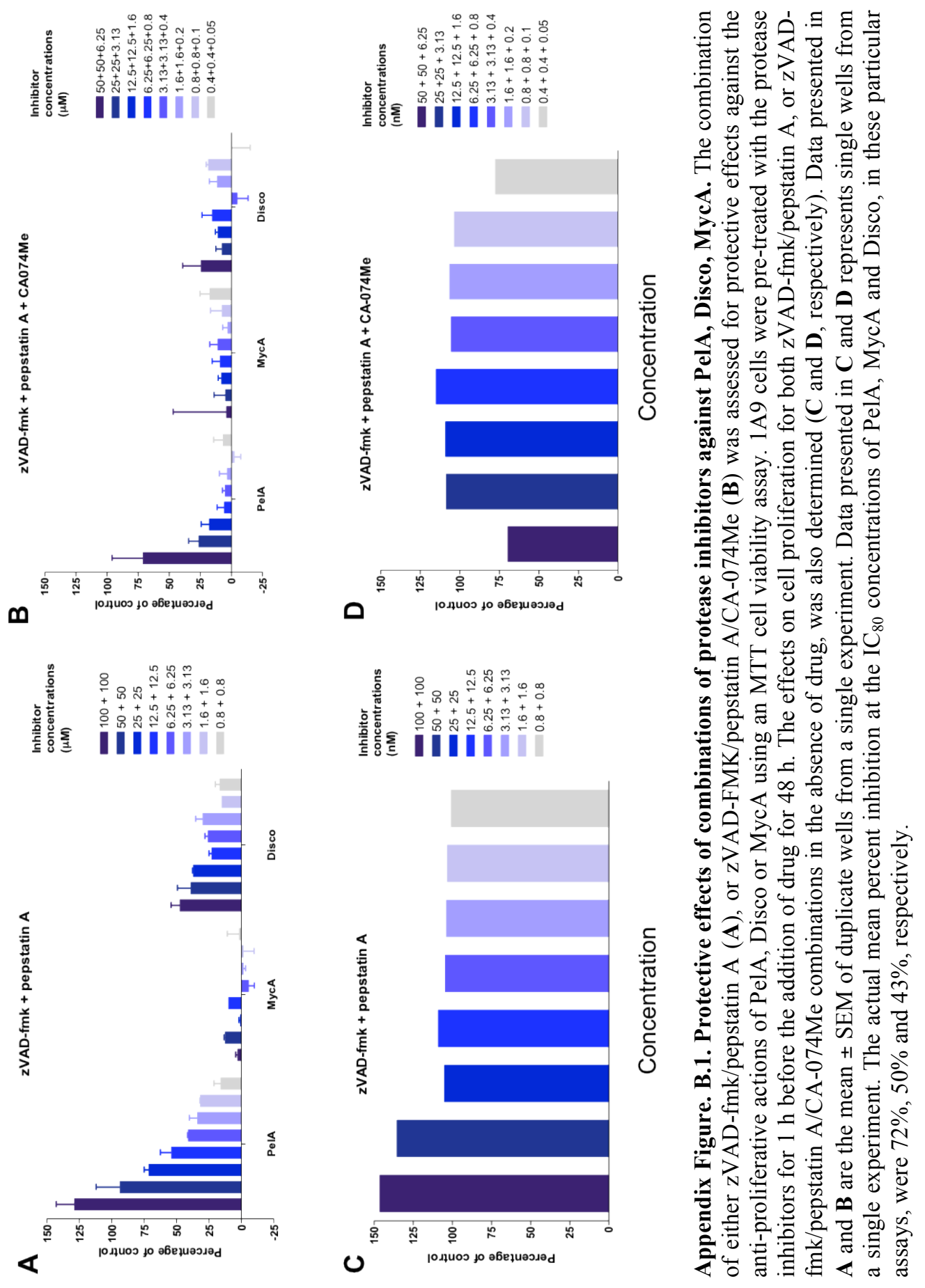



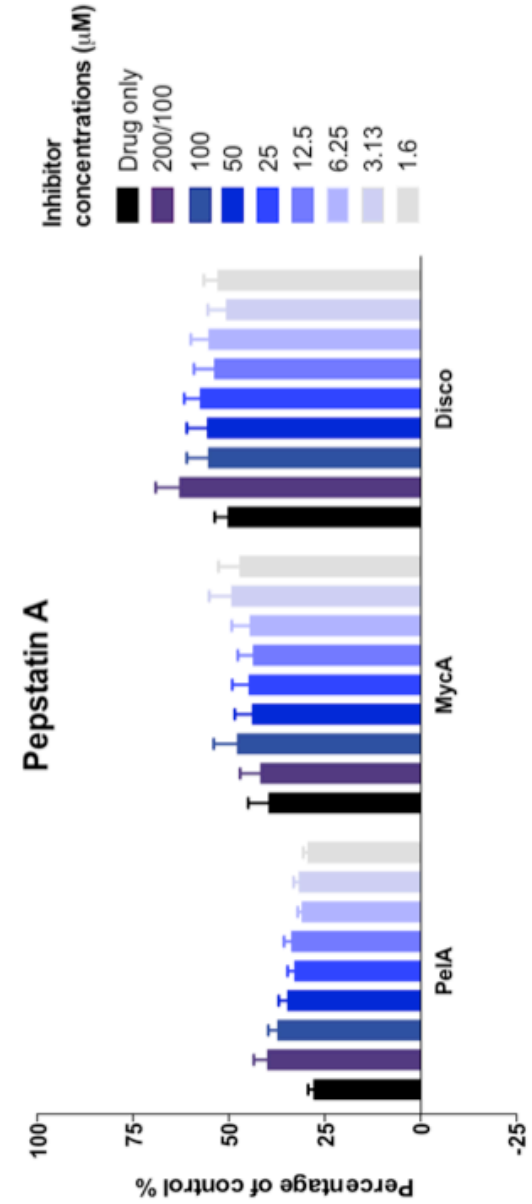

m

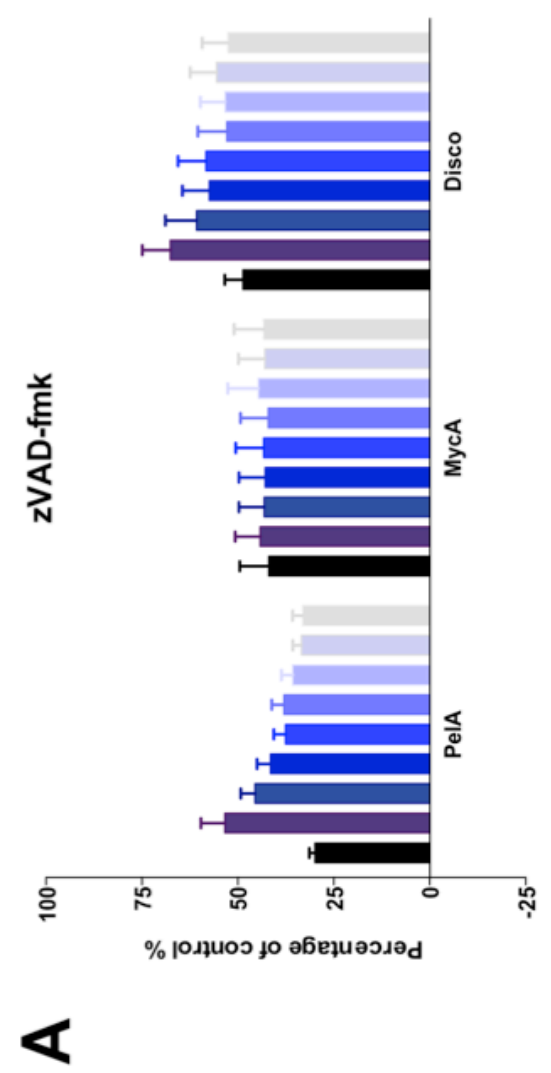

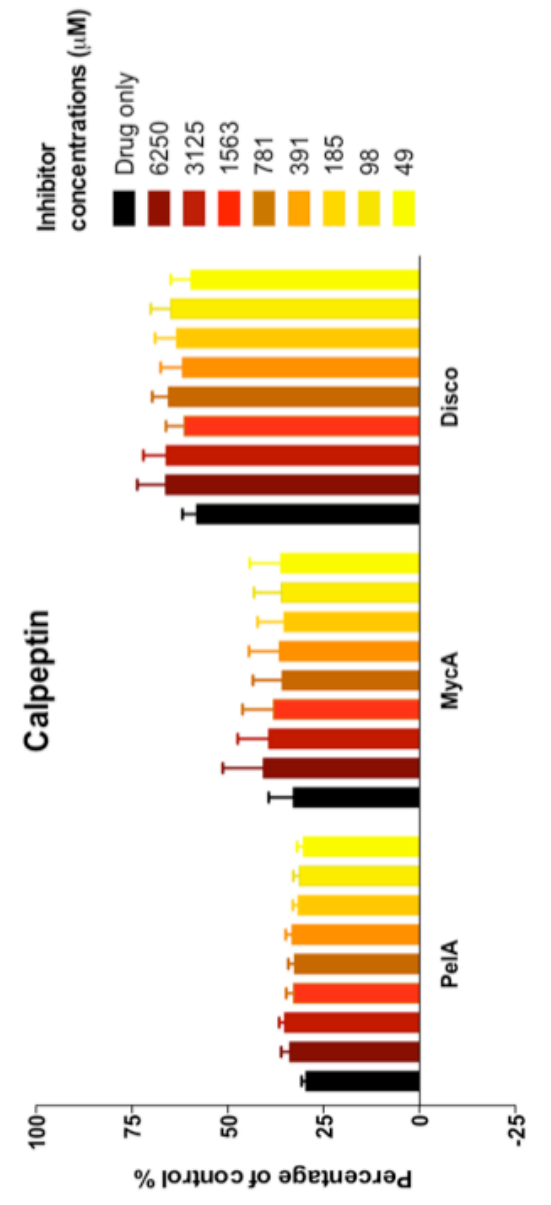
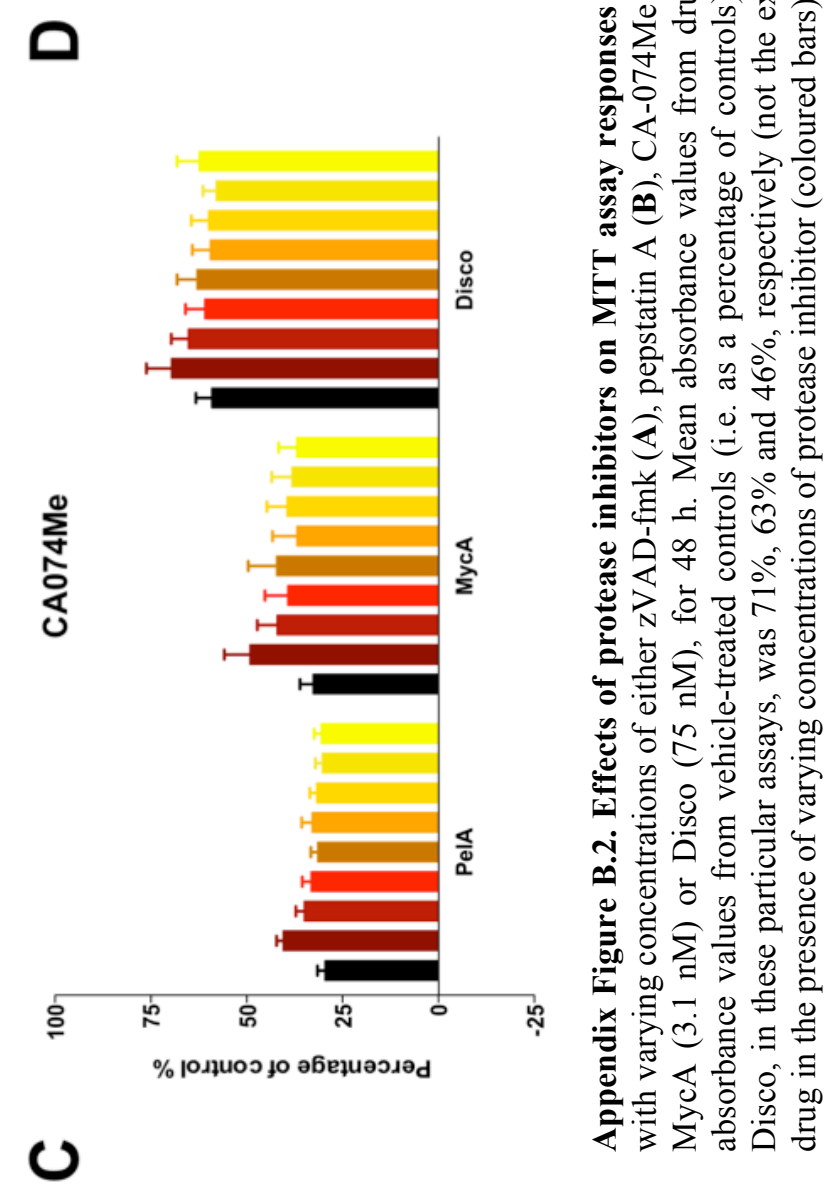

० क ते

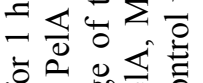
政造 o 0

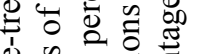

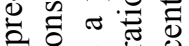
일 3 过 券 政 $\leq 0$ 呵

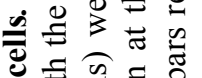
过记 焉

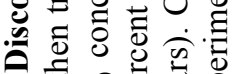
สิอย

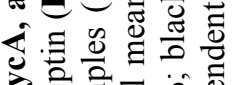
है 웜 त्य 过吉告 Q0 0 on

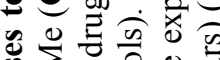

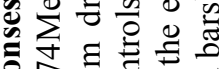

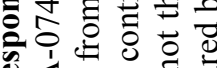

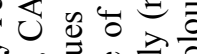

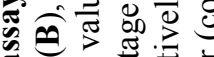
$\checkmark 8 \overline{0}$ 表 w 의 क्ष

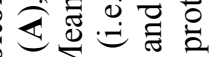
更 퍼응

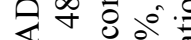

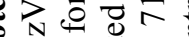
a

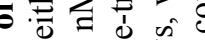
o in क

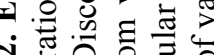

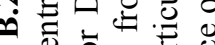
过

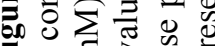
品- 8 政

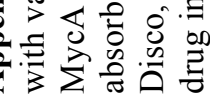

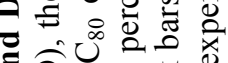




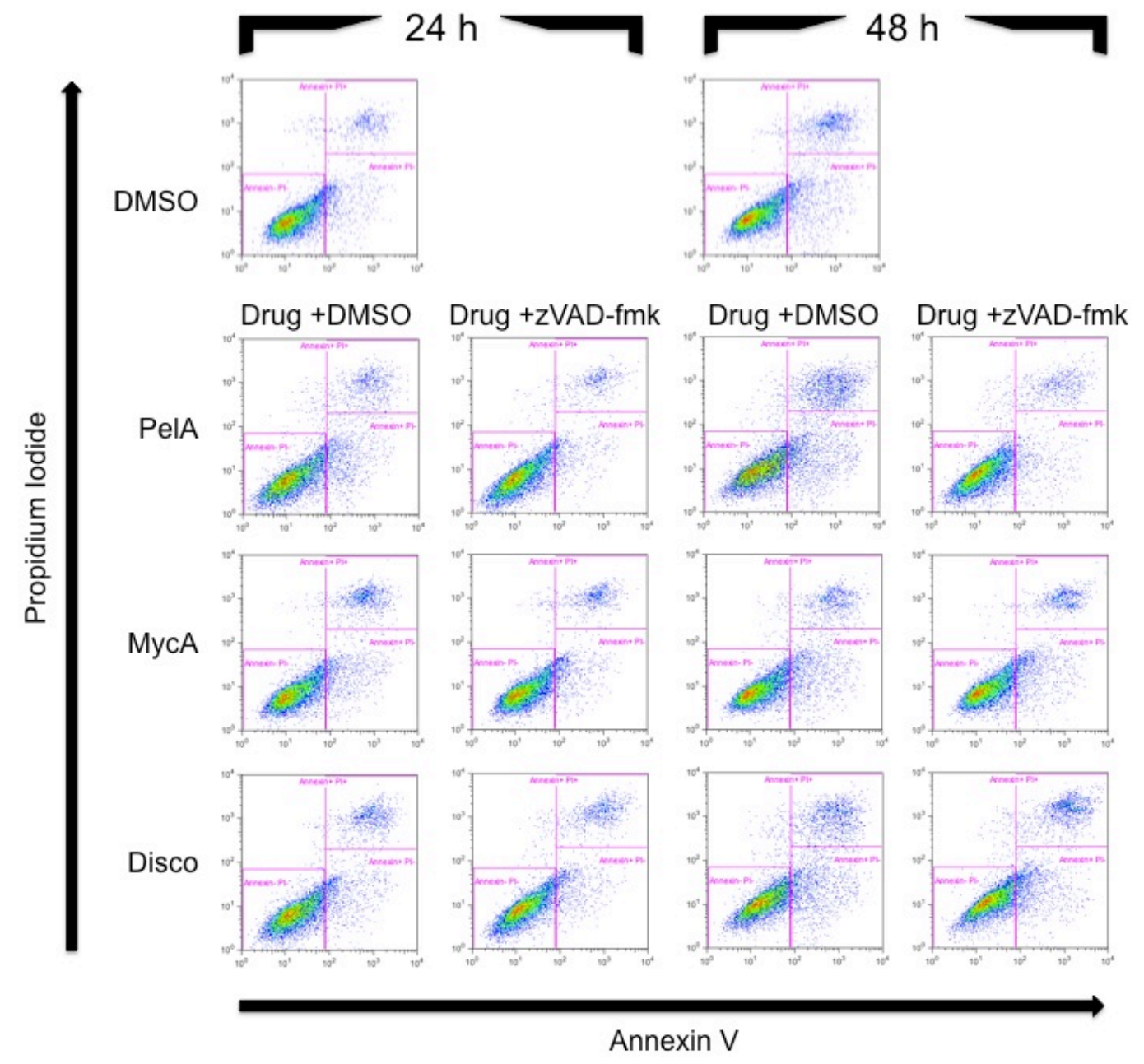

Appendix Figure B.3. Protective effects of zVAD-fmk against PelA, MycA and Disco. 1A9 cells were pre-treated with zVAD-fmk for $1 \mathrm{~h}$ before being treated with the $\mathrm{IC}_{80}$ concentrations of PelA, MycA, and Disco. At various timepoints, cells were harvested and stained with annexin $\mathrm{V}$ and propidium iodide and analysed by flow cytometry. Figure shows representative scatter plots collected at $24 \mathrm{~h}$ and $48 \mathrm{~h}$, indicating apoptosis in drug + DMSO, or drug $+\mathrm{zVAD}$-fmk samples. Plots also indicate the gating that was used for the analysis, with the lower left, lower right and upper right quadrants representing Annexin $\mathrm{V}^{-\mathrm{ve}} / \mathrm{PI}^{-\mathrm{ve}}$, Annexin $\mathrm{V}^{+\mathrm{ve}} / \mathrm{PI}^{-\mathrm{ve}}$ and Annexin $\mathrm{V}^{+\mathrm{ve}} / \mathrm{PI}^{+\mathrm{ve}}$ cells, respectively. 

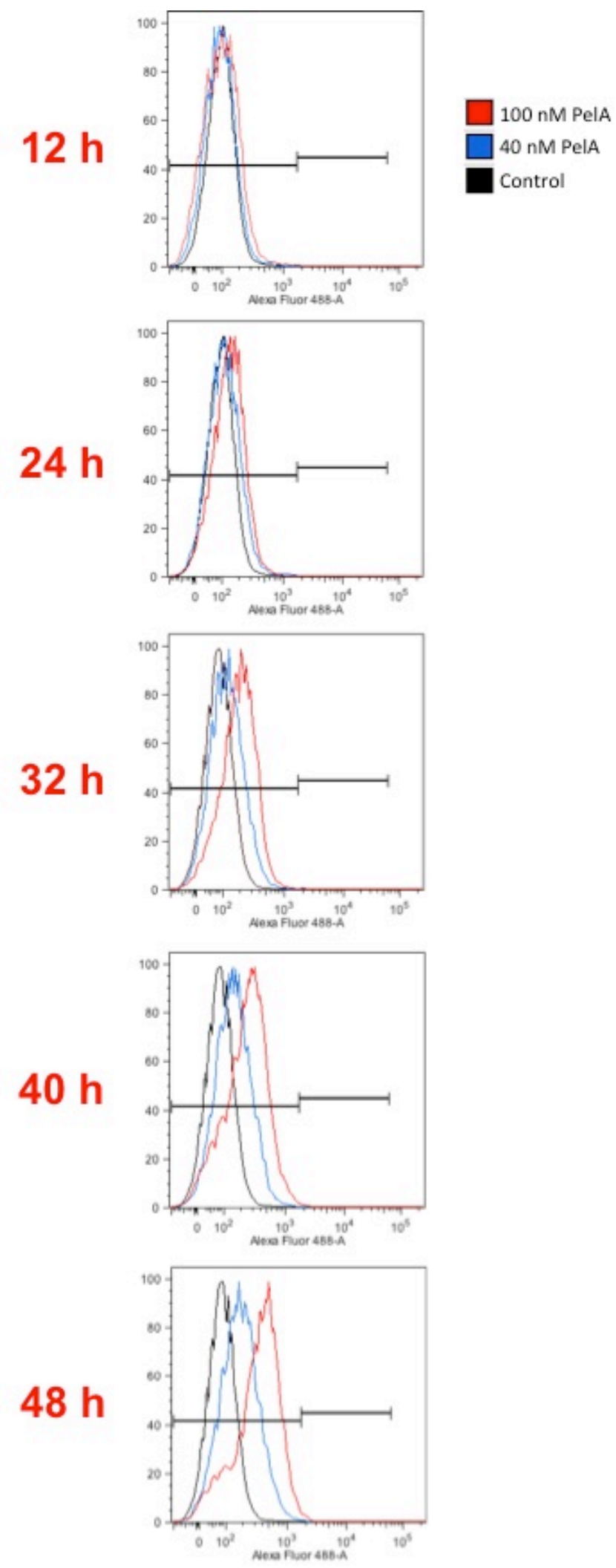

Appendix Figure B.4. Positive shift in autofluorescence in response to PelA. 1A9 cells were treated with medium (black curves), or medium containing 40 or $100 \mathrm{nM}$ PelA (red and blue curves, respectively). At various timepoints, cells were harvested and fixed as for the FLICA-stained samples but were left unstained to assess the degree of autofluorescence in response to PelA treatment so that the appropriate negative and positive gates could be set. Histograms indicate the number of cells versus fluorescence intensity on the Alexa Fluor $488 \mathrm{~nm}$ channel. Gates indicate negative (left-hand gate) and positive (right-hand gate) FLICA gates that were set, based on the shift in autofluorescence. 


\section{FLICA 8}

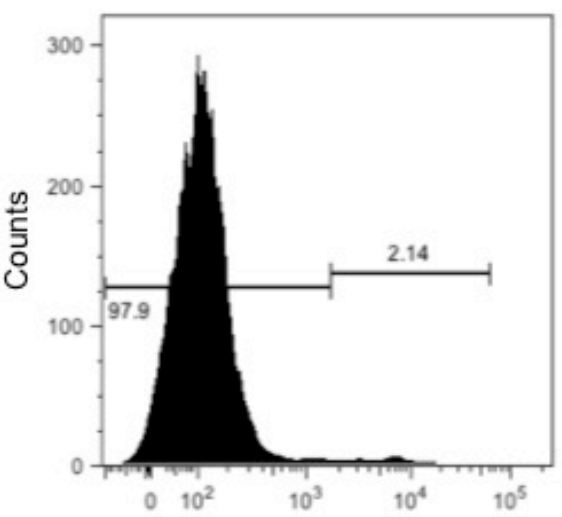

$10 \mu \mathrm{M}$ CPT

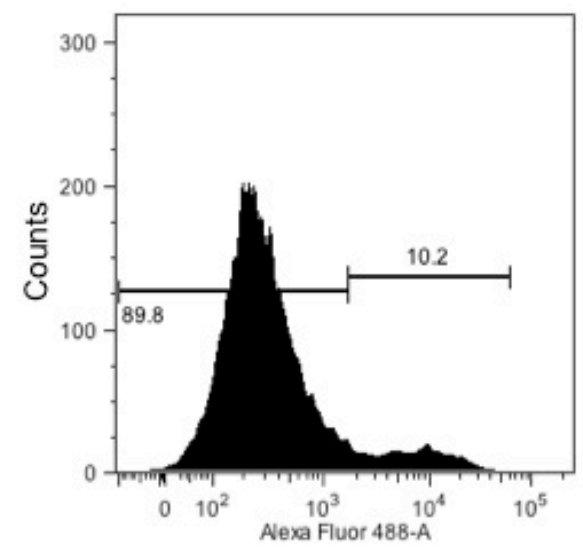

FLICA 9
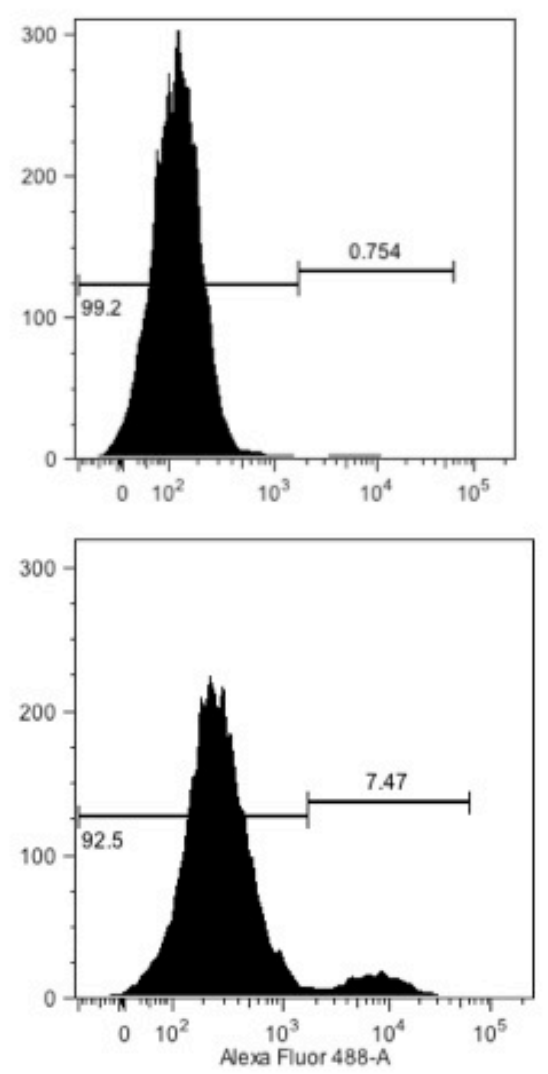

Appendix Figure B.5. Positive control for caspase-8 and -9 activation. 1A9 cells were treated with plain medium (Control) or medium containing $10 \mu \mathrm{M}$ camptothecin (CPT) for $16 \mathrm{~h}$ before being harvested, fixed and probed for either caspase- 8 or caspase- 9 activation by staining with the appropriate FLICA probes. Histograms indicate the percentage of FLICA negative (left-hand gates) and FLICA positive (right-hand gates) cells for each of the treatments. 


\section{Caspase-8 Caspase-9}

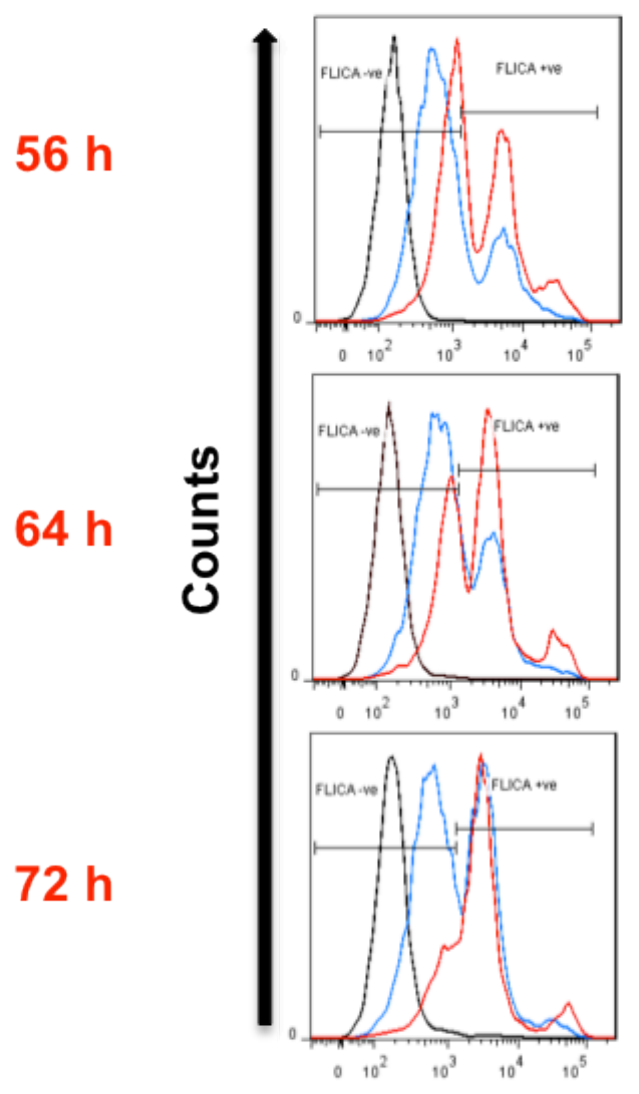

AF 488
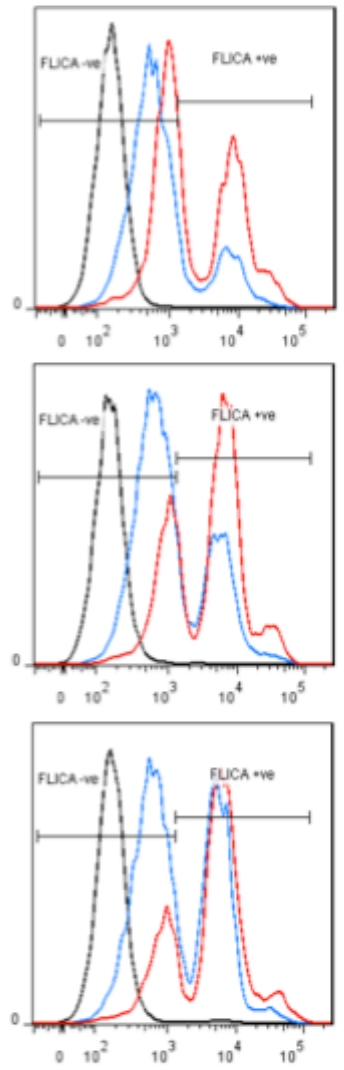

AF 488

Appendix Figure B.6. Caspase-8 and $\mathbf{- 9}$ activation by PelA at later timepoints. 1A9 cells were treated with medium only (black curves), or medium containing 40 or $100 \mathrm{nM}$ PelA (red and blue curves, respectively). At 56, 64, and $72 \mathrm{~h}$ timepoints, cells were harvested, stained with either caspase- 8 or -9 FLICA probes, fixed and then analysed by flow cytometry. Negative FLICA gates (left-hand gates) were set based on the autofluorescence of PelA. Positive FLICA gates indicate the presence of multiple FLICA $^{+v e}$ peaks that appear at later timepoints. 


\section{Appendix C: Chapter 4}
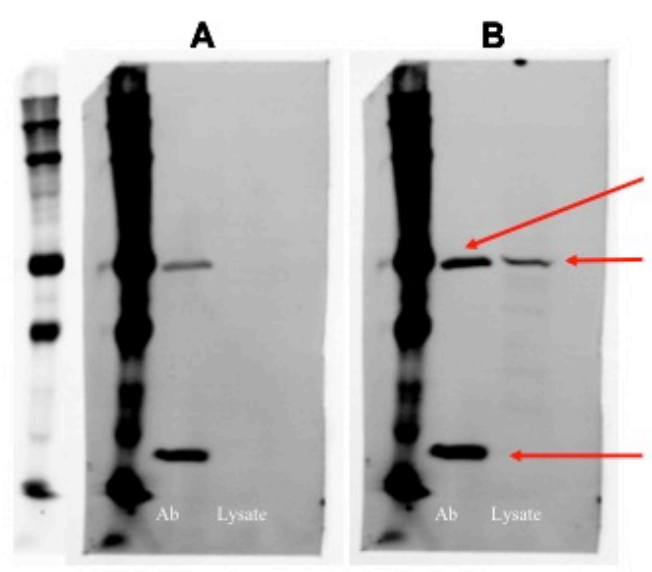

$\mathrm{Ab} \mathrm{HC}$

p55CDC

C

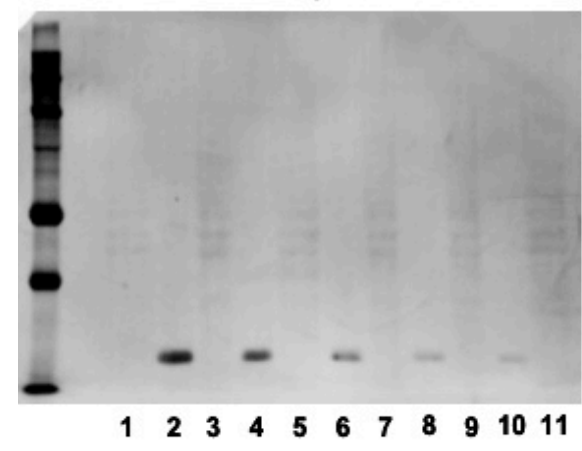

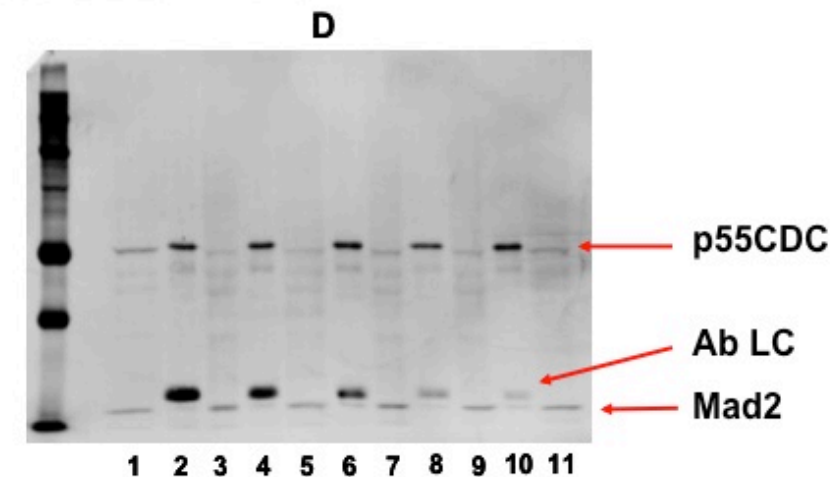

Appendix Figure C.1. Co-immunoprecipitation (Co-IP) optimization. To determine whether the mouse anti-p55CDC antibody heavy chain and p55CDC protein could be resolved by SDS-PAGE, 4 $\mu \mathrm{g}$ of antibody (Ab lanes in A and B) and $60 \mu \mathrm{g}$ of $1 \mathrm{~A} 9$ cell lysate (Lysate lanes in A and B) were loaded onto a $10 \%$ acrylamide gel and electrophoretically transferred to PVDF membranes. The membrane was first probed with anti-mouse Cy5 secondary antibody (A), followed by anti-p55CDC antibody and secondary antibody (B). The membrane scans in A and B indicated that the secondary antibody detects the antibody heavy/light chains ( $\mathrm{Ab} \mathrm{HC} / \mathrm{Ab} \mathrm{LC}$ ), and that the heavy chain and p55CDC migrate at the same speed. To assess the effectiveness of crosslinking the anti-p55CDC antibody to magnetic dynabeads, a series of immunoprecipitations using various amounts of crosslinked antibody were carried out. The proteins were eluted using denaturing conditions, separated by SDS-PAGE and transferred to PVDF membranes (as above). Membranes were first stained with secondary antibody only (anti-mouse Cy5) (C) and then stained with anti-p55CDC and anti-Mad2, followed by another round of secondary staining (D). Lane 1 - 1A9 cell lysate; lanes 2, 4, 6, 8 and 10 - IP using 4, 2, 1, 0.5 and $0.25 \mu \mathrm{g}$ anti-p55CDC, respectively; lanes 3, 5, 7, 9, 11 - supernatant after Co-IP of preceding lane. The secondary antibody only stain shows that after crosslinking, the heavy chain does not elute under denaturing conditions (only the light chain elutes). Subsequent staining using anti-p55CDC and anti-Mad2 primary antibodies, followed by anti-mouse Cy5, indicates that p55CDC and Mad2 can be distinguished from the heavy and light chains of the p55CDC antibody by first crosslinking anti-p55CDC to the magnetic dynabeads. 


\section{Appendix D: Chapter 5}

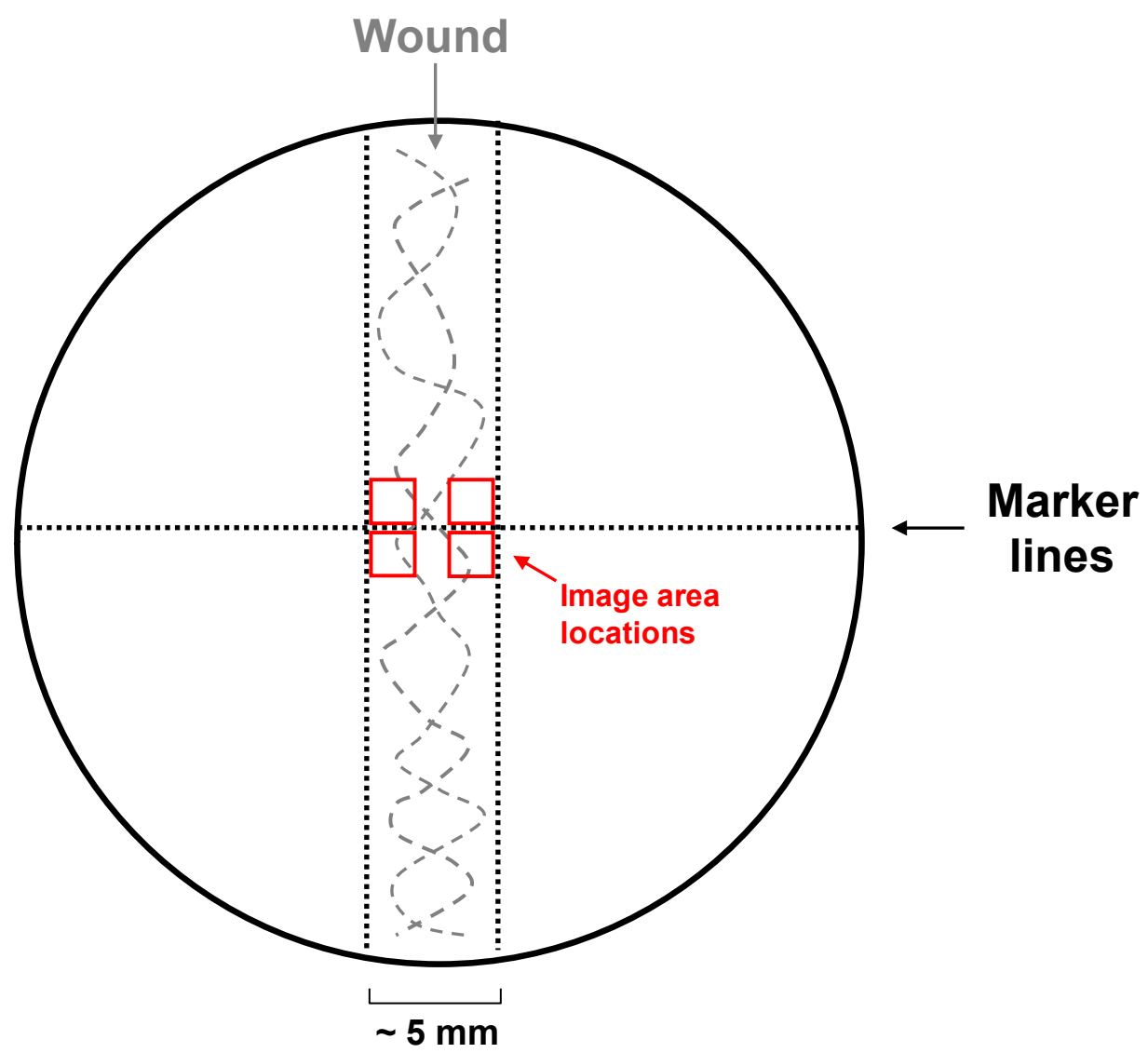

Appendix Figure D.1. Mulitwell plate layout for wound scratch assay. In order to orientate images of the wound at $0 \mathrm{~h}$ and $18 \mathrm{~h}$, two lines are drawn in parallel at the centre of each well, approximately $5 \mathrm{~mm}$ apart. A perpendicular line bisecting the two parallel lines is also drawn. The wound was created down the centre of the well, such that it falls between the two parallel lines. Depending on whether the wound is closer to the left or right marker line, images are taken at either the inner upper left or right sections, or inner lower left or right sections (as indicated by the red squares). 


\section{A}

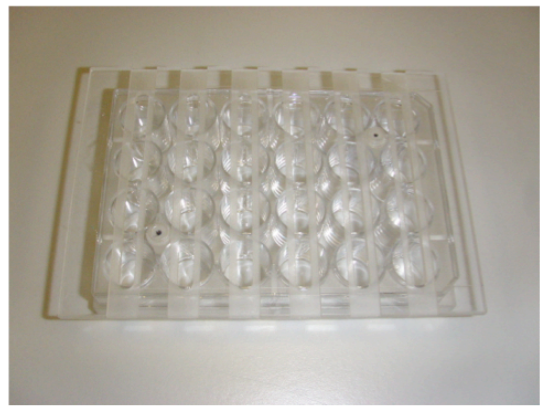

B

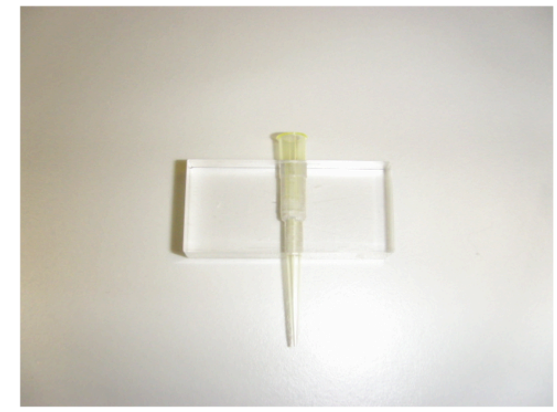

C

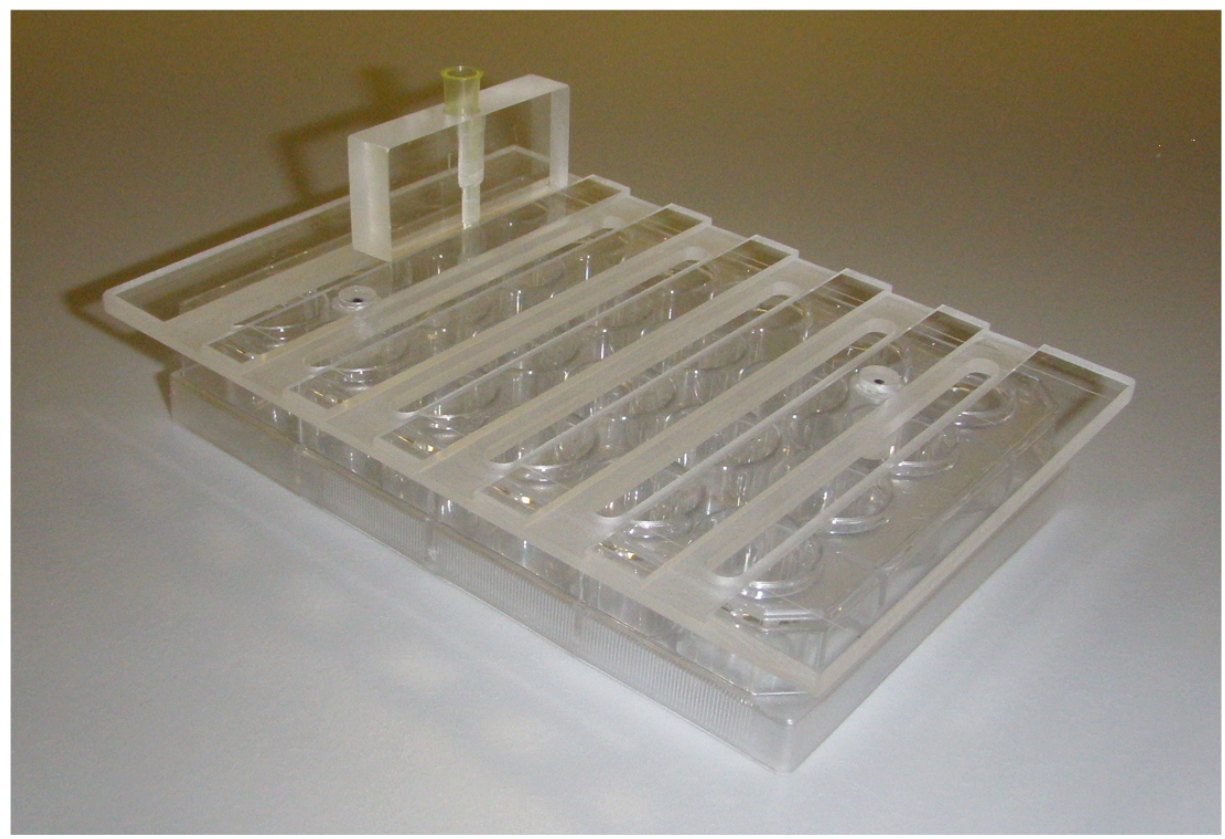

Appendix Figure D.2. Plate insert for generating more consistent wound scratches. (A) A plate insert was designed for use with a 24-well plate, with grooves aligned with the centre of each well. (B) A small plastic block was used to hold a $200 \mu \mathrm{L}$ pipette tip, which could then be run along the grooves of the plate insert $(\mathbf{C})$. This method created straighter wounds and more consistent initial wound widths (average error between triplicates was $4.1 \%$ ). 

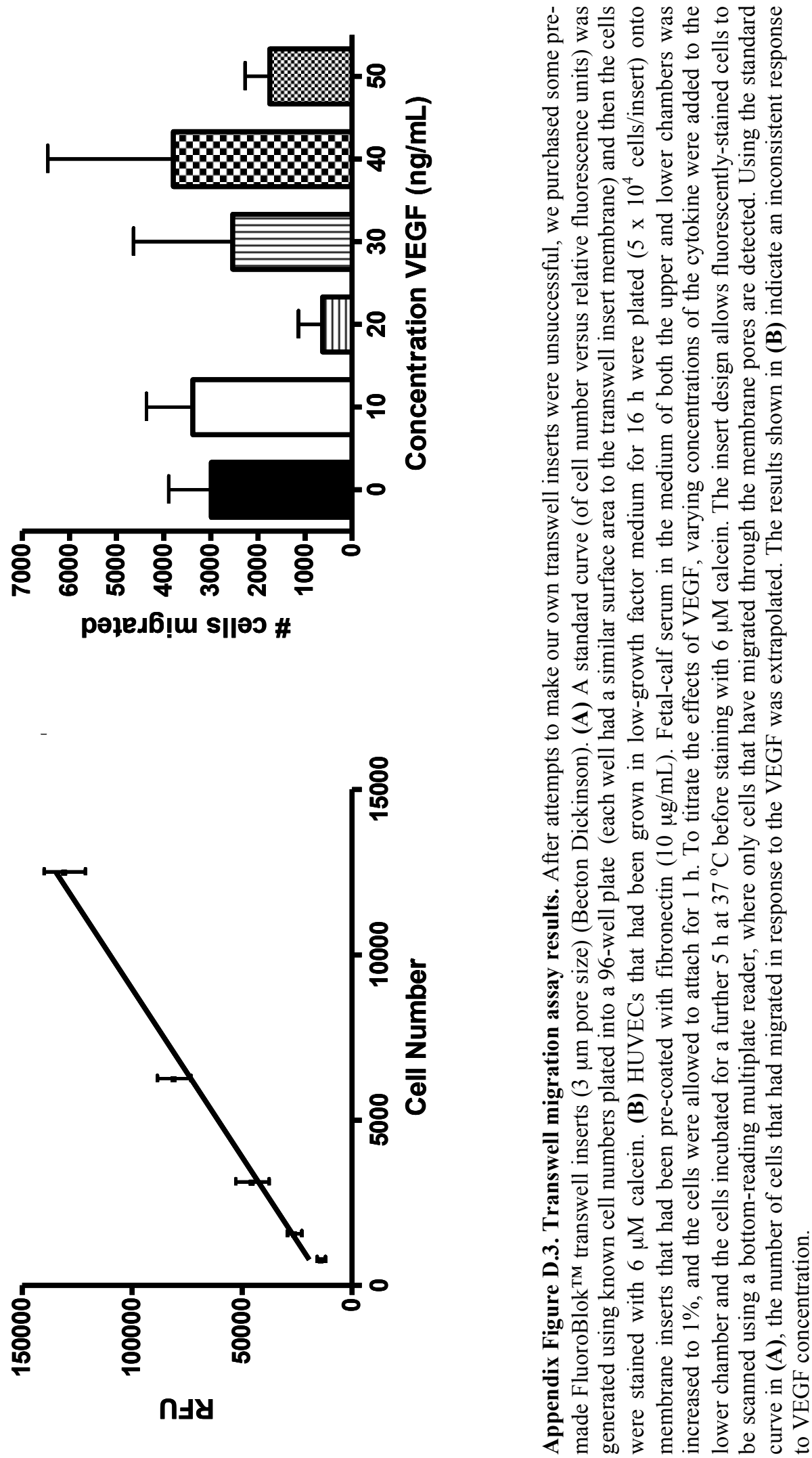

m

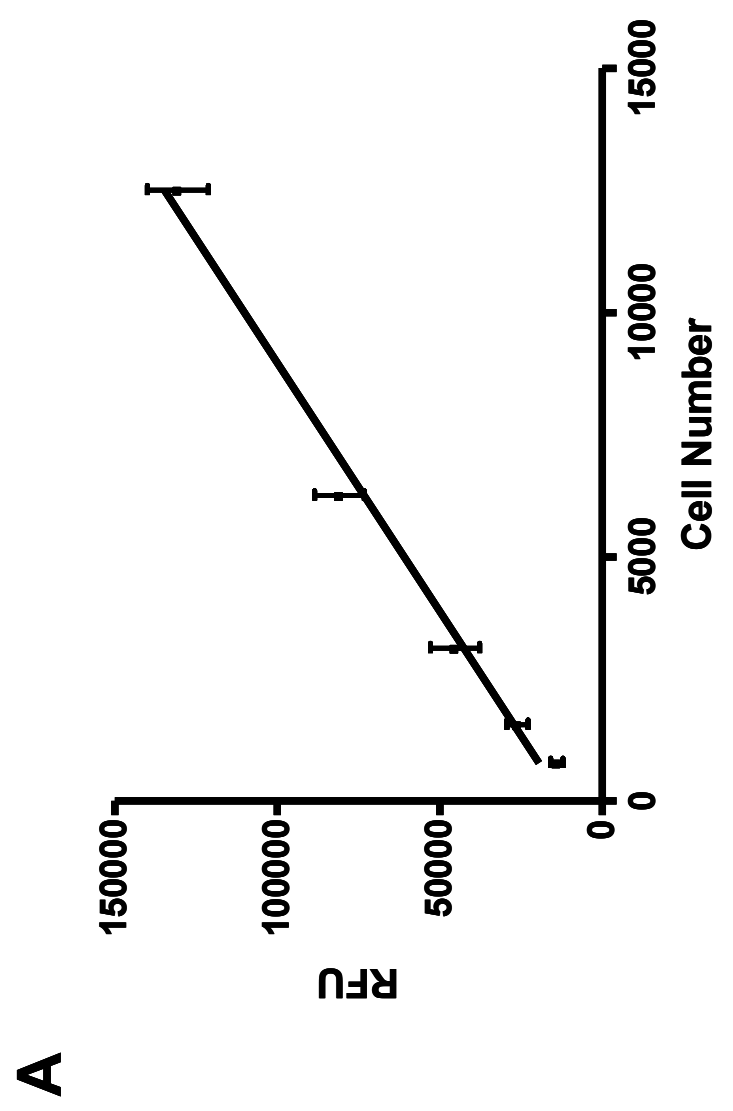




\section{Appendix E: Chapter 6}

\section{Recipes for SA- $\beta$-gal assay}

Fixative (in PBS) - stable for 1 month at RT

Formaldehyde $\quad 2 \%$

Glutaraldehyde $\quad 0.2 \%$

$\underline{\text { X-Gal Stock solution }}-$ stored at $-20{ }^{\circ} \mathrm{C}$ for up to 2 weeks

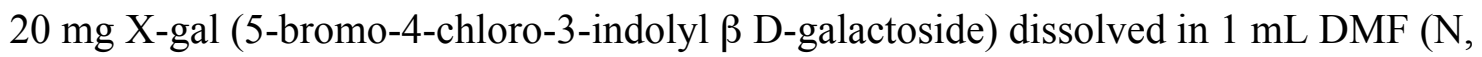
$\mathrm{N}$-dimethylformamide)

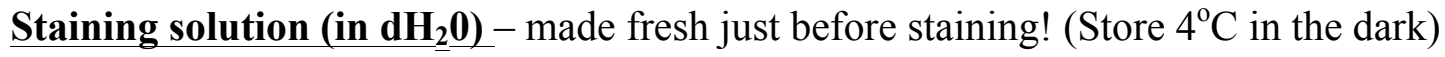

Citric acid/Na phosphate buffer (pH 6.0) $\quad 40 \mathrm{mM}$

$\mathrm{K}_{4}\left[\mathrm{Fe}(\mathrm{CN})_{6}\right] \cdot 3 \mathrm{H}_{2} \mathrm{O} \quad 5 \mathrm{mM}$

$\mathrm{K}_{3}\left[\mathrm{Fe}(\mathrm{CN})_{6}\right] \quad 5 \mathrm{mM}$

$5 \mathrm{M} \mathrm{NaCl}$ solution $\quad 150 \mathrm{mM}$

$1 \mathrm{M} \mathrm{MgCl}_{2} .6 \mathrm{H}_{2} 0$ solution $\quad 2 \mathrm{mM}$

$\mathrm{X}$-Gal $\quad 1 \mathrm{mg} / \mathrm{mL}$

Phosphate buffer (500 mM), pH 7.4 - stable several weeks at $4^{\circ} \mathrm{C}$

$500 \mathrm{mM} \mathrm{K}_{2} \mathrm{HPO}_{4} .3 \mathrm{H}_{2} \mathrm{O}$

or $500 \mathrm{mM} \mathrm{KH}_{2} \mathrm{PO}_{4}$

Citric acid solution- stable several weeks at RT

$100 \mathrm{mM} \mathrm{C}_{6} \mathrm{H}_{8} \mathrm{O}_{7} \cdot \mathrm{H}_{2} \mathrm{O}$

Sodium phosphate (dibasic) solution - stable several weeks at RT

$200 \mathrm{mM} \mathrm{NaH}_{2} \mathrm{PO}_{4} \cdot \mathrm{H}_{2} \mathrm{O}$

or $\mathrm{Na}_{2} \mathrm{HPO}_{4} .2 \mathrm{H}_{2} \mathrm{O}$

0.2 $\mathrm{M}$ citric acid/Na phosphate buffer, $\mathbf{p H} 6.0$ - stable for 1 month at RT $36.85 \mathrm{~mL}$ of $100 \mathrm{mM}$ citric acid

$63.15 \mathrm{~mL} 200 \mathrm{mM}$ sodium phosphate (dibasic solution)

Potassium hexacyano-ferrate (II) trihydrate solution - stable several months at $4^{\circ} \mathrm{C}$ $100 \mathrm{mM} \mathrm{K}_{4}\left[\mathrm{Fe}(\mathrm{CN})_{6}\right] .3 \mathrm{H}_{2} \mathrm{O}$

Potassium hexacyano-ferrate (III) solution - stable for several months at $4^{\circ} \mathrm{C}$

$100 \mathrm{mM} \mathrm{K}_{3}\left[\mathrm{Fe}(\mathrm{CN})_{6}\right]$

Sodium Chloride solution

$5 \mathrm{M} \mathrm{NaCl}$

Magnesium Chloride hexahydrate solution

$1 \mathrm{M} \mathrm{MgCl}_{2} \cdot 6 \mathrm{H}_{2} \mathrm{O}$ 

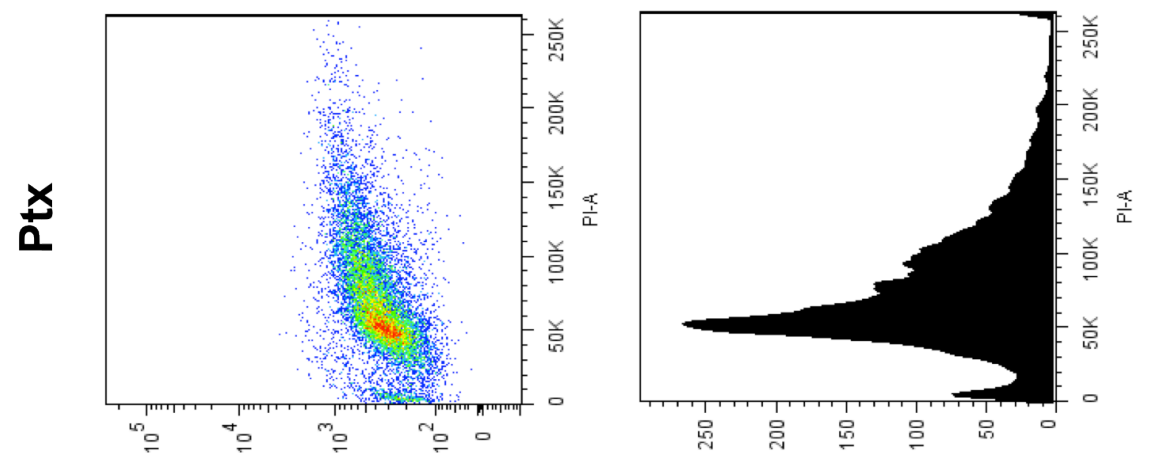

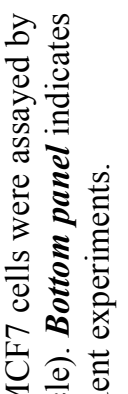
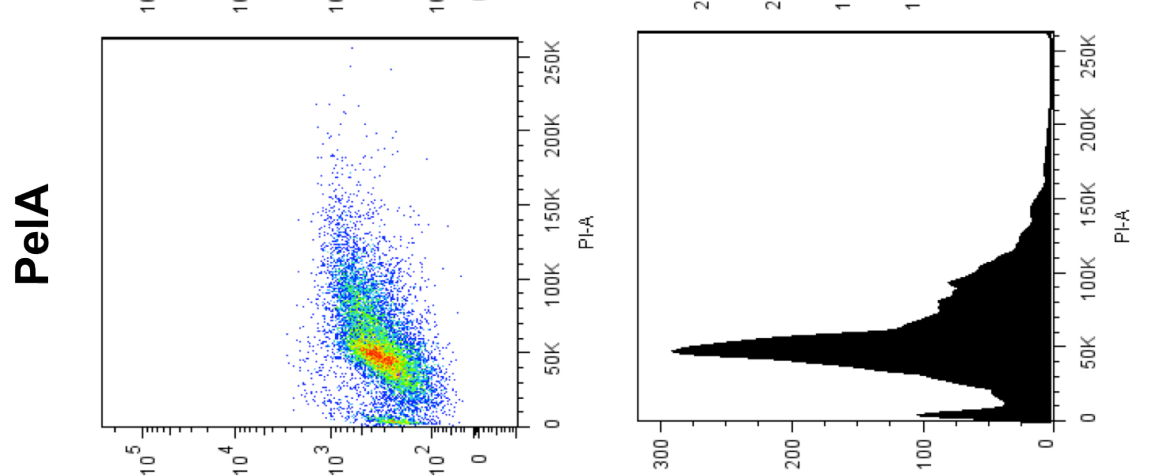

잉

过宇

$x$

के के

क人

乎

过近苛

$\therefore \approx$

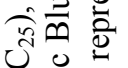

象
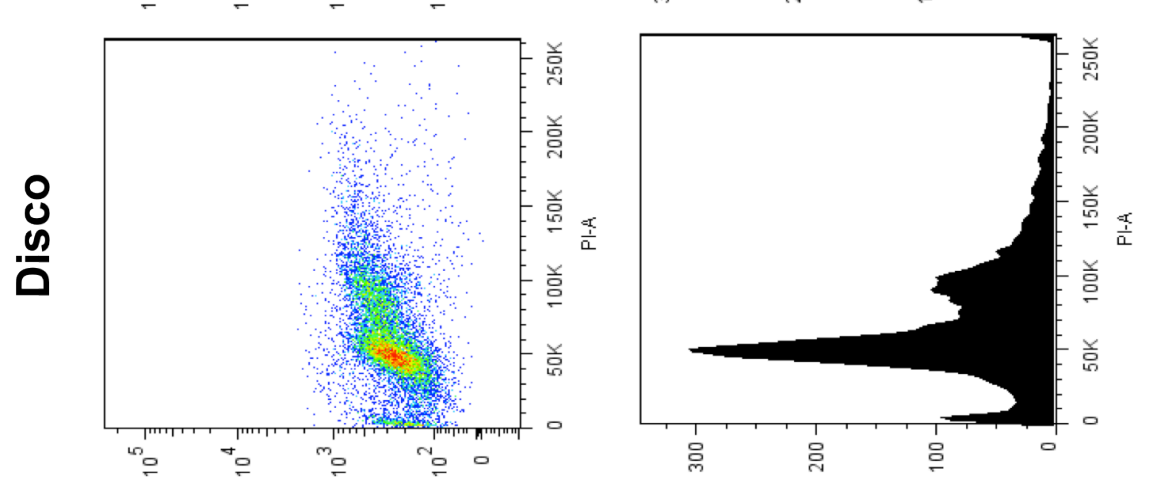

8 .

吕范

in 용 क

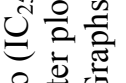

额

คิ

जै

政

త্

올
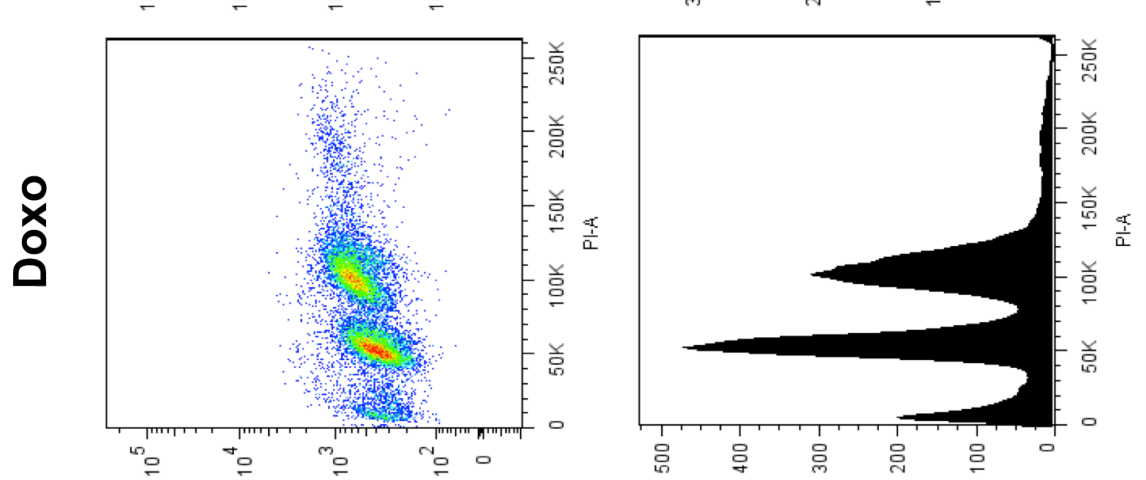

ङ

융

윰 욤

.

这迹就

응

:

苛

贾

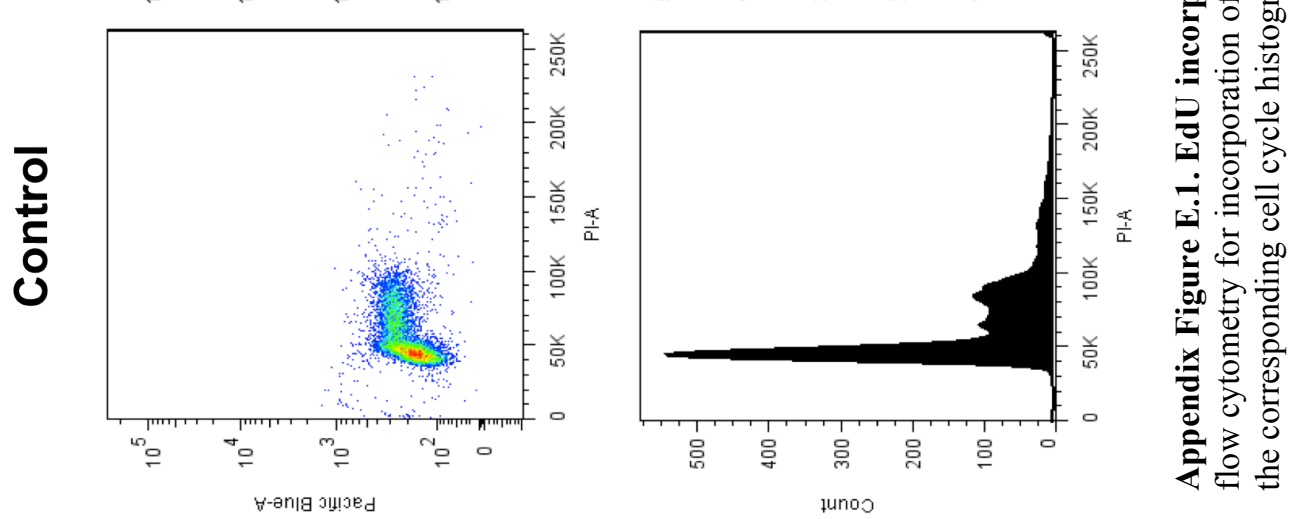



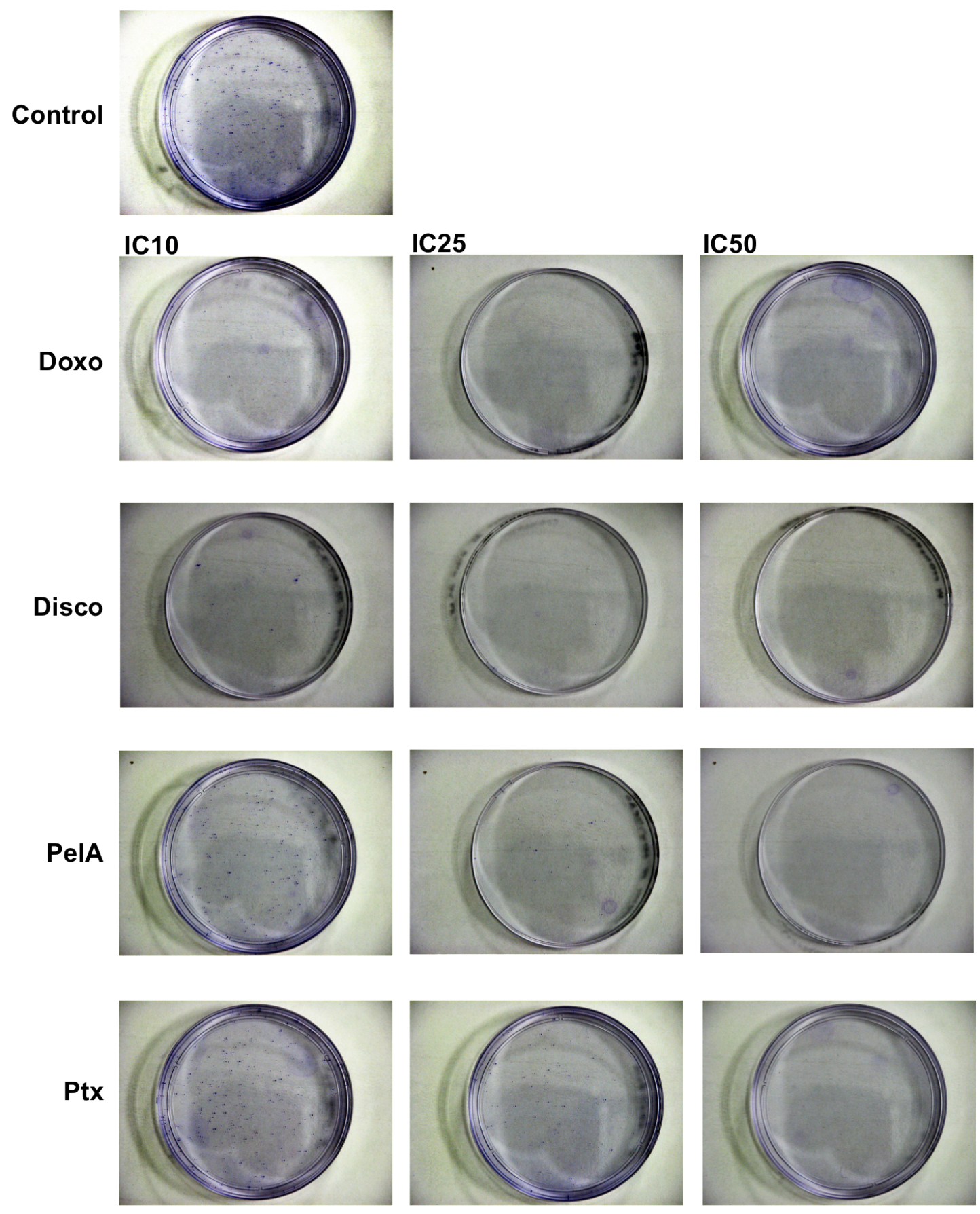

Appendix Figure E.2. Clonogenic assay plates. MCF7 cells were treated with Doxo, Disco, PelA or Ptx for 6 days. Cells were then re-plated and grown for an additional 10 days in drug-free medium, before being stained with $1 \%$ crystal violet. Colonies can be seen as purple-stained islands of cells but only plates from the control or lower concentrations of drug contained obvious colonies. Each colony is expected to have formed from a single viable cell. Images are representative of four independent experiments. 


\section{Appendix F: Chapter 7}

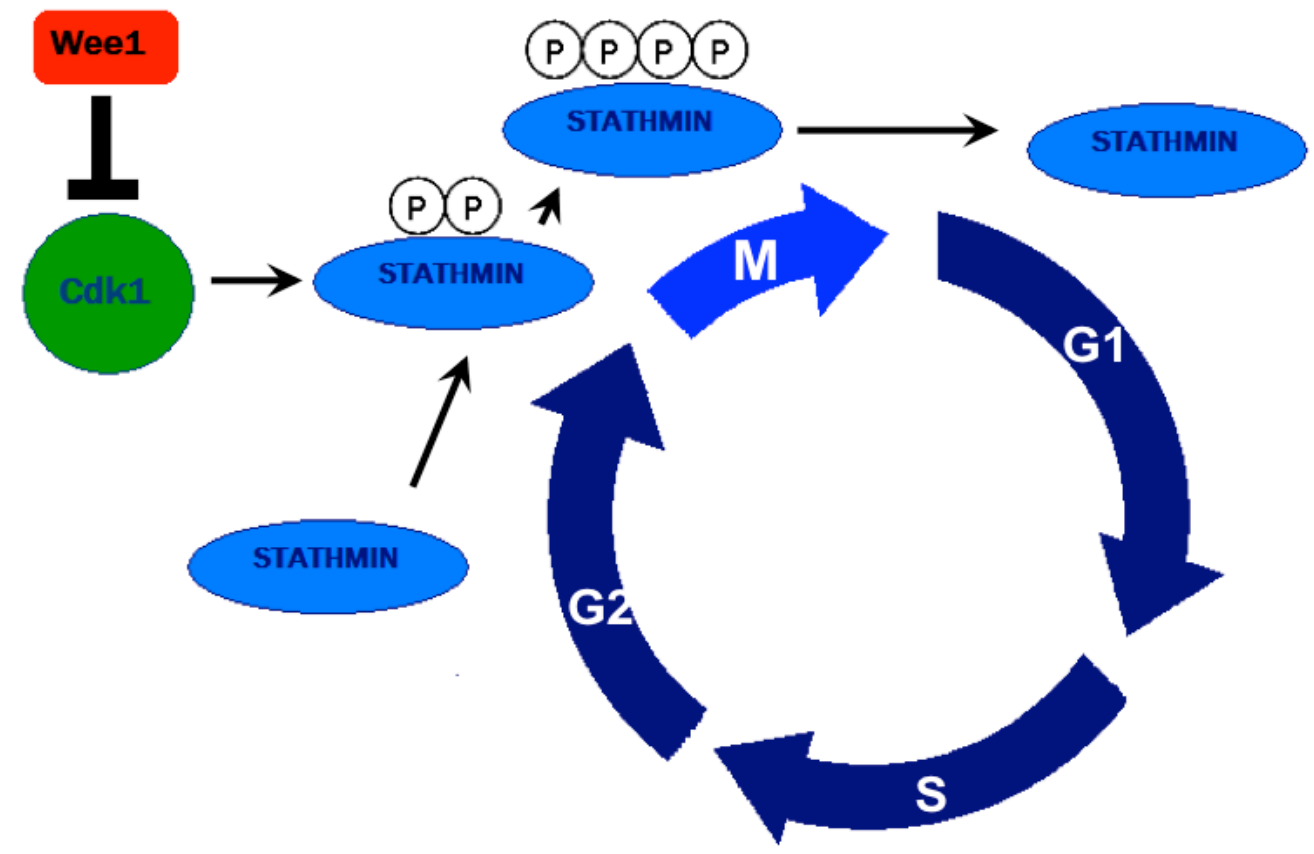

Appendix Figure F.1. Stathmin regulates mitotic entry. Stathmin initially becomes phosphorylated by cyclin-dependent kinase 1 (CDK1) at two serine residues during $\mathrm{G}_{2} / \mathrm{M}$ phase of the cell cycle. For mitotic entry, stathmin must be inactivated by phosphorylation at two additional sites. Wee- 1 can prevent the CDK1-mediated phosphorylation of stathmin to prolong $\mathrm{G}_{2} / \mathrm{M}$ phase and delay mitotic progression. Adapted from Alli et al. (2007). 


\section{Appendix G: Topoisomerase I methods, results and discussion}

\section{Methods}

\section{Topoisomerase relaxation assay}

The TOPO relaxation assay can be used to assess the conversion of supercoiled DNA into relaxed topoisomers in the presence or absence of drug. We tried two different protocols, one designed by Dr Anja Wilmes and another protocol from a commercially available topoisomerase I drug screening kit as per the manufacturer's instructions (TopoGEN, Inc. Port Orange, FL). The protocols are briefly described:

\section{Topogen protocol}

Recombinant TOPO I enzyme (Topogen, Inc.) was first titrated (using a 2-fold serial dilution of enzyme under the conditions described below) to determine the concentration of enzyme activity in units/mL (1 unit (U) is defined as the amount of enzyme needed to relax $250 \mathrm{ng}$ supercoiled DNA under our assay conditions). Assay reaction volumes were $20 \mu \mathrm{L}$ and comprised the following reagents (reactions were assembled on ice and enzyme was added last): RNase- and DNase-free $\mathrm{dH}_{2} 0$ to make up the volume, $2 \mu \mathrm{L} 10 \mathrm{x}$ assay buffer (100 mM Tris-HCl (pH 7.9), $10 \mathrm{mM}$ EDTA, $1.5 \mathrm{M}$ $\mathrm{NaCl}, 1 \%$ bovine serum albumin, $1 \mathrm{mM}$ spermadine, 50\% glycerol), $250 \mathrm{ng}$ supercoiled DNA (pHOT1 DNA in TE buffer (10 mM Tris (pH 7.5), $1 \mathrm{mM}$ EDTA), $2 \mu \mathrm{L}$ of solvent/test drug (i.e. PelA, Ptx or Disco, and camptothecin (CPT) as a positive control), and $1 \mu \mathrm{L}$ containing $1 \mathrm{U}$ enzyme diluted in TOPO dilution buffer. Drugs were reconstituted in EtOH or DMSO to a final concentration of $10 \mathrm{mM}$ and then further diluted in $\mathrm{dH}_{2} \mathrm{O}$ or $10 \%$ solvent such that the final solvent control was never greater than $2 \%$ in the final assay mix. Immediately after the addition of TOPO I (addition was staggered), reaction tubes were placed in a $37^{\circ} \mathrm{C}$ waterbath for $30 \mathrm{~min}$. Reactions were terminated by the rapid addition (while the tubes were still in the waterbath) of $2 \mu \mathrm{L}$ $10 \%$ SDS and $2.2 \mu \mathrm{L}$ proteinase $\mathrm{K}$ (final concentration of $50 \mu \mathrm{g} / \mathrm{mL}$ ), and incubated for a further $30 \mathrm{~min}$. Two $\mu \mathrm{L}$ of $10 \mathrm{x}$ stop buffer $(0.25 \%$ bromophenol blue and $50 \%$ glycerol) was then added to each sample, followed by extraction using an equal volume of chloroform:isoamyl alcohol (24:1). Samples were briefly vortexed and then centrifuged at 13, $000 \mathrm{rpm}$ for $5 \mathrm{~min}$. The top aqueous blue layer $(10 \mu \mathrm{L})$ was loaded onto a $1 \%$ agarose gel and run at $50 \mathrm{~V}$ for $5 \mathrm{~h}$ in TAE buffer. A relaxation marker provided in the kit was also run $(0.1 \mu \mathrm{g} / \mathrm{lane})$ for comparison. Gels were stained for 15 min in ethidium bromide staining solution $(1 \mu \mathrm{g} / \mathrm{mL})$ and destained for at least $30 \mathrm{~min}$ 
in $\mathrm{ddH}_{2} \mathrm{O}$. Photos of the stained gels were taken using an AlphaImager ${ }^{\circledR}$ Mini UV transilluminator system (Alpha Innotech).

\section{Dr Wilmes' protocol}

Assay reactions were assembled in ice in a total volume of $20 \mu \mathrm{L}$ using a relaxation buffer (50 mM Tris (pH 7.5), $20 \mathrm{mM} \mathrm{KCl,} 1 \mathrm{mM}$ EDTA, $1 \mathrm{mM}$ DTT and $0.1 \mathrm{mg} / \mathrm{mL}$ BSA) to make up the volume. Two $\mu \mathrm{L}$ of diluted supercoiled plasmid DNA (pGEM4Z diluted in relaxation buffer; In Vitro Technologies) was added such that the final amount in each reaction was $300 \mathrm{ng}$. Two $\mu \mathrm{L}$ of drug (diluted in 33\% DMSO) or solvent equivalent was added, with enzyme added last. TOPO I enzyme (Invitrogen) was added to each tube and incubated for $1 \mathrm{~h}$ at $37{ }^{\circ} \mathrm{C}$. Enzyme was not titrated to determine the actual unit/mL concentration; thus, enzyme dilutions were prepared that gave 0.1-0.4 units based on the manufacturer's stated approximate enzyme concentration. The reaction was stopped by the addition of $2.5 \mu \mathrm{L}$ SDS (10\%) and 6.5 $\mu \mathrm{L}$ loading dye (2\% SDS, $14 \%$ Ficoll and $0.1 \%$ bromophenol blue). Samples (10 $\mu \mathrm{L})$ were loaded onto a $1 \%$ agarose gel using TBE running buffer, and electrophoresed at 20 $\mathrm{V}$ for $14 \mathrm{~h}$. Gels were stained and photographed as described above.

\section{Results}

\section{Topoisomerase I activity}

Despite it being a relatively simple assay, and one that is widely used, we were unable to generate any consistent results regarding the ability of PelA to interfere with the function of TOPO I. Initially when we switched to the commercially available TOPO I assay kit, we received several defective batches of enzyme with inadequate enzyme activity (due to inappropriate shipping conditions). There was a 4-6 week shipping delay between each shipment that interfered with our ability to conduct the assay properly.

The titration of the TOPO I enzyme was fairly straightforward (Appendix Fig. G.1 A), and allowed us to determine the enzyme activity (units/ $\mu \mathrm{L}$ ) under our assay conditions. The enzyme was titrated to determine the amount needed to just relax all of the supercoiled DNA. As an example, Appendix Figure G.1 A shows that this particular batch of enzyme titrated to 8 units $/ \mu \mathrm{L}$. The faster migrating supercoiled plasmid DNA can be seen compared to the slower migrating relaxed topoisomers and the nicked, open-circular DNA. CPT was used as a positive control for TOPO I inhibition. A range 
of CPT concentrations $(25-200 \mu \mathrm{M})$ caused a concentration-dependent increase in the amount of supercoiled DNA. The DMSO vehicle control showed minimal TOPO I inhibition (Appendix Fig. G.1 B).

Unfortunately, when this assay was repeated using various test MSAs, we did not observe a consistent drug or vehicle effect (Appendix Fig. G.1 C and D). For example, we observed an increase in the amount of supercoiled DNA in the presence of PelA, but this "inhibition" was also observed with the equivalent vehicle controls (Appendix Fig. G.1 C). Additionally, the inhibitory effects of CPT in this particular case (Appendix Fig. G.1 C) were only marginally greater than the vehicle. In another assay (Appendix Fig. G.1 D), we did not observe an inhibitory effect by DMSO (used to reconstitute CPT) but we did observe an inhibitory effect with ethanol (used to reconstitute PelA and Disco) at the highest vehicle equivalent (100 $\mu \mathrm{M}$ drug equivalent). However, TOPO I activity in the presence of $100 \mu \mathrm{M}$ PelA and Disco appeared to be greater than the vehicle control (suggesting a possible stimulatory effect). This was despite a similar level of supercoiled DNA at $50 \mu \mathrm{M}$ compared to the $100 \mu \mathrm{M}$ vehicle equivalent. For comparison, a gel showing the results of an assay using the protocol established by Dr Wilmes (note that the recombinant enzyme and DNA plasmid were from a different company) further added to the inconsistent results (Appendix Fig. G.1 E). In this assay there appeared to be greater levels of supercoiled DNA in the presence of Disco (at 100 $\mu \mathrm{M}$ ), PelA (at 25 and $100 \mu \mathrm{M}$ ) and CPT (at 25, 50 and $75 \mu \mathrm{M}$ ), indicating that all three of these drugs inhibited TOPO I activity. Thus, different results were obtained with repeat assay, within the same assay, and using different protocols, making it impossible to draw any firm conclusions. The reasons for the inconsistent results using a standard relaxation assay are not known, but after an expenditure of close to $\$ 7500$, the study was temporarily abandoned as it was not worth the cost and time. In all, three different graduate students tried to answer the question of whether TOPO I was a secondary target of PelA, and none of us succeeded. The answer was important to us, as it would have been the first validation of a yeast microarray hit for PelA and would have confirmed the value of using yeast chemical genetics to determine the mode of action of novel drugs. 
A

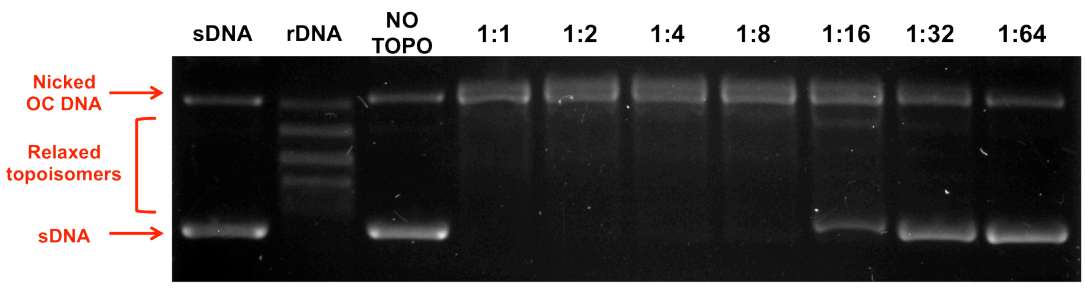

B

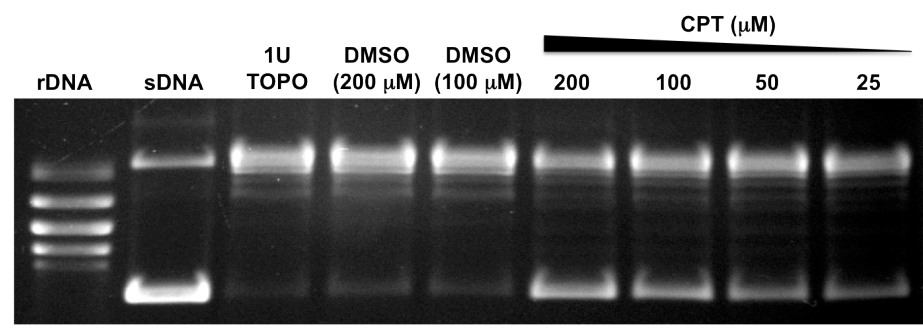

C

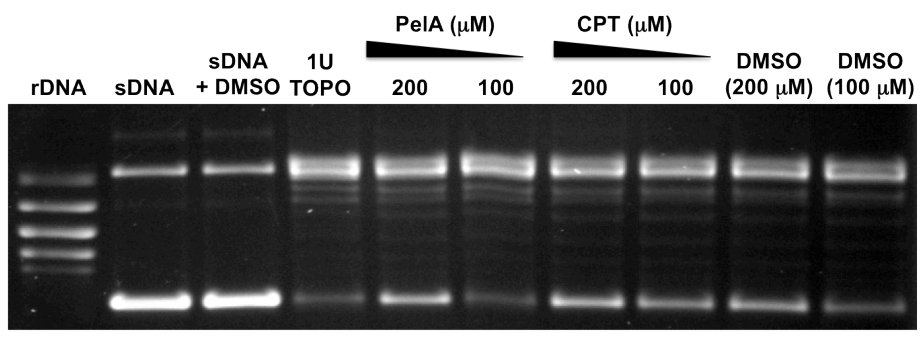

D

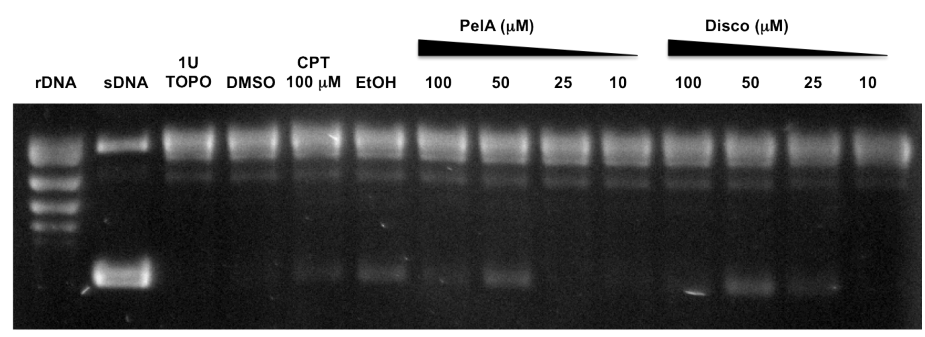

E

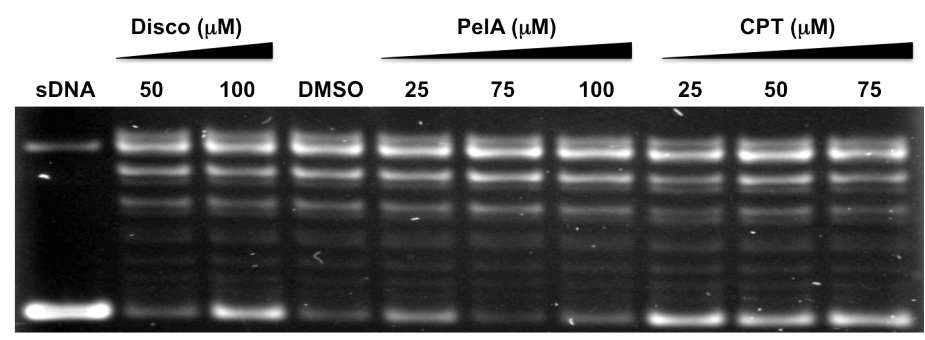

Appendix Figure G.1. Topoisomerase I (TOPO I) relaxation assay. We assessed the ability of PelA, Disco and CPT to inhibit TOPO I activity by measuring the ability of the enzyme to convert supercoiled DNA (fastest migrating band) into its relaxed topoisomers (slower migrating bands). (A) A representative gel showing the titration of TOPO I (serial 2-fold dilution) with $250 \mathrm{ng}$ supercoiled plasmid DNA (PHOT1), incubated for $30 \mathrm{~min}$ at $37{ }^{\circ} \mathrm{C}$. This particular batch of enzyme titrated to a concentration of $8 \mathrm{U} / \mu \mathrm{L}$ (i.e. an 8 -fold dilution of $1 \mu \mathrm{L}$ TOPO I was the minimum amount of enzyme required to completely relax the supercoiled plasmid DNA). Relaxed DNA marker (rDNA) and supercoiled DNA (sDNA), without enzyme, are run as controls. Bands corresponding to the migration of nicked, open circular (OC) DNA, relaxed topoisomers and supercoiled DNA are also indicated on the gel image. (B), (C) and (D) illustrate the effects of various drugs on the relaxation activity of TOPO I (purchased from Topogen). 1 unit of enzyme was incubated with $250 \mathrm{ng}$ plasmid DNA for $30 \mathrm{~min}$ at 37 ${ }^{\circ} \mathrm{C}$ in the presence or absence of drug (or vehicle concentrations equivalent to 100 or $200 \mu \mathrm{M}$ of drug if no concentration is given on the gels, then vehicle was tested at the highest drug concentration equivalent). (E) For comparison, a different relaxation assay protocol designed by Anja Wilmes was carried out using TOPO I enzyme (Invitrogen) incubated with $300 \mathrm{ng}$ supercoiled plasmid (PGEM4Z), as described in the methods. 


\section{Discussion}

\section{Effect of MSAs on topoisomerase I activity}

Despite a significant effort, including that of previous $\mathrm{PhD}$ and $\mathrm{MSc}$ students, we have so far been unable to definitively validate whether TOPO I is a secondary target of PelA (only a few of the many assays run were presented here). We observed inconsistent results with all of the drugs (PelA, Ptx, Disco and CPT) that were tested in the assay and in some cases the vehicle itself appeared to increase the amount of supercoiled plasmid DNA remaining, thus showing inhibition of TOPO I. With MSAs, we did observe an increased supercoiled band density (above vehicle levels) in some cases, suggesting that there may be an inhibitory effect by the two MSAs (albeit not always), but we first need to improve the reproducibility of the assay and of our controls for us to draw any valid conclusions from this assay. The TOPO I relaxation assay manufactured by Topogen, Inc. seems to be the most widely used assay to screen potential TOPO I inhibitors, so we are unsure why we could not get this assay to work properly. The assay seemed to be quite sensitive to the amount of enzyme (which itself was not very stable), and because we were working with quite small volumes, this may contribute to the variability in our results. It is important for this assay not to add too much enzyme per reaction to prevent saturation of the enzyme response and masking the inhibitory activity of the test drugs. Another potential issue is the fact that the vehicle concentration needs to be kept within a certain threshold (usually less than 1-2\%), which means the working stocks of the drugs need to be diluted in $\mathrm{dH}_{2} \mathrm{O}$ or diluted vehicle (i.e. 10-30\% DMSO or EtOH). Another confounding factor is that the low aqueous solubility of MSAs, which are notoriously hydrophobic, could lead to precipitation of the drugs as they are diluted with buffer, and this may contribute to the inconsistencies we observed, especially in cases where a higher drug concentration had less of an inhibitory effect than a lower concentration.

Topoisomerase inhibitors can act by directly inhibiting the catalytic activity of the enzyme, or by stabilizing the intermediate cleavage complexes that form when topoisomerase binds to DNA. Stabilization of cleavage complexes by CPT prevents reannealing of the cleaved DNA strand (Rothenberg, 1997) and should lead to an increased production of open circular (OC) DNA; however, we did not see an increase in OC DNA, but rather an increase in supercoiled DNA. The effects of CPT on the relaxation of DNA have been shown previously to be dependent on the concentration of TOPO I that is present, as it is only at higher concentrations of enzyme that significant 
amounts of nicked DNA are observed (Jaxel et al., 1989). CPT has also been shown to increase the degree of DNA damage by generating double-strand breaks; hence, TOPO I inhibition as a secondary effect of PelA offers an attractive explanation as to why PelA triggers premature senescence. It is a shame that we have not been able to get a definitive answer, one way or the other, on whether TOPO I is a target of PelA, and work is ongoing in this regard. 